

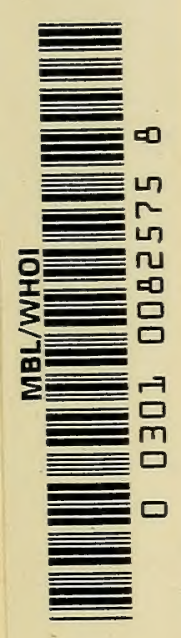



UNDERWATER ACOUSTICS 


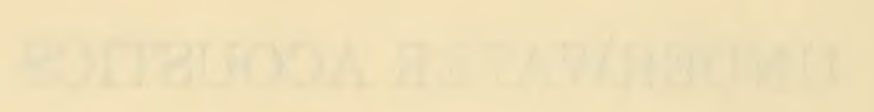




\title{
UNDERWATER ACOUSTICS
}

Proceedings of an Institute sponsored by the Scientific Affairs Committee of the North Atlantic Treaty Organization and conducted by The Pennsylvania State University at The Imperial College of Science and Technology of The University of London

July 31-August 11, 1961

\section{Edited by \\ V. M. ALBERS}

The Ordnance Research Laboratory The Pennsylvania State University

University Park, Pennsylvania

\author{
$\Phi$ \\ Distributed by \\ PLENUM PRESS \\ NEW YORK
}


Library of Congress Catalog Card Number 62-8011 (19193 Pennsylvania State University University Park, State College, Pennsylvania All rights reserved

No part of this publication may be reproduced in any form without written permission from the publisher

Printed in the United States of America 


\section{FOREWORD}

This book represents a compilation of the lectures presented at the Institute on Underwater Acoustics held at the Department of Physics of The Imperial College of Science and Technology of The University of London between July 31 and August 11, 1961. The institute was conducted by the Continuing Education Services of The Pennsylvania State University under a grant from the Scientific Affairs Division of the North Atlantic Treaty Organization.

At the institute, eighteen papers were presented by seventeen outstanding scientists in the field of Underwater Acoustics. In addition, eighty-five scientists, representing eleven nations, attended the institute and participated in the discussions following the presentation of each paper. Since each of the lecturers is an authority in his field, these proceedings, which include the bibliographies supplied by the lecturers, should provide a valuable source of information for workers in the field of Underwater Acoustics. The discussions following the lectures, where available, have been included to make these proceedings more meaningful to the reader.

Dr. R. W. B. Stephens of The Imperial College, Co-Chairman of the institute, assisted in the preparation of the program and made all arrangements for the lecturers and participants. The contribution of Dr. Stephens in the formulation of the program and in arranging for the housing of both the lecturers and the participants was an important factor in the success of the institute.

I wish to thank all of the speakers for their efforts in preparing the lectures as well as for preparing manuscripts in a form suitable for publication. I am grateful to the staff of the Department of Physics of The Imperial College and to the staff of Continuing Education Services at The Pennsylvania State University for their assistance in carrying out the program. I am also indebted to Dr. Merritt A. Williamson, Dean of the College of Engineering and Architecture, and Dr. John C. Johnson, Director of the Ordnance Research Laboratory, at The Pennsylvania State University for their support and interest.

Vernon M. Albers Chairman 



\section{CONTENTS}

Introduction The Scientific Program Sponsored by NATO

H. R. Baker .....................

Lecture 1 TRANSDUCERS

D. Schofield ..................... 5

1.1 Introduction . . . . . . . . . . . . . 5

1.2 Electroacoustic Conversion Principles . . . . . . 6

1.3 Equivalent Electrical Circuits . . . . . . . . . 7

1.4 Electromechanical Coupling Factor . . . . . . . 9

1.5 Transducer Materials .............. 10

1.6 Examples of Transducers . . . . . . . . . . 13

1.6.1 Projectors . . . . . . . . . . . . . 13

1.6.2 Resonant Frequency .............. 13

1.6.3 Electromechanical Coupling Coefficient ..... 14

1.6.4 Power-Handling Capabilities . . . . . . . . 16

1.6.5 Deep Hydrophones . . . . . . . . . . . . 20

1.7 Conclusion . . . . . . . . . . . . . 24

1.8 Acknowledgment . . . . . . . . . . . . 25

Lecture 2 SONAR ARRAYS, SYSTEMS, AND DISPLAYS

D. G. Tucker . . . . . . . . . . . . . . .

2.1 Introduction . . . . . . . . . . . . 29

2.2 Arrays and Directionality . . . . . . . . . 30

2.2.1 Multiplicative Arrays . . . . . . . . . . . 30

2.2.2 Superdirective Arrays . . . . . . . . . . 32

2.2.3 Multifrequency Two-Element Arrays ..... 32

2.2.4 Wide-Band Arrays . . . . . . . . . . . 35

2.3 Sonar Systems . . . . . . . . . . . . 37

2.3.1 Within-Pulse Scanning Systems Using

Multielement Arrays ............ 37

2.3.2 Within-Pulse Scanning Systems Using

Multifrequency Arrays ........... 40

2.3.3 Double-Transducer FM System ........ 42

2.4 Displays . . . . . . . . . . . . . 43

2.4.1 Integration and Correlation ......... 44 
2.4.2 Pattern Recognition and Size of Target .... 45

2.5 Echo-Formation and Randomization ......... 46

2.6 Conclusions and Acknowledgments ......... 48

\section{Lecture 3 EXPLOSIVE SOURCES}

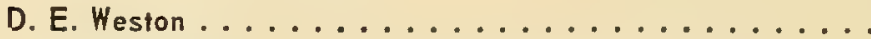

3.1 Introduction $\ldots \ldots \ldots \ldots \ldots \ldots \ldots \ldots \ldots \ldots$

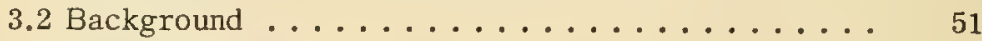

3.3 General Ideas on Scaling Laws and

Spectrum Slopes .................. 52

3.4 Description of Underwater Explosions . . . . . . . . 54

3.5 Measurements and Theory for Underwater

Explosions ............................ 57

3.6 Comparison with Underground Explosions ...... 59

3.7 Uses in Research .................. 61

3.8 Relative Advantages of Underwater

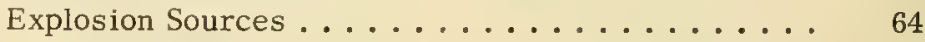

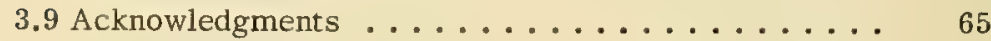

\section{Lecfure 4 THE IMPROVEMENT OF VIBRATION ISOLATION}

G. G. Parfitt . . . . . . . . . . . . . .

4.1 Introduction . . . . . . . . . . . 67

4.2 Additional Considerations . . . . . . . . . 68

4.3 Damping of Spring Isolators . . . . . . . . 73

4.4 Additional Mass in Vibrating Structures . . . . . 76

4.5 Summary . . . . . . . . . . . . 83

4.6 Acknowledgments ............. 84

Lecture 5 A SING-AROUND VELOCIMETER FOR MEASURING THE SPEED OF SOUND IN THE SEA

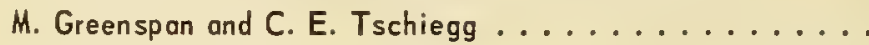

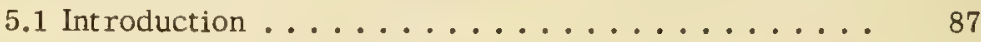

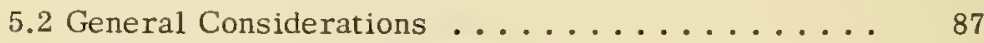

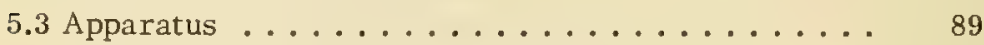

5.3.1 The Transducers and the Path ........ 89

5.3 .2 The Electronics ............... 92

5.3.3 Frequency Measurement ............ 94

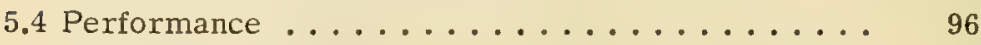

5.4 .1 Stability ...................... 96

5.4.1.1 Supply-Voltage and Ambient-Temperature Fluctuations ............ 96

5.4.1.2 Frequency Stability .......... 96

5.4 .2 Calibration ..................... 96

5.5 History and Distribution . . . . . . . . . . . 98

5.6 Acknowledgments .................... 99 
M. P. Foüche . . . . . . . . . . . . . . .

6.1 Hydrophone Calibration . . . . . . . . . . . 103

6.1.1 Electrodynamic Calibration by Discharge into a Ballistic Galvanometer. . . . . . . . . . 103

6.1.2 Absolute Electrostatic Calibration . . . . . . 104

6.1.3 Relative Electrostatic Calibration ....... 105

6.1.4 Closed-Tank Pressure-Calibration Technique ................ 106

6.1.5 Calibration by Comparison Using Filtered Noise. . . . . . . . . . . . . . . . . 106

6.2 Transducer Calibration ............... 108

6.2.1 Impedance Measurements . . . . . . . . . . 109

6.2.2 Principle of the Pulse Method ......... 110

6.2.2.1 Separation Distance . . . . . . . . . . 111

6.2.2.2 Pulse Length. . . . . . . . . . . . 111

6.2.2.3 Pulse Rate ............... 111

6.2.2.4 Size of the Anechoic Tank ....... 112

6.2.2.5 Instrumentation . . . . . . . . . . . 113

6.2.3 Application of the Pulse Method for

Reciprocity Calibration............. 114

6.2.3.1 Calibration by Comparison . . . . . . 115

6.2.3.2 Directivity Measurements . . . . . . 115

6.2.3.3 Transparency Measurements ...... 116

6.3 Test-and-Calibration Barge Moored in the

Harbour of Le Brusc . . . . . . . . . . . . . . 116

6.3 .1 Mission . . . . . . . . . . . . . 116

6.3.2 Physical Features and Layout ......... 117

6.3.3 Electrical Installation. . . . . . . . . . . . . . 119

6.3.4 Water Depth . . . . . . . . . . . . . . . . 119

6.4 Test-and-Calibration Barge of the Lake of Castillon . . . . . . . . . . . . . . . . 119

6.4.1 Introduction: History . . . . . . . . . . . . 119

6.4.2 General Description . . . . . . . . . . . . 120

6.4.3 The Test-and-Calibration Barge........ 121

6.4.3.1 General Layout . . . . . . . . . . . 121

6.4.3.2 Mechanical Operating Gear . . . . . . 121

6.4.3.3 Electrical Installation. . . . . . . . 121

6.4.3.4 Acoustical Test-and-Calibration

Facilities .............. 122

Lecłure 7 SOME AREAS IN WHICH UNDERWATER ACOUSTICS RESEARCH IS NEEDED

H. R. Baker . . . . . . . . . . . . . . .

7.1 Sound Transducers $\ldots \ldots \ldots \ldots \ldots \ldots \ldots \ldots$ 


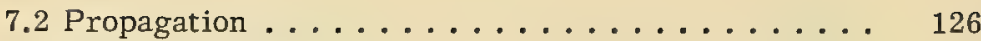

7.3 Signal Processing . . . . . . . . . . . . . . . . . . . 127

7.4 Countermeasures ................... 127

Lecłure 8 INTERNAL WAVES

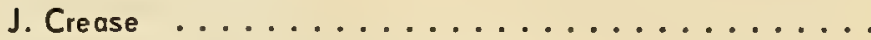

Lecture 9 HIGH-INTENSITY SOUND IN LIQUIDS

E. Meyer . . . . . . . . . . . . . . .

9.1 Generation of High Acoustic Power. . . . . . . . 139

9.1.1 Continuous Wave Transducers . . . . . . . 139

9.1.2 Pulse Transducers............... 140

9.2 Measurement of High Sound Intensities . . . . . . . 142

9.3 Properties of Liquids Exposed to High Sound

Intensities . . . . . . . . . . . . . . . 146

9.3.1 Increase of Sound Absorption . . . . . . . 146

9.3.2 Generation of Higher Harmonics . . . . . . . 146

9.3.3 Generation of Shock Waves by Collapsing

Cavities .................... 148

9.3.4 Steepening of Shock Waves............ 151

9.3.5 Sonoluminescence ............... 154

Lecture 10 SCALE-MODEL STUDY OF PROPAGATION IN SHALLOW SEAS:

A VISUAL METHOD OF REPRESENTATION OF LOW-INTENSITY SOUND FIELDS

A. B. Wood ....................... . 159

10.1 Introduction . . . . . . . . . . . . . . . 159

10.1.1 The Scale of the Model Experiments ..... 161

10.2 Experimental-"Point-by-Point" Method ....... 166

10.2.1 Directional Properties and Depth

of Transmitter............... 167

10.2.2 Range from Transmitter to Receiver

Varied ................... 167

10.2.3 Effects of Varying Nature of Bottom

and Frequency of Sound ............ 167

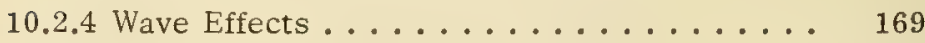

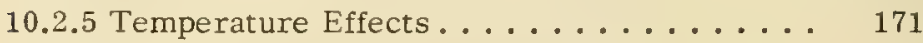

10.2.6 Depth of Water............... 172

10.3 Visual Observation of Sound Distribution

on the Bottom and Surface............. 173

10.4 "Picture" Records of Sound Distribution in

Vertical Cross Sections of "Open" Water-Scanning 175

10.4.1 Nature of Bottom . . . . . . . . . . . 180

10.4.2 Depth of Water. . . . . . . . . . . . 181

10.4.3 Wavelength of Sound . . . . . . . . . . 187

10.4.4 Temperature of Water.................. 187

10.4.5 Directional Transmission .......... 187 
10.4.6 Calibration of Scan Pictures of

Sound Fields ................ 190

10.5 Theoretical Work-Full-Scale Trials ........ 190

Lecture 11 NONSPECULAR SCATTERING OF UNDERWATER SOUND BY THE SEA SURFACE

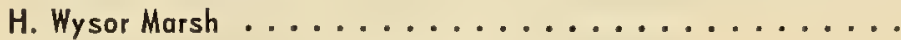

11.1 Introduction . . . . . . . . . . . . . . . . 193

11.2 Nonspecular Scattering . . . . . . . . . . . . . . . . 193

11.3 Back Scattering. . . . . . . . . . . . . . . 194

11.4 Conclusions ....................... 196

Lecłure 12 THERMAL MICROSTRUCTURE IN THE SEA AND ITS CONTRIBUTION TO SOUND LEVEL FLUCTUATIONS

E. J. Skudrzyk . . . . . . . . . . . . . . . . .

12.1 The Temperature Structure of the Sea . . . . . . . 199

12.2 The Characteristics of the Temperature

Structure of the Sea ................ 209

12.3 The Scattered Pressure and Scattered

Intensity ...................... 211

12.3.1 The Rayleigh Integral ............ 211

12.3.2 Spherical Scatterers .............. 213

12.3.3 Scattering Described by the Correlation

Function . . . . . . . . . . . . . 217

12.3.4 Scattering Described by the Power

Spectrum of the Sound Velocity

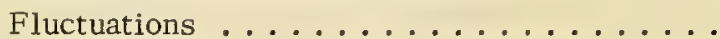

12.4 Fluctuation of the Transmitted Signal . . . . . . . 221

12.4.1 Elementary Theory ............... 221

12.4.2 Computation of Amplitude and Phase of Fluctuations for Spherical Patches. . . . . 224

12.4.3 Continuous Patch Distribution........ 226

12.5 Experimental Results . . . . . . . . . . . . 228

12.5.1 Standard Deviation of the Transmitted

Signal . . . . . . . . . . . . . . 228

Lecfure 13 AMBIENT NOISE IN THE SEA AND ITS MEASUREMENT

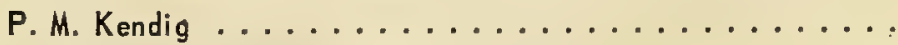

13.1 Ambient Noise .................... 235

13.1.1 Introduction. . . . . . . . . . . . . 235

13.1.2 Deep Water-Thermal and Surface

Noise ................... 236

13.1.3 Shallow Water-Man-Made and Biological

Noise . . . . . . . . . . . . . . . 238

13.1 .4 Rain Noise . . . . . . . . . . . . . . 240

13.1.5 Directional Characteristics .......... 241 
13.2 The Measurement of Ambient Noise ..........

13.3 Factors That Determine the Equivalent Noise

Pressure, Free-Field Voltage Response, and Efficiency of a Transducer at Low

Frequencies ...................

13.3.1 The Equivalent Circuit and the Efficiency ..................

13.3.2 The Free-Field Voltage Response and Equivalent Noise Pressure .........

\section{Lecture 14 FLOW NOISE, THEORY AND EXPERIMENT}

E. J. Skudrzyk and G. P. Hoddle ...............

14.1 Introduction . . . . . . . . . . . . . . . . 255

14.2 The Nearfield Flow Noise Generated by a

Turbulent Boundary Layer . . . . . . . . . . . 256

14.3 The Radiated Flow Noise . . . . . . . . . . . 259

14.4 Effect of Size and Shape of Sound Receiver

on Amplitude-vs-Frequency Curve of

Flow Noise. . . . . . . . . . . . . . . . . . . 259

14.5 Noise Generated by the Surface Roughnesses . . . . 264

14.6 Experimental Results . . . . . . . . . . . . 266

14.7 Radiated Pressure and Shell Vibration Level ..... 273

14.8 Effect of Size and Shape of Hydrophone on

the Received Noise Level . . . . . . . . . . . . 273

14.9 The Reduction of Flow Noise . . . . . . . . . 275

14.10 The Reproducibility of the Measurements

and the Effect of Shell Vibrations on the Result ...

Lecture 15 SOME CONTRIBUTIONS FROM AERONAUTICS TO THE FIELD OF UNDERWATER NOISE

E. J. Richards, J. L. Willis, and D. J. M. Williams ........

15.1 Introduction ...................... 279

15.2 Boundary-Layer Pressure Fluctuations . . . . . . . 280

15.2.1 The Radiated Sound from a Small

Flat Plate ................ 286

15.3 Structural Response and Reradiation ......... 287

15.4 Applications to Underwater Noise Problems . . . . . 290

15.4.1 Radiated Noise. . . . . . . . . . . . 290

15.4.2 Noise from the Turbulence........... 291

15.4.3 Turbulent Boundary-Layer Wall

Pressure Noise ... . . . . . . . . . . 292

15.4.4 Noise from Roughness. . . . . . . . . . 293

15.4.5 Reradiated Noise from Body Vibrations .... 293

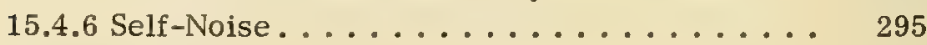

15.5 Conclusion ........................ 298 
Lecłure 16 UNDERWATER ACOUSTICS AS A TOOL IN OCEANOGRAPHY

M. J. Tucker and A. R. Stubbs . . . . . . . . . . .

16.1 Introduction . . . . . . . . . . . . . . 301

16.2 Marine Geological Applications . . . . . . . . 301

16.2.1 Precision Echo-Sounding . . . . . . . . . . 301

16.2.2 Multiple-Beam and Scanning Echo-

Sounders.................. 304

16.2.3 Echo-Sounding for Subbottom Strata . . . . 305

16.2.4 Seismic Refraction Shooting . . . . . . . . 307

16.2.5 Sea-Bed Survey Using Asdic . . . . . . . . 307

16.3 Biological Applications . . . . . . . . . . . 311

16.3.1 Fish Detection . . . . . . . . . . . . 311

16.3.2 Scattering Layers. . . . . . . . . . . . 312

16.4 Acoustic Telemetering . . . . . . . . . . . . 314

16.4 .1 General . . . . . . . . . . . . . . 314

16.4.2 The "Pinger"................ 315

16.4.3 Acoustic Telemeters with Continuous

Transmission .............. 316

16.5 Miscellaneous Applications . . . . . . . . . . 317

16.5.1 Wave Recording . . . . . . . . . . 317

16.5.2 Internal Waves. . . . . . . . . . . 318

Lecture 17 SOME EXPERIMENTS ON THE REDUCTION OF STRUCTURE. BORNE NOISE

J. H. Jonssen . . . . . . . . . . . . . .

17.1 Introduction $\ldots \ldots \ldots \ldots \ldots \ldots \ldots \ldots \ldots \ldots \ldots . \ldots \ldots$

17.2 Units and Levels . . . . . . . . . . . . . 321

17.3 Resilient Mounts . . . . . . . . . . . . . . . 324

17.4 Bending Waves in Structures . . . . . . . . . 326

17.5 Mechanical Impedance . . . . . . . . . . . 328

17.6 Radiation of Sound . . . . . . . . . . . 334

17.7 Conclusions . . . . . . . . . . . . . . 337

Lecture 18 SIGNAL PROCESSING OF UNDERWATER ACOUSTIC FIELDS

P. L. Stocklin . . . . . . . . . . . . . .

18.1 Introduction . . . . . . . . . . . . 339

18.2 Rationale . . . . . . . . . . . . . . . 339

18.3 Space-Time Decision Theory Applications ..... 341

18.3.1 Introduction. . . . . . . . . . . 341

18.3.2 Sampling Theorems . . . . . . . . . . . 342

18.3.2.1 Theorem I (Uniform Space Sampling of a Monochromatic Field) ...... 342

18.3.2.2 Theorem II (Band-Limited Frequency Spectrum, Finite Time) ....... 343

18.3.3 Space-Time Likelihood Ratio for Detection 343 18.3.3.1 Examples ............. 344

Appendix: Attendance at the Institute $\ldots \ldots \ldots \ldots \ldots \ldots$ 



\title{
INTRODUCTION
}

\section{THE SCIENTIFIC PROGRAM SPONSORED BY NATO}

\author{
H. R. Baker \\ The North Atlantic Treaty Organization \\ Scientific Affairs Division \\ Paris, France
}

Greetings from the Division of Scientific Affairs of the North Atlantic Treaty Organization, the sponsor of this Advanced Study Institute on the subject of Underwater Acoustics. The Assistant Secretary General for Scientific Affairs, Dr. William A. Nierenberg, has asked me to convey to Dr. Albers of The Pennsylvania State University and to Dr. Stephens of The Imperial College, London, his appreciation for their work in organizing and administering this Institute. It is gratify ing to see so many scientists in the field of underwater acoustics from so many nations taking part in the Study.

My purpose in speaking to you is to attempt to acquaint you with the organization of NATO and, in particular, with the organization, purpose and program of its Division for Scientific Affairs.

NATO is an organization of fifteen sovereign nations in Europe and North America united in a treaty of mutual security. NATO is, however, more than a military alliance; it is dedicated also to the political, economic, social, and scientific cooperation of its member nations. The governing body of NATO, the North Atlantic Council, is composed of an Ambassador from each member nation and is chaired by the Secretary-General, Dr. Dirk U. Stikker of the Netherlands. Dr. Stikker's predecessor was Mr. Paul-Henri Spaak of Belgium who resigned this year. Each Ambassador heads a delegation from his own nation and, in addition, there is an international staff reporting to the Secretary General. On this international staff is the Assistant Secretary General for Scientific Affairs, at present Dr. William A. Nierenberg, who is Chairman of the Science Committee. The Science Committee, constituted of one eminent scientist from each member nation, meets three times a year, usually in the permanent headquarters in Paris. The purpose of the Science Committee, which came into being in 1958, is to increase the effectiveness of western science. I shall pass out copies of a report prepared by a special study group for the Science Committee entitled "Increasing the Effectiveness of Western Science," which will give a more complete picture of the purpose of the Science Committee than I could do in the brief time allotted to me. 
The program of the Scientific Affairs Division which I shall briefly describe is more completely explained in another pamphlet with the title "Facts about NATO Scientific Cooperation," which is available for those who wish a copy.

The Advanced Study Institute program ị one of the most successful ventures sponsored by the Scientific Affairs Division. This program has been in operation for three years. In 1959 five institutes were held at a total cost of $\$ 100,000$. In 1960 , with a budget of $\$ 200,000$, ten institutes were conducted. In 1961 , a total of nineteen institutes will be sponsored with an expenditure of approximately $\$ 300,000$; this institute is one of the nineteen. To qualify for NATO support, both the lecturers and participants must be drawn from several NATO countries; however, persons from non-NATO lands also attend. Three members of the Science Committee serve as an expert Advisory Panel to screen the applications. Subjects discussed vary widely and may include any branch of the physical, biological, or mathematical sciences. The institutes fulfill several purposes. International understanding is improved by allowing scientists of different nations to meet and talk with one another; the education of young scientists is appreciably stimulated by the high level of instruction; and more senior scientists have the opportunity to engage in a frank exchange of views. Many of the studies undertaken are of vital importance if measured by their potential benefit to mankind.

\section{SCIENCE FELLOWSHIP PROGRAM}

One of the earliest actions of the Science Committee was to launch the NATO Science Fellowship Program. In 1959, its first year, the program allowed over two hundred students to study in countries other than their own; fields of study included an impressive variety of topics in mathematics, physics, chemistry, biology, astronomy, and engineering. The fellowship program is administered by national agencies of the various countries. The NATO countries provided $\$ 1,000,000$ to support the program in $1959, \$ 1,750,000$ in 1960 , and $\$ 2,500,000$ in 1961.

The fellowships help to meet the needs of not only the less developed NATO nations, whose lack of opportunities places them in a special category, but also of the more technologically advanced countries whose students may be faced by an insufficiency of grants for study in neighboring lands. Only the most qualified applicants are encouraged, yet the number of qualified applicants has always been greater than the number of fellowships available.

\section{RESEARCH GRANTS PROGRAM}

Scientific advances depend upon research; to sponsor specific research projects the Research Grants Program was started in 1960. Though the Science Committee takes the view that scientific research is most appropriately financed on a national basis, it recognizes that there are particular instances when the resources of NATO should be used. For example, some scientific fields such as oceanography are inadequately investigated because the subject is of an international nature and for this reason unduly neglected by individual countries. Again, in a few of the NATO countries, funds devoted to research are meager and the Research Grants program has been used to sponsor a few active research projects in the hope that these may have a catalytic effect and encourage governments to devote more resources to science. 
The Research Grants Program was established with an initial fund of $\$ 1,000,000$, of which the greater part was allocated in 1960 . To continue the program, the Council approved expenditure of a second sum of $\$ 1,000,000$, of which $\$ 250,000$ will be spent on oceanography and meteorology.

There is not sufficient time to describe all of the ways in which the Science Committee seeks to increase the effectiveness of Western science. The most advanced and successful programs to date have been described. There is some work in Operations Research being sponsored by NATO as well as work in Defense Psychology.

The main thoughts which I wish to leave with you are: NATO is a healthy growing alliance, not only for mutual defence but for political, social, economic, and scientific cooperation. In each of these areas much progress has been made. It is perhaps unfortunate that day-by-day advances in international cooperation are not always considered news by the press, rather the failures or disagreements are the things which appear in headlines. It is my belief, based on some direct contact with the program, that scientific cooperation within the Alliance is in an extremely healthy state and that western science is advancing for the good of the Alliance and for the good of all mankind. 



\section{LECTURE 1}

\section{TRANSDUCERS}

\section{Schofield}

Naval Research Establishment Halifax, Nova Scotia, Canada

\subsection{INTRODUCTION}

Research into underwater acoustics could be carried out using purely acoustic links but the limitations would be many. In a purely acoustic system, there are very few practical instruments for detecting sound other than the ear. Although the ear is a beautiful instrument and in many ways surpasses electronic devices, it is not adequate when accurate quantitative measurements are required. The conversion of acoustic energy to electrical energy immediately allows the employment of a wide variety of electrical measuring and recording instruments. Similarly, electrical to acoustic conversion devices give a greater measure of control than the use of bells, explosive charges, etc., would permit in the generation of sound.

Electroacoustic transducers for use in air, i.e., loudspeakers and microphones, are familar devices. However, waterproofed loudspeakers and microphones designed to couple to air are quite inadequate to couple to water. In technical terms, the acoustic mismatch is great and the loss in conversion efficiency is high. The characteristic acoustic impedance of water is nearly 4000 times the characteristic acoustic impedance of air, and a simple calculation indicates that the efficiency of conversion of a transducer designed for operation in air would drop by a factor of about 10,000 . An underwater projector is required to produce about 60 times the force and $1 / 60$ the displacement of an equivalent loudspeaker projecting the same energy into air.

The other immediately obvious difference is the static pressure exerted by the environment. Greater interest is developing in the deep ocean, and a trans ducer at $10,000 \mathrm{ft}$ is subjected to a static pressure of $4457 \mathrm{psi}$. The problem posed by the hydrostatic pressure is not only one of mechanical strength. It also places limitations on the type of active component and on the acoustic design of the unit; for example, sponge rubbers cannot be used as pressure-release materials. Changes in the parameters of transducer materials may also be caused by the stresses produced by the hydrostatic pressure.

This paper is restricted to electroacoustictransducers; i.e., projectors which convert electrical energy to acoustic energy and, vice versa, hydrophones which convert acoustic energy into electrical energy. Most of the units are reciprocal 
in the sense that they can be used for either purpose. Interesting possibilities, such as hydrodynamic oscillators, will not be considered. The principles of electroacoustic conversion will be only very briefly discussed since these are covered in well-known texts [1]. Piezoelectricity, electrostriction, and magnetostriction are the most widely used phenomena in underwater acoustic transducers, and the advantages and disadvantages of materials exhibiting these phenomena will be discussed. Much of the foregoing is covered in the literature but a gap exists between the fundamental principles and the application of these principles to practical transducers. About one-half of the paper is devoted to an examination of the design of two typical transducers, a multielement compound-bar projector unit and a hydrophone designed for operation deep in the ocean.

\subsection{ELECTROACOUSTIC CONYERSION PRINCIPLES}

Examining all the theoretically possible direct methods of converting electrical to acoustic energy means determining the possible forces that electrical and magnetic energy exert on matter.

Basically the force effects due to an electric field are dependent on the fact that a force $\mathbf{F}$ is exerted on an electric charge $q$ in an electric field $\mathbf{E}$ given by the expression $\mathbf{F}=q \mathbf{E}$. This fundamental relation is only true if $q$ can be regarded as a point charge. Such conditions do approximately exist in crystal lattices; and if the ions in the lattice move easily relative to one another, the crystal will deform on the application of an electric field. Conversely if a mechanical force is applied to such a crystal and the lattice deforms, electric charges appear on the surface of the crystal. This phenomenon is called piezoelectricity and has been widely used in underwater transducers. However, due to the anisotropy inherent in their crystal structure, the relationship between the electric field and mechanical stress in piezoelectric crystals is not simple, and in the general case, not only normal but also shear stresses are generated. The stresses can be represented by six equations of the type:

$$
\begin{aligned}
& T_{x x}=e_{11} E_{x}+e_{21} E_{y}+e_{31} E_{z} \\
& T_{y y}=e_{12} E_{x}+e_{22} E_{y}+e_{32} E_{z} \\
& T_{z x}=e_{13} E_{x}+e_{23} E_{y}+e_{33} E_{z} \\
& T_{y z}=e_{14} E_{x}+e_{24} E_{y}+e_{34} E_{z} \\
& T_{z x}=e_{15} E_{x}+e_{25} E_{y}+e_{35} E_{z} \\
& T_{x y}=e_{16} E_{x}+e_{25} E_{y}+e_{36} E_{z}
\end{aligned}
$$

where $E_{\dot{x}}, E_{y}$, and $E_{z}$ are the electric field strength components; $T_{x x}, T_{y y}$, and $T_{z z}$ the normal stresses; $T_{y z}, T_{z x}$, and $T_{x y}$, the shear stresses; and $e_{i k}$, the piezoelectric constants, which are different for the various piezoelectric materials. Some simplification is possible by grinding plates from the crystals at particular orientations to the crystallographic axes. In quartz, for example, all the e coefficients are zero with the exception of $e_{11}, e_{12}, e_{14}, e_{25}$, and $e_{26}$. If a rectangular plate is ground from the crystal, with the planes of the plate perpendicular to the coordinate axes of quartz, and a voltage $V$ is applied in the $x$ direction, the equations representing the stresses are reduced to three. Moreover, if our 
attention is restricted to a frequency region around a mechanical resonance, only this force need be considered, and the force law reduces to $F=\gamma V$ where $\gamma$ is a constant. It is easy to show that the displacement law is given by $q=\gamma \xi$ where $\xi$ is the displacement.

In all other force effects on matter due to the application of electric fields, the stress is proportional to the square of the electric field. There are three separate force effects:

a. Dielectric stress, the attractive force between condenser plates

b. $\nabla \epsilon$ stress, where $\epsilon$ is the dielectric constant

c. Electrostrictive stresses

In magnetism there are analogs of the $\nabla \epsilon$ and the electrostriction effects, both proportional to the square of the magnetic induction, but analogs of the dielectric stress or piezoelectricity do not exist since there are no true free magnetic charges. There is also one force effect which has no analog in electricity. This is the force exerted on a conductor of length $l$ carrying a current $i$ in a magnetic field of induction $B$ given by $F=B l i$ and is usually called the electrodynamic effect. It is a linear function of both magnetic induction and current.

In many underwater acoustic experiments, it is important that the transducer not introduce any changes in the waveform and this dictates the use of a linear conversion effect. Therefore, only the piezoelectric and electrodynamic effects are immediately suitable for transducers. Compensation for any variation in response with frequency can usually be made in the electronics.

It is easy to show that, if the mechanical stress is dependent on the square of the electrical or magnetic field, the frequency of the developed stress is twice that of an impressed sinusoidal variable. However, if a dc electrical quantity is added to the small alternating electrical quantity, it is possible, within limits, to obtain energy conversion without frequency change. This is the so-called polarized method of operation. In some electrostrictive and magnetostrictive materials the remanent electric displacement or magnetic field of induction provides almost optimum polarization and an external dc field is not required. It is also important to note that with quadratic force effects the conversion of mechanical to electrical energy is associated with the existence of an electric or magnetic field. During the remainder of the paper, electrostrictive and magnetostrictive materials will always be considered polarized, e.g., ferroelectric materials such as barium titanate and lead zirconate titanate will be considered as pseudopiezoelectric.

\subsection{EQUIVALENT ELECTRICAL CIRCUITS}

Every electroacoustic transducer is an electric storage element, e.g., capacitor, or inductance, combined with an acoustic oscillator. The latter affects the input impedance of the transducer, and the analysis of a transducer as a component of an electrical network is greatly facilitated if the unit is replaced by an equivalent electrical circuit. It can be shown by relatively simple analysis [2] that, neglecting losses, a piezoelectric bar excited in a longitudinal mode of vibration can be represented by the equivalent electrical circuit of Fig. 1.1. The parameters for the equivalent circuit are: 


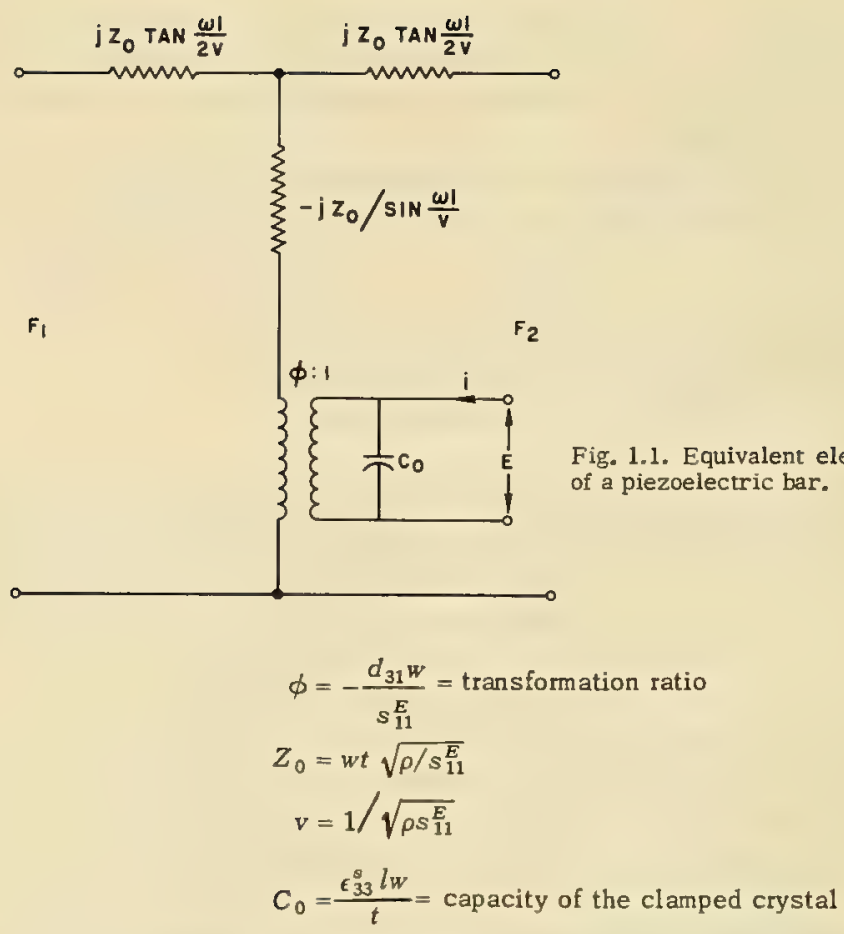

where $l$ is the length, $w$ is the width, $t$ is the thickness, $d_{31}$ is the piezoelectric constant, $s_{11}^{E}$ is the compliance at constant electric field, $\rho$ is the density, and $\epsilon_{33}^{s}$ is the dielectric constant at constant strain.

More complex acoustic oscillators can be analyzed by adding the appropriate acoustic components to the terminals $F_{1}$ and $F_{2}$. In the immediate neighborhood of resonance, a considerably simplified circuit, Fig. 1.2, can be used. $C_{0}$ and $R$ are the clamped capacity and resistance; and $K, N, S_{I}$, and $S_{R}$ are the motional capacity, inductance, internal loss resistance, and radiation resistance, respec-

Fig, 1.2. Simple lumped equivalent electrical circuit of a piezoelectric transducer.

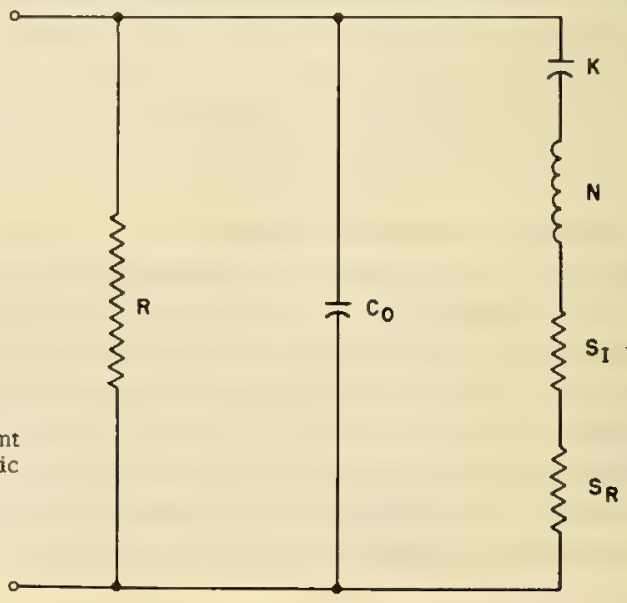


tively. It is to be noted that in this circuit the motional capacity and inductance are lumped constants while in the mechanical system the stiffness and mass are distributed. The immediately obvious difference is that, whereas the mechanical system can resonate at higher harmonics, the electrical circuit has only one resonant frequency. It is, nevertheless, a very useful circuit for any piezoelectric transducer provided attention is limited to the region about the fundamental resonant frequency. Similar equivalent circuits can be developed for transducers based on other force effects.

One of the important characteristics of a transducer is the bandwidth. It has become customary to consider two $Q$ factors in transducers: the mechanical or motional $\ell, Q_{m}$, which can be calculated from the mechanical characteristics of the unit or from the motional arm of the equivalent electrical circuit and the electrical $Q, Q_{e}$, which from Fig. 1.2 is $\omega_{0} C S_{T}$, where neglecting the dielectric loss resistance $S_{T}=S_{I}+S_{R} \cdot Q_{m}$ controls the frequency response in both projecting and receiving modes of operation if either the driver or the receiver has a high impedance. However, in transmission the electrical storage element is usually tuned out and the amplifier approximately matched to the motional resistance. From Fig. 1.2 it can be shown that, parallel-tuning a piezoelectric transducer with an inductance, neglecting the dielectric loss resistance, and matching the impedances of the amplifier and transducer, the bandwidth is given by $2 /\left(Q_{m}+Q_{\Theta}\right)$. This quantity is a maximum when $Q_{m}=Q_{0}$. It will be shown later that the product $Q_{m} Q_{e}$ is related to the electromechanical coupling factor, and that for a wide bandwidth a high coupling factor is required.

\subsection{ELECTROMECHANICAL COUPLING FACTOR}

The importance of the ratio of the clamped capacity to motional capacity as a measure of activity in a piezoelectric transducer was first noted by Dye [3] in 1926, but it was not until 1935 that Mason [4] introduced the idea of an electromechanical coupling coefficient closely related to this capacitance ratio. Since that time there have been a number of definitions and approaches $[1,2,3,6-9]$ almost all of which were both consistent and correct, but there remained a need for a general definition from which all such definitions could be derived. Hersh [10] presented such a general approach in 1957: if the equations defining a coupled reciprocal system can be written as

$$
\begin{aligned}
& y_{1}=a_{11} x_{1}+a_{12} x_{2} \\
& y_{2}=a_{21} x_{1}+a_{22} x_{2}
\end{aligned}
$$

and are homogeneous in the variables of generalized force and displacement, where the coefficients $a_{m n}$ are coefficients or ratios of coefficients from the same energy function, the coupling coefficient $k$ of the coupled system is defined by

$$
k^{2}=\frac{a_{12} a_{21}}{a_{11} a_{22}}
$$

If the equations of state are mixed in the variables of generalized force and dis placement, the coupling factor is defined by

$$
k^{2}=\frac{a_{12} a_{21}}{a_{11} a_{22}+a_{12} a_{21}}
$$


The piezoelectric equations of state for the length extensional mode of a bar excited by a transverse field are

$$
\begin{aligned}
& S_{1}=s_{11}^{E} T_{1}+d_{31} E_{3} \\
& D_{3}=d_{31} T_{1}+\epsilon_{33}^{T} E_{3}
\end{aligned}
$$

When we compare these equations with Eqs. (2), and use Eq. (3), we find that the coupling coefficient is given by the equation

$$
k^{2}=\frac{d_{31}^{2}}{s_{11}^{E} \epsilon_{33}^{T}}
$$

This equation is frequently used to define the electromechanical coupling coefficient.

Starting from Eqs. (2), an expression can be derived for the coupling coefficient in terms of the input open- $\left(x_{2}=0\right)$ and short-circuit $\left(y_{2}=0\right)$ impedances:

$$
k^{2}=\frac{Z_{\mathrm{oc}}-Z_{\mathrm{sc}}}{Z_{\mathrm{oc}}}
$$

where the impedances are either all resistances or all reactances of the same sign.

Again starting from Eqs. (2), it can be shown that

$$
k^{2}=\frac{\text { Total energy stored unclamped }\left(x_{2}=0\right)-\text { Total energy stored clamped }\left(y_{2}=0\right)}{\text { Total energy stored unclamped }\left(x_{2}=0\right)}
$$

In the case of a piezoelectric transducer, this is equivalent to

$$
k^{2}=\frac{\text { Energy stored in mechanical form }}{\text { Total electrical energy input }}
$$

Using the simple lumped equivalent circuit, Fig. 1.2, the electromechanical coupling coefficient derived from Eq. (7) reduces to

$$
k_{a}^{2}=\frac{K}{C+K}
$$

However, since the equivalent circuit of Fig. 1.2 is not exact, $k_{a}$ is not the true electromechanical coupling factor.

It can be shown that for a longitudinally resonant piezoelectric bar

$$
k_{a}^{2}=\frac{k^{2}}{\pi^{2} / 8-k^{2}\left(\pi^{2} / 8-1\right)}
$$

A useful approximation in transducer design is

$$
k_{a}^{\prime 2} \simeq k_{a}^{2}=\frac{K}{C}=\frac{8}{\pi^{2}}\left(\frac{k^{2}}{1-k^{2}}\right)=Q_{m} Q_{\text {e }}
$$

and hence, it can be seen from the previous section, the tuned bandwidth is dependent upon the coupling factor.

\subsection{TRANSDUCER MATERIALS}

Although some use has been made of the electrodynamic and $\nabla \mu$ effects in transducers, most underwater acoustic units have made use of the piezoelectric, 
TABLE 1. I

\begin{tabular}{|c|c|c|c|c|c|c|c|c|}
\hline Material & Mode & $\epsilon / \epsilon_{0}$ & $k, \%$ & $\begin{array}{c}d \cdot 10^{12} \\
\mathrm{~m} / \mathrm{v}\end{array}$ & $\begin{array}{l}\mathrm{g} \cdot 10^{3}, \\
\mathrm{v}-\mathrm{m} / \mathrm{N}\end{array}$ & $\tan \delta$ & $k^{2} / \tan \delta$ & $\begin{array}{c}\tan \delta \text { at } \\
2 \mathrm{kv} / \mathrm{cm}\end{array}$ \\
\hline Quartz, X-cut & 33 & 4.5 & 11 & 2.3 & 58 & 0.0002 & 60 & \\
\hline $\mathrm{ADP} 45^{\circ} \mathrm{Z}$ & 31 & 15.3 & 28 & 24 & 177 & 0.024 & 3.3 & \\
\hline Lithium sulphate & 33 & 10.3 & 38 & 16 & 175 & & & \\
\hline Rochelle salt & & & & & & & & \\
\hline $45^{\circ} \mathrm{X}$-cur & 14 & 200 & 54 & 140 & 79 & 0.05 & 6 & \\
\hline $\mathrm{BaCaTiO}_{3}$ & 33 & 1300 & 50 & 150 & 13 & 0.007 & 36 & $>0.10$ \\
\hline $\mathrm{BaCaCoTiO}_{3}-\mathrm{NRE} 4$ & 33 & 1400 & 46 & 145 & 12 & 0.005 & 42 & 0.02 \\
\hline $\mathrm{PZT}-4^{*}$ & 33 & 1200 & 64 & 256 & 24 & 0.003 & 135 & 0.02 \\
\hline PZT $-5^{*}$ & 33 & 1500 & 68 & 320 & 24 & 0.013 & 35 & 0.11 \\
\hline $\mathrm{Pb}\left(\mathrm{NbO}_{3}\right)_{2}$ & 33 & 2500 & 59 & 290 & 13 & 0.006 & 58 & \\
\hline
\end{tabular}

electrostriction, or magnetostriction phenomena. The most important characteristics for transducer applications of piezoelectric, polarized electrostrictive, and magnetostrictive materials are summarized in Tables 1.I and 1.II. The relative importance of the various material parameters depends upon the particular requirement of the transducer incorporating the material, but the parameters presented do indicate some of the advantages and disadvantages of the various materials. Furthermore, since the materials are not isotropic, the magnitude of the parameters depends upon the mode of operation: e.g., the piezoelectric constants depend on the cut of the sample and the coupling factor changes for different modes of vibration.

Undoubtedly the most important single characteristic of a transducer material is the electromechanical coupling factor. It has already been shown that the coupling factor is related to the ratio of stored mechanical energy to total electrical input energy for electrical to mechanical conversion and that it controls the maximum bandwidth of a projector. In addition, Kendig [11] recently emphasized that for detecting very small acoustic signals, it is the equivalent noise pressure of the receiver that is of prime importance, not the sensitivity of the hydrophone. He developed expressions for the efficiency and equivalent noise pressure of three different shapes of hydrophones, and $k^{2} / \tan \delta$, in which $\delta$ is the dielectric loss angle, is a common factor in each equation. Thus, $k^{2} / \tan \delta$ appears to be a good criterion for hydrophone materials. This factor is

TABLE 2. I

\begin{tabular}{|c|c|c|c|c|c|}
\hline Material & Type & $\frac{\mu_{\mathrm{r}}}{\mu_{\mathrm{o}}}$ & $k, \%$ & $\begin{array}{c}\lambda, \\
N / w\end{array}$ & $\begin{array}{c}\rho_{\mathrm{c}}, \\
\text { ohm-m }\end{array}$ \\
\hline \multirow[t]{2}{*}{ Nickel } & Polarized & 41 & 31 & $-20 \cdot 10^{6}$ & $7 \cdot 10^{-8}$ \\
\hline & Remanent & 160 & 23 & -25 & $7 \cdot 10^{-8}$ \\
\hline $2 \mathrm{~V}$ Permendur & Remanent & 54 & 20 & $36 \cdot 10^{6}$ & $8.5 \cdot 10^{-8}$ \\
\hline 13 Alfer & Polarized & 190 & 27 & $6.7 \cdot 10^{6}$ & $9 \cdot 10^{-7}$ \\
\hline $4 \% \mathrm{Co}-\mathrm{Ni}$ & Polarized & 130 & 59 & $32 \cdot 10^{6}$ & $10.3 \cdot 10^{-8}$ \\
\hline Ferroxcube A & Remanent & 430 & 5.5 & $21 \cdot 10^{6}$ & $10^{6}$ \\
\hline
\end{tabular}


highest for the ferroelectric ceramics, particularly PZT-4, and for quartz. However, with low-dielectric-constant materials such as quartz, the shunting effect of even short lengths of cable significantly reduces the effective sensitivity of the unit. Care should be exercised in the use of any simple criterion such as $k^{2} / \tan \delta$; in practical cases the whole receiving and recording system must be considered.

For projectors again the coupling factor and tan $\delta$, particularly at high driving fields, are important. In addition, it is desirable to have a low-impedance device to avoid high voltages, and this is achieved by using high-dielectric-constant materials. With quartz, the impedance of the units is so high that voltage breakdown across the crystal surfaces limits the power output. The most interesting materials for high-power projectors are PZT-4 and the cobalt additive barium titanate, NRE-4 [12]. Among the other properties which affect the choice of material are the mechanical strength, the aging of the parameters, the variation of parameters with temperature, and the maximum temperature at which the material can be used.

There is one big difference between the crystal piezoelectric materials and the ceramic pseudopiezoelectric materials. Because of the crystalline nature of the former, the impurities are small and the values of the parameters are reasonably constant and subject to little modification. The ceramics are not pure materials, and compositions consisting of mixtures of chemical compounds have been developed with particular characteristics. Small percentages of additives can have a pronounced effect on some characteristics. For example, Fig. 1.3 shows the effect of adding small quantities of cobalt to a barium titanate composition on the dielectric loss tangent as a function of exciting field. A corollary is, of course, that small changes in composition and manufacturing technique can have large effects on the material parameters. Reproducibility on a production scale has presented problems with some compositions.

Some characteristics of the more important magnetostrictive transducer materials are given in Table 1.II. Only the $4 \%$ cobalt-nickel alloy [13] has a coupling coefficient comparable with the coupling factors of the ferroelectric ceramics. With the exception of the ferrites, the main advantage of the magnetostriction unit is ruggedness. It is a low-impedance device and there are

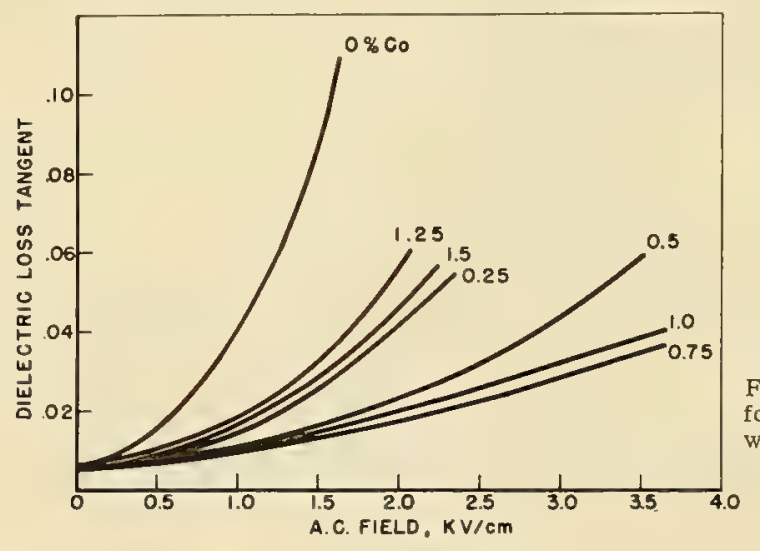

Fig. 1.3. Tan $\delta$ vs ac field for barium-calcium titanate with additions of cobalt. 
no insulation problems; it does not have to be enclosed in a watertight container. The disadvantages are that the unit, if made of metal, must be laminated to keep the eddy-current loss low, and this affects the useful top frequency (about 50 kcps); for optimum operation magnetostrictive materials require a bias field, necessitating an auxiliary magnetic circuit.

\subsection{EXAMPLES OF TRANSDUCERS}

The remainder of the paper will be devoted to a consideration of two typical transducer designs: the first is a design of a high-power projector, the basic ideas of which can be used from a few kcps to over $100 \mathrm{kcps}$; the second is a hydrophone for operation deep in the ocean.

\subsubsection{Projectors}

Simple resonant bars do not have optimum characteristics for projectors. For example, the mechanical $Q$ of a longitudinal resonant bar of barium titanate radiating into water is about 50 ; whereas for the maximum bandwidth condition $\left(Q_{e} \simeq Q_{m}\right)$ and with a coupling factor of $0.2, Q_{m}$ should be 5 . A more complex acoustic oscillator design has a larger number of variables with which to achieve the required characteristics.

\subsubsection{Resonant Frequency}

Consider an idealized compound-bar resonator $[14,15]$, of the type shown in Fig. 1.4. The center of the ceramic section is a velocity node and each half of the element may be considered separately as a quarter-wave vibrator, the head being 3-ply and the tail 2-ply. By setting up the equations of the dynamic displacements in the various plys of the element and substituting the various boundary conditions at the interfaces, the conditions for resonance can be determined. For the 3-ply vibrator we have

$$
\frac{Z_{3} A_{3}}{Z_{2} A_{2}} t_{2} t_{3}+\frac{Z_{3} A_{3}}{Z_{1} A_{1}} t_{1} t_{3}+\frac{Z_{2} A_{2}}{Z_{1} A_{1}} t_{1} t_{2}=1
$$

where $Z=\rho_{i} c_{i}$ is the characteristic impedance; $\rho_{i}$ is the density of the ply $i$; $c_{i}$ is the velocity of sound in ply $i ; t_{i}=\tan k_{i} l_{i} ; k_{i}$ is the wave number of ply $i$ $l$ is the length of the ply $i$; and $a$ is the cross-section area of ply $i$.

If $l_{3} \ll \lambda_{3}, \tan k_{3} l_{3} \simeq k_{3} l_{3}$ and $Z_{3} A_{3} t_{3}=\omega M$, where $M$ is the mass of section 3 .

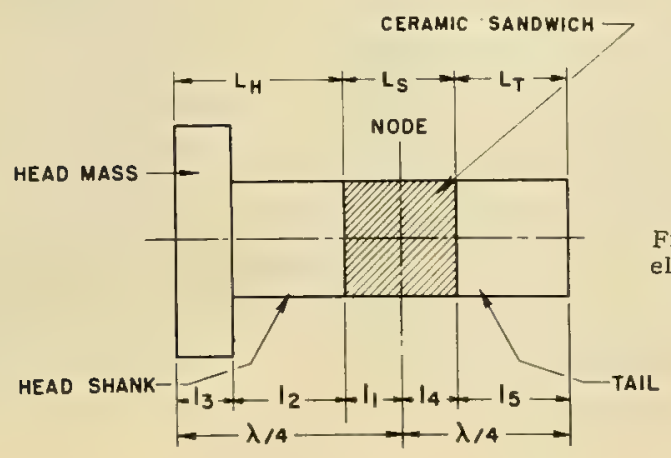

Fig. 1.4. Compound-bar piezoelectric element. 
Also, $A_{1}=A_{2}$ and Eq. (13) simplifies to

$$
\frac{\omega M t_{2}}{Z_{2} A_{2}}+\frac{\omega M t_{1}}{Z_{1} A_{1}}+\frac{Z_{2}}{Z_{1}} t_{1} t_{2}=1
$$

For the tail section the condition for resonance is

$$
\frac{Z_{5}}{Z_{4}} t_{4} t_{5}=1
$$

\subsubsection{Electromechanical Coupling Coefficient}

An expression for the coupling factor of a compound bar can be developed by considering the complete equivalent circuit and using Eq. (7). For the resona tor in Fig. 1.4 in which $l_{1}=l_{4}$, the expression [16] is

$$
k_{B}^{2}=\frac{k^{2}}{1+\frac{l_{2} s_{2}}{2 l_{1} s_{1}}+\frac{l_{3} A_{1}}{2 l_{1} A_{3}} \frac{s_{3}}{s_{1}}+\frac{l_{5} s_{5}}{2 l_{1} s_{1}}}
$$

where $k$ is the coupling coefficient of the active material alone and $s_{i}$ is the compliance of the $i$ th ply. The significance of the various material parameters can be more easily appreciated by simplifying the compound vibrator so that the front and back masses are of the same material and are of the same diameter as the active component. Equation (15) reduces to

$$
k_{B}^{2}=\frac{k^{2}}{1+\left(l_{T} / 2 l_{1}-1\right) s_{2} / s_{1}}
$$

where $l_{T}$ is the total length of the element.

Equation (16) shows that for a high coupling coefficient, the compliance of the loading mass should be small compared with the compliance of the active material. The theoretical and experimental variations of coupling factor with the ratio of active length to total length of the vibrator are shown in Fig. 1.5. The agreement between the experimental points (whichare for a brass-loaded barium titanate sandwich) and theory is good.

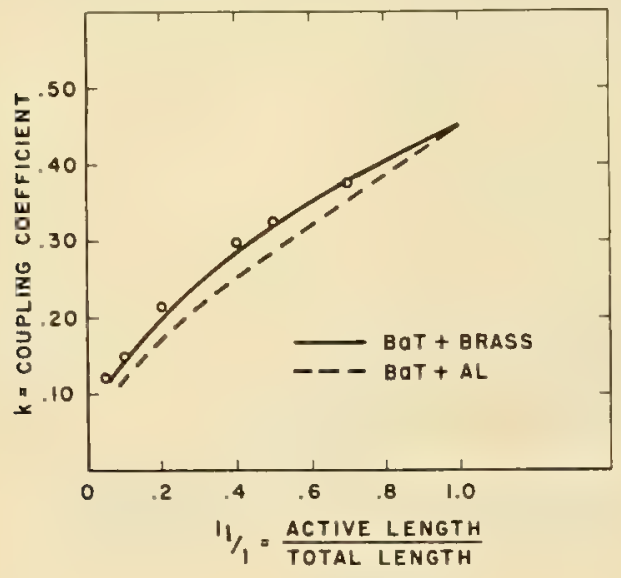

Fig. 1.5. Variation of coupling coefficient with the ratio of active to inactive length for a compound-bar element. 
It has already been stated that for maximum bandwidth the practical problem is to achieve the condition $Q_{m} \simeq ?$. The mechanical $\varrho$ can be calculated from the equation

$$
\bigcap_{m}=\frac{\omega_{0} M_{E}}{\rho_{0} c_{0} A}
$$

where $V_{E}$ is the effective mass of the element and can be calculated from kinetic energy considerations, $\rho_{0} c_{0}$ is the characteristic impedance of sea water, $A$ is the effective radiating area, and $\omega_{0}$ is the resonant angular frequency. There should also be an additional small term in the denominator to take into account the internal mechanical losses in the transducer element. With this type of element there are a large number of possible variables with which to obtain optimum characteristics for the particular requirement. The mechanical $Q$ can be adjusted by altering the effective mass or the acoustic loading. It is usually easier to adjust the acoustic loading by changing the radiating area of the head.

In a projector, only the behavior in the neighborhood of resonance is usually important, and it is a simple matter using the foregoing equations to predict the lumped equivalent circuit when the electrostatic capacity is specified. Although the compound-bar transducer has been described in terms of a mass-loaded piezoelectric element, a very similar analysis can be carried out for longitudinal mass -loaded magnetostriction units.

Figure 1.6 is a photograph of four barium titanate mass-loaded compound elements ranging in resonant frequency from $120 \mathrm{kcps}$ to $5 \mathrm{kcps}$. The construction is similar in all cases: a barium titanate sandwich loaded with a head and a tail.

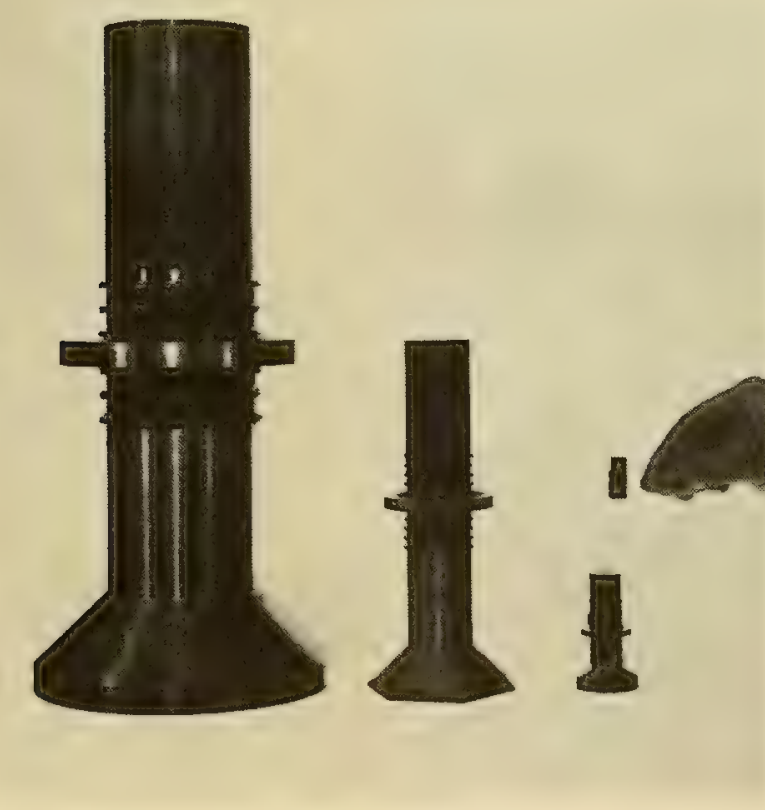

Fig. 1.6. Typical transducer elements. 
All but the $120 \mathrm{kcps}$ unit have aluminum heads with a head-to-shank area ratio of 4 to 1 and a brass tail. The components of an individual unit are cemented together with a strong epoxy adhesive, electrical contact being made to the electrodes of the barium titanate discs by copper gauze in the joints.

The elements a re usually mounted in air-filled cases, radiation being admitted through a neoprene window which has the same characteristic impedance as sea water and is bonded to the radiating surface of the heads. Figure 1.7 is a typical box and Fig. 1.8 shows an array of boxes in a towed body for use in propagation studies.

The calibration results of a 4 -element, 5 -kcps transducer having a radiating area of about one wavelength by one wavelength are shown in Fig. 1.9.

\subsubsection{Power-Handling Capabilities}

Transducers such as the ones just described are capable of radiating powers of several watts per square centimeter of radiating area, and with the development of such projectors the factors which limit the power-handling capabilities of transducers become important.

The onset of cavitation determines the limit imposed by the medium, i.e., the maximum power that can be transmitted by a projector of a certain radiating area. Cavitation occurs when the instantaneous acoustic pressure exceeds the sum of the static pressure and any inherent cohesive pressure of the liquid. Under this condition small cavities filled with water vapor are formed. When applied to transducers cavitation can be described physically as the rupture of the water in front of the projector face caused by the negative-pressure excursions exceeding the sum of the hydrostatic pressure and the tensile strength of

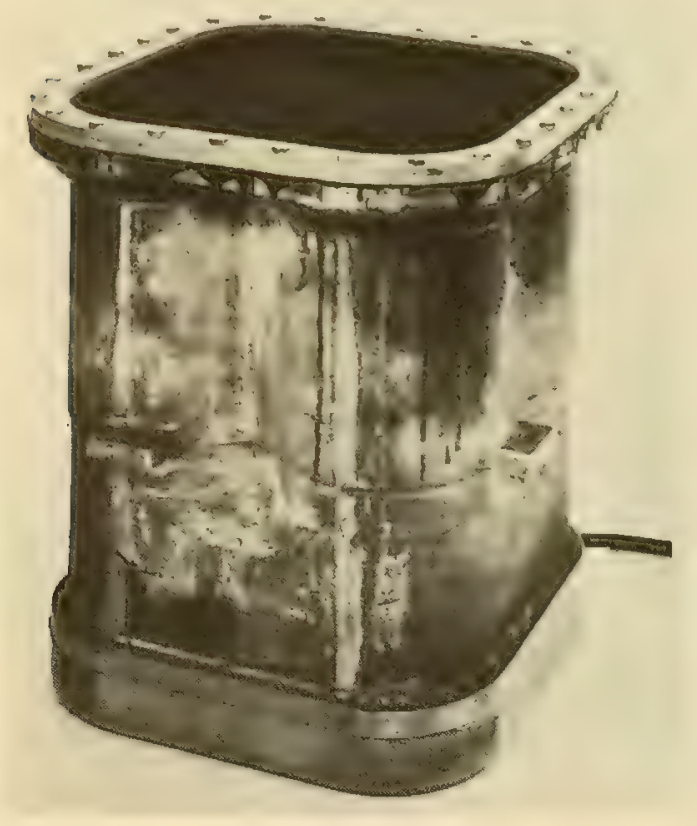

Fig. 1.7. Five-kcps transducer. 


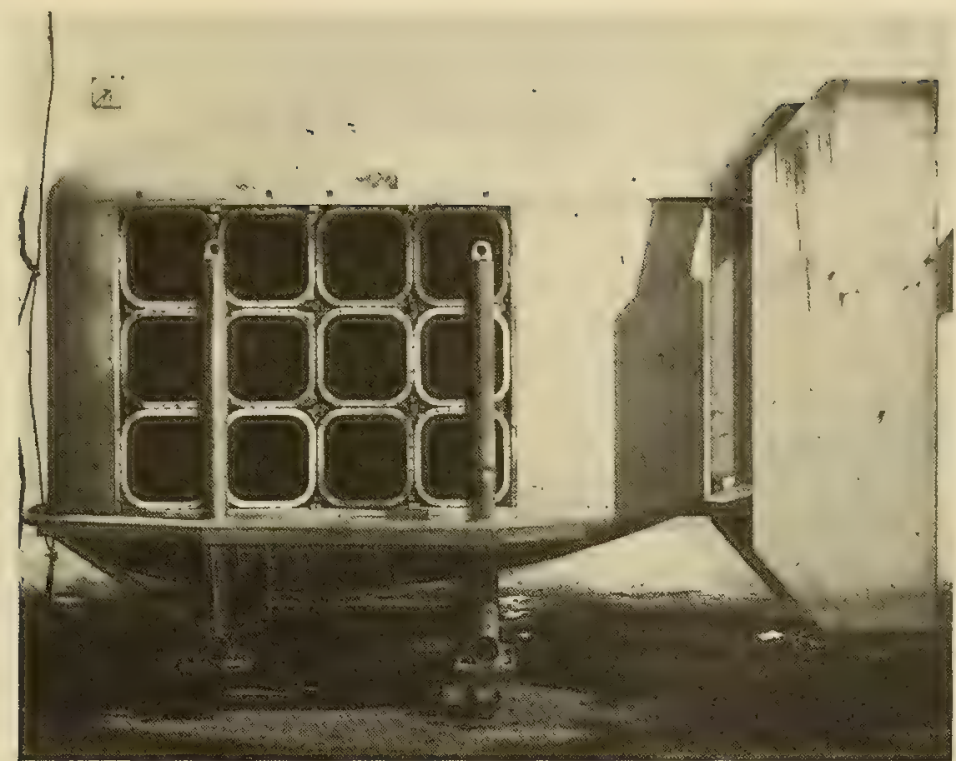

Fig. 1.8. Array of 5-kcps transducers.

the water. The presence of these cavities or bubbles sets an upper limit to the power transferred from a vibrating piston to the water since they reflect, scatter, and absorb the incident energy. At the same time, the radiation resistance changes and the acoustic waveform is distorted. The latter occurs because the amplitude of the acoustic signal is not symmetrical about the zero axis and a form of rectification takes place. Considerable erosion of the transducer face can occur.

Assuming that water does not exhibit a cohesive force and equating the atmospheric pressure to the peak acoustic pressure, we find that the intensity to produce cavitation at shallow depths is $0.3 \mathrm{w} / \mathrm{cm}^{2}$. As is well known, a sound intensity

Fig. 1.9. Calibration results of 5-kcps transducer.

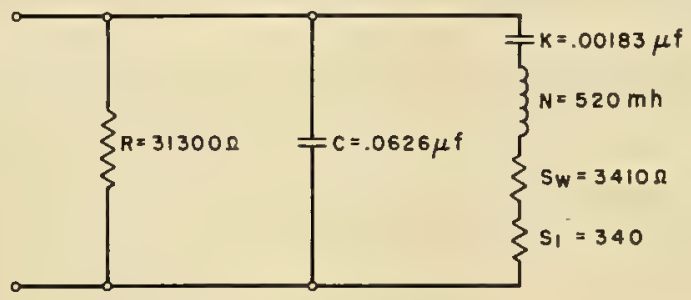

$f_{0}=5.1 \mathrm{kC} / \mathrm{s}$

$Q m=4.9$

$k=0.17$

PRO SENS fO $=217 \mu$ BAR / VOLT AT 3'

$H / P$ SENS $f_{0}=370 \mu$ VOLT $/ \mu$ BAR

$\eta$ eo $=81 \%$

$\eta \mathrm{ma}=90 \%$ 
of about $1 \mathrm{w} / \mathrm{cm}^{2}$ is required to produce cavitation in sea water. This implies that the inherent tensile strength of sea water is about 0.8 atm. The relationship between intensity to produce cavitation $l\left(\mathrm{w} / \mathrm{cm}^{2}\right)$ and the depth of the projector in feet $H$ is therefore of the type

$$
l=0.3\left(\frac{H}{32}+1.8\right)^{2}
$$

If such a relation is reliable, the cavitation limit is raised to about $7 \mathrm{w} / \mathrm{cm}^{2}$ at a depth of $100 \mathrm{ft}$. No published work on the effect of depth or hydrostatic pressure on the cavitation limit has been found; however, the limit of $7 \mathrm{w} / \mathrm{cm}^{2}$ at $100 \mathrm{ft}$ is in agreement with measurements carried out by the Naval Research Establishment which will be described later.

The above discussion of cavitation has been oversimplified; the presence of dissolved gases, impurity nuclei, temperature, and the pulse length of the acoustic signal will all affect the intensity at which cavitation is initiated. The radiating face of the transducer can also affect the inception of cavitation and "hot spots" can occur at levels considerably below the average level of cavitation. Further studies on cavitation as applied to transducers are desirable.

Although cavitation is the ultimate factor limiting the power output, many transducers reach their maximum power-handling capacity before the onset of cavitation even at shallow depths. Crystal piezoelectric transducers of the ADP and quartz type are inherently high-impedance devices requiring very high voltages to excite them to high powers. The limiting factor in this class of trans ducers is not cavitation but the voltage at which breakdown occurs across the surface of the crystals.

The important factors in the barium titanate compound-bar type of element are considered to be: (a) the dynamic strength of the element and (b) the heating associated with the dielectric and mechanical losses. The dielectric losses are kept as low as possible by using a specially developed barium titanate composition with additions of cobalt [12]. The mechanical losses are not a problem since the elements have a high motional-to-acoustic conversionefficiency. The powerhandling capacity therefore appears to be limited by the dynamic strength of the element.

Tests were carried out on a 10 -kcps transducer containing four elements at a depth of about $100 \mathrm{ft}$ with $75-\mathrm{msec}$ sinusoidal pulses at a repetition rate of 1 per sec. Figures 1.10 and 1.11 and Table 1 .III summarize the results. It is evident that the change in admittance and the decrease in efficiency is due to an increase in the internal mechanical losses. An examination of the conductance vs frequency curves shows that the clamped value of conductance, which is proportional to dielectric loss, changes little with increasing driving voltage as was expected with the barium titanate composition used.

The elements fractured at about $6 \mathrm{w} / \mathrm{cm}^{2}$. It can be readily calculated that at this peak power output, the stress at the node is about 670 psi. Tensile-strength tests showed that the static strength was about 3000 psi or four times the dynamic strength. This discrepancy has never been satisfactorily explained. Care was taken to eliminate transients, and fatigue is not a satisfactory explanation since one pulse at the high level would cause the element to break. The fracture took 


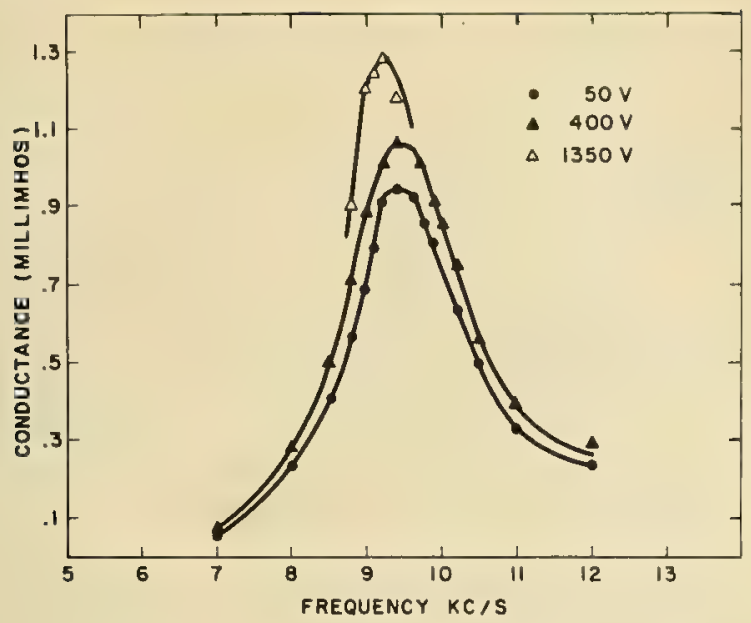

Fig. 1.10. Conductance vs frequency for various exciting voltages.

Fig. 1.11. Power output and efficiency vs input power.

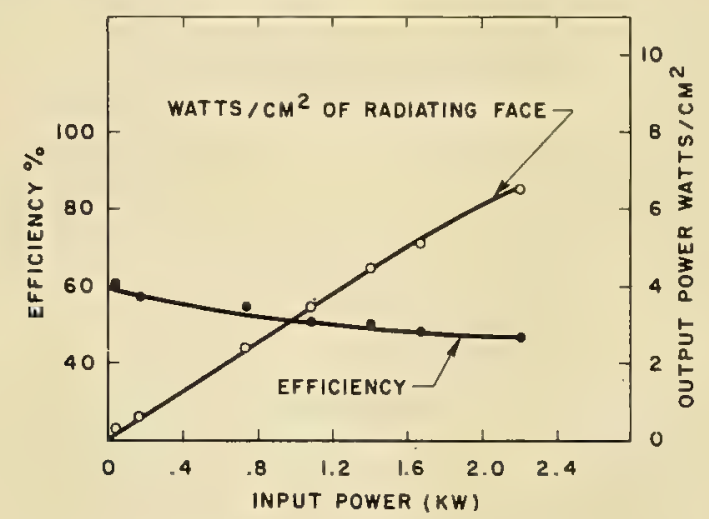

TABLE 3. III

\begin{tabular}{|c|c|c|c|c|}
\hline \multirow{2}{*}{ Quantity } & \multirow{2}{*}{ Units } & \multicolumn{3}{|c|}{ Driving voltage rms volts } \\
\hline & & 50 & 800 & 1350 \\
\hline $\begin{array}{l}\text { Resonant frequency, } f_{0} \\
\text { Mechanical } Q \\
\text { Coupling factor } \\
\text { Clamped capacity } \\
\text { Clamped resistance } \\
\text { Motional capacity, } K \\
\text { Motional inductance, } N \\
\text { Motional resistance, } S_{T} \\
\text { Radiation resistance, } S_{W} \\
\text { Internal loss resistance, } s_{1} \\
\text { Projector sensitivity at } 3 \mathrm{ft} \\
\text { Over-all conversion efficiency, } \eta_{e a} \\
\text { Motional to acoustic conversion } \\
\text { efficiency, } \eta_{\text {ma }}\end{array}$ & $\begin{array}{l}\mu \mathrm{f} \\
\text { ohms } \\
\mu \mu \mathrm{f} \\
\mathrm{mH} \\
\text { ohms } \\
\text { ohms } \\
\text { ohms } \\
\mu \mathrm{bar} / \mathrm{v} \\
\% \\
\%\end{array}$ & $\begin{array}{c}9.4 \\
5.5 \\
0.20 \\
0.069 \\
12,500 \\
2,680 \\
107.1 \\
1,150 \\
725 \\
425 \\
332 \\
59 \\
63\end{array}$ & $\begin{array}{c}9.3 \\
5.8 \\
0.20 \\
0.0705 \\
11.200 \\
3,050 \\
96.5 \\
970 \\
573 \\
397 \\
320 \\
54 \\
59\end{array}$ & $\begin{array}{c}9.2 \\
5.5 \\
0.22 \\
0.072 \\
10,000 \\
3.700 \\
81.0 \\
850 \\
430 \\
420 \\
322 \\
47 \\
51\end{array}$ \\
\hline
\end{tabular}


place either in the barium titanate or in the joints. Since with present techniques the joint strength can be made at least equal to the tensile strength of the barium titanate ceramic and since it is unlikely that the strength of the ceramic can be increased significantly, a method was sought which would overcome the inherent tensile weakness of the elements. Use was made of the high compressive strength of ceramics: a mechanical bias or compressional prestressing was applied to the ceramic sandwich so that the dynamic tensile forces would be eliminated or at least held within the dynamic tensile strength of the ceramic [17].

Figure 1.12 is a cross-section sketch of a typical element. The stress is applied by means of a bolt which passes through a hole along the center line of the element and is threaded into a tapped hole in the head piece. The bolt is then tightened with a torque wrench to apply compressional stress to the ceramic.

Calibration of stress-rod elements showed that the introduction of the stress rod had little effect on the performance of the elements; the electroacoustic efficiency remains sensibly the same regardless of stress. Power outputs of $7 \mathrm{w} / \mathrm{cm}^{2}$ were achieved but tests at higher powers were eliminated by the inception of cavitation. Driving these units in a highly cavitating state at shallow depths or in air did not change their characteristics.

The principles of prestressing are, of course, applicable to any transducer in which the amplitude of vibration of the element is limited by the mechanical strength of the active material or by the joint strength. Since all ferroelectric ceramics and magnetostrictive ferrite ceramics are weak in tension but inherently strong in compression, prestressing should allow transducers to be built with higher power-handling capability and with greater resistance to mechanical shock.

\subsubsection{Deep Hydrophones}

To illustrate the problems associated with the design of transducers for operation at great depths, a hydrophone designed to operate at depths down to $15,000 \mathrm{ft}$ will be described. The first requirement of a deep transducer is that it be mechanically capable of withstanding the hydrostatic pressure. A spherical shell is an excellent shape to withstand hydrostatic pressure since the stress is evenly distributed and no stress concentrations occur.

Consider a thin spherical shell of a ferroelectric material which is polarized in the radial thickness. The low-frequency sensitivity of the unit is given by

$$
\frac{V}{P}=\frac{-b}{2\left(1+\rho+\rho^{2}\right)}\left[\xi_{33}\left(2-\rho-\rho^{2}\right)+g_{31}\left(4+\rho+\rho^{2}\right)\right]
$$

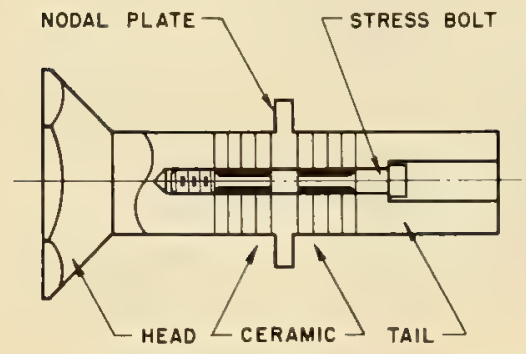

Fig. 1.12. Sketch of stress-rod element. 
where $\rho=a / b$ and $a$ and $b$ are the internal and external radii of the shell, respectively. For a thin shell $\rho \rightarrow 1$ and the equation reduces to

$$
\frac{V}{P}=-b g_{31}
$$

The sensitivity of a thin spherical-shell hydrophone is therefore directly proportional to the diameter.

The sensitivity as a function of frequency should be constant until the sphere is no longer a small fraction of a wavelength, say $\lambda / 8$. When this occurs, there will be a phase difference across the sphere and cancellation will cause a reduc tion in the sensitivity.

Resonance occurs in air at a frequency given by

$$
f_{0}=\frac{1}{\pi b}\left[\frac{Y_{11}}{2 \rho(1-\sigma)}\right]^{1 / 2}
$$

where $Y_{11}$ is the elastic-stiffness constant, $\sigma$ is the Poisson ratio, and $\rho$ is the density of ceramic. Only for very thin-walled spherical shells is the resonant frequency appreciably lower in water than in air.

Spherical shells have been assembled by cementing together carefully selected hemispheres with a nonconducting adhesive such as an epoxy resin. The lead to the inner electrode passes through a ceramic-to-metal seal in the shell. The hydrophone is waterproofed by several dippings in a liquid neoprene solution.

The sensitivity as a function of frequency is given in Fig. 1.13 for a 3-in. diameter $1 / 4$-in. wall thickness barium titanate sphere. The experimental sensitivity is in good agreement with the theoretical value of $17 \mu$ volt/ $\mu$ bar. As suggested previously, the sensitivity is flat with frequency until the sphere is about $\lambda / 8$, i.e., at about $3 \mathrm{kcps}$. Theoretically, a spherical transducer should be omnidirectional at all frequencies but, since the practical units are not perfect spheres, deviations from true omnidirectionality occur in the neighborhood of resonance and at higher frequencies. At resonance the directivity pattern is omnidirectional to about $1 \mathrm{db}$ and at $50 \mathrm{kcps}$ to about $\pm 2 \mathrm{db}$.

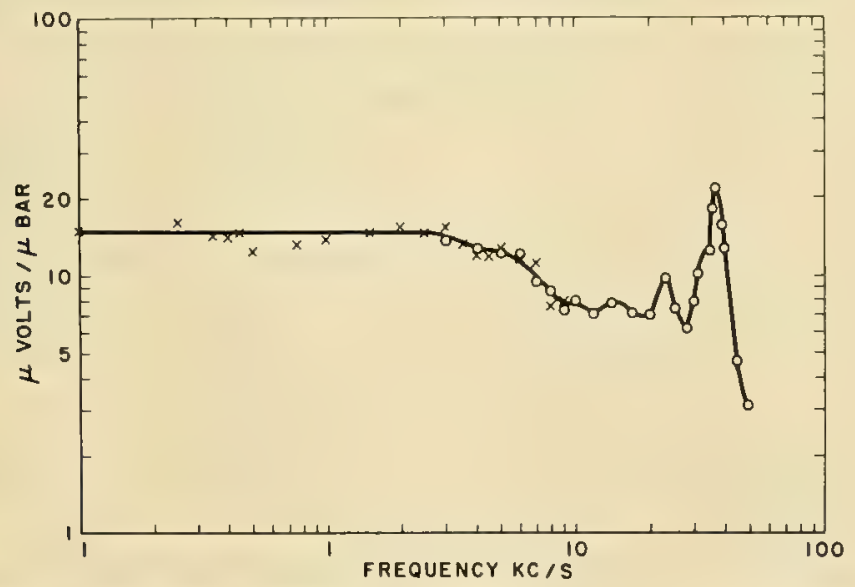

Fig. 1.13. Hydrophone sensitivity vs frequency for a 3-in. diameter and $1 / 4-i n$. wall barium titanate sphere. 


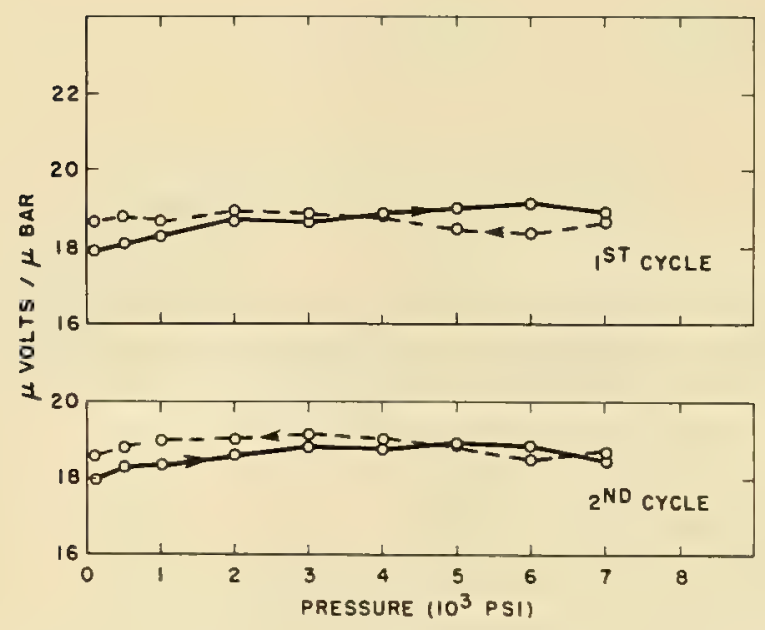

Fig. 1.14. Sensitivity at 200 cps vs hydrostatic pressure.

Figures 1.14 and 1.15 show the effect of pressure on the sensitivities of a barium titanate and a PZT-4 spherical hydrophone at $200 \mathrm{cps}$ for the first and second cycles of pressure; there is little change in the barium titanate but the sensitivity of the PZT -4 unit is reduced from approximately $24 \mu$ volts $/ \mu$ bar to $20 \mu$ volts/ $\mu$ bar.

Very little work has been published on the effect of pressure on the important parameters of transducer materials. However, some work has been published on the effect of stress on the dielectric properties of ceramic ferroelectrics [18] and it has been shown that large changes in dielectric constant can occur. The effect of stress is complicated; and the results depend on a number of variables in addition to the stress; the state of polarization of the samples, their history, time of measurement after the application of stress, and whether the stress is unidimensional, planar, or hydrostatic. These facts probably account for some of the disagreement among the papers.

Fig. 1.15. Sensitivity at 200 cps vs hydrostatic pressure of $\mathrm{PZT}-4$ sphere.

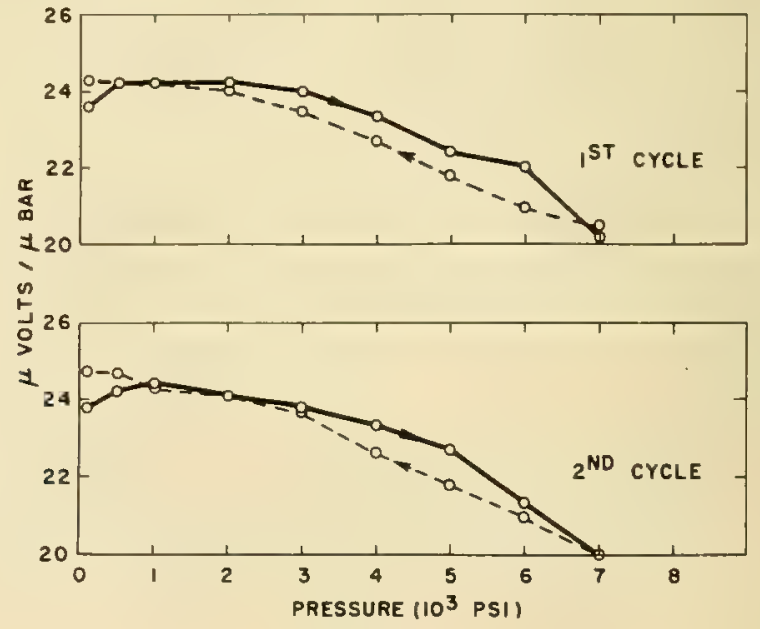


Fig. 1.16. Variation of dielectric constant with hydrostatic pressure.

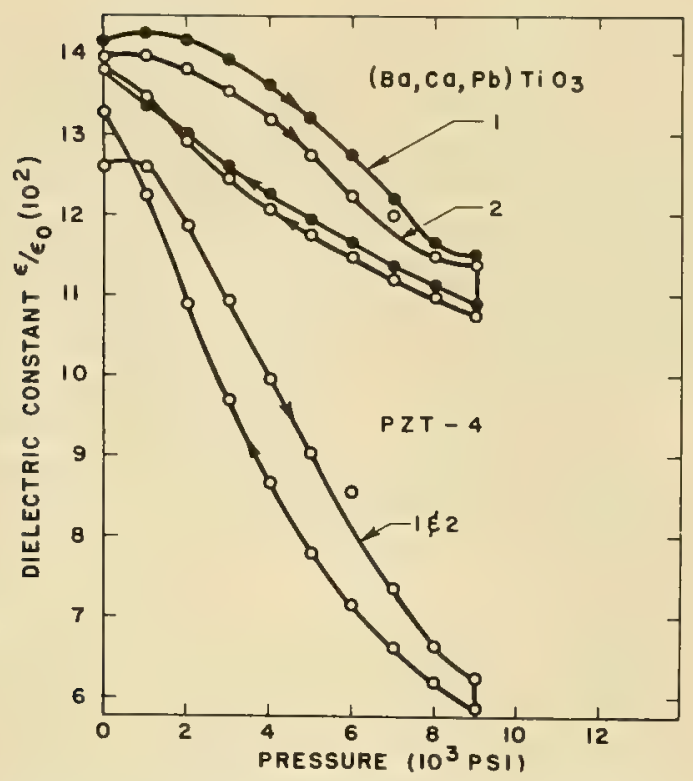

Barium titanate has a tetragonal structure, the elongated axis being the direction of polarization. The application of planar compressional stress to ceramic barium titanate tends to align the elongated axis, or c axis, normal to the plane of the stress. Since the intrinsic permittivity is considerably smaller along the $c$ axis, than in the a direction, this domain alignment should result in a reduction of the contribution to the total dielectric constant from the intrinsic permittivity of the domains. There are other contributions to the total dielectric constant (see [18]), but this is sufficient to illustrate one effect of stress and to suggest that other parameters of the materials may also be affected. Figure 1.16 shows the

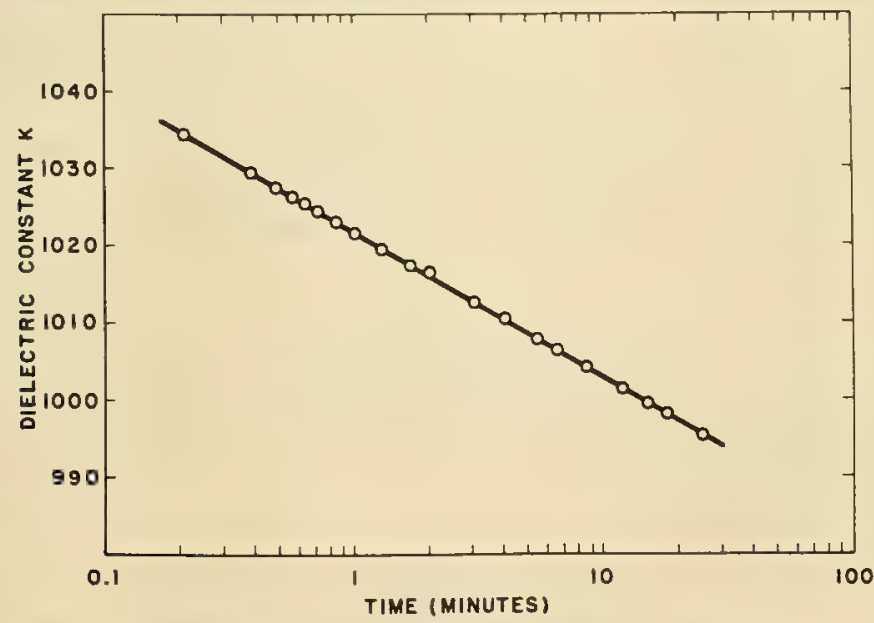

Fig. 1.17. Aging of dielectric constant following application of 9000 psi to a barium titanate sphere. 
effect of pressure on the dielectric constant of 3 -in. diameter, $1 / 4$-in. wall thickness spheres of barium titanate and PZT-4. Each experimental point was taken $10 \mathrm{~min}$ after the application of stress. In addition to the pressure effect, there is a time effect shown in Fig. 1.17. The capacity decays linearly with the logarithm of time after the application of the stress. This is an aging phenomenon similar to the aging obtained following the poling of ferroelectric ceramics. Figure 1.18 shows the effect of pressure on the dielectric constant, with $d$ constant and the coupling coefficient of NRE-4 $\left(\mathrm{BaCaCoTiO}_{3}\right)$; the material was in the form of a 3-in.-diameter, $1 / 4$-in.-wall thickness sphere.

It should be emphasized that all the parameters (e.g., dielectric constant, coupling), need to be examined when considering the effect of pressure on trans ducers. It should also be noted that these results are only applicable to trans ducers subjected to two-dimensional stresses, the components of which are approximately equal.

\subsection{CONCLUSION}

It has not been possible to cover even superficially the whole of the field of electroacoustic transducers and no attempt has been made to do so. High-power projectors, highly efficient over a relatively narrow bandwidth, can be designed for the frequency region of a few kcps to over $100 \mathrm{kcps}$. These longitudinally resonant projectors are not satisfactory at low frequencies, say less than $1 \mathrm{kcps}$, because of their physical size and consequent difficulties in handling. Flexural resonances and designs incorporating relatively soft mechanical springs can be used and are effective in reducing the physical size. However, low-frequency projectors, particularly for deep operation, remain a problem.

Hydrophones for particular applications at shallow depth are fairly readily designed.

Both projectors and hydrophones for operation at great depth present interesting problems. At N.R.E. a study has been undertaken of the effect of stresses, unidirectional, bidirectional, and three-dimensional, on the parameters of ceramic

Fig. 1.18. Variation of dielectric constant, piezoelectric constant, and planar coupling coefficient with hydrostatic pressure for NRE4 in the form of a 3 -in.-diameter sphere.

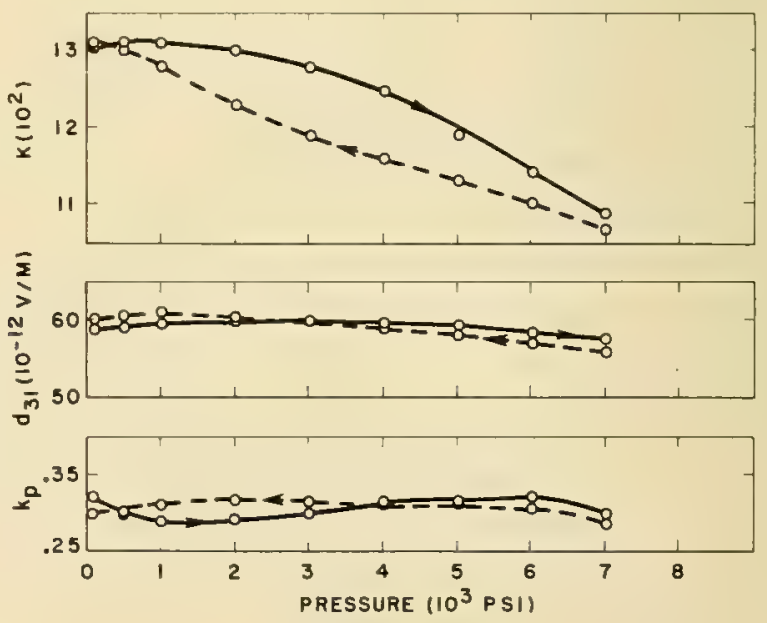


ferroelectric transducer materials. Not only will information pertinent to the design of transducers be produced, but also data which must be explained by any comprehensive theory of ferroelectricity.

To date great use has been made of pressure release materials, e.g., sponge rubber, or air, in transducers. These conventional pressure-release materials are not suitable for use at great depths: sponge rubber collapses and the use of air requires cases which are mechanically very strong, and hence heavy and bulky. It may, of course, be possible to design elements which will only radiate unidirectionally. Techniques using spatially separated units and time delays can be used to obtain unidirectional hydrophone arrays.

Knowledge of cavitation applicable to transducers is limited and further work is desirable.

Although in the past decade with the introduction of ferroelectric ceramics, there has been much improvement in transducer materials, further improvements in coupling factor and loss are desirable.

\subsection{ACKNOWLEDGMENT}

This work is published by permission of the Defence Research Board.

\section{DISCUSSION}

DR. W. N. ENGLISH asked the lecturer how closely the performance of a lowfrequency transducer followed the design performance figures and, secondly, whether the production of a transducer operating at 1 to $2 \mathrm{kcps}$ and giving, say, $110 \mathrm{db}$ above $1 \mathrm{~d} / \mathrm{cm}^{2}$ at 1 yard was now feasible without having a weight of several tons.

DR. SCHOFIELD: The physical principles of low-frequency transducers are the same as for transducers at high frequencies. Therefore, provided that all the important parameters are taken into account, the design performance of lowfrequency transducers can be predicted to the same order of accuracy as transducers at higher frequencies.

Low-frequency resonant projectors are large and heavy for two reasons:

1. Low-frequency mechanically resonant systems are inherently larger than for frequencies in the range of tens of kiljcycles

2. To obtain a good transfer of energy from a vibrating piston to the water, the dimensions of the radiating face must be at least the order of one wavelength

For a highly efficient projector at $1 \mathrm{kcps}$, the dimensions of the radiating face must be about $5 \mathrm{ft}$. To keep the weight of the projector low, care must be taken in designing the mechanically resonant system, and some compromise in characteristics such as frequency bandwidth and depth of operation may be necessary. I think that a $1 \mathrm{kcps}$ projector with a weight of less than one ton is practical.

DR. S. WENNERBERG suggested that the reduction of the dynamic tensile strength in a composite transducer could originate in the excess stresses caused by differences between the Poisson coefficient of the different materials, and inquired whether any investigation had been made of this possibility. 
DR. SCHOFIELD: No investigations into this possibility have been made.

PROFESSOR R.E.H. RASMUSSEN referred to a formula quoted by Dr. Schofield giving the maximum intensity of radiation $(l$, in $w$ per square centimeter) from a barium titanate transducer in terms of the depth $(H)$ of immersion in feet, when cavitation is the limiting restriction as $I=0.3(H / 32+1.8)^{2}$.

DR. SCHOFIELD: This formula should only be used as a "rule-of-thumb" indication of the cavitation level. The presence of dissolved gases and impurity nuclei could cause large deviations from the levels predicted by this formula.

MR. J.S. M. RUSBY commented that the pattern of cavitation observed on the radiating face of the $10-\mathrm{kcps}$ longitudinal projector mentioned in the lecture could result from the regional lines of high displacement arising from flexural vibrations, and so lowering the cavitation threshold as was observed. He also enlarged upon the suggestion concerning planar arrays of projectors in which it was stated that a method of overcoming the pressure-release problem in deep water was to make the rear ends of the individual elements inefficient as radiators compared with their front faces. Mr. Rusby said that this could be achieved by reducing the cross-sectional areas of the rear ends and separating them; in this way, the value of the total radiation impedance as seen by the rear of the array would be lowered.

DR. SCHOFIELD: It is agreed that the cavitation erosion marks could be caused by "flapping" of the radiation heads. NRE have been considering methods of mismatching the tails of elements to water as a means of reducing back radiation.

DR. H. A.J. RYNJA following upon a point raised in the discussion of lowfrequency transducers on the use of magnetostrictive devices, spoke of his own experience with a scroll of $1 \mathrm{~m}$ diameter of a nickel-four-percent cobalt material forming a resonator of $1.5 \mathrm{kcps}$ with a tuned bandwidth of one octave. With two such rings mounted coaxially and driven with $90^{\circ}$ phase difference and with pressure-release material on the outside surface of the rings, he had succeeded in obtaining quite sufficient power in unidirectional radiation. Dr. Rynja agreed, however, with Dr. Schofield on the difficulties of manufacturing the scrolls.

\section{REFERENCES}

1. F.A. Fischer, Fundamentals of Electroacoustics (Interscience Publishers Inc., New York, 1955).

2. W. P. Mason, Electromechanical Transducers and Wave Filters (D. Van Nostrand Co. Ltd., 1942, 1948).

3. D.W.Dye, "The Piezoelectric Quartz Resonator and Its Equivalent Electrical Circuit," Prac. Phys. Soc., Vol. 38, 339-457 (1926).

4. W.P. Mason, "An Electromechanical Representation of a Piezoelectric Crystal Used as a Transducer," Proc. Inst. Radio Eng., Vol. 23, 1252-1263 (1935).

5. W.P. Mason, Piezoelectric Crystals and Their Applications to Ultrasonics (D. Van Nostrand Co. Ltd., 1950).

6. P. Vigoureux and C. F. Booth, Quartz Vibrators (H. M. Stationery Office, 1950).

7. H. Hecht, Die Elektroakustischen Wandler (Johann Ambrosius Barth, Leipzig, 1954).

8. F. V. Hunt, Electroacoustics (Harvard Univ. Press, 1954). 
9. R. Beckmann, "Some Applications of the Linear Equations of State," Trans. Inst, Radio Eng. PGUE, Vol. 3, 43-62 (1955).

10. J.F. Hersh, "Coupling Coefficients," Acoustics Research Lab. Harvard, Tech. Memorandum No. 40.

11. P. M. Kendig, J. Acoust, Soc. of America, Vol, 33, 674-676 (1961)

12. D. Schofield and R. F. Brown, Can. J. of Phys., Vol. 35, 594-607 (1957).

13. C. A. Clark, Brt. J. of Applied Physics, Vol. 7, 356-360 (1956).

14. Kinsler and Frey, Fundamentals of Acoustics, Chapter 3 (1950).

15. H. Nodtvedt, Electromechanical Analogues Applied to Magnetostrictive Systems, Forsvarets Forkningsinstitutt Arbok (1947). In English.

16. A. Mohammed (unpublished work).

17. R. F. Brown (unpublished work).

18. R. F. Brown, Can. J. of Phys., Vol. 39, 741-753 (1961). (Contains list of references on the effect of two-dimensional stress on the dielectric constant of ferroelectrics.) 



\title{
LECTURE 2
}

\section{SONAR ARRAYS, SYSTEMS, AND DISPLAYS}

\author{
D. G. Tucker \\ Department of Electrical Engineering \\ University of Birmingham \\ Birmingham, England
}

\subsection{INTRODUCTION}

The echo-location group of the University of Birmingham has been concerned primarily with active sonar systems. Apart from general considerations of long ranges and oceanic propagation (with which we are not able to deal) it has seemed to us that the main directions in which sonar research must progress are:

1. The gathering of more information about what lies within the area of search, and

2. The processing and presentation of this information sothat classification of targets is quick and reliable.

The more progress that is made with 1 , the more difficult quick classification becomes, since with a human operator the sorting out of the increased information becomes too large a task for immediate visual decision. Yet without the increased information, the process could not possibly become more reliable. It is not possible to separate completely these two lines of work, 1 and 2, and a coordinated approach to them is the one which is most likely to succeed.

To gather more information about what lies within the area of search, it is necessary to achieve one or more of the following: (a) higher directionality and (b) higher range resolution to give geometrical information-shape and size of targets; (c) knowledge of frequency response of targets; and (d) more rapid scanning.

The direct approach to (a) is to use larger transducer arrays, but, like many other people, we have been looking closely into the possibilities of obtaining higher directionality from a given size of array by such methods as superdirectivity, multiplicative processing, multifrequency operation, and double-FM operation. The last two methods, together with (b) and (c) above, demand the use of wider frequency bands in the water, and so we have had to think about both transducers and arrays suitable for operation over a wide frequency band. The need for more rapid scanning is not only obvious in itself, but follows more emphatically from (a); and we have therefore done a great deal of work on the development of within-pulse electronic scanning systems. 
The approach to quick and reliable classification is not so obvious. The combination of (a), (b), and (d) above evidently gives rise to the possibility of an accurately delineated visual display of target shape; but in typical operational situations the ratio of the number of unwanted (or irrelevant) targets to the number of wanted targets is so large that the human task becomes too great, and reliability suffers. In the corresponding radar case, this ratio is, in contrast, usually very small, and automatic detection, holding, and tracking of targets is already feasible. Progress in this direction, however, for any but the most simple of sonar situations is likely to be difficult and slow. One step toward more reliable detection and classification is the improvement of the amplitude ratio of genuine signals to random signals by display integration methods, and we have given much attention to this. The effect of shape and size of the target display on the ability of an operator to detect and recognize it is another very relevant matter which has been under investigation.

Classification by knowledge of frequency response of targets ( $c$ above) is very problematical at present, but we are trying to develop a wide-band system, having a 10 to 1 frequency band and constant directivity over this width, which can display this information and which will enable us to assess the potentialities of the method.

Propagation of underwater acoustic signals is obviously a fundamental matter requiring continuous research in order that system improvements may be coordinated and exploited. Long-range propagation studies are beyond our facilities, but we have some interesting results on short-range echo formation which point to other limitations on the use of improved systems.

\subsection{ARRAYS AND DIRECTIONALITY}

Throughout this section, directionality will be considered in terms of a line or strip array, so that only two-dimensional directional patterns are involved.

\subsubsection{Multiplicative Arrays}

In the search for higher directionality for a given size of array, the replacement of addition by multiplication in the combining of signals from different sections of the receiving array has some very definite attractions $[1,2]$. For instance, if a strip array is divided into two equal sections, and the output signals from these (assumed of narrow bandwidth) are multiplied together, the output of the multiplier comprises two parts (as far as the wanted signals are concerned), one a dc signal and the other a signal at twice the original frequency. The amplitudes of both these parts are functions of the direction from which the signal was received, although the double-frequency part is less sensitive to direction than the dc part. The directional response of the dc signal, plotted as the variation of amplitude as the array is rotated in the plane of its length, has a main lobe, or beam, which is only half the width of the main beam of the same array when used in the normal manner, i.e., with the signals from its sections merely added together. It is therefore attractive to regard multiplication (with a low-pass filter to remove the high-frequency output) as a way of doubling the directivity of the array. There are other advantages, too. In the ordinary (additive) system, the output of the array is at signal frequency, and it is necessary 


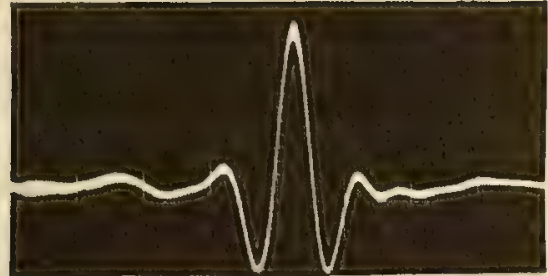

(a)

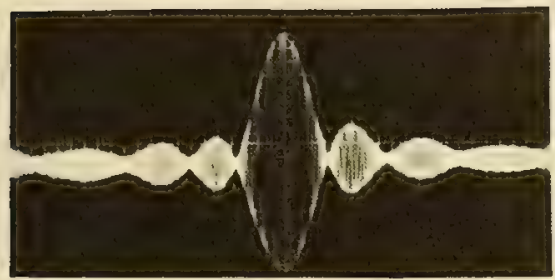

Fig. 2.1. Multiplicative and additive directional patterns for the same array of eight elements: (a) multiplicative (b) additive.

(b)

to rectify this for display. Thus all the lobes in the directional pattern become of the same polarity (say positive). But in the multiplicative array, the output is $\mathrm{dc}$ and the largest secondary lobes of the directional pattern are of negative polarity as is shown in Fig. 2.1. Thus, response from signals arriving from directions corresponding to these lobes can be eliminated by the use of a rectifier following the multiplier and its low-pass filter; or with an intensity-modulated display on a cathode-ray tube, signals from these directions will not brighten the screen.

There are, of course, complications and disadvantages. One complication is that the doubled directionality strictly applies only to the directional variation of output from a single source as the array is rotated; it is not the same thing as directional discrimination. Whereas in the linear additive system directional response and directional discrimination are essentially the same thing, in the multiplicative (and nonlinear) system they are not. Welsby has shown, however, at least for some important practical situations, that resolution of two targets at the same range is approximately doubled [3]. The limited experimental experience we have so far had of within-pulse scanning sonar using the multiplicative system (see below, Section 2.3) adequately confirms this. The reduced secondary response of the multiplicative system has also been shown to be genuinely realized in a multitarget situation. One of the disadvantages referred to is the fact that signal-noise discrimination is $3 \mathrm{db}$ worse than in the additive system.

Welsby has recently discovered that some use can be made of the doublefrequency output of the multiplier when the signals are of coherent type [4]. If this output is rectified and added to the dc discussed above, then the signal-noise discrimination at the peak of directional response can be partially restored-to the extent of $1.6 \mathrm{db}$; and if subtracted instead of added, it can lead to a further narrowing of the main beam.

The use of multiplicative reception has been known for a long time in con- 
nection with wide-band signals of continuous spectrum (i.e., noise emissions); in this case its use is natural since it provides the process of cross-correlation which is used so extensively in the mathematical consideration of such signals. But the use of the process in narrow-band (and coherent) echo ranging seems to be a new development and we are not aware that anyone else has been inves tigating its practical problems.

\subsubsection{Superdirective Arrays}

There is copious literature on superdirective arrays [5], to which we have contributed [6], but practical results are not conspicuous. There is no doubt that the academic interest of superdirectivity rather exceeds its practical utility. Naturally, the idea of obtaining a greatly narrowed beamwidth is very attractive, but there are numerous disadvantages and difficulties in this method of attaining the desired result:

a. The signals must be restricted to a very narrow band.

b. The sensitivities (or excitations, since the method is applicable also to radiators) of the various elements of the array must be set to extremely close limits.

c. On reception, the signal-noise performance (in relation to receiver noise) is very poor; on transmission, the efficiency is very low.

d. The calculation of a practical array is made very difficult-at present, perhaps impracticable in most cases-by interelement coupling, which is inevitably a major effect in an array where adjacent elements must be less than half a wavelength apart and must usually operate in antiphase.

It thus seems unlikely that there will be much serious development of superdirective arrays of the normally considered single-frequency multielement type. But it will be shown in the next section that multifrequency two-element arrays may encourage trials of superdirectivity for limited applications, since they almost eliminate the difficulty of interelement coupling.

It seems fairly certain that multiplicative arrays are superior in most respects to superdirective arrays, since they are quite easily realized and give a much better signal-noise performance. Figure 2.2 shows how a particular directional improvement can be obtained in both ways, but while the multiplicative method costs only $3 \mathrm{db}$ in worsening of the noise factor, the superdirective method costs $20 \mathrm{db}$.

\subsubsection{Multifrequency Two-Element Arrays}

The idea of "space-frequency equivalence," where the effect of a multielement array is obtained from two elements used simultaneously at a number of frequencies, appears to be very recent $[7,8]$. The basic reasoning is simple; the spacing of the two elements is a different number of wavelengths at each frequency, so that the use of a harmonic series of frequencies can give the effect of a uniformly spaced multielement array. Althoughthis kind of array is in some respects analogous to an ordinary multielement array, it has been shown that it is certainly not properly analogous [9]. Even to make equivalent transmitting and receiving arrays separately stretches the analogy somewhat, and it fails completely when one tries to associate a multifrequency two-element transmitter 


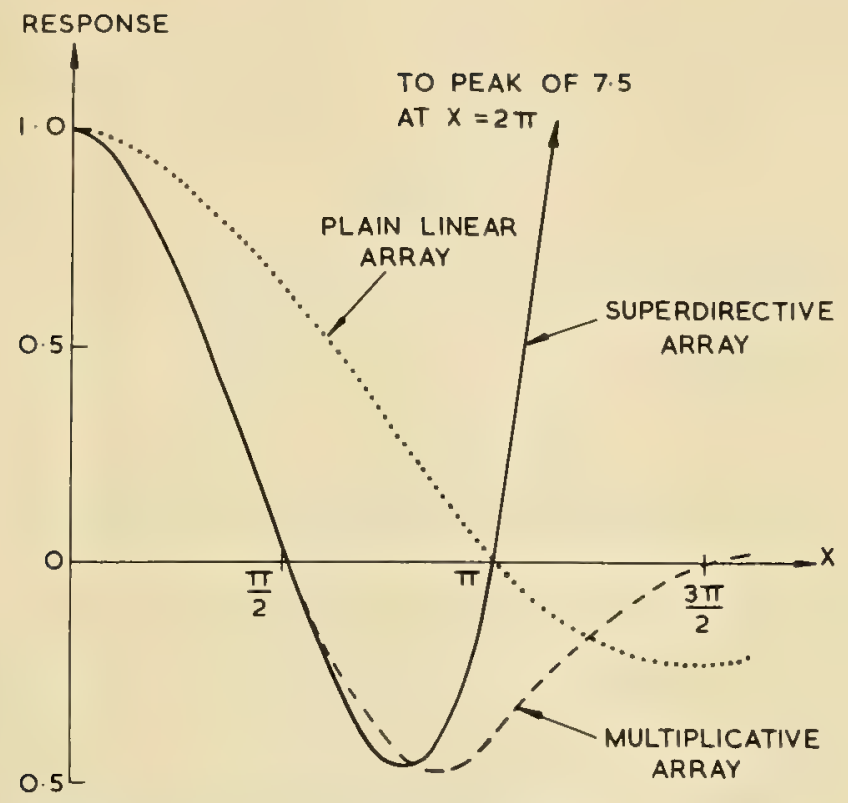

Fig. 2.2. Comparison of (a) normal additive, (b) multiplicative, and (c) superdirective directional responses for the same array.

with a similar receiver; they just cannot work together. There is not space to deal with this question here (a full account is being published elsewhere [9]), and we must accept multifrequency two-element receivers as a special kind of array to be studied separately. Nevertheless, with care, some of the methods of ordinary arrays, e.g., the synthesis of superdirective directional responses, can be applied.

The practical form of this kind of receiving array is based on the multiplicative system, in which the outputs of the two multifrequency receiving elements are multiplied together, as shown in Fig. 2.3. The multifrequency signal sent from the transmitter comprises a group of $r$ harmonics. On reception at the two elements, these harmonics contain directional information in the form of the phase angles $\pm m \phi$, where $\phi=(\pi d / \lambda) \sin \theta, d$ is the spacing between the elements, and $\lambda$ is the wavelength at the fundamental angular frequency $p$. On multiplication (and ignoring the "special filter" for the moment), the dc output becomes a function of signal direction, thus

$$
1 / 2 \sum_{m=1}^{5} E_{m}^{2} \cos 2 m \phi
$$

If only odd values of $m$ are used, and all $E_{m}$ (for $m$ odd) are equal, this becomes

$$
\frac{1 / 4 E^{2} \sin (r+1) \phi}{\sin \phi}
$$

which is the well-known form of directional response for a linear array of uniform sensitivity, with $r+1$ elements (which is an even number), except for a factor of 2 in the angular scale. 


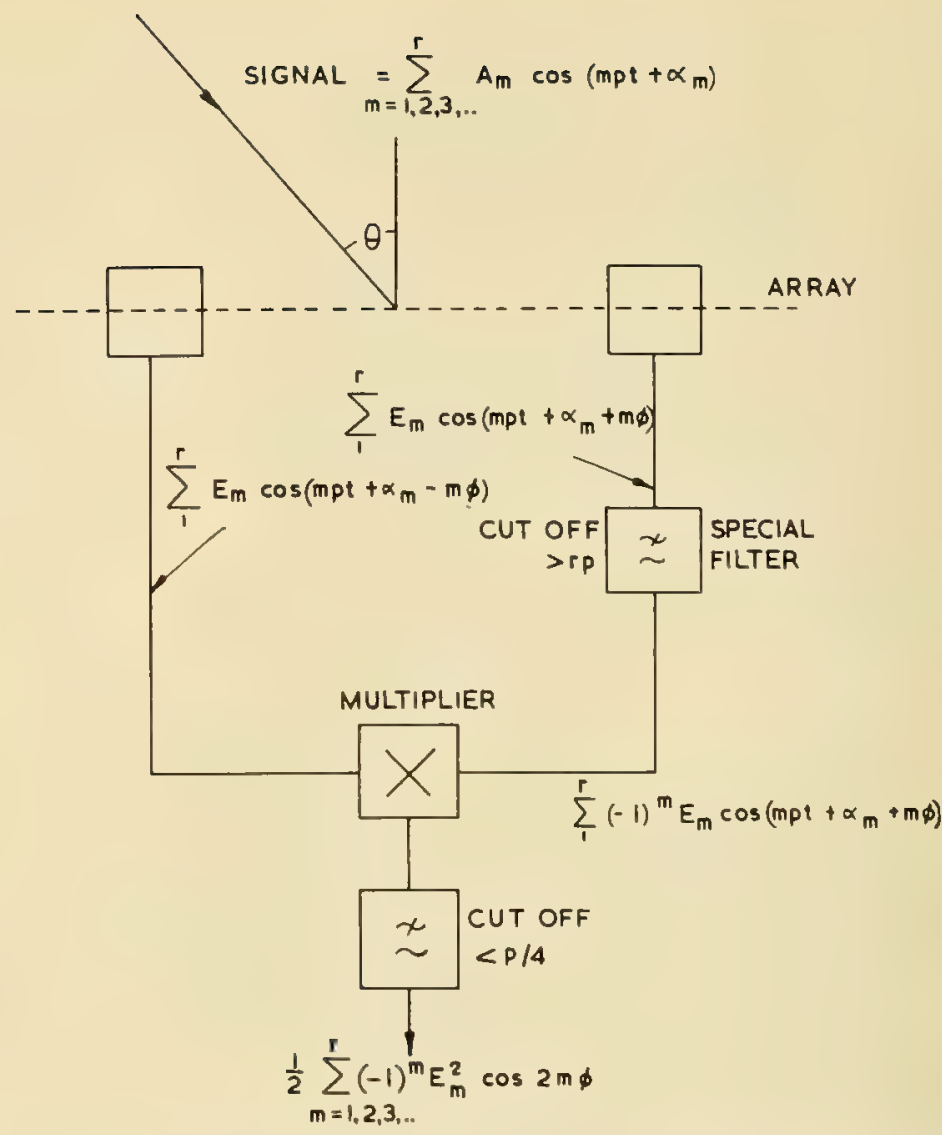

Fig. 2.3. Multifrequency multiplicative pair (with provision for superdirectivity).

Now the amplitude $E_{m}^{2}$ determines the "taper" of the array, and it is clear that, if desired, this taper (although effective at the receiver) may be imposed at the transmitter by giving different amplitudes to the transmitted signal components. But it should also be noted that if the transmission properties of the medium (or the target strengths in an echo-ranging system) are different at different frequencies, then an unwanted taper function will be imposed.

When all harmonics are used (i.e., $m=1,2,3, \ldots$ ), then, in the space-frequency equivalence, this corresponds to a multielement array with an odd number of elements but with the central element omitted. The effect of the central element can be obtained by adding to the output of the multiplier a dc voltage derived from the square-law rectification of the output of one of the array elements.

Superdirectivity can be obtained with this system; and if approached from the point of view that a particular number of frequencies (or pairs of elements in the corresponding "spatial," or multielement, array) is specified and that a superdirective response is to be obtained from it, then superdirectivity can be obtained easily. On this basis the problem reduces to (a) choosing $p$ and $d$ so that the number of wavelengths in $d$ progresses by less than one wavelength for 


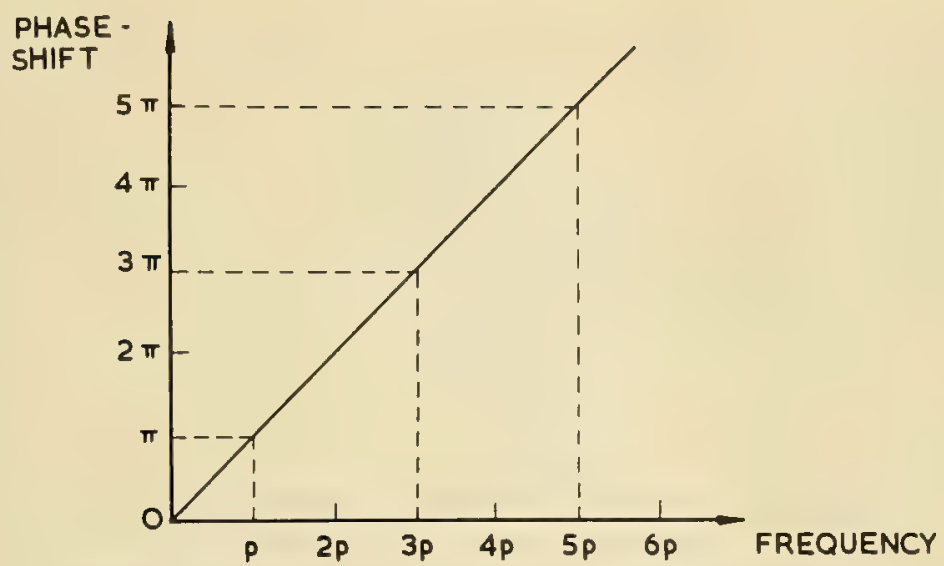

Fig. 2.4. Phase-frequency requirement of special filter in Fig. 2.3.

each step in $m$, i.e., the equivalent spatial elements are less than half a wavelength apart and (b) reversing the polarity of alternate frequency components. The latter can be done by inserting the special filter in one channel, as shown in Fig. 2.3, having a phase shift-frequency characteristic as shown in Fig. 2.4. The values of $E_{m}$ are then chosen to give the required directional response. For more complicated cases of superdirectivity, it is always possible to separate the various frequency components before multiplication, multiply them separately, and then adjust amplitudes and polarity as required in the separate outputs before they are finally added together.

It is clear that superdirectivity obtained with a multifrequency array removes much of the difficulty of interelement coupling that was mentioned in Section 2.2.2, since it is probably only at the lowest frequency that the elements are less than half a wavelength apart.

The system so far described involves frequencies spread over a rather wide band; it may be useful at low frequencies where a full array would be too large for handling, but where a two-element array could have its transducers mounted, for example, on two different ships or on two different towed bodies. For narrowband use at higher frequencies, the series of harmonics may be replaced by a series of frequencies

$$
(f+S \delta f) \quad-r \leq S \leq r
$$

where $\delta$ is small compared with unity. The frequency $t$ should be transmitted at a relatively high level or be suitably enhanced or reinserted at the receiver [10]. The complex signals from the two transducers are separately envelopedetected before being multiplied together-see the example in Section 2.3.2.

\subsubsection{Wide-Band Arrays}

Normally, echo-ranging systems have fractional bandwidths, although it is well known that detection is improved as the bandwidth is widened-and properly utilized. We are developing a really wide-band system-9 to 1 ratio of upper to lower frequency-so that frequency response of the target as well as its range and bearing may be displayed. One of the more important problems to be solved 
is that of obtaining a directional receiving transducer with its beamwidth constant over this wide frequency range. It will probably be satisfactory for the transmitter to be omnidirectional, and for the system to rely only on the receiver for directionality; however, the method to be described can apply to a transmitter if necessary. Since target bearing is to be determined, directionality in the system is clearly essential; but it must obviously also be constant at all the frequencies concerned, since it is hardly possible to correct for the angular position of the target in the beam.

There are several ways of achieving a constant beamwidth. The Birmingham method [11,12] is to have an array divided into sections, which are connected, via decoupling circuits, into a number of phase-shifting networks (or delay lines) as shown in Fig. 2.5. These have a phase shift which increases with frequency, so that at each end the effective directional pattern is deflected by an amount which increases with frequency. Successive delay lines have a successively larger range of phase shift. Then, after correcting the phase of the outputs from the various lines so that they are all in phase at all frequencies, the outputs are added together. Now, at the lowest frequency of the range, the phase shifts in the lines are small (ideally zero) and the directional patterns all very nearly coincident; their addition therefore produces only the ordinary $(\sin x) / x$ pattern, corresponding to the number of wavelengths in the length of the array at that frequency. As the frequency is increased, the individual patterns begin to separate, so that their addition leads to a wider beam in terms of wavelength, but it

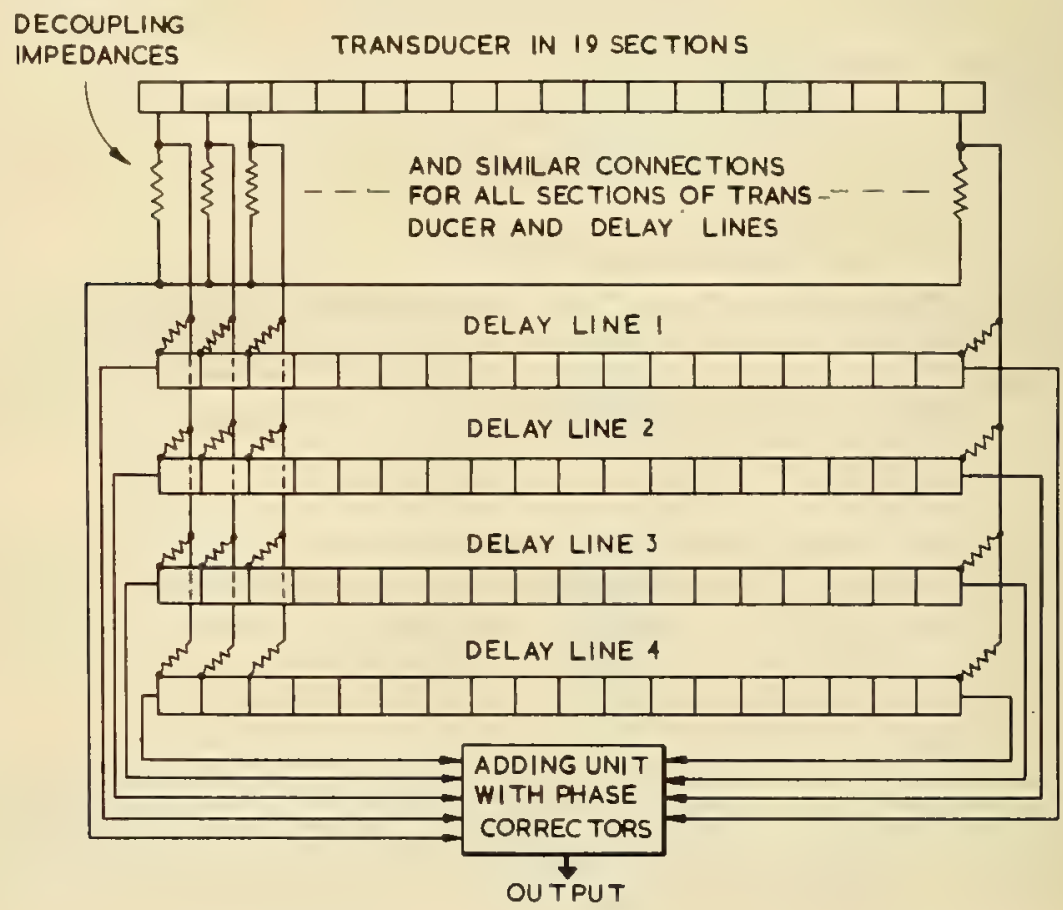

Fig. 2.5. Wide-band constant-beamwidth array: block schematic for frequency band of 9 to 1 . 
is arranged that the beamwidth in terms of physical angles remains very nearly constant; about $\pm 10 \%$ variation over a 9 to 1 frequency ratio was achieved. Figure 2.6 shows the state of affairs at the maximum frequency. Above this frequency, dips occur and the pattern begins to break up.

An experimental array covering the frequency range 9 to $81 \mathrm{kcps}$ has been built and tested in water. The theoretical results have been matched very closely.

It is, of course, difficult to operate the transducers at good sensitivity over this wide frequency range, but we are trying to develop a satisfactory capacitance transducer.

\subsection{SONAR SYSTEMS}

\subsubsection{Within.Pulse Scanning Systems Using Multielement Arrays}

The idea of electronic sector-scanning sonar systems in which the receiving beam is repeatedly swung across the search sector, insonified by a wide-beam transmitter, at least once during each period of time equal to the duration of the transmitted pulse, is by now probably quite familiar. By this method, a virtually simultaneous examination is made in all directions within the search sector. For a sector equal to $n$ times the 3 - $\mathrm{db}$ beamwidth of the receiving array, the array has to be divided into $n$ sections. Alternative arrangements for swinging the beam have been published; the system developed at Birmingham $[13,14]$ uses phase-shift networks to link the sections of the receiving arrays, with frequency changers interposed in the leads from each array section. The local oscillation supplied to the frequency changers is swept through a range of frequencies with a repetition rate equal to the desired scanning rate; and since the phase-shift networks have a phase shift varying with frequency, the beam direction is swept over a sector in sympathy with the sweep of frequency.

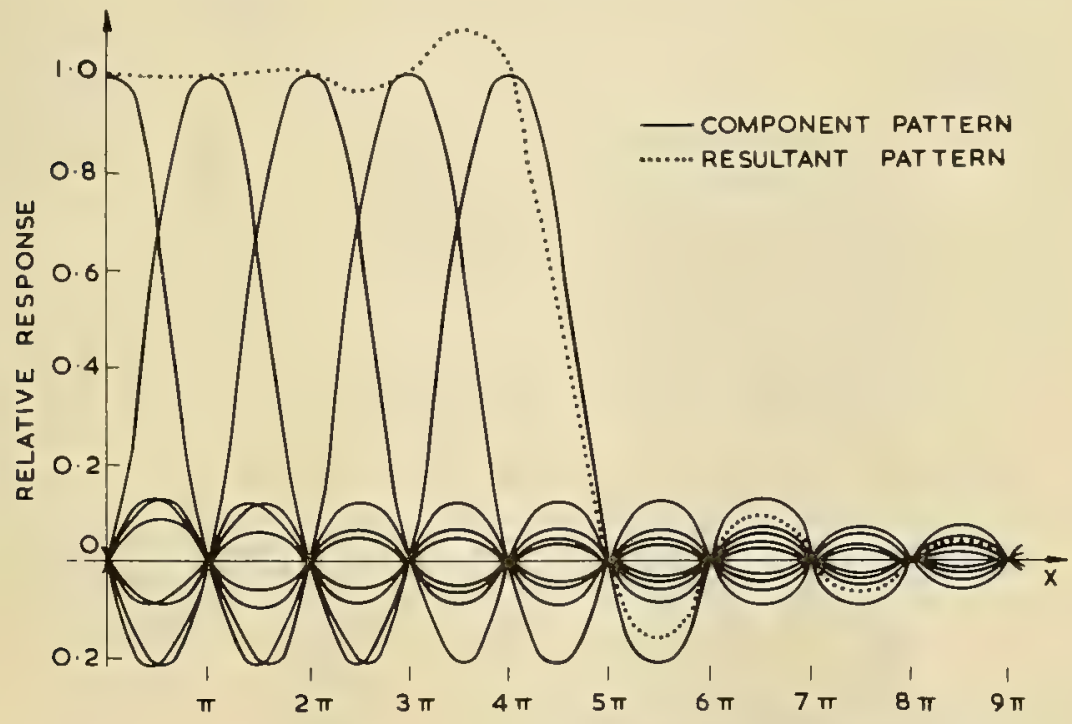

Fig. 2,6. Wide-band constant-beamwidth array: component and resultant directional patterns at upper frequency limit. 


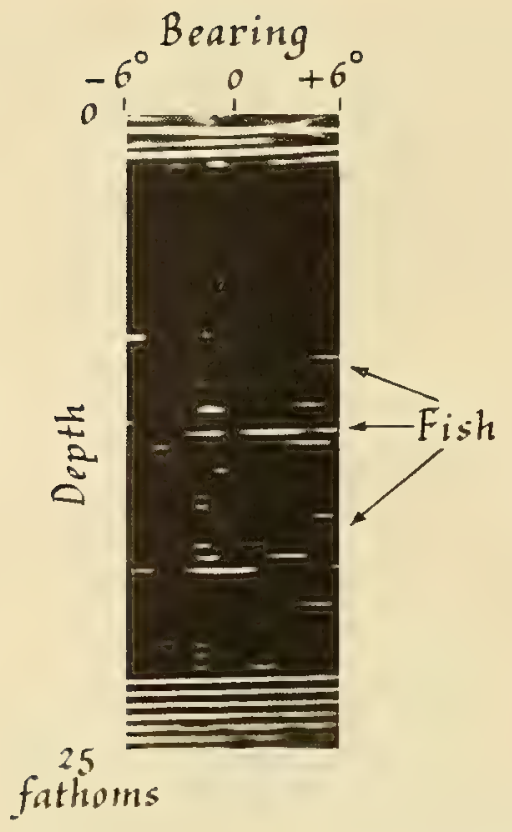

Fig, 2.7. Scanning echo-sounder display with fish passing through sector. (Southern North Sea, depth about 20 fathoms; maximum range on scale, 25 fathoms.) (Courtesy, Institute of Navigation, London.)

This method of scanning has been very successful, and the experimental equipment has been useful in examining fish shoals in connection with a fisheries research program of the Fisheries Laboratory at Lowestoft (see Fig. 2.7). The equipment has also been effectively demonstrated [15] as an aid to hydrographic and oceanographic surveying, both with horizontal beam (Fig. 2.8) and with vertical beam (Fig. 2.9); in the latter application it shows a complete bottom profile on a single pulse transmission, and this proves useful in bad weather when "quenching," by suppressing nearly all the transmissions, makes ordinary echo sounders useless. In these experiments, the beam was about $1.5^{\circ}$ wide between $3-\mathrm{db}$ points in the plane of scanning, and $12^{\circ}$ at right angles to this plane. The frequency was $37 \mathrm{kcps}$, and of 1 -msec pulse duration.

The analysis and experimental investigation of the factors which limit the performance of this type of scanning system have been taken a long way [16], partly with the help of an electromagnetic analog [17] (i.e., a radar version of it), and currently a high-resolution system is nearing completion which exploits the system fully. This has 35 sections to the array, and with an operating frequency of $400 \mathrm{kcps}$ will give a resolution of $1 / 2^{\circ}$ in bearing, $6 \mathrm{in}$. in range, over a $17^{\circ}$ sector, with a maximum range approaching 100 yards; it is intended, among other things, to allow observation of the movements of individual small fish in a reservoir.

The most striking achievement in this development at Birmingham is the successful application of the maltiplicative principle to the scanning system [3]. Figure 2.10 shows in block form how it is done. This has resulted in a genuine doubling of the angular resolution for a given length of array, as illustrated in Fig. 2.11. In the experimental trial, of which this is one of the records obtained, 


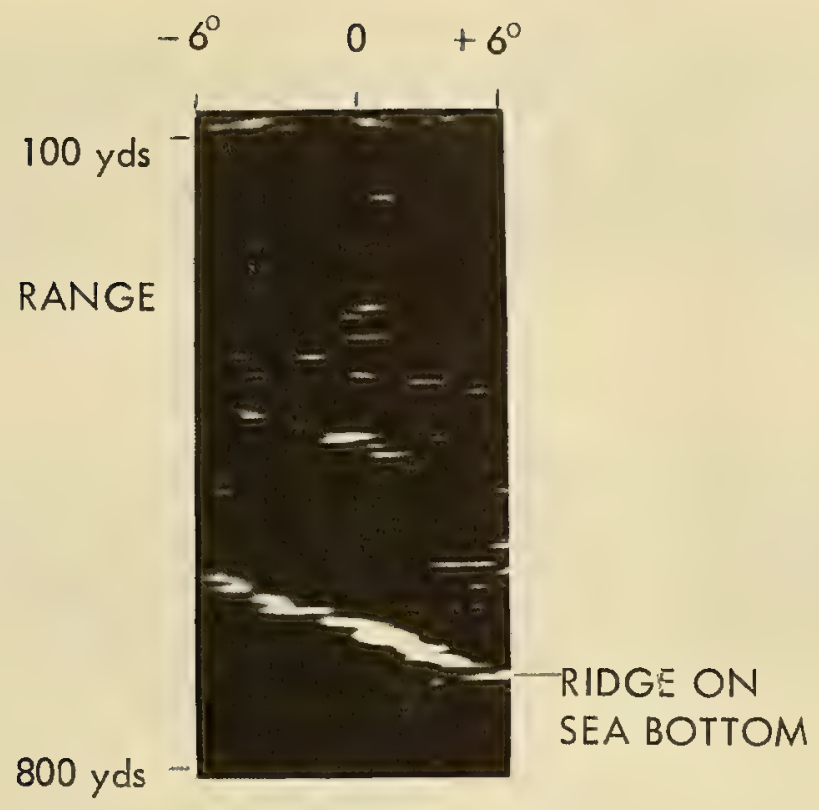

Fig. 2.8. Scanning sonar display with horizontal beam, showing ridge on sea bottom. (Shallow coastal waters, Southern England.)

Fig. 2.9. Scanning echo-sounder display, showing bottom slope of approximately $45^{\circ}$ on Continental slope, Eastern Atlantic, (Courtesy, Institure of Navigation, London,)

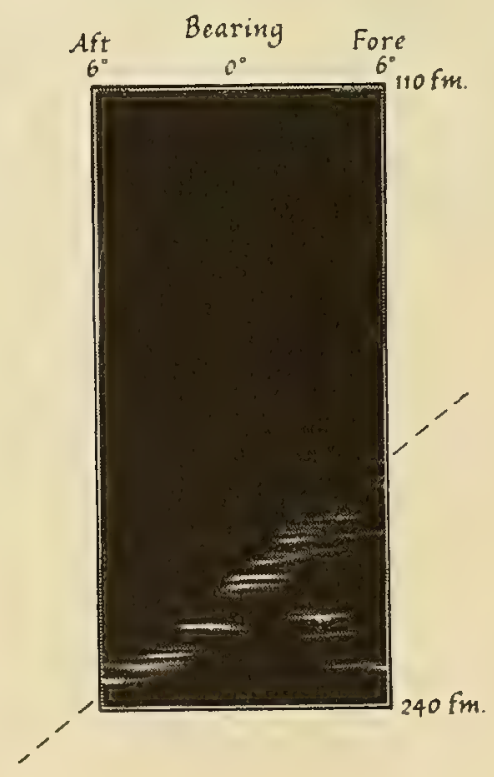




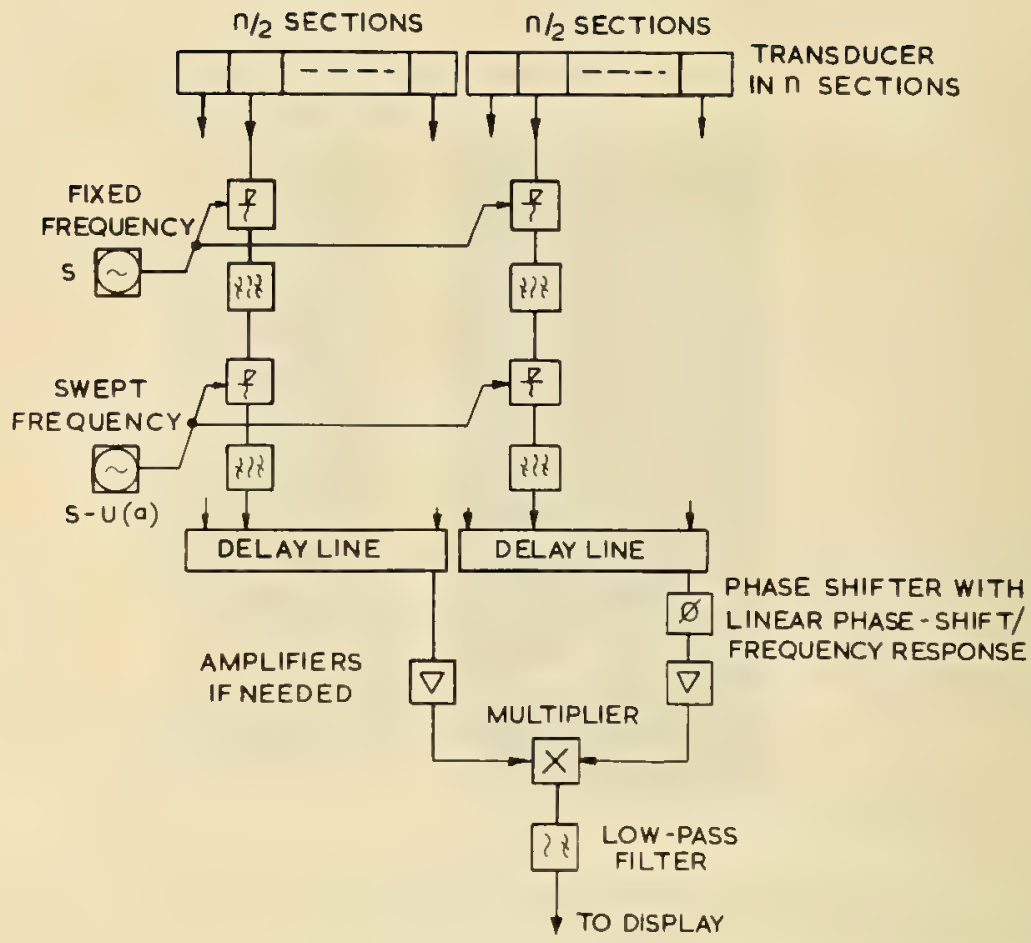

Fig. 2.10. Block schematic diagram of scanning sonar system with multiplicative operation.

two 18-in.-diameter air-filled steel spheres were suspended in the water, and one was carefully moved relative to the other. When on exactly the same range, they were moved together slowly while photographs of the B-scan display were taken. This was done for both ordinary additive and for multiplicative operation. The figure shows the sphere echoes just clearly resolved in each case, and it is quite obvious that the multiplicative system not only has half the beamwidth, but twice the angular resolution as well. The effect of the reduced secondary responses of the multiplicative system is also shown clearly in Fig. 2.11, where the receiving gain was greatly increased. With the additive system, the beam and its secondaries have spread right across the screen, due to the high intensity of the signal relative to the display threshold, but with the multiplicative system an interpretable display is still obtained.

\subsubsection{Within-Pulse Scanning Systems Using Multifrequency Arrays}

In the search for both better and cheaper scanning sonar systems, it is clear that the multifrequency two-element array (discussed in Section 2.2:3) has a potential attraction since it permits beam scanning to be done with a single variable time delay in one channel, in place of the complex series of phase shifters needed for the multielement array. Various kinds of variable timedelay networks are under consideration, but one which Welsby [10] has proposed for the narrow-band multifrequency system (mentioned at the end of Section 2.2.3) is of particular interest. His system is shown in block form in Fig. 2.12, 

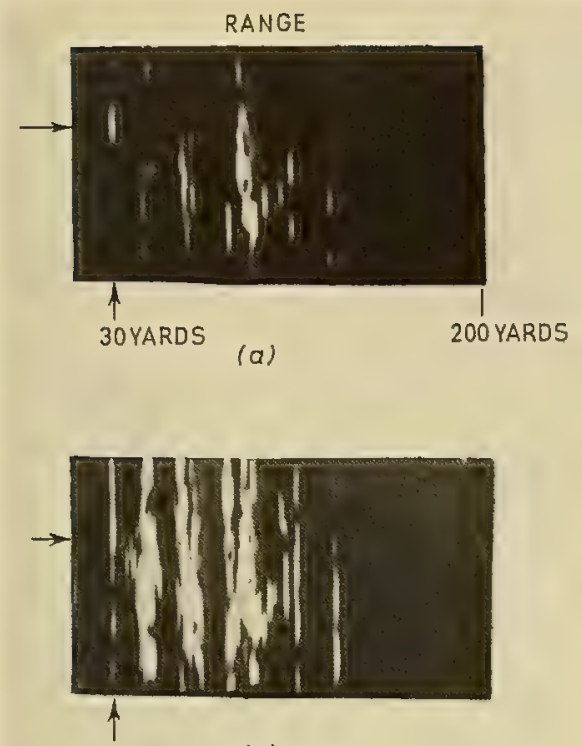

(c)

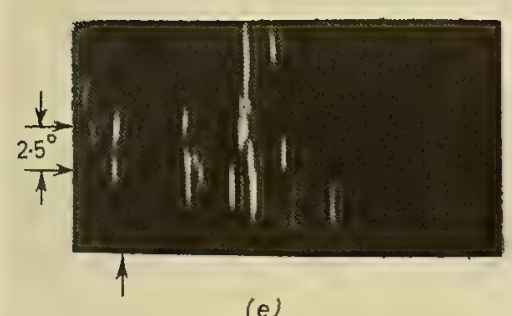

Fig. 2.11. Comparison of multiplicative and additive scanning sonars using same array (eight channels from array). (Courtesy, British Institution of Radio Engineers.) (a,b) Single 18-in.-diameter sphere at 30 yd: (a) additive system (b) multiplicative system. (c, d) Same as (a, b), but with receiver gain greatly increased to show effect of side lobes: (c) additive system (d) multiplicative system. (e,f) Two 18-in.diameter spheres at same range of $30 \mathrm{yd}$ (e) additive system (f) multiplicative system.

for the case where the frequencies transmitted consist of the center frequency $\omega_{0}$ and the two odd-harmonic difference pairs, $\omega_{0} \pm \omega_{1}$ and $\omega_{0} \pm 3 \omega_{1}$, where $\omega_{1} \ll \omega_{0}$. The output of one of the receiving transducers is translated in frequency to $\omega_{s}$, $\omega_{s} \pm \omega_{1}$, and $\omega_{s} \pm 3 \omega_{1}$ by means of a frequency changer fed with a frequency-swept local oscillation; thus $\omega_{s}$ is being swept through a range of frequency during every interval of time equal to the duration of the transmitted pulse. The narrow group of frequencies $\omega_{s}, \omega_{s} \pm \omega_{1}$, and $\omega_{s} \pm 3 \omega_{1}$ is thus swept up and down the delay frequency characteristic (Fig. 2.13) of the "quadratic phase network" and receives a different delay at different instants in the sweep. The sonar information, being carried by the envelope amplitude of the complex signal, is then recovered by normal detection before being multiplied by the signal from the other trans- 

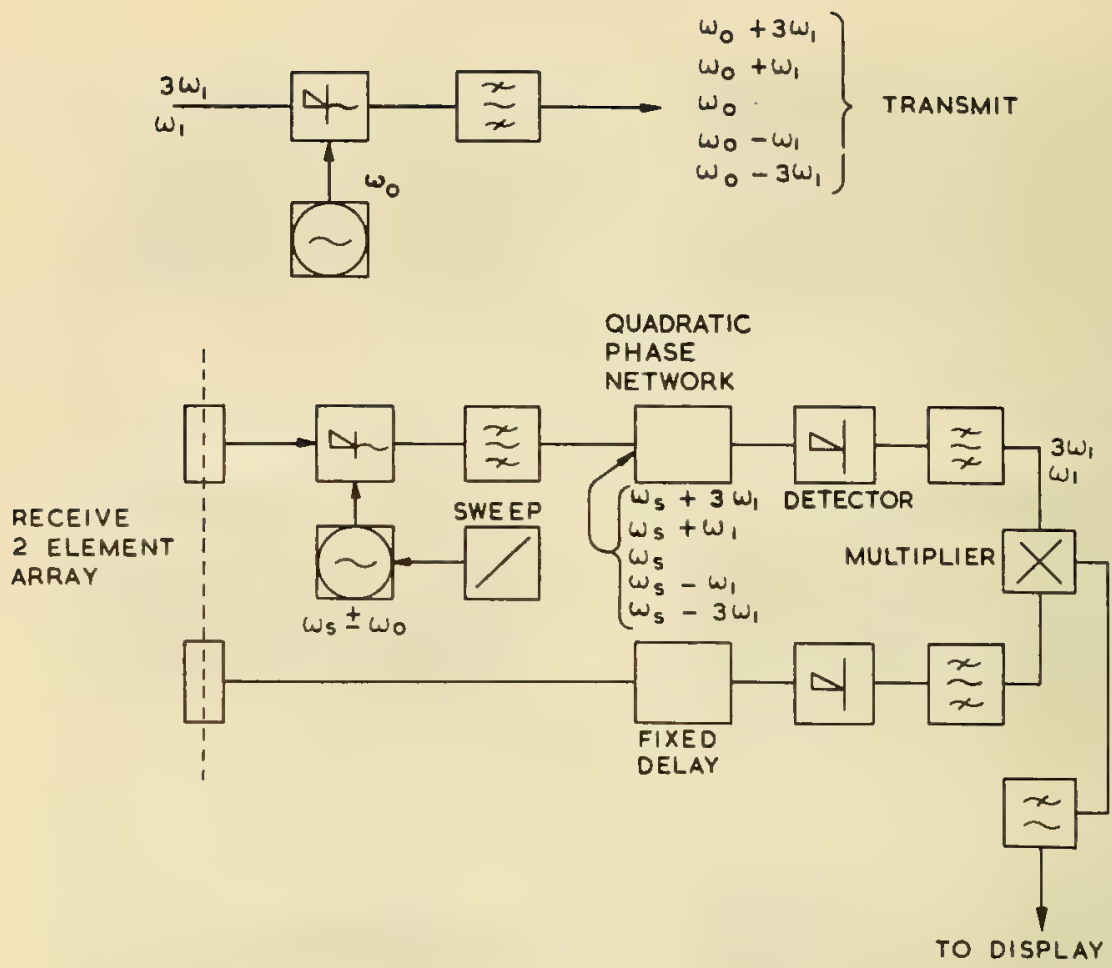

Fig. 2.12. Multifrequency sector-scanning system.

ducer channel. Since, in the channel from the other transducer, only a fixed delay is provided, it is clear that effective scanning of the beam has been obtained.

The design of the delay network is based on the phase-frequency characteristic

$$
\phi=a \omega^{2}+b \omega+c
$$

which can be obtained with reasonable accuracy by a low-pass filter section with an abnormally high value $(\simeq 3$ ) of the derivation parameter $m$.

\subsubsection{Double-Transducer FM System}

As a result of his studies of echo location by bats, Kay has proposed a sonar system which, in addition to giving a possible explanation of the bat's echolocation acuity, indicates a line of development for man-made sonars which may be very profitable $[18,19]$.

Suppose that the sector of search is insonified by a frequency-swept (or FM) continuous (or quasi-continuous) tone, and that two spaced transducers are used as receivers. If each of the latter is connected to its own conventional FM sonar receiver, then at the output of each receiver the range of any particular target is indicated by the frequency of the output echo signal. But if the target lies in a direction making an angle to the normal axis of the two transducers, the output frequencies will be different in the two receivers, since the ranges are different. 


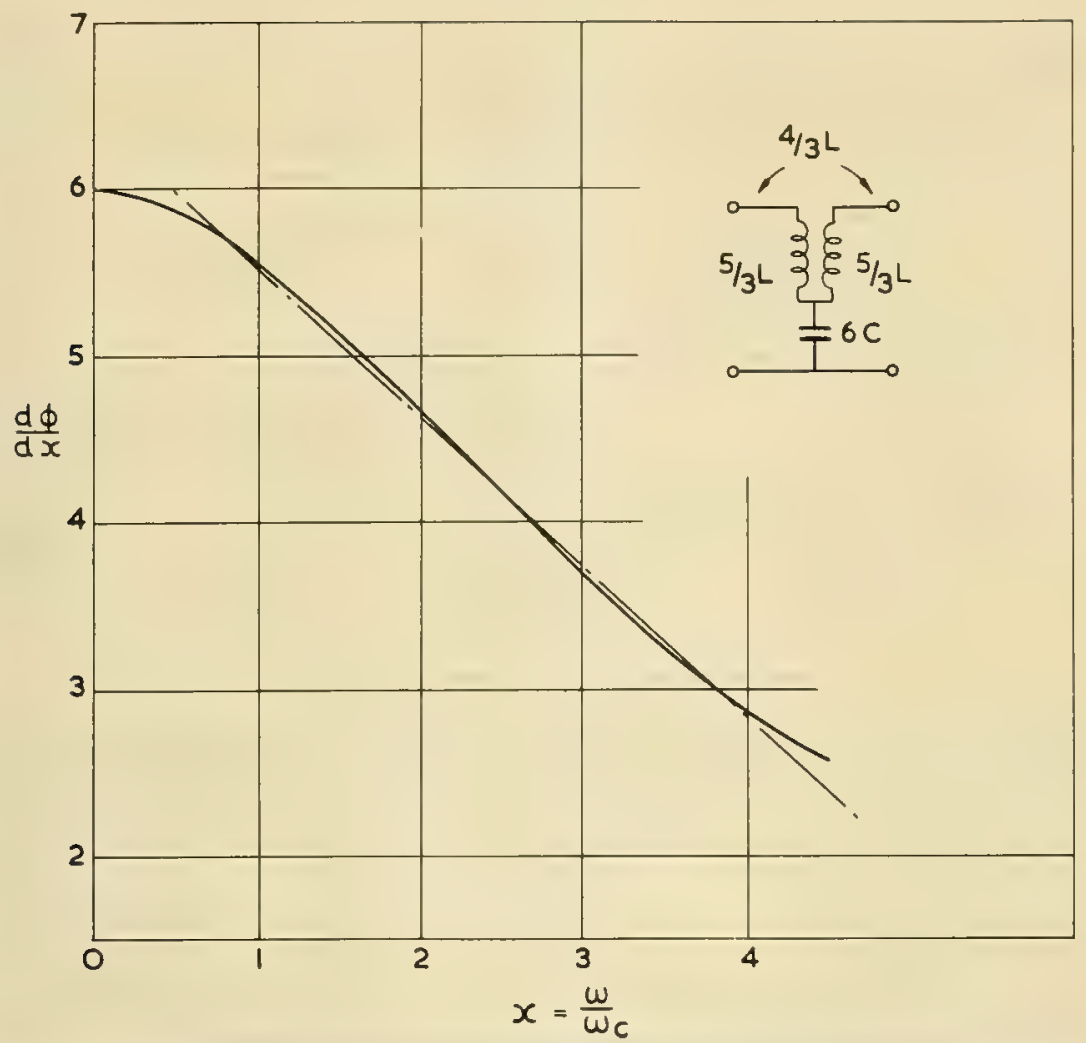

Fig. 2.13. Graph showing the shape of the delay/frequency characteristics for an $m$-derived low-pass filter section with $m=3$. The curve is a reasonable approximation to a straight line over the range $x=0.1$ to 0.4 . A filter section with $m>1$ can be realized by the use of mutual inductance as shown.

If then the difference frequency is obtained, this will be a measure of target bearing. (The sum frequency will be a measure of mean range.) Thus, two transducers will give a full sector search with no secondary-lobe effect, although in the simplest case there will be a left-right ambiguity. Even this latter can be removed by using a polyphase circuit for obtaining the difference frequency.

The block schematic of the system is shown in Fig. 2.14. Kay suggests that this is the way bats obtain accurate obstacle-and prey-location with only two small ears.

\subsection{DISPLAYS}

The theoretical investigation of displays has always been bedeviled by the difficulty of finding a criterion of performance which is both realistic and suitable for mathematical analysis. Experimental work has been hindered by the subjective nature of the measures of performance. Thus, progress has been slow, as far as any fundamental improvement in detection thresholds is concerned. One contribution from Birmingham is the improvedintegrator described below. It is also thought that the work on pattern recognition to be described 


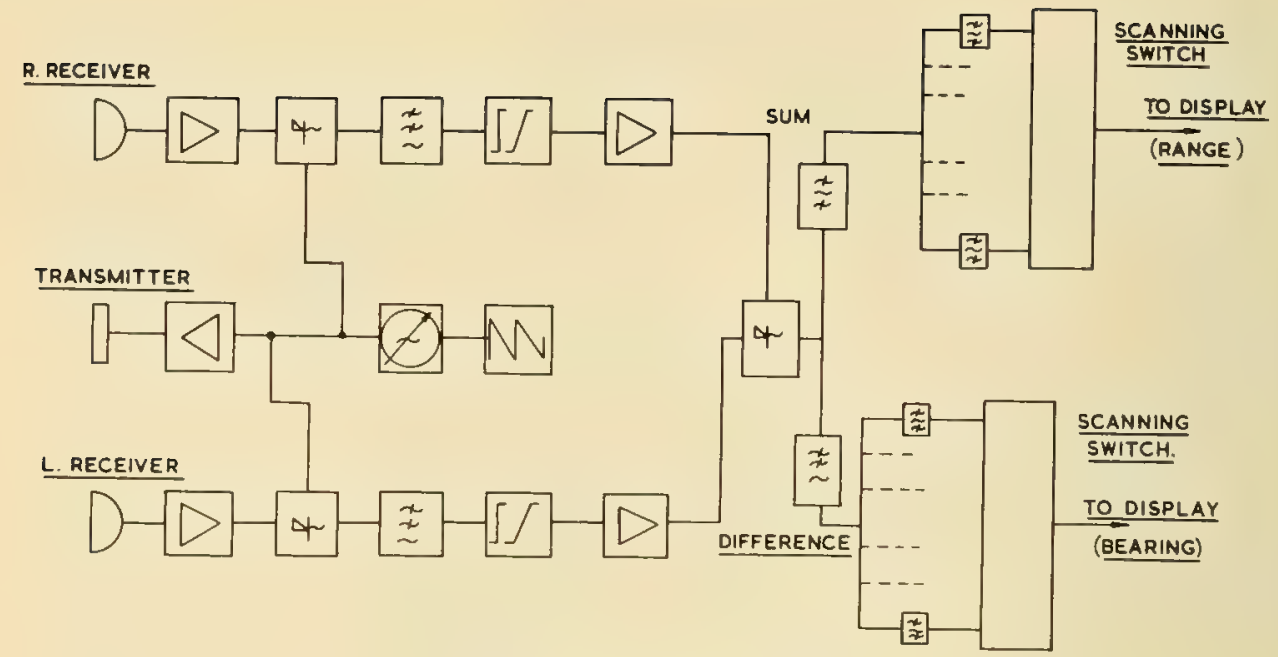

Fig. 2.14. Double FM sonar system.

may eventually improve the use of visual displays. Of course, great progress has been made in what may be called the mechanics of displays, e.g., the writing of strobes, markers, and numbers in between signal scans, but we have not been concerned with this.

\subsubsection{Integration and Correlation}

Sonar systems are only too often concerned with threshold detection, and it is therefore important to make the maximum use of information which may be repeated in successive range or bearing scans. The improvement of detection obtained with a paper (chemical) recorder when a target appears on several successive range scans, and thus appears as a line on the record, is well known [20]. The improvement of threshold signal-noise level has been shown to be about $2.4 \mathrm{db}$ per doubling of the number of scans. The same method can be applied in cathode-ray displays of similar type, but it is, of course, not suitable for P.P.I. or sector-scan displays. For these latter, some improvement of threshold arises from either integration of successive signals on the phosphor itself, or from a human memory effect when successive pictures are mentally superposed. A more satisfactory basis of improvement, however, is properly designed instrumental integration.

Integrators using a delay line (e.g., mercury) as the storage element are well known, and used occasionally. Cooper and Griffiths [21] have not only established a basis for optimum design, but have also developed an improved integrator using $t$ wo loop circuits, as shown in Fig. 2.15. Working on the assumption of a P.P.I. display obtained from a single-beam echo-ranging system, with the beam rotated at a relatively slow rate (giving several echoes from any particular target on each sweep), and assuming a directional pattern of Gaussian shape, they have determined the proper loop gain for single - and double-1oop integrators. These factors lead to the following optimum performance figures; all are expressed as decibels-improvement in video signal-noise ratio relative to that of 


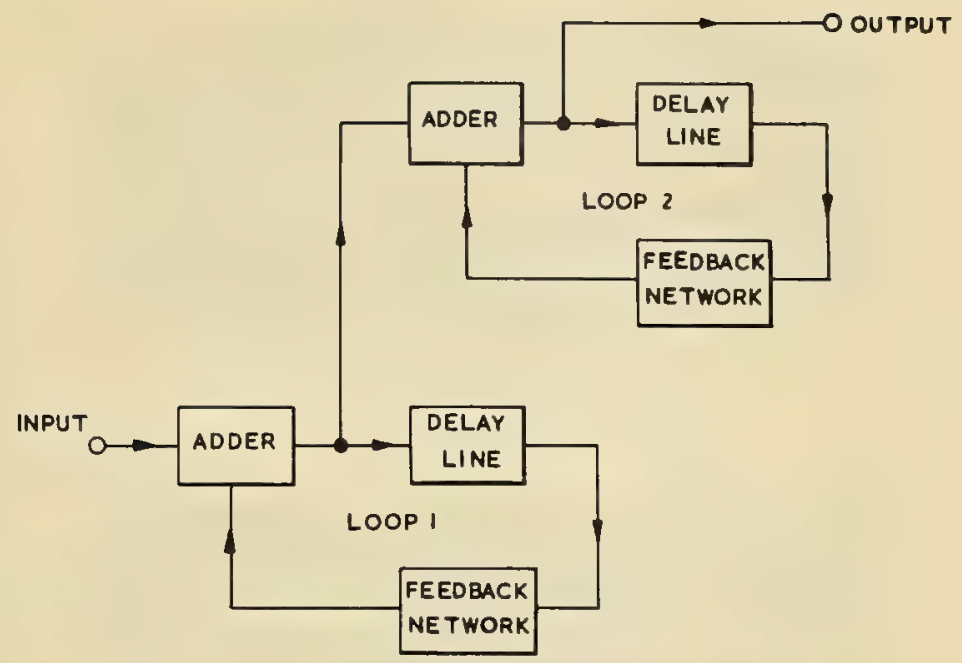

Fig. 2.15. Double-loop signal integrator system.

a hypothetical (ideal) system in which the best weighting is given to every component signal at all times.

a. Uniform weighting of the optimum number of signal returns, the system input being cut off before and after these returns $-0.5 \mathrm{db}$ worse than the ideal system

b. Single-loop continuous integrator with optimum loop gain $-0.95 \mathrm{db}$ worse than the ideal system

c. The new double-loop integrator with optimum loop gains $-0.3 \mathrm{db}$ worse than the ideal system

In view of the fact that the double-loop integrator is thoroughly practical, its performance is so close to the ideal that its use seems well justified. It is somewhat surprising, however, that the investigation shows the form of the integrator to be so uncritical. What we do not know at present is how far the performance of ordinary P.P.I. displays (where there is some integration on the phosphor) falls below the performance of these properly designed integration systems.

\subsubsection{Pattern Recognition and Size of Target}

It has been mentioned previously that the detectability of a signal in noise is improved by causing the signal to trace out a line on successive returns, as in the chemical recorder. It would be expected that, correspondingly, a signal pattern of any shape would show increasing detectability as its area increased relative to the "grain" of the noise background. Probably, the shape itself would have some influence on detectability.

Recent experimental work by Nagaraja [22] at Bírmingham, using a cathoderay display, has shown that:

a. Circular patches show a decrease in threshold of detection of about $2.3 \mathrm{db}$ per doubling of area, provided the area is fairly small $\left(<100 \mathrm{~min}^{2}\right.$ as 


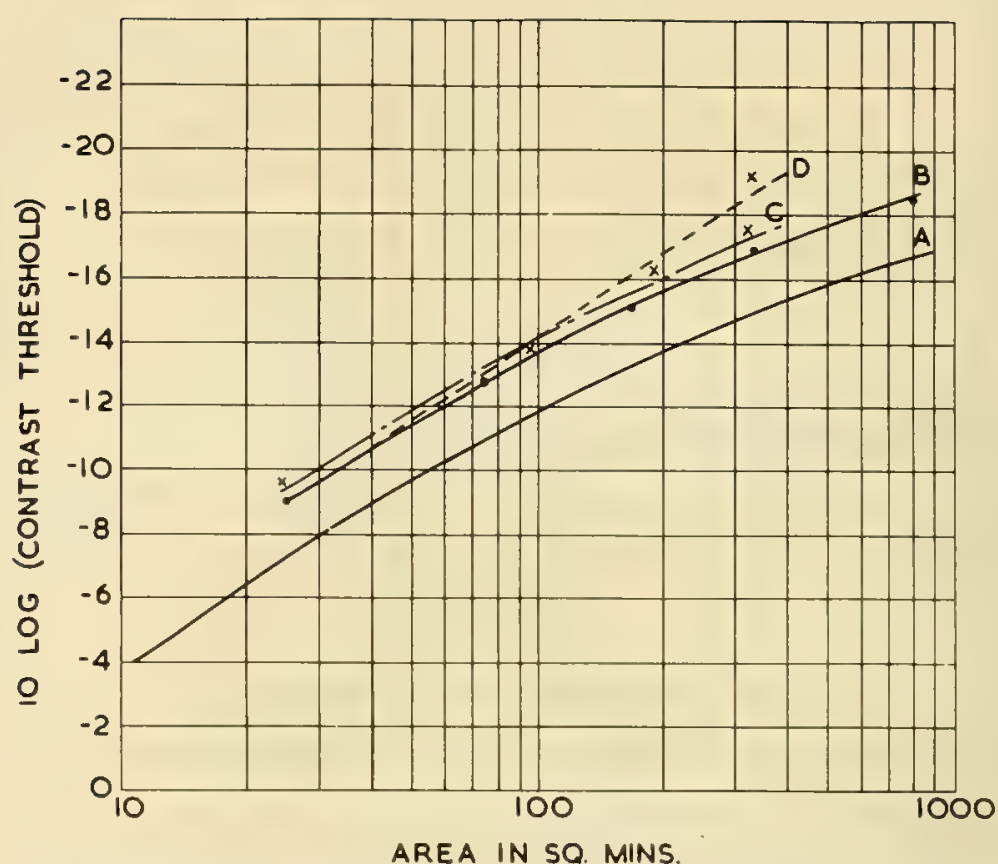

Fig. 2.16. Area/contrast threshold relationship at background luminance of $0.1 \mathrm{ft}-\mathrm{L}$. (A) Blackwell's data (B) With noise background-circular patch (C) With noise-free background-circular patch (D) With noise-free background-line patch.

seen by the eye); this is the same rate as for a line (or slit) target patch. With larger areas, the rate falls off.

b. With fairly low background luminance $(0.1 \mathrm{ft}-\mathrm{L})$, the threshold is almost the same whether the background is noise or merely a uniform bright ness, but at higher background luminance (say, $1 \mathrm{ft}-\mathrm{L}$ ) the threshold is much poorer for noise than for uniform brightness. This is consistent with the conception of internal noise in the human optical system.

Figure 2.16 shows the results for a background of $0.1 \mathrm{ft}-\mathrm{L}$, and also shows that these results agree well with those obtained with different experimental methods by Blackwell [23].

\subsection{ECHO.FORMATION AND RANDOMIZATION}

One of the most serious difficulties in sonar development is that, however good the array and system generally, there is always a limitation on performance imposed by the medium through which the system operates. In the medium, the sea, there are variations in temperature, salinity, aeration, etc., as well as currents, turbulence, and inhomogeneities generally, which affect to a greater or lesser extent the transmission of signals. There are reflections and scattering at the bottom and surface of the sea which produce multipath transmission as well as an interfering background. The environmental conditions which have an adverse effect on sonar performance include the instability of transducer position when the equipment is shipborne. These various factors are very hard 

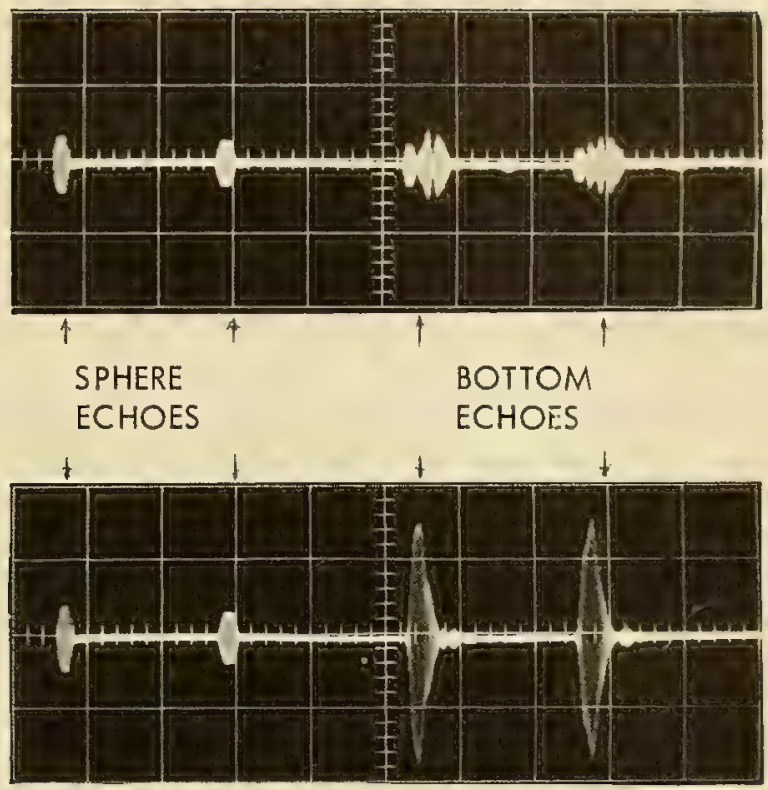

Fig. 2.17. Echoes from sphere and sea bottom on different transmissions. Duration of first and second pulses in each transmission $=0.25 \mathrm{msec}$; separation $=2.5 \mathrm{msec}$.

to evaluate. It is doubtful if they have received anything like their proper share of research effort.

An illustration of the sort of thing that may make higher resolution an embarrassment, and automatic recognition of targets impracticable, is an effect which has been studied at Birmingham recently [24]. It is the rather startling influence of small transducer movements on the echo amplitude (and waveform) from complex targets. One particular result may be quoted to demonstrate this. A 50 -kcps beam of about $12^{\circ}$ by $14^{\circ}$ was pointed vertically downwards from the ship Discovery II anchored in about 12 fathoms of water in a tideway. The trans ducer was stabilized against roll, although the water was dead calm. An 18-in.diameter sphere was suspended a few feet above the bottom. The sonar equipment emitted, on every cycle of repetition, two 0.25 - msec pulses spaced a few milliseconds apart. Figure 2.17 shows the oscilloscope record of the returned signals for two different emissions. The echoes from the sphere (which is a simple single target) remained substantially constant throughout the test, but the echo from the bottom (which represents a complex target) was completely randomized in amplitude and waveform from one emission to another. Indeed, as can be seen from the records in Fig. 2.17 the lower amplitude return is partially randomized even between the two pulses which are only $2.5 \mathrm{msec}$ apart. The only possible explanation for this effect is horizontal movement of the ship due to yaw, but the magnitude of this movement cannot even approach the wavelength in $2.5 \mathrm{msec}$, although from one pair of pulses to another it could be several wavelengths. Laboratory tests confirm this explanation, as can be seen from the results of a tank test at $500 \mathrm{kcps}$ with different kinds of bottom configuration, shown in Fig. 2.18. 
(A)

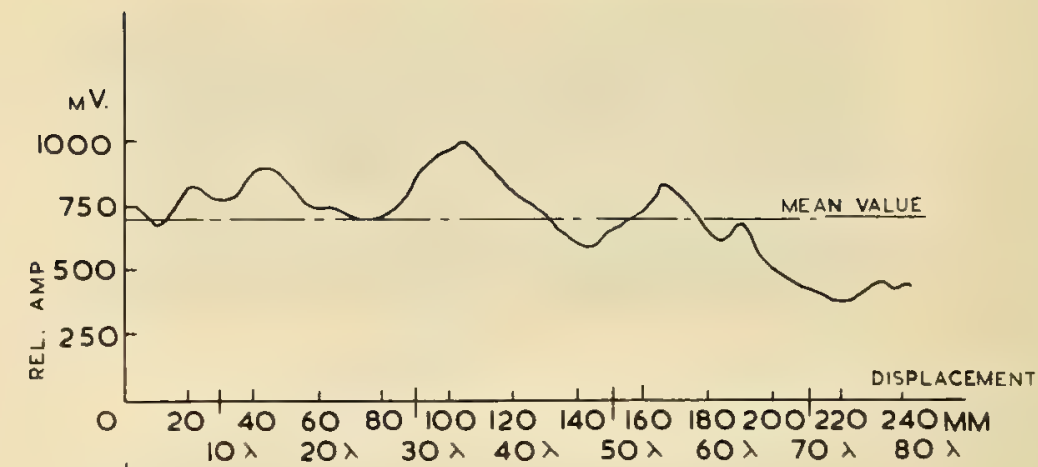

(B)

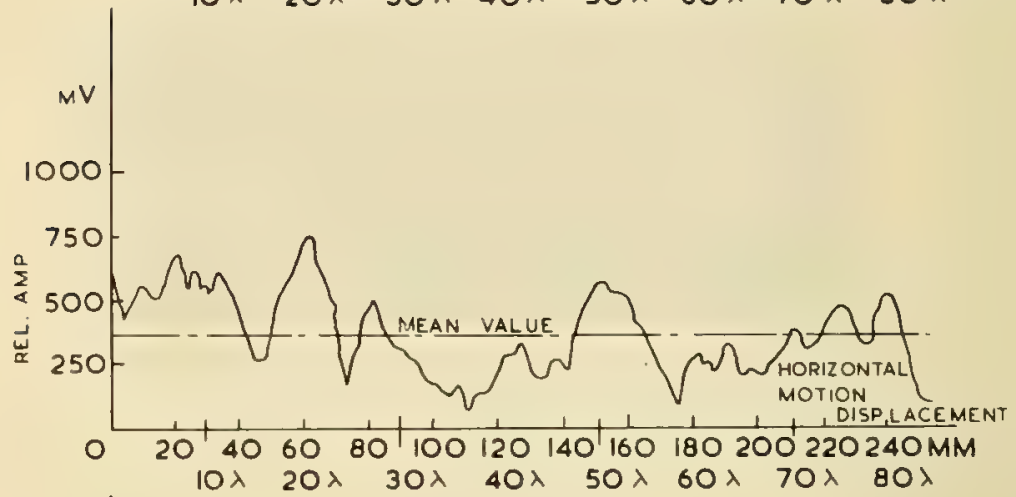

(c)

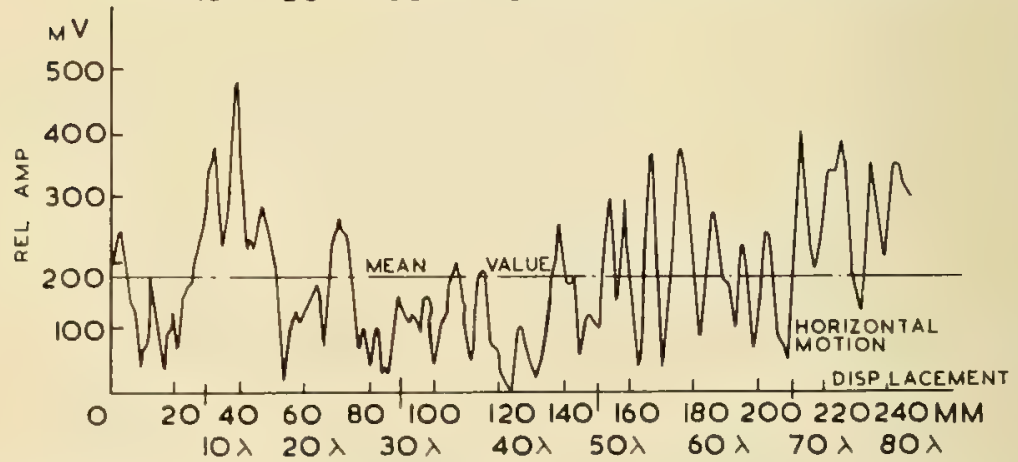

Fig. 2.18. Amplitude fluctuations due to lateral motion of the ransducers in tank test at $500 \mathrm{kcps}$. Pulse duration $0.1 \mathrm{msec}$. Amplitudes recorded at fixed time-point in returned pulse. (a) Tank bottom covered with plaster of paris, grain size $<\lambda / 12$, undulations less than 0.5 in, high. (b) Tank bottom covered with sand, grain size $\lambda / 12$ to $\lambda / 3$, undulations less than 0.5 in. high. (c) Tank bottom covered with sand and gravel, grain size $\lambda / 12$ to $3 \lambda$, undulations less than 0.5 in. high. Abscissa shows lateral displacement of transducer in a plane parallel to mean level of bottom.

\subsection{CONCLUSIONS AND ACKNOWLEDGMENTS}

It is hoped that this paper has shown some ways in which useful progress may be achieved in the development of sonar systems. The work and ideas described owe much to the author's colleagues mentioned in the text, and to others (especially research students) too numerous to mention. The cooperation of the National Institute of Oceanography in providing ship facilities (including a trans - 
ducer) and financial assistance has been of the greatest value. The Fisheries Laboratories at Lowestoft and Aberdeen have also given help and stimulation.

\section{REFERENCES}

1. D. G. Tucker, "Signal-Noise Performance of Electroacoustic Strip Arrays," Acustica, Vol, 8, 53 (1958).

2. V.G. Welsby and D.G. Tucker, "Multiplicative Receiving Arrays," J. Brit. Inst. Radio Engrs., Vol. 19. 369 (1959).

3. V.G. Welsby, "Multiplicative Receiving Arrays: The Angular Resolution of Targets in a Sonar System with Electronic Scanning," J. Brit. Inst. Radio Engrs., Vol, 22, 5 (1961).

4. V.G. Welsby, "A Note on a Possible Method of Improving the Directional Response of a Single-Frequency Multiplicative Receiver," Electrical Eng. Dept., University of Birmingham, Memorandum No. 89 (1961).

5. A. Bloch, R. G. Medhurst, and S.D. Pool, "Superdirectivity," Proc. Inst. Radio Engrs., Vol. 48, 1164 (1960). (This paper gives very comprehensive bibliography of the subject.)

6. D.G. Tucker, "Signal-Noise Performance of Super-Directive Arrays," Acustica, Vol. 8, 112 (1958).

7. W. E. Kock and J. L. Stone, "Space-Frequency Equivalence," Proc. Inst. Radio Engrs., Vol. 46, 499 (1958).

8. V.G. Welsby, "Two-Element Aerial Array: Use of Multifrequency Carrier Waveform to Improve Directivity," Electronic Technology, Vol. 38, 160 (1961).

9. D. G. Tucker, "Space-Frequency Equivalence in Directional Arrays, with Special Reference to SuperDirectivity and Reciprocity," Inst. Elect. Engrs. (London) Monograph 479 E (1961).

10. V. G. Welsby, "Electronic Sector Scanning Arrays," Electronic Technology, Vol. 39, 13 (1962).

11. D. G. Tucker, "Arrays with Constant Beamwidth over a Wide Frequency Range, " Nature, Vol, 180,496 (1957).

12. J. C. Morris and E. Hands, "Constant Beamwidth Arrays for Wide Frequency Bands," Acustica, Vol, 11, 342 (1961).

13. D. G. Tucker, V.G. Welsby, and R. Kendell, "Electronic Sector Scanning," J. Brit. Inst. Radio Engrs., Vol. 18, 465 (1958)

14. D. G. Tucker, V.G. Welsby, L. Kay, M. J. Tucker, A.R. Stubbs, and J. G. Henderson, "Underwater Echo-Ranging with Electronic Sector Scanning: Sea Trials on R.R.S. Discovery II," J. Brit. Inst. Radio Engrs., Vol, 19, 681 (1959).

15. E. A. Howson and J.R. Dunn, "Directional Echo-Sounding," J. Inst. Navigation (London), Vol, 14, 348 (1961).

16. B.S. McCartney, "An Improved Electronic Sector-Scanning Sonar Receiver," J. Brit. Inst. Radio Engrs., Vol. 21,481 (1961).

17. D. E. N. Davies, "A Fast Electronically Scanned Radar Receiving System," J. Brit. Inst. Radio Engrs., Vol. 21, 305 (1961).

18. L. Kay, "A Plausible Explanation of the Bat's Echo-Location Acuity," Animal Behaviour, Vol, 10, 34 (1962).

19. L. Kay, "The Orientation of Bats and Men by Ultrasonic Echo-Location," Brit. Communications and Electronics, Vol. 8, 582 (1961).

20. D.G. Tucker, "Detection of Pulse Signals in Noise: Trace-to-Trace Correlation in Visual Displays," J. Brit. Inst. Radio Engrs., Vol. 17, 319 (1957).

21. D. C. Cooper and J.W.R. Griffiths, "Video Integration in Radar and Sonar Systems," J. Brit. Inst. Radio Engrs., Vol. 21, 421 (1961).

22. N.S. Nagaraja, "Intensity-Modulated Displays: Contrast Thresholds in the Presence of Noise," Electrical Eng. Dept., University of Birmingham, Memorandum No. 87 (1961).

23. H. R. Blackwell, "Contrast Thresholds of the Human Eye," J. Opt. Soc. Am., Vol. 36, 624 (1946).

24. L. Kay, "Progress in Underwater Echo-Ranging: Recent Sea Trials in R.R.S. Discovery II," Brit. Communications and Electronics, Vol. 8, 753 (1961). 



\section{LECTURE 3}

\section{EXPLOSIVE SOURCES}

\section{E. Weston}

Admiralty Research Laboratory

Teddington, Middlesex

England

\subsection{INTRODUCTION}

This paper deals with transducers, though of a rather special sort. In this type of "transducer" there is a rapid and irreversible conversion of chemical energy into acoustic energy. I will not be much concerned with explosions as such, but will deal with explosions as acoustic sources - particularly underwater explosions. Only so-called point sources such as simple blocks of TNT will be described, and various special sources such as explosive gas mixtures will not be considered. A good deal of the ground covered will be familiar to many.

This paper falls naturally into three parts. The first (section 3.2) gives some historical background. The second or main part (sections $3.3,3.4,3.5$, and 3.6) concerns the characteristics of explosive sources, and draws heavily on two recent papers $[9,10]$ which give more detailed information than can be presented here. Reference [10] concerns underwater explosions, and [9] presents some more general ideas which are applied in particular to underground explosions. The approach here differs slightly from that in $[9,10]$. The third part (sections 3.7 and 3.8 ) describes some of the uses of explosions in underwater acoustics research and touches upon interpretation of results.

\subsection{BACKGROUND}

Underwater explosions have been used as acoustic or seismic sources in many connections, in geophysics, navigation, communications, etc. They were first used for underwater acoustics research about the turn of the century and received fresh impetus at the time of the First World War. In this early work the pressure levels very near the charges were investigated, but charges as acoustic sources were used mainly for timing. More work was started during the Second World War, and since then much as been done in many countries. It was at this time that acoustic levels were first measured and used for the quantitative estimation of transmission loss [4]. Why use explosions for this? Briefly, it is because they produce a nice loud bang with energy in a wide frequency range, as discussed further in section 3.8 . 
At the Admiralty Research Laboratory the main use of explosion sources has been in the investigation of transmission loss. In order to deduce the absolute transmission loss from the signal level it is necessary to know the source level. One also needs to know the source differences between the charges of different sizes and types that may have to be used, together with the depth dependence of source level. Thus it became necessary to make a general study of explosion sources, including the measurement of absolute levels and differences at various depths.

It may be noted that the most important parameter is often the acoustic energy rather than the peak pressure, since the pulse energy should obey the same acoustic transmission laws as the intensity from a continuous wave source. The spectrum level of the acoustic energy which has flowed through a unit area is often quoted in $\mathrm{db}$ re $1 \mathrm{erg} / \mathrm{cm}^{2}-\mathrm{cps}$. Actually, it is usually $\int p^{2} d t$ ( $p$ is the pressure) which is measured, comparable to $p^{2}$ for the C.W. source, and strictly speaking these quantities do not obey quite the same transmission law as energy or intensity (e.g., near a free surface).

\subsection{GENERAL IDEAS ON SCALING LAWS AND SPECTRUM SLOPES}

There is surprisingly little in the literature on charge spectrum levels or on scaling laws, and what there is contains many misleading statements. Thus, the second part of the paper starts off with some general ideas which are applicable to any type of disturbance in a three-dimensional medium, and it is not essential to consider the mechanisms involved. However, to be specific, think of an explosion with charge weight $W$, so that total energy is proportional to $W$. Volume is also proportional to $\mathrm{W}$, so that the linear scaling factor for both distance and time is $w^{1 / 3}$. More precisely one has identical pressure-time curves when these are plotted against reduced time $t W^{-1 / 3}$ and measured at corresponding values of reduced range $\mathrm{rW}^{-1 / 3}$. This explosion-similarity principle is semitheoretical but confirmed by experiment.

The scaling law may be applied to explosion spectrum levels, e.g., for a change from $1 \mathrm{lb}$ to $50 \mathrm{lb}$ where $w^{1 / 3}=3.68$. It is necessary to make the additional assumption of spherical spreading, which leads to some appreciable though calculable errors, especially at the shorter ranges. Fig. 3.1 shows that the whole spectrum is shifted back in frequency by $W^{1 / 3}$ (3.68 in the example), corresponding to the $W^{1 / 3}$ time scaling. It is also raised in level by $W^{4 / 3}$ (or $23 \mathrm{db}$ in the example); the factor $W$ comes from the change in total energy and the factor $W^{1 / 3}$ from the change in the bandwidth of any given portion of the spectrum.

Consider now how the spectrum level depends on $W$ at a given frequency. This dependency is a function of the spectrum shape, so that in general there is no simple scaling law for a fixed frequency. There is a simple law only in a region of constant spectrum slope (assuming a logarithmic plot), the index being the sum of the above $4 / 3$ for general-level change plus the product of $1 / 3$ (frequency change) times the index of the spectrum slope. Table 3.1 shows some of the important practical laws.

To make use of the laws given in Table 3.I, it is necessary to know what spectrum slopes to expect, and Table 3 .II illustrates some useful general relations between pulse shape and spectrum slope, together with the predicted scal- 


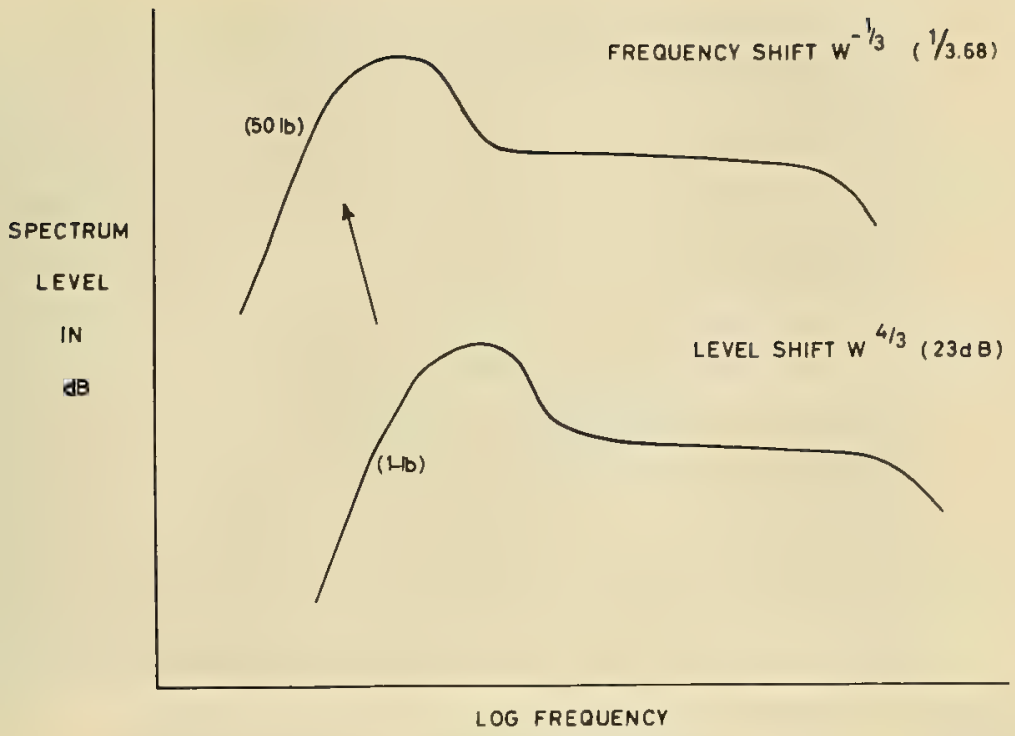

Fig. 3.1. Spectrum scaling.

ing laws. It is best to start with the delta function in example 4 which is well known as having a flat spectrum; the other pulses are derived from the one above by differentiation. At least the middle five of these examples have important applications to explosions. Example 5 is perhaps not very obvious but is actually the most generally occurring case. It is possible to show from the wave equation that

$$
\int_{-\infty}^{+\infty} p d t
$$

falls off with range faster than p itself, and at long ranges may be taken as zero. It follows that at sufficiently low frequencies the $f^{2}$-spectrum slope holds, as does the $W^{2}$ scaling law. These two laws are of great generality and, besides holding true for the radiated longitudinal elastic waves, will apply to both shear waves and electromagnetic waves. The arguments are developed in more detail in [9], together with the differing laws for two- and one-dimensional propagation.

TABLE 3.I. Scaling Law at a Given Frequency as a Function of Spectrum Slope

\begin{tabular}{cc}
\hline $\begin{array}{c}\text { Frequency dependence } \\
\text { of spectrum level }\end{array}$ & $\begin{array}{c}\text { Weight dependence } \\
\text { of spectrum level }\end{array}$ \\
\hline$f^{2}$ & $W^{2}$ \\
$f^{0}$ & $W^{4 / 3}$ \\
$f^{-2}$ & $W^{2 / 3}$ \\
\hline
\end{tabular}


TABLE 3.II. Pulse-Shape Relations

\begin{tabular}{|c|c|c|c|c|c|}
\hline \multirow[t]{2}{*}{$\begin{array}{l}\text { Example } \\
\text { number }\end{array}$} & \multirow[t]{2}{*}{$\begin{array}{l}\text { Schematic shape of } \\
\text { rypical pressure pulse }\end{array}$} & \multirow[t]{2}{*}{$\begin{array}{l}\text { Lowest derivative } \\
\text { that is zero at }\end{array}$} & \multicolumn{2}{|c|}{$\begin{array}{c}\text { Spectrum level } \\
\text { proportional to }\end{array}$} & \multirow{2}{*}{$\begin{array}{c}\text { Erequency } \\
\text { band }\end{array}$} \\
\hline & & & frequency & weight & \\
\hline 1 & & $d^{3} p / d t^{3}$ & $f^{-6}$ & $W^{-2 / 3}$ & $\mathrm{HF}$ \\
\hline 2 & & $d^{2} p / d t^{2}$ & $f^{-4}$ & $w^{0}$ & $\mathrm{HF}$ \\
\hline 3 & & $d p / d t$ & $f^{-2}$ & $w^{2 / 3}$ & $\mathrm{HF}$ \\
\hline 4 & & $p$ & $t^{0}$ & $w^{4 / 3}$ & all $f$ \\
\hline 5 & & $\int p d t$ & $f^{2}$ & $w^{2}$ & LF \\
\hline 6 & & $\iint p d t$ & $t^{4}$ & $w^{8 / 2}$ & $\mathrm{LF}$ \\
\hline 7 & $-\|$ & $\iiint p d t$ & $f^{6}$ & $w^{10 / 3}$ & LF \\
\hline
\end{tabular}

\subsection{DESCRIPTION OF UNDERWATER EXPLOSIONS}

The characteristics of underwater explosions are well established. Cole [3] and Underwater Explosion Research [8] may be given as general references and Weston [10] as a reference for acoustic effects. Upon detonation, the explosive is converted to incandescent gas at a very high pressure, and a shock wave is radiated into the water. The gas bubble expands and due to water inertia overshoots its equilibrium radius; at its maximum radius the radiated pressure is slightly negative. The bubble now contracts, and in fact undergoes a damped radial oscillation. At each bubble minimum a further positive-pressure pulse is radiated, though only the first two of these bubble pulses are of much significance.

When the shock wave reaches the surface it is reflected as a tension wave, which may cause cavitation. For moderate shocks the surface shows corrugations, sometimes known as the black ring, and for stronger shocks a spray dome is formed. The precise mechanisms are still uncertain, but the critical depth for corrugations over a $1-1 \mathrm{~b}$ TNT charge is $7 \frac{1}{2}$ fathoms, suggesting a water-breaking 


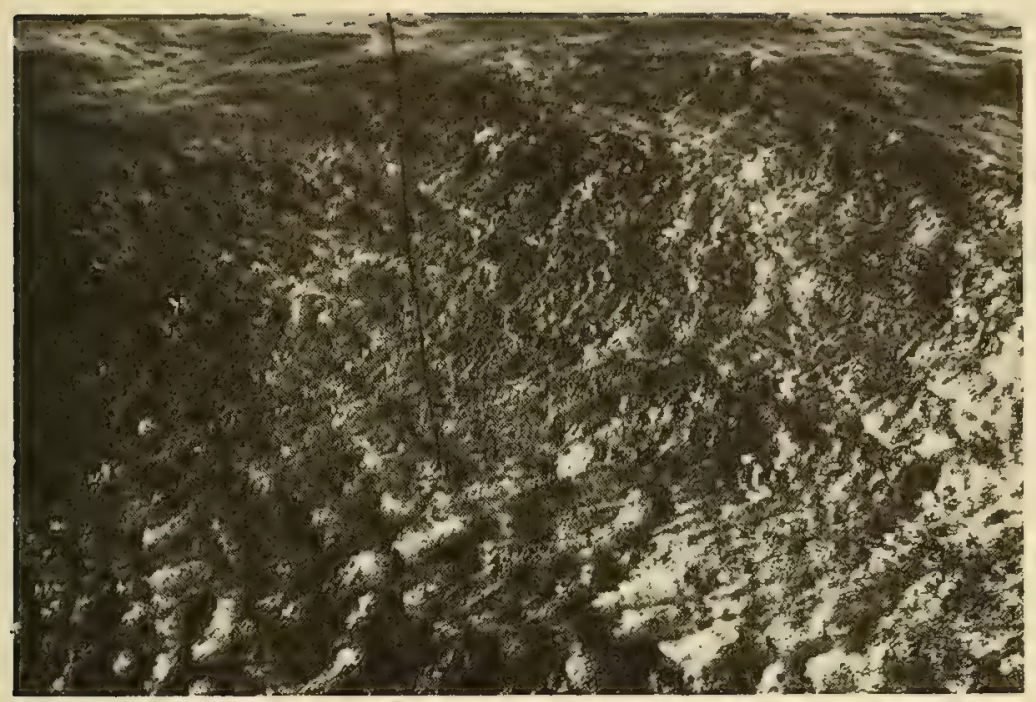

(a)

(b)

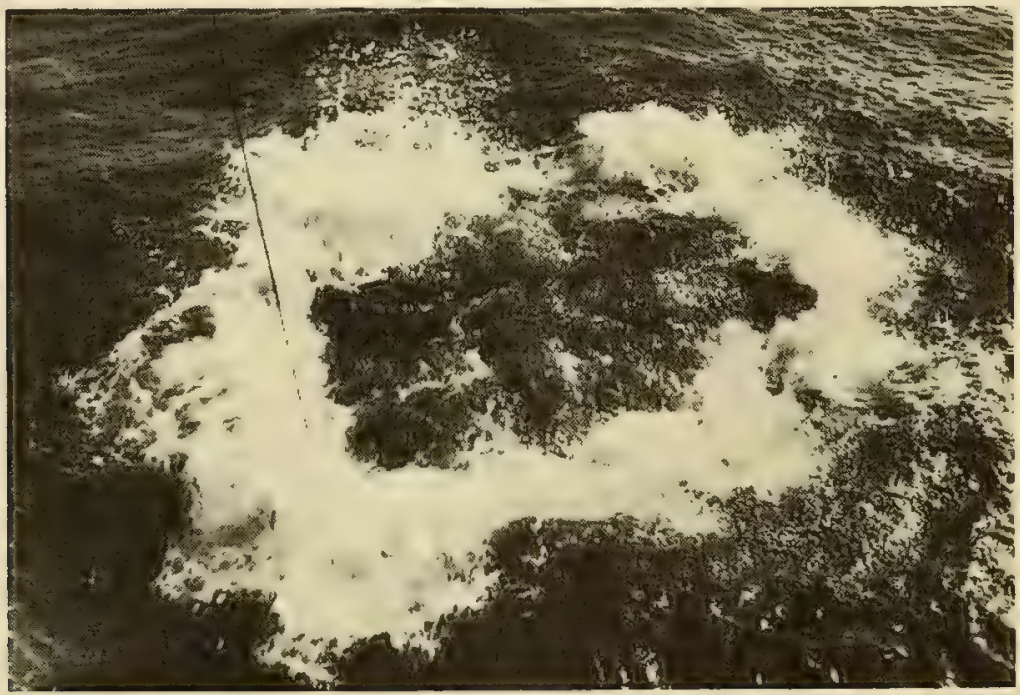

Fig. 3.2. Surface effects for 1 -lb charges at 5 fathoms; (a) corrugations (b) plume.

tension of about $300 \mathrm{lb} / \mathrm{in}^{2}$. There is a separate and sometimes more impressive surface effect known as the plume, which occurs when the oscillating explosion gases reach the surface. Figure 3.2 illustrates the phenomena of corrugations and plume for a 1-1b charge at 5 fathoms, and indicates that for most shots used in transmission experiments the surface effects are not spectacular.

Acoustically, an underwater explosion does not behave precisely like a fixed low-amplitude source, both because of the high pressure generated and because the explosion source may move while radiating. It is worth listing the differences. 


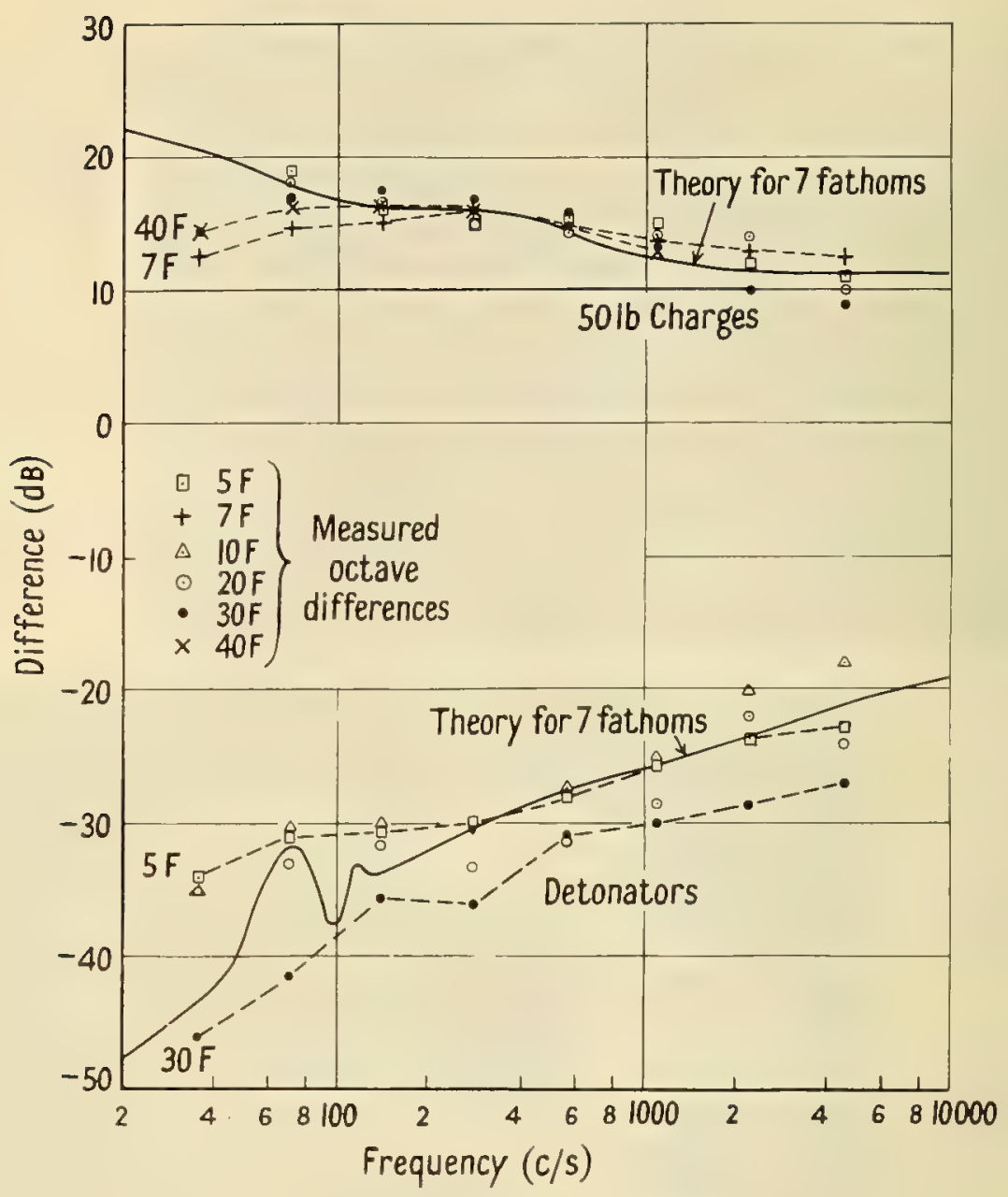

Fig. 3.3. Differences from a 1-1b charge.

1. There is a Riemann broadening and sharpening of the shock pulse; the latter keeps the viscous losses at the shock front at a high level.

2. Reflection at the sea surface is imperfect due to cavitation.

3. Some of the lost energy may be reradiated eventually; due, for example, to water droplets falling back into the sea.

4. At small glancing angles a strong shock wave suffers an irregular type of reflection at the sea surface, which may be compared or contrasted with Mach reflection.

5. Due to migration under gravity the bubble pulses may be radiated from a reduced depth.

Most of these points are usually unimportant but it is necessary to watch them. 


\subsection{MEASUREMENTS AND THEORY FOR UNDERWATER EXPLOSIONS}

Experimental results have been arrived at using octave filters followed by small analog computers, which compute $\int \nu^{2} d t$, where $\nu$ is the input voltage. The results in Fig. 3.3 show some measured differences between charges, with an average accuracy of $\pm 1 \mathrm{db}$. They show that there is no simple law, that there is a real variation with depth, and that there is general agreement with the theory (to be described later). It may be noted that at high frequencies the $50-\mathrm{lb}$ results are in the region of the $w^{2 / 3}$ or $11^{1 / 2}-\mathrm{db}$ law, and that at low frequencies the detonator results are approaching the $W^{2}$ or 54 - $\mathrm{db}$ region.

For close shots it is possible to estimate the transmission loss theoretically and deduce absolute source levels from the measured signals. Some results are shown in Table 3.III; there is little depth dependence above $140 \mathrm{cps}$. The accuracy is a little worse than that for the differences.

Results for other charge sizes may be obtained by adding in the measured differences, and it then becomes possible to test the $W^{1 / 3}$ and $W^{4 / 3}$ scaling laws for the whole spectrum by shifting these spectra as in Fig. 3.1. It may be noted that the similarity and scaling laws of section 3.3 should apply to the bubble pulses as well as to the shock, provided that the depth is constant and there are no appreciable secondary effects due to gravity, etc. The result is shown in Fig. 3.4, all points lying well on a single line. Some of the minor discrepancies can be explained, e.g., the low-frequency 50 -lbvalues are low in level because bubble migration under gravity suppreses the bubble pulse. The spectral peak at the reciprocal of the bubble-pulse time is evident for the detonator results in both Fig. 3.3 (70 to $160 \mathrm{cps}$ ) and Fig. 3.4 ( $8 \mathrm{cps}$ ). The agreement with theory in Fig. 3.4 may be noted.

So far it has been demonstrated that the scaling laws work, and now the more detailed theory for the spectrum will be presented. This comes from a Fourier analysis of the shot pressure-time curves given by Arons $[1,2]$. The shock wave may be represented as a sharp rise in pressure $P_{0}$, followed by an exponential decay with time constant $t_{0}$. Either bubble pulse may be approximated by two similar back-to-back exponentials, with peak pressure $P_{1}$ and time constant $t_{1}$ for the first bubble. The pulse shapes and the resulting energy-spectrum equations are shown in Table 3.IV (where $\rho \mathrm{c}$ is the characteristic impedance). The average total spectrum at high frequencies is obtained by incoherent addition of the shock and bubble contributions, as shown in Fig. 3.5. At low frequencies the shock and bubbles may be replaced by their impulse values $I_{0}, I_{1}$, and $l_{2}$ occurring at time intervals $T_{1}$ and $T_{3}=T_{1}+T_{2}$ together with a steady negative pressure. The results axe also shown in Table 3.IV and Fig. 3.5.

TABLE 3.III. Free-Field Source Spectrum Levels for a 1-1b TNT Charge at 60 Fathoms

\begin{tabular}{c|c|c|c|c|c|c|c|c}
\hline Frequency in cps & 35 & 70 & 140 & 280 & 560 & $1.1 \mathrm{k}$ & $2.2 \mathrm{k}$ & $4.5 \mathrm{k}$ \\
\hline $\begin{array}{c}\text { Energy flux density in db } \\
\text { re } 1 \text { erg/cm }\end{array} \mathrm{cms}^{2}$ at 100 yards & +17.2 & +12.8 & +9.9 & +5.4 & +2.8 & -0.5 & -5.0 & -13.9
\end{tabular}




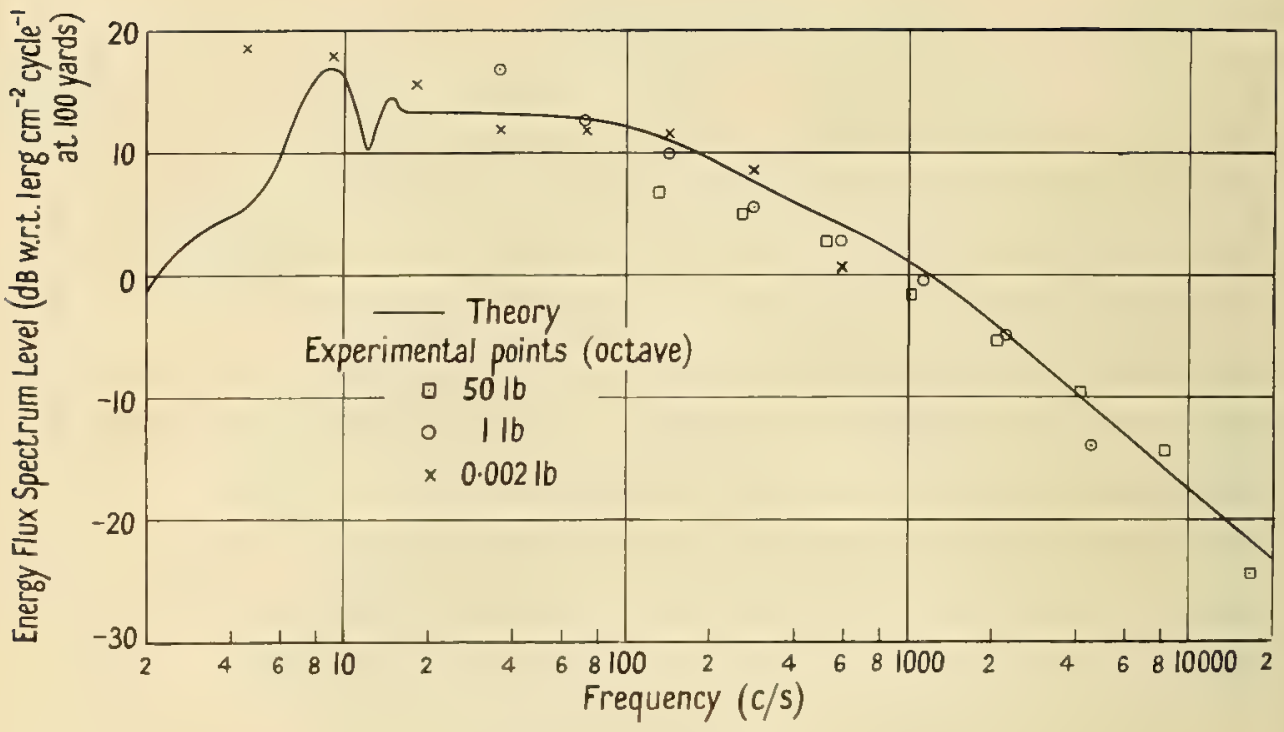

Fig. 3.4. Source levels for 7-fathom charges scaled to $1 \mathrm{lb}$.

Note that there are regions in Fig. 3.5 with dependence on $f^{2}, f^{0}$, and $f^{-2}$. The high-frequency bubble pulse gives an $f^{-4}$ law, and just below the spectral peak at the reciprocal of the bubble-pulse frequency there is a region with a law close to $f^{4}$. The latter arises because of the particular values of the impulses $l$ and intervals $T$; the last illustration in Table 3.IV is closer to example 6 of Table 3 .II than to example 5. There is a sort of cancellation which in practice is both imperfect and variable, so that the experimental levels in this region are higher than the simple theory (see Fig. 3.4) and also rather variable.

TABLE 3.IV. Underwater-Explosion Pulse Shapes and Spectrum Equations

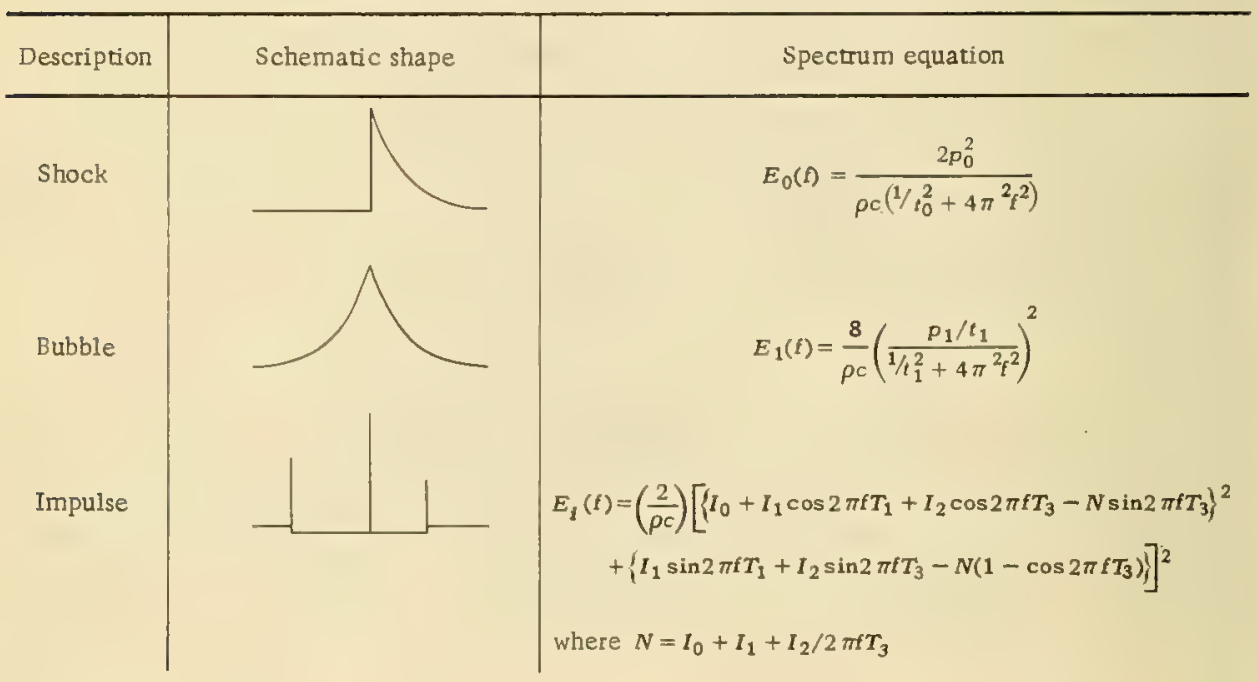




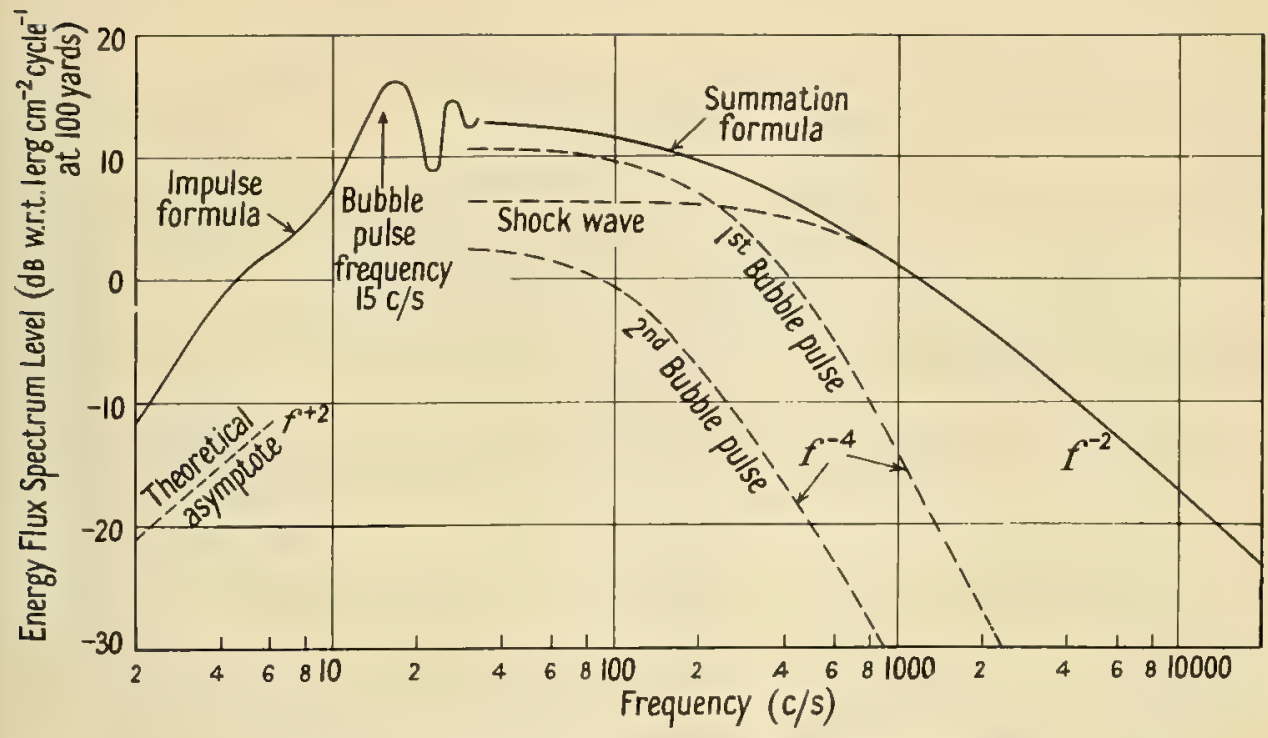

Fig. 3.5. Theoretical source spectrum for 20-fathom, 1-lb charge.

It may be mentioned that Weston [10] shows some measure of agreement between theory and experiment for depth dependence. This dependence is mainly due to the changing bubble-pulse frequency and is only important below, say, $140 \mathrm{cps}$ for 1-lb charges where, however, it can be very large.

The position has been reached where the acoustic spectra of underwater explosions are generally well understood, though many small points remain to be explained. It is still usually better to use experimental rather than theoretical figures for differences and for source levels.

\subsection{COMPARISON WITH UNDERGROUND EXPLOSIONS}

It is interesting to compare explosions underwater and underground, though the latter are much less well understood. Their mechanisms have been broadly described as a spreading explosive pulse which causes shattering or plastic flow of the solid material out to a critical radius, beyond which there is normal elastic wave propagation. The most notable thing about the transmitted signal is that seismic amplitude is experimentally found to be directly proportional to charge weight (O'Brien [6], Weston [9]). This is of course the same as the lowfrequency $W^{2}$ energy law of section 3.3, which applies because all the highfrequency energy is attenuated in passing through the ground. It should be noted that this law with low-frequency energy proportional to the square of the total energy can work only if the low-frequency energy is a small fraction of the total. Efficiency as a seismic source improves as the size of the charge increases.

There are very few measurements on underground-source spectrum levels, though the work of McDonal et al. [5] provides experimental verification of the $f^{2}$ law. The source spectrum may, however, be calculated from the model as sumed [9]; Fig. 3.6 shows a much-simplified comparison of the spectra in the two media. The total energy radiated does not change much whether the explo- 


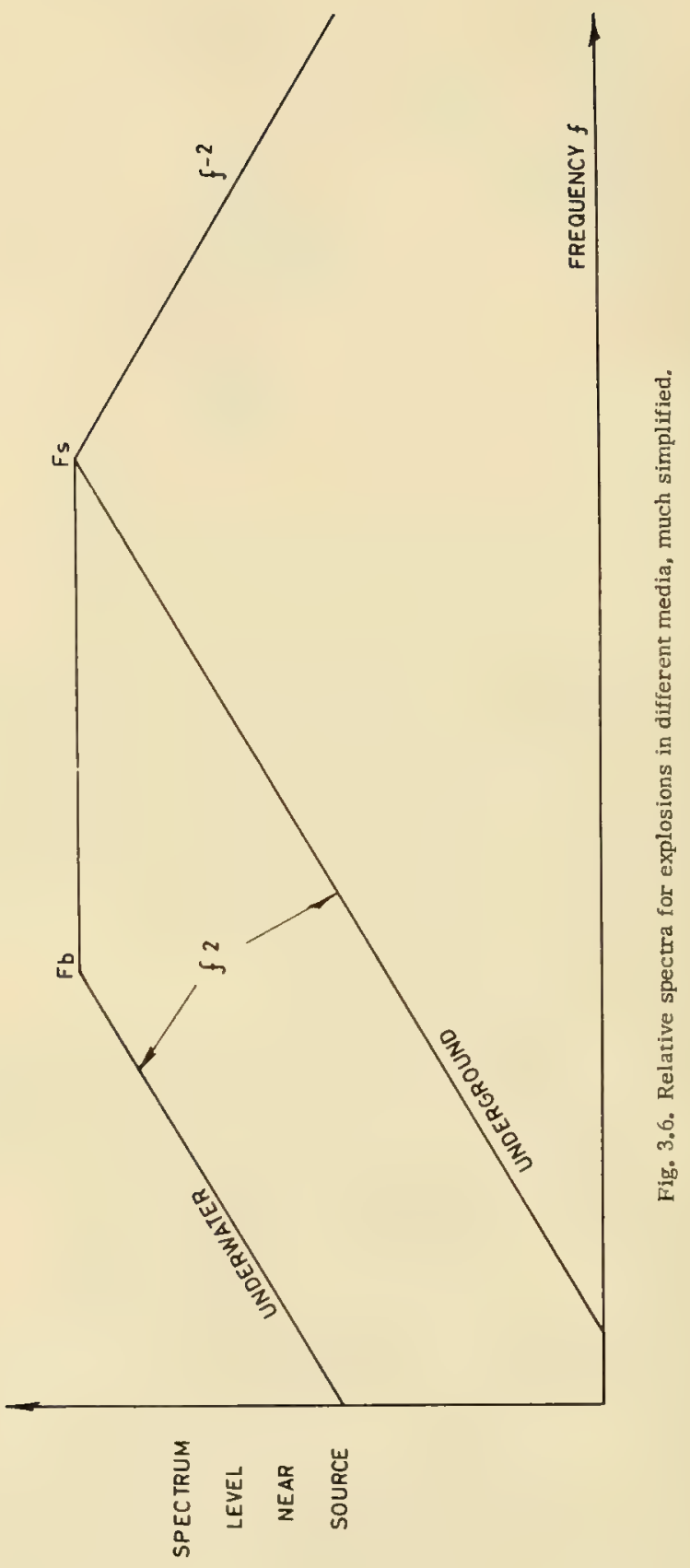


sion is underwater, underground, or even in the air. The shock duration is similar underwater and underground, so that both spectra show a turning point near the reciprocal $F_{s}$ of this shock duration, and a similar high-frequency spectrum. However, the low-frequency spectra are quite different since the average underwater spectrum is flat down to about $F_{b}$, the bubble-pulse frequency. This arises because the restoring force underwater is the hydrostatic pressure, leading to a negative pressure phase of much greater duration than the shock. Underground, the restoring force is the rock rigidity, the positive and negative phases are of comparable length, and the $f^{2}$ spectrum law starts just below $F_{s}$. These differences mean that the underwater explosion is a much better seismic source; a typical calculation suggests $10 \%$ efficiency underwater and $0.05 \%$ underground. There is experimental evidence for this underwater superiority.

\subsection{USES IN RESEARCH}

A very important aspect which should be included in any account of underwater explosions is the use of these explosions in acoustic research. The uses are manifold, even excluding seismic investigations of the sea bed, so I will not attempt to give a full list. One use is to investigate transmission loss, including such things as reflectivity and fluctuation measurements. Another is to measure reverberation: volume reverberation including that from biological scatters, surface reverberation, bottom reverberation, and reflections from distant topographic features. There are several other possible uses, many little explored. (Shots were fired in a wide range of conditions - four shots to measure trans mission and three shots near the receiver to measure reverberation.)

A little more will be said about the investigation of what may be considered the central problem in underwater acoustic research, i.e., transmission loss. Most investigators apparently use similar techniques, which are not particularly novel. Typically, one goes to sea with two ships. One ship is used for sound reception, with one or two hydrophones suspended in the sea. The second ship opens range while firing charges, possibly using a safety fuse. Alternatively, there may be serials at constant range, varying the depth of the charge or the hydrophone. Normally, the signal energy levels are measured using analog computers, but there are possibilities for other processing methods, such as the digital computer. For some purposes, peak pressures may be measured. Worthwhile precautions include monitoring overload in all channels, scheduling some close shots as a check on source level and on the whole system, and measuring rather than estimating any differences between different charge types. In the typical trial, a large quantity of information is collected and a large analysis headache is generated. Lastly, note that one should beware of overload; in shot work this is of extreme importance and cannot be stressed too often.

Figures 3.7 and 3.8 show two examples of results achievable with explosion sources, both taken with 1 -lb charges in water of constant depth. Only a very little will be said about the physical meaning of these plots. Figure 3.7 shows a composite frequency plot for a range run in the Norwegian Sea, with 58-fathom source and 140-fathom receiver. Signal level itself has been plotted, and this is a first stage in presentation which is useful when source level may not be accurately known. Note that at low frequencies there is hardly any departure 


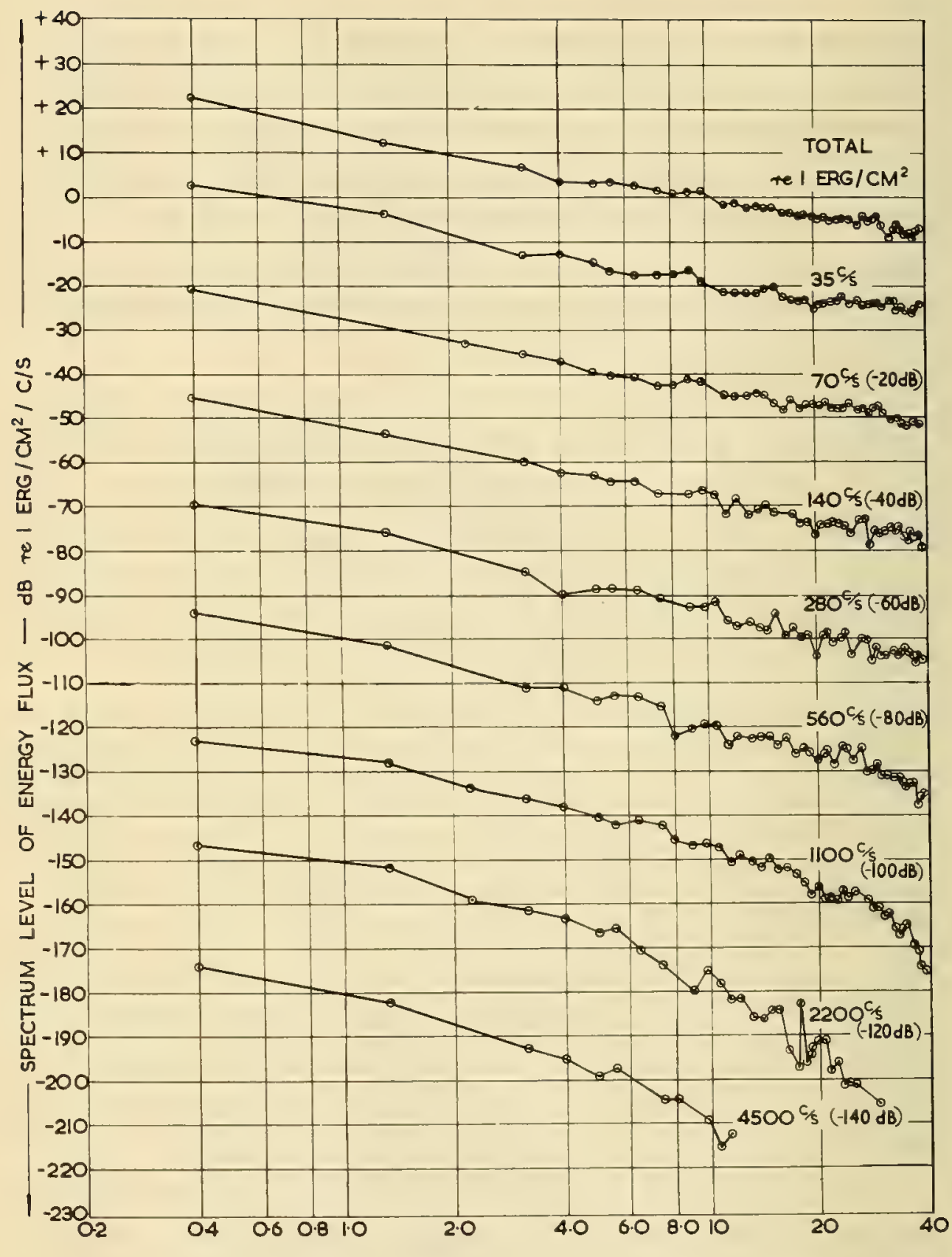

RANGE FROM HYDROPHONE IN NAUTICAL MILES

Fig. 3.7. Sample contour transmission run in 666 fathoms. 

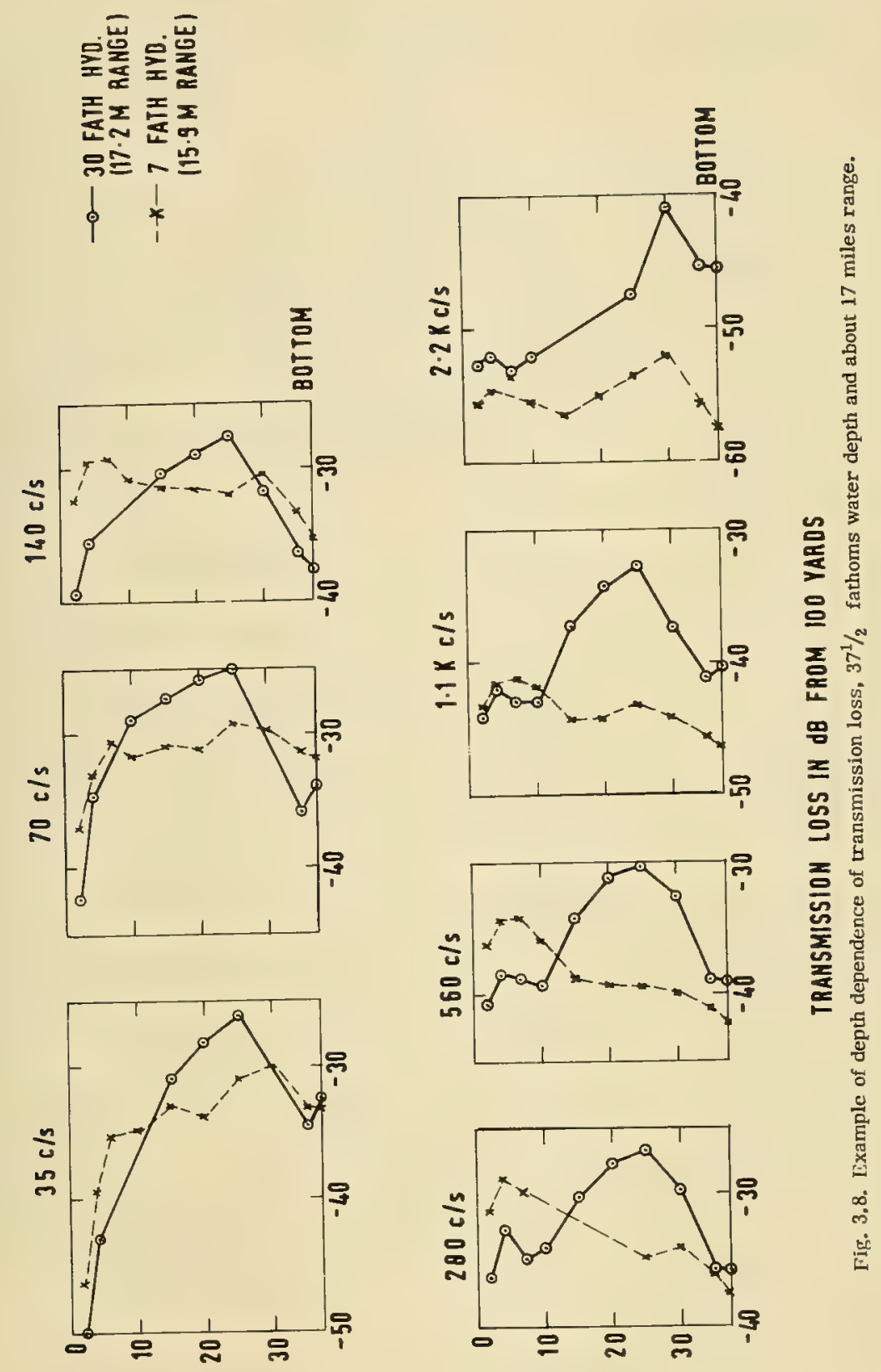

SWOHLYJ KI HLdJO 39 YHHJ 
from the cylindrical spreading law right out to the extreme 40 -mile range. However, at the higher frequencies there is additional attenuation. Figure 3.8 illustrates depth dependence in a North Sea area and shows the second stage of presentation when transmission loss itself is plotted. This is an improvement, but it is recommended that in such cases the assumed source level always be quoted. (Figure 3.8 assumed source levels and depth dependence based on Weston [10]; see also Table 3.III.) It may be seen that the transmission loss increases as either boundary is approached, because each boundary acts as a free surface. This is true for the bottom because the measurements were made in August when there is apparently a large quantity of gas held there. The details of the curve shapes may be explained as due to the addition of a number of modes. In August there is a strong thermocline at 15 fathoms. Thus the lowest modes are trapped below the thermocline and tend to produce a signal maximum at about 25 fathoms: some intermediate modes produce a maximum above the thermocline near 7 fathoms, and the higher modes show little depth dependence. It is possible to make the arguments for Figs. 3.7 and 3.8 quantitative, and learn about bottom character, etc.

\subsection{RELATIVE ADVANTAGES OF UNDERWATER EXPLOSION SOURCES}

The competitor to the underwater explosion source is the projector radiating continuous waves. Comparative measurements of transmission loss have been made at various times using projectors and explosives, and when done carefully they agree on average. However, a pure-tone continuous wave transmission will often show very large fluctuations due to interference effects, which are smoothed out when using the larger bandwidth in the charge experiment. Thus, to study average transmission loss one should use shots or a projector radiating bandlimited noise; whereas to study fluctuations one would probably use a pure tone from a projector.

In summary, most measurements may be made with either source. There are many measurements that can only be made with a continuous wave source. There are a few measurements which virtually can only be made with explosions; for example, to study low-level arrivals one needs the very high pulse power of an explosion. In practice, experimental convenience plays a big part; a shot needs no auxiliary equipment and is almost unrestricted in depth.

In passing, I want to comment on Dr. Schofield's [7] remarks on the necessary sizes of projectors for reasonable efficiency, and to consider production of acoustic energy at $1000 \mathrm{cps}$. Here the electroacoustic device needs to be about 2 ft across, whereas a spherical charge of only about 2 in. in diameter (say, $1 / 2 \mathrm{lb}$ TNT) converts about half its chemical energy into acoustic energy centered at $1000 \mathrm{cps}$. This high efficiency at low frequencies is only possible because of the finite amplitude effects near the source. I could have stretched my case further since the same 2-in. charge would have a bubble-pulse frequency of the order of $20 \mathrm{cps}$, but this would be cheating since the $20 \mathrm{cps}$ is really associated with the maximum bubble diameter of a few feet.

In conclusion, to be well equipped for research one needs to be able to use both continuous wave and explosion sources, depending on the experiment. Personally, I consider this likely to be the position for a long time to come. 


\subsection{ACKNOWLEDGMENTS}

Figures 3.3, 3.4 and 3.5 are reproduced from the Proceedings of the Physical Society published by the Institute of Physics and the Physical Society [10]; and Fig. 3.6, from the Geophysical Journal published by the Royal Astronomical Society [9].

\section{REFERENCES}

1. A.B. Arons, "Secondary Pressure Pulses Due to Gas-Globe Oscillations in Underwater Explosions: II. Selection of Adiabatic Parameters in the Theory of Oscillation, " J. Acoust. Soc. Am., Vol, 20, 277282 (1948).

2. A.B. Arons, "Underwater Explosion Shock Wave Parameters at Large Distances from the Charge," J. Acoust. Soc. Am., Vol. 26, 343-346 (1954).

3. R.H. Cole, Underwater Explosions (Princeton Univ. Press, Princeton, New Jersey, 1948).

4. C. Herring, "Physics of Sound in the Sea, Part I, Transmission," NDRC Summary Technical Report, Div, 6, Vol. 8 (1946).

5. F. J. McDonal, F. A. Angona, R. L. Mills, R. L. Sengbush, R. G. van Nostrand, and J. E. White, "Attenuation of Shear and Compressional Waves in Pierre Shale," Geophysics, Vol. 23, 421-439 (1958).

6. P. N.S. O'Brien, "Seismic Energy from Explosions," Geophysics, Vol. 3, 29-44 (1960).

7. D. Schofield, "Transducers," Inst. on Underwater Acoustics (1961).

8. "Underwater Explosion Research," Office of Naval Research, Joint Anglo-American Compendium of Reports in three Volumes (1950).

9. D. E. Weston, "The Low-Frequency Scaling Laws and Source Levels for Underground Explosions and other Disturbances," Geophysics, Vol. 3, 191-202 (1960).

10. D. E. Weston, "Underwater Explosions as Acoustic Sources," Proc. Phys. Soc. (London), Vol. 76, 233249 (1960).

\section{DISCUSSION}

PROFESSOR M. FEDERICI asked whether the change of shape of the shock wave, as it progresses, had any influence on the scale law.

MR. WESTON: The explosion-similarity principle takes account of the main changes in the shock-wave shape; but, to proceed from there to the $W^{1 / 3}$ and $W^{4 / 3}$ spectrum scaling laws for a fixed range, it is necessary to assume spherical spreading. In fact, the peak pressure falls off faster than this (with an extra factor of $\mathbf{1 . 1 3}$ in the index) and, at the same time, the shock-wave duration increases slowly. This necessitates some small corrections to the above laws, leading, for example, to a slight increase in the expected dependence of highfrequency spectrum level on charge weight. At still higher frequencies or at long range, there is, in addition, a falling-off in spectrum level due to a lengthening of the shock rise time. This last effect is not taken into account in the explosionsimilarity principle, since the latter implicitly assumes an instantaneous rise.

PROFESSOR T.S. KORN asked the order of velocity change in the shock wave as compared with the normal sound wave and whether the normal laws of diffraction were applicable. He also inquired if a slow-burning explosive material led to some improvement in the over-all efficiency as a source.

MR. WESTON: Very near the charge, the shock velocity is a few times greater than the normal sound velocity; but, at distances of several charge radii, it is only fractionally greater. I think that some modifications to normal diffraction theory might be necessary. On the second point, the underwater acoustic outputs of slow-burning and high explosives are very different. The main wave from the former is of rounded form; compare the sharp rise of the 
high explosive shock. The total energy in the pulse from the slow-burning explosive is much less than for the high explosive. Thus, the former may be regarded as a low-efficiency acoustic source, whereas it is unlikely the efficiency value of about 50\% for a high explosive could be much improved. The pulses from the two explosive types have a comparable impulse and, therefore, comparable low-frequency energy, but the rounded pulse produces negligible high-frequency energy. Also the gas bubble from the slow material does not greatly overshoot its equilibrium radius, so there is little bubble oscillation and very little energy in the bubble pulse. Since the bubble pulses contribute a good proportion of the low-frequency energy for high explosives, the net result is that the slow material is down over most of the spectrum (very low frequencies have not been investigated and could be an exception). 


\title{
LECTURE 4
}

\section{THE IMPROVEMENT OF VIBRATION ISOLATION}

\author{
G. G. Parfitt \\ Imperial College of Science and Technology \\ London, England
}

\subsection{INTRODUCTION}

The noise and vibration generated by a ship's engines and machinery will to a greater or lesser degree be transmitted to its hull plating by air-borne and, particularly, structure-borne paths and be radiated into the surrounding sea, thus helping to disclose the ship's presence to passive listening devices. Effective isolation of machinery vibration from its supporting structure may thus be of especial importance in naval applications. The present paper examines the elementary principles of vibration isolation and discusses various potential means of improving on the simplest form of spring isolator. This discussion is based mainly on work done at Imperial College and the University of Michigan by Dr. J.C. Snowdon.

The simplest idealized resilient isolation system may be depicted as in Fig. 4.1a by a simple spring of stiffness (force-to-displacement ratio) $s$ supporting a machine, represented by a simple mass $M$, above an infinitely rigid foundation. The mass is supposed free to move only in the vertical direction, under the action of an applied force $F$. A force $F_{t}$ is transmitted to the foundation, and the ratio $F_{t} / F$ is termed the transmissibility $T$ of the system, or in decibels

$$
T=20 \log \frac{F_{t}}{F}
$$

Here, $1 / T$ or $-T$ in decibels is a measure of the isolation. The system has a natural resonance frequency $\omega_{0} / 2 \pi$ given by

$$
\omega_{0}^{2}=\frac{S}{M}
$$

If the spring stiffness $S$ is linear and frequency-independent, the downward deflection $d$ of the spring under the weight $\mathrm{Mg}$ of the supported body is given by $S d=M g$, whence

$$
\omega_{0}^{2}=\frac{S}{M}=\frac{g}{d}
$$




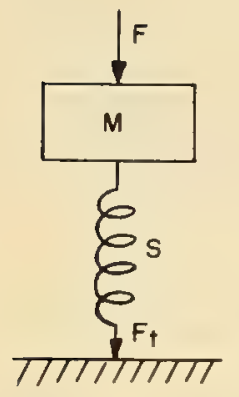

$\omega_{0}^{2}=S / M$

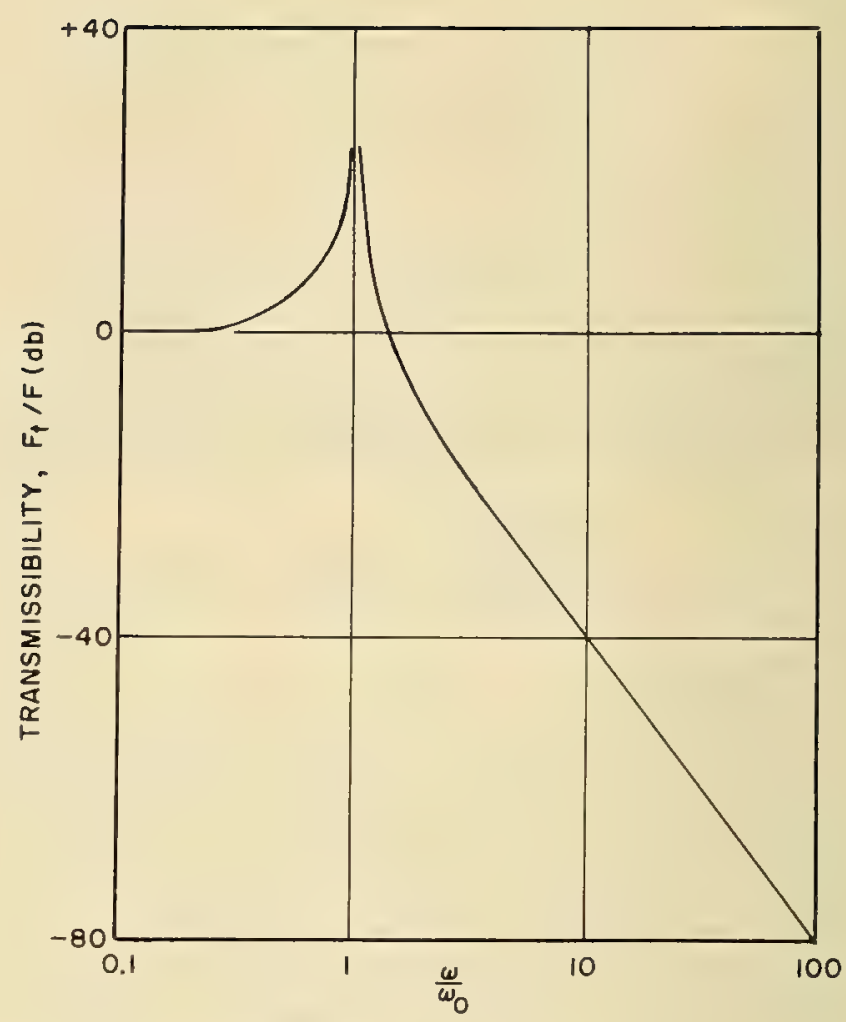

(b)

Fig. 4.1. The idealized simple mounting system and its transmissibility curve.

If the forces concerned are sinusoidal, with amplitudes $F$ and $F_{t}$ and frequency $\omega / 2 \pi$, the transmissibility as a function of $\omega$ is as given in Fig. 4.1b. No isolation is achieved unless $\omega>\sqrt{2} \omega_{0}$. At high frequencies $T$ varies as $1 / \omega^{2}$, as in Eq. (8), i.e., decreases at $12 \mathrm{db}$ per octave. It is therefore necessary that $\omega_{0}$ fall below all significant frequency components present in the spectrum of the exciting force $F$. If it is not practicable to make the springs soft enough to achieve this-and Eq. (3) shows that irrespective of the nature of the spring, so long as it is linear and frequency-independent as stated, a low natural frequency necessarily requires a large static spring deflection-then $\omega_{0}$ must fall between and not close to any strong low-frequency spectrum components. The process of isolation is of course a purely reactive one, and no means of a bs o r bing vibrational energy is necessarily involved.

\subsection{ADDITIONAL CONSIDERATIONS}

The foregoing simple theoretical predictions are in general well borne out by measurements on mountings of a mechanically simple character [1]. Effects of finite damping, to be discussed below, of course modify the transmissibility 
curves, and an additional feature in practice is the presence at high frequencies of so-called "wave effects." These are the result of standing waves within the body of the resilient material and lead to some loss in isolation. They are not usually large in rubber mounts, but may be serious in, say, steel coil springs with very low damping. For this reason it is common to insert a rubber pad under a coil spring mount to give additional high-frequency isolation.

In spite of the fact that it forms a very useful starting point for discussion, the system of Fig. 4.1a is nevertheless a substantial oversimplification of a practical mounting system in several respects. First, practical mechanisms do not normally have only one degree of freedom, but many degrees. There will thus be many natural modes of oscillation, and hence many resonances; in general there will be coupling between them. These effects are likely to complicate rather than invalidate the discussion which follows, and they will be ignored henceforth. They are of course extensively dealt with in standard texts [2 to 5].

Secondly, the foundation on which the isolator rests will never be infinitely rigid, particularly in marine applications, and this will modify the transmissibility. In such cases of nonrigid foundations, it is often more instructive to use in place of $T$ some other measure of isolation describing the extent to which the motion of the foundation is reduced by the isolator. One which has been used [6] is the so-called response ratio $R$, namely,

$$
R=\frac{\text { amplitude of foundation under isolator }}{\text { amplitude of foundation with } M \text { rigidly attached to it }}
$$

The same exciting force at the same frequency is assumed applied to $M$ in each case. "Amplitude" here may refer to the displacement, velocity, or acceleration of the foundation, or even to the force upon it, for at a given frequency force will be proportional to motion. If the mechanical impedance of the foundation is at all frequencies very large compared with that seen looking into the base of the mount, then $R$ is identical with $T$ as shown in Fig. 4.1b.

If the foundation consists of a pure mass $M_{i}$ or a pure spring of stiffness $S_{f}$, the resulting response-ratio curves are those shown in Figs. 4.2 and 4.3, respectively. The curve for the mass foundation is of the same form as Fig. $4.1 \mathrm{~b}$, but the resonance frequency is increased, leading to a reduction of isolation (increase in $T$ ) at high frequencies in the ratio

$$
\frac{M+M_{f}}{M_{f}}
$$

With an elastic foundation the resonance frequency is somewhat less than with a rigid foundation (negligibly so in the case shown in Fig. 4.3), and the curve contains a trough due to removal of the original resonance of the main mass $M$ on the elastic foundation [which gives a maximum for the denominator of Eq. (4)]. Above this frequency the response ratio reaches the constant value $S /\left(S+S_{i}\right)$. Thus, in both these cases the response ratio, i.e., the gain from inserting a spring mount, is less at high frequencies than it would be with a rigid foundation. However, it is worth noting that this is not so much due to an impairment of the action of the mount, but to the fact that before the mount is inserted the inertia of the body to be supported is already relieving the foundation of part 


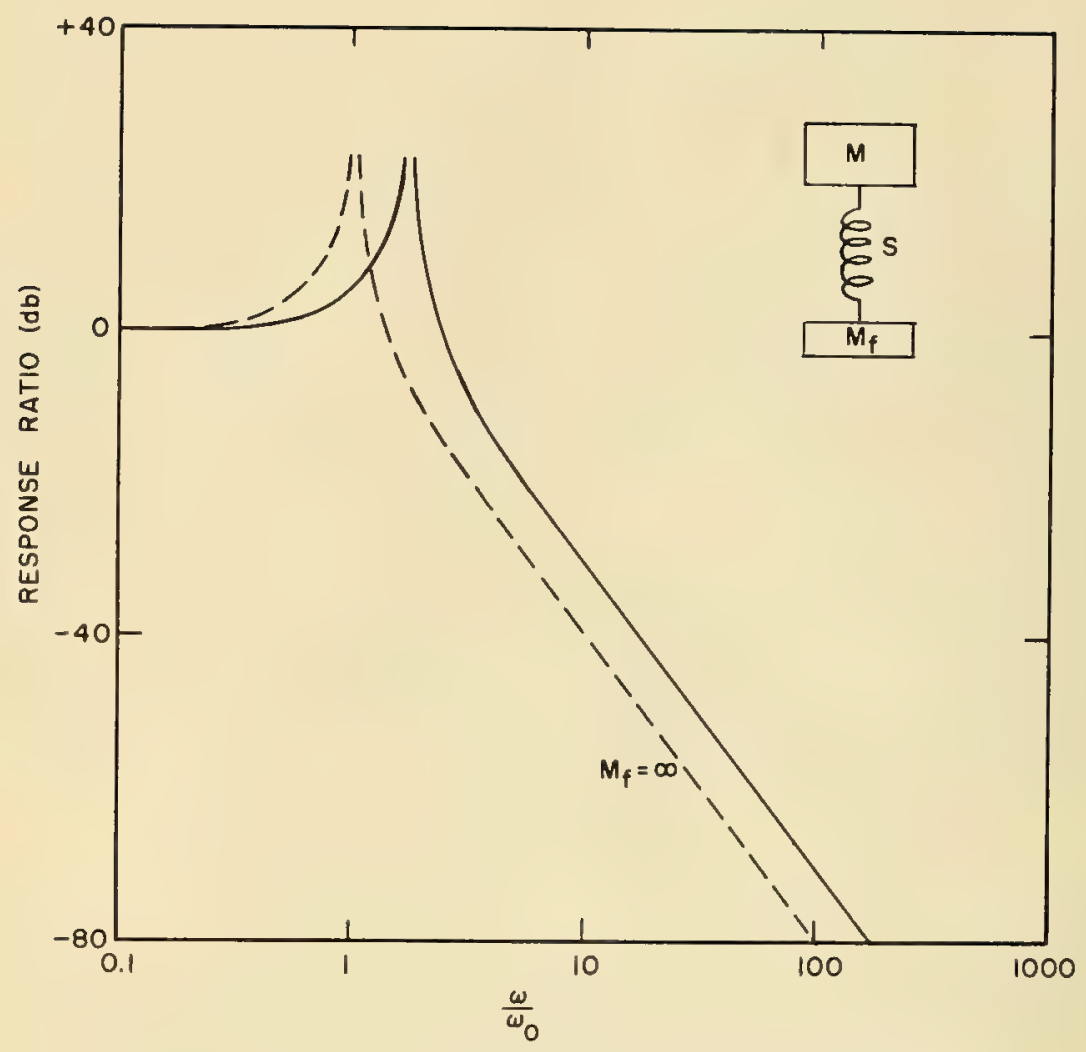

Fig. 4.2. Response ratio of a mount on a masslike foundation (schematic).

of the applied force, i.e., some isolation is already present. This effect vanishes as the foundation becomes increasingly rigid.

In practice, foundations would have both spring- and masslike properties, with one or many internal resonances. Thus a foundation with one resonance gives a response-ratio curve (Fig. 4.4) which is essentially a combination of those of Figs. 4.2 and 4.3 , but it has a new peak corresponding to the foundation resonance. Above this the curve shows a shift to the right compared with that for a rigid foundation, as in Fig. 4.2 , and the loss in isolation is again $\left(M+M_{f}\right) / M_{f}$. With a multiresonant foundation such as a metal beam, the response ratio is as in Fig. 4.5, showing now a progressive shift to the right.

A word of warning about the interpretation of such curves may be in order. In general, apart from the basic mounting resonance, they will show pairs of adjacent troughs and peaks, representing the positions of the foundation resonance before and after inserting a mount. Thus the existence of such a peak, i.e., a region of poor isolation, is not necessarily an indictment of the mount's performance, but simply indicates that a shift of a resonant frequency has occurred which may or may not be deleterious depending on whether the peak moves closer to or further from any strong components in the excitation spec- 


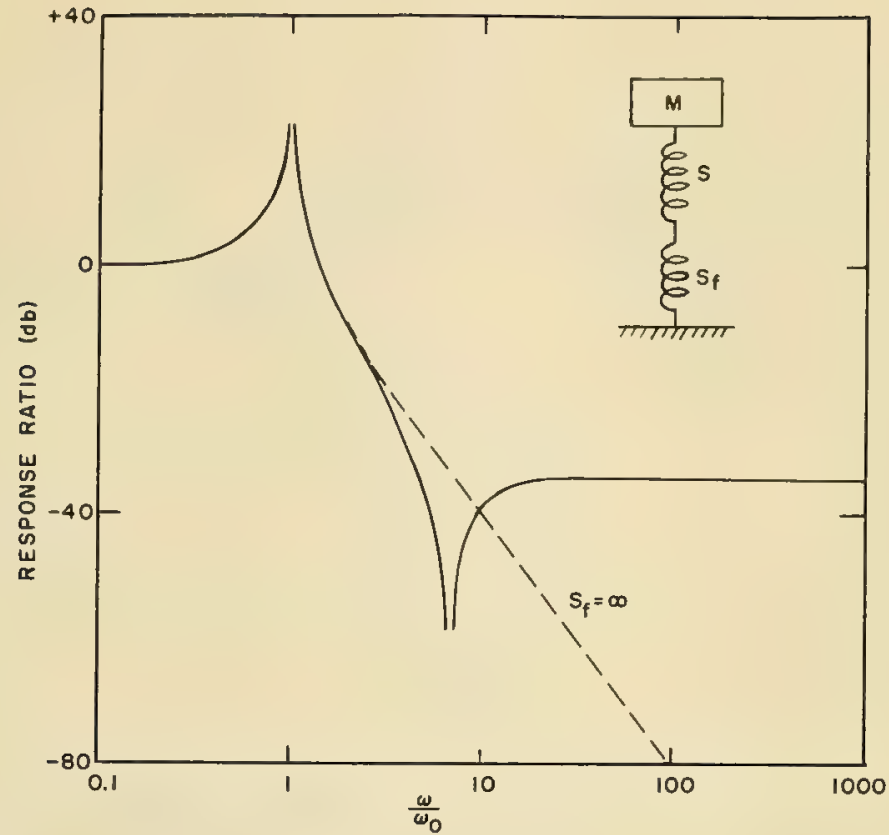

Fig. 4.3. Response ratio of a mount on an elastic foundation (schematic).

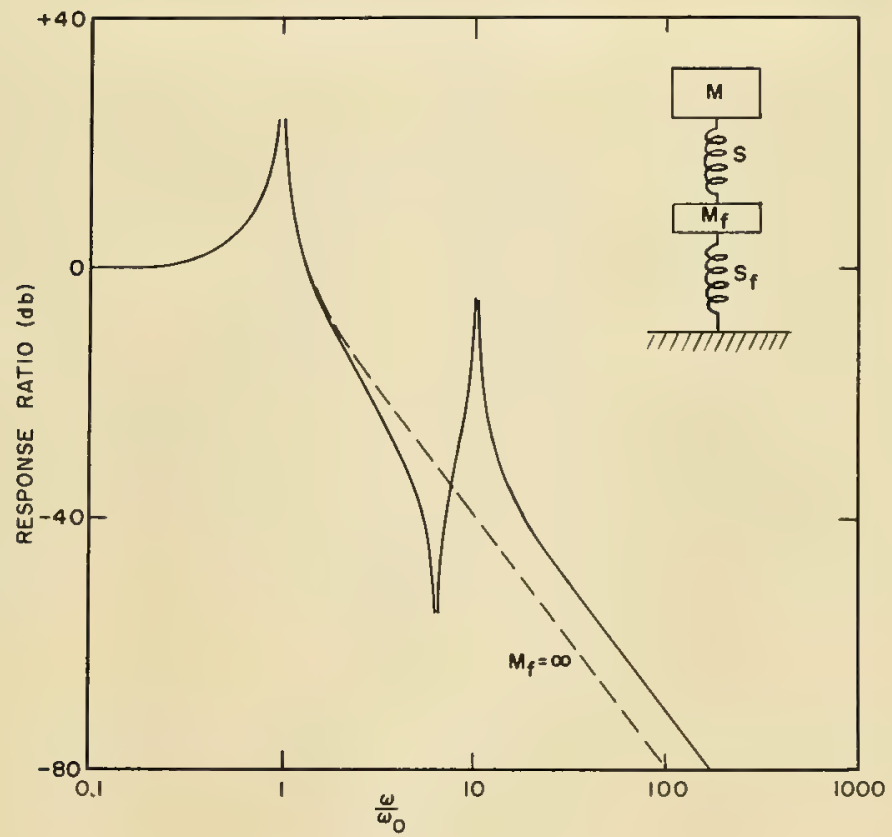

Fig. 4.4. Response ratio of a mount on a resonant foundation (schematic). 


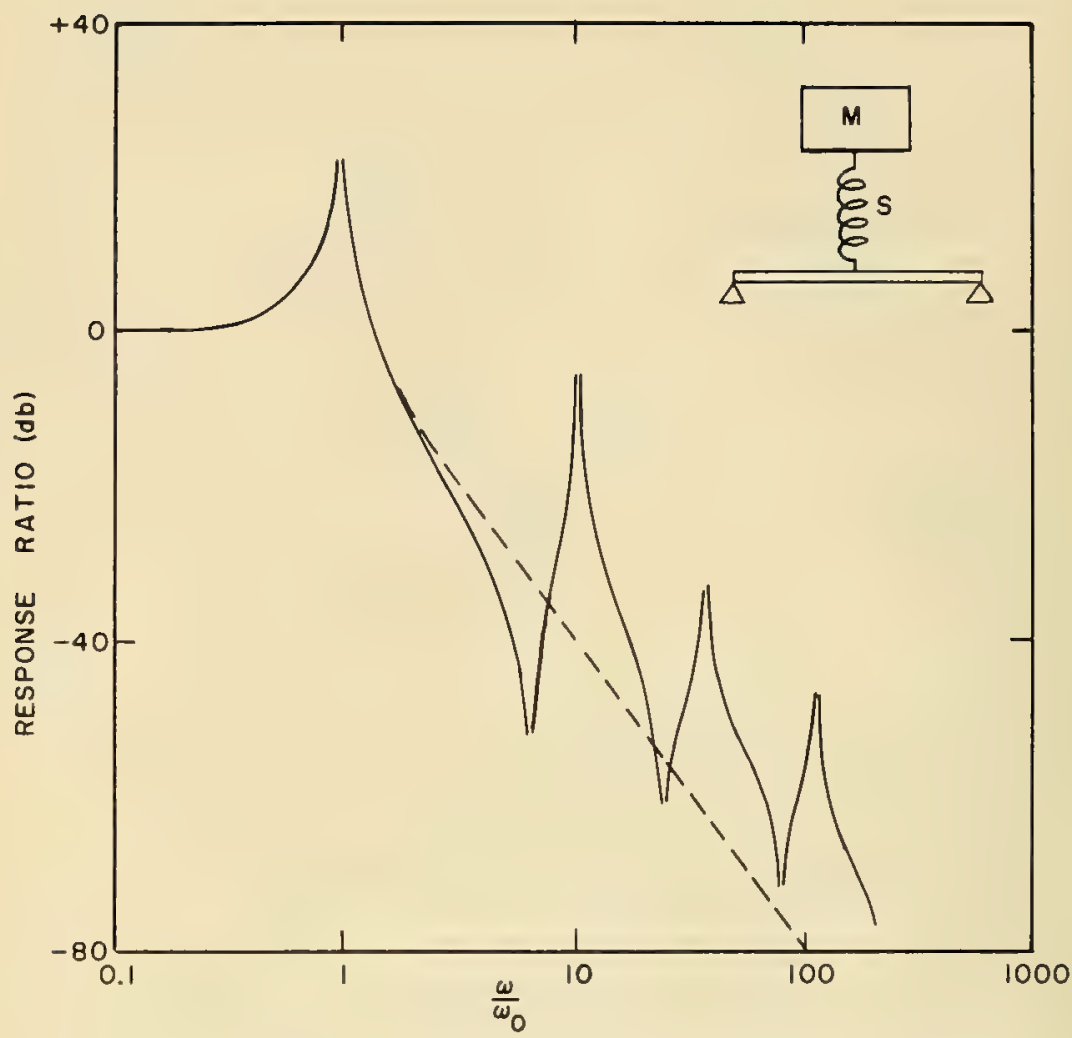

Fig. 4.5. Response ratio of a mount on foundation with multiple resonances (schematic).

trum. A more generally significant feature of the response-ratio curves is the presence of any upward or downward shift of general level.

A third way in which Fig. 4.1a is an oversimplification is in that the mounted item will not in general behave as a pure mass except at low frequencies. This effect has not been widely studied in the literature, but might be expected to produce effects qualitatively rather similar to those of nonrigid foundations.

Three main directions are apparent in which the performance of a simple spring isolator of given static stiffness could be improved. The first is to reduce its resonance frequency with a given mass. This can only be done by making the dynamic stiffness decrease with increasing frequency or with increasing load. Unfortunately, in all real materials where stiffness varies with frequency, the change is in the undesired direction and so merely detrimental. Systems can, however, be devised which are nonlinear in such a way that the dynamic stiffness for small vibratory motion is well below the average stiffness controlling static or quasi-static deflection. One such system is a strut which is buckled under the load of the machine; another employs two conventional springs, one tending to pull the mass away from its equilibrium position due to the other [2]. One difficulty with such systems is that of making them effective for motion in more than one direction. 
A second improvement in a mount is to reduce the height of the basic resonance peak in its transmissibility curve by damping, or otherwise. One obvious reason for this is that, particularly in the presence of random vibrational forces, it may not be entirely possible to avoid excitation at the resonance frequency. Other secondary reasons are the avoidance of temporary resonance during the run-up or stopping of a rotary machine, the minimization of oscillation following shock or other stray disturbance, and, as will be shown below, a possible damping of foundation resonances.

A third beneficial step is to increase the rate at which isolation increases above the resonance, so as, for instance, to reduce the levels attained by the foundation resonance peaks of Fig. 4.5.

Such ends can be achieved by damping or by adding mass to the system in various ways, and the resulting effects will be considered in what follows.

\subsection{DAMPING OF SPRING ISOLATORS}

If the isolator spring is damped, its performance for sinusoidal forces can be represented by treating its stiffness as a complex quantity $S^{x}$, viz.,

$$
S^{x}=S(1+j \delta)
$$

where $\delta$ is the damping coefficient. Both $S$ and $\delta$ may be functions of frequency in general. One important case, to which many fairly lightly damped solid materials conform approximately, is that where $S$ and $\delta$ are constants. Another is the case of pure viscous damping, in which $S$ is constant and $\delta$ is proportional to frequency. Writing

$$
\delta=2\left(\frac{\omega}{\omega_{0}}\right)^{\bar{\delta}}
$$

then defines the constant $\bar{\delta}$, the damping ratio, which is equal to the ratio of the actual damping present to that required to give critical damping of the system at its resonant frequency.

The transmissibility $T$ for a damped mount on a rigid foundation is given in general by

$$
T^{2}=\frac{1+\delta^{2}}{\left(1-\omega^{2} S_{0} / \omega_{0}^{2} S\right)^{2}+\delta^{2}}
$$

where $S$ is the stiffness at frequency $\omega$ and $S_{0}$ that at the resonance frequency $\omega_{0}$. This equation is plotted for $\delta=0$ in Fig. 4.1b.

Introduction of heavy damping obviously will reduce the resonance peak, but in general will detract from the high-frequency isolation. Thus, with $S=S_{0}$ equal to a constant and $\delta$ constant, Eq. (7) reduces for $\omega \gg \omega_{0}$, to

$$
T=\left(1+\delta^{2}\right)^{1 / 2}\left(\frac{\omega_{0}}{\omega}\right)^{2}
$$

or for $S$ and $\bar{\delta}$ constant [Eq. (6)] to

$$
T=2 \bar{\delta}\left(\frac{\omega_{0}}{\omega}\right)
$$




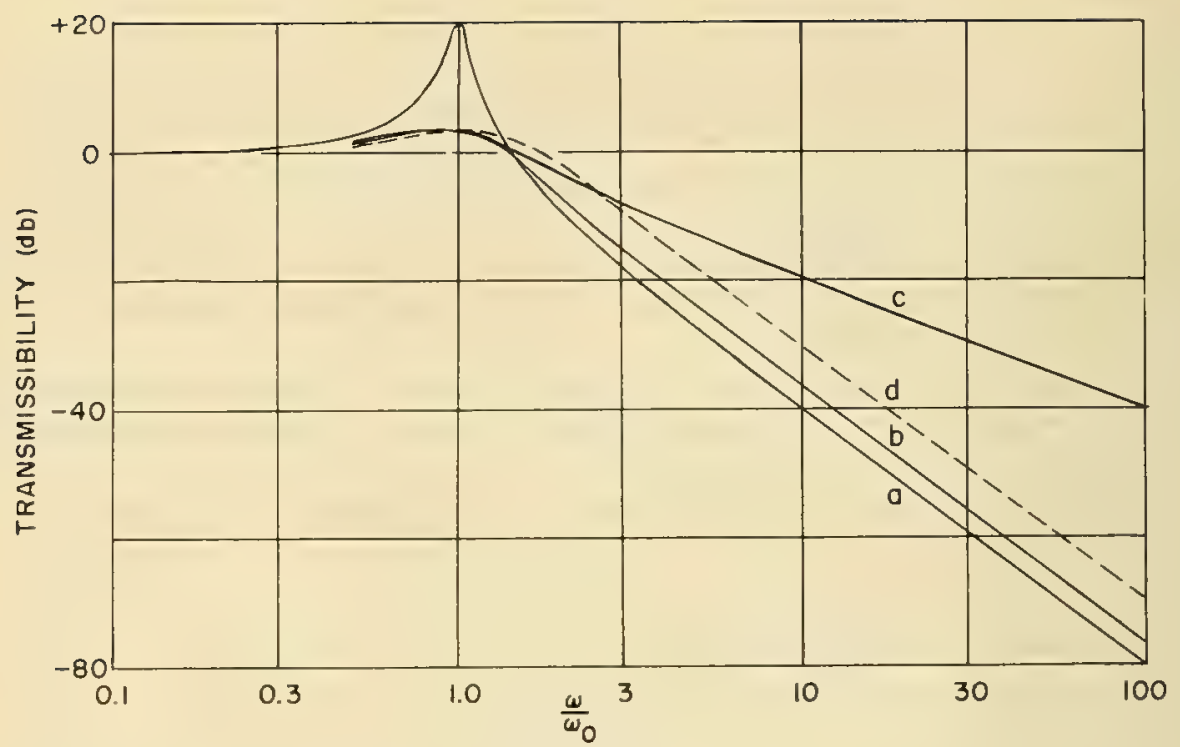

Fig. 4.6. Transmissihility curves of simple mounts with various types and degrees of damping. (a) $\delta=0.1$, (b) $\delta=1.0$, (c) $\delta=0.5$, (d) modulus and damping as in Fig. 7c.

Equation (8) represents only a relatively small loss of isolation compared with an undamped mount, but Eq. (9) gives a serious loss due to the large viscous stress set up at high rates of shear (or high frequencies). The full transmissibility curves are shown in Fig. 4.6, curves b and c. High damping of the order of $\delta=1$ can be obtained in high polymers, and though there it may not be greatly frequency-dependent, it tends to be accompanied by an elastic modulus which increases with frequency. In fact, it can be shown [7] that in a typical polymer having a wide spread of viscoelastic relaxation times there is an intrinsic relation between the two, viz.,

$$
\delta \div \frac{\pi}{2} \frac{d(\log S)}{d(\log \omega)}
$$

Thus, for example, $\delta=1$ implies an increase of some 19 times in stiffness over a frequency range of two decades. The increasing stiffness leads to a loss in isolation (relative to that of an undamped mount) which in practice is similar to that caused by large viscous damping (curve $c$ of Fig. 4.6). Thus, for example, if in Eq. (7) $S / S_{0}=\omega / \omega_{0}$ and $\delta$ is constant, then for $\omega \gg \omega_{0}$

$$
T=\left(1+\delta^{2}\right)^{1 / 2}\left(\frac{\omega_{0}}{\omega}\right)
$$

which has the same frequency dependence as Eq. (9). In addition, polymers with high damping are rather prone to show substantial creep or "compression-set" effects under a static load.

The frequency dependence of viscous damping, such as may be obtained from a simple dashpot device, may be mitigated by the use of a multiple isolator element containing two springs and a dashpot, as in Figs. 4.7a or 4.7b. These two forms are identical in performance and have an effective stiffness and a 
(a)

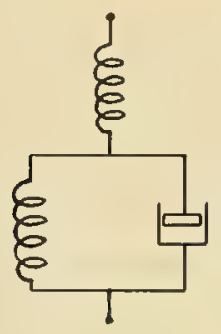

(b)

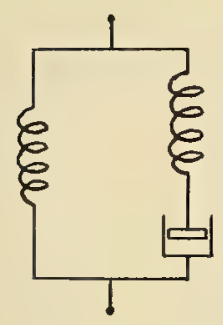

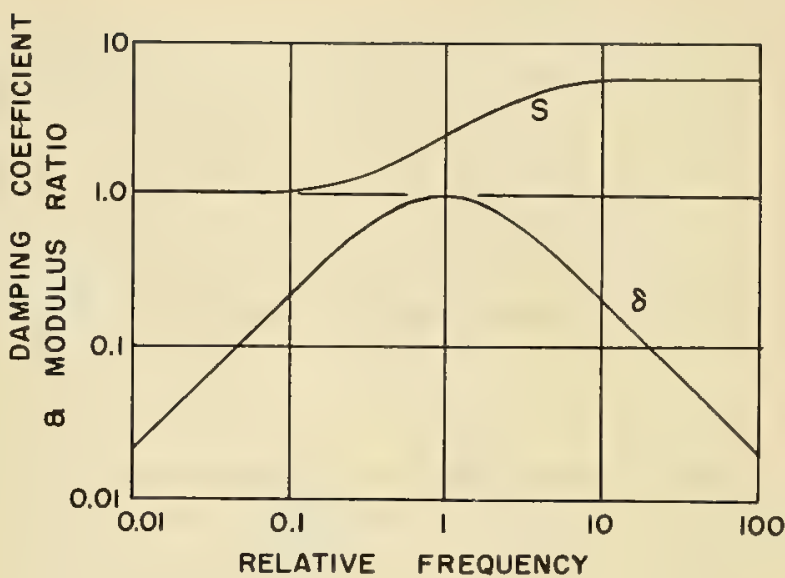

(c)

Fig. 4.7. A damped spring system with a single relaxation time $(a, b)$ and its modulus and damping spectra (c).

damping factor both of which vary as in Fig. 4.7c. Stiffness still increases with frequency but does so now only over a limited range of frequencies. For a maximum damping factor of unity the over-all increase of modulus between very low and very high frequencies is 5.8 fold. If such a system is arranged to have a maximum damping factor of magnitude unity placed at the resonance frequency of the mounting system, then the resulting transmissibility curve would be that given by curve $d$ in Fig. 4.6. It is seen to achieve a low resonant transmissibility with less sacrifice at high frequencies than the simple viscous damper or the high-damping polymer. The systems of Fig. 4.6 are compared on the basis of a common resonance frequency. A fairer basis of comparison would be that of a common static stiffness, as this determines the static stability of the equipment. In this case the relaxation curve d would be shifted to the right by a frequency factor of about 1.6. However, the curve for a high-damping polymer, at present indicated as coinciding approximately with the viscous-damping curve, would be shifted considerably more owing to the larger ratio of high-frequency to static moduli.

Snowdon [8] has studied the properties of a mount consisting of sections of a lightly damped natural rubber (hevea) and of a highly damped polymer placed mechanically in parallel. By suitable choice of material and relative cross section it can be arranged that the natural rubber provides most of the stiffness, which is therefore not strongly dependent on frequency and not too subject to static creep, while the other polymer contributes considerable damping. In this way a transmissibility curve combining substantial resonance damping with little loss in high-frequency performance can be obtained. Such a mount does not 
escape from the dilemma represented by Eq. (10), but represents a means of obtaining a good practical compromise between the conflicting requirements with available materials.

Snowdon has also shown $[8,9,10]$ that substantial damping in a mount at high frequencies is useful in suppressing any peaks occurring in this region due to foundation resonances (as in Fig. 4.5). This comes about since resonant vibration of the foundation with an antinode below the mount will involve substantial deformation in the mount, the mounted mass remaining almost stationary. Those mounts having high damping at high frequencies may therefore be useful here. A similar effect is of course achieved by adding damping material to the foundation structure [11], and may in particular be more effective for the higher-order modes of the foundation. Moderate damping of the foundation has relatively little effect except near the foundation resonance frequencies.

\subsection{ADDITIONAL MASS IN VIBRATING STRUCTURES}

As might be expected, the transmission of vibration through a system may be reduced by making certain parts of it more massive, particularly in such a way as to introduce or emphasize differences in mechanical impedance between adjoining parts of a structure. Taking the idealized case of a mass m supported by an isolation spring at the center of a beam (Fig. 4.8), an additional mass can usefully be added either to $y$ itself, to the beam either distributed along its length or concentrated at the base of the spring, to the spring at some point along its length, or attached to $/ /$ via an additional spring. These possibilities will be considered in turn.

Increasing the mass of the mounted item merely reduces the resonance frequency $\omega_{0}$ as $M^{-1 / 2}$, with a corresponding gain in high-frequency isolation (for a rigid foundation) proportional to $/$. Alternatively, a stiffer spring, giving greater static stability, may be used for the same resonance frequency. The extra mass also serves to reduce the motion of the machine or equipment itself at high frequencies, and may be used to provide a more rigid base for the machine.

The effect of adding a mass $m$ to the base of the spring has been investigated by Snowdon $[9,10]$ from whose work Figs. 4.9 and 4.10 are taken. Figure 4.9 shows the response ratio for a system in which the mass $\mu_{i}$ of the supporting beam foundation is $1 / 50$ of the supported mass $M$, and in which masses $m$ equaling $0,0.1,0.2$, and 1.0 times $M$ are attached at the beam center. The natural fre-

Fig. 4.8. The idealized simple mount and resonant foundation.

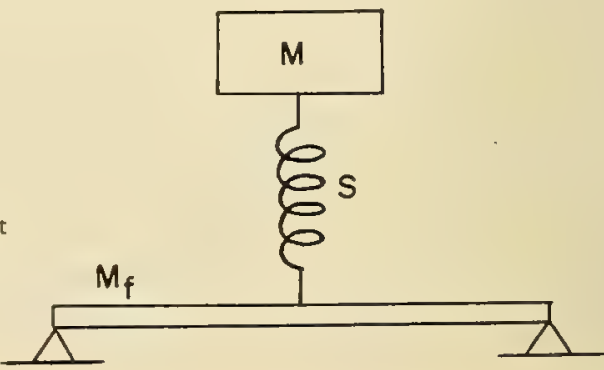




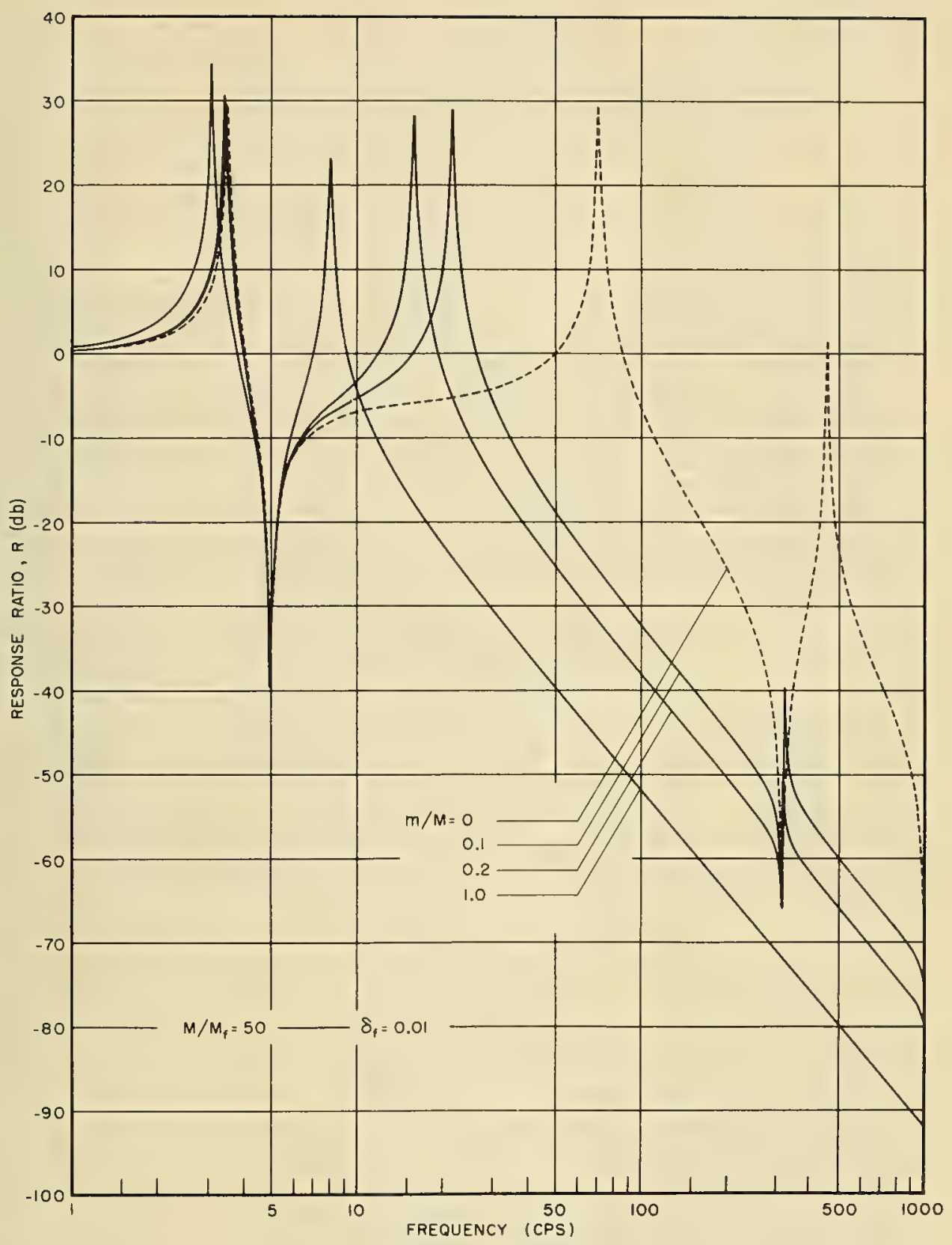

Fig. 4.9. Response ratio of the system of Fig. 4.8 with masses $m$ added at the beam center. $M=50 M_{f}, \delta_{f}=$ $0.01[10]$.

quency of $M$ on the spring mount on a rigid foundation has been chosen as 5 cps, and the beam damping factor $\delta_{t}$ as 0.01 . Response ratio is here taken as the ratio of amplitudes or forces at the beam center after and before the spring 


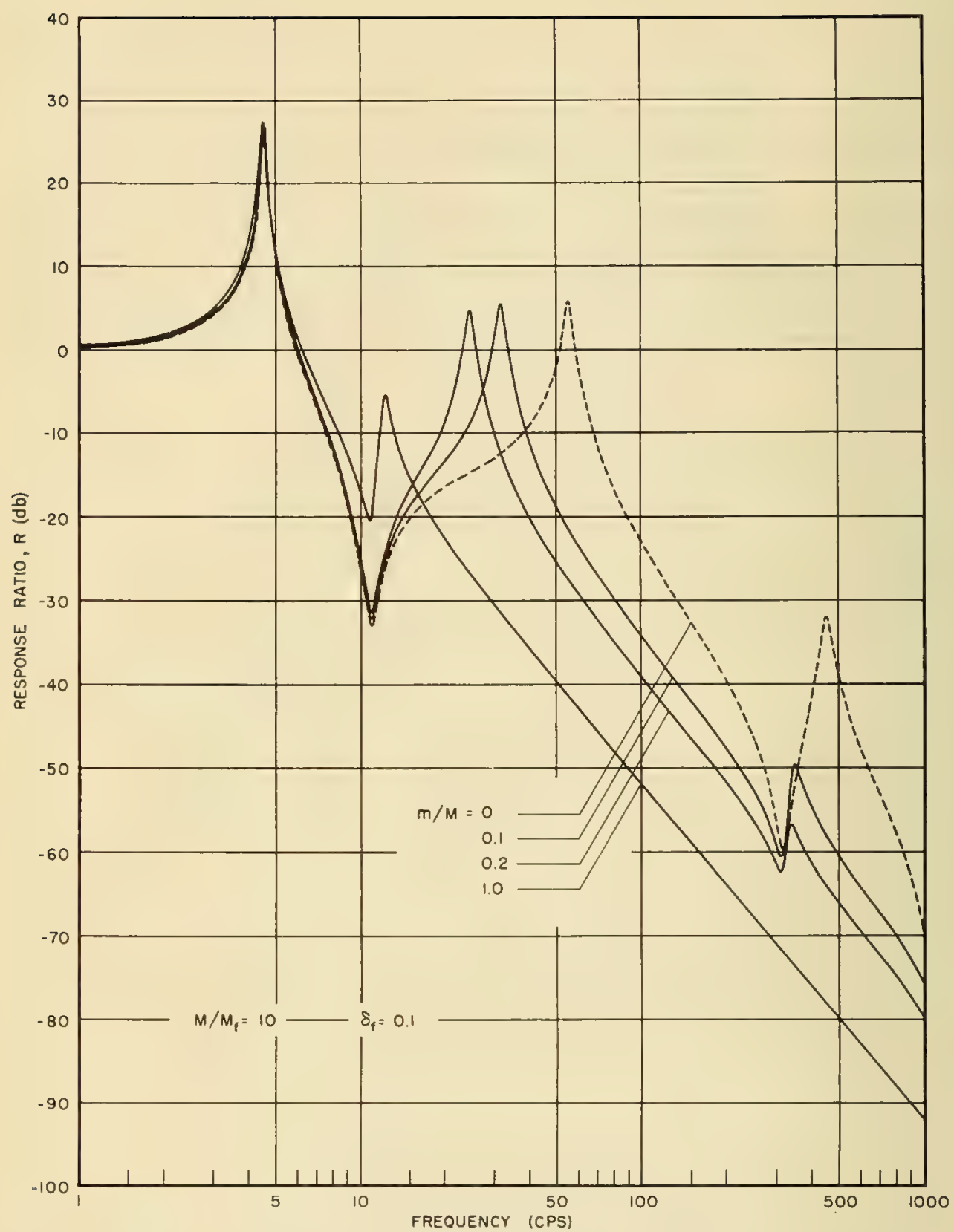

Fig. 4.10. Response ratio of the system of Fig. 4.8 with masses $m$ added at the beam center. $M=10 M_{l}$, $\delta_{i}=0.1[10]$.

mount and the mass $m$ are inserted together. Since the ratio of force applied to the end supports of the beam to the amplitude or force at its center is the same with or without the spring and mass present, the response ratio is also the ratio of support forces after and before the spring and mass are inserted. 
It may be seen that at high frequencies there is a large gain in the effectiveness of the isolation if a mass comparable with the supported mass is added to the beam. For the simple case of the single-resonance foundation used in Fig. 4.4 the response ratio at high frequencies is given by

$$
R=\left(\frac{M+M_{f}}{m+M_{i}}\right)\left(\frac{\omega_{0}}{\omega}\right)^{2}
$$

This equation also describes the high-frequency behavior in Fig. 4.9 tolerably well when $m$ is appreciable. In particular it shows that when $m=M$, then $R=\left(\omega_{0} / \omega\right)^{2}$, which is the value for an undamped mount on a rigid foundation. The process at work here is of course simply that in which the inertia of the mass $m$ relieves the beam of much of the force transmitted through the mount.

Another very apparent effect is that the effective foundation resonance is brought down in frequency by the mass $m$, in this case from some $70 \mathrm{cps}$ to $8 \mathrm{cps}$ for $m=M$. (More exactly, the mass largely nullifies cile effect of the spring mount in detaching the mass $M$ from the beam and allowing the foundation resonance to rise from its loaded value of $5 \mathrm{cps}$ to $70 \mathrm{cps}$.) This could be detrimental if there are strong excitation components in the low-frequency region, such as the rotor frequency of a machine. Figure 4.10 , where the mass $M$ is ten times the beam mass $M_{f}$ and the beam damping factor has been increased to 0.1 , shows the same general effects, but the frequency shifts are of course smaller and the resonant peaks due to beam resonance lower.

It will be observed that the curves for $m / M=1.0$ show no indication of beam resonances other than the lowest, and in fact at higher frequencies agree closely with the transmissibility curve for a mount on a rigid foundation. This should not be taken to imply that the amplifying effects of the higher-order resonances have been eliminated from the system, but only that they are similar with and without the spring mount and mass inserted. Other results of Snowdon show that applying the same additional mass distributed along the foundation beam is less effective than adding a concentrated mass under the mount.

The third possible position for additional mass in the system is at a point subdividing the isolator spring, typically into two equal sections. The isolator can then be regarded as a two-stage unit and has been termed a compound mounting (Fig. 4.11a). Being a two-degree-of-freedom system, it has two natural resonances, and typical transmissibility curves will appear as in Fig. 4.12 [6,9]. These are calculated for a typical vulcanized rubber (hevea). As the ratio $\beta$ of the secondary mass halfway down the spring to the main supported mass $M$ increases, the secondary resonance frequency moves downtowards the first. Above the secondary resonance, the isolation (for little or no damping) increases as $\omega^{4}$ instead of $\omega^{2}$ as for the simple mount $(\beta=0)$. If strong damping is applied to the springs to suppress the resonances, some penalty is paid in high-frequency loss, just as for the simple mounting. It will be seen that unless the secondary mass is of a magnitude comparable with the main mass, any advantage of the compound system over the simple is not realized until a frequency is reached where the isolation of either is already very good.

When used on a nonrigid foundation the effect of adding extra mass in a compound mounting is broadly similar to that of adding it at the base of a simple 
mount, with some additional advantage appearing at very high frequencies. For substantial masses and a light foundation, the secondary resonance peak for a compound mounting appears on the response-ratio curve at a position only a little above that of the foundation with a simple mount and loading mass. The response-ratio curve for high secondary masses is rather less smooth at high frequencies than that for the simple mount with added mass, but, as indicated above, this disadvantage may be more apparent than real.

Figure 4.13 represents a comparison due to Snowdon [9] of various methods of mounting on a light foundation beam of low damping, and employing an added mass of $0.2 \mathrm{~m}$. The "hevea" mount referred to is of a representative vulcanized natural rubber, while the "parallel" mount uses this rubber in parallel with a section of a high-damping polymer as discussed in section 4.3 above. On balance it appears that the simplest and broadly the most effective technique in these circumstances is to use the simple mounting with a substantial mass at its base and moderate damping to minimize the foundation and mount resonances.

A fourth method of employing extra mass to minimize vibration is to attach this mass via a damped spring to the primary vibrating mass. If the added mass and spring are tuned to some resonance frequency of the primary system, then they absorb energy strongly at this frequency and so help to suppress the resonance of the primary system. The device is normally termed a dynamic absorber. Its behavior has been considered by a number of investigators, and Snowdon [12] in particular has recently examined the values of tuning and damping which give optimum suppression when various types of idealized viscoelastic media are used for the absorber spring. Figure 4.14 shows a typical set of transmissibility curves which he derived for the system shown in Fig. 4.11b. The secondary mass $1 / 2$ is attached via a spring with viscous damping to the spring-mounted main mass ${H_{1}}_{1}$. Curves are plotted for various values of mass ratio $\mu=M_{1} /\left(M_{1}+M_{2}\right)$. Thus, for example, $\mu=1 / 2$ corresponds to $y_{1}=y_{2}$. It is seen that, owing to the frequency selectivity of the device, the use of relatively large absorber masses

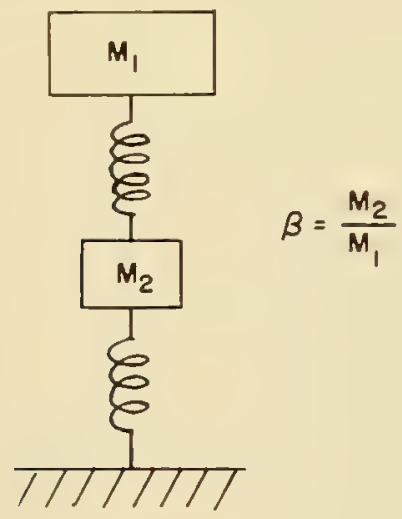

(a)
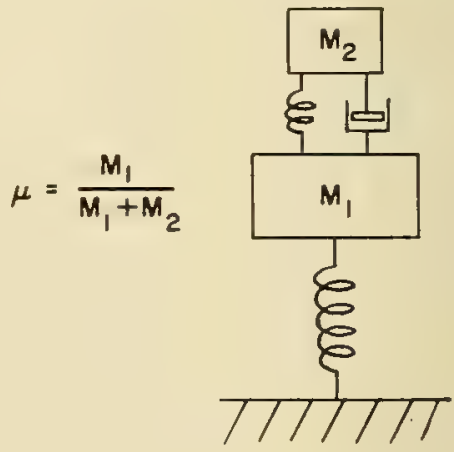

Fig. 4.11. (a) The compound mount and (b) the dynamic absorber. 


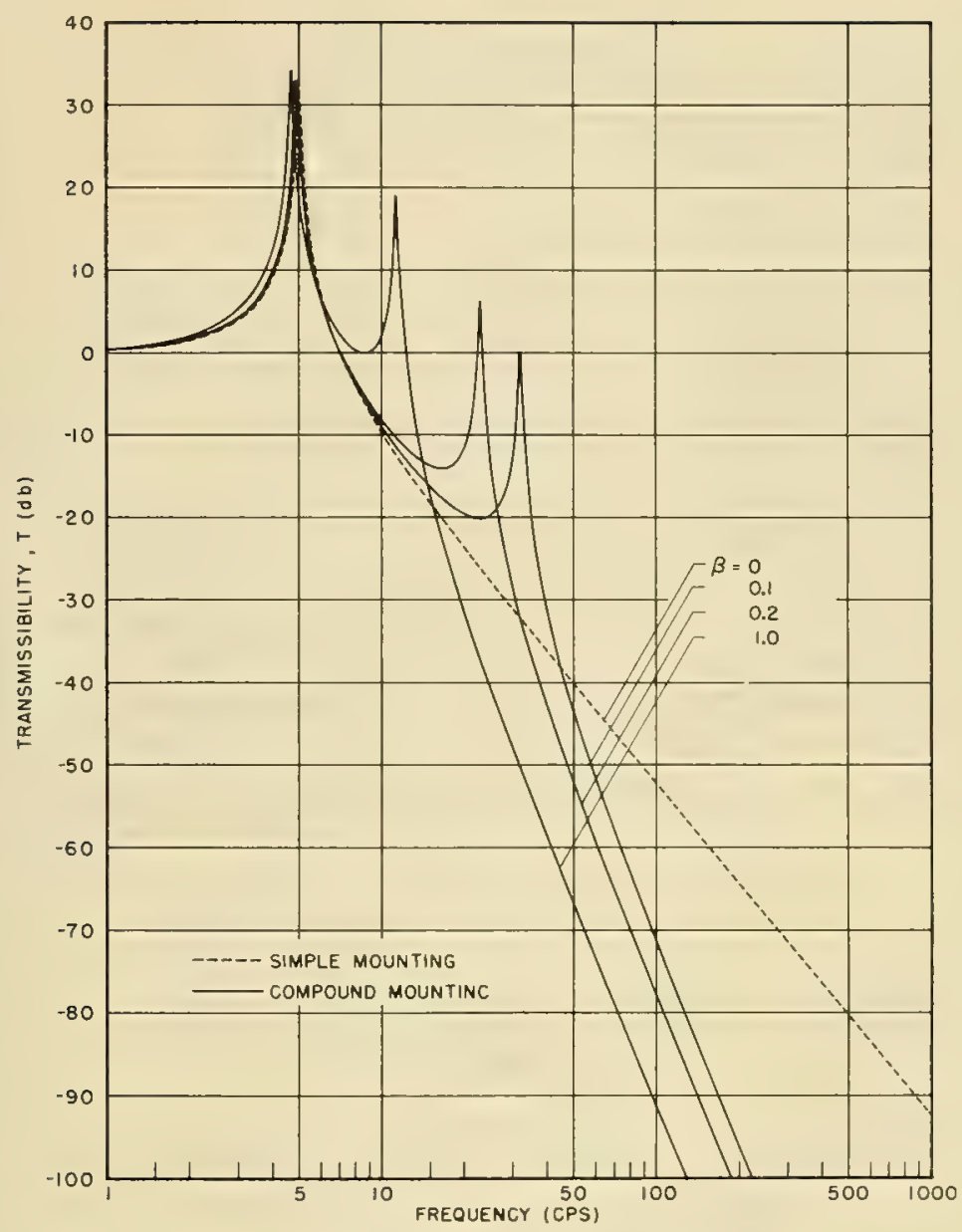

Fig. 4.12. Transmissibility of compound mounts using rubber springs [10].

(with the appropriate optimum damping value) can lead to a suppression of the main resonance comparable with that achieved by heavy damping of the main spring mount, but without the loss of isolation at high frequencies which the latter causes. Snowdon has also shown that a further improvement in performance can be obtained by replacing the viscously damped absorber spring by a viscoelastic material having a high and constant damping factor and a modulus which increases quite rapidly with frequency. Such a material is quite practical to realize.

At frequencies well above resonance, the absorber mass remains almost stationary and the performance of the system becomes virtually independent of its presence. If the three cases considered in Fig. 4.14 has been compared on the basis of a common stiffness in the main spring instead of a common resonance frequency of the complete coupled system, then the system trans- 


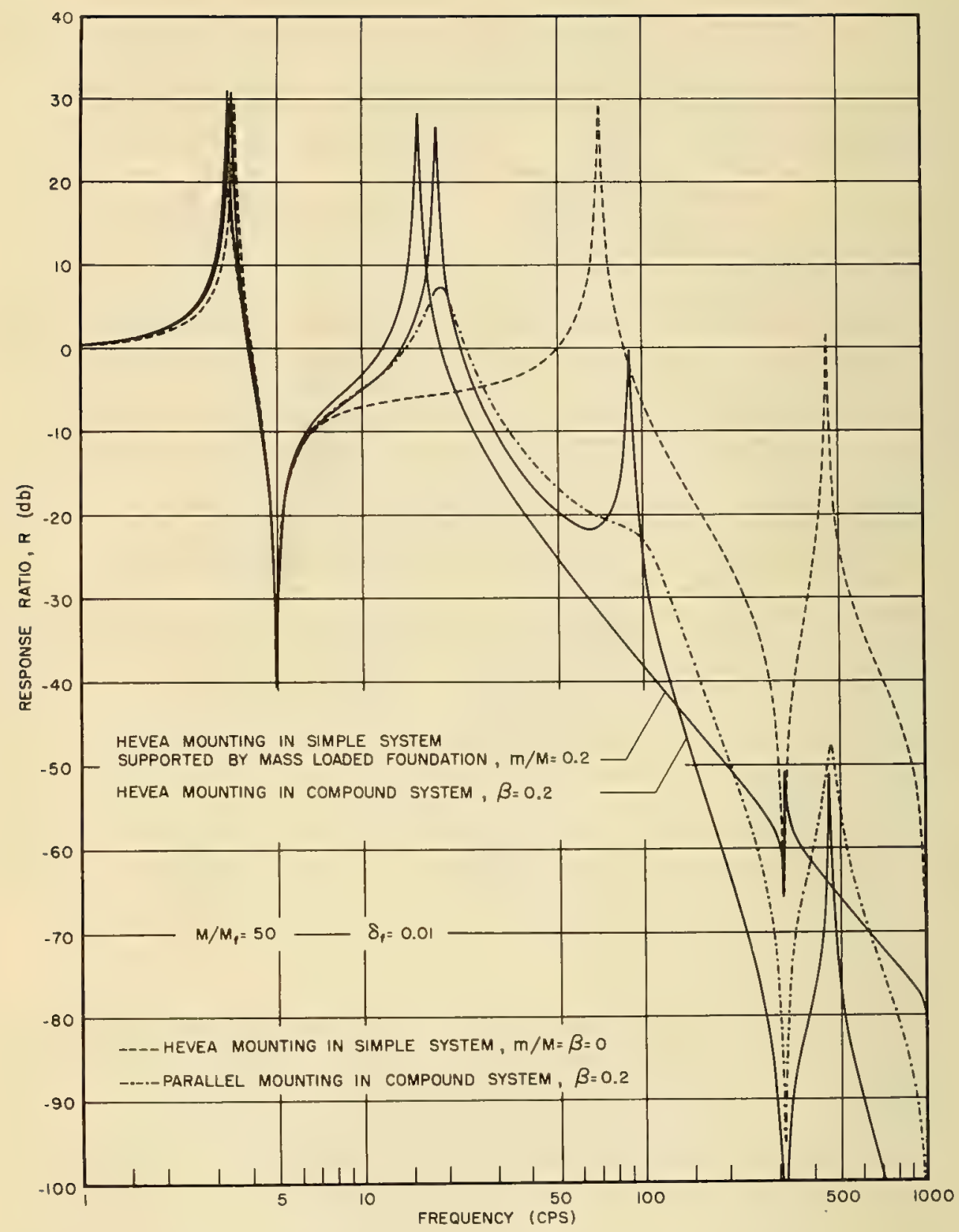

Fig. 4.13. Comparison of various mounting systems on a resonant foundation.

missibility curves would have coincided at high frequencies with each other and with that of an undamped simple mounting system. In view of this it is not to be expected that the dynamic absorber as described would offer any additional 
protection against foundation resonances occurring well above the main resonance frequency of a spring-mounted equipment. However, the device might well be considered for use attached to a foundation beam at the base of the main mounting spring, or elsewhere. A detailed study of this case has not yet been made.

\subsection{SUMMARY}

A review has been given of the performance of the simple resilient antivibration mounting and of means of improving its performance, particularly when it is set upon a foundation which is nonrigid, by addition of damping or mass to the system. It appears that, by suitable choice of mount materials or arrangement, a fairly good compromise between low resonant peaks and good high-frequency isolation can be obtained. Damping of the foundation members may be useful but only at and near the frequencies of foundation resonance.

The addition of substantial masses to the system as force barriers can be beneficial. Probably the simplest method to reduce the excitation of a foundation by this means is to attach the mass to the foundation at the base of the mount. The mass needs to be larger than that of the foundation to have a substantial effect. Although addition of large idle masses in a ship is not an appealing idea, it may occasionally be possible to use for the purpose the mass of other equipment which is already present.

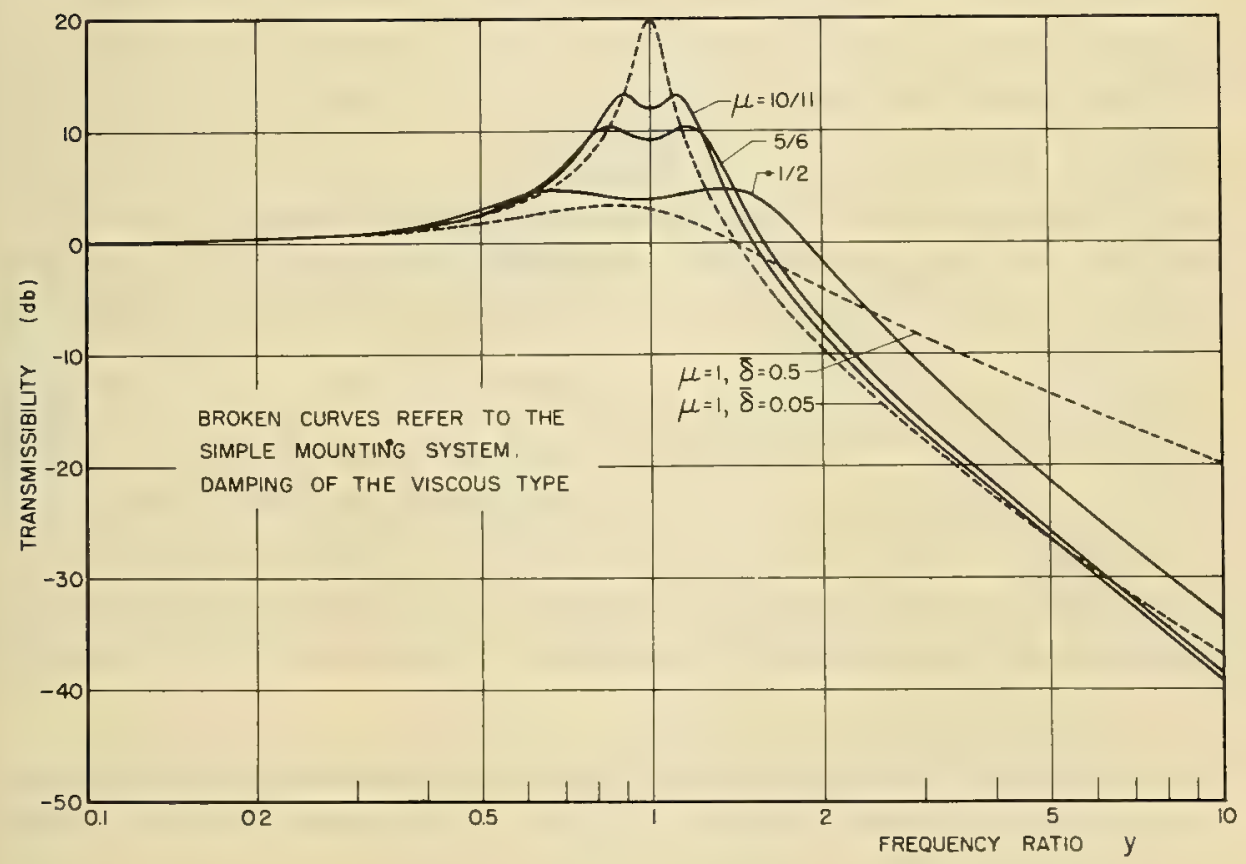

Fig. 4.14. Transmissibility of the dynamic absorber with viscous damping [12]. 


\subsection{ACKNOWLEDGMENTS}

The author is indebted to Dr. J. C. Snowdon and to the Director of The University of Michigan Institute of Science and Technology for permission to reproduce Fig. 4.13.

\section{REFERENCES}

1. J.C. Snowdon, "The Choice of Resilient Materials for Antivibration Mountings," Brit. J. Appl. Phys., Vol. 9, 461-469 (1958).

2. J.N. Macduff and J.R. Curreri, Vibration Control (McGraw-Hill Book Co., Inc., New York, 1958).

3. C. E. Crede, Vibration and Shock Isolation (J. Wiley and Sons, New York, 1951).

4. J.P. den Hartog, Mechanical Vibrations, 3rd ed. (McGraw-Hill Book Co,, Inc., New York, 1947).

5. R.E.D. Bishop and D.C. Johnson, The Mechanics of Vibration (Cambridge Univ. Press, Cambridge, 1960).

6. J. C. Snowdon, "The Reduction of Structure-Borne Noise," Akust, Beih., Vol. 6, 118-125 (1956).

7. J. Heyboer, P. Dekking, and A. J. Staverman, "The Secondary Maximum in the Mechanical Damping of Polymethyl Methacrylate: Influence of Temperature and Chemical Modification," Proc. Second Inter. Cong, on Rheology, Oxford, Vol. 26 to 31, 123-133 (1953).

8. J. C. Snowdon, "Reduction of the Response to Vibration of Structures Possessing Finite Mechanical Impedance, Part 1," Rep. 2892-4-T, Fluid and Solid Mech. Lab., Willow Run Lab., Univ, of Michigan (November, 1959).

9. J. C. Snowdon, "Reduction of the Response to Vibration of Structures Possessing Finite Mechanical Impedance, Part II, " Rep. No. 2892-T, Fluid and Solid Mech. Lab., Willow Run Lab., Univ, of Michigan (January, 1960).

10. J.C. Snowdon, "Reduction of the Response to Vibration of Structures Possessing Finite Mechanical Impedance," J. Acoust. Soc. Am., Vol. 33, 1466-75 (1961).

11. R. N. Hamme, Handbook of Noise Control, C. M. Harris (ed.), Chapter 14 (McGraw-Hill Book Co., Inc. New York, 1957).

12. J. C. Snowdon, "Steady-State Behavior of the Dynamic Absorber," J. Acoust. Soc. Am., Vol. 31, 10961103 (1959); and, "The Steady-State and Transient Behavior of the Dynamic Absorber," Rep. 1941-3-F, Willow Run Lab., Univ, of Michigan (November, 1958).

\section{DISCUSSION}

PROFESSOR T.S. KORN drew attention to the usefulness of the concept of mechanical impedance or of electroacoustical analogies generally in the study of mechanical vibrations. He emphasized the importance of the behavior of elastic mountings when the input impedance of the base is reactive and not infinite. He discussed the concept of the transmissibility factor as the ratio of forces and the possibility of using the velocity ratio or the concept of apparent power transmitted to the base.

DR. PARFITT: I, personally, find the use of mechanical circuit elements of considerable help in visualizing and sometimes in idealizing the behavior of a complex mechanical system, but their application does not, of course, alter the problem or otherwise assist in its solution. The same is true of representation in terms of equivalent electric circuit elements, though there is, here, at least the possibility of actually building the electrical equivalent network and making with it electrical measurements which are often simpler than the analogous mechanical ones.

I certainly agree that calculations such as are given in the paper do not tell the whole story of sound emission from a ship. What has been presented is an attempt to isolate one aspect of the whole complex problem of noise transmission from engine to sea and to see what factors in it are the significant ones, at least in idealized cases. The beam foundation considered in the paper would in practice be attached at its ends to further members of finite impedance 
through which vibrational energy would escape to the ship's outer structure. These terminations would modify the resonance frequencies and damping of the beam and, if they represented tight couplings to further resonanting systems, could introduce further resonances. However, it does not seem likely that these changes would invalidate the general ideas enunciated. Accordingly, it seems reasonable that almost any measure of the beam's motion or of the force upon it can be employed when one wishes to estimate the change in the final noise radiated due to the isolating measures considered. I would admit, however, that although response ratio as used in the paper provides accurate values for the effects of inserting an isolating system, it is probably safer, owing to certain slightly misleading characteristics mentioned in the paper, to calculate the end-to-end transmissibility (e.g., force on beam over force applied by machine) for each case in reviewing the over-all performance of the system.

MR.S. BYARD emphasized the importance of the mechanical impedance concept in assessing the performance of resilient mountings. He asked the lecturer to comment further on the use of nonlinear springs to reduce the natural frequency of a mounting system for a given deflection under static load.

Mr. Byard pointed out that Dr. Parfitt had suggested that the over-all weight of a compound mounting might be kept down by arranging that the intermediate mass comprise in part at least some of the auxiliary components associated with the main engine. He thought it would be dificult to ensure that such an intermediate load behaved as a true mass and not as a complex resonant structure. Regarding the question of force "pick-ups," Mr. Byard said that there was no real difficulty in constructing pick-ups which effectively measure force and, in the practical measurement of the mechanical impedance of a structure, such a pick-up is used in conjunction with a velocity pick-up.

DR. PARFITT: Mr. Byard is very right to question the practicability of using auxiliary equipment for the loading masses considered in the paper. The main requirement here is that the loading be masslike in impedance over the frequency range where foundation resonances are important. Inasmuch as foundation beams may be relatively long, and hence of low frequency, whereas items of equipment may be relatively compact, there is a possibility of achieving this limited requirement. However, every individual case must certainly be treated with great care and on its own merits.

I have not had any personal experience with nonlinear spring mounts for reducing resonance frequency. I understand that they have been used successfully in large engineering installations, though I suspect that for mobile ship-borne and service use they may be a little critical in adjustment and maintenance.

Dr. F.M.V. FLINT said that the question of the advantages of the equivalent circuit approach to the analysis of structural vibrations merited further dis cussion. He pointed out that with underwater radiated noise, it was not so much the disturbance of the base of the mounting which was of interest; for the ultimate current-carrying network (in the electrical analog) was the entire surrounding medium and one wished to know the energy dissipated in the resistive element 
oi that medium. If the disturbances of the base followed a simple pattern, i.e., a piston motion, the medium could be represented by a very simple pendent network. In ships, the base was liable to be stimulated into a very complicated pattern which might be significantly influenced by the existence of the outside medium. Dr. Flint did not see clearly, however, that to resort to electrical analogs was going to give any clearer qualitative understanding of the problems involved in reducing energy transmission into the medium or any greater facility in quantitative analysis of particular situations.

PROFESSOR R.E.H. RASMUSSEN complimented the lecturer on his clear exposition and wondered whether he had studied the effect of mechanical disturbances, due to traffic, on the stability of laboratory instruments. He also asked Dr. Parfitt for practical results obtained by using mechanical isolating mounts.

DR. PARFITT: Unfortunately, I have no figures on vibration caused by traffic. Actual field results on the benefit obtained from isolation systems are very difficult to come by since comparable before-and-after measurements are hardly ever possible. I have no reason, however, to think that the simple theory is not applicable, except insofar as the foundation impedance is not usually well known. 


\title{
LECTURE 5
}

\section{A SING-AROUND VELOCIMETER FOR MEASURING THE SPEED OF SOUND IN THE SEA}

\author{
M. Greenspan and C.E. Tschiegg \\ National Bureau of Standards \\ Washington, D. C., U.S.A.
}

\subsection{INTRODUCTION}

The so-called sing-around velocimeter is by now a well-established instrument used mostly for the in situ measurement of the speed of sound in the sea and other natural waters. It has been applied to a lesser extent to measurement problems in the laboratory; there is reason to believe that with suitable modifica tions the laboratory use of the instrument could be greatly extended.

The instrument is automatic, has fast response, and is easily adapted to recording. The models with which we are here concerned are restricted to use with liquids which show no appreciable frequency dispersion except possibly at very high frequencies. They must be designed, adjusted, and calibrated for a particular class of liquids within which the total variation of the speed and attenuation of sound is not too large. These changes are commonly caused by changes in temperature, pressure, or composition. The velocimeters have high stability and are therefore especially adapted to differential measurements, such as that of sound-speed gradients in the sea. Other examples of differential measurement are the determination of the effect of dissolved air on the speed of sound in water [1] and the measurement of the temperature coefficient of the speed of sound in water near the turning point (approximately $74^{\circ} \mathrm{C}$ ) [2].

\subsection{GENERAL CONSIDERATIONS}

A sing-around velocimeter is outwardly similar to the ultrasonic delay line employed in digital computers for information storage. It may be thought of as a cylindrical tank the ends of which are electroacoustic transducers, and the whole filled with the liquid under test. A voltage pulse is applied to the "sender," and a corresponding pulse of sound travels through the sample liquid and is received and converted to an electrical pulse by the receiver. In order to define uniquely the time interval between the pulses and to specify their location in time, some characteristic must be selected that will still be recognizable after the pulse has been distorted by transmission through the liquid and by the bandwidth limitations of the transducers. In the present instrument, the pulse position is specified by the instant at which it begins to rise from the noise. 
This choice has several important consequences. To begin with, a pulse-modulated carrier has now no advantage over the much simpler video pulse even though the distortion of the former would be much less* Further, it becomes essential that the pulse rise rapidly. This is no problem so far as the input pulse is concerned, but the output rises, relatively, very slowly. The fast rise is restored by amplification; nevertheless there is introduced an unknown delay equal to the time which the output pulse spends below the noise. This delay depends on the attenuation characteristics of the liquid. It is primarily for this reason that the velocimeter must be calibrated and used on a class of liquids within which the attenuation characteristics are not too variable.

The timing is automatic. The received pulse after suitable amplification and reshaping is again applied to the sender; thus the device regenerates and the pulse repetition frequency (prf) depends upon the speed of sound in the liquid and to some extent upon electrical and other delays. The principle is not new. The earliest description we have found occurs in a patent [3] filed in 1937 by Shepard. Similar systems are described in later patents by Kock [4] and by Larsen [5]. The designation "sing-around" appears to have been coined by Hanson [6]. Hanson, Barrett and Suomi [7], and several others (for references see a recent paper by Ficken and Hiedemann [8]) have constructed apparatus similar to that described here. These instruments were not of high precision.

A major source of difficulty is the existence of multiple echoes between the transducers. The various sets of echoes, each set arising from a different primary pulse, are not synchronous because of the electrical time delay. Various means of eliminating the reflections have been used. In the case of a straight path, the transducers are tilted slightly out of parallel so that all received pulses but the first are lost in the noise. In the case of a bent path, where the sound is reflected back nearly on itself, the transducers and reflector occupy their geometrically correct positions, but the reflection coefficient is rather small. The first received pulse is attenuated by reflection once and the second three times; the result is that all received pulses after the first are negligible. Hard rubber, Teflon, and perforated metal are suitable materials for the reflector.

A bent path minimizes errors which arise from mass motion of the liquid and is preferred for field models, and in the laboratory in cases where a large volume of sample, which necessitates vigorous stirring formaintenance of thermal equilibrium, is used. In cases where the liquid is contained in a small tank immersed in a temperature-controlled bath the straight path is satisfactory.

The advantages of a bent path are even greater for a doubly bent path with two reflectors. This arrangement, which is used on all recent models, will be described later in more detail.

We recapitulate briefly the principle of operation. A block diagram of the sing-around principle is shown in Fig. 5.1. The input transducer is energized by a trigger-type, pulse-forming circuit which produces short fast pulses. This circuit is adjusted to run free at a prf somewhat less than the expected minimum operating prf. The pulses of pressure produced by the input transducer travel

However, the choice of a video pulse restricts operation to nondispersive liquids. An instrument based on a pulse-modulated carrier could, in principle, be used on dispersive liquids, but at a single frequency only. 
down the sample liquid in a time $l / c$, where $c$ is the speed of sound and $l$ is the path length. The received pulses are amplified and shaped and are used to synchronize the original pulse-forming circuit. If $t_{\theta}$ is the sum of the electrical delays and the time lost in the noise, the total time delay is

$$
\frac{1}{f}=t_{e}+\frac{l}{c}
$$

The prf, $t$, is measured and perhaps recorded. Both $t_{\theta}$ and $l$ are obtained by direct calibration with a liquid in which the speed of sound is known. If the velocimeter is to be used in the sea, for example, a suitable calibration liquid is distilled water. Readings of $f$ with distilled water at various temperatures between 0 and $60^{\circ} \mathrm{C}$ cover the range which would be obtained in the sea where the extremes of temperature are 0 and $40^{\circ} \mathrm{C}$ and the salinity reaches perhaps $4 \%$. Corresponding to each temperature of the distilled water is a known speed and an observed prf, $f$. These determine the unknowns $t_{e}$ and $l$ in Eq. (1). It is also possible to determine $t_{\mathrm{e}}$ by measuring $f$ for two different known values of $l$. This method is both more cumbersome and less accurate; the length $l$ in Eq. (1) is only an effective length and is difficult to define in an absolute sense, especially in the case where the receiving transducer is not accurately parallel to a wavefront.

\subsection{APPARATUS}

\subsubsection{The Transducers and the Path}

An external view of the velocimeter is presented in Fig. 5.2. The structure on top is a protecting plate for the sound head; when it is removed, the essential parts are as shown in Fig. 5.3. The inner structures are the transducer mounts; on the left-hand mount the transducer is visible; the reflectors are on the periphery. The sound ray traverses a zigzag path about $20 \mathrm{~cm}$ long. The transducer mounts are fixed in their geometrically correct positions, but the reflectors are adjustable. The proper adjustment is made by trial while observing waveforms on an oscilloscope; once it is attained, the reflectors are locked in place with the cap screws shown. For some applications the reflectors should also be pinned.

Good results have been obtained with both $\mathrm{x}$-cut quartz and with ceramic transducers. The latter are now preferred because they operate in conjunction with simpler electronic circuits. The transducers are made as thin as is consistent with ease of handling; thicknesses from 0.2 to $1 \mathrm{~mm}$ are satisfactory, the best value depending on the type of mount.

Mounts of the type shown in Fig. 5.3 have become more or less standard. With these are used transducers of barium-calcium-lead titanate $\left(\mathrm{Ba}_{0.80} \mathrm{Ca}_{0.12}\right.$ $\mathrm{Pb}_{0.08} \mathrm{TiO}_{3}$ ), a material not overly sensitive to temperature, devised some years ago by W. P. Mason of the Bell Telephone Laboratories. The discs are $1.25 \mathrm{~cm}$ in diameter and $0.66 \mathrm{~mm}$ thick, corresponding to a fundamental thickness resonance of about $3.6 \mathrm{Mc}$, with surfaces flat and parallel to about $25 \mu$. The electrodes are composed of fired-on silver-ceramic paste; the outer (ground) electrode covers the entire area and the inner (hot) electrode is about $6 \mathrm{~mm}$ in diameter and has two \#30 silver-plated copper wires attached to it with 63-33-4 tin-lead- 


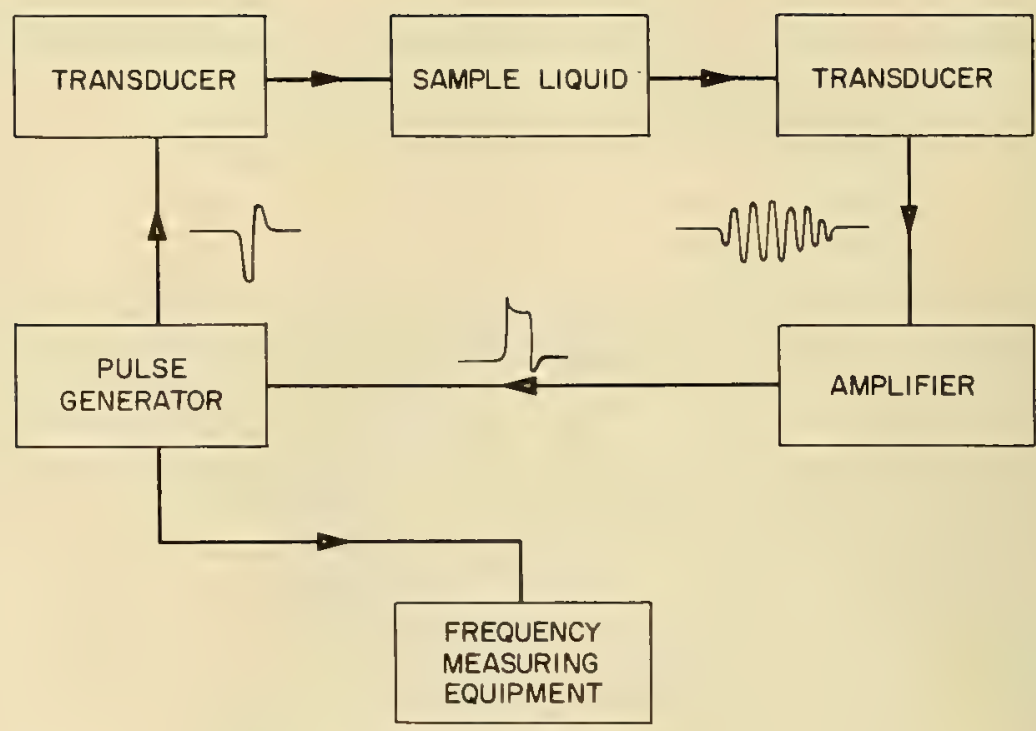

Fig. 5.1. Block diagram showing sing-around principle.

Fig. 5.2. General view of velocimeter. 


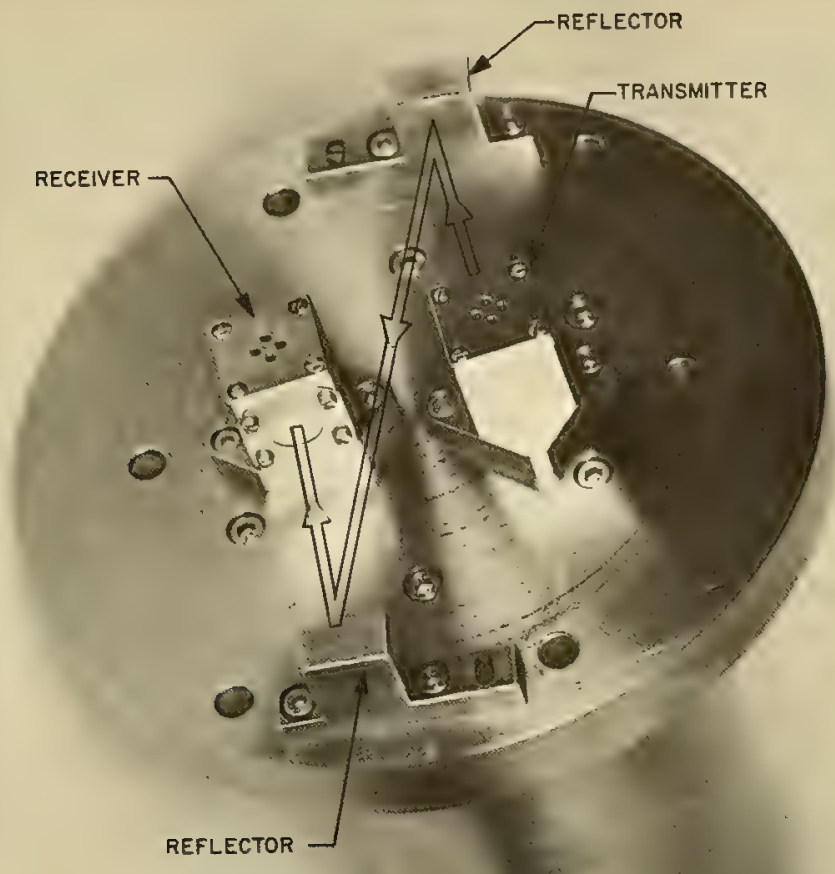

Fig. 5.3. The sound head.

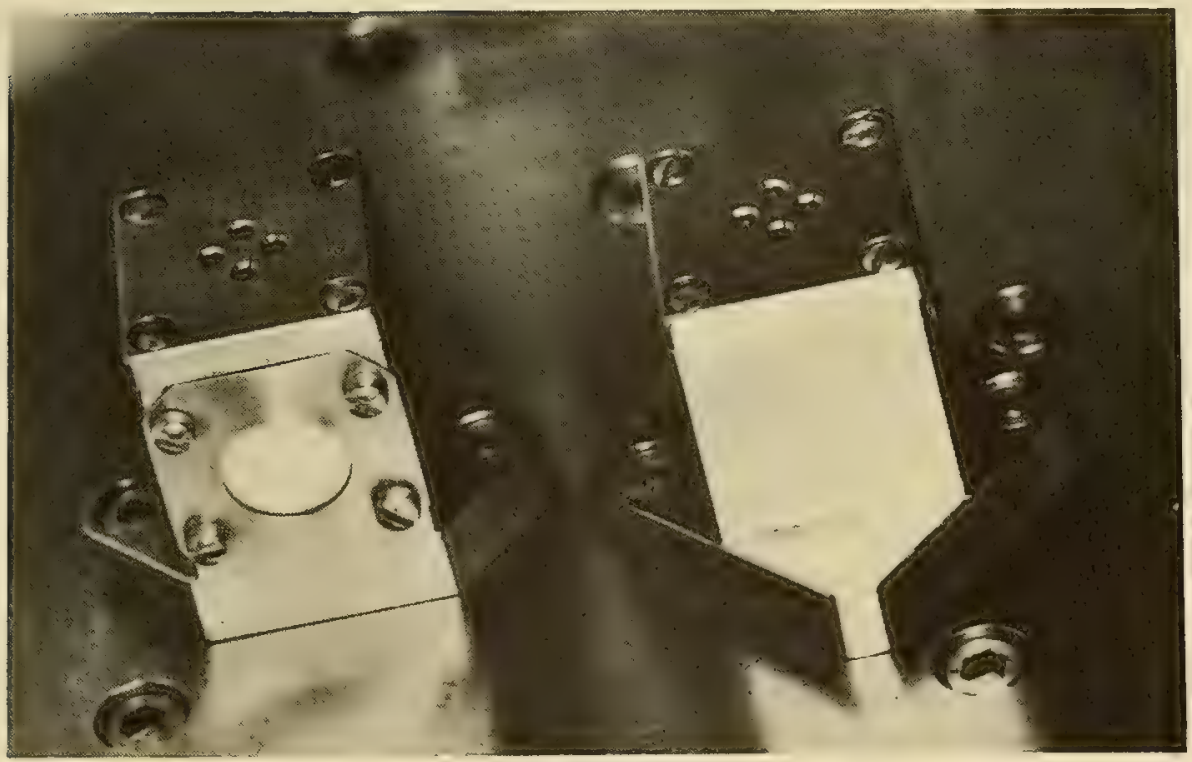

Fig. 5.4. The transducer mounts. 
silver solder. The two transducers in each set are oppositely poled for proper operation with the circuit to be described. If another inverting stage of amplification were added, then the transducers would have to be similarly poled.

The transducer mounts are better seen in Fig. 5.4. They are hollow and contain only the electrical connections and silicone grease (DC-4). The transducers are held in place by the spring retainers shown, but are not cemented or otherwise fastened. The grease is centrifuged into the mount to eliminate aix bubbles and the final seal is made using a thin neoprene gasket under the perforated top cap. The ceramic disc is thus under substantially hydrostatic pressure so that there is no tendency for water to leak in at the edges of the disc or to percolate through it. We have never been able to fabricate a rigidly backed transducer, even using heavily plated material especially processed for low porosity, which is completely impervious at pressures above a few hundred atmospheres. The hollow mounts have been repeatedly tested to $1500 \mathrm{~atm}$ without evidence of leakage.

\subsubsection{The Electronics}

As may be seen from the schematic diagram, Fig. 5.5, the circuit employs 8 microalloy transistors, all of the same type. The power required is $8 \mathrm{ma}$ at $6.5 \mathrm{v}$ and may be obtained from any sufficiently stable supply. For instruments that are to be lowered to great depths, the most practical supply is a pair of mercury batteries, visible at the top of Fig. 5.6.

The blocking oscillator (Fig. 5.5) utilizes a miniature, ferrite-core pulse transformer of one-to-one turns ratio. It is adjusted to run free at about $6 \mathrm{kc}$, somewhat lower than any synchronized prf which will occur in practice. The blocking oscillator applies to the sender a positive pulse of amplitude about $6 \mathrm{v}$ and width somewhat less than $1 \mu \mathrm{sec}$. The sendor oscillates briefly at its natural frequency of $3.6 \mathrm{Mc}$ and a corresponding pressure variation is transmitted through the water to the receiver. It is only the initial transient, confined to less than the first quarter-cycle of this disturbance, which is of interest here. Suppose the rising part of the positive $B O$ pulse expands the sender, then a pressure wave travels through the water and compresses the receiver. It follows from the piezoelectric equations that the receiver would put out a positive-going voltage if the transducers were poled alike; in our case, they are oppositely poled and the input to the base of common emitter stage Q1 is negative-going.

The amplifier section consists of common emitter Q1, common collector Q2, and common emitter Q3. Stage Q3 is heavily saturated with negative-going output. Detector $\mathrm{Q} 4$ rectifies and amplifies; the output is about $3.6 \mathrm{v}$ rf positivegoing. It also removes most of the base line noise. The first four stages Q1-4 are intended as a rise-time amplifier; for example, corresponding to an input slope of $5 \mathrm{mv} / \mu \mathrm{sec}$ is an output slope of $75 \mathrm{v} / \mu \mathrm{sec}$. The relevant gain in the linear range is thus about 15,000 . In operation, however, stage Q3 is heavily saturated, and the output of $\mathrm{Q} 4$ is at its limiting value, $150 \mathrm{v} / \mu \mathrm{sec}$. Note that it is possible to apply the output of Q4 directly to the sender, thus obviating the necessity for stages Q5-8. This arrangement has been tried out in the laboratory. It has the disadvantage that it is not self-starting, and also that one or more independent series of pulses may arise adventitiously.

The remainder of the circuit removes these inconveniences. Schmitt trigger 


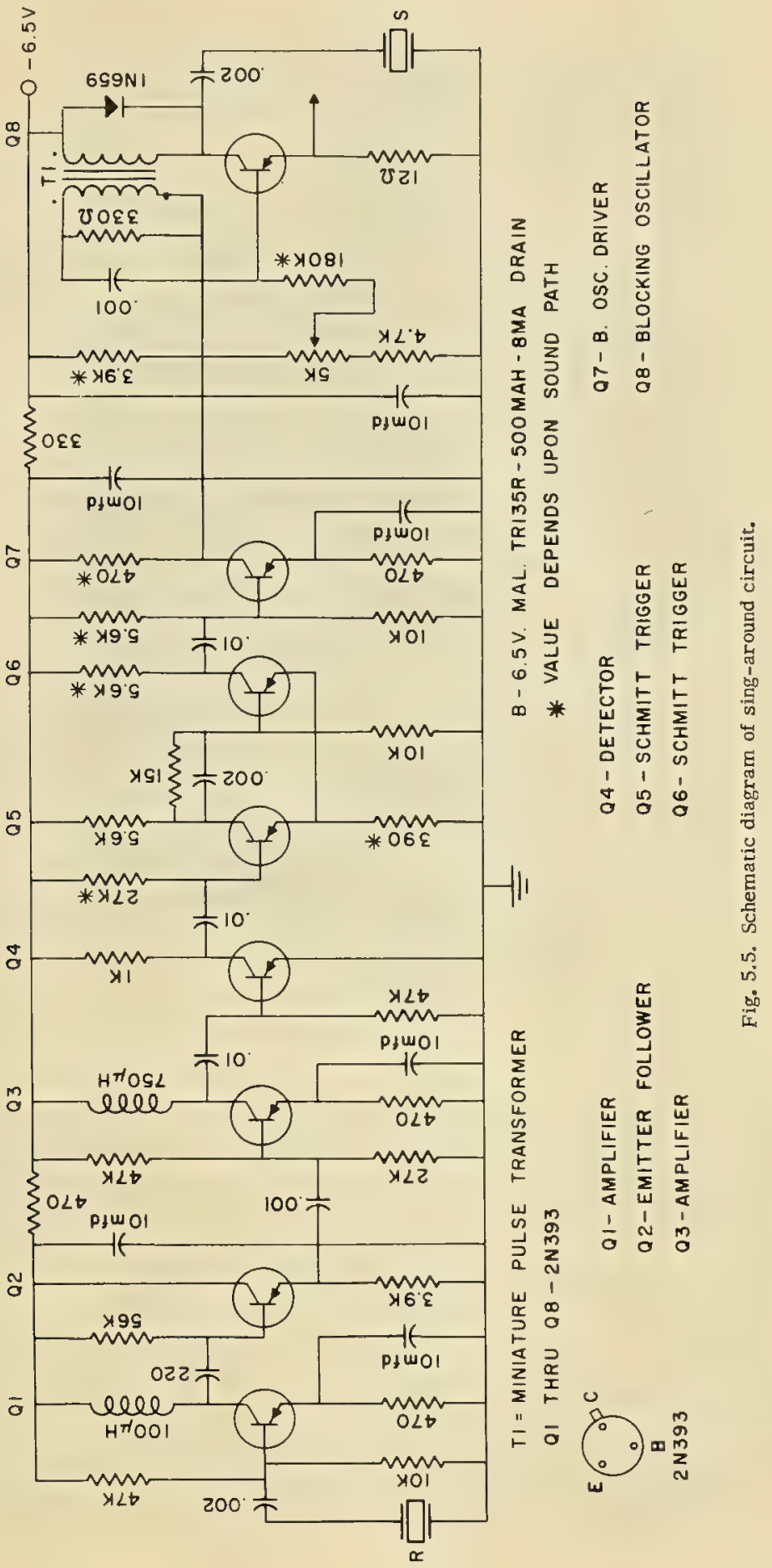




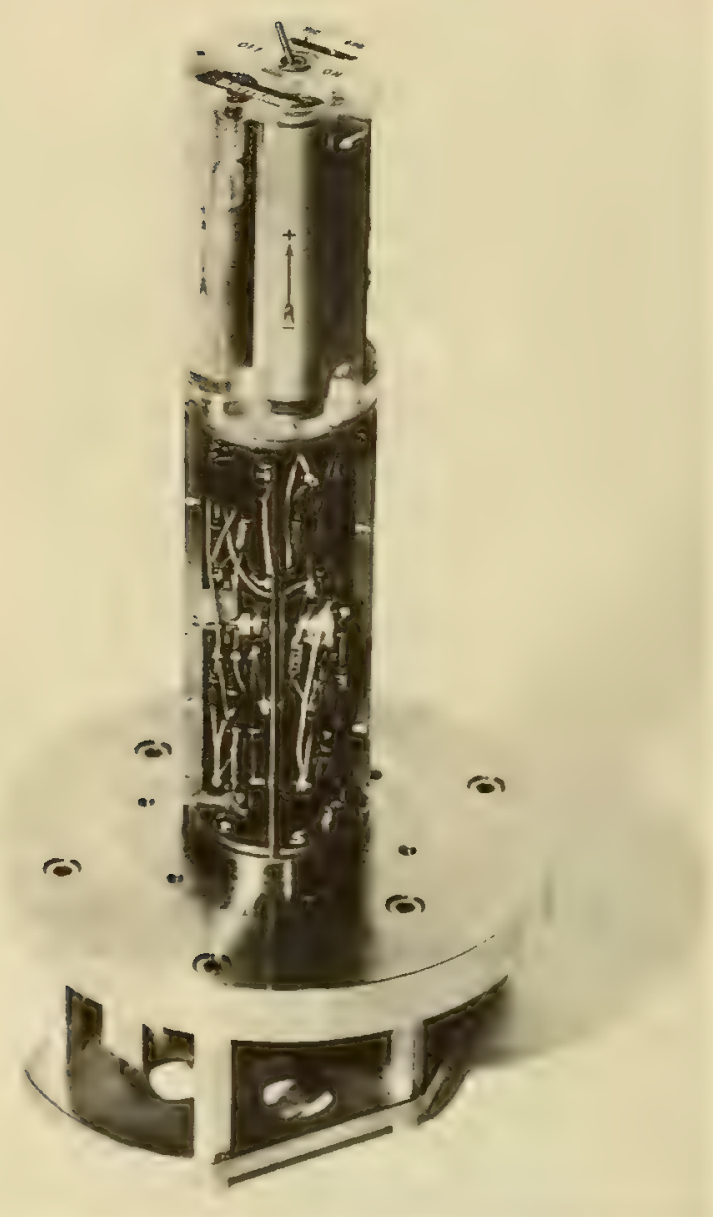

Fig. 5.6, Velocimeter with case removed.

Q5-6 further cleans up the base line and removes most of the $\mathrm{rf}$. The output here is a rectangular positive pulse of about $3 \mathrm{v}$ amplitude and $8 \mu \mathrm{sec}$ width. Stage Q7 is an inverting, low-impedance-output power amplifier which produces the negative-going pulse necessary to synchronize the blocking oscillator. It is adjusted to provide a pulse large enough to trigger the blocking oscillator reli ably, but not so large that there is any possibility of doubling.

\subsubsection{Frequency Measurement}

The input to the frequency measurement equipment is taken from the emitter of the blocking oscillator where a narrow rectangular pulse of amplitude $-1 \mathrm{v}$ is available. The prf, which is in the neighborhood of $7 \mathrm{kc}$, may be measured directly, but in the case of a very long cable connecting the sing-around circuit to the measuring equipment, as in deep-sea work, the signal is first converted to a square wave (about $3.5 \mathrm{kc}$ ) by means of an Eccles-Jordan type of divider. The 


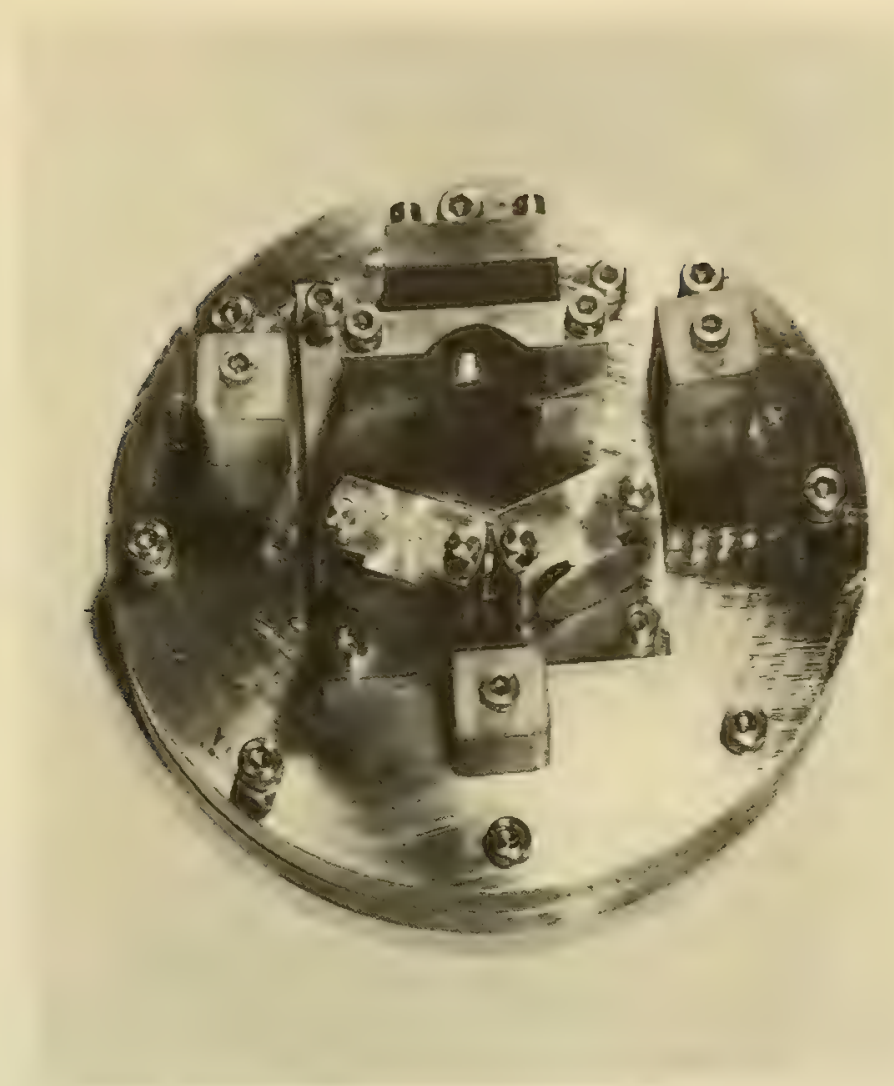

Fig. 5.7. Sound head of a velocimeter built by the U.S. Naval Ordnance Laboratory.

impedance is matched to the cable by an emitter-follower amplifier. For oceanographic applications, the cable need only be an insulated single conductor; the sea acts as the return.

At the output end of the cable appears an attenuated, nearly sinusoidal wave. This is amplified and doubled. In any case, then, we have to measure small variations in a frequency of about $7 \mathrm{kc}$.

The prf of the system may be measured by any of the standard techniques depending on the particular requirements and the available equipment. In the laboratory an electronic counter is very convenient, but a stable radio receiver tuned to a high-order harmonic (say 200) of the prf gives equally good results.

In many field applications automatic operation and/or recording is required. In such cases the multiplied frequency is converted to an audio-frequency which may be fed to an ordinary frequency meter having a dc output suitable for operation of a recorder. The local oscillator is preferably crystal controlled. 


\subsection{PERFORMANCE}

\subsubsection{Stability}

5.4.1.1. Supply-Voltage and Ambient-Temperature Fluctuations

The transistor velocimeters built at NBS exhibit changes in output prf of from 5 to $11 \mathrm{ppm}$ for a $1 \%$ change in supply voltage.

The effect of ambient temperature changes on the circuit itself is rather small, amounting to a total change in prf of about 1 part in 35,000 over a temperature interval 0 to $42^{\circ} \mathrm{C}$. The thermal expansion of the sound path is more serious. Most of the transistor velocimeters have a base plate of stainless steel for which the mean thermal expansion is $16.4 \cdot 10^{-6} /{ }^{\circ} \mathrm{C}$. If the standard path length is taken as that at $20^{\circ} \mathrm{C}$, then the error at 0 or $40^{\circ} \mathrm{C}$ is about 1 part in 3000 , unless corrections for temperature are made. Most users of the instrument require temperature measurements in any case, and their practice is to correct the velocimeter readings for temperature. The base plate can be made of invar, as it was for the dozen or so vacuum-tube type of instruments made before the present instrument was developed. This procedure effectively solves the thermalexpansion problem, but makes it necessary to take steps to prevent the serious corrosion which occurs wherever the invar touches stainless steel and also at tool marks and scratches in the invar.

\subsubsection{Frequency Stability}

The term "frequency stability" here denotes the degree to which the prf of the velocimeter remains constant if the speed of sound in the liquid remains constant. In water, the speed of sound is a maximum at about $74^{\circ} \mathrm{C}[2]$. At this temperature a variation of $1^{\circ} \mathrm{C}$ changes the speed of sound by only $13 \mathrm{ppm}$, and a variation of $0.1^{\circ} \mathrm{C}$ by only $0.1 \mathrm{ppm}$. The stability is therefore conveniently measured in water at $74^{\circ} \pm 0.1^{\circ} \mathrm{C}$; the prf is obtained by counting for ten seconds three times a minute. Such a test was made for $6.3 \mathrm{hr}$ on a transistor velocimeter. The data scattered about a line representing a drift rate of 4.7 $\mathrm{ppm} / \mathrm{hr}$ with a standard deviation of 0.47 count in about 75,500 , or $6.2 \mathrm{ppm}$. Vacuum-tube type velocimeters [9], equipped with an invar path and solidly backed transducers, are better than this. A 10.5-hr run on such an instrument showed a drift of only $0.3 \mathrm{ppm} / \mathrm{hr}$ with a standard deviation of 0.72 count in about 153,000 , or $5 \mathrm{ppm}$. In both cases the standard deviation includes the effect of the \pm 1 -count inherent counter error, and possibly some errors due to accidental counts. Figure 5.7 shows the sound head of such a velocimeter. Note the singly bent path and the hard rubber reflector. Figure 5.8 shows the construction of the transducer mounts.

\subsubsection{Calibration}

The standard liquid is outgassed distilled water; we use as standard values of $c$ those we measured by a pulse technique in 1957 [10]. The water, rapidly stirred, is held at each of several temperatures, measured with a platinum resistance thermometer, while the prf, $f$, is measured by counting pulses for $10 \mathrm{sec}$. Corresponding values of $\mathrm{c}$ and $f$ are fitted by least squares to Eq. (1), which we repeat for convenience

$$
\frac{1}{f}=t_{0}+\frac{l}{c}
$$


TABLE 5.I. Calibration of Laboratory Velocimeters

\begin{tabular}{l|c|c}
\hline Model & $\begin{array}{c}\text { II } \\
\text { tube } \\
\text { invar }\end{array}$ & $\begin{array}{c}\text { TR-2(NBS) } \\
\text { transistor } \\
\text { Circuit type }\end{array}$ \\
$\begin{array}{l}5 \text { Path } \\
\text { No. of points }\end{array}$ & 9 \\
Temperature extremes, ${ }^{\circ} \mathrm{C}$ & 0.8 to 83.2 & 0.6 to 50.4 \\
Effective length, $l$ & & \\
Value, cm & 14.6941 & 20.4848 \\
St. dev., cm & 0.0010 & 0.0068 \\
Time delay, $t$ e & & \\
Value, $\mu$ sec & 0.7188 & 0.3713 \\
St. dev., $\mu$ sec & 0.0004 & 0.0464 \\
Std. dev. of $1 / f$ data & & \\
$\mu$ sec & 0.0018 & 0.0045 \\
Ppm & 18 & 32 \\
Std. dev. of prediction ppm & 2.4 & 11
\end{tabular}

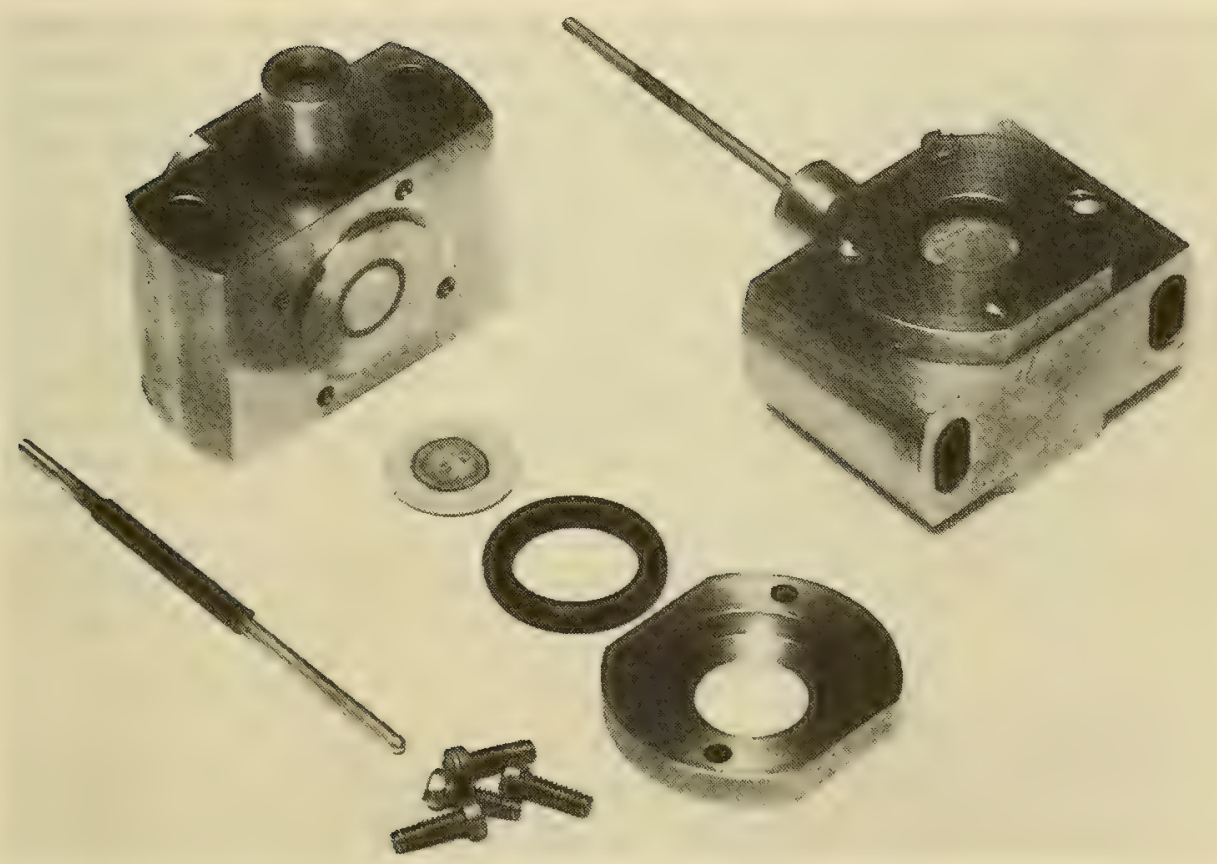

Fig. 5.8. Transducer mounts for the sound head of Fig. 7. 
TABLE 5.II. Calibration of Field Velocimeters

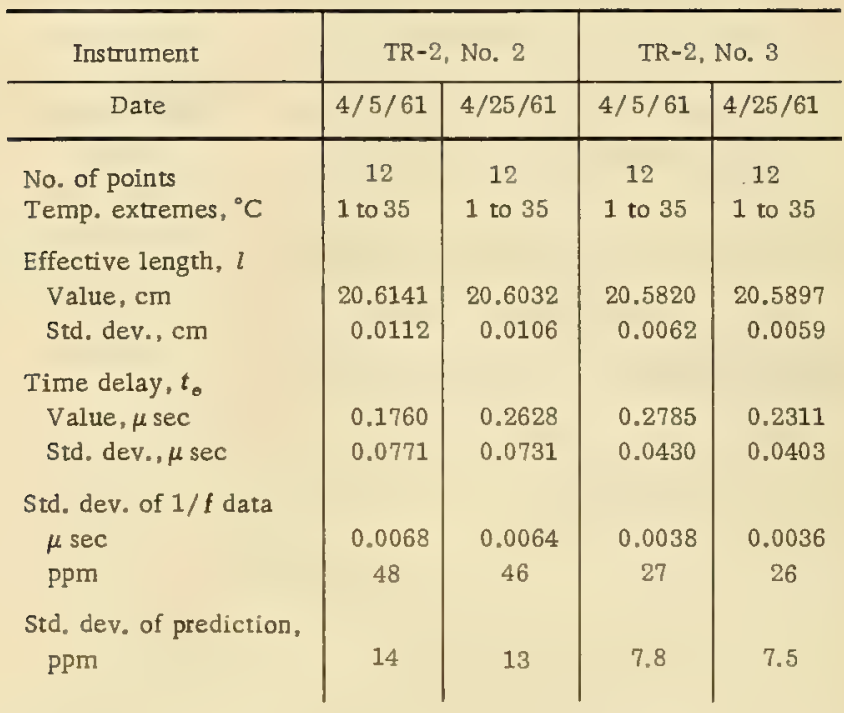

This computation yields the time delay $t_{*}$ and the effective path length $l$. (If the path is not of low-expansion material, the length $l$ in Eq. (1) should be replaced by $l_{0}\left[1+a\left(T-T_{0}\right)\right]$, where $l_{0}$ is the length at temperature $T_{0}, T$ is the temperature, and $a$ the coefficient of thermal expansion.) Table 5.I shows the results for two instruments made at NBS. Again, the old instrument is much superior to the new, but the new one is sufficiently accurate for the field use for which it is intended. It should be borne in mind that the uncertainty in $f$ due to the inherent \pm 1 counter error is $\pm 10 \mathrm{ppm}$ for the tube model and $\pm 13 \mathrm{ppm}$ for the transistor model, and that at the low temperatures, an uncertainty of $0.01^{\circ} \mathrm{C}$ corresponds to an error of $30 \mathrm{ppm}$ in $t$.

Some interesting calibration data are available for twotransistorized instruments made by NBS and in constant use for more than two years by the U.S. Naval Electronics Laboratory in San Diego. Mr. K. V. Mackenzie of NEL kindly made available to us some calibration data taken before and after a cruise during which the instruments were subjected to severe treatment in a storm so violent that it was barely possible for the crew to work. The results are given in Table 5.II. Note that these instruments are roughly equivalent to the TR-2 instrument used only in the laboratory. The differences in $l$ and $t_{e}$ barely exceed the standard deviations and are probably not highly significant. For these calibrations the counter errors and the errors in temperature measurement are probably insignificant.

\subsection{HISTORY AND DISTRIBUTION}

The earliest mention we have found of the sing-around principle is in the US patent [3] filed in 1937 and granted in 1943 to F.H. Shepard, Jr., of RCA. As mentioned in a FIAT review [11], Freund and Hiedemann filed a German patent application in 1940. Shortly thereafter, in 1941, W. Kock, then of the Baldwin 
Piano Company, filed for a similar US patent; this was granted in 1946 [4]. Huntgren and Hallman [12] discussed possible applications to radar (they used the term "ring-around") in 1947; in the same year, M.J. Larsen [5] filed for a US patent, granted in 1949, covering a sing-around echo-location system for the blind. In 1948, R. D. Holbrook [13], working at Brown University, made what so far as we know was the first serious laboratory application of the principle; this was for measurement of small changes in the speed of sound in solids. It was in 1948, also, that R. L. Hanson [6] read his paper, based on Kock's patent, in which the term "sing-around" was coined; and at the same meeting, W. E. Kock and F.K. Harvey demonstrated a system using loud speakers in air. This demonstration and Hanson's paper introduced the subject to us. Barrett and Suomi [7] in 1949 experimented with a balloon-borne sing-around device for the measurement of air temperature. They used a thyratron pulser and a 16 -in. path. The electrical time delay was $34 \mu \mathrm{sec}$. Holbrook's work at Brown was continued by Cedrone and Curran [14], who by 1954 had produced an instrument employing a pulse-modulated $10-\mathrm{Mc}$ carrier with an accuracy in liquids of about $0.1 \%$. A much simpler instrument, utilizing video pulses and good to about $1 \%$, was described in 1956 by Ficken and Hiedemann [8].

Our own work began in 1952 and by the end of the year a prototype model of high stability, described in NBS Report 2702, Jan. 2, 1953, was in operation. The first operational model had a straight path and quartz transducers, and was field tested in June, 1953. This instrument was used by the Chesapeake Bay Institute for several years. All succeeding vacuum-tube velocimeters, of which more than a dozen were built, had ceramic transducers and a singly bent path with a reflector of hard rubber or perforated metal. These were described in 1957 [9] but were first announced in 1955 [15]. The transistorized version was developed in 1957 to meet the need for a deep-sea instrument. At the present time (August, 1961), 65 of these instruments have been manufactured and we know of current invitations to bid on 61 more. Of the 65 , three were made by NBS, two by US Coast and Geodetic Survey, two by the Woods Hole Oceanographic Institute, and the remainder by three different commercial manufacturers. Two instruments are in England, two in Norway, one is at the Saclant ASW Research Center in Italy; most of the rest are owned by various naval or oceanographic installations in the United States.

In addition, about 25 instruments are being made with the same sound head but a different timing mechanism. There are also three instruments with a 10 cm path for $1 \frac{1}{2}-\mathrm{v}$ operation.

In 1958, A. Lutsch of the NPL of the Union of South Africa reported on an instrument [16] similar to that of Cedrone and Curran [14], but of much higher accuracy.

\subsection{ACKNOWLEDGMENTS}

We are indebted to our section's shop support group headed by Henry A. Schmidt, Jr, and particularly to Marshall A. Pickett who performed many of the necessary pressure tests.

The initial phases of the work were supported by the Office of Basic Instrumentation of NBS. After 1952, most of our support came from the ONR. Numerous 
field tests were performed by the Chesapeake Bay Institute of Johns Hopkins University (Martin Pollack), by the U.S. Navy Underwater Sound Laboratory (Harry Sussman), and by the Woods Hole Oceanographic Institution (Earl Hays).

\section{REFERENCES}

1. M. Greenspan and C. E. Tschiegg, "Effect of Dissolved Air on the Speed on Sound in Water," J. Acoust. Soc. Am., Vol. 28, 501 (1956).

2. M. Greenspan, C. E. Tschiegg, and F.R. Breckenridge, "Temperature Coefficient of the Speed of Sound in Water Near the Turning Point," J. Acoust. Soc. Am., Vol. 28, 500 (1956).

3. F.H. Shepard, Jr., U. S. Patent No. 2,333,688 (November 9, 1943).

4. W. E. Kock, U.S. Patent No. 2,400,309 (May 14, 1946).

5. M. J. Larsen, U.S. Patent No. 2,580,560 (January 1, 1952).

6. R. L. Hanson, "Applications of the Acoustic Sing-Around Circuit," J. Acoust. Soc. Am., Vol. 21, 60-61 (1949).

7. E. W. Barrett and V.E. Suomi, "Preliminary Report on Temperature Measurement by Sonic Means," J. Meterol., Vol. 6, 273-276 (1949).

8. G. W. Ficken, Jr., and E.A. Hiedemann, "Simple Form of the Sing-Around Method for the Determination of Sound Velocities," J. Acoust. Soc. Am., Vol. 28, 921-923 (1956).

9. M. Greensapn and C.E. Tschiegg, "Sing-Around Ultrasonic Velocimeter for Liquids," Rev. Sci. Inst., Vol. 28, 897-901 (1957).

10. M. Greenspan and C. E. Tschiegg, "Speed of Sound in Water by a Direct Method," J. Research NBS, Vol. $59,249-254$ (1957)

11. E. Hiedemann, FIAT Rev. Ger. Sci,, 1939-1946, Part 1, 178 (1947).

12. Huntgren and Hallman, "The Theory and Application of the Radar Beacon," Proc. Inst. Radio Engrs., Vol. 35, 716-730 (1947).

13. R.D. Holbrook, "A Pulse Method for Measuring Small Changes in Ultrasonic Velocity in Solids with Temperature," J. Acoust. Soc. Am., Vol. 20, 590 (1948).

14. N.P. Cedrone and D.R. Curran, "Electronic Pulse Method for Measuring the Velocity of Sound in Liquids and Solids," J. Acoust. Soc, Am., Vol, 26, 963-966 (1954).

15. Tech. News Bull. NBS 39, 89 (1955).

16. A. Lutsch, "An Apparatus for Measuring and Recording the Velocity of Sound and Temperature Versus Depth in Sea Water," Acustica, Vol, 8, 387-391 (1958).

\section{DISCUSSION}

MR. J. CREASE enquired about the effect of dispersion on the velocity of sound in sea water between low and high frequencies and asked whether it was possible to make an estimate of the difference between Wilson's sound velocity data and the phase velocity at low frequencies.

MR. GREENSPAN: In reply to Mr. Crease, I have already stated that the principle of operation of the velocimeter is such that the readings have a precise meaning only in liquids which are practically nondispersive. In sea water, the only dispersion for which there is positive evidence is that associated with dissolved $\mathrm{MgSO}_{4}$. As Dr. Sette has pointed out, Fox and Marion* have measured the dispersion in $\mathrm{MgSO}_{4}$ solutions by a differential method. Their data could best be fit by a single relaxation process centered at $150 \mathrm{kc}$, independent of concentration. The total dispersion is about $13.6 \cdot 10^{-4}$ parts per mole per liter, and as the concentration of $\mathrm{MgSO}_{4}$ in sea water is about 0.028 molar, the total dispersion in sea water should be about four parts in $10^{5}$, a value too small for Fox and Marion to detect reliably in sea water itself.

If it should turn out that other and larger sources of dispersion exist, as sociated, for example, with plankton or microbubbles, then new problems will arise. For one thing, most devices which operate at low frequency measure

*F. E. Fox and T.M. Marion, J. Acoust, Soc. Am. 25, 661 (1953). 
phase velocity; but, in many cases, it is the signal velocity or perhaps the group velocity which is desired. In any case, the appropriate velocity will have to be measured at a frequency near that of interest.

In view of what has just been said, the second part of Mr. Crease's question cannot be answered at present.

MR. L. KAY, in connection with near-field irregularities, drew attention to the work by Christie* on the near-field characteristics of a circular transducer which had been set ringing by an applied impulse. This work had revealed variations in the near-field pattern which are not predicted by continuous wave theory; and, depending upon the position of the receiving transducer, the received signal obtained was of varying wave shape. The fact mentioned by Mr. Greenspan that two instruments did not operate satisfactorily following a slight change in position of the transducers would tend to confirm this. Mr. Kay, then, commented upon the stress laid by Mr. Greenspan on the use of an impulse rather than a driving wave as being better for the reliable operation of the system. He thought that there would be no difference in the received signal, since the transducers are free to ring under the damping effect of the water only, giving a ?, say, of 15 . Even with an impulse driving force, there will be several cycles of the resonant frequency which in the medium will decay exponentially but at the output of the receiver will appear as a typical rf pulse.

MR. GREENSPAN: I believe that Mr. Kay has misconstrued my remarks on the relative merits of a video-pulse and pulsed-carrier drive. The velocimeter operates on the portion of the signal received earliest; what follows the first quarter-cycle is ignored. Either type of drive would give the same result; it is only that use of a video pulse simplifies the electronics. In either case, we have to cope with the fact that the receiving circuit recognizes the leading wavefront somewhat later than it would in the ideal case of zero attenuation and zero noise. Some of this delay is accounted for in the calibration; but there is a variable part, associated with the diffraction effects mentioned by Mr. Kay, which remains as a source of error difficult to estimate, but thought to be small.

DR. W. N. ENGLISH commented upon the discrepancies in the values of the sound velocities calculated by various methods for the deep ocean. He pointed out that C. D. Maunsell, of Pacific Naval Laboratory, British Columbia, following a suggestion of M. Peterson (San Diego), has compared the observed and calculated positions of the first convergence zone for the Kuwahara and Wilson formulations. The former gives a range less than that observed while the latter, even after applying a sphericity correction, gives a greater range by two miles or so. Dr. English said that the discrepancy they have obtained is in the same direction as that found by Hays (Wood's Hole) in his experiments in the Mediterranean at a depth of 2200 meters.

PROFESSOR T.S. KORN pointed out that in the standing-wave method it was the measured phase velocity of sound which, in certain circumstances, was obviously different from the required propagation velocity.

* Progress in Nondestructive Testing, Vol. 1. 

LECTURE 6

\title{
UNDERWATER SOUND CALIBRATION STATIONS AT LE BRUSC LABORATORY
}

\author{
M.P. Foäche \\ Laboratoire de Detection Sous Marine \\ Le Brusc (Var.), France
}

The Laboratoire du Brusc has at its disposal four different calibration stations for hydrophones and underwater sound transducers, each one using a different calibration technique. The joint operations of these calibration facilities permit measurements covering a range from $0.1 \mathrm{cps}$ to $100 \mathrm{kcps}$.

Depending on the frequency range to be covered, the following procedures are used: (1) electrodynamical calibration, (2) electrostatic calibration, (3) closedtank alternating pressure technique, (4) calibration by comparison of filtered noise, (5) pulse technique, and (6) free-field reciprocity calibration.

\subsection{HYDROPHONE CALIBRATION}

The calibration of hydrophones at low frequencies is generally conducted in a range below $1 \mathrm{kc}$. The hydrophones being of different types, the procedure of measurement depends not only on the frequency range to be covered but also on the hydrophone design.

The hydrophones used in the frequency range from 0.1 to $1000 \mathrm{cps}$ have a rigid metal diaphragm vibrating in contact with a piezoelectric element, for instance, a slab of titanate or zirconate ceramic. Electronic calibration is particularly well adapted to this kind of design. The hydrophone used in higher frequency ranges is an assembly of mounted ADP crystals or ceramic elements sheathed in a rubber boot; the elements are either in direct contact or bathed in castor oil which fills the boot. Certain techniques used with diaphragm-hydrophones may not be applied to these structures.

\subsubsection{Electrodynamic Calibration by Discharge into a Ballistic Galvanometer}

The approximate sensitivity of a hydrophone can be measured by the deflection of a ballistic galvanometer when a sudden change of pressure is applied. This pressure variation is produced by applying a weight to the rigid diaphragm. For other types of hydrophones, a decompressional chamber may be used.

This kind of measurement, when used for any of several types of production hydrophones, is liable to cause considerable errors, as the capacitance of some kinds of ceramics depends upon the frequency. We are using this method primaxily for a rapid check of production hydrophones. 


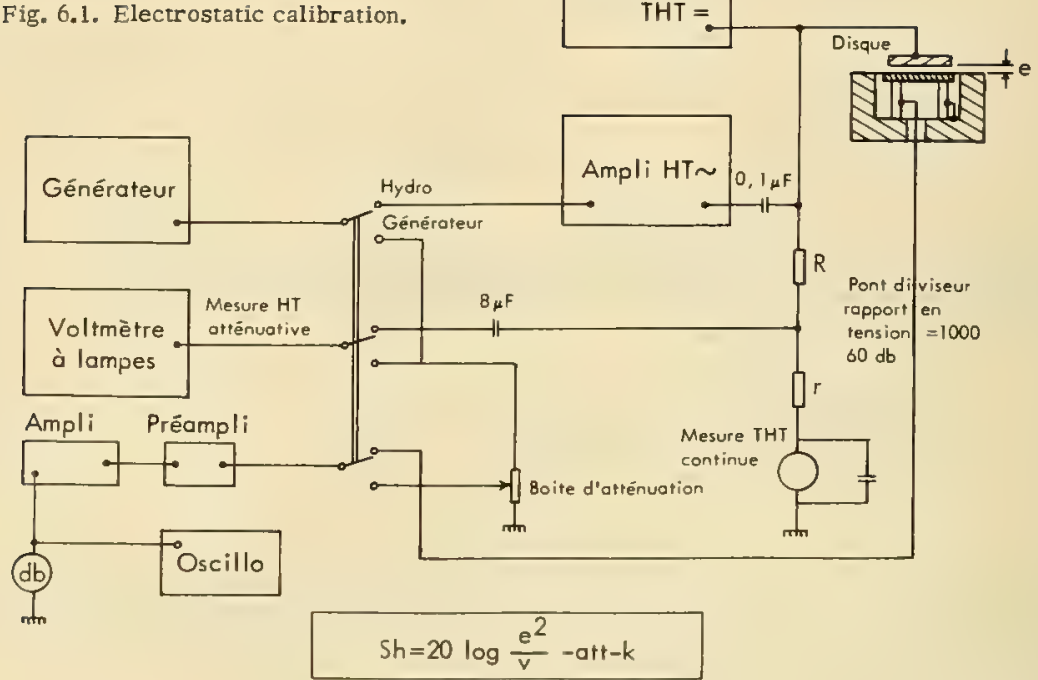

\subsubsection{Absalute Electrostatic Colibration*}

This is our basic technique adopted for the low frequency range, and we constantly endeavor to improve it. Covering a range from 1 to $1000 \mathrm{cps}$, it may be used only for hydrophones having a rigid and absolutely plane diaphragm. It works on the following principle: a driving force generated by an ac field is applied between the diaphragm and another plate representing the electrode. This technique is currently used for small standard microphones; it may also be used for hydrophones, but the technological conditions are not the same. For instance, the diaphragms of our hydrophones have a diameter of $640 \mathrm{~mm}$ and are $8 \mathrm{~mm}$ thick.

The value of the pressure applied to the diaphragm can be found as follows: if the voltage applied to the plates of a plane capacitor contains a dc component $V$ and an ac component $v$, we have for the electrostatic force driving the electrode, e being the distance between the plates,

$$
F=\frac{\partial C}{\partial e}\left(V v+\frac{v^{2}}{2}\right)
$$

If $V$ is much higher than $v$, and if the electrode displacements are negligible, we can write

$$
F=\frac{\partial C}{\partial e} V v
$$

Denoting the electrode area by $s$, and the area of the diaphragm by $s$, the pressure due to the electrostatic force is

$$
p=\frac{\epsilon}{4 \pi e^{2}} \frac{S}{S} V v
$$

In practice, we have adopted the following values: 


$$
\begin{aligned}
& V=1000 \mathrm{v} \quad \mathrm{e}=1 \mathrm{~mm} \\
& V=100 \mathrm{v} \quad \mathrm{s}=80 \mathrm{~cm}^{2}
\end{aligned}
$$

The signal output voltage developed across the terminals of the hydrophone by this pressure is measured with the same voltmeter used for measuring $v$, with the help of a precision attenuator, in order to have the same deflection for $v$ as for the signal output voltage. The errors due to the instrument are thus eliminated.

The hydrophone response is given by

$$
S_{h}=20 \log \frac{e^{2}}{V}-20 \log \frac{\epsilon s}{4 \pi S} \text { - attenuation }
$$

Accuracy of measurement may be affected by fringe effects, but classical cor rection factors will reduce this effect to a negligible value.

The distance between the electrode and the diaphragm may prove to be a limiting factor for the accuracy of the method. This distance being of the order of $1 \mathrm{~mm}$, it will have to be measured with an accuracy better than $0.015 \mathrm{~mm}$ in order to avoid a resulting error greater than all the other errors involved. Provided that the diaphragm be absolutely plane, good precision can be obtained by using a comparator.

The total error involved in one measurement is less than $1 \mathrm{db}$. Taking the average of a series of measurements we may assume the error to be less than $0.5 \mathrm{db}$.

The holding device for hydrophone and electrode has to be completely rigid, free from vibration and natural resonance, and it must be set up on resilient mountings. We have mounted this device on a concrete pillar erected on the rock foundation and isolated from the laboratory building.

The upper limit of the frequency range covered by this calibration method is given by the natural resonance of the holding device or of the diaphragm. In this case, free-field calibration will be called for.

Basically, this method has no limits in the low frequency region, but in fact ac voltages below $1 \mathrm{cps}$ are not easy to produce. On the other hand, it is not necessary in this range that the driving force be distributed evenly over the whole area of the rigid diaphragm; a drive in the center is sufficient. In this case, simpler mechanical methods can be applied; for instance, stretching a spring periodically by means of a crank. Another method consists of gluing to the diaphragm a coil which is placed in a magnetic field. The driving force being proportional to the current in the coil, the calibration is easy to achieve.

\subsubsection{Relative Electrostatic Calibration}

In the case of a diaphragm that is not rigid and plane, the absolute electrostatic calibration technique does not work but may be applied in a relative way for measurements up to $10 \mathrm{kcps}$. It then works on the following principle. The electrode surrounds the diaphragm, the latter being made of rubber and having a cylindrical or any other convenient shape. The conductivity of the rubber is assured by a thin coating of silver paint. Although the value of the distance e between the diaphragm and the electrode is not known with an accuracy adequate for absolute measurements, the shape of the frequency curve can be plotted with sufficient accuracy. We use this technique for achieving calibration of the hydro- 


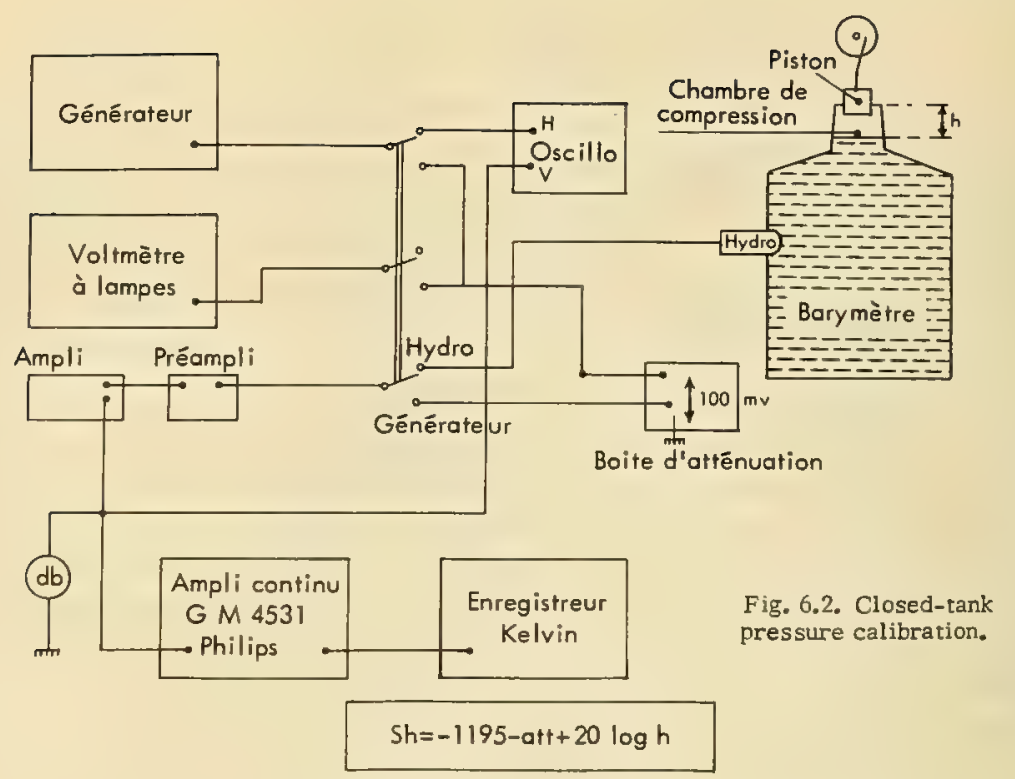

phones in the 100 to $3000 \mathrm{cps}$ range which cannot be easily obtained under freefield conditions. Free-field calibration is carried out in a range from 2 to 10 kcps. It is thus possible, by using the value measured in the 2 to $3 \mathrm{kcps}$ band, to obtain the over-all response between $100 \mathrm{cps}$ and $10 \mathrm{kcps}$. Provided some precautions are taken, this method works with a good accuracy.

\subsubsection{Closed-Tank Pressure-Colibration Technique*}

Although preferring on the whole the method of electrostatic calibration, we also apply the well-known closed-tank measuring technique for occasional checks.

Our closed-tank system consists of a small cylindrical tank, thick-walled in order to minimize the excitation by flexure modes. It is filled with water and a small volume of air of known value. The hydrophones having been placed in the water, the air is compressed mechanically by a small piston. This device works in the range below $50 \mathrm{cps}$. In the very low frequency region $(3 \mathrm{cps})$, the heat exchange between tank wall and air would call for correction factors to the adiabatic law, but we have never exploited this technique to its utmost limits. We are, however, aware of the fact that other laboratories have succeeded in improving this calibration procedure in a very satisfactory manner. In Fig. 6.3 are summarized the results of various methods for the calibration of a H6 Tcl-type hydrophone.

\subsubsection{Colibration by Comparison Using Filtered Noise}

The hydrophones used in acoustic ranging are calibrated in the range 2000 to $100,000 \mathrm{cps}$ by comparison with a standard hydrophone calibrated by the reciprocity method. The most highly perfected anechoic tank still presents a certain

*Figure 6.2 . 


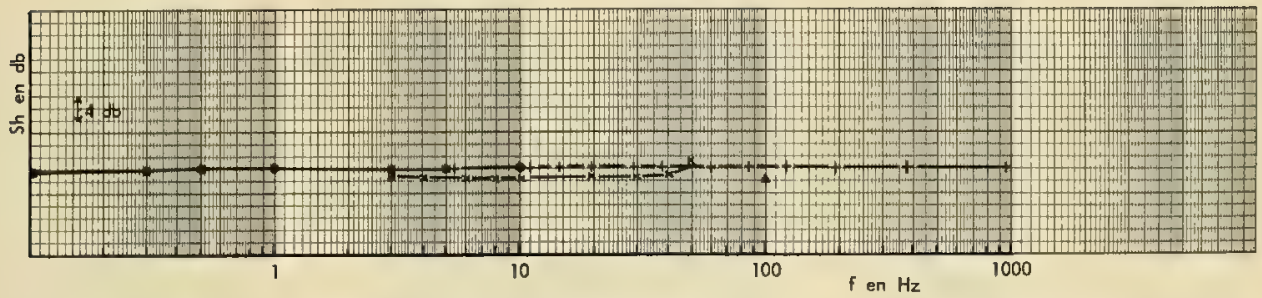

Fig. 6.3. Comparison of different calibration methods. (Type H6 Tel. No, 23 hydrophone): +Electrostatic Method, Electromagnetic method, 1 Ballistic galvanometer method, $\times$ Barymetric method.

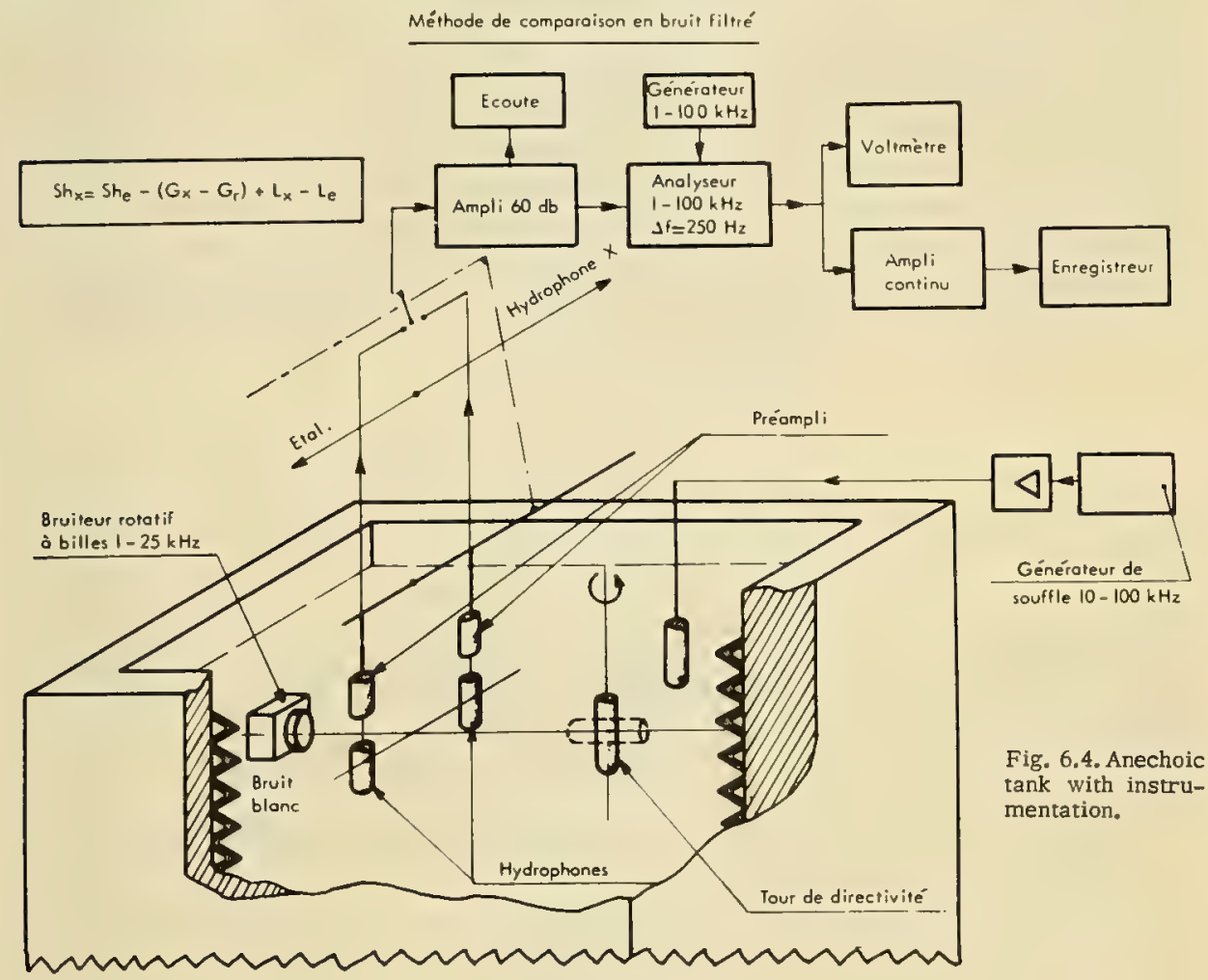

standing-wave ratio; therefore the calibrating signal used consists of a white noise which is filtered at the receiving end by a narrow-band filter only wide enough for filtering out the standing waves. Sometimes the noise source is a mechanical set consisting of a rotating drum filled with bearing balls producing a noise in a spectral band from 1 to $35 \mathrm{kcps}$ and sometimes a ceramic projector driven by a white-noise source.

The hydrophone to be calibrated and the standard hydrophone are placed successively in the same position, their signals being picked up by a heterodyne analyzer between 2 and $100 \mathrm{kcps}$. An automatic processing device permits simultaneous analysis and recording of the response curve. 


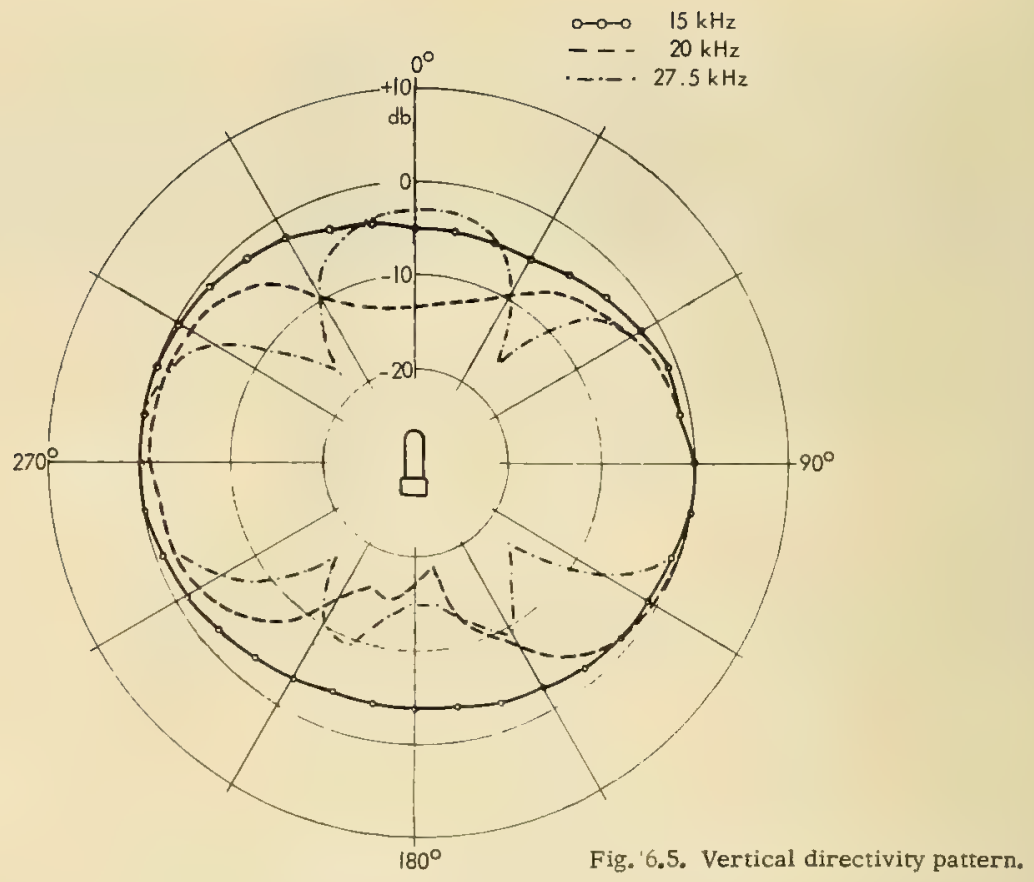

HP50 AN 011 directivité en site

These measurements are carried out in an anechoic tank $3.5 \mathrm{~m}$ long, $2.2 \mathrm{~m}$ wide, and $1.5 \mathrm{~m}$ deep. The inner tankwalls are lined with wedges of a cork-andrubber mixture especially effective in the range above $10 \mathrm{kcps}$ where a reflection factor of less than $-10 \mathrm{db}$ has been measured. Figure 6.4 shows the experimental arrangement.

A shaft rotating by remote control is used for the recording of the horizontal and vertical directional patterns of the hydrophones shown in Fig. 6.5.

By averaging the results obtained by a series of successive measurements, an accuracy better than $1.5 \mathrm{db}$ has been achieved, supposing that the frequency response of the standard hydrophone is known to $\pm 1 \mathrm{db}$.

\subsection{TRANSDUCER CALIBRATION}

For some purposes transducers are calibrated by comparison with a standard transducer using the filtered noise method, but most commonly we apply the pulse technique or the reciprocity method. Measurements are carried out in an anechoic tank $4.60 \mathrm{~m}$ long, $3.15 \mathrm{~m}$ wide, and $3.15 \mathrm{~m}$ deep.

The inner walls and bottom are lined with the same cork-and-rubber wedges described above for the filtered noise method. The damping value of this lining measured at normal incidence is given by Fig. 6.6 in the range from 8 to $40 \mathrm{kcps}$. Through the use of this lining the inside size of the tank has been reduced to 3.80 by 2.40 by $2.80 \mathrm{~m}$. 


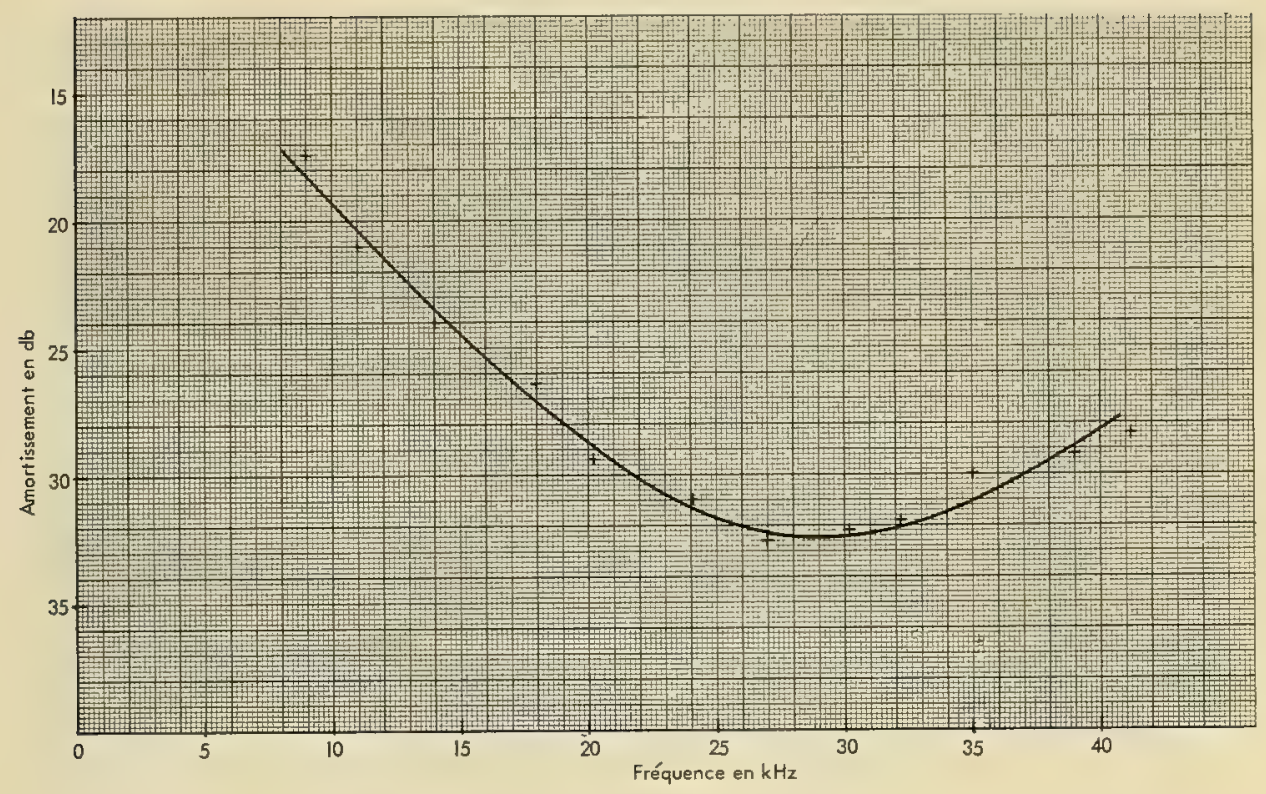

Fig. 6.6. Damping value of the tank lining.

The tank, shown in Fig. 6.7, is fitted with two movable and rotatable vertical shafts, with weight-handling capacities of 150 and $300 \mathrm{~kg}$, rotated by a servomotor designed to give a bearing accuracy of $0.10^{\circ}$.

Because the transducers to be calibrated were of different shapes, a special device for fixing them on the shafts had to be provided for each transducer. Some of these devices, shown in Fig. 6.8, are designed in such a way as to permit the rotation of the transducer using different rotating axes in order to measure the directivity in several planes.

The electronic instrumentation of this test tank is of an all-purpose design allowing complete measurements of transducers, i.e., impedance, response, and directivity.

\subsubsection{Impedance Measurement}

An automatic recorder for complex inpedances, the SEXTA 4912, is provided for measuring and recording the value of transducer impedances as a function of frequency. This instrument is of the continuously recording type and has an accuracy of $\pm 5 \%$. The impedance-frequency plot is displayed on a CRT screen in the frequency range 5 to $500 \mathrm{kcps}$ in two bands. Positive and negative reactances from 5 to 10,000 ohms can be measured with this set, and the transducer can be biased by a direct current during this measurement. An additional device permits observation on the CRT of the parallel reactances and resistances. The remanence of the tube is long enough to allow the plotting of Kennelly curves.

This device may very conveniently be used for matching transformers with transducers by observing the impedance value when the tuning is completed. 


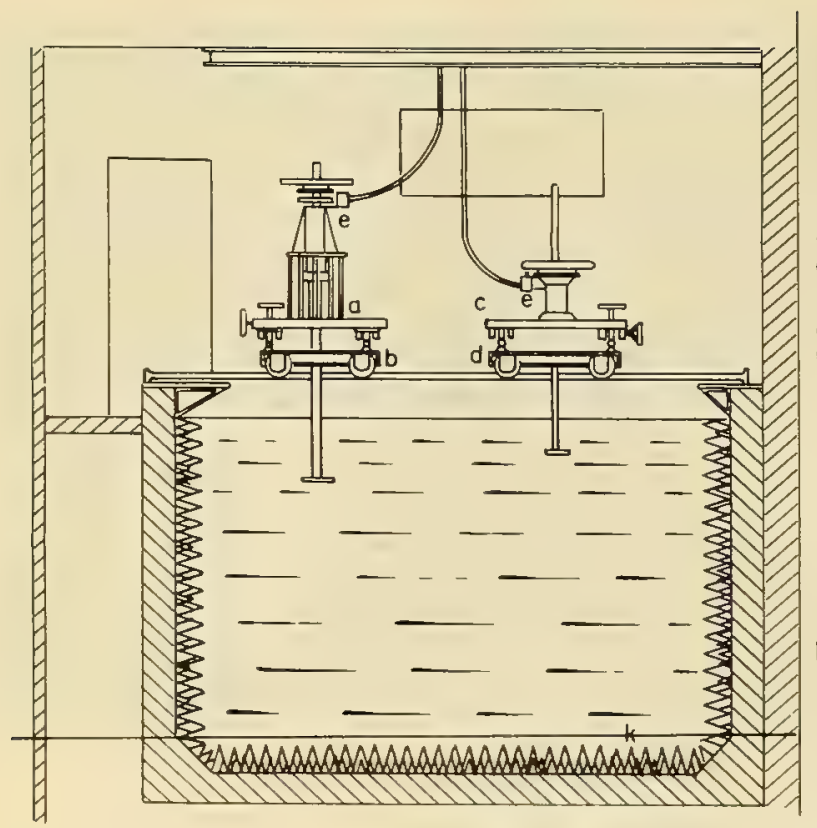

APPAREILLAGE MECANIQUE d' EXPLOITATION de la CUVE

a) petit chariot

b) petit pont

c) grand chariot

d) grand pont

e) selsyn d'orientation

f) entrainement des chariots

g) volant d'orientation

h) frein des chariots

i) frein des ponts

i) entrainement des ponts

k) absorbeurs

l) rail du palan

Dimentions interieures

longueur 4570

largeur 3140

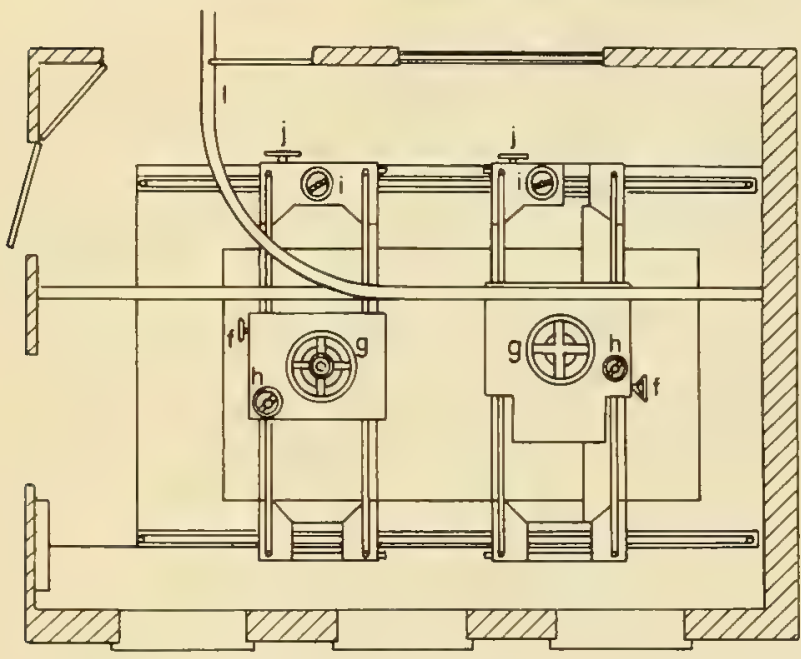

hauteur 3150

Fig. 6.7. Schematic of a transducer-calibration tank with fittings.

\subsubsection{Principle of the Pulse Method}

Acoustic calibration of transducers must be carried out in a free acoustic field. When using the pulse method, each single pulse has to be considered as traveling in such a field at the exact moment when it is measured, and it must not be disturbed by interference produced by reflection on the tank boundary wall. This condition imposes a proper separation distance between projector and receiver, and a determined value of pulse length and repetition rate. 


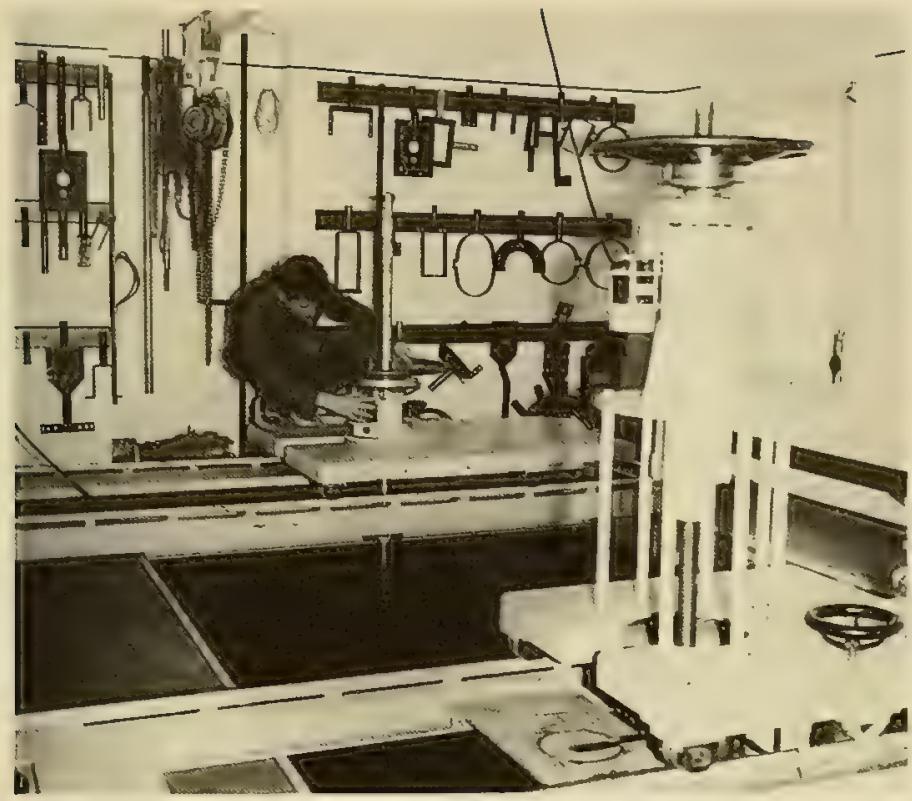

Fig. 6.8. Transducercalibration tank with fittings.

\subsubsection{Separation Distance}

In the vicinity of the transducer, intensity fluctuations due to the Fresnel zone do not allow measurement of the sound level. For a plane transducer, with maximum dimension $d$, the distance has to be more than $d^{2} / \lambda$ in order to have a discrepancy of less than $3 \%$ from the spherical divergence law.

Figure 6.9 gives the minimum separation distance to be used as a function of frequency and transducer size for a given accuracy to be obtained.

\subsubsection{Pulse Length}

When the pulse length $T$ is such that $T$ equals $2 D / c$, where $D$ is the separation distance between transducers and $c$ is the velocity in the medium, interferences due to reflections between the surfaces of transducer and hydrophone are avoided. We have plotted for our measuring tank the basic diagram of Fig. 6.10 establishing a pulse length such that the direct pulse has arrived before the pulses due to reflection.

On the other hand, the $Q$ factor of the transducer calls for a minimum pulse length, the duration of the transient state increasing with the value of $\varrho$. The diagram in Fig. 6.11 determines the pulse length for a given value of $Q$ corresponding to an amplitude equal to 87,95 , and $99 \%$ of the signal value on continuous waves. When measuring transducers at a frequency far from the resonance, the pulse length has to be twice as great as that fixed by Fig. 6.11.

\subsubsection{Pulse Rate}

Pulse rate must be such as to introduce an appreciable difference from continuous wave measurement. It is therefore a function of cathode-ray-tube remanence or of the integration time constant of the meter.

We commonly use a pulse separation of $20 \mathrm{msec}$ for measurements carried 


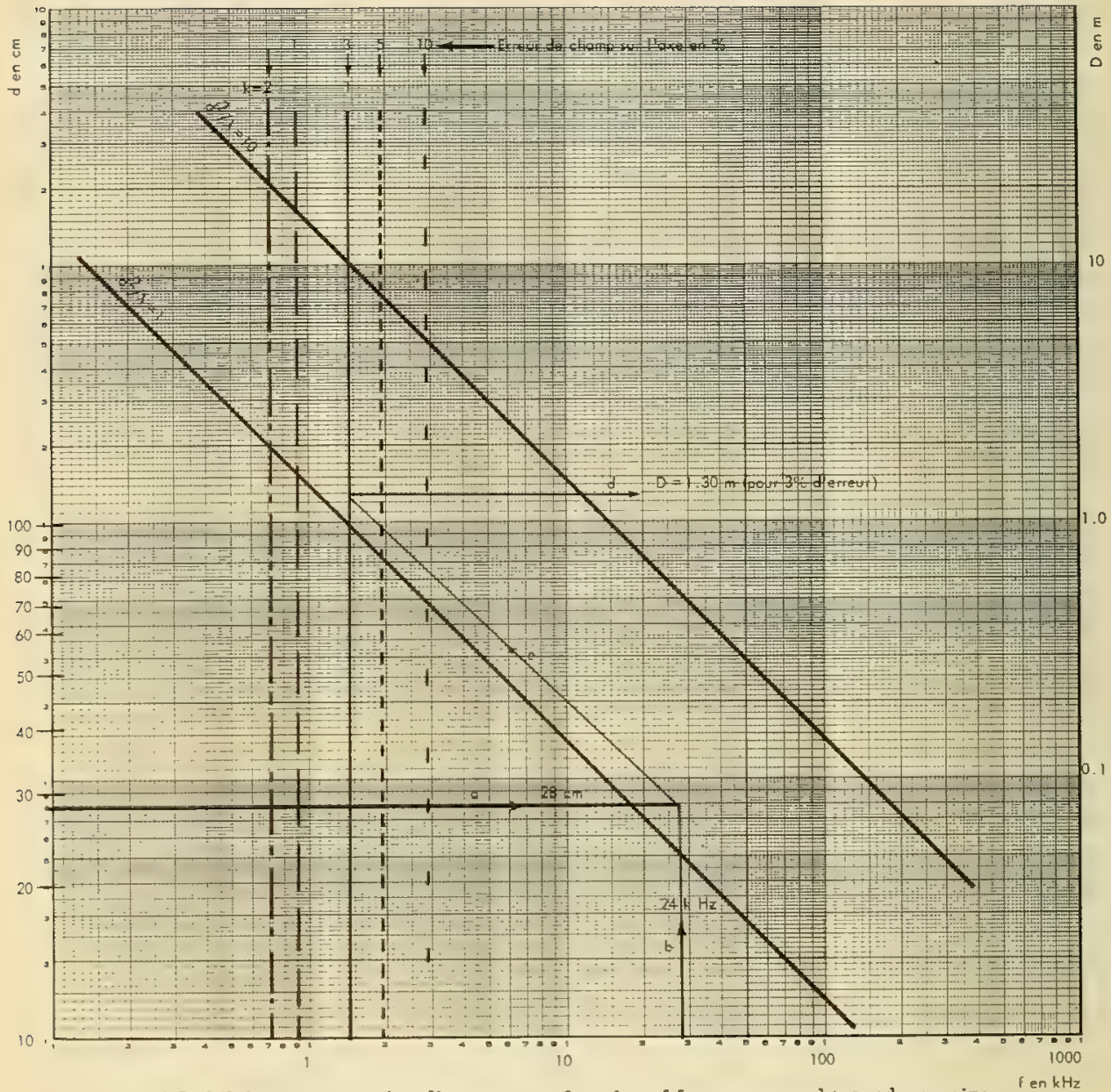

Fig. 6.9. Minimum separation distance as a function of frequency, and transducer size.

out in our tank. This value permits adequate signal integration on a B.K. Level Recorder for a convenient speed of directivity recording.

\subsubsection{Size of the Anechoic Tank}

It appears from the diagrams that the tank we now have in use is adequate for transducer measurement at frequencies above $10 \mathrm{kcps}$, provided that its largest dimension is less than $1 \mathrm{~m}$.

In our opinion, the best proportions for measuring tanks are: length equal to 1.5 times the width, and a width equal to the depth.

When $d$ is the largest dimension of a transducer to be calibrated and $\lambda$ is the wavelength of the lowest frequency wanted, we choose the maximum separation distance between transducers equal to the width of the tank and to $d^{2} / \lambda$. If, for example, a transducer with a maximum dimension of $1.5 \mathrm{~m}$ has to be measured at $5 \mathrm{kcps}$, we have $d^{2} \lambda=7.5 \mathrm{~m}$, and the tank size would have to be 11.25 by 7.5 


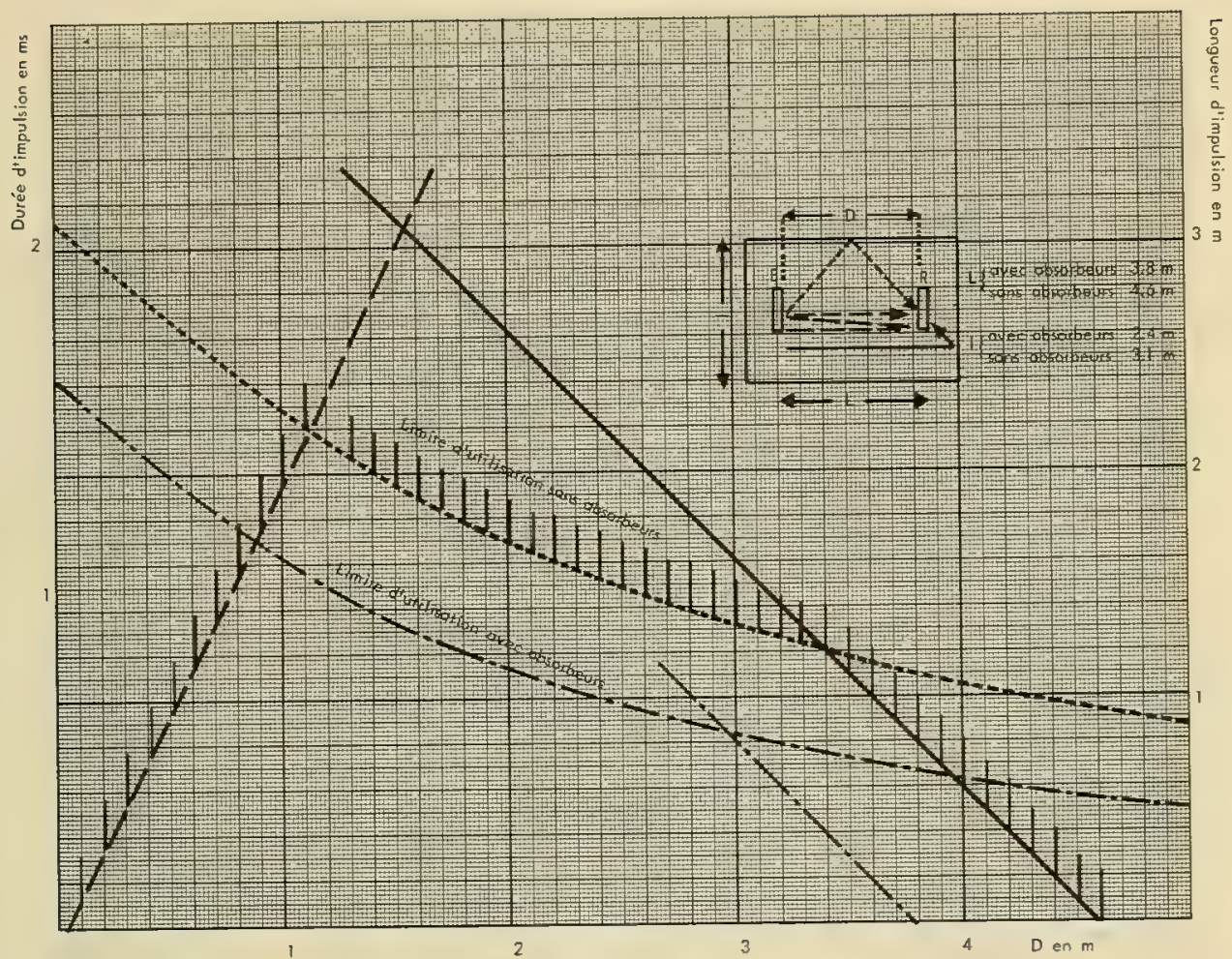

Fig. 6.10. Basic diagram plotted for measuring tank.

by $7.5 \mathrm{~m}$. As a matter of fact, a new tank is to be installed at the Laboratory, measuring 16 by 8 by $8 \mathrm{~m}$.

\subsubsection{Instrumentation}

The electronic equipment, shown in Fig. 6.12, consists of an ac generator and a broad-band power amplifier delivering a driving power of $1 \mathrm{kw}$ for pulses and $500 \mathrm{w}$ for continuous waves. The frequency range covered is 7 to $27 \mathrm{kcps}$. A tuned circuit provides the possibility of driving either magnetostrictive or piezoelectric transducers. The power driving the transducer is measured by a voltage divider for very high potentials. Pulses with a length from $2 \mathrm{msec}$ to $1.5 \mathrm{sec}$ are delivered by the generator.

The receiver-amplifier is gated to measure only the part of the signal corresponding to steady state. A B.K. Level Recorder is also provided for plotting signals received as a function of varying elements.

In normal or routine measurements, the receiver response is obtained by us ing two standard projectors, the PP25 and the P30P, their response curve, determined by the reciprocity method, being flat enough in the frequency band explored. The receiving standards are the omnidirectional hydrophones BRUSH BM 101 and MASSA M $115 \mathrm{~B}$, the response of which is nearly flat between 1 and 100 kcps. 


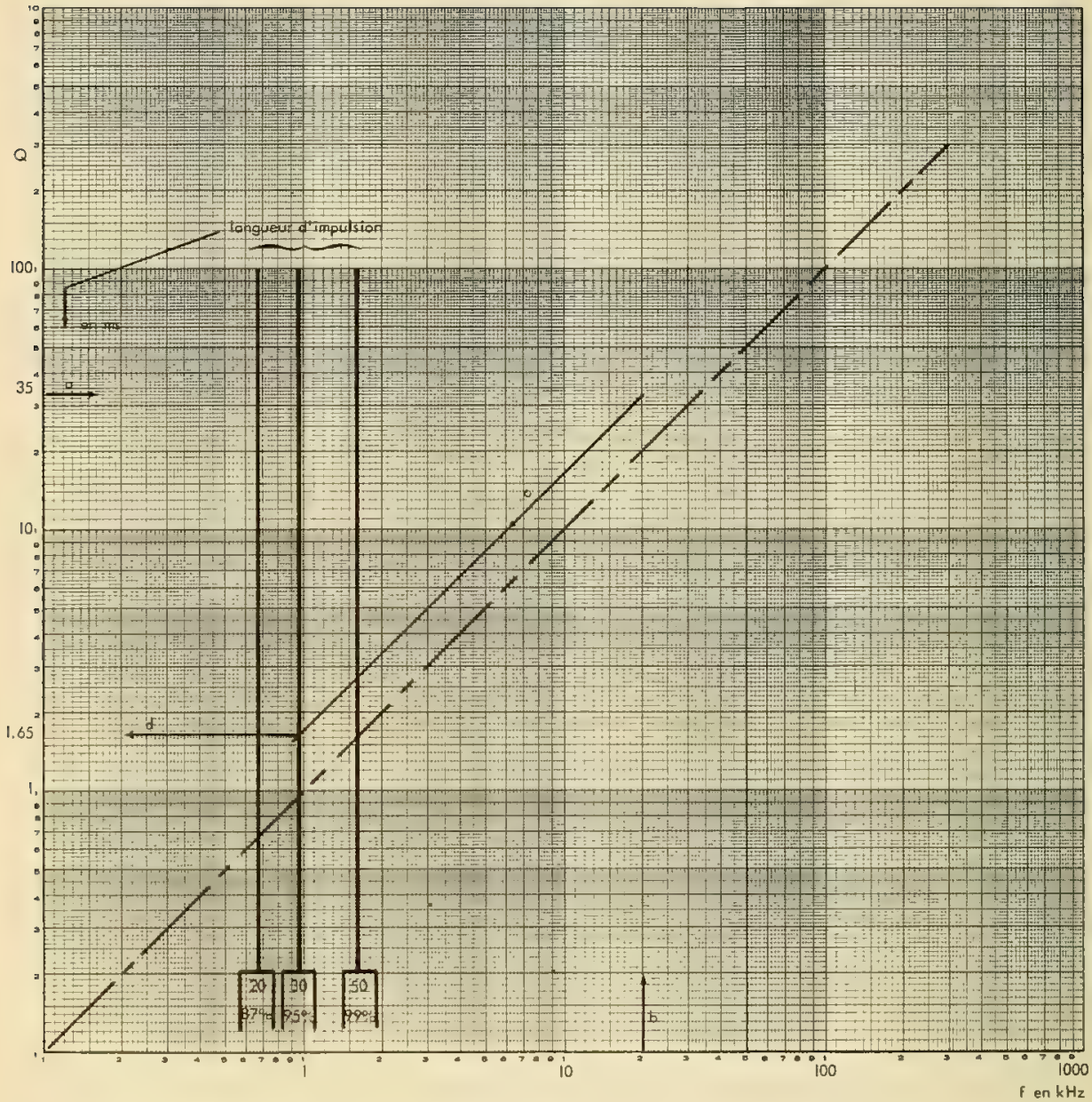

Fig. 6.11. Pulse length vs $Q$ for given amplitudes of the signal value on continuous waves.

\subsubsection{Application of the Pulse Method for Reciprocity Calibration}

We will call $T_{1}$ and $T_{2}$ the two transducers to be calibrated, $T_{3}$ being a third auxiliary transducer. Their receiving sensitivities are $M_{1}, M_{2}$, and $M_{3}$; their transmitting responses, $s_{1}, s_{2}$, and $s_{3}$. To summarize the results:

$$
\begin{gathered}
\text { Reciprocity factor, } J=\frac{M_{1}}{S_{1}}=\frac{M_{2}}{S_{2}}=\frac{2 \cdot 10^{-5}}{f} \\
\text { Transfer impedance, } Z=M_{2} S_{1}=M_{1} S_{2}=\frac{e_{2}}{l_{1}}
\end{gathered}
$$

where $e_{1}$ is the voltage on $T_{1}, e_{2}$ is the voltage on $T_{2}$, and $l_{1}$ is the intensity through $T_{1}$. The ratio of sensitivity between $T_{1}$ and $T_{2}$ is

$$
R=\frac{e_{1}}{e_{2}}=\frac{M_{1}}{M_{2}}
$$




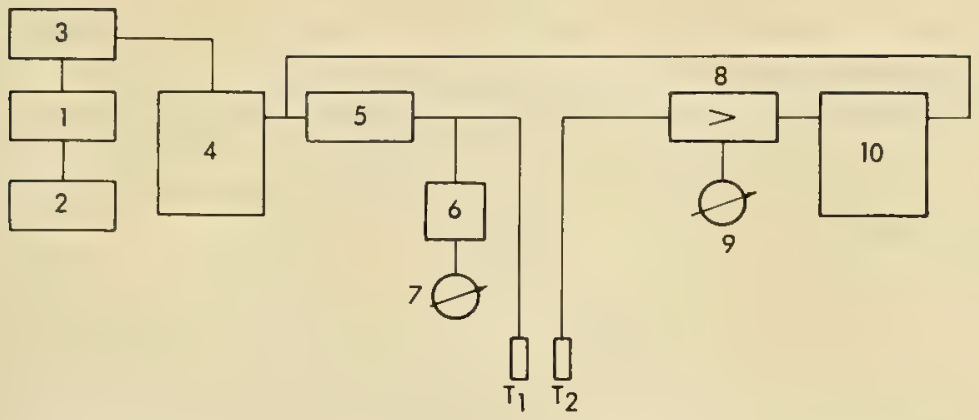

1) Générateur Bruël et Kjœer

5) Boite d'accord des transducteurs

2) Fréquencemètre Rochar

6) Diviseur T.H.T.

3) Impulseur-cadenceur

7) Voltmetre Philips 6015

4) Ampli puissance Jaubert

8) Ampli réception

(500 $w$ en régime continu

9) Philips 6017

I kw en impulsion)

10) Oscillo double trace Cossor

Fig. 6.12. Instrumentation for response measurements.

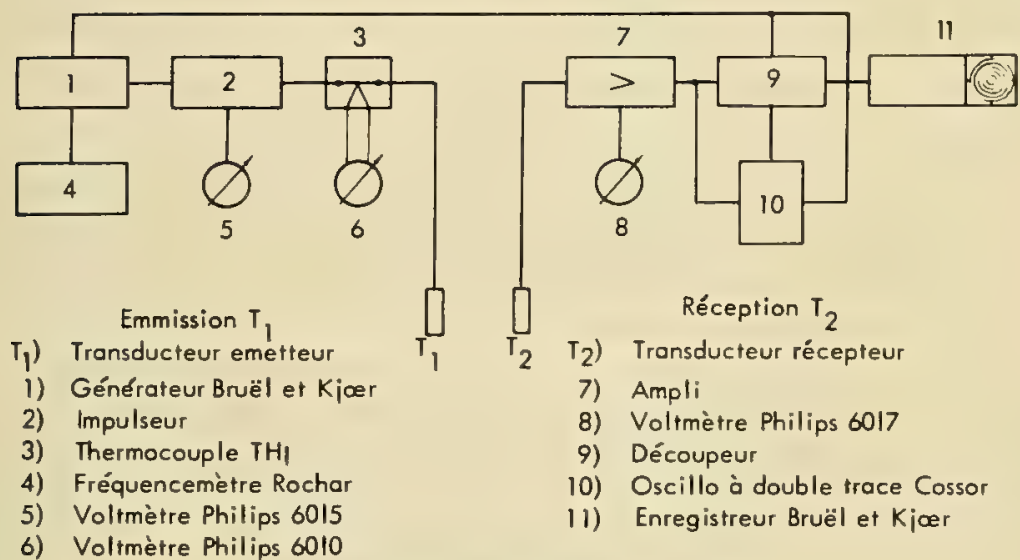

Fig. 6.13. Stand for reciprocity calibration.

and $Z$ is obtained by measuring $e_{2}$ and $l_{1}, R$ by measuring $e_{1}$ and $e_{2}$. The block diagram in Fig. 6.13 shows the instrumentation used for these measurements.

\subsubsection{Calibration by Comparison}

This is a much simpler calibration procedure than the reciprocity method. We have only to compare the transducers to be calibrated with a standard model.

\subsubsection{Directivity Measurements}

The tank instrumentation furnishes a polar plot of received pulses in $\mathrm{db}$ level as a function of transducer bearing and frequency. A $360^{\circ}$ directivity diagram can be plotted in two minutes. For special purposes, in order to get complete details about a transducer, we have had to carry out up to 500 directivity measurements for different aspect angles in bearing and tilt and at different fre- 
quencies. Directivity measurements are used to check off the identity of the different units of a split transducer.

\subsubsection{Transparency Measurements}

When designing sonar transducers, it is sometimes necessary to use for the construction of the radiating area a material having a high degree of transparency. Materials of this kind are tested in the tank by the pulse method. Tests are also carried out on sonar-dome materials.

For this purpose, we use a directional transducer transmitting pulses received by an omnidirectional hydrophone located at a distance of two meters. The sample is placed halfway between transducer and hydrophone, and the comparison of the signals obtained with and without interposition of the sample furnishes the transparency value. These measurements can be made at different angles of incidence, while the signal level is recorded as a function of the angle chosen.

In this way, we measure rubber, plastic, and steel plates. A convenient size for the sample to be measured is 1 by $1 \mathrm{~m}$. The accuracy of this kind of meas urement is largely dependent on the way the inclination can be measured.

As the results obtained are not very accurate, these measurements have only a relative value, but they are very convenient for comparing various materials.

\subsection{TEST.AND.CALIBRATION BARGE MOORED IN THE HARBOUR OF LE BRUSC}

\subsubsection{Mission}

The mission of the field station of Le Brusc is to provide a test-and-calibration facility for underwater sound transducers, arrays, and sonar domes under free-field conditions and by continuous wave measurements, on a large scale and more effectively than would be possible in an anechoic tank. Some of the measurements conducted in this barge are:

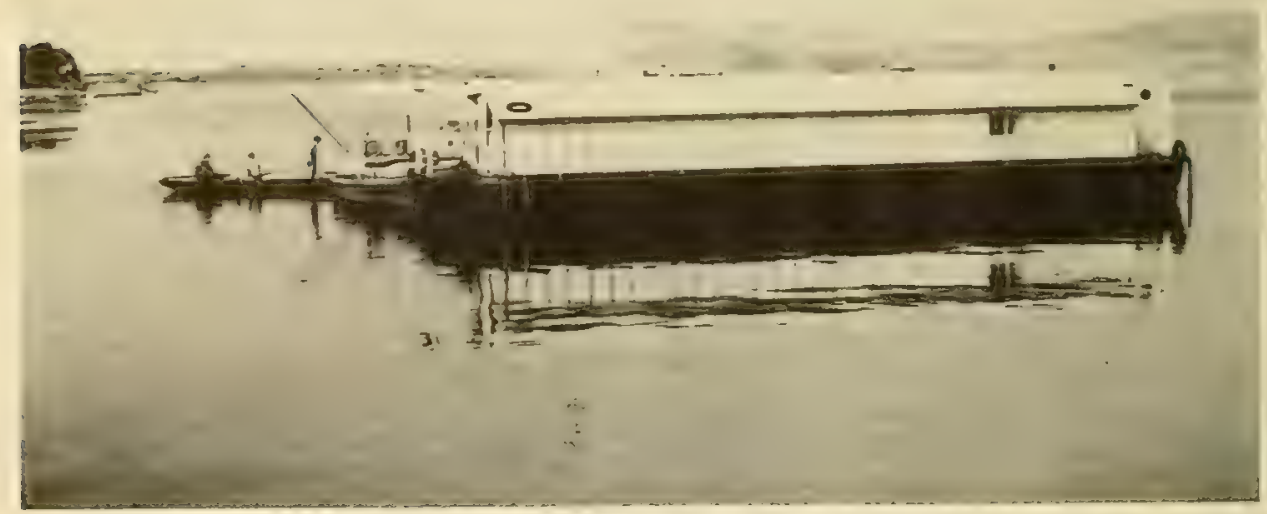

Fig. 6.14. Test-and-calibration barge of Le Brusc. 

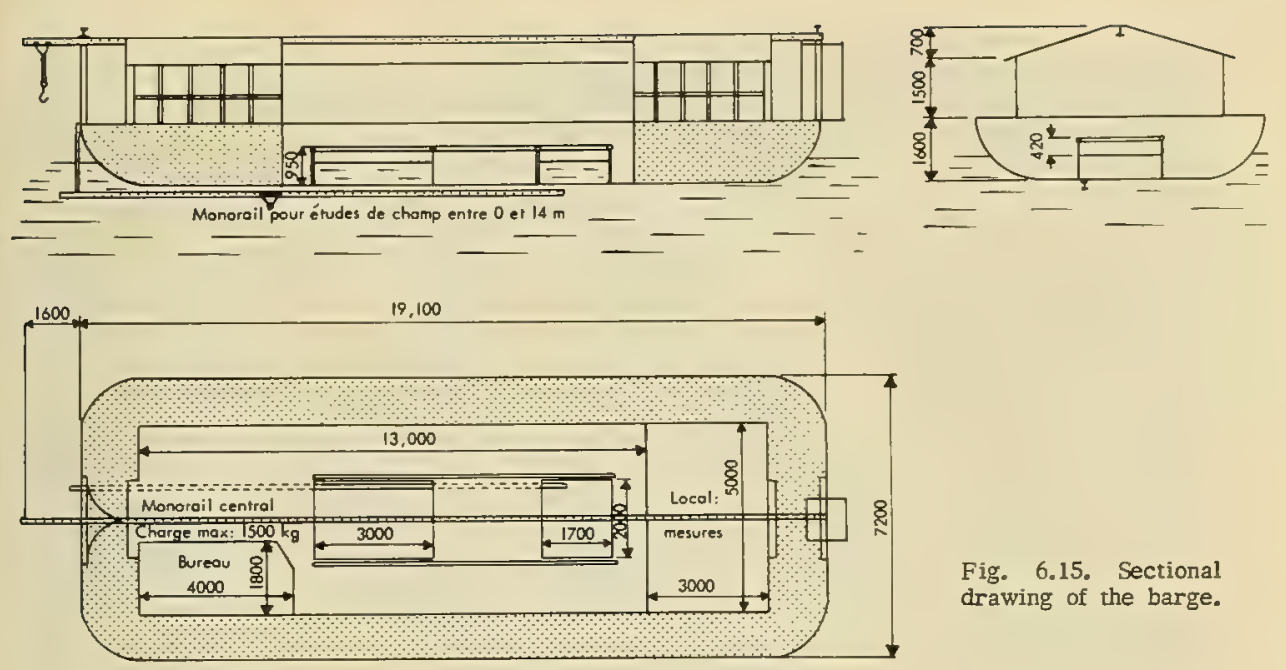

Fig. 6.15. Sectional drawing of the barge.

1. Primary reciprocity calibration by sine wave or in a narrow band.

2. Secondary calibration by sine wave by comparison with a standard projector.

3. Calibration and recording of directivity patterns of transducers larger than the wavelength where the dimensions of the anechoic tank do not allow working out of the Fresnel zone.

4. Calibration of small transducers where surface reflection due to the small value of the directivity could not be avoided in an anechoic tank.

5. Study and tests of sonar domes.

\subsubsection{Physical Features and Layout}

A pit $30 \mathrm{~m}$ long, $15 \mathrm{~m}$ wide, and $4.5 \mathrm{~m}$ deep has been dredged in the harbor bottom of Le Brusc, and the testing barge, shown in Figs. 6.14 and 6.15, has been moored over it $200 \mathrm{~m}$ off shore. This barge has been converted into a test facility by the installation of two test wells permitting a maximum separation distance from projector to receiver of $6.5 \mathrm{~m}$. The barge has an over-all length of $19.10 \mathrm{~m}$ and a width of $7.2 \mathrm{~m}$. It has a glass-walled structure covered with a roof, and is divided into calibrating, measuring, and office rooms. For hoisting the transducers and lowering them into the well, there is a monorail carrying a hoist with a weight-handling capacity of 1.5 tons.

The wells are surrounded by a gliding rail for measuring instruments. A bridge is mounted across carrying a rolling carriage and a shaft which rotates by remote control, while a polar ink recorder driven by a servosystem records the directivity patterns. By means of this carriage, transducers of up to 1.5 tons can be displaced in all directions, the bearing being adjustable to $1 / 4^{\circ}$, while the separation distance between the two shafts is known with an accuracy of $5 \mathrm{~mm}$. Less weighty transducers (up to $300 \mathrm{~kg}$ ) and projectors to be tested are displayed by two smaller carriages.

Another rolling carriage gliding on a rail fixed below the keel of the barge carries transducers for continuous measurement of the acoustic field between 0 


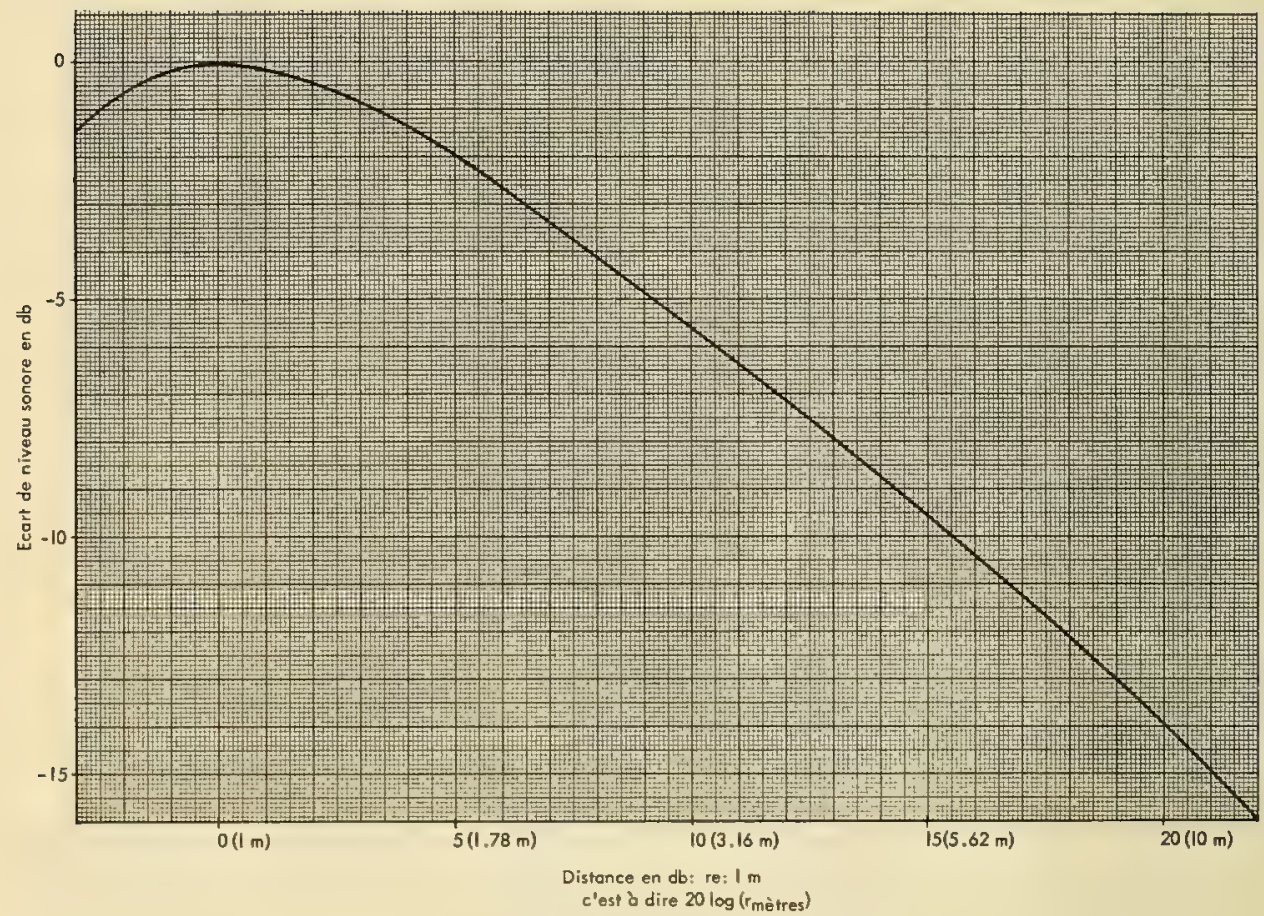

Fig. 6.16. Acoustic field between two transducers: Panoramic Projector OUM-15 and P30P-209.

and $14 \mathrm{~m}$. Between 0 and $20 \mathrm{~m}$ the measurements have to be made point by point. Thus, heavy and large-sized transducers can be calibrated free from the Fresnel zone. Figure 6.16 shows the accoustic field between the omnidirectional transducer OUM 15 and P3OP at 15 kcps.

Two concentric shafts are available for the testing of sonar domes. The central shaft carrying the transducer is rotated by remote control in the same way as described above. When studying vertical directivity patterns, depth and tilt of the test transducer can be adjusted by a rack gearing.

The standard transducers are checked by reciprocity calibration every three months. They are used in the frequency range from 6 to $34 \mathrm{kcps}$.

The instrumentation is shown in Fig. 6.17. The Test Stand provides facilities for the following measurements: (1) reciprocity calibration, (2) calibration by comparison, (3) field measurements, (4) measurement and recording of directivity patterns, and (5) study and tests of sonar domes. This stand consists of: (1) A Muirhead generator, (2) a 20 -w power amplifier, (3) a milliammeter with thermocouple for reciprocity calibration, (4) three Philips voltmeter-amplifiers, (5) a calibration amplifier with an amplifier and a series of octave-band filters covering a range from 3 to $70 \mathrm{kcps}$. This amplifier can be replaced by another with a bandwidth of $450 \mathrm{cps}$ containing tuning circuits and covering a range from 3 to $50 \mathrm{kcps}$.

The Impedancemeter is used for measuring in the range from 3 to $50 \mathrm{kcps}$ : (1) impedances $\left(R_{p}\right)$ up to 500 kohms and $(2)$ capacitances $\left(C_{p}\right)$ up to $1 \mu \mathrm{f}$. 


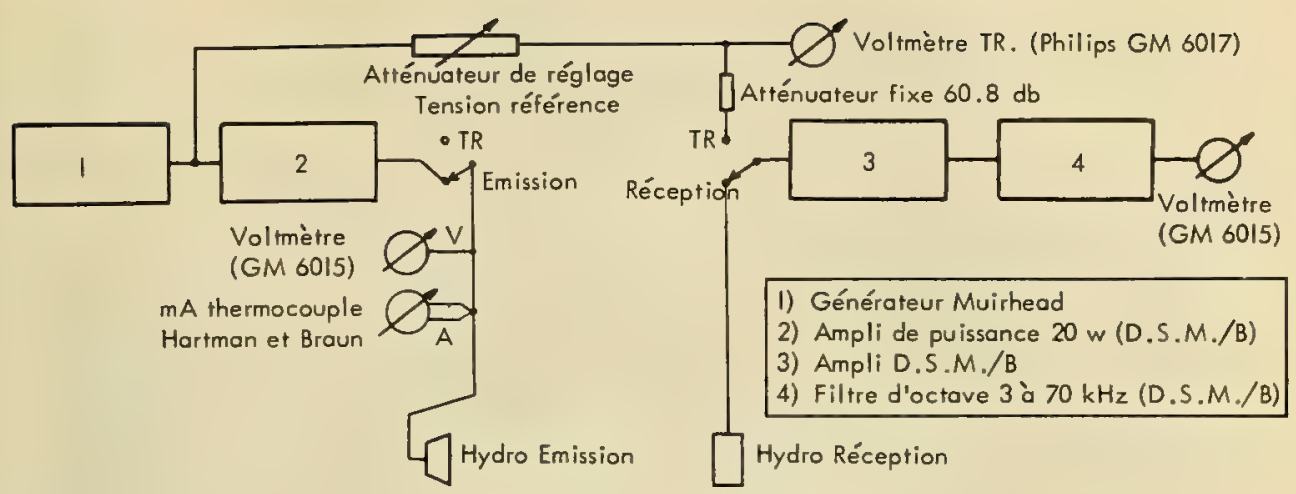

Fig. 6.17. Instrumentation on the barge of Le Brusc.

\subsubsection{Electrical Installation}

Power of $9.5 \mathrm{kv}-\mathrm{a}$ is provided by electric supply from the shore through a submarine cable.

\subsection{4, Water Depth}

One limitation on measurements is imposed by the water depth under each well area available for the realization of free-field conditions. By way of illustration, Fig. 6.18 shows some field curves measured at various frequencies between standard projectors P3OP.

It can be said that the test and calibration barge of Le Brusc provides means for precision measurements in a frequency range down to $6 \mathrm{kcps}$.

\subsection{TEST-AND-CALIBRATICN BARGE OF THE LAKE OF CASTILLON}

\subsubsection{Introduction: History}

The enlarging of the field of underwater acoustics research by measurements in the low and very low frequency range emphasized, several years ago, the necessity of an acoustic range suitable for these frequencies.

As it had not been possible to discover a point along the Mediterranean coast sheltered enough to make measurements at great depths, the necessity of finding a deep lake became evident. All the natural and artificial lakes in the near and far countryside of Toulon were surveyed during the years of 1956 and 1957, when at last the choice fell on the artificial lake of Castillon.

The ambient underwater noise level in this lake is very low, corresponding to sea state zero $(-16 \mathrm{db})$, and a water depth varying from 50 to $80 \mathrm{~m}$ can be obtained for a test facility afloat in the middle of the lake.

The project was studied and carried out by the Repair Group of the Naval Shipyard (DCAN) of Toulon, with the help of the Naval Artillery Section and the General Workshop of the Yard.

The steel frame and the floats were sent by rail from Toulon to St. Andréles-Alpes, and from there hauled by truck to the improvised assembly yard on the bank of the lake on May 12, 1959. The assembly work and the launching preparations were carried out with all desirable speed by the Sociéte Industrielle Toulonnaise, and the barge, shown in Fig. 6.19, was launched on August 13, 1959. 


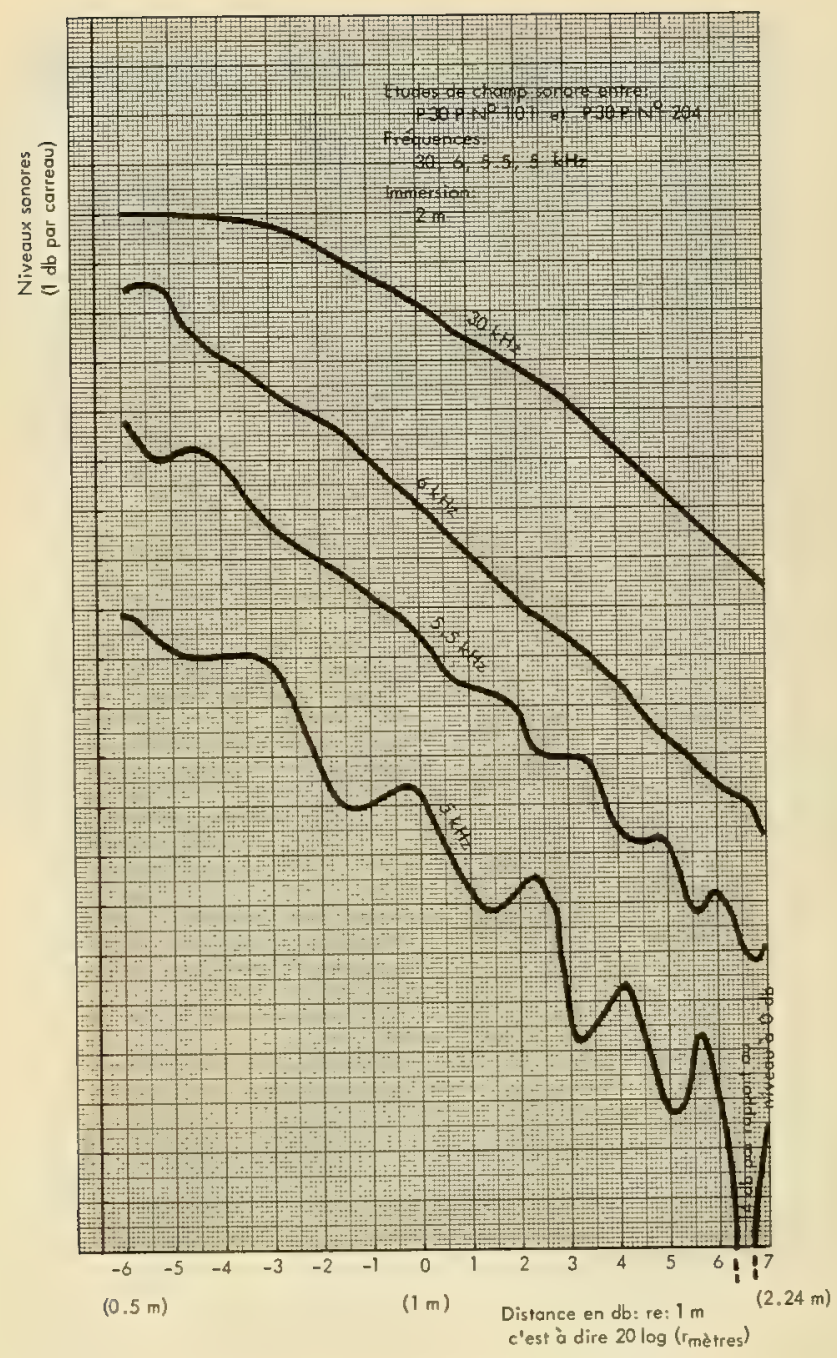

Fig. 6.18. Acoustic field at different frequencies.

\subsubsection{General Description}

The artificial lake of Castillon was created by a dam erected across the valley of the Gorges de Verdon, $6 \mathrm{~km}$ to the north of Castellane and at a distance of $146 \mathrm{~km}$ by road from Toulon.

The lake depth varies with the seasons, attaining a maximum of $80 \mathrm{~m}$ during December and January and again during June and July, while the lowest depth of $40 \mathrm{~m}$ occurs in April and September. The crest of the dam has a height of $85 \mathrm{~m}$.

The test-and-calibration barge is moored in the middle of the lake nearly $400 \mathrm{~m}$ from the dam in an area measuring 200 by $400 \mathrm{~m}$ and corresponding to the lowest bottom level. 


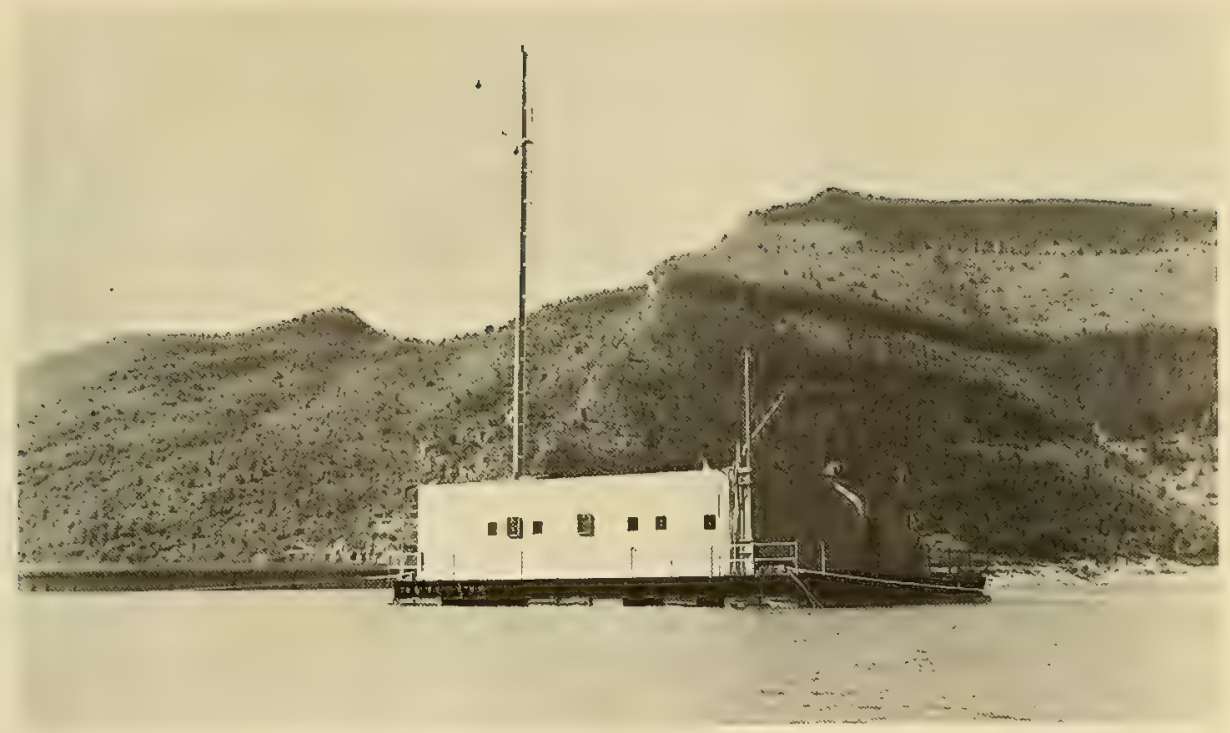

Fig. 6.19. Test-and-calibration barge of Castillon.

The mooring assembly consists of steel hawsers and nylon cables going to four buoys which are linked by a chain to four concrete blocks weighing one ton each.

\subsubsection{The Test-and-Calibration Barge}

The barge, constructed entirely of welded steel plates, consists of a roof house erected on a platform borne by 31 floats, three of which can be used to control the maintenance of a horizontal position. With a displacement of 110 tons, the barge measures $17 \mathrm{~m}$ in length and $14 \mathrm{~m}$ in width.

\subsubsection{General Layout}

In the interior of the roof house a central hall, equipped with a monorail carrying a hoist, is surrounded by a space divided into office, laboratory, test stand, workshop, and living accommodations.

In the back part of the hall, a test well has been installed measuring 9.70 by $2.80 \mathrm{~m}$. It is surrounded by a hatchway $10 \mathrm{~cm}$ high, the upper part forming a gliding rail for the testing and measuring apparatus connected with this well. By opening a gate the well can be approached by an amphibious landing craft.

\subsubsection{Mechanical Operating Gear}

A mast $20 \mathrm{~m}$ high provides the facility for handling long shafts to which the transducers are fixed. Other handling facilities consist of two davits with a weighthandling capacity of $500 \mathrm{~kg}$ each and a derrick with a capacity of one ton. The central monorail has a capacity of one ton, but it has been reinforced at the rear end in order to allow the hoisting of transducers weighing five tons.

\subsubsection{Electrical Installation}

A transformer station on the bank provides the power by means of a submarine cable. The maximum power available is $25 \mathrm{kw}$. 


\subsubsection{Acoustical Test-and-Calibration Facilities}

The facilities currently provided allow the acoustical testing and calibrating of transducers weighing less than 5 tons, by means of a bridge with rolling carriage and a hand-operated rotating shaft mounted across the well.

The standard projectors used are of the types TP8E, IK4O, and P3OP, permitting a continuous wave calibration down to $1.5 \mathrm{kcps}$ at depths attaining $30 \mathrm{~m}$.

The following improvements are already provided for in the budget:

1. Remote control for the training of the shaft

2. Bridge and rolling carriage for the handling of 8-ton transducers

3. A set of two concentric shafts allowing the testing of sonar domes.

For the lowering of 8-ton transducers into the well, the structure around the point of suspension will have to be stiffened.

The installation of the test-and-calibration barge on the lake of Castillon, at a cost of nearly $300,000 \mathrm{NFr}$, has provided a research facility where continuous wave measurements and ranging down to $1.5 \mathrm{kcps}$ are possible under very acceptable free-field conditions. Systematic studies are under way to improve the measuring conditions, such as transducer depth, influence of the water level, and so forth.

Improvement of the test facilities will have to proceed with a view to making the equipment suitable for the handling of very large transducers and capable of testing them completely and rapidly.

\section{DISCUSSION}

DR. H. A. J. RYNJA commented, regarding the electrostatic method of determining sensitivity, on the measurement of the distance between the hydrophone membrane and the capacitor plate using an interferometer. He thought that a more straightforward method was to measure the actual capacitance of the air gap because the thickness of the gap occurs in the formula for the calculation of the capacitance. This latter method offers some advantages when calibrating hydrophones having a rubber-covered face or a curved sensitive surface.

DR. FO $\overparen{C H E}$ : It is certainly possible to measure the distance between the hydrophone membrane and the capacitor plate by a capacitance measurement, but this method does not allow one to check the parallelism of the plates. As the capacitance is a linear function of this distance and the attraction strength a quadratic function, the result may be a slight error if the plates are not parallel.

DR. D. SCHOFIELD said that the reciprocity calibration of hydrophones at low frequencies can be made in water-filled rigid tanks. The only measurement required, other than what is normal for the reciprocity technique, is the compressibility of the tank, pipes, and contents. This procedure provides a simple means of determining the sensitivity of the hydrophone as a function of the hydrostatic pressure.

Dr. FOÄCHE: The reciprocity method for low-frequency measurements is an excellent one, but further work still needs to be done, and it has, therefore, not yet been possible for us to use it. 
DR. D. E. WESTON: During the discussion, mention was made of a technique of hydrophone calibration in which the instrument is moved up and down in the water. The method was at a disadvantage if the hydrophone was sensitive to acceleration, but Dr. Weston drew attention to two variants of the procedure, in one of which the problem of hydrophone acceleration did not occur. In this variant, the water, in a suitable container, is moved up and down past the hydrophone when the total acoustic pressure will be due to the changing hydrostatic pressure plus the known pressures exerted by the acceleration of the water.

DR. FOACHE: There are so many methods of calibrating hydrophones at very low frequencies that it would demand too much time to try each of them. The suggestion by Dr. Weston is certainly interesting, but we have never used that method. 

LECTURE 7

\title{
SOME AREAS IN WHICH UNDERWATER ACOUSTICS RESEARCH IS NEEDED
}

\author{
H. R. Baker \\ The North Atlantic Treaty Organization \\ Scientific Affairs Division, Paris, France
}

It is with some hesitation that I speak to this group of experts in underwater acoustics on the subject of areas of underwater acoustics where research is particularly needed. Most of you have contributed greatly to the present state of knowledge in this field and will continue to contribute, as the papers presented at this Institute demonstrate. I need not elaborate on the importance of the problem of submarine detection to the NATO Alliance. We are all aware that underwater sound techniques are the only promising means for detecting submarines at acceptable ranges. I warn you that my remarks are likely to be biased in the direction of submarine detection, classification, and identification; at the same time, I am aware that the solution to these problems depends upon a basic understanding of acoustic phenomena in the oceans.

Almost every area in which I intend to suggest the need for research has been or will be touched upon in talks given at this Insitute, but let us proceed in the hope that I may stimulate your thinking about a few areas where basic and applied research are needed.

\subsection{SOUND TRANSDUCERS}

Basic research on transducing materials should continue, but perhaps we need most to concentrate on the effective use of the knowledge we already possess in this area. First, I must point out that research could profitably be done into the conversion of mechanical energy directly to sound. Hydraulic devices are capable of exerting a great force through a small distance; and this is exactly what is needed, if we can find ways to exert this force in a controlled periodic manner. This approach seems very attractive for low-frequency sound sources because it offers high power capability, ruggedness, and reliability.

Large open arrays of transducer elements seem to be the answer to many sonar problems, yet the criteria for designing such arrays to a given specification are not well understood. Perhaps what is not understood is the interaction between elements of an array when it is driven at high power or when delay lines are used to form and steer acoustic beams. This problem requires both theo- 
retical and experimental work involving a great many measurements. The number of measurements is likely to be great, and the calculations many and extensive, but modern instrumentation and computers should make the problem more attractive. A few researchers are attacking this problem, but it will require the efforts of the ablest people in the field to obtain the knowledge required for reliable design of open arrays.

For some applications it seems desirable to operate transducers at great depth; thus, there is a need to know the effects of high pressures on various types of transducer elements.

Calibration facilities for large low-frequency arrays is another major problem. Because of the long wavelengths involved, free-field measurements are not possible at most present-day calibration facilities. Pachner and others have done theoretical work on the extrapolation of many near-field measurements to the far-field pattern. Another approach is the design of a stable platform for use in deep water, so that free-field measurements can be made. This approach is certain to be very costly and cumbersome. Pachner's work must be carefully evaluated and subjected to experimental test.

\subsection{PROPAGATION}

In the past ten years the use of low-frequency sound which propagates to great distances with little attenuation has stimulated investigations of numerous propagation paths, namely: propagation in surface-bounded ducts, propagation by bottom-reflected paths, by refracted paths to convergence zones in deep waterand in recent years some investigation has been made of propagation paths from deep sound sources. Much of the propagation data is not susceptible to rigid analysis because the conditions under which it was taken could not be rigidly controlled, and it is impossible to simulate the ocean adequately in laboratory tanks. Some facilities and techniques recently developed make it possible to remedy the situation in part. These facilities are fixed, high-power, low-frequency sound sources, sensitive hydrophone arrays, and vastly improved signal processing equipment. This equipment, combined with modern recording techniques and electronic computers, makes it possible to collect and process data on a continuous basis. All of this, however, is expensive and will require the cooperation of national governments, navies, and scientists of many nations. For example, underwater sound sources and receiving arrays set up on either side of the English Channel and cabled to shore could be operated on a yearround basis. Properly instrumented, these stations could continuously record signal levels, reverberation levels, and noise background levels, and could correlate them with weather and oceanographic conditions. Data in quantity will make valid statistical analysis possible. At the sametime, such a pair of stations could provide a continuous surveillance for the passage of submarines through these narrow waters. Other sets of sound stations in restricted shallow water areas, such as the Danish Straits, the Bosphorous, the Straits of Gibraltar, etc., could provide surveillance of strategic areas and at the same time yield valuable propagation data to sound physicists. The stations described, of course, could only provide data for shallow water situations.

The political and physical geography of Europe is an asset to the NATO nations in the matter of submarine surveillance. To capitalize on this asset, it is 
necessary to accumulate data on sound propagation through the shallow waters of strategic locations. These data can best be obtained by a cooperative effort of the nations bordering the waters of these strategic areas. Some fixed installations for research purposes are highly desirable. It is entirely possible that information gained from relatively modest fixed installations may serve to guide the development of operational installations.

There are several locations near islands where deep-water sound stations could be installed and cabled to shore. From these stations, continuous data on reverberation and noise levels could be recorded. In cooperation with a submarine equipped to transmit and receive, transmission loss could be measured over a great range in any kind of weather.

Propagation data for the cold water of the North Atlantic, the Norwegian Sea, and the Arctic Basin are meager. While the severe climate would increase the difficulty, fixed stations in these strategic areas could be established; and the data are badly needed.

\subsection{SIGNAL PROCESSING}

Fixed sonar stations can be used for experimental signal processing with little additional equipment. Such matters as signal coherence in time and space can be analyzed on a statistical basis. By careful attention to recording procedures, data can be preserved and distributed on magnetic tape to the various laboratories, where it can be used many times and with different signal processing equipment. Ideas for new research in the area of signal processing will be covered in another paper.

\subsection{COUNTERMEASURES}

A submarine is blind when denied the use of the surface of the ocean and depends upon its ears for information. Little effort has been exerted in research and development to find effective means to deceive submarines. While acoustic countermeasures $i_{i}$ themselves cannot destroy submarines, under the right conditions they can be used effectively to lure them to areas susceptible to attack or to keep them away from convoys or naval task forces. Acoustic torpedoes can likewise be deceived and directed away from their intended victims by well-designed countermeasures. Carefully planned work on acoustic countermeasures in peacetime could be of great value in time of war.

I have deliberately pointed out areas where international cooperation is desirable in the research and development program. The Division for Scientific Affairs in NATO attempts to stimulate interest and sponsor research in areas where the efforts of more than one nation are required for achieving best results. 



\section{LECTURE 8}

\section{INTERNAL WAVES}

\section{J. Crease \\ National Institute of Oceanography \\ Wormley, Surrey, England}

The intention of this paper is to review our knowledge of internal waves in the ocean, selecting particularly those aspects which may be relevant to underwater acoustics. For present purposes, internal waves may be roughly defined as gravity waves having greater amplitude in the body of the sea than at the surface. Noteworthy is their effect in disturbing the lower boundary surface of an isothermal surface layer and the consequent variation in horizontal distance to the shadow zone. Further, the periodic variation in curvature of the isovelocity lines will result in some degree of focusing and defocusing of the sound rays. Lee [1] recently computed the ray paths for a source in an isothermal region overlying another and separated from it by a wavy surface. Figure 8.1, from [1], illustrates the resulting variation in intensity. As the height of some internal waves is known to be several tens of meters, it will be of some importance acoustically to discover their properties.

Interest in the subject was first stimulated by the observations of Scandinavian oceanographers at the beginning of the century. The "Fram" expedition

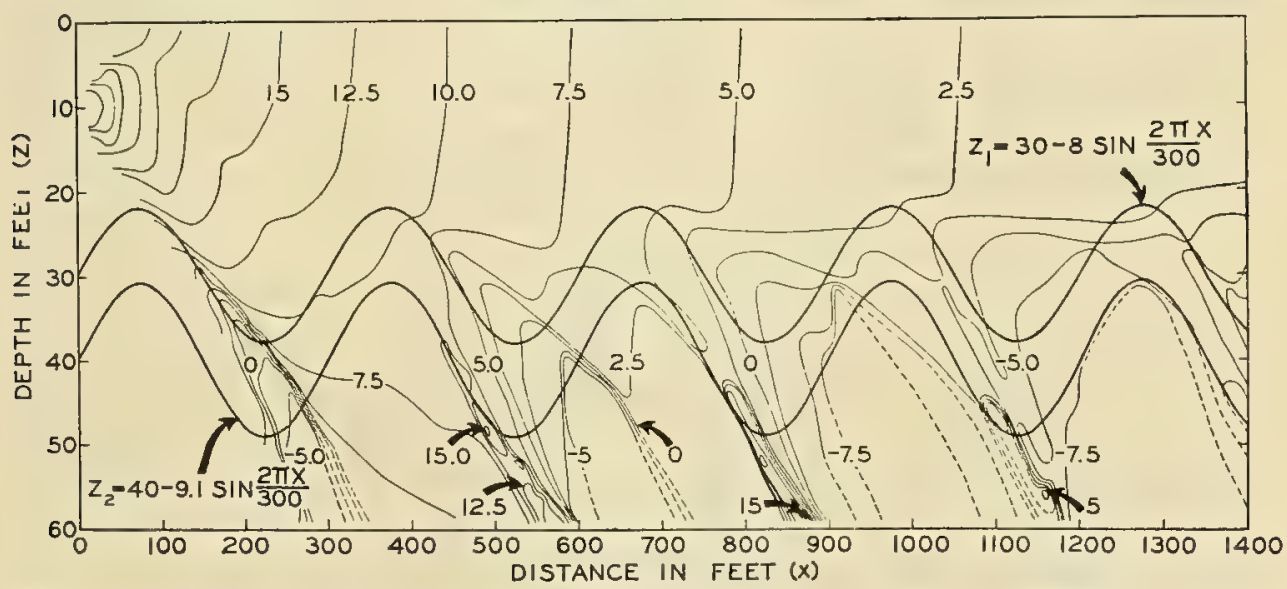

Fig. 8.1. Focusing of sound from a source by internal waves on two internal discontinuities. Heavy lines are the interfaces, light lines the sound level (from Lee) [1]. 
under Nansen's leadership, on approaching the ice edge in northern waters, encountered the phenomenon of Dead Water, well known to seamen, but not previously investigated scientifically. Ships encountering it found themselves able to make little or no headway. Ekman was able to show with an excellent series of model experiments that Dead Water arose when a ship running into a layer of fresh water overlying salt creates ship waves on the internal interface between the layers; the extra power required to generate the internal waves was sufficient to reduce the headway drastically. It was at about the same time that the repeated use of the more accurate deep-sea thermometers then becoming available indicated naturally occurring oscillations within the sea unaccompanied by visible surface displacement.

Rather than catalog here the historical record of the subject, it is now proposed to give a qualitative account of the theory and the most significant observa tions. Stokes (cf. Lamb [2], p. 370) was the first to consider waves on the interface between two liquids of different densities. We need only note here that in a homogeneous fluid there is a unique frequency-wave number relationship $\sigma^{2} / k^{2}=(g / k)$ tanh $k h$, which for short waves becomes $\sigma^{2} / k^{2}=g / k$, and for long waves is $\sigma^{2} / k^{2}=g h$, where $h$ is the depth of water, $\sigma$ is the frequency, and $k$ is the wavenumber. For a two-layer system on the other hand, there are essentially two degrees of freedom (analogous in some ways to the motion of a double pendulum) and the $\sigma-\mathrm{k}$ relation is no longer single-valued. In fact, for liquids of almost equal densities the relation factors into $\sigma^{2} / k^{2}=g / k$, and into $\sigma^{2} / k^{2}=$ $g h(\Delta \rho / \rho)$ for the special case of an upper layer of depth $h$ overlying a deep lower layer whose density is greater by $\Delta \rho$ - where $h$ is small compared to the internal wavelength. The first relation is appropriate to ordinary deep-water surface waves and the second to a wave with maximum amplitude at the interface. The latter has the appearance of a long surface wave with gravity reduced by a factor $1 \rho / \rho$. This may be seen to arise from the reduced potential energy required to deform the interface between liquids of almost the same density, as opposed to that required for the deformation of the free surface. By introducing successively more interfaces, one may approximate to a continuous distribution of density; and it should be clearthat this will be associated with an infinite sequence of $\sigma-k$ relations.

The following equation for the vertical velocity (and displacement) was derived by Fjeldstadt [3] in 1933 for an ideal, incompressible, rotating fluid with a continuous distribution of density; the notation is somewhat different from his:

$$
W^{\prime \prime}+\left[k^{2} \frac{N^{2} / \sigma^{2}-1}{1-f^{2} / \sigma^{2}}-\frac{N^{4}}{4 g^{2}}-\frac{N N^{\prime}}{g}\right] W=0
$$

where $W=\rho^{1 / 2} \omega ; \omega e^{l\left(k x-\sigma_{t}\right)}$ is the vertical velocity (or displacement); the derivatives are with respect to the vertical coordinate $z ; k$ and $\sigma$ are the horizontal wavenumber and frequency; $f$ is the Coriolis parameter equal to $2 \Omega \sin \theta$, where $\Omega$ is the earth's angular velocity and $\theta$ is the latitude. Finally, $N$ is the Brunt Vaisala frequency equal to $[(g / \rho)(\partial \rho / \partial z)]^{1 / 2}$. This is clearly a stability parameter and is the frequency of oscillation of a parcel of fluid displaced vertically from its position of equilibrium in a gravitational field. The last two terms in the equation are generally small in the ocean and will be neglected in the subsequent 
remarks. For a temperature gradient of $1^{\circ} \mathrm{C} / \mathrm{m}, N=4.4 \cdot 10^{-2} \mathrm{sec}^{-1}$ for $1^{\circ} \mathrm{C} / 10 \mathrm{~m}$, $N=1.4 \cdot 10^{-2} \mathrm{sec}^{-1}$. The first figure is more appropriate to the diurnal thermocline, and the latter to the seasonal thermocline at a depth of $150 \mathrm{ft}$ or so. Together with the boundary conditions, Eq. (1) constitutes an eigenvalue problem. The boundary condition at a flat bottom is $W=0$ and the same condition may be used at the free surface, bearing in mind that the solutions of most interest in the present context have maximum amplitude in the interior with little deformation of the free surface. By this artifice the surface wave solutions are, of course, lost.

With the equation in this form it is clear that there is fundamentally different behavior of the solution in different ranges of $\sigma$. First, if $\sigma$ is greater than the maximum value of $N$ (i.e., $N_{\max }$ ), all solutions will be of exponential type throughout the depth range; and in view of the boundary conditions this means that in fact no solutions at all are possible. If $N_{\max }>\sigma>f$ there is at least a range of $Z$ in which the solution is oscillatory, and there is then the possibility of a solution satisfying the boundary conditions. Finally, for $\sigma<f$ there are again no solutions (other than the unallowable exponential ones). I have tacitly assumed, as is generally true in the ocean, that $N>f$. Eckart [4] (1960) has given a full treatment of this eigenvalue problem for a compressible fluid.

From these remarks we may conclude that $N$ and $f$ are upper and lower bounds to the frequencies of free internal waves in the sea, although there are further free oscillatory motions which have periods greater than a week or so which are quasi-geostrophic in character and will not be considered here. Groen [5] in 1948 first drew explicit attention to the upper limit $N$ on the frequency, but there has been little in the way of observations (in the open literature) to confirm it. Only now are the techniques becoming available to make the required observations in deep water at sufficiently close time intervals. An appropriate reference is the paper of Haurwitz, Munk, and Stommel [6] (1958). A long series of temperature measurements were made with thermistors on the sea floor at depths of 50 and $500 \mathrm{~m}$ offshore from Bermuda. From Fig. 8.2, a section of the record, it is immediately clear that the deep thermistor shows the presence of higher frequencies. This is confirmed by the respective spectra. They both approach a low level rapidly with increasing frequency, and the shallower record differs from the deeper record by a factor of ten. Figure 8.3, showing the variation of $N$ with depth, indicates $N_{\max }$ at $5 \frac{1}{2}$ cycles/hr; at the upper thermistor, $N$ equals $1 / 2$, while at the lower it is $1 \frac{3}{4}$. It would seem likely that herein lies the explanation of the observed difference in frequency response. The two peaks in $N$ correspond to the seasonal and main thermoclines-often one may also expect a diurnal thermocline responding to meteorological conditions with possibly large values of $N$. The development of chains of thermistors at some laboratories, notably at Woods Hole Oceanographic Institution, for rapid sampling of the vertical profile of temperature in the top $400 \mathrm{ft}$ of the sea, would seem to be of the greatest importance in investigating this part of the spectrum.

It is possible to make some statements about the velocity of propagation without getting too involved in equations. Fjeldstadt [3] in 1933 and Groen [5] in 1948 treated the analytical details in special cases. At frequencies away from either limit, Eq. (1) becomes 


$$
W^{\prime \prime}+\frac{k^{2}}{\sigma^{2}} N^{2} W=0
$$

and with the boundary conditions will lead to a wave velocity $c=N h / \pi$ for the first mode if $N$ is constant (i.e., $c=1 / \pi[(\Delta \rho / \rho) g h]^{1 / 2}$, where $\Delta \rho$ is the total density variation in a depth $h$. In the case of the deep thermocline (and sound channel) where large stability is confined to a limited part of the depth, one may expect intuitively that the $\Delta \rho$ and the $h$ appropriate to the problem are the change in density and the thickness of the thermocline itself. Thus, if there is a temperature gradient of $8^{\circ} \mathrm{C}$ between, say, 400 and $1000 \mathrm{~m}$, the velocity is approximately $100 \mathrm{~cm} / \mathrm{sec}$, and for an $8^{\circ} \mathrm{C}$ change at a depth of $50 \mathrm{~m}$ (using the simple Stokes formula for two layers), we find that $\mathrm{c}=90 \mathrm{~cm} / \mathrm{sec}$. There is rather little differ ence between these two apparently dissimilar cases of internal waves, but on the other hand the velocity of surface waves in a total depth of, say, $3000 \mathrm{~m}$ is $\mathrm{c}=2 \cdot 10^{4} \mathrm{~cm} / \mathrm{sec}$, far greater. We shall return to this disparity later. Using this velocity $(100 \mathrm{~cm} / \mathrm{sec})$, we find the wavelength equal to $3 \frac{1}{2} \mathrm{~km}$ for a wave of period of $1 \mathrm{hr}$. As the frequency approaches $N$ the velocity goes to zero. It is on such time and space scales that information is badly needed. The paper of Haurwitz et al. [6] has been referred to, and there is one further set of observations by Ufford [7] (1947) which is of particular interest. He worked with a triangle of three temperature elements moored to the bottom up to $300 \mathrm{~m}$ apart. By correlation techniques the phase lag was computed between the records and, hence, the wavelength was deduced. The apparent period of the waves is about $6.8 \mathrm{~min}$, but this is affected by the mean current present. In most cases he was able to explain the $\sigma-k$ relation satisfactorily as the first mode of a simple Stokes two-layer theory or a simple extension of it. Recent progress reports from Scripps indicate that Cox has been getting satisfactory results also. It seems possible that work on acoustic propagation could yield much useful information about the waves (cf. Lecture 16).

Alongside the development of experimental techniques there is room for some extension of the theory. The theory has been primarily concerned with the eigensolutions of the equations. It would seem fruitful to apply statistical methods akin to those now being explored for surface waves in view of the "noisy" appearance of the spectrum and the apparent lack of discrete lines over most of it. There is the added complication that the $\sigma-k$ relation is not single-valued. A further problem is that in many cases, one of which will be illustrated shortly, the disturbance to the isotherms is too large to satisy a linear theory. As with long surface waves, this will lead to change of form and possible breaking. This could well increase the spectrum level at frequencies beyond the upper limit $N$ (i.e., the region of microstructure). The only other work of which the author is aware regarding the nature of the spectrum will also serve to introduce a discussion of the lower frequencies which are susceptible to observation by repeated dips of a standard bathythermograph. Two ships of the USHO occupied an anchor station to the north of Bermuda and made $B / T$ observations every half hour for the whole period of 25 days. In generai, the spectrum of the fluctuations in level (Fig. 8.4) of an isotherm does not contradict the idea that it may be regarded as noise. However, between 20 - and $30-\mathrm{hr}$ periods there is a resolved 

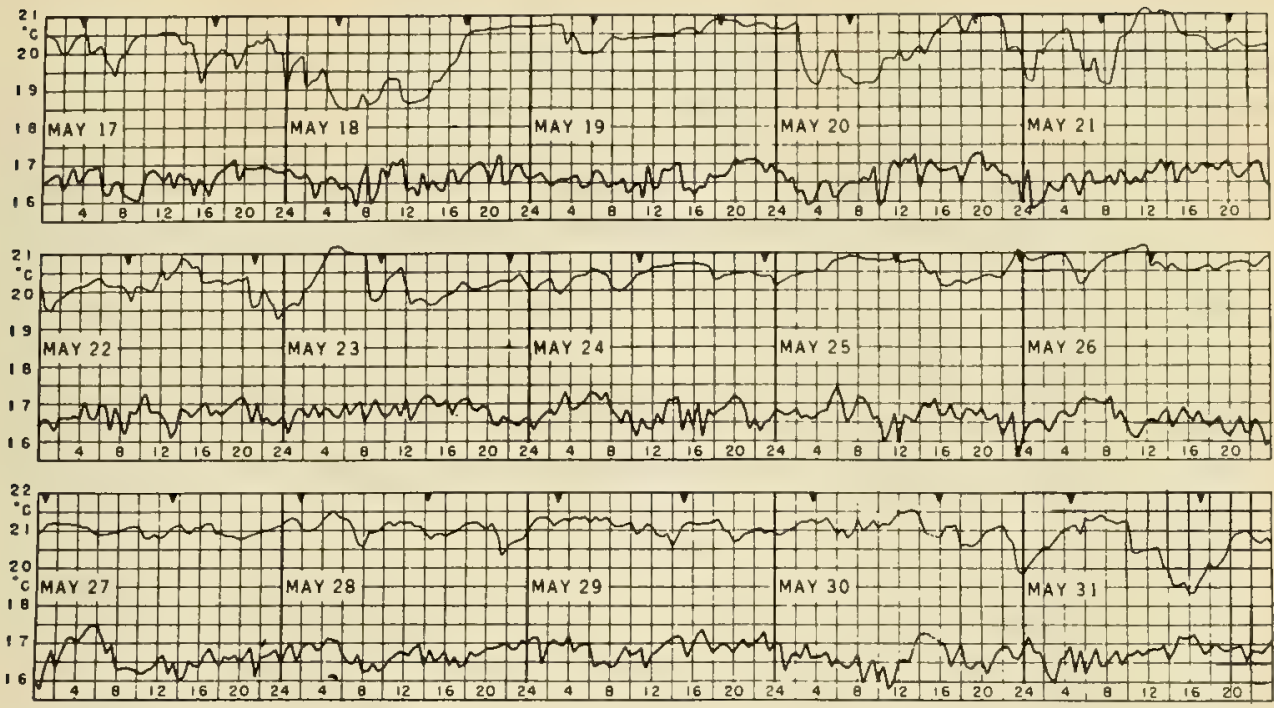

Fig. 8.2. Temperature record at $50 \mathrm{~m}$ (upper trace) and $500 \mathrm{~m}$ (lower trace) off Bermuda (from Haurwitz et al.) [6].

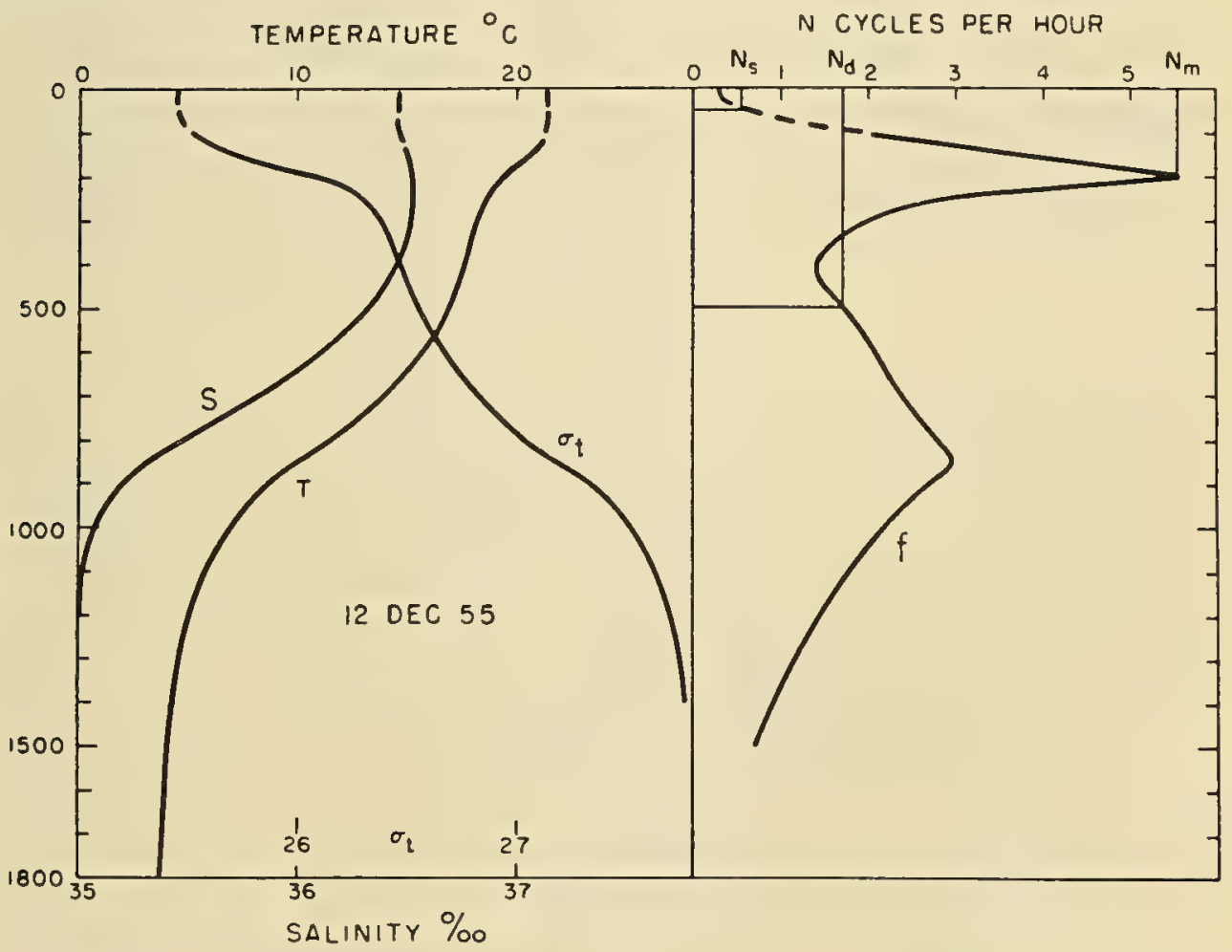

Fig. 8.3. Mean density $\left(\sigma_{t}\right)$ and $N$ as a functions of depth (meters) off Bermuda (from Haurwitz et al.) [6]. 


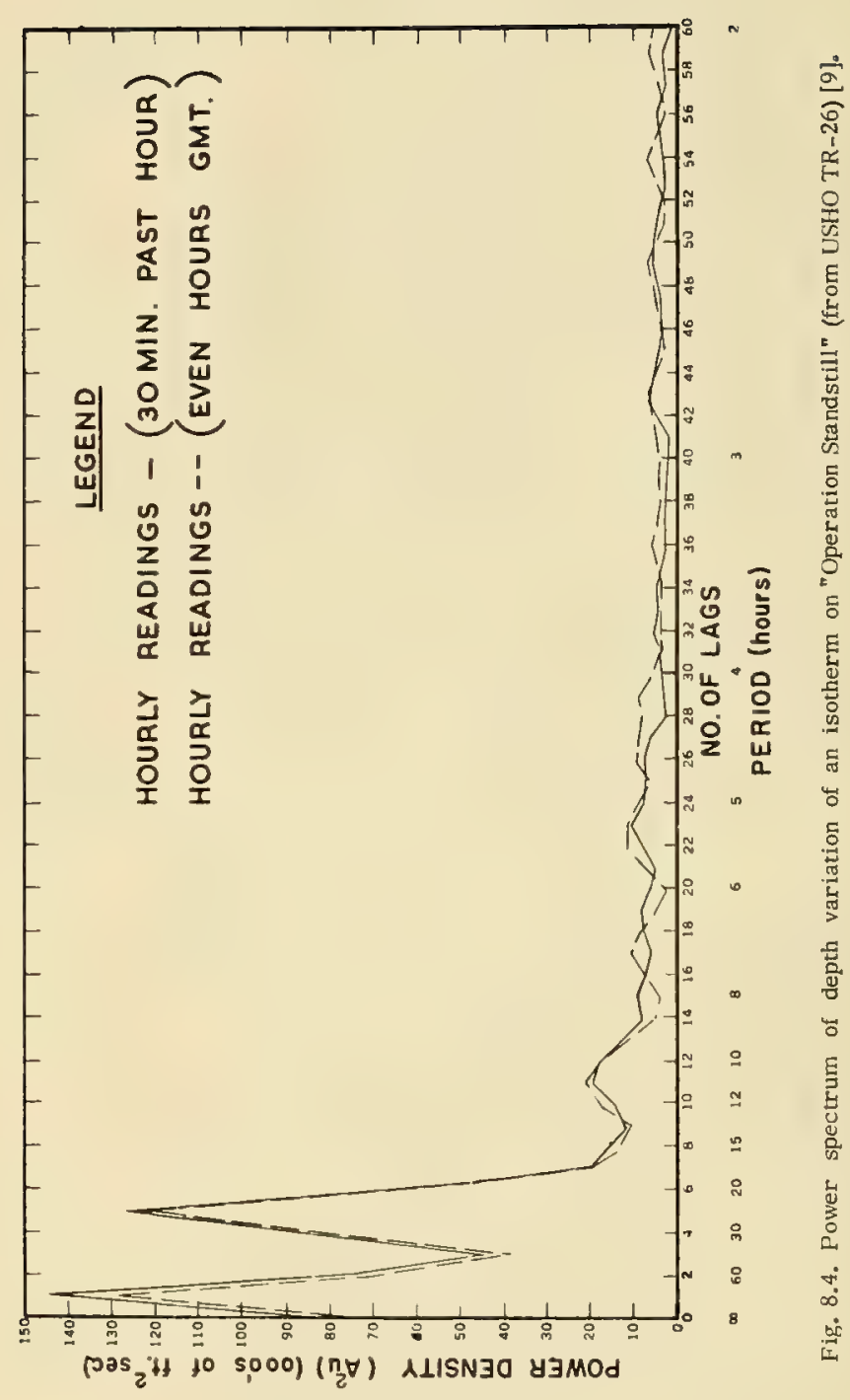


peak. This is more obvious in the correlogram from which the spectrum was made. It is at these longer periods observable by $B / T$ and hydrographic station work that most observations have been made, and there are many reports in the literature of the presence of definite periodicities. Defant [8] (1961) describes a number in which there appear to be semidiurnal and diurnal waves. Anomalies in long oceanographic sections across the sea are often attributed without any direct evidence or justification to the transient effect of the waves. If they are present, they will seriously affect the interpretation of the mean temperature field and, as a consequence, of the acoustic regime as well. The fault of most of these measurements is that they were of relatively short duration, perhaps two or three days, and thus contained only a small number of waves of the suspected period. Haurwitz (1954) has shown, in a statistical treatment of four of the open ocean cases reported in the literature, that only one (by the German research ship Meteor) definitely indicated a semidiurnal tidal component that could not equally well be attributed to chance variations. Even though they may be random, these variations of long period are likely to be of considerable importance, as they may have amplitudes approaching $100 \mathrm{~m}$.

There are some difficulties in accounting fordiurnal and semidiurnal internal waves. It is natural to look for their cause in the tide-raising force that creates the surface tides but this does not immediately seem possible-the tidal force is acting on the whole body of the fluid, and without some resonance mechanism nothing much is to be expected in the way of internal waves. It has been noted that typical velocities in the midrange of the spectrum are $100 \mathrm{~cm} / \mathrm{sec}$, much lower than the speed of the tide, and there wouldn't seem to be much hope of resonance. However, to look at the possibilities, consider again Eq. (1) and note that for waves whose frequency $\sigma \rightarrow f$ the internal wave velocity is given by the same expression as before except for a factor $\left(1-t^{2} / \sigma^{2}\right)^{-1 / 2}$ i.e.,

$$
\frac{\sigma}{k}=c=\left[g h \frac{\Delta \rho}{\rho} \frac{1}{\left(1-t^{2} / \sigma^{2}\right)}\right]^{3 / 2}
$$

Thus the rotation of the earth implies that for $\sigma \rightarrow f$ the velocity becomes large and the wavelength long. There is now the possibility of some degree of resonance. However, $f$ varies with latitude and the diurnal tide will strictly achieve resonance only at about $30^{\circ}$ latitude, and for semidiurnal tide the resonance latitude will be closer to the poles. It is interesting that the USHO [9] observations off Bermuda in $33 \mathrm{~N}$ should show the possible presence of diurnal waves and no semidiurnal waves. Thus there is noexplanation so far for the semidiurnal waves except perhaps in high latitudes. Regarding these, a most significant set of observations were made by Reid [10] (1956) off California (Fig. 8.5). These are the observations referred to earlier in connection with breaking. The semidiurnal period is very pronounced and the important point is that this record was obtained just off the continental borderland in deep water and records made farther offshore revealed nothing like this regularity. What appears to be happening here is that while the surface and internal waves are effectively uncoupled when moving over a flat bottom, the presence of an abrupt change of depth at the continental slope enables a transfer of energy to take place from the sur- 


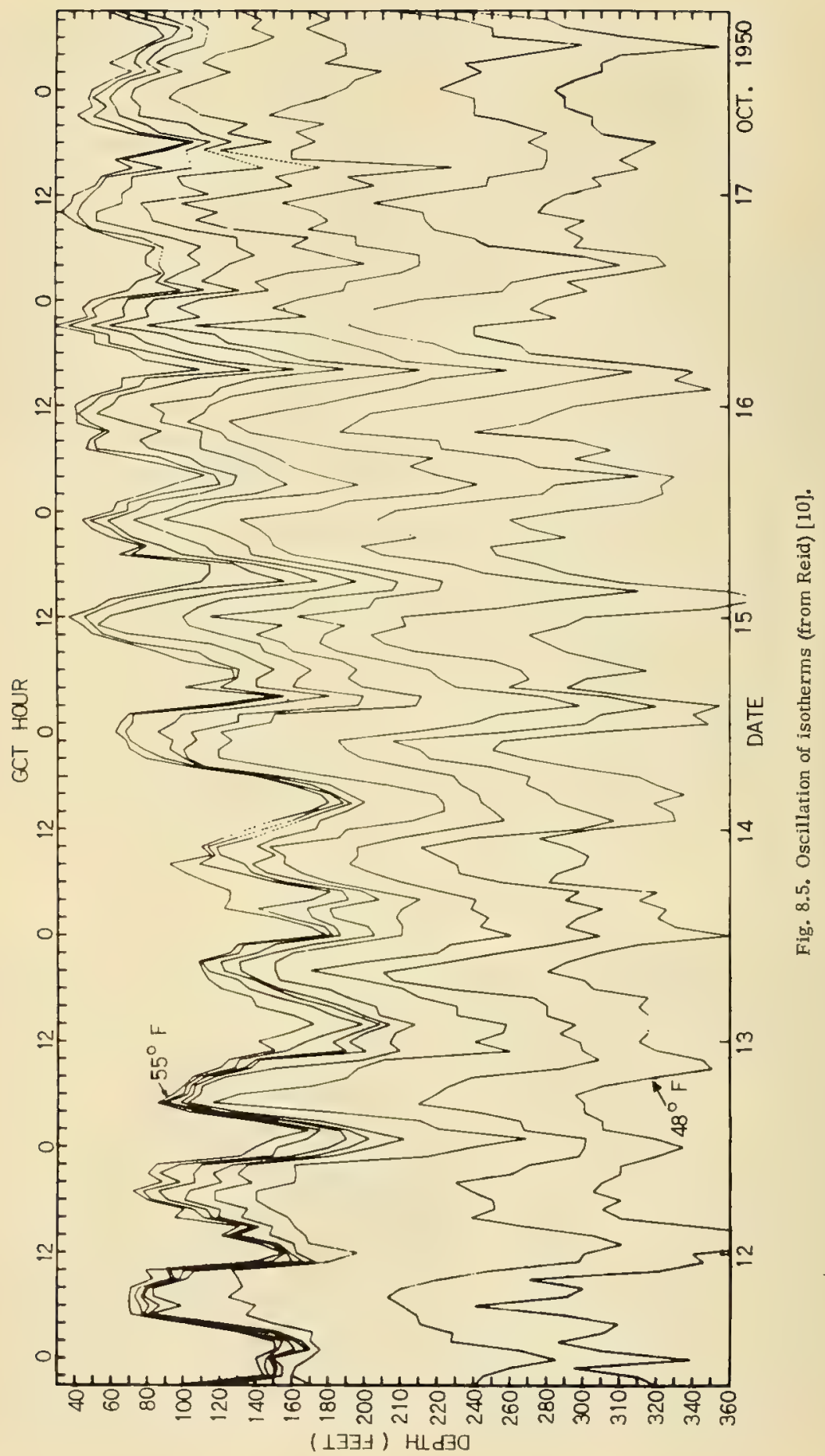


face tide to the internal tide. Rattray [11] (1960) has considered this problem in some detail and given numerical examples. It is perhaps worth quoting Nansen (1909), who said soon after the first observations were made that tidal waves crossing from the North Atlantic into the polar basin across a ridge might be expected to generate internal waves. The presence of semidiurnal waves in open ocean at the Meteor station might now perhaps be accounted for by its location, the mid-Atlantic ridge. Further effects of submarine topography will be found in the production of lee waves. Frassetto [12] (1960), for example, has obtained records with a thermistor chain in the straits of Gibraltar which suggest the possible presence of lee waves.

In conclusion, theory suggests that free internal waves will exist in a band of frequencies $f<\sigma<N_{\max }$. It appears that the spectrum has the character of noise with discrete tidal frequencies and lee waves appearing at abrupt changes in depth, such as the continental shelves. Significantly, the outermost of Reid's stations referred to above, although showing no coherent picture, did contain as much mean square fluctuation as the innermost station with its regular oscillations. This could be explained by the waves' breaking and distributing their energy over a broad band of frequencies.

\section{REFERENCES}

1. O.S. Lee, "Effect of an Internal Wave on Sound in the Ocean," J. Acoust. Soc. Am., Vol. 33,5 (1961).

2. H. Lamb, Hydrodynamics, 6th ed. (Cambridge, 1932).

3. J. E. Fjeldstadt, "Interne Wellen," Geofys. Publikasjoner, Vol. 10, No. 6 (1933).

4. C. Eckart, Hydrodynamics of Atmospheres and Oceans (Pergammon, 1960).

5. P. Groen, "Contribution to the Theory of Internal Waves," Kon. Ned. Met. Inst., B, II, 11 (1948).

6. B. Haurwitz, H. Stommel, and W.H. Munk, "On the Thermal Unrest in the Ocean," Rossby Memorial Volume, 74-94 (1958).

7. C. W. Ufford, "Internal Waves Measured at Three Stations," TAGU, Vol. 28, 1 (1947).

8. A. Defant, Physical Oceanography, VoI, II (Pergammon, 1961).

9. "Power Spectrum Analysis of Internal Waves from Operation Standstill, " USHO, TR-26 (1955).

10. J. L. Reid, "Observations of Internal Tides in October 1950," TAGU, Vol. 37, 3 (1956).

11. M. Rattray, "On Coastal Generation of Internal Tides," Tellus, Vol. 12, 1 (1960).

12. R. Frassetto, "A Preliminary Survey of the Thermal Microstructure in the Straits of Gibraltar," DeepSea Research, Vol, 7, 152 (1960).

13. B. Helland-Hansen, and F. Nansen, "The Norwegian Sea," Report on the Norwegian Fisheries and Marine Investigation, II, No. 2, Bergen (1909).

\section{DISCUSSION}

MR. D.E. WESTON commented upon the considerable fluctuations in level found in most sound propagation experiments which could arise from multiple-path interference effects of various kinds, effects of reflection from a moving surface, and inhomogeneities in the water, of which internal waves are a special case. Because it is difficult to state the precise cause of the fluctuations, in any investigation of the effects of internal waves on acoustic fields care should be taken to arrange special experiments in the regions where high-amplitude internal waves are known to exist. Mr. Weston also sought information on the usefulness of biological markers. He mentioned the existence of the sound scatterers which congregate at thermoclines, so permitting the latter to be recorded on echo sounders. There was also the phenomenon of "slicks", which have recently been shown to be associated with the periodic convergences over internal waves; these may possibly be rendered visible by biological effects. 
MR. CREASE: Mr. Weston is quite right to emphasize the difficulties that will arise in interpretation of internal wave effects on sound propagation. A good example of internal waves, disclosed by biological scatterers, is to be found in Lecture 16, and I agree again that slicks are often evidence of internal waves, although they might well be the result of convective processes.

PROFESSOR R. E.H. RASMUSSEN asked whether the theory of internal waves in sea water was also applicable to the atmosphere. He suggested that if such were the case, then the theory might be checked more easily by observations in the atmosphere.

MR. CREASE: In reply to Professor Rasmussen, the internal was theory should be applicable in the atmosphere, but I would not expect verification to be any easier. 


\title{
LECTURE 9
}

\section{HIGH-INTENSITY SOUND IN LIQUIDS}

\author{
E. Meyer \\ III Physikalisches Institut \\ der Universität Göttingen \\ Gortingen, West Germany
}

When speaking of the generation of high sound intensities, we are inclined to think of explosions and atom bombs. These produce enormous intensities in water as well as in air. We will, however, exclude such generators from this report because it is still rather difficult and dangerous to explode atom bombs in the laboratory. We will limit ourselves to conventional methods of sound generation and will discuss the generation and measurement of high acoustic power in water and the effects observed, when intense sound waves are propagated through liquids.

\subsection{GENERATION OF HIGH ACOUSTIC POWER}

\subsubsection{Continuous Wave Transducers}

In the generation of sound, it is appropriate to distinguish between sinusoidal, more or less stationary excitation, and pulse excitation. It is obvious that sinusoidal sound sources radiate high power only if operated at resonance. It is also evident that only magnetostrictive and piezoelectric transducers are of interest in this connection. We will not consider the generation of the lower frequencies up to some kcps. High frequencies, or rather small wavelengths, are preferred since still another condition must be fulfilled for the generation of high intensities, i.e., the radiated sound has to be focused. This is achieved either by using large radiating areas or, even better, spherical or cylindrical radiating areas.

If we use a quartz plate as a sound source and if we are able to prevent cavitation in the liquid, we can generate sound intensities of up to about $60 \mathrm{w} / \mathrm{cm}^{2}$. It has been reported that, under exceptionally favorable conditions, intensities of up to $300 \mathrm{w} / \mathrm{cm}^{2}$ may be generated at $1.5 \mathrm{Mcps}$ [1]. These figures show, by the way, that the maximum attainable sound intensities do not nearly reach the theoretical limit given by the tensile strength of the quartz itself. A practical limit is determined by the fact that it is impossible to completely prevent breakdown of the high electric tension at the boundaries of the quartz plate.

With barium titanates as piezoelectric transducer material, the intensities which can be obtained are much smaller than can be obtained with quartz; 
namely, 2 to $5 \mathrm{w} / \mathrm{cm}^{2}$. If additional cooling is used, up to $15 \mathrm{w} / \mathrm{cm}^{2}$ may be reached. The reason for the low output of the barium titanates is the internal heating of the transducer by mechanical and dielectric losses. At high input power, the temperature of the transducer may exceed the Curie temperature.

Pulse modulation permits somewhat higher peak sound intensities because of the smaller mean output. With quartz, values of up to $500 \mathrm{w} / \mathrm{cm}^{2}$ (at $1.5 \mathrm{Mcps}$ ) and with barium titanate about $20 \mathrm{~W} / \mathrm{cm}^{2}$ are reported in the literature [2]

For frequencies below $200 \mathrm{kcps}$, magnetostrictive transducers are very often used. These are, in most cases, composed of superficially oxidized nickel sheets. In spite of the rugged design of these transducers, the maximum intensity is limited to about 7 to $16 \mathrm{w} / \mathrm{cm}^{2}$ by friction losses between the sheets and by the $\Delta E$ effect.

The new piezomagnetic ferrites (nickel-copper-cobalt ferrites, "Ferroxdure") developed by Philips, Eindhoven, can generate maximum intensities of 3 to $6 \mathrm{w} / \mathrm{cm}^{2}$ in continuous operation [3].

In order to concentrate high energies into very limited space, it is much more efficient to use cylindrical or even spherical shells as radiators. The first to propose such radiators was Johannes Gruetzmacher [4], who used a concave quartz shell with a resonant frequency of several hundred kcps. If the point of convergence is near the surface of the liquid, the well-known bubbling water or oil spring caused by Langevin's radiation pressure is observed. The height of the spring is an indication of the extreme energy densities. The attainable power at the focus of the quartz radiator is estimated to be of the order of $20 \mathrm{kw} / \mathrm{cm}^{2}$. For barium titanate shells, $300 \mathrm{w} / \mathrm{cm}^{2}$ was reported. Even if cavitation is prevented (e.g., by applying excess static pressure), a natural limit is set for the attainable energy densities because the energy is prevented from focusing by diffraction, and also because nonlinear strains in the transmitting medium increase the absorption.

In the lower frequency ranges often many small sound-radiating elements are arranged mosaiclike on a large spherical surface (A. Williams). Such instruments are manufactured with barium titanate transducers by the Clevite Company in Cleveland, Ohio.

L. D. Rozenberg and M. G. Sirotyuk [5] have proposed an especially interesting construction of this kind. They have used a spherical aluminum shell with an inner radius of $314 \mathrm{~mm}$; the thickness of the shell is exactly equal to $\lambda / 2$ at the operating frequency of $500 \mathrm{kcps}$. Distributed over the outer surface of the shell are 160 to 200 spherically polished quartz thickness vibrators immersed in oil. The attainable intensity in the focus area is 60 to $70 \mathrm{kw} / \mathrm{cm}^{2}$ with peak pressures of up to $500 \mathrm{~atm}$.

An arrangement consisting of 21 magnetostrictive transducers (14.6 kcps) was used in the III Physikalisches Institut of the University of Göttingen for the investigation of cavitation in liquids in the absence of disturbing boundary layers $[6]$.

\subsubsection{Pulse Transducers}

The sinusoidal vibration of electroacoustic transducers may be pulse-modulated in order to keep the time average of the power small. This is facilitated by a very simple method used with magnetostrictive and barium titanate trans- 
ducers; the fundamental vibration of the system is excited by a single current or voltage pulse producing a damped wave train. This method of excitation is a transition from stationary sinusoidal excitation to a single compression or rarefaction pulse, which has rather interesting qualities. The three methods of exciting such single pulses will be discussed: (1) the collapse of a cavity in liquid, (2) an underwater spark, and (3) an electrodynamic pulse transducer.

As is well known, collapsing bubbles play an important part in cavitation. Cavitation is observed, for example, as "flow cavitation" at the tip of ships' propeller blades or when a stream of liquid impinges on a sharp edge. It can also be generated in the underpressure phase of a sound radiator as "sonic or ultrasonic cavitation." The collapse of the cavity produces a shock wave, the intensity of which increases as the gas pressure in the cavity just before the collapse becomes smaller. Therefore, the collapsing of gas-free or almost gasfree cavities in degassed water produces rather intense shock waves. This is often easily observed by just listening to the event.

It is not necessary for the cavity to be formed by underpressure inflow or by the underpressure phase of an oscillation. It is also possible to introduce "ready-made" cavities into the liquid by blowing hot steam from a nozzle into cold water, where the hot steam bubbles collapse. A very elegant method is to completely evacuate a thin-walled glass sphere, such as a Christmas-tree decoration, or to fill it with a certain gas at a given low pressure. This sphere is then immersed in water or another liquid and destroyed by mechanical shock. The liquid streaming into the cavity is stopped abruptly, radiating intense shock waves. Such experiments were made at the III Physikalisches Institut at Göttingen [20] as a simple means of studying sonoluminescence described in section 9.3.5.

Shock waves can also be generated by the use of an underwater spark. Fringel and Bailites [8] studied this method using the very simple circuit shown in Fig. 9.1. A condenser of 0.01 to $0.05 \mu \mathrm{f}$ is charged to 7 to $9 \mathrm{kv}$ and then dis charged through a series circuit containing the underwater spark gap, an air spark gap, and the circuit inductance of about $1 \mu \mathrm{h}$. In order to increase the durability of the system, one electrode of the spari gap consists of a resilient strip of metal and the other of a brass or copper block. The sudden evaporation

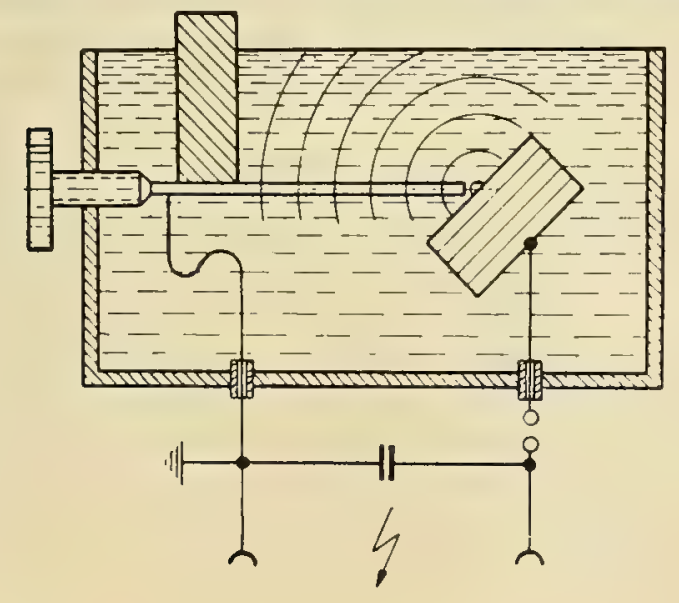

Fig.9.1. Sound generation by underwater sparks. 


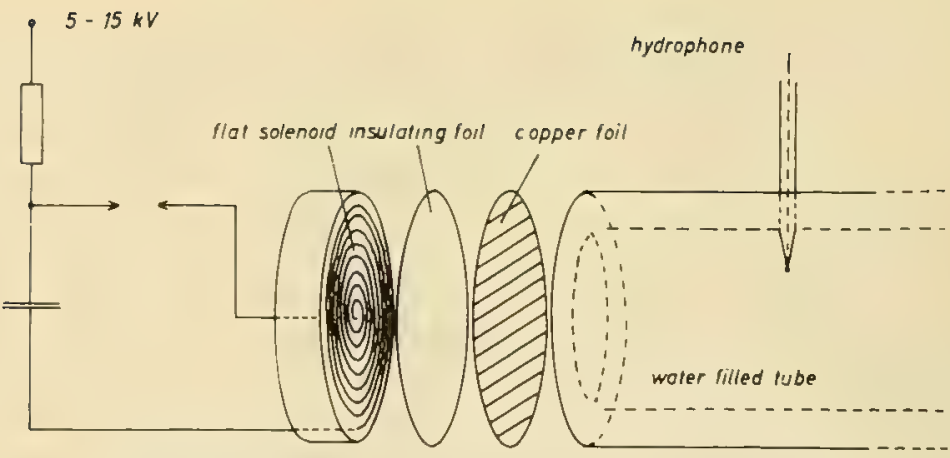

Fig. 9.2. Electromagnetic generation of pressure pulses.

of the liquid wall of the spark discharge duct generates a high-pressure impact in the surrounding water, which propagates as a shock wave.

Even more efficient is a method known from the electrolytic interrupter. The two electrodes in the liquid are separated from one another by means of an insulating plastic plate. If there are one or more holes in this plate, the liquid in the holes is evaporated after connecting the condenser with the electrodes and the discharge then passes through the vapor. The authors, mentioned above, report that a mean acoustic power of $200 \mathrm{w}$ with peak powers of up to $300 \mathrm{kw}$ was obtained with a plate with 10 holes. The reproducibility of the cavity shock transmitter is low due to the nonspherical collapse of the bubbles, even if the initial sizes and gas contents are equal.

These difficulties are overcome in an electromagnetic shock transducer developed by W. Eisenmenger [9], shown in Fig. 9.2. Opposite to a flat solenoid and separated from it by thin plastic foil is a copper membrane, which terminates the water column into which a shock wave is to be radiated. A current discharge through the flat solenoid induces strong eddy currents in the copper membrane. The resulting magnetic fields produce very strong repulsive forces between the flat solenoid and the copper membrane. Typical data are: A current of $4000 \mathrm{~A}$ flows through a solenoid consisting of 50 turns, when a condenser with a capacity of $0.8 \mu \mathrm{f}$ charged to $20 \mathrm{kv}$ is discharged through it. The duration of the current pulse is only $2 \mu \mathrm{sec}$ and pressures of up to $200 \mathrm{~atm}$ are produced. A condenser with $8 \mu \mathrm{f}$ capacity and a charging voltage of $12 \mathrm{kv}$ produces shock waves with peak pressures of up to $700 \mathrm{~atm}$.

\subsection{MEASUREMENT OF HIGH SOUND INTENSITIES}

In the last decade, water-borne sound measuring technique has reached the same high standard as has existed for a long time in the measurement of air borne sound fields. There is a great variety of hydrophones available. They are usually calibrated like air-borne sound microphones by the reciprocity method. We will discuss only those types of microphones for the measurement of high intensities which are sufficiently insensitive, overload-resistant, and either very small or very large compared to the wavelength to be measured. They should have a very high upper limiting frequency and should, furthermore, be of a very rugged design. Many conventional types of microphones fail to meet these requirements. 


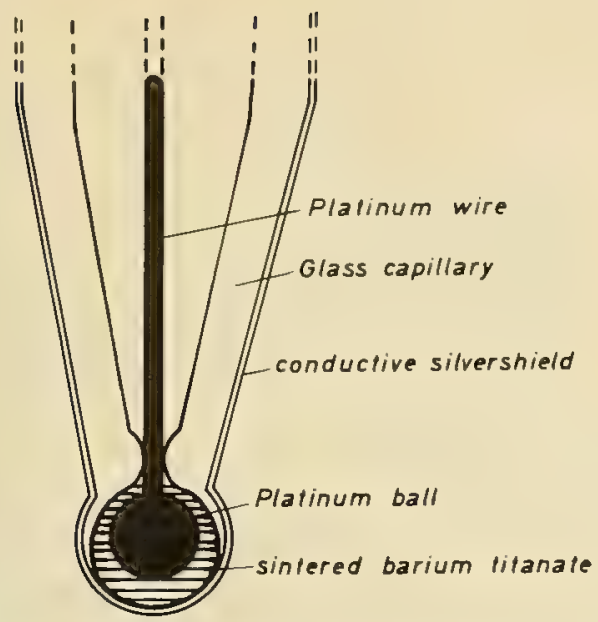

Fig. 9.3. Barium titanate probe hydrophone.

One of the smallest microphones known today is the harium titanate "pearl" which was first developed by N.A. Roi and E.V. Romanenko [10] and is shown in Fig. 9.3. A thin platinum wire is drawn through a very thin glass tube. A small quantity of barium titanate powder is then made to adhere at the end of the platinum wire and is afterwards sintered in a Bunsen flame. The wire inside the pearl is used as one electrode and the other electrode is produced by silverplating the hydrophone on the outside. A polarization voltage is applied between the electrodes. The natural frequencies of such hydrophones are in the Mcps range; the frequency response curve of a pearl microphone is presented in Fig. 9.4. At 10 Mcps the sensitivity is reduced by about 30 per cent of its value in the midrange.

According to a proposal by Eisenmenger the well-known condenser microphone may also be used. The design differs, however, from the types used in ordinary water- or air-borne sound technique. Since the sound pressure to be measured is very high, a thin plastic foil metalized on one side has the rear side glued to a solid back-plate such as a metal disk. The sensitive microphone surface of such an instrument is large compared to the wavelength. The compressibility of the plastic foil used as a solid dielectric is very small, but since the acoustic pressure is very high, sufficient changes of the thickness of the

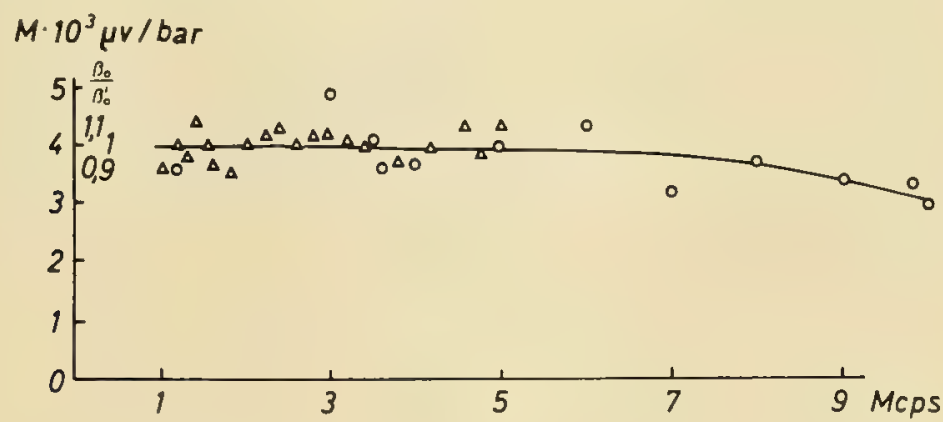

Fig. 9.4. Frequency response of miniature barium titanate hydrophone. 


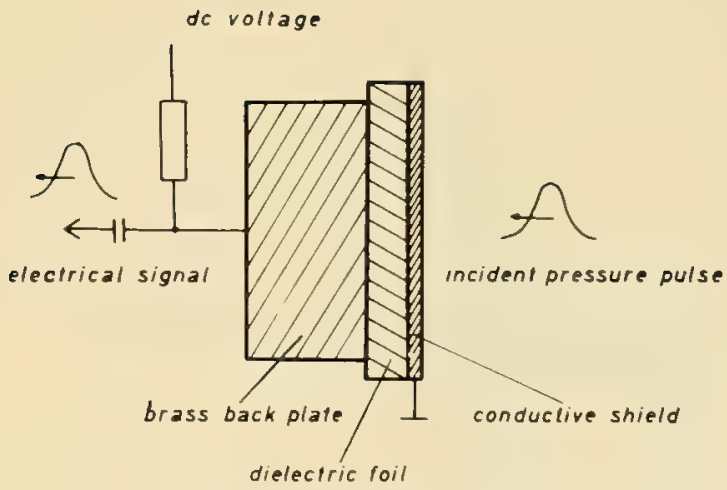

Fig. 9.5. Electrostatic hydrophone with rigid dielectric.

foil are obtained. With a dc voltage applied, an alternating signal voltage is obtained across a load resistance as in conventional condenser microphones. The highly damped resonant frequencies occur in the Mcps-range. Figure 9.5 shows the configuration of such a microphone.

If the sound wave to be measured hits a free water surface, it is possible to measure directly the elongation of the surface, for example, with an electrostatic pick-up arrangement. At higher frequencies it is, however, more expedient to measure the particle velocity. According to Eisenmenger, this is achieved by covering the free water surface with a thin light plate with a conductive strip of silver paint on the rear side. This conductive strip is placed in a homogeneous magnetic field. The arrangement is similar to a ribbon microphone measuring the particle velocity of the free surface. The frequency response of this microphone depends more upon the electric circuit characteristics than upon the mechanical design which is illustrated in Fig. 9.6. It is obvious that this microphone operates only at high sound energy levels and that it will always be relatively large compared to the wavelength.

Due to the high sound energy levels, it is also possible to measure the density variation of the water in the sound field or, more exactly, to determine the density gradient with a photoelectric means. For this purpose, a beam of light is sent through the water at a given test point and Schlieren optics are used to mask out the beam when there is no applied sound field. When the sound field

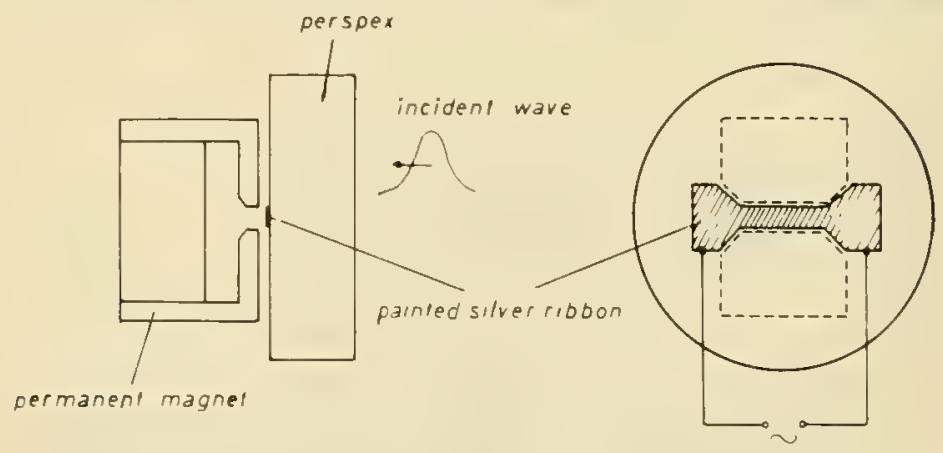

Fig. 9.6. Ribbon-type hydrophone. 
is present, light is diffracted by the density gradients produced by the sound wave, so it passes the sides of the Schlieren diaphragm and is measured with a photomultiplier. The density gradient can be determined, if the width of the beam of light is very small. If the beam is wide, the arrangement integrates over the respective area and the compression is measured directly. This microphone seems to be rather attractive, but it is very difficult to obtain frequency resolution up to 1 Mcps. This is caused by insufficient parallel adjustment of the diaphragm gap and by discontinuities at the gap edges. On the other hand, the above described method is perfectly suitable for photographing the spatial structure of the entire incident wavefront as indicated in Fig. 9.19.

For time or frequency analysis of a shock front, a special microphone is required. The frequency response curve should exceed, by far, the fundamental frequencies of the smallest quartz microphones, since these fundamental frequencies are only of the order of magnitude of several Mcps. Eisenmenger [21] proposed the design of a microphone similar to the hypersonic quartz transducer reported by Bömmel and Dransfeld, and Jakobsen. In his design, the water-borne sound wave hits the front surface of a longitudinal expander quartz rod shown in Fig. 9.7. The front surface is, for this purpose, adjusted exactly parallel to the wavefront. The dilatational wave excited in the quartz rod is propagated through the quartz until it is reflectedat the free rear surface, which is polished plane and parallel to the front surface. Simultaneously with the reflection, the electric space-charge wave traveling with the acoustic wave is coupled out by means of a low-resistance coaxial line circuit. Apart from the attenuation in the quartz, the frequency response curve is almost entirely dependent upon the parameters of the electrical circuit. The lower limiting frequency is given by the length of the quartz rod. Towards higher frequencies, the frequency range extends into the $\mathrm{kMc}$ range.

Finally, still another microphone must be mentioned, which has the advantage that measurements may be carried out even at hard-to-reach points by proper shaping of the sensitive element. In principle, this microphone is closely related to the quartz coaxial line microphone, but utilizes the magnetostrictive effect. According to Koppelmann [11], the sound pressure of a sound wave incident on the tip of a thin nickel wire immersed in water generates an extensional wave which propagates along the wire. A coil wound around the nickel

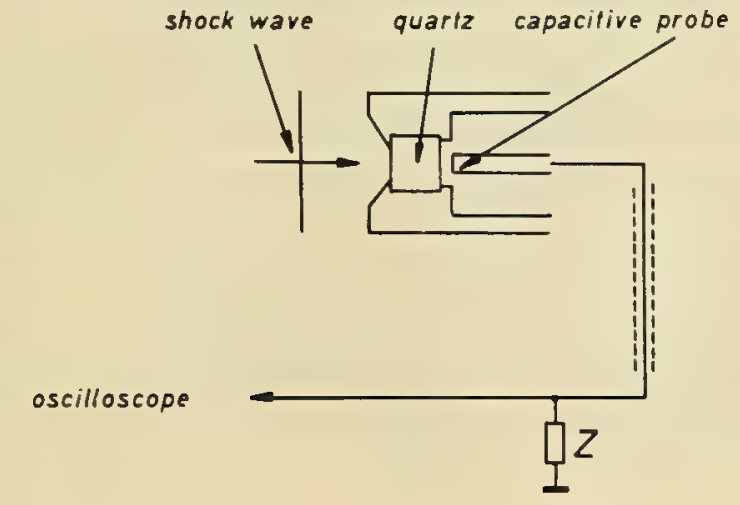

Fig. 9.7. Quartz hydrophone for shock wave measurement. 


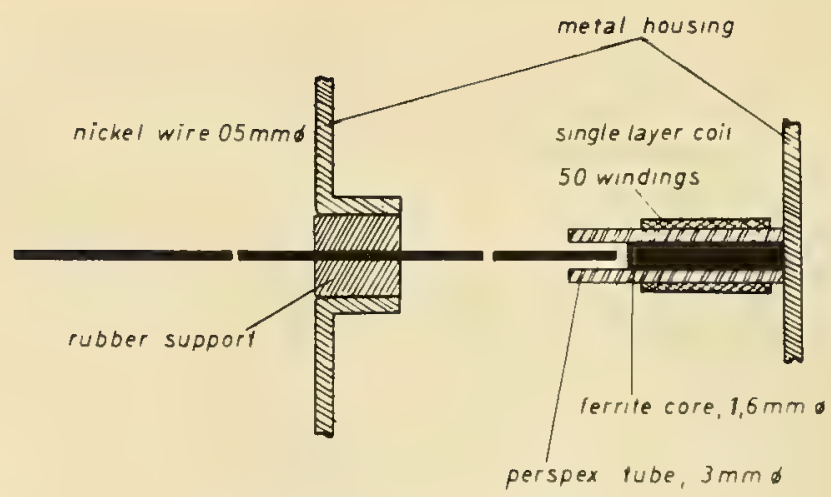

Fig. 9.8, Nickel wire hydrophone.

wire in the presence of a permanent magnetic field transduces the extensional wave in the wire into an ac voltage. The frequency-resolving power of this type of nickel wire hydrophone depends upon the "gap-width" of the coil relative to the wavelength in the wire. An arrangement similar to the piezoelectric microphone described above was, therefore, used by Kuttruff [22] and is illustrated in Fig. 9.8. The magnetization wave propagating in the wire is detected by a ferrite body at the end of the wire. A corresponding signal voltage is induced in a coil wound around the ferrite body. The sensitive part of this microphone, the nickel wire, is easily deformed. The microphone has a rather large frequency bandwidth. However, mechanical and magnetic losses in the nickel wire which increase with frequency play a more decisive part in the characteristics of this arrangement than do the corresponding effects in quartz.

\subsection{PROPERTIES OF LIQUIDS EXPOSED TO IIIGH SOUND INTENSITIES}

\subsubsection{Increase of Sound Absorption}

The first effect to be discussed is the increase of sound absorption in liquids which are exposed to high sound intensities. The physical reason is obvious. Because of the nonlinearity of certain properties of the liquid, higher harmonics are generated due to acoustic overload under a sinusoidal strain. The absorption is naturally higher for the higher harmonic frequencies than for the fundamental frequency which leads to an increase of the observed total loss. Figure 9.9 is taken from a paper by R.T. Beyer and V. Narasimhan [12]. In this figure, $a / \gamma^{2}[a=$ absorption coefficient, $\gamma=$ frequency] (atm/Mcps) as proposed by R. B. Lindsay. At low sound levels, the normal absorption coefficients are indicated; but at higher sound levels, a significant increase in the absorption is observed. The absorption rises approximately in proportion to the quotient of pressure divided by frequency.

\subsubsection{Generation of Higher Hormonics}

With sinusoidal excitation of sound, higher harmonics are generated by the nonlinear behavior of the liquid medium. Direct measurement of these overtones can provide information on the nonlinear characteristics of the liquid. Such experiments have been carried out in many laboratories in recent years. Es- 


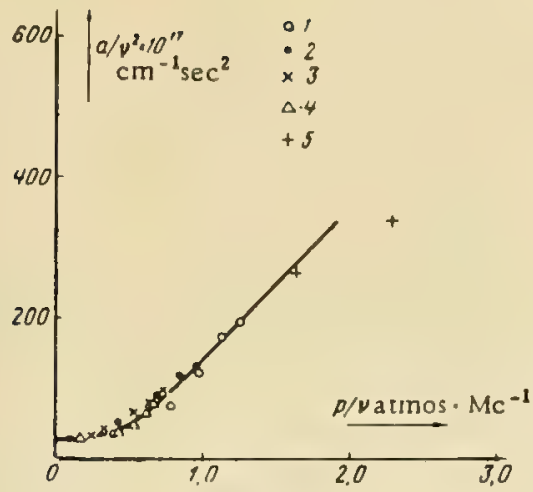

Fig. 9.9. Attenuation in water as a function of the ratio of sound pressure and frequency.
1) $3.85 \mathrm{Mc}$,
2) $5.85 \mathrm{MC}$
3) $6.8 \mathrm{MC}$,
4) $8.74 \mathrm{MC}$;
5) $1.5 \mathrm{MC}$,

pecially, E. Hiedemann and his co-workers in East Lansing and I. G. Mikhailov and V.A. Shutilov in Moscow have studied these problems. Figure 9.10 displays diffraction spectra obtained at $1.76 \mathrm{Mcps}$ taken from a recent publication by E. A. Hiedemann and M. A. Breazeale [13]. The spectra are arranged horizontally according to the distance between test point and sound source and vertically according to increasing sound intensity given by the Raman-Nath parameter $V=2 \pi \mu \cdot L / \lambda$, where $L$ is the depth of the sound field, $\lambda$ is the wavelength, and $\mu$ is the amplitude of fluctuation of the index of refraction. $V=7.5$ corresponds approximately to a pressure amplitude of 1 atm in the vicinity of the sound source. The dissymmetry is a characteristic feature of the spectra which are symmetrical at low intensities; this dissymmetry increases with increasing intensity and increasing distance from the sound source. The explanation of this effect is rather simple. At high intensities and large distances, the sinusoidal sound wave in the liquid is increasingly distorted to form a saw-toothed curve. Consequently, the

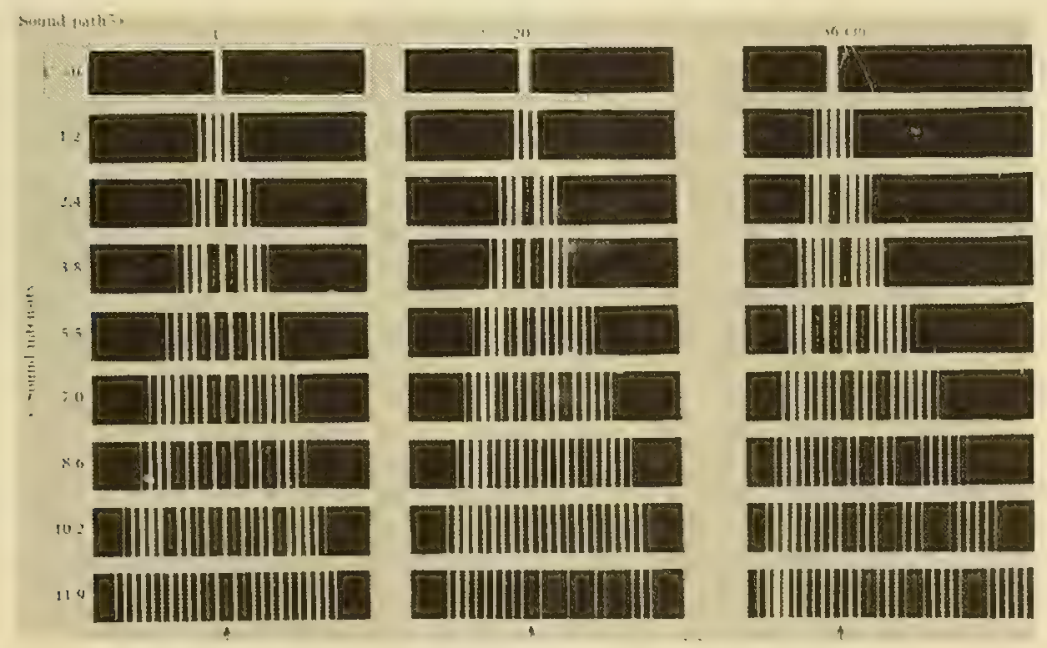

Fig. 9.10. Light diffraction by ultrasonic waves in water at $1.76 \mathrm{Mc}$. 

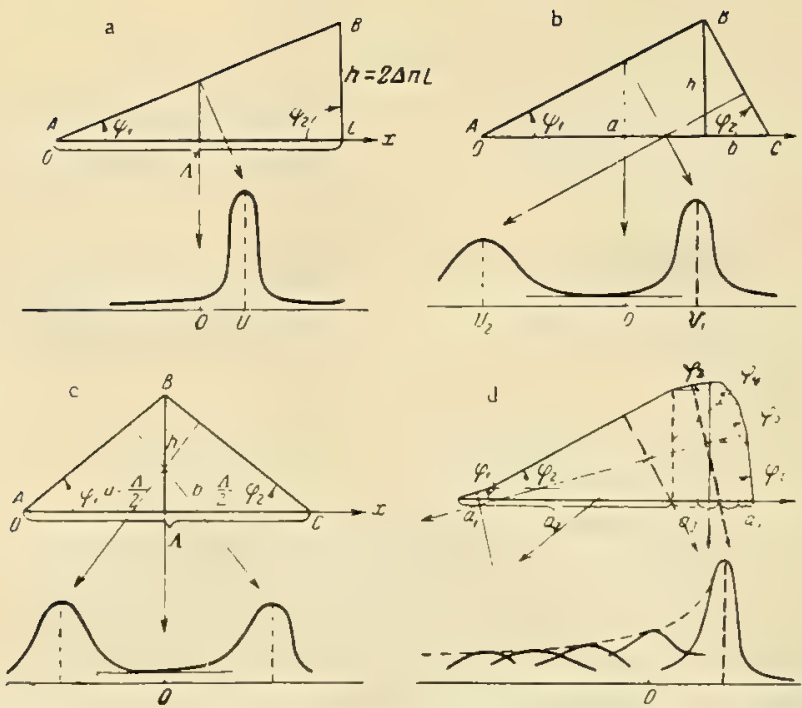

Fig. 9.11. Relation between pressure curve and optical diffraction pattern.

index of refraction in the sound field also follows a saw-tooth curve. The phase surface of the light wave entering the sound beam is plane; but, after penetrating the sound beam, the phase surface becomes sinusoidal if the amplitudes are small. But at high amplitudes, the phase surface of the light wave will also be saw-toothed in shape. This is similar to the diffraction of light by a specially made amplitude grating ("Echelette" grating), which is dissymmetric and diffracts more light into certain orders. The envelope of the spectral distribution of the different orders of diffraction resembles the phase surface of the light beam which, in turn, reflects the shape of the sound pressure wave.

Figure 9.11 was taken from the paper of I. G. Mikhailov and V. A. Shutilov [14]. It displays the theoretical intensity distribution in the diffraction spectra for four different sound pressure patterns or optical index-of-diffraction patterns respectively. Dissymmetric sound pressure curves produce dissymmetric spectra and, furthermore, a distinct preference for certain orders in the spectrum is observed, and it is also possible from the shape of the spectrum to deduce the shape of the sound wave.

It should be mentioned in this connection that by using $\lambda / 2$-plate filters certain harmonics may be filtered out and indicated separately (Mikhailov and Shutilov [15]).

\subsubsection{Generation of Shock Waves by Collapsing Cavities}

This paragraph will treat the question of how a collapsing cavity filled with more or less gas generates a shock wave. Starting with Rayleigh's theory, W. Güth [16] gave a simple explanation of this process. A segment of a cavitation bubble is shown in Fig. 9.12, where the pressure inside the bubble is $p_{0}$, the out side atmospheric pressure is $p_{a}$, and the initial radius is $R_{0}$. The flow velocities in the water streaming into the cavity are indicated in Fig. 9.12 at an arbitrary moment. The distribution of flow velocities as a function of the distance from 
Fig. 9.12. Velocity of water around the cavitation bubble.
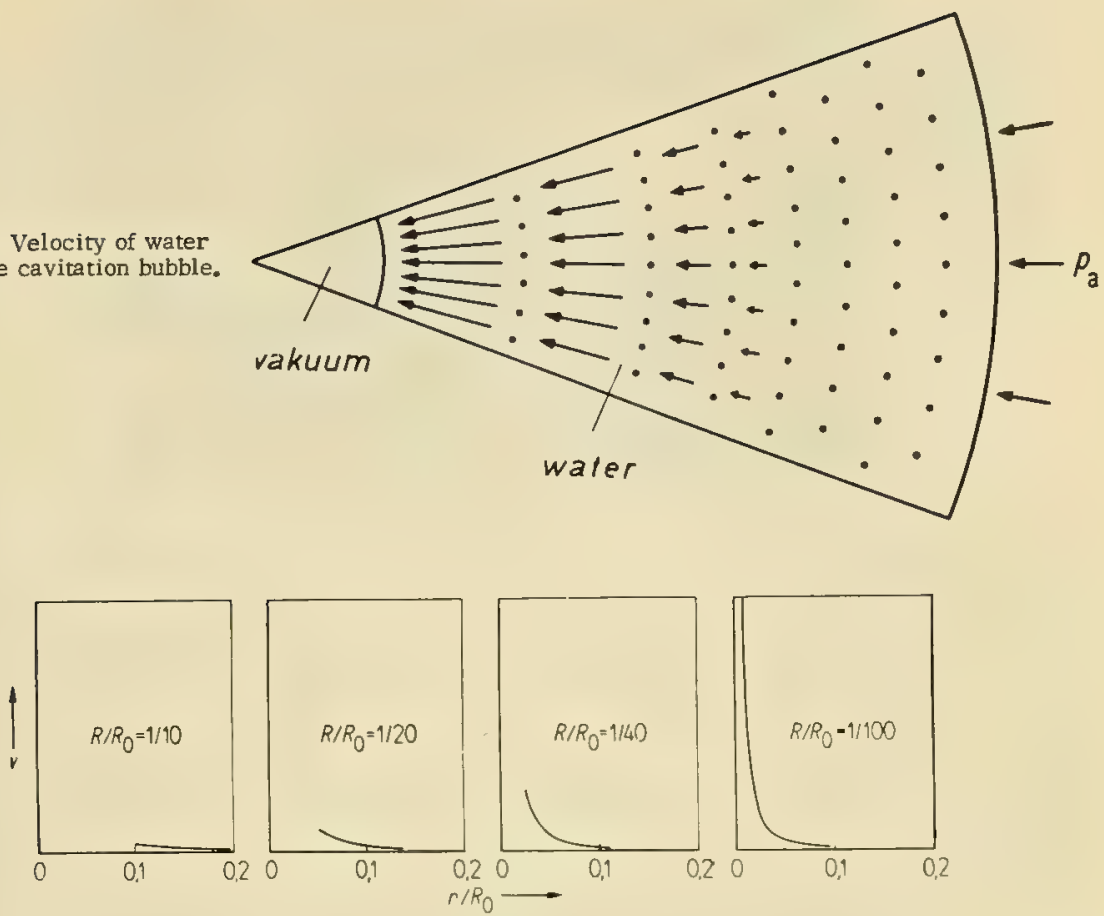

Fig. 9.13. Flow velocity for different implosion states.

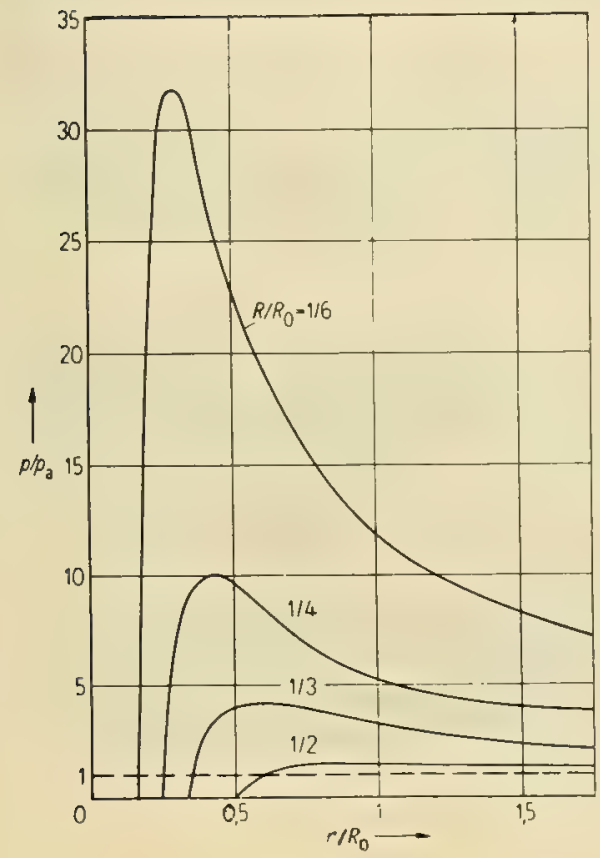

Fig. 9.14. Dynamic pressure distribution for different implosion states. 


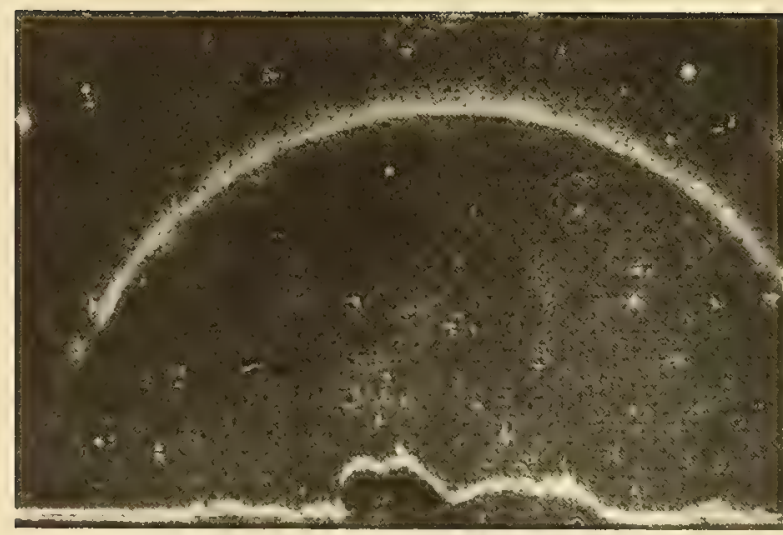

Fig. 9.15. Schlieren photograph of the shock wave.

the center of the spherical cavity is plotted in Fig. 9.13 for several instances during the collapse of the bubble where $R / R_{0}=1 / 10$ to $1 / 100$. Under favorable circumstances, the velocity of the surface of the bubble reaches the velocity of sound. The corresponding pressures in the "pressure head wave" at different stages of the implosion are plotted in Fig. 9.14. These also determine the pressure in the radiated shock wave. At the front of the shock wave the pressure gradually increases up to very high values; this is confirmed by the Schlieren photograph of Fig. 9.15. The shock wave pressure is obviously higher the smaller the gas content of the initial cavity. With such collapsing cavities, extremely powerful sound waves may be generated. At the time of formation, the cavity is almost spherical, but no one has ever succeeded in having the bubble collapse with a spherical shape throughout. The shape of the surface is unstable on account of the surface tension, and therefore the sphere, at the end phase of the collapse, usually disintegrates into a number of smaller bubbles of various sizes and closely neighboring centers. Each of these bubbles generates individual shock waves separated with respect to time and space. An example illustrating this effect, shown in Fig. 9.16, is taken trom the work of J. Schmid [7]. In his work, the movement of a water-filled vessel is suddenly stopped and the water column torn apart by inertial forces at a bubble nucleus in the water, forming a cavity
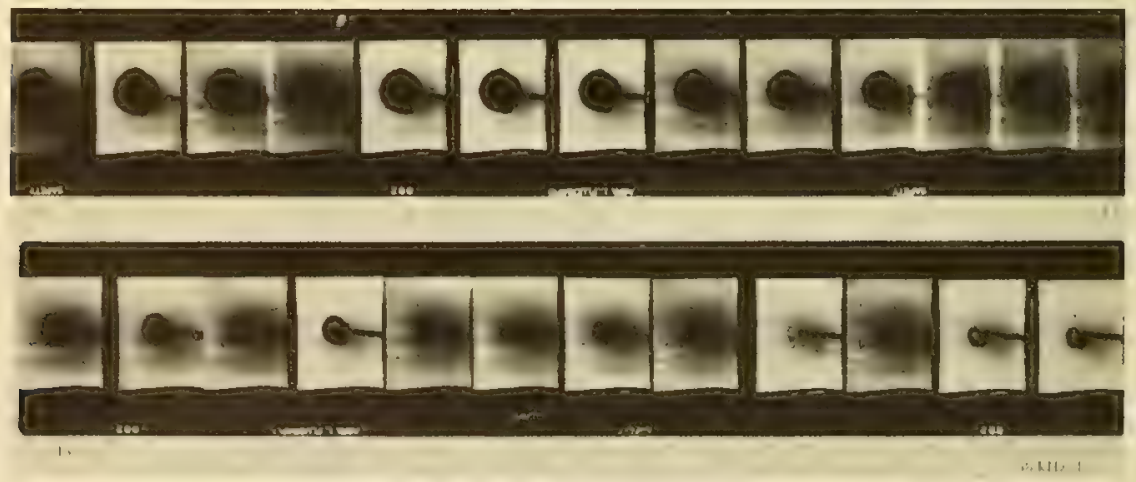

Fig. 9.16. Implosion of a bubble. 

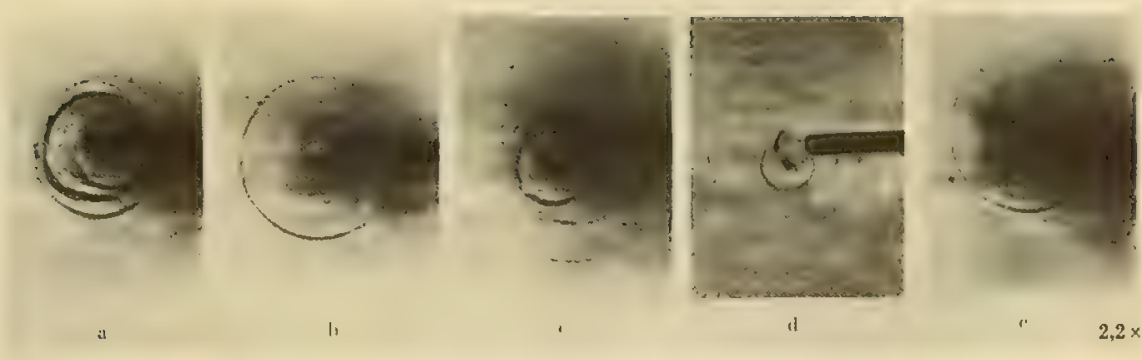

Fig. 9.17. Shock waves generated by an imploding bubble.

about $1 \mathrm{~cm}$ in diameter. Following the collapse, this bubble radiates shock waves shown in frame 22 in Fig. 9.16, which are strong enough to be visible without Schlieren optics just by the shadows they cast. More detail can be seen in Fig. 9.17 which shows the results of several series of measurements. It is clearly seen that each time many shock waves are radiated from neighboring centers in closely succeeding intervals. The same observations are made when hot steam is blown into cold water, where the hot steam bubbles collapse [17].

\subsubsection{Steepening of Shock Waves}

An intense compressional wave has a higher velocity than a weak sound wave in water as well as in air; the higher the compression, the higher the velocity. In air, one of the main reasons for this effect is the higher temperature in the superpressure range. In water, however, the high particle velocity plays an important part. For example, with a sound pressure of $5000 \mathrm{~atm}$, the corresponding particle velocity is $250 \mathrm{~m} / \mathrm{sec}$, which is large relative to the velocity - of sound even at the lower compressibility and, therefore, results in higher velocity in pressurized water.

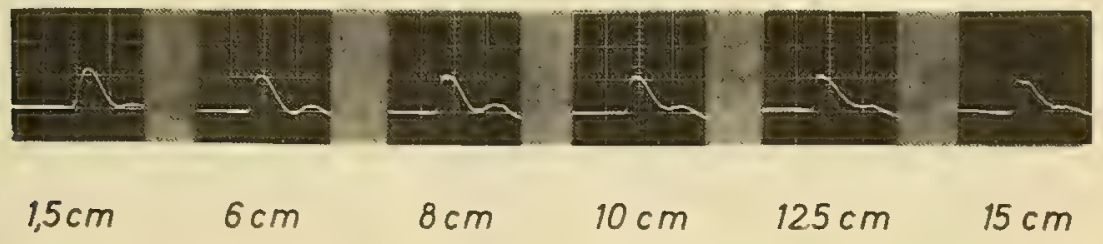

Dislance from sound pressure source

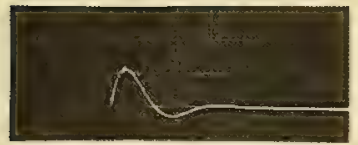

• 5 usec

discharge current
Shock front development of a pressure pulse in water

Peakpressure 75 al.

Fig. 9.18. Variation of shock front with distance from transducer. 


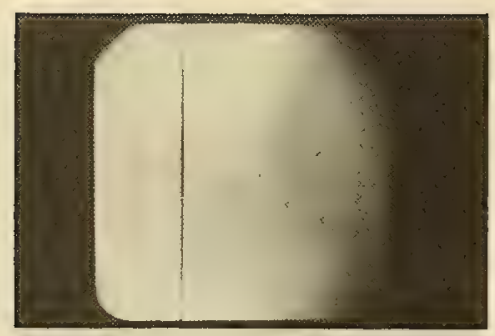

Fig. 9.19. Schlieren shadow picture of shock wave in water.

\section{$\longmapsto 1 \mathrm{~cm}$}

The physical effects are illustrated with a number of oscillograms recorded with the electrodynamic shock wave transducer of W. Eisenmenger. A copper membrane terminates a water column as described in Section 9.1.5, through which the shock wave generated by the impact of the membrane propagates. The sound pressure is measured with a small piezoelectric hydrophone described in Section 9.2. Figure 9.18 demonstrates the formation of the shock front with increasing distance from the transducer (pressure, $75 \mathrm{~atm}$ ). The lower diagram displays the plot of the current in the sound generator.

A Schlieren photograph of the shock wave is presented in Fig. 9.19. When such a shock wave hits the rear end of the water column with a pressure-release termination, it is reflected with phase reversal and the resulting high underpressure causes strong cavitation in the entire water column like the unbottling of soda water.

Now the question arises, how to measure the rise time of the pressure or the thickness of the shock front more exactly. For this purpose, the quartz coaxial line microphone described in Section 9.2 is the proper instrument because it provides the ultimate resolution with respect to time. The oscillogram presented in Fig. 9.20 was obtained from the quartz coaxial line microphone with a laboratory oscilloscope with maximum sweep magnification. The front surface of the quartz rod is exactly parallel to the incident shock front. The shock wave is repeatedly reflected inside the quartz as shown in Fig. 9.20b. It is inferred from such oscillograms that the shock process lasts less than $10^{-8} \mathrm{sec}$, so the bandwidth of the oscilloscope is obviously insufficient (upper

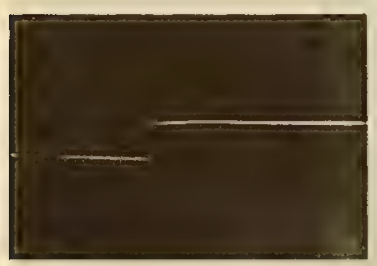

$\longmapsto 0,1 \mu \mathrm{sec}$

(a)

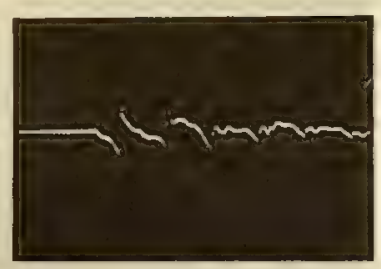

Fig. 9.20. Shock front oscillogram with quartz hydrophone. $\longmapsto 5 \sec$

(b) 


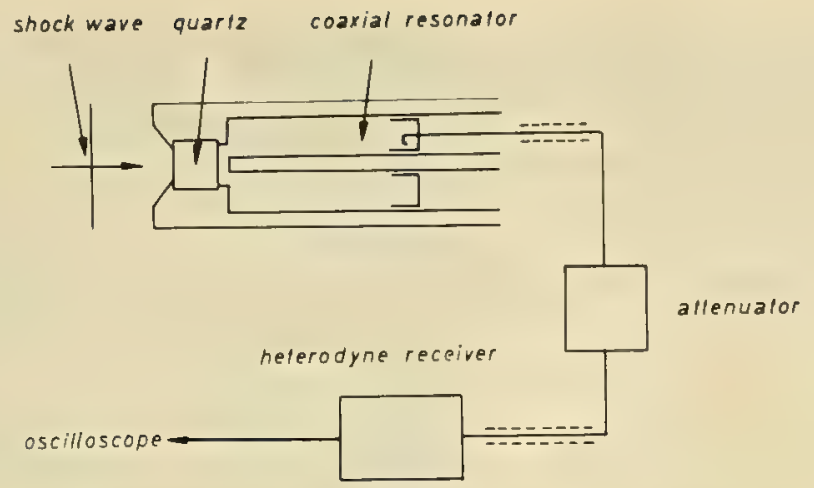

Fig. 9.21. Frequency analysis of shock front spectrum with quartz hydrophone.

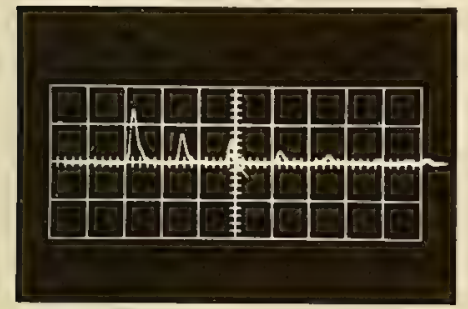

$\longmapsto 2 y \sec 280$ mcps

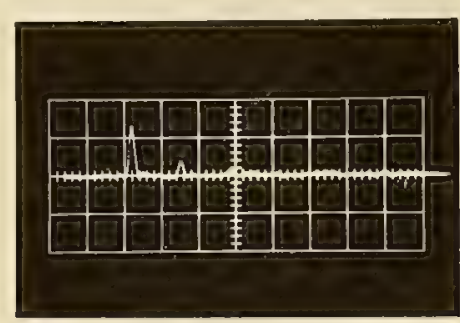

$\longmapsto 2 \mu \mathrm{sec} 950$ mcps

Fig. 9.22. Frequency components of shock front as function of time.

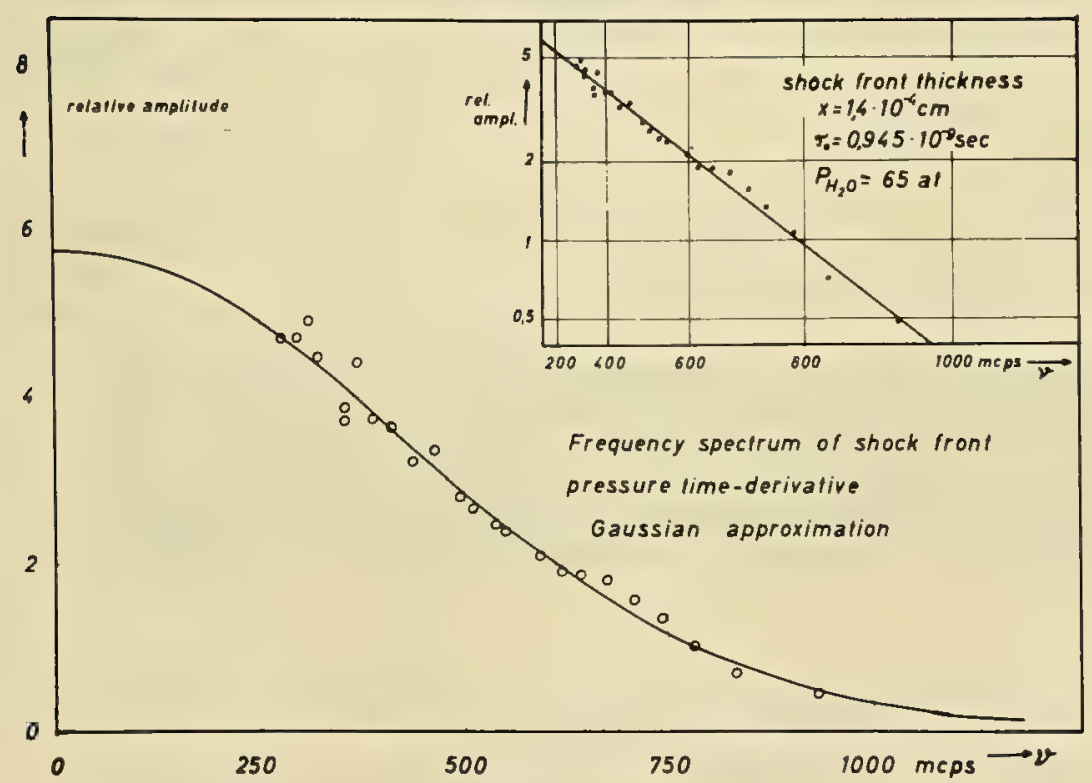

Fig. 9.23. Frequency spectrum of shock pressure-time derivative, Gaussian approximation. 


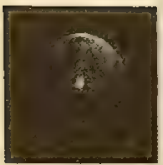

$180^{\circ}$

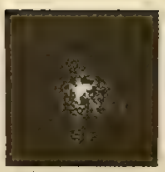

$210^{\circ}$

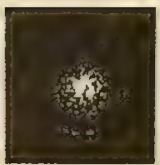

$240^{\circ}$

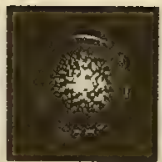

$270^{\circ}$

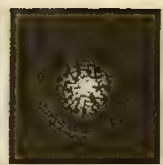

$300^{\circ}$

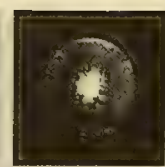

$330^{\circ}$

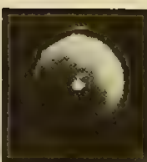

$360^{\circ}$

Fig. 9.24. Cavitation bubbles at differentphases in front of a vibrating piston made at $30^{\circ}$ intervals.

limiting frequency is only $30 \mathrm{Mcps}$ ) and traveling wave oscilloscopes available now are not sensitive enough.

Therefore, a Fourier analysis of the event is suggested. For this purpose, the coaxial line is replaced by a coaxial resonator, which is successively tuned to different frequencies as shown in Fig. 9.21. The resonant frequency filtered out from the shock wave is magnetically coupled from the resonator and is applied to a superheterodyne receiver. Oscillograms of the output signal are shown in Fig. 9.22 for the frequencies 280 and 950 Mcps. The multiple reflections of the shock wave in the quartz can be clearly seen. If such measurements are carried out in the frequency range from 200 to $1000 \mathrm{Mcps}$, the curve displayed in Fig. 9.23 results. The time derivative is plotted against the frequency of the measured pressure component, that is, the product of "spectral intensity times frequency." The small diagram in the upper right-hand corner with a logarithmic ordinate scale and square-law frequency scale demonstrates that the measured values may be approximated by a Gaussian distribution. From this, it follows that, for a shock wave with a peak pressure of 65 atm and a rise time of $0.95 \cdot 10^{-9} \mathrm{sec}$, the shock front thickness is $1.4 \cdot 10^{-4} \mathrm{~cm}$. It is interesting to note that the sensitivity of the quartz microphone used in these measurements was $-180 \mathrm{db}$ relative to $1 \mathrm{v} / \mu \mathrm{b}$. It was $20 \mathrm{~mm}$ in diameter and $8 \mathrm{~mm}$ thick.

\subsubsection{Sonoluminescence}

We will now treat a physical effect which is closely related to the collapse of cavities in liquids and, thus, also with the generation of high sound intensities, namely, sonoluminescence. This effect is observed when a single cavity, for example, the Christmas-tree-decoration sphere mentioned in Section 9.1.2, col-
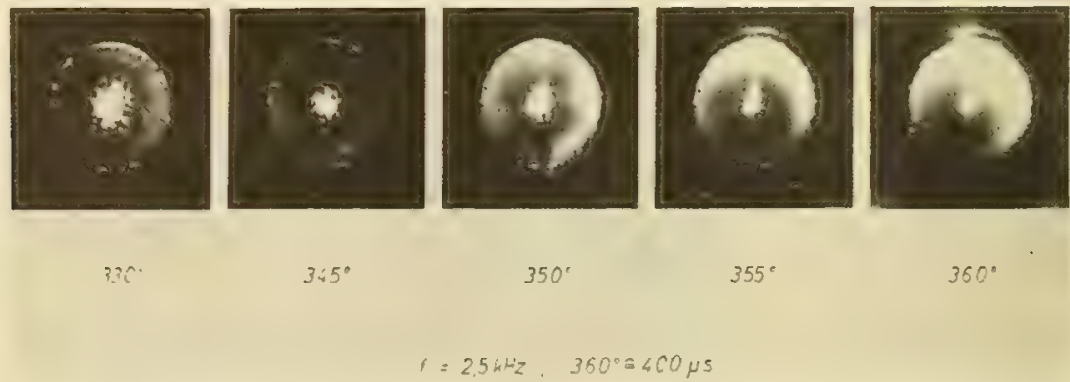

$360^{\circ}$

Fig. 9.25. Cavitation bubbles at different phases in front of a vibrating piston at $5^{\circ}$ intervals. 


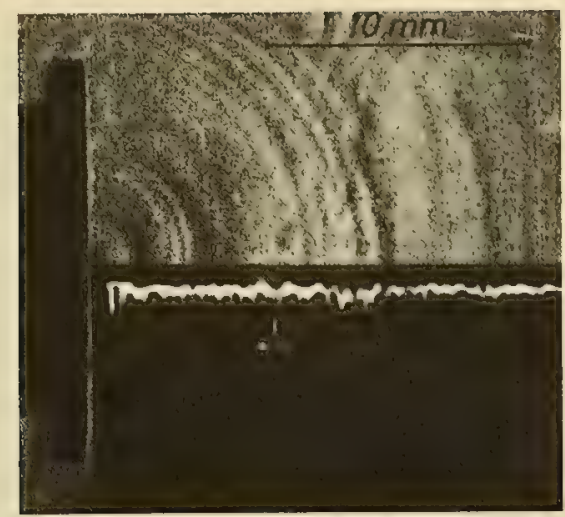

Fig. 9.26. Luminescence pulses and shock waves.

lapses. In the last stages of the collapse, the cavity disintegrates into a great number of smaller cavities, and therefore a large number of light pulses is also observed. Luminescence requires the presence of small quantities of gas which are excited to emit light. If the initial pressure in the glass spheres exceeds about 20 torrs, the luminescence almost entirely disappears; the luminescence pulses are very small for water with small gas contents, but relatively large for glycerin with krypton as the gas and higher gas contents.

Another interesting effect is the sonoluminescence occurring together with ultrasonic cavitation. Such experiments were made by Meyer and Kuttruff [18], and Fig. 9.24 shows instantaneous photographs of cavitation bubbles. The photographs were made at $30^{\circ}$ intervals of the phase of the vibration of the exciting system which was a nickel rod vibrating at $2.4 \mathrm{kcps}$. Phase zero indicates

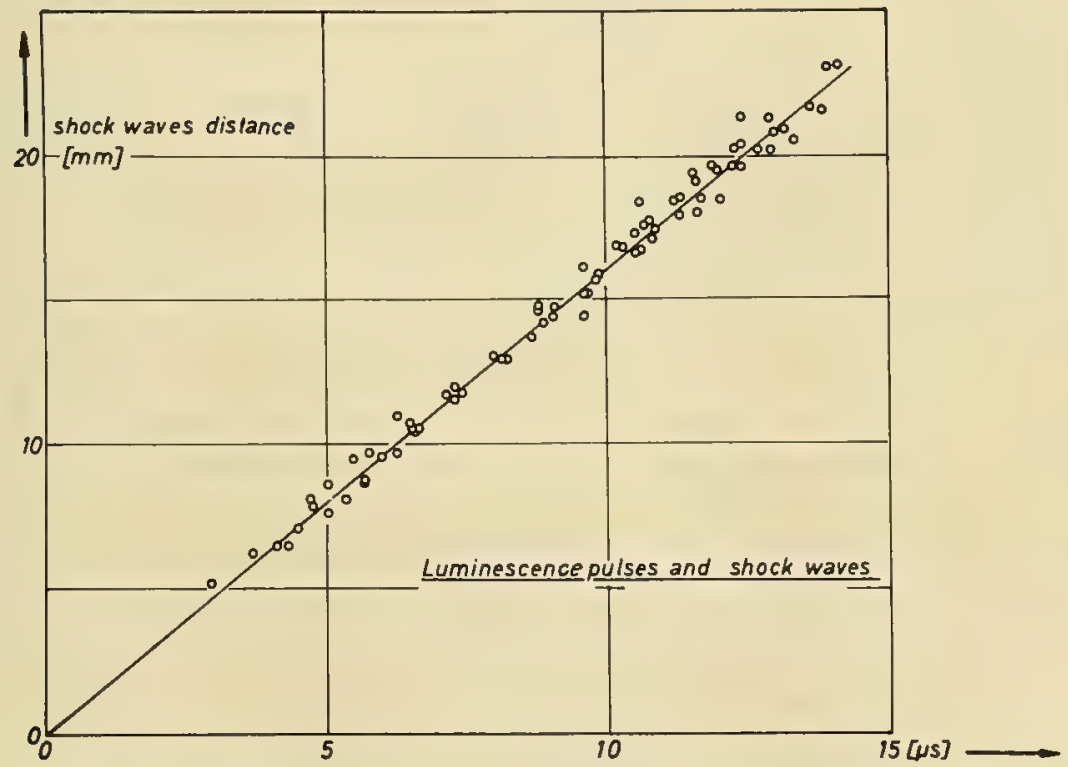

Fig. 9.27. Time delay between spark illumination and luminescence pulse. 
emission of the luminescence pulse which, in these experiments, is generally composed of a great number of closely succeeding individual pulses. During one half-period, that is, in the phase interval $0^{\circ}$ to $180^{\circ}$, there are no bubbles at all. They occur quite suddenly between $180^{\circ}$ and $240^{\circ}$. Just before the phase reaches $360^{\circ}$, their number and volume rapidly decrease as shown in Fig. 9.25. It can be inferred from such experiments, together with measurements of the spectral distribution of the luminescence light [19], that luminescence is caused by thermal excitation of the highly compressed gas in the bubble in the last stage of collapse.

Luminescence occurs just at the end of the collapse and simultaneously the shock wave mentioned in Section 9.1.2 is radiated. This coincidence is especially emphasized in a photograph made by Kuttruff displaying the luminescence pulse and the shock wave shown in Fig. 9.26. The luminescence pulses were recorded with an oscilloscope, and simultaneously the luminescence pulse triggers an illumination spark for photographing the shock wave with Schlieren optics. If the time delay between these events is known, both effects (luminescence pulse emission and shock wave generation) are easily correlated. A great number of such measurements have been made and the results are plotted in Fig. 9.27. They show the close relation between shock wave generation and sonoluminescence.

\section{DISCUSSION}

PROFESSOR D. SETTE questioned whether, in the measurements of acoustic absorption at different sound intensities using light diffraction, the effect of nonplanarity in the waves had been taken into account. He and his colleagues, in 1948, had noted at low intensities a distortion of the diffraction pattern with increasing distance from the source, which they had attributed to the divergence of the beam.

PROFESSOR MEYER: Experimental data on sound absorption in liquids obtained at various sound intensities with the light diffraction method were taken from the literature. I believe that the effect of nonplanarity of the sound waves has been properly considered in the respective studies.

DR. H. MEDWIN commented upon the uncertainty of the magnitude of the acoustic pressure in the front and the tail of the shock disturbance in a liquid. When the shock impinges on the face of the quartz detector, we can neither assume that the quartz is a rigid boundary when immersed in a liquid nor that the pressure is doubled, as we are no longer dealing with linear acoustics. When using a quartz detector for shock waves in a gas, the assumption of its behavior as a rigid boundary for reflection is justified, and, for shock reflection, the resultant acoustic pressure is somewhat greater than twice the value for the incident disturbance. For shock reflection in liquids, the amplification factor would require experimental determination. The use of the reciprocity techinique for the calibration of microphones assumes that operations are in the field of linear acoustics, where it is possible to calculate the attenuation caused by divergence and energy absorption (by the medium) between the source and receiver.

PROFESSOR MEYER: With respect to the very interesting remark of Dr. Medwin, I would like to confirm that there are considerable differences in the 
reflection of shock waves of approximately equal pressures in air and water. Shock waves in gaseous media are a problem of nonlinear acoustics; while with the shock waves generated in water, it is probably correct to assume linear relations. This is also indicated by the respective Mach numbers (shock front velocity divided by velocity of sound) for equal pressures.

DR. S. WENNERBERG pointed out that a possible way of checking the steepness of the shock front was to utilize the directivity of the receiving crystal. Assuming the rise time for the shock front to be $10^{-9} \mathrm{sec}$, the velocity of propagation to be $1500 \mathrm{msec}^{-1}$, and the diameter of the receiving face of the crystal to be $1 \mathrm{~cm}$, then a rough calculation shows that the crystal has to be aligned to about 0.01 of a degree to obtain maximum response. He wondered whether experiments had been made to substantiate this.

PROFESSOR MEYER: The question of Dr. Wennerberg is perfectly justified. The adjustment of the detector quartz is carried out in such a way that the indication on the CRT screen goes to a maximum. Indeed, very accurate adjustment is necessary. The detector quartz, has a relatively large surface area, but only the size of the scanning electrode, on the rear side of the quartz, is of significance. The size of this electrode is about $2.5 \mathrm{~mm}$.

\section{REFERENCES}

1. A. K. Burov, "Obtaining High Ultrasonic Intensities in Liquids," Sov. Physics Acoust., Vol. 4, 326-330 (1958).

2. I.N. Kogan and L.I. Menes, "A Barium Titanate High Intensity Ultrasonic Radiator," Sov. Physics Acoust., Vol. 3, 64-66 (1957).

3. C. M. van der Burgt, "Piezomagnetic Ferrites," Electronic Tech., Voì. 37, 330-341 (1960).

4. J. Gruetzmacher, "Piezoelektrischer Kristall mit Ultraschallkonvergenz," Z. Phys., Vol. 96, 342-349 (1935).

5. L.D. Rozenberg and M.G. Sirotyuk, "Apparatus for the Generation of Focused Ultrasound of High Intensity," Sov. Physics Acoust., Vol. 5, 206-210 (1959).

6. L. Bohn, "Schalldruckverlauf und Spektrum bei der Schwingungskavitation," Acustica, Vol. 7, 201-216 (1957).

7. J. Schmid, "Kinematographische Untersuchung der Einzelblasen-Kavitation," Acustica, Vol. 9, 321$326(1959)$.

8. F. Frürgel, Impulstechnik, 273 (Leipzig, 1960).

9. W. Eisenmenger, "Eine elektromagnetische Impulsschallquelle zur Erzeugung von Druckstössen in Fliussigkeiten und Festkörpern," Proc. Third Internat'I Congress of Acoust. (1961).

10. E. V. Romanenko, "Miniature Piezoelectric Ultrasonic Receivers," Sov, Physics Acoust., Vol. 3, 364370 (1957).

11. J. Koppelmann, "Beiträge zur Ultraschallmesstechnik in Flüssigkeiten," Acustica, Vol. 2, 92-96 (1952).

12. R.T. Beyer and V. Narasimhan, "On the Absorption of Ultrasonic Waves of Finite Amplitude in Liquids," Sov. Physics Acoust., Vol. 4, 196-197 (1958).

13. M. A. Breazeale and E. A. Hiedernann, "Diffraction Patterns Produced by Finite Amplitude Waves, " J. Acoust. Soc. Am., Vol. 33, 700-701 (1961).

14. I. G. Mikhailov and V.A. Shutilov, "Diffraction of Light by Large-Amplitude Ultrasonic Waves," Sov. Physics Acoust., Vol. 4, 174-184 (1958).

15. I.G. Mikhailov and V.A. Shutilov, "Diffraction of Light by the Harmonics of an Ultrasonic Wave Which Is Distorted in the Course of Propagation in a Liquid," Sov. Physics Acoust., Vol. 5, 75-78 (1959).

16. W. Guith, "Zur Entstehung der Stosswellen bei der Kavitation," Acustica, Vol. 6, 527-531 (1956).

17. W. Güth, "Kinematographische Aufnahmen von Wasserdampfblasen," Acustica Ak. Beiheft, Vol. 4. 445455 (1954).

18. E. Meyer and H. Kuttruff, "Zur Phasenbeziehung zwischen Sonolumineszenz und Kavitationsvorgang bei periodischer Anregung," Z. Angew. Phys ". Vol. 11, 325-333 (1959).

19. P. Günther, E. Heim, and $\mathrm{H} U$. Borgstedt, "Über die kontinuierlichen Sonolumineszenzspektren wässriger Lösungen," Z. Elektrochem., Vol. 63, 43-47 (1959).

20. J. Schmid, "Gasgehalt und Lumineszenz einer Kavitationsblase (Modellversuche an Glaskugeln)," Acustica (1962).

21. W. Eisenmenger, "Elektromagnetische Erzeugung von ebenen Druckstössen in Flüssigkeiten," Acustica (1952).

22. H. Kuttruff, "Über den Zusammenhang zwischen der Sonolumineszenz und der Schwingungskavitation in Flüssigkeiten," Acustica (1962). 

LECTURE 10

\title{
SCALE-MODEL STUDY OF PROPAGATION IN SHALLOW SEAS: A VISUAL METHOD OF REPRESENTATION OF LOW-INTENSITY SOUND FIELDS
}

\author{
A. B. Wood \\ Admiralty Research Laboratory \\ Teddington, Middlesex, England
}

\subsection{INTRODUCTION}

It has long been recognized in engineering practice that the considerable cost and bulk of the structures with which the engineer is concerned frequently make comprehensive research upon them extremely laborious and prohibitively expensive. The conditions of operation of such full-scale structures also are not conducive to the acquisition of reliable information. On the other hand, experiments with small scale models have shown that they are capable of demonstrating physical effects of a general character and of providing data which can be applied with some confidence in full-scale practice [1]. Aeronautics, naval architecture, hydrodynamics, and building acoustics have long furnished examples of successful small-scale research, and it is increasingly evident that the experimental scale-model technique can be applied to a much wider range of subjects than interests the civil or mechanical engineer. A model can be constructed for a very small fraction of the cost of its full-scale counterpart, and it need only include those features which are known to influence the efiects under consideration. A model is easy to manipulate, and its cost in labor and time is relatively low. Further, success is more likely to be achieved under controlled conditions in the laboratory. Many of the variable physical quantities which affect a particular problem can be simulated in a laboratory experiment and are capable of adequate, and in some cases separate, control. On a model scale modifications of apparatus or method can be made with an ease and rapidity which is quite unattainable at full scale. Model apparatus is also ideal for checking novel theories and data predicted theoretically. These advantages of model over full-scale research techniques are, of course, now common knowledge. The alternative, a purely mathematical treatment of a problem, is within certain limitations often a possibility, but in some practical cases is either very difficult or quite intractable. In acoustical problems, as is well known, it is often advantageous to consider analogous optical cases $[2,3]$ when the wavelengths and linear dimensions involved can be suitably scaled. 
Particularly in the special case of underwater acoustics with which I am concerned, the propagation of sound in shallow waters, full-scale methods are expensive, lengthy, and laborious; and, experimental sea conditions of common occurrence are not under our control. Thus, when it is required to test a particular aspect of the theory of sound propagation, it is rarely possible to find conditions at sea which enable the test to be made free from ambiguities. In practice, perturbing factors are often present which in one way or another mask the effect it is desired to detect. Theoretical treatment of propagation problems is also in many cases very difficult, and simplifying assumptions have to be made. Even skilled mathematicians, with the aid of modern electronic computers and invoking idealized conditions, have hitherto found the answers to propagation problems in shallow water slow and tedious to obtain. Small-scale experiments, however, provide information much more rapidly and, even if this information is not always as accurate quantitatively as we should like, it gives at least a reasonably good guide to what we may expect to find under full-scale conditions.

My interest in the subject of sound propagation in the sea was first aroused in 1915-16 when F.B. Young and I made some observations with an audio-frequency source $(580 \mathrm{cps})$ of the sound field in water and around obstacles placed in the water [4]. During the course of our experiments, we noticed definite indications of a pattern in the sound field in the water, which we ascribed to the interference between the direct sound and that reflected from the surface and the bottom-somewhat similar to the Lloyd's mirror interference effect in the optical case, although more complicated due to the presence of the bottom. We also noted differences over soft mud and hard rock bottoms. It was not until a later date, 1934-35, however, that I had the opportunity for further study of the underwater interference problem. I then selected the relatively simple case of deep-sea propagation where there is only one surface reflection and no bottom reflection to consider. In this case it is easily shown that lines of maxima occur when the path difference $s$ between the direct ray and the surface-reflected ray is an odd multiple of half a wavelength $(n \lambda / 2)$. This indicates of course that the interference maxima can be represented by a family of hyperbolas, or, at ranges that are large compared with the depths of the transmitter and receiver, by the corresponding asymptotes of these hyperbolas. The experiments which I made at $300 \mathrm{kcps}$ (wavelength, $5 \mathrm{~mm}$ ) in a tank $80 \mathrm{ft}$ long, $15 \mathrm{ft}$ wide, and $10 \mathrm{ft}$ deep, confirmed the simple interference theory, and in addition revealed temperaturegradient effects which caused bending of the lines of interference maxima. A receiver moving at constant depth $d$ away from the transmitter at depth $D$ passes through a series of maxima spaced at intervals increasing with range up to a critical distance $4 D d / \lambda$ where the path difference is equal to half a wavelength, $\lambda / 2$, and beyond which the signal decreases steadily to zero at infinity. It was interesting to see these model experiments confirmed more recently in full-scale experiments by F.H. Sanders and R.W. Stewart $[5,6]$ in very deep water off British Columbia.

The main purpose of the experiments which I propose to describe is to examine the case of propagation of continuous sound waves in shallow water. Here we have to deal with the theoretically more difficult case which involves interference between the direct sound wave and waves reflected possibly many 
times between surface and bottom. Some of the more important factors likely to influence sound propagation are contour, slope and nature of the sea bed, state of the sea surface, temperature and salinity gradients, water movements, and depth of water relative to the sound wavelength. The major cause of variation of signal strength in shallow water is, however, due to interference between the direct sound and that reflected from surface and bottom. The theoretical approach to the problem is very difficult even when this cause of variation is considered alone and all other variables are eliminated. The normal mode theory due to C. L. Pekeris [7] and various "ray" theories [7] have been developed to explain the main phenomena under idealized conditions. The present paper is concerned, however, with a new experimental technique which gives a picture of the sound field under various prescribed conditions [9]. A brief reference is also made to a preliminary attempt by a mathematical computer method to reproduce such a sound field theoretically [8]. As was anticipated, the sound fields in shallow water are often very complex, but certain broad features depending on the ratio of depth to wavelength, the directional properties of the source, and the nature of the bottom are now apparent.

\subsubsection{The Scale of the Model Experiments}

The scale of the model experiments has been chosen somewhat arbitrarily and results from a compromise between full-scale requirements and available laboratory facilities. The shallow seas around the British Isles vary considerably in depth but 100 to $200 \mathrm{ft}$ is a reasonable average. The frequency range of present interest lies around 500 to $1000 \mathrm{cps}$, the corresponding wavelengths in sea water being about 10 to $5 \mathrm{ft}$ (or 3 to $1.5 \mathrm{~m}$ ). Considerations of this nature have led to a choice of tank dimensions $20 \mathrm{ft}$ long, $5 \mathrm{ft}$ wide, and $6 \mathrm{in}$. deep, approximately equivalent at full scale to 4 miles by 1 mile by $500 \mathrm{ft}$ and representing a scale factor of the order of $1 / 1000$. Using the same medium, water, on the model as on the full scale, we regard $C=\lambda N=\lambda / T$ as constant. This is achieved by scaling down both wavelength $\lambda$ and periodic time $T$, or simply by increasing frequencies $N$ by $1000 / 1$. Consequently the frequencies $500 \mathrm{kcps}$ and $1 \mathrm{Mcps}$, wavelengths 3 and $1.5 \mathrm{~mm}$, on the model correspond to full-scale conditions when water, in which $C=1500 \mathrm{~m} / \mathrm{sec}$, is the medium employed. The mean ratio of depth to wavelength would under these conditions be around $20 / 1$ in both full scale and model, the depth of water corresponding to a sea depth of $200 \mathrm{ft}$, being about $2 \mathrm{in}$. in the model. During the course of the experiments, however, the depth-to-wavelength ratio has been varied over the range from $30 / 1$ to $4 / 1$.

The effects of loss of sound intensity due to attenuation or to increasing range have either been neglected or automatically compensated (reference is made to this later).

The scale-model tank referred to above was made of sheet steel $1 / 8 \mathrm{in}$. thick suitably mounted and leveled on tables and fitted with side rails of Dexion girders on which a motor-driven platform carrying one of the transducers could move smoothly and quietly along the tank, as shown in Figs. 10.1 and 10.2. Provision was also made for driving the transducers across the tank and for raising and lowering them at any desired constant speed. In subsequent developments, to which I shall refer later, arrangements were made for raising and lowering a transducer at a controlled repetition rate (say, up and down three times per 


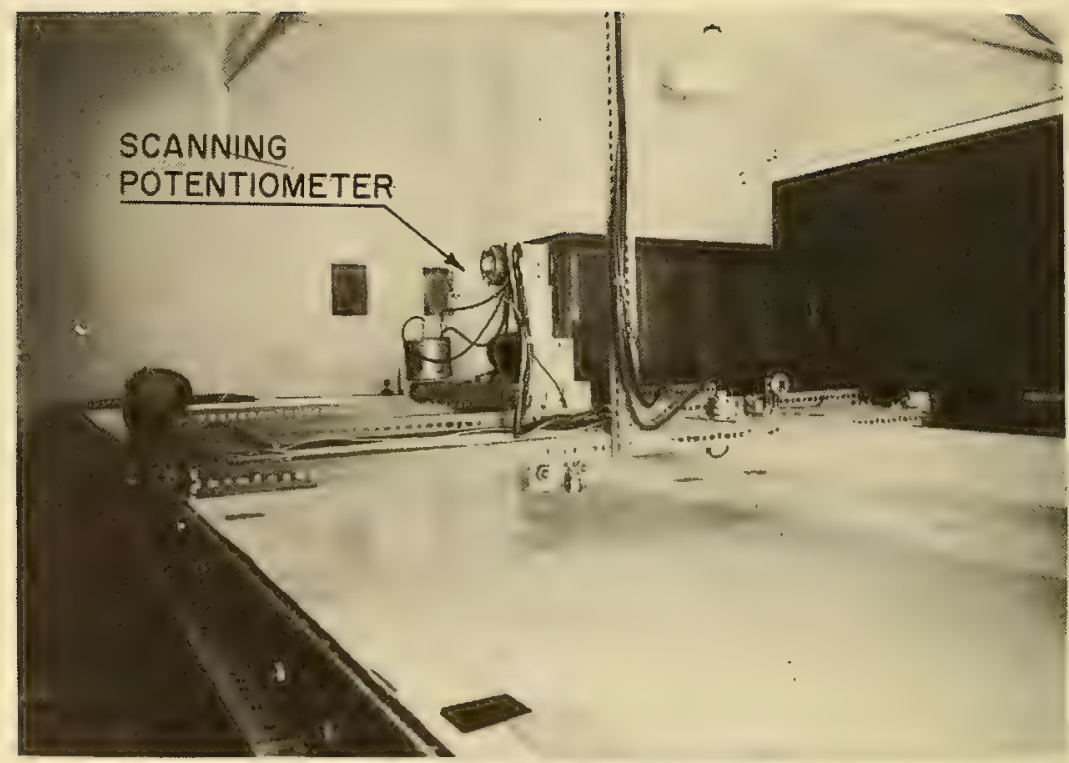

Fig. 10.1. View of model tank and movable trolley.

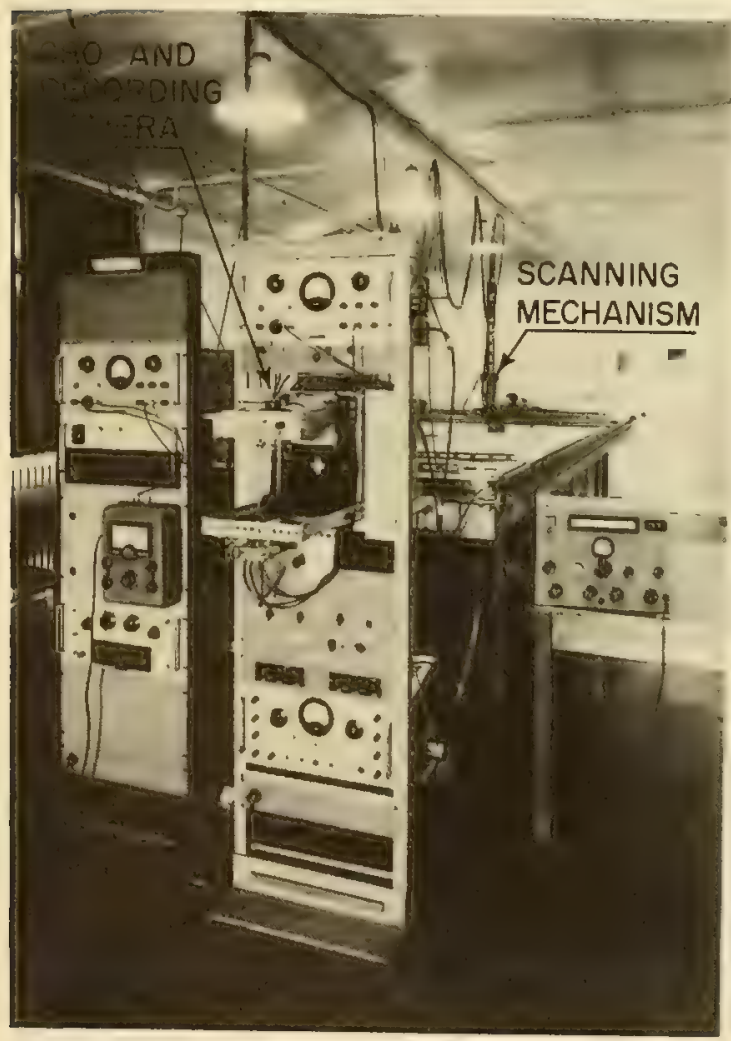

Fig. 10.2. Model tank showing transmitter panels (left) and receiver and control panel (right). 
second) between the bottom and surface of the water. Transducers could also be rotated at a constant angular speed if required for the purpose of recording their directional characteristics. In some of the earlier experiments, use was made of a small concrete tank as a more realistic approach to solid rock than is the case with the bottom of the steel tank. The dimensions of this tank were 38 by 33 by 4 in., the solid concrete bottom being 24 in. thick. It was used mainly in studies of temperature gradients and of variation of water depth, and also served in a comparison with the steel bottom of the large tank.

As had been anticipated, the sides and ends of the steel tank were found to be good sound reflectors. Using directional pulse transmissions at $1 \mathrm{Mcps}$, a regular succession of end-to-end reflections was observed. Various methods were tried to prevent these reflections, and the one ultimately used was very effective. This method employed sheets of thin rubber covered with "spikes" about $3 / 8$ in. high and spaced about $0.3 \mathrm{in}$. apart in an equilateral triangle arrangement. The material, shown in Fig. 10.3, is a commercial product used in the manufacture of batting gloves, soap mats, etc. These spiked rubber mats, $15 \mathrm{in}$. long and $4 \frac{1}{2} \mathrm{in}$. wide, were used with the spikes vertical in piles equal to the depth of water so as to screen the sides and ends of the tank. In this way a reduction of echo strength of about 25 to $30 \mathrm{db}$ was obtained. The mats are easily removable for cleaning and remain constant in acoustic properties for long periods.

Both directional and omnidirectional (point) transducers have been used with quartz, barium titanate, or PZT as the piezoelectric material. The directional transducers, varying in diameter from 1.0 to $2.4 \mathrm{~cm}$ and in thickness according to the frequency required, produced primary beams of semiangles ranging between $5^{\circ}$ and $30^{\circ}$, approximately, according to diameter and frequency. These directional transducers, although having large diameters as compared with the scale we are considering, served a useful purpose in providing known directional characteristics of transmission in certain propagation tests which will be mentioned later. An approximation to an omnidirectional transmitter required that the diameter of the active face should not appreciably exceed one wavelength of the transmitted sound, i.e., $1.5 \mathrm{~mm}$ at 1 Mcps or $3.0 \mathrm{~mm}$ at $0.5 \mathrm{Mcps}$. This was ultimately achieved to a sufficient approximation in the following way. A short length (say, about 4 in.) of a metal wire having a flat conical end, as shown in

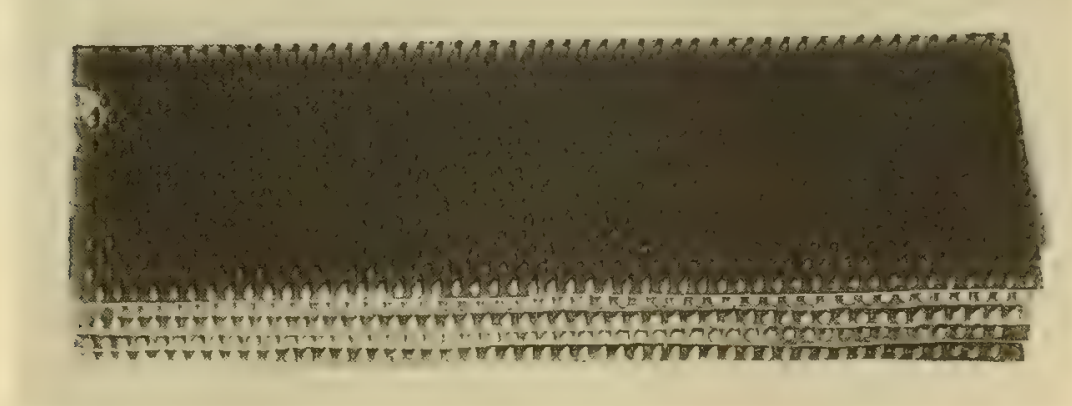

Fig. 10.3. Pile of spiked rubber mats used to suppress reflection from sides of steel tank. 

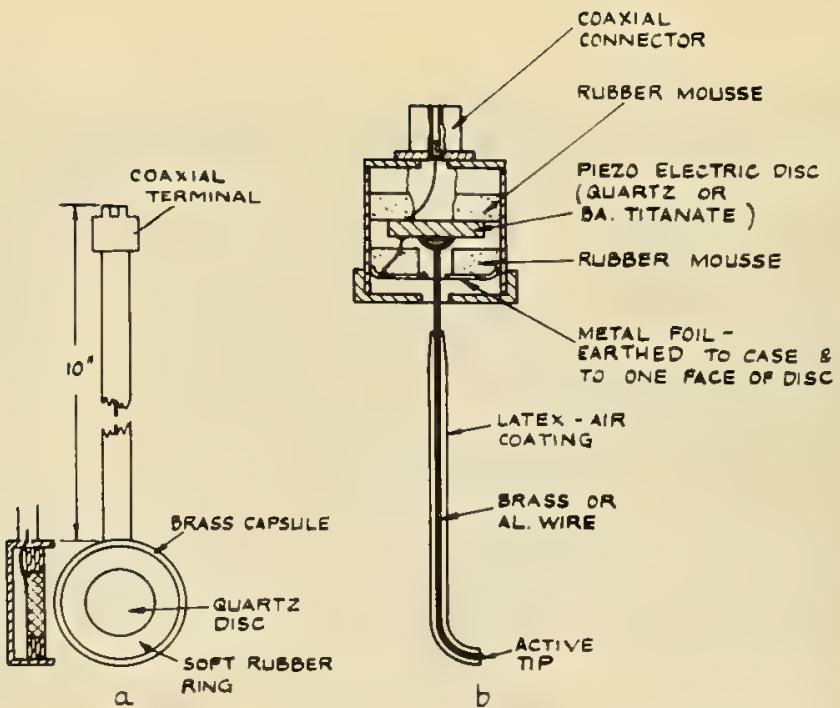

Fig. 10.4. Transducers: (a) directional transducer, (b) nondirectional transducer.

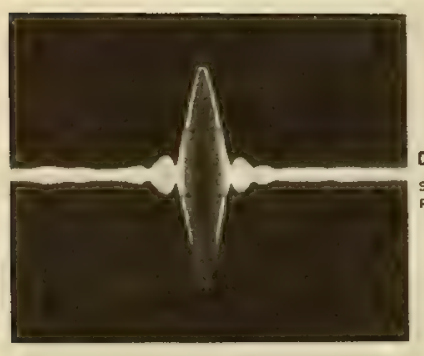

OLAMETER $2.4 \mathrm{cms}$

LATEX - AIR

COATING

BRASS OA

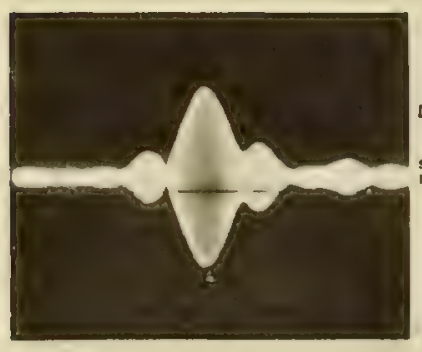

SEMI - ANGLE OF

PRIMAAY $4 . \mathbf{B}^{\circ}$

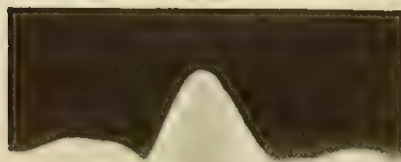

DIAMETER $1.0 \mathrm{cms}$

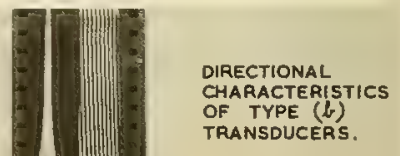

DIAMETER $1.5 \mathrm{cms}$

SEMI - ANGLE OF

- ACtIVE

b

OF TYPE (b)

TRANSDUCERS.

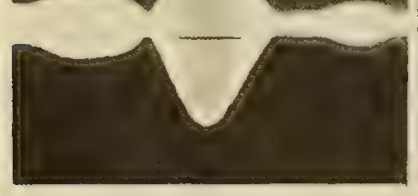

SEMI- ANGLE OF

DIRECTIONAL CHARACTERISTICS

OF TYPE (a) TRANSDUCERS.

Fig. 10.5. Directional characteristics of transducers at one megacycle per second. 
Fig. 10.4, is cemented to the center of one face of the piezoelectric disc, the vibrations of the disc being transmitted though the wire to the water (or conversely in the case of the receiver). The active tip of the wire is bent at a right angle to send or receive preferably in a horizontal direction in the water layer. The cylindrical side of the wire is coated with a pressure-release material to ensure that only the tip takes part in the transmission or reception of sound. The coatings are various thin, watertight layers containing free gas films or bubbles, e.g., (a) rubber latex to which small quantities of $\mathrm{MnO}_{2}$ and $\mathrm{H}_{2} \mathrm{O}_{2}$ have been added, (b) "microballoons" in " pliobond" adhesive, or (c) plastic tube containing an air film. Such wire-tip transducers are good "point" sources or receivers in all practical cases encountered in the model experiments. The directional properties of the types of transducers used, directional and omnidirectional, are shown in the records of Fig. 10.5, the frequency in these cases being $1 \mathrm{Mcps}$, approximately.

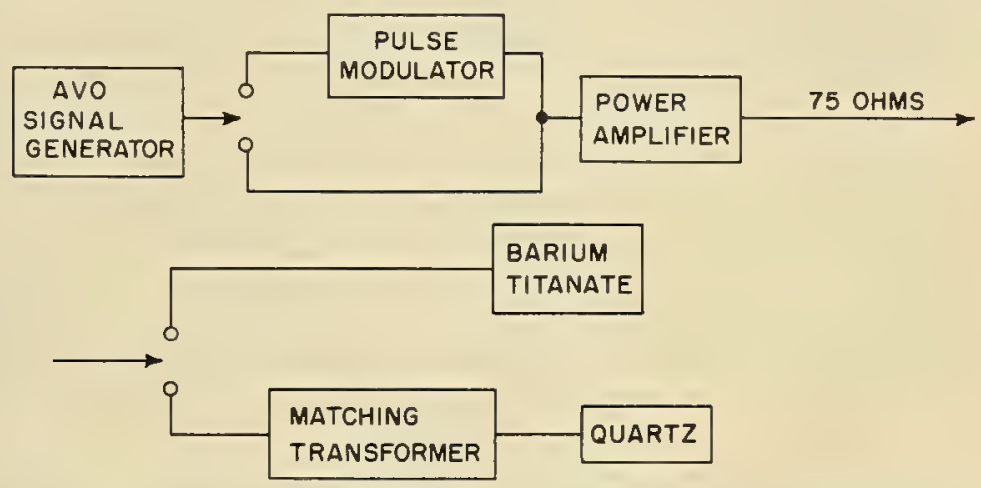

(a) TRANSMITTER SUPPLY CIRCUIT C.W. AND PULSE

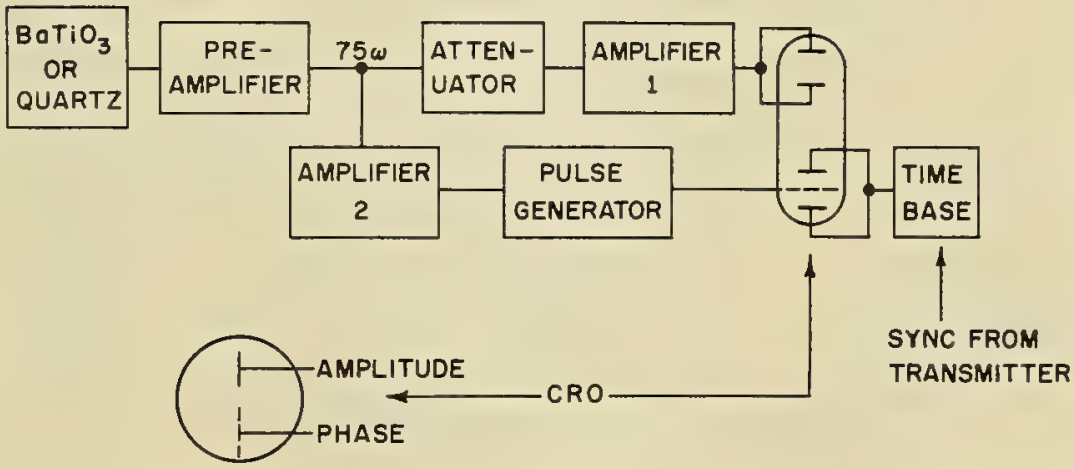

(b) RECEIVER AMPLITUDE AND PHASE RECORDER

Fig. 10.6 Receiver amplitude and phase recorder. 
In the earlier experiments, both amplitude and phase of received signals were recorded using a two-gun CRO. Figure 10.6 shows the circuits used (a) for continuous wave and pulse transmission, and (b) for amplitude and phase recording of the received signal. In the case of the phase recording, the time base on the $Y$ axis is synchronized with the transmitted signal and appears as a line of bright spots spaced one cycle apart whose vertical position indicates the phase of the received signal with respect to the transmitted signal. When only amplitude recording is required, a Bruel and Kjaer logarithmic (db) recorder has been used as well as the CRO.

\subsection{EXPERIMENTAL - "POINT-BY-POINT"METHOD}

With the transmitter emitting continuous waves at a chosen fixed depth and position in the tank, oscillographic records were made showing the variation of pressure amplitude when the receiver was moved slowly at constant velocity from the bottom to the surface of the water, the records being repeated at different ranges along the tank. In other experiments the variation of pressure amplitude was recorded as the range of the receiver was continuously decreased, the depths of transmitter and receiver being constant. These experiments indicated clearly that the sound distribution in the water showed a number of maxima and minima between surface and bottom, the spacing and amplitude of these varying in a somewhat irregular fashion and depending on a number of factors of more or less importance. The number of maxima between bottom and surface was clearly dependent on the directional properties of the transmitter and receiver, on the depth of the water, the wavelength of the sound, and on the nature of the bottom. It was also modified by waves on the water surface and by temperature gradients.

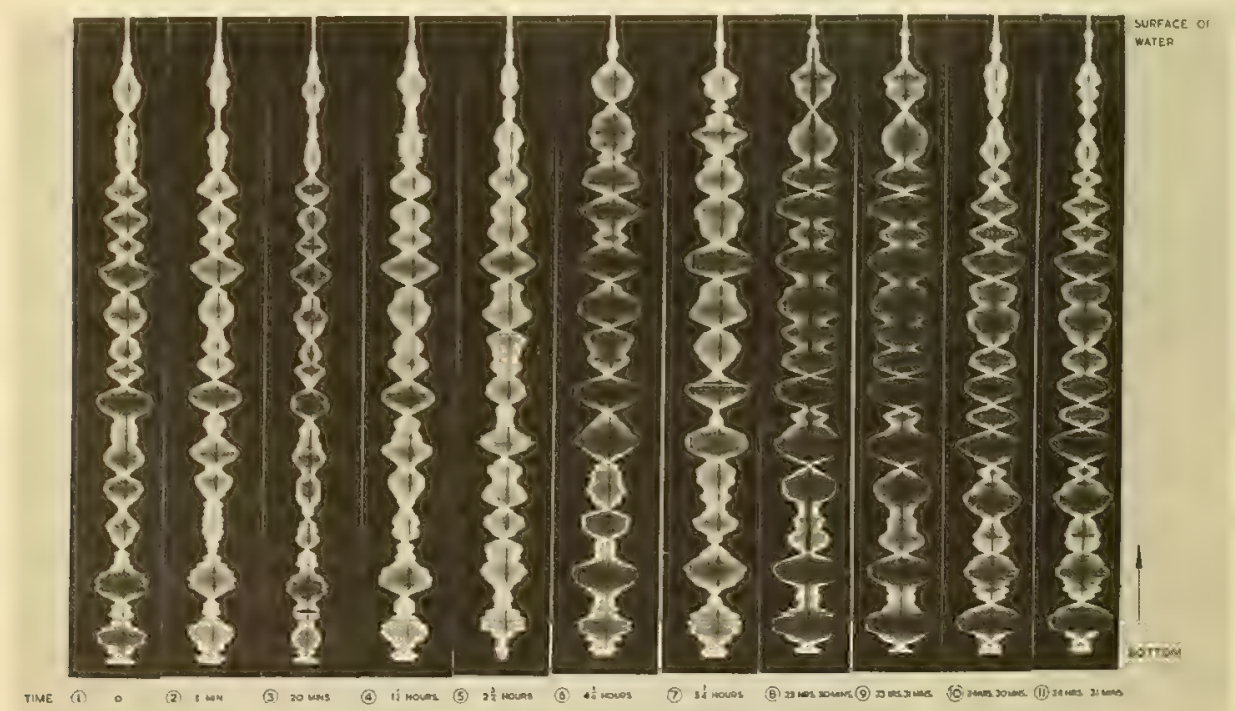

Fig. 10.7. Pressure-amplitude records for constancy tests showing records of received signal as receiver is raised slowly from bottom to surface of water. 
As a preliminary to the experiments to be outlined, a test was made of the "constancy" or "repeatability" under prescribed conditions, extending over a period of 24 hours. In these tests a "point" (omnidirectional) transmitter emitting a continuous wave was arranged at a fixed depth below the water surface while a point receiver was slowly raised from bottom to surface. Records of the pressure-amplitude variations, shown in Fig. 10.7, with maxima and minima of somewhat irregular spacing and amplitude, were made at various intervals of time. For short periods of three or four hours, the repetitions of the bottom-to-surface records were reasonably consistent, but for longer periods extending overnight to the following day considerable variations were noted, maxima and minima positions being often interchanged. At first, temperature fluctuations were suspected, but temperature control and stirring to prevent gradients of temperature made no appreciable difference. Eventually the trouble was traced to a small change in the depth of the water due to evaporation. This effect, which may be very critical, will be dealt with later. When a directional transmitter was used, however, the effect of variation with time was much less noticeable.

\subsubsection{Directional Properties and Depth of Transmitter}

A comparison of directional and omnidirectional transmitters when bottomto-surface records are made at various depths and ranges of the transmitter is very striking. When a transmitter of semiangle $10^{\circ}$ is used and the bottom is steel, there may be only 3 or 4 maxima between bottom and surface, whereas the number is increased to 10 or 12 if a point transmitter is used. The positions and amplitudes of the maxima vary as the depth or the range of the transmitter is changed, but the general characteristics of the records are the same, considerably more maxima being observed when the transmitter is a point than when it is directional. As the range is varied, unexpected variations in location of the maxima are liable to occur, however, and it is practically impossible to associate a particular maximum at one range with a "corresponding" maximum at any other range. If any order exists between maxima at different ranges, there is no obvious means of connecting them one with another by this point-by-point method of recording.

\subsubsection{Range from Transmitter to Receiver Varied}

In this case the receiver and transmitter are fixed in depth while the range between them is varied. Here again the directional properties of the transmitter are very important. There is a gradual decrease of amplitude as the range is increased from, say, 1 to $4.5 \mathrm{~m}$, but superposed on this are irregular fluctuations in amplitude. With directional transmission these fluctuations are much less noticeable than in the case where the transmitter is a point. In the latter case, when the bottom is steel, the fluctuations are very numerous indeed, but are much less serious when the steel bottom is covered with sound-absorbent material. In all cases the difference between narrow-beam and wide-beam transmission is very striking.

\subsubsection{Effects of Varying Nature of Bottom and Frequency of Sound}

It might appear that a scale-model tank having a "sea bed" of $1 / 8$-in. steel sheet would have little resemblance to a real sea bed consisting of mud, sand, gravel, or rock. When it is considered, however, that the sea bed in shallow 
coastal areas is subject not only to considerable variation in the size of its particles (from mud to pebbles) but also to irregularities such as sand waves due to undercurrents, it would seem somewhat pedantic to attempt to scale down sand and mud particles. It was considered reasonable to use fine sand, particle size 0.1 to $0.3 \mathrm{~mm}$, linear, which is small compared with the shortest wavelength of sound (about $1.5 \mathrm{~mm}$ ) employed in the experiments. In the early stages of the investigation it was found that the bottom-to-surface oscillograph records were very similar whether the steel bottom of the tank was covered with fine wellwetted sand (free from entrapped air) or with a $1 / 8$-in.-thick layer of rubber sheet. This was very fortunate, for the removal or insertion of the rubber sheet was a very simple matter compared with the corresponding operation with a layer of sand, and incidentally was much more reproducible. In what follows, I shall therefore refer to the bottom covered with the rubber sheet as equivalent to that covered with well-wetted, air-free sand or mud. The steel bottom has been regarded as acoustically hard, equivalent to rock, but this of course was not obvious and required some experimental proof. In the first instance it was necessary to find out how much high-frequency sound (0.5 to $1.0 \mathrm{Mcps}$ ) was actually transmitted along the bottom of the steel tank containing water. A small $(1 \mathrm{~cm}$ in diameter) quartz receiver was attached in good acoustical contact to the underside of the steel bottom, while a similar quartz transmitter was arranged face down in midwater on the movable trolley. As the transmitter was moved away from a point directly above the receiver, the received signal strength was meas ured by the CRO and an attenuator. At a frequency of 1 Mcps, the attenuation was found to be $40 \mathrm{db} / \mathrm{m}$. With the steel bottom covered by a sheet of $1 / 8-\mathrm{in}$.thick rubber, the attenuation increased to $300 \mathrm{db} / \mathrm{m}$. These attenuation values relate to sound incident normally on the steel or rubber surface, and it is reasonable to assume that they may be even greater at small grazing angles. As a further check on the advisability of using $1 / 8$-in. steel as equivalent to hard rock bottom, comparative observations were made when the bottom was sheet steel $1 / 8$ in. thick or concrete 24 in. thick (equivalent to $2000 \mathrm{ft}$, full scale). Bottom-tosurface records in bothcases, the former in the large $20 \mathrm{ft}$ steel tank and the latter in the small concrete tank, showed similar characteristics as regards the number of maxima in the same depth of water at the same frequency. Similar records were also obtained when the bottom of the steel tank was covered with $1 / 4$-in.-thick plate glass. In all the above tests point (omnidirectional) transducers were used, and both cathode-ray-oscillograph photographic recordings and logarithmic (db) pen recordings were made. In Fig. 10.8 is shown a series of comparative bottom-to-surface records, using a Bruel and Kjaer logarithmic recorder, in which the nature of the bottom and the frequency are varied. In all four series of records, a point source was used; the bottom was successively $1 / 8-i n$. steel, $0.1-i n$. rubber covering $1 / 8$-in. steel, 0.1 -in. rubber covering concrete, and bare concrete. The frequencies used are, left to right, 430, 189, 160 (approximate), $92,52.5$, and $25.6 \mathrm{kcps}$. These records were all made in the small concrete tank so that all coverings mentioned, rubber and/or steel, were overlying the thick concrete bottom. Important features to be noted are (1) the general reduction in the number of maxima or minima as the frequency decreases (or wavelength increases) and (2) the reduction in the number of maxima and minima when 


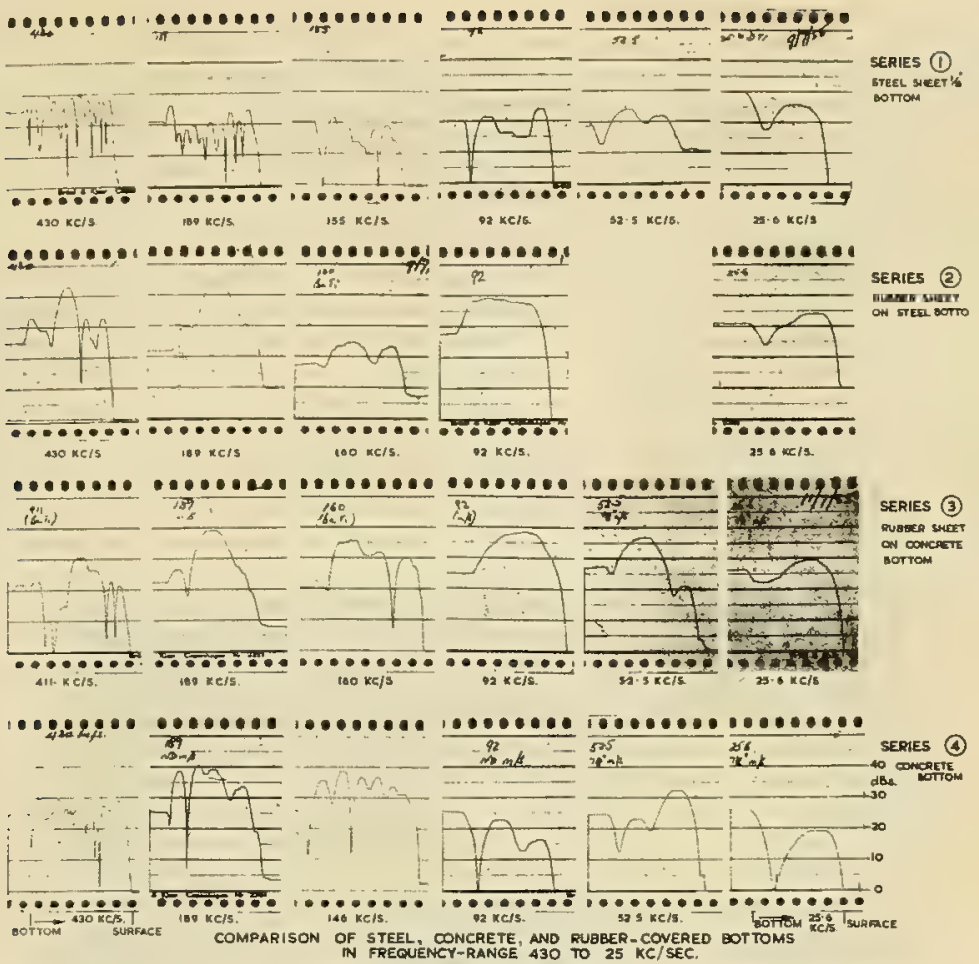

Fig. 10.8. B. and K. logarithmic records showing effect of varying the nature of the bottom and the frequency of the sound.

either the steel or the concrete bottom is covered with a thin sheet $(0.1$ in.) of rubber. A further series of records using the concrete tank was made when the bottom was covered with fine sand $3 / 8$ in. deep in an aluminum tray. These records confirmed that covering the steel sheet or the concrete bottom with thin sheet rubber was equivalent in general features to covering the bottom with fine wellwetted sand free from air inclusions.

\subsubsection{Wave Effects}

Neglecting the case of surface tension waves or ripples and considering only so-called gravity waves, we have two cases:

Deep-water waves

$$
\lambda=\S T^{2} / 2 \pi
$$

Shallow-water waves

$$
\lambda=T(g h)^{1 / 2}(\lambda \gg h)
$$

where $\lambda$ is the wavelength and $T$ the periodic time of the waves, $g$ is the gravitational constant, and $h$ is the depth of water. In dealing with small-scale waves in the model tank, however, attention has been paid only to the scaling of wavelengths and wave heights. The time factor, involving $T$ and $g$, has not been considered. 
On a model scale, the effect of waves on the water surface in modulating the received sound amplitude is easily demonstrated. A wave $1 \mathrm{~m}$ high, full scale, will be $1 \mathrm{~mm}$ on the model scale we are considering. Various methods of generating waves (of lengths lying between 5 and $25 \mathrm{~cm}$ ) and measuring their height have been tried, and very simple techniques have been used. Shaking the side of the tank proved very satisfactory for generating waves having wavefronts parallel to the line of sound propagation along the tank, while a simple "linedipper" at the end of the tank would produce waves with fronts at right angles to the direction of sound propagation. A simple and convenient method of recording the wave amplitude and frequency was the use of an air condenser. This consisted of a strip of metal mounted parallel to the water surface about a centimeter above it, forming a condenser with the water surface, and varying in capacity as the water waves parallel to the length of the metal strip pass under it. This
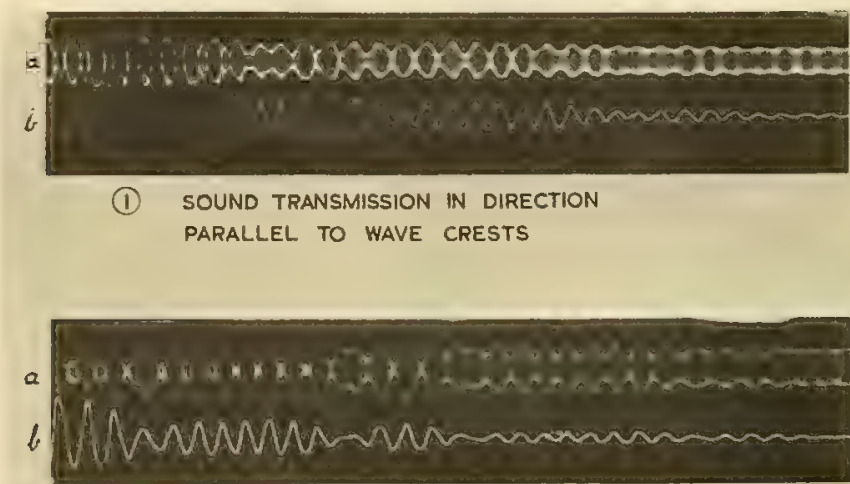

(2) SOUND TRANSMISSION IN DIRECTION PARALLEL TO WAVE CRESTS
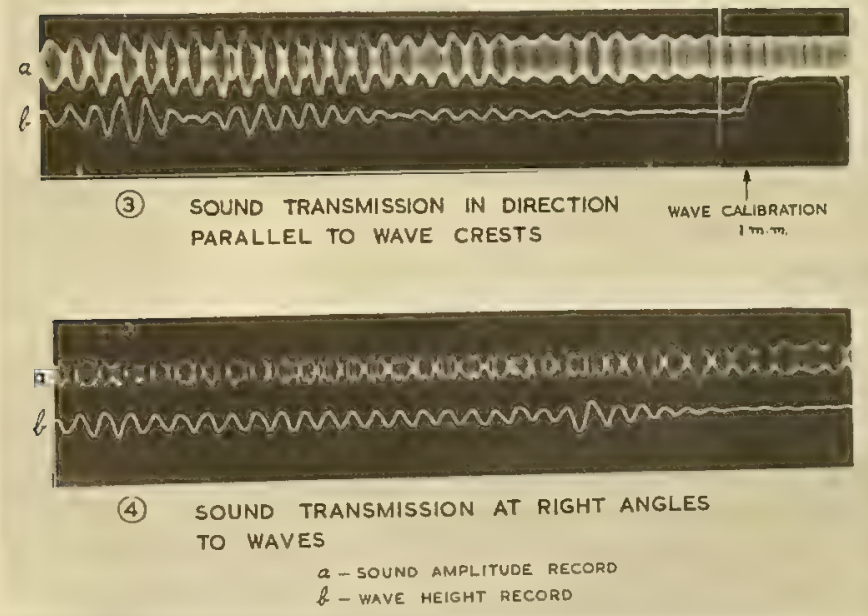

Fig. 10.9. Modulation of the sound amplitude by surface wives. 
arrangement could easily be calibrated by a displacement of the metal strip $1 \mathrm{~mm}$ from its normal position above the water surface. Using the double-beam cathode ray oscillograph, records have been made illustrating how waves of small amplitude modulate the received sound signal when running with their wavefronts parallel or at right angles to the direction of sound propagation (see Fig. 10.9). Using omnidirectional transducers, as in the examples illustrated, the wave effects are very striking, but are barely observable when directional transducers $\left(4.5^{\circ}\right.$, semiangle) are used.

\subsubsection{Temperature Effects}

Two cases arise: (a) isothermal effects-change of uniform temperature and (b) temperature-gradient effects. In case (a), small changes of temperature, of the order of a few degrees centigrade such as are experienced between winter and summer conditions in the sea around the British Isles, result in corresponding changes of sound velocity $C$ in the water. At a constant transmission frequency $N$ this implies a corresponding change of wavelength $\mathrm{C} / N$. The same result may of course be obtained at a constant water temperature by an equivalent small

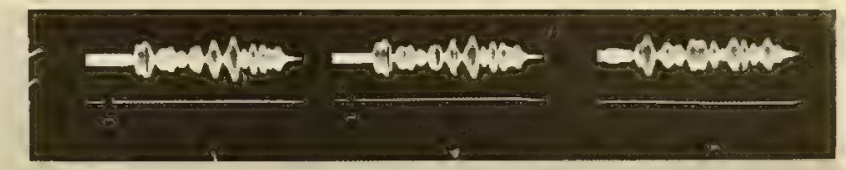
(1)
$15 \cdot 2^{\circ} \mathrm{C}$
(2) $15.25^{\circ} \mathrm{C}$
(3) $16.0 \mathrm{c}$

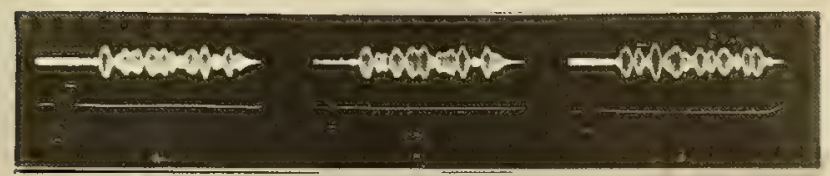
(4) $17 . \% \mathrm{C}$
(5) $1 \cdot x^{\circ} \mathrm{C}$
(6) $20 \cdot 4^{\circ} \mathrm{C}$
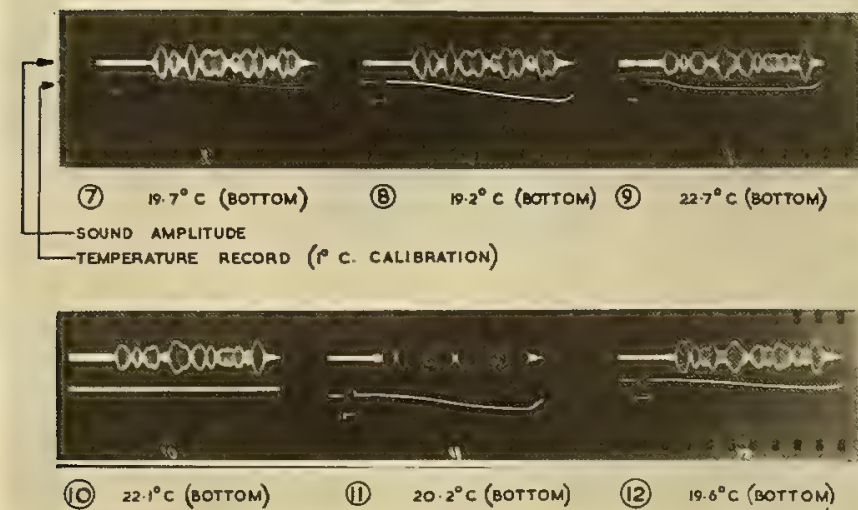

Fig. 10.10. Isothermal temperature, and temperature-gradient effects. 
change of frequency (wavelength) of transmission. This has been confirmed by making a series of bottom-to-surface records at a mean frequency of $410 \mathrm{kcps}$ and varying by about $1.6 \mathrm{kcps}$ on either side of the mean. The small but definite variation in appearance of the records is equivalent to that which is produced by a total isothermal temperature variation of $3.5^{\circ} \mathrm{C}$. This has been verified by experiments in the small concrete tank. In case (b), when temperature gradients are established in the water layer, the first problem is to produce the required temperature gradient. This is simply achieved by inserting an electric immersion heater in the water for the appropriate time, quick stirring, then allowing the water to stratify for a few minutes; the warm water rises to the surface and leaves the colder water in contact with the concrete bottom of the tank. A calibrated point thermistor mounted near the point transducer is raised from bottom to surface with the transducer, and a two-beam cathode ray oscillograph records bottom-to-surface sound amplitude with the corresponding temperature variation (and its calibration marks). Many such records (see Fig. 10.10) have shown that, although changes in the sound distribution occur due to small temperature gradients, the records are still much the same in general character as those due to correspondingly small isothermal temperature changes.

\subsubsection{Depth of Water}

Reference has been made earlier to constancy tests of bottom-to-surface records using point transducers, when it was shown that changes could occur overnight when the experimental setup was fixed. After suspecting temperature efiects, small frequency changes, etc., it was eventually demonstrated that a small change of the order of a fraction of a millimeter in a total water depth of about $50 \mathrm{~mm}$ could sometimes result in a change of about $30 \mathrm{db}$ in the received signal, both transmitter and receiver having been kept at a constant depth and distance apart, and the wavelength of the sound constant at near $3.5 \mathrm{~mm}$. In pursuance of such observations, a series of records was made in the small concrete tank to discover the effect of varying the water depth over a much wider range, e.g., from 0 to $5 \mathrm{~cm}$ maximum depth. In these experiments the bottom was varied from "soft" to "hard" and "absorbent." These three cases were represented by "air" (in the form of rubber mousse cemented to the upper side of a metal-plate sinker), steel, and rubber sheet. In all cases two point transducers are placed a fixed distance apart on the bottom of the tank, and water is allowed to fill the tank slowly (without making waves or ripples) while a logarithmic record of received sound intensity as a function of water depth is being made. Three typical records are shown in Fig. 10.11. Record (a), where the bottom is rubber-covered, indicates a fairly regular series of maxima and minima differing by 20 or $30 \mathrm{db}$ in intensity. It will be noted that at certain critical depths the sound intensity changes very rapidly. Records of this type, the bottom being rubber (sound-absorbent and equivalent to mud and sand) have been made at various frequencies, the spacing of the intensity "crevasses" becoming progressively smaller as the sound frequency increases. Records (b) and (c) in Fig. 10.11 were made in a similar manner when the bottom was soft or hard. Deep crevasses in intensity level are again observed, occurring much more frequently than when the bottom is rubber-covered and sound-absorbent* Such rapid variations of *Note: the frequency is $430 \mathrm{kcps}$ in records (a), (b), and (c). 

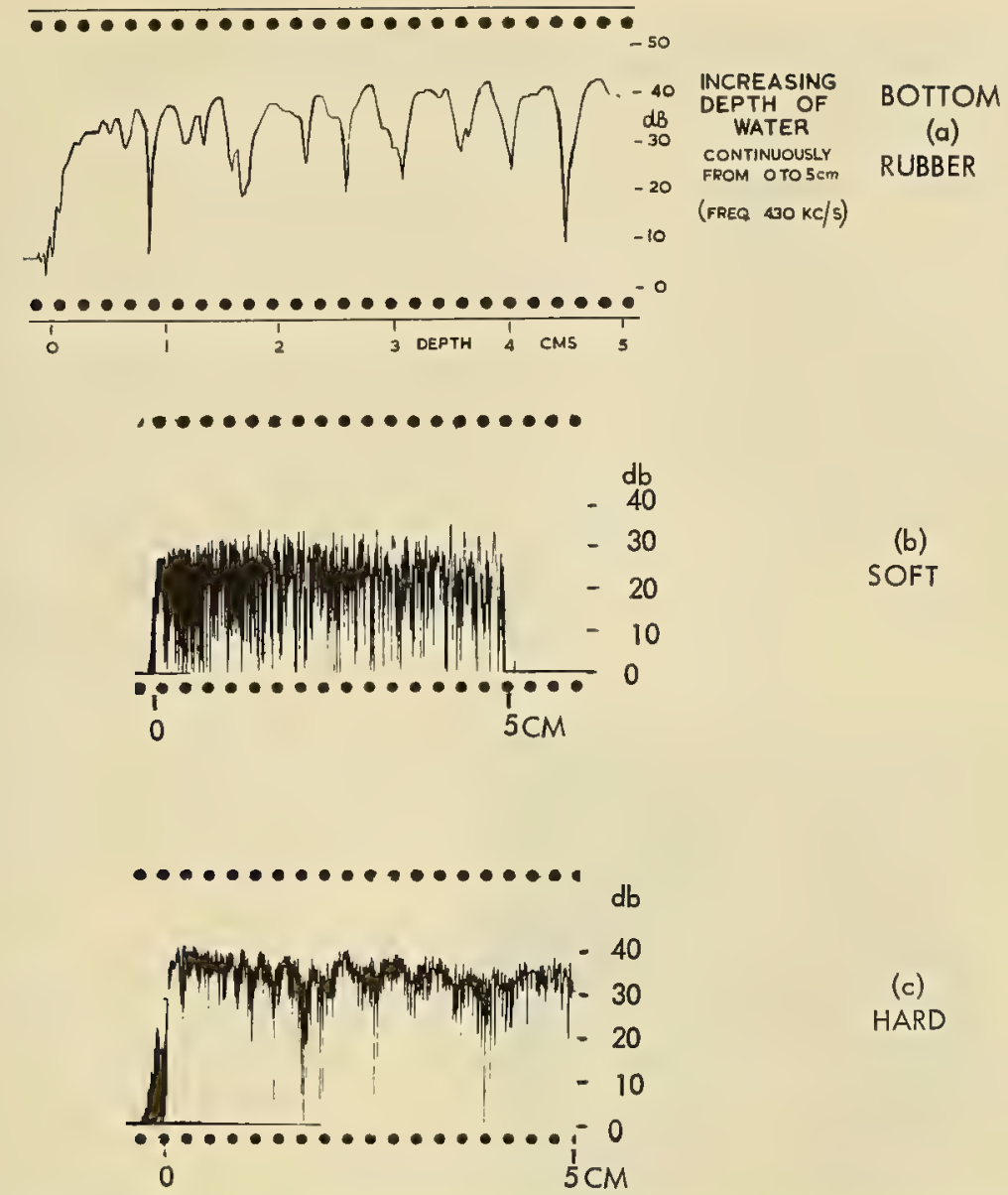

(c) HARD

Fig. 10.11. Records of sound transmission over different bottoms (frequency constant at $430 \mathrm{kcps}$ ) as the water depth is varied. (a) Absorbent (rubber) bottom, $430 \mathrm{kcps}$, (b) soft bottom, and (c) hard bottom.

sound intensity with very small increases in depth have an important bearing on sound observations in tidal water where the sea level may vary over a range of 10 to $15 \mathrm{ft}$ with the tidal changes. As we have seen, on the model scale a change of depth of the order of a millimeter, corresponding to a meter at full scale, may result in a change of $30 \mathrm{db}$ in sound intensity at a receiver.

\subsection{VISUAL OBSERVATION OF SOUND DISTRIBUTION ON THE BOTTOM AND SURFACE}

The small-scale observations made so far can only be regarded as preliminary and qualitative. A few general pointers have been established regarding the nature of the bottom and depth of water relative to wavelength. The one out standing feature, however, is the great difficulty of forming a mental image of the general sound distribution in the water. It has become obvious that a very large number of bottom-to-surface records taken at very short range intervals 
would be necessary to produce in this way a complete picture of the sound field in a vertical plane along the midline of the 20 -ft steel tank. Such a large number of records would take a long time to produce and many disturbing changes could possibly occur during that time, making the resulting very complicated picture untrustworthy. From such considerations as these, the incentive has arisen to find a method which will give a complete and permanent picture of the sound field in the water in the shortest possible time. As a first step in this direction experiments were made to obtain a picture of the sound distribution on the bottom, in the hope that a satisfactory technique for this might give a lead to its application in midwater.

After trying various chemical methods using gelatine-coated plates soaked in dyes and adding bleach to the water in the tank, a much simpler and more effective method was ultimately employed. In this method, and working in the small concrete tank, a false bottom of plate glass or metal sheet is used. This is first sprayed with a thin coat of "water paint" (e.g., distemper, walpamur, etc.) and allowed to dry, but not so long as to set or become bone-dry. The glass plate is then placed in the required position in the water and exposed for a few minutes, sometimes less, to the sound. A quartz transmitter of frequency $250 \mathrm{kcps}$ is

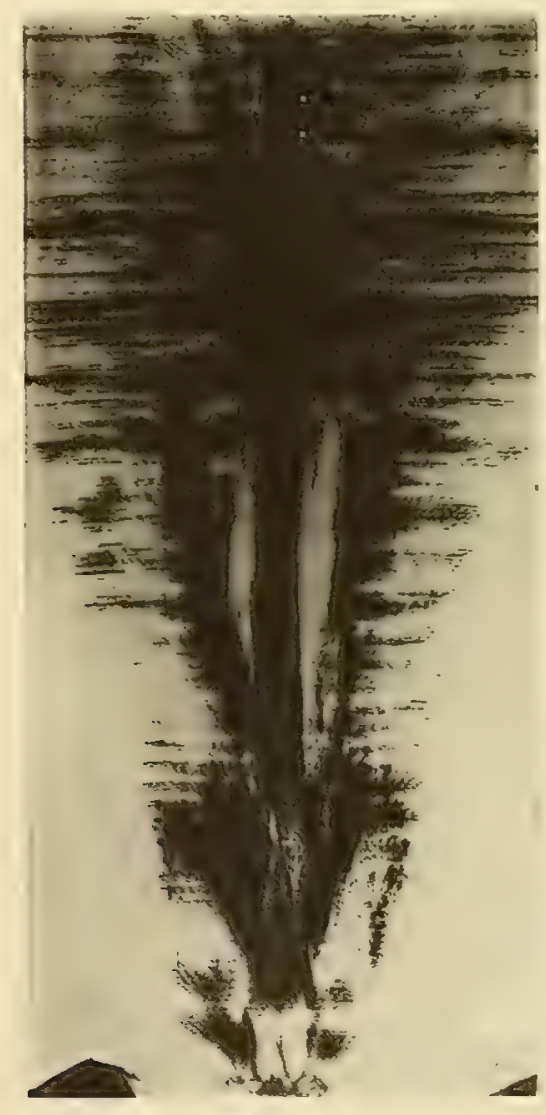

Fig. 10.12. Sound pattern on a hard sloping bottom. 
used, emitting sound energy at the rate of about $5 \mathrm{w}$ (estimated). In a short time, with much less sound intensity than required by the chemical methods, a pattern appears on the surface of the coated glass or metal plate. The most striking results were noticed when one end of the plate rested on the concrete bottom of the tank while the other, remote edge was arranged to be fairly close to the water surface. In this arrangement with a hard sloping bottom, the water forms a wedge above the glass plate. A typical bottom picture obtained in this way is shown in Fig. 10.12. In addition to the primary and secondary beams from the transmitter, parallel interference lines can be seen at right angles to the axis of the primary beam. The more closely spaced equidistant interference lines are due to stationary waves a half-wavelength apart. Attempts made to obtain patterns of the longitudinal (horizontal and vertical) cross sections of the sound beam were not very successful. With certain illuminations of the water surface, however, it was observed that a stationary pattern of parallel lines was visible on the surface of the water. As the angle of the wedge formed by the bottom plate and the surface of the water is increased, these interference lines on the surface get closer together and when the angle of tilt is small the lines are widely spaced. The interference lines occur at intervals corresponding to half-wave increases of water depth and are analogous to the case of Newton's interference rings in optics. The observations on model scale just described apply, of course, to the full-scale case in shallow water where the sea bed is sloping. A variable slope in two dimensions could result in the acoustical equivalent of an oil-film optical interference pattern.

\section{4. "PICTURE" RECORDS OF SOUND, DISTRIBUTION IN VERTICAL CROSS SECTIONS OF "OPEN" WATER - SCANNING}

In what follows a description will be given of a method of recording a "picture" of the sound field in the body of free undisturbed water, with sound of relatively low intensity. As a first step, with this aim in view, a series of cathode-ray oscillographic records were made, using point transducers (as described in Section 10.1 above) to show the distribution of pressure amplitude (a) in a vertical plane and (b) in a horizontal plane along the midline of the tank between the ranges 1 and $2 \mathrm{~m}$ ( $\mathrm{km}$, full scale) from a point transmitter at a fixed depth. In the first series (a) the point receiver travelled on the midline of the tank on parallel courses displaced vertically at 0.1 -in. intervals from near surface to near bottom. These records laid closely together are shown in Fig. 10.13. It will be seen that progressive changes with depth are revealed. A characteristic "V" or "Diamond" structure of the sound field is indicated. (This should be compared with later records made by the method to be described below.) In the second series (b) the parallel courses were in the same horizontal plane in midwater, these courses being spaced horizontally $1 \mathrm{~cm}$ apart up and to +10 and $-30 \mathrm{~cm}$ on each side of the midline. The resulting series of records shows no significant changes from one to another, the sound amplitude on any of the courses being the same as on any other.

As a means of delineating a picture of the sound field in a cross section of the water, the following method has given very encouraging results and is much simpler and more efiective than the one just described. The new method is es- 


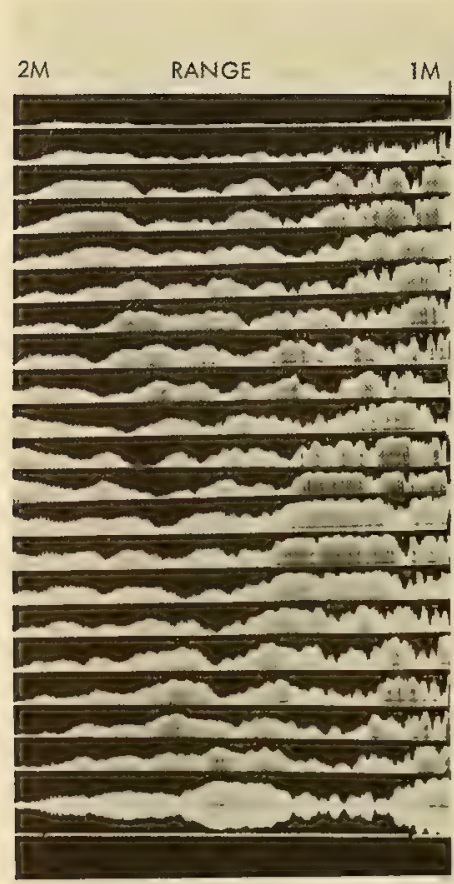

(c)

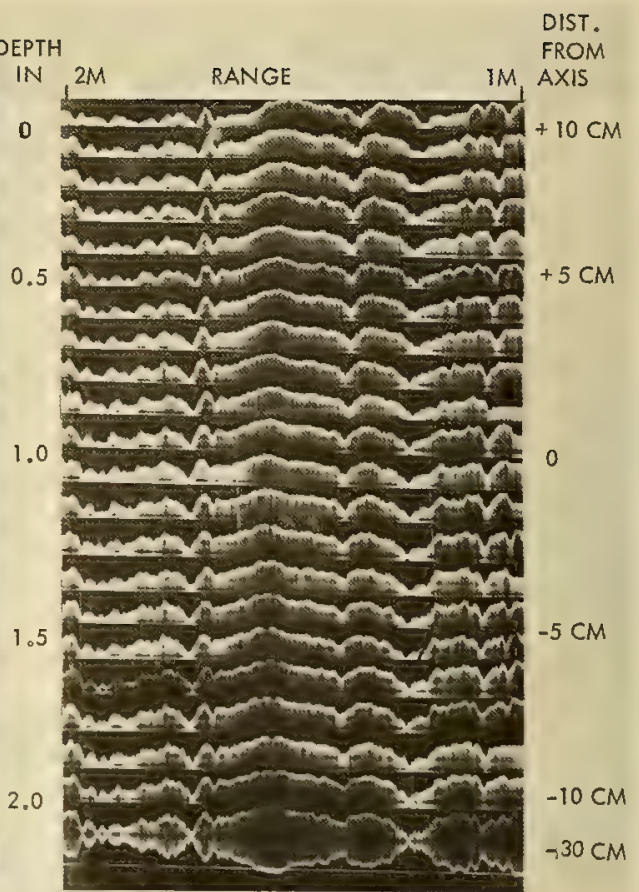

(b)

Fig. 10.13. Sound distribution in vertical cross sections over a range of one to two meters from the projector. (a) Each scan is made with the transducer at a different depth, the top is at the surface, and each succeeding scan is made at an interval of depth of 0.1 in. (b) Each scan is made at constant depth on a line parallel to the axis of the projector, the center scan is on the axis, the top scan is $10 \mathrm{~cm}$ on one side, the next-to-bottom scan is $10 \mathrm{~cm}$ on the opposite side, and the bottom scan is $30 \mathrm{~cm}$ from the axis.

sentially a scanning technique which gives a complete picture on a single record of the sound distribution in a vertical cross section of the water either along the full length of the tank or across it at selected ranges. In this method, one of the transducers, either the transmitter or the receiver, is mounted on the scanning mechanism shown in Fig. 10.14. A metal plate to which it is attached is driven up and down vertically between smooth guides by means of a rotating arm of adjustable length so that its double amplitude of motion in a vertical direction is equal to the depth of the water. The repetition rate at which the transducer moves between the surface and the bottom is under control, an average speed being approximately three times per second in each direction. The motion is adjusted so that the transducer point does not break the water surface nor strike the bottom of the tank. Experiments have shown that the result is the same whether the "scanning" transducer is the transmitter or the receiver, or whether the scanning mechanism is mounted on the fixed platform near one end of the tank or on the moving platform (trolley) which travels along the rails at the side of the tank. A chain passing over a sprocket wheel connects the rotating arm to the sliding plate which supports the transducer, and the shaft of the sprocket wheel drives the brush contact of a potentiometer which is supplied by a $3-v$ battery. The voltage on the brush is amplified and applied to the $Y$ plates of the cathode ray 


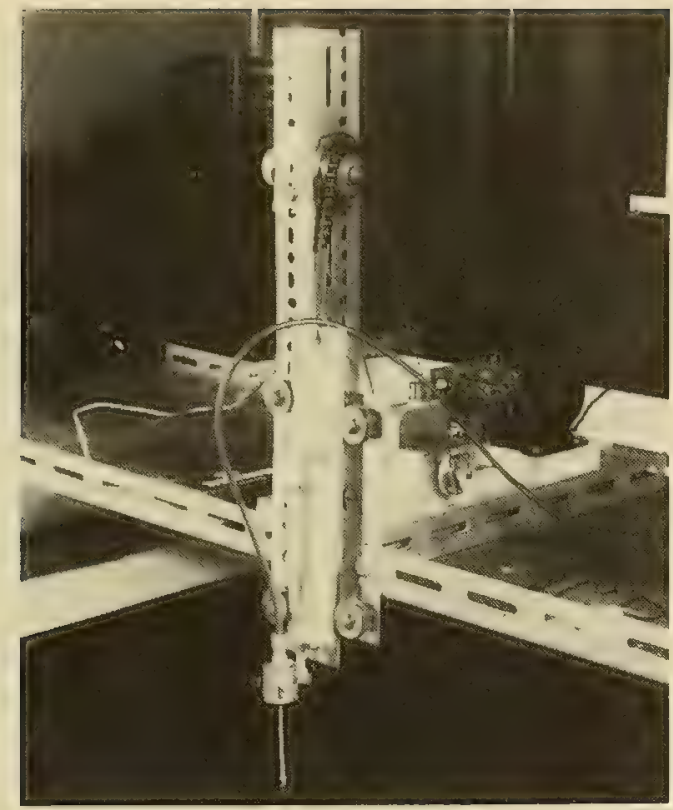

Fig. 10.14. Scanning mechanism.

oscillograph, causing the spot to move up and down on the screen in synchronism and in phase with the transducer point in the water. The received signal is used to modulate the brightness of the cathode ray spot after suitable amplification by a preamplifier and a three-stage tuned amplifier. The hand-operated brightness control of the CRO is adjusted until the spot is barely visible at the surface and bottom turning points when no sound is being transmitted. When the transmitter is switched on, the cathode-ray spot moving up and down on a vertical line on the screen appears as a series of irregularly spaced bright dots and/or dashes due to the fluctuations of sound intensity as the scanning transducer moves between the surface and the bottom of the water. If now in addition the trolley carrying the receiver moves along the tank, the arrangement of bright and dark spots on the CRO changes according to the position of the receiver. Consequently, if a record is made by a film moving slowly in a direction at right angles to the line of dots on the CRO screen, while the trolley moves from one end of the tank to the other, a continuous picture is recorded of the whole sound pattern in a vertical section along the tank. It is important, however, if a more or less uniform density of pattern is required, to compensate for the general decrease of sound intensity with range, due to spreading and attenuation in the water and by reflection at the bottom. This is achieved automatically by means of resistances shunting the output of the receiver preamplifier, one of which is automatically varied by the moving trolley and the other preadjusted by hand according to the circumstances pertaining when a record is to be made.

Using barium titanate point transducers, good records can be made by the method described above when the transmitting voltage is as low as one volt. The sound intensity in the water in this case is of course very low indeed compared with that which was used to produce the bottom records in Fig. 10.12. 
With this new technique numerous records have been made in a study of sound propagation in shallow water. With either the mode or ray theories of propagation, where interference between direct and reflected sound is the main feature, the important influences which must be studied are the depth of the water, the wavelength of the sound, and the ratio of these two quantities; the depth of the source of sound; and the nature of the bottom. Other factors which have already been mentioned, such as temperature effects and state of sea surface and bottom, must of course also be considered, but for the present it will be sufficient to deal with these main considerations. In the earlier experiments which were described at a meeting of the Acoustical Society of America at Chicago in November 1958 [9], records were made using the sheet steel bottom of the model tank as representative of a flat, acoustically hard, reflecting bottom, and when covered with sheet rubber, as an acoustically absorbent or relatively poor reflecting bottom. The records obtained, particularly those on the hard steel bottom, were very complicated and it was thought at the time that this was partly due to the fact that the steel bottom was not sufficiently flat, irregularities in some parts amounting to as much as 2 or $3 \mathrm{~mm}$, i.e., comparable with the wavelengths of the sound. Since then the bottom has been covered with plate glass $1 / 4$ in. thick of good quality, carefully leveled by ebonite wedges around the edges and made parallel to the water surface by the use of a number of metal cones accurately turned to have heights equal to the water depths required (viz., $2,1,3 / 4$, and $1 / 2$ in.), the tip of each cone just touching the underside of the water surface. Care was taken to ensure that the space between the plate glass and the steel bottom was water-filled and free from air bubbles. Before making records the water was well stirred, by dragging a long "comb" through it, to ensure isothermal conditions.

Scan records have been made covering a wide variety of conditions affecting the propagation of sound in shallow water. As in the point-by-point technique, it was found in the early stages of the investigation that there was a very marked contrast in the sound fields according to the nature of the bottom (i.e., acoustically hard and a good reflector like steel or rock, or a poor reflector like rubber or mud and sand). Consequently, when other factors influencing propagation have been examined, e.g., depth of water, wavelength (frequency) of sound, directional properties of transmitter, depth of transmitter, and so on, these have been considered in relation to the nature of the bottom, reflecting or absorbent. The "picture" records of sound fields to which reference is made later therefore generally relate to the variable factor considered as it applies to the two bottom types just mentioned. It will be necessary therefore to refer to the same records in different connections.

Most of the illustrations of picture records of sound fields were made with a point (omidirectional) transmitter, but a few are reproduced to show the markedly different characteristics when a directional transmitter is used. The scanning receiver is a point in all cases. Records have been made showing longitudinal vertical cross sections of the sound fields extending to the full length of the model tank, and of transverse vertical sections at a series of ranges from the transmitter. A simple method has been found useful for the intensity calibration of records by the introduction of a series of known db steps in the transmission voltage or in the circuit of the receiver. 


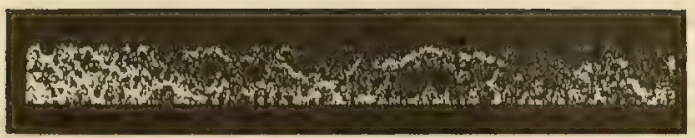

(1) $640 \mathrm{KC}, 24 \mathrm{~V}$. ON TRANSMITTER

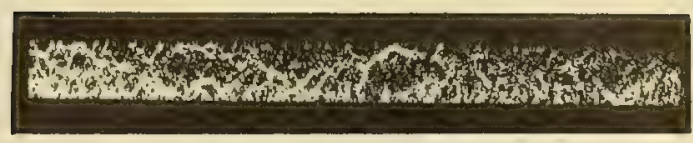

(2) $600 \mathrm{KC}, 2.5 \mathrm{~V}$. ON TRANSMITTER

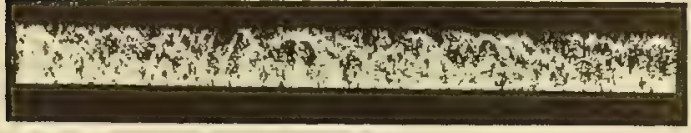

(3) $500 \mathrm{KC}, 5.5 \mathrm{~V}$. ON TRANSMITTER

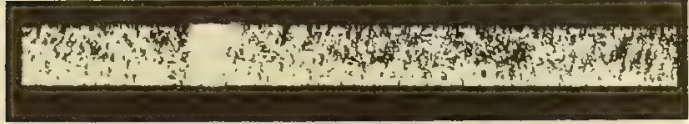

(4) $400 \mathrm{KC}$

Fig, 10.16. Transverse vertical scan records at $1-, 2-, 3-$, and 4-m range. The bottom is steel, water is 2 in. deep, and the frequency is $440 \mathrm{kcps}$.
Fig. 10.15. Vertical scan over the range from 0.6 to $4.3 \mathrm{~m}$ at frequencies of 640 , 600,500 , and $400 \mathrm{kcps}$. Water is two inches deep and the bottom is steel.

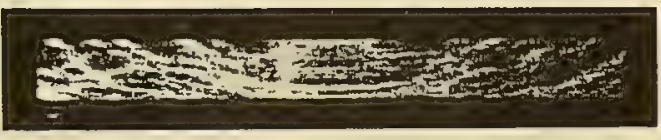

$d=1$ METER

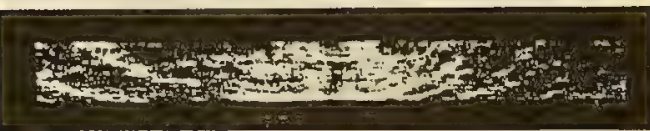

$d=2$ METERS

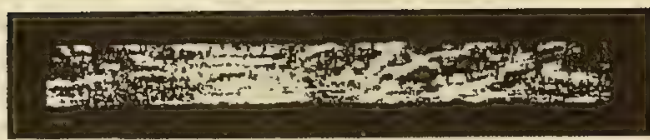

$d=3$ METERS

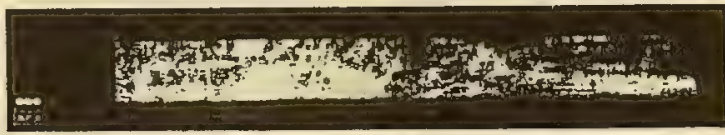

$d=4$ METERS 


\subsubsection{Nature of Bottom}

Reference was made in Section 10.2.3 above to the use on the model scale of sheet steel or plate glass as equivalent to solid rock on the full scale-supported by observations in a model tank having a more realistic thick concrete bottom. Similarly it was found that a sheet of rubber $1 / 8 \mathrm{in}$. thick covering the bottom of the tank had an effect comparable to a layer of fine sand. In what follows, therefore, steel, concrete, and plate glass bottoms are regarded as equivalent to rock and as good reflectors of the incident sound in the frequency range under consideration, while a rubber sheet covering these surfaces corresponds to a layer of mud or sand under full-scale conditions.

When the bottom is acoustically hard (equivalent to rock), and the source is a point, the average scan line of a record consists of, say, 10 or 12 small dots indicating sound-pressure maxima. This confirms the observations made by the oscillographic amplitude-recording techniques described in Section 10.2 above. When the bottom is steel sheet $(1 / 8-i n$.$) , the sound picture as a whole is$ very complex (see Fig. 10.15), particularly when the depth-to-wavelength ratio is large, e.g., around 20 to 1 . If, however, the bottom is plate glass carefully leveled parallel to the water surface, there is more evidence of regularity in the pattern, a characteristic V pattern being noticeable. There is considerable indication in these hard-bottom records of the propagation of the higher modes, or of many reflections between surface and bottom if we regard the indications in the light of the ray theory. Transverse records at ranges of $1,2,3$, and $4 \mathrm{~m}$, in water $2 \mathrm{in}$. deep at a frequency of $440 \mathrm{kcps}$ with a point transmitter and bottom of steel are shown in Fig. 10.16. These show clear evidence of a stratified pattern in the sound field with many maxima in a scan.

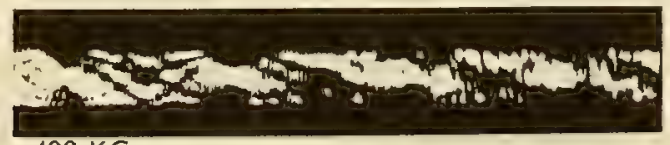

$400 \mathrm{KC}$

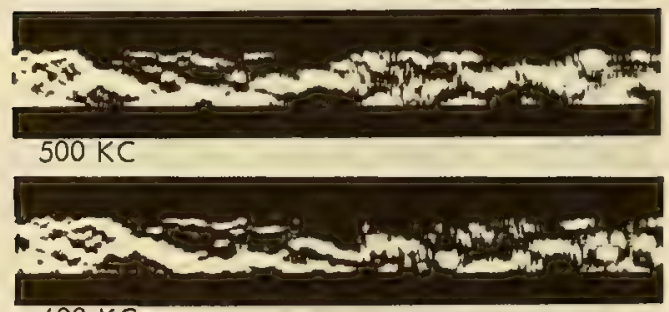

$600 \mathrm{KC}$

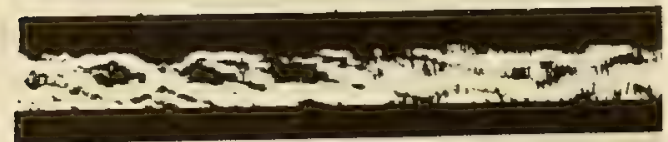

$640 \mathrm{KC}$

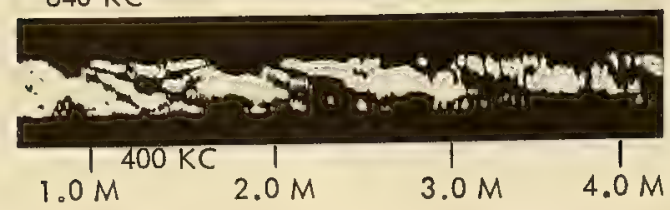

Fig. 10.17. Vertical scan between 0.6 and $4.3 \mathrm{~m}$ at various frequencies. Water is 2 in. deep and the bottom rubber-covered. 


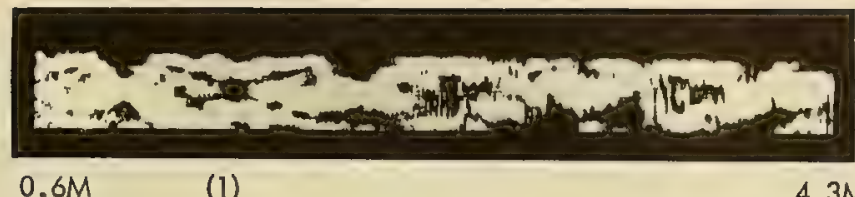

$0.6 \mathrm{M}$

$4.3 \mathrm{M}$

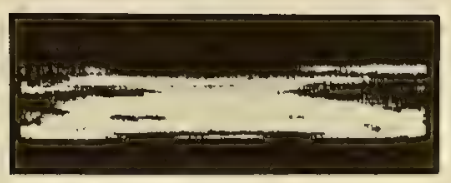

(2) RANGE IM

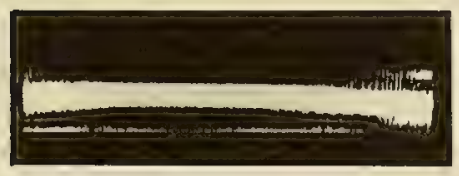

(3) RANGE $2 M$

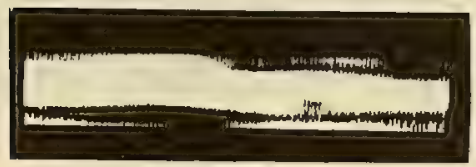

(4) RANGE $3 M$
Fig. 10.18. Longitudinal vertical scan and three transverse vertical scans at 1-, 2-, and 3-m range. Frequency is 450 keps, the water $2 \mathrm{in.}$ deep, and the bottom is rubber-covered.

When the bottom is acoustically absorbent, rubber-covered (equivalent to mud and sand), and the source is an omnidirectional point, the picture of the sound field is entirely changed. There are now fewer maxima in each scan line-perhaps only two or three dots (or dashes). The over-all pattern is much simpler, but for depth-to-wavelength ratios around $20 / 1$ is still somewhat complicated. Examples of such records are shown in Fig. 10.17. The effect of the sound-absorbent or poorly reflecting bottom is to reduce the number of surface-to-bottom reflections and some of the higher modes are absent. Figure 10.18 shows a longitudinal scan over a rubber bottom, and three transverse scans at ranges of 1,2 , and $3 \mathrm{~m}$. The reduced number of maxima is strikingly shown in these records also.

Picture records of sound fields such as these serve to show the weakness of the point-by-point method where it was impracticable to obtain more than a small proportion of the data required to plot the complete sound field. By the scanning method the complete picture can be recorded in a matter of a few minutes.

\subsubsection{Depth of Woter}

As already pointed out, the effect of varying the depth of the water must always be considered in relation to the wavelength of the sound. The nature of the bottom plays an important part, and the record obtained depends also on the depth of the transmitter.

Many picture records have been obtained in which these factors have been varied. In Figs. $10.19,10.20$, and 10.21 are shown three sets of records, the water 

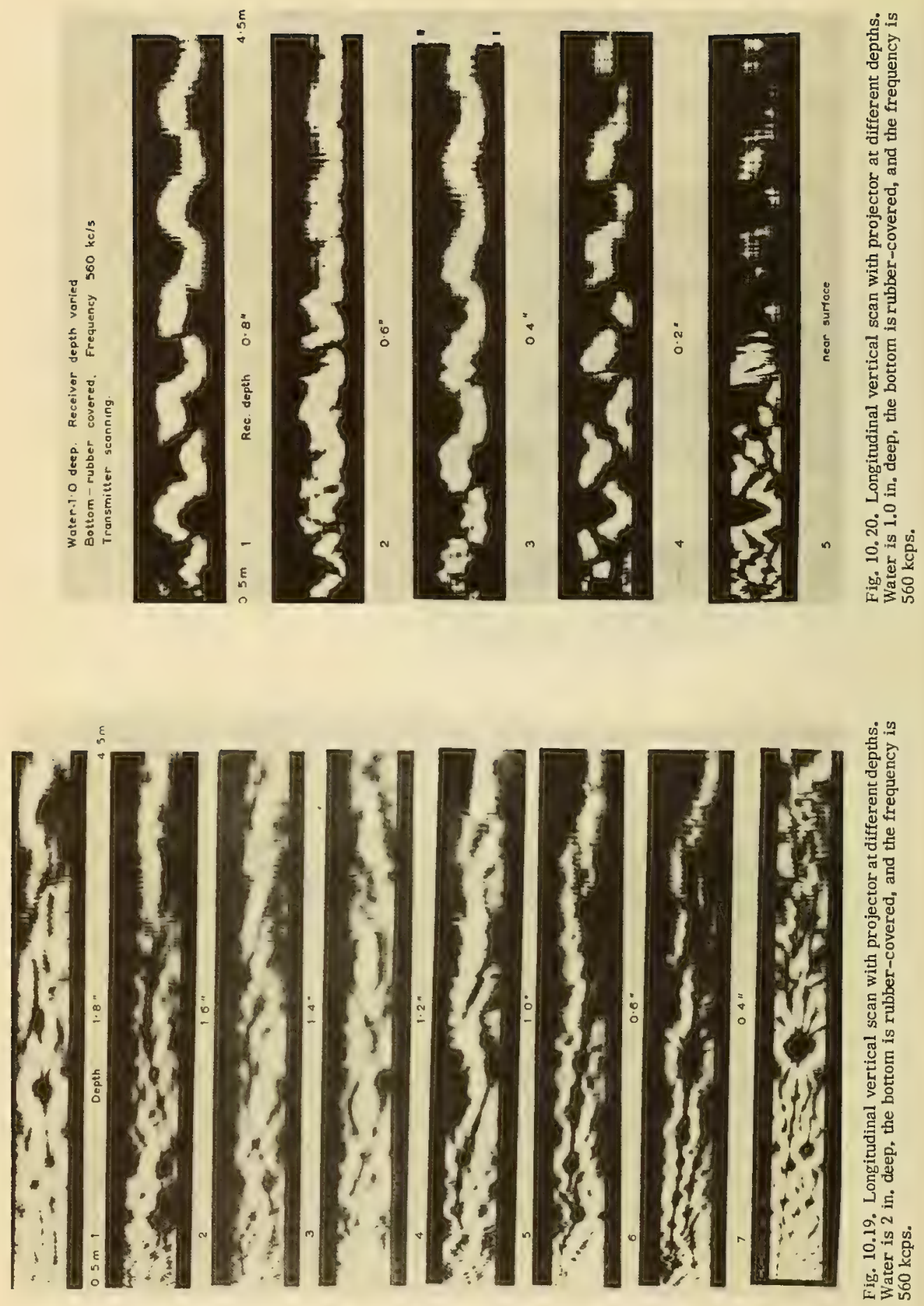

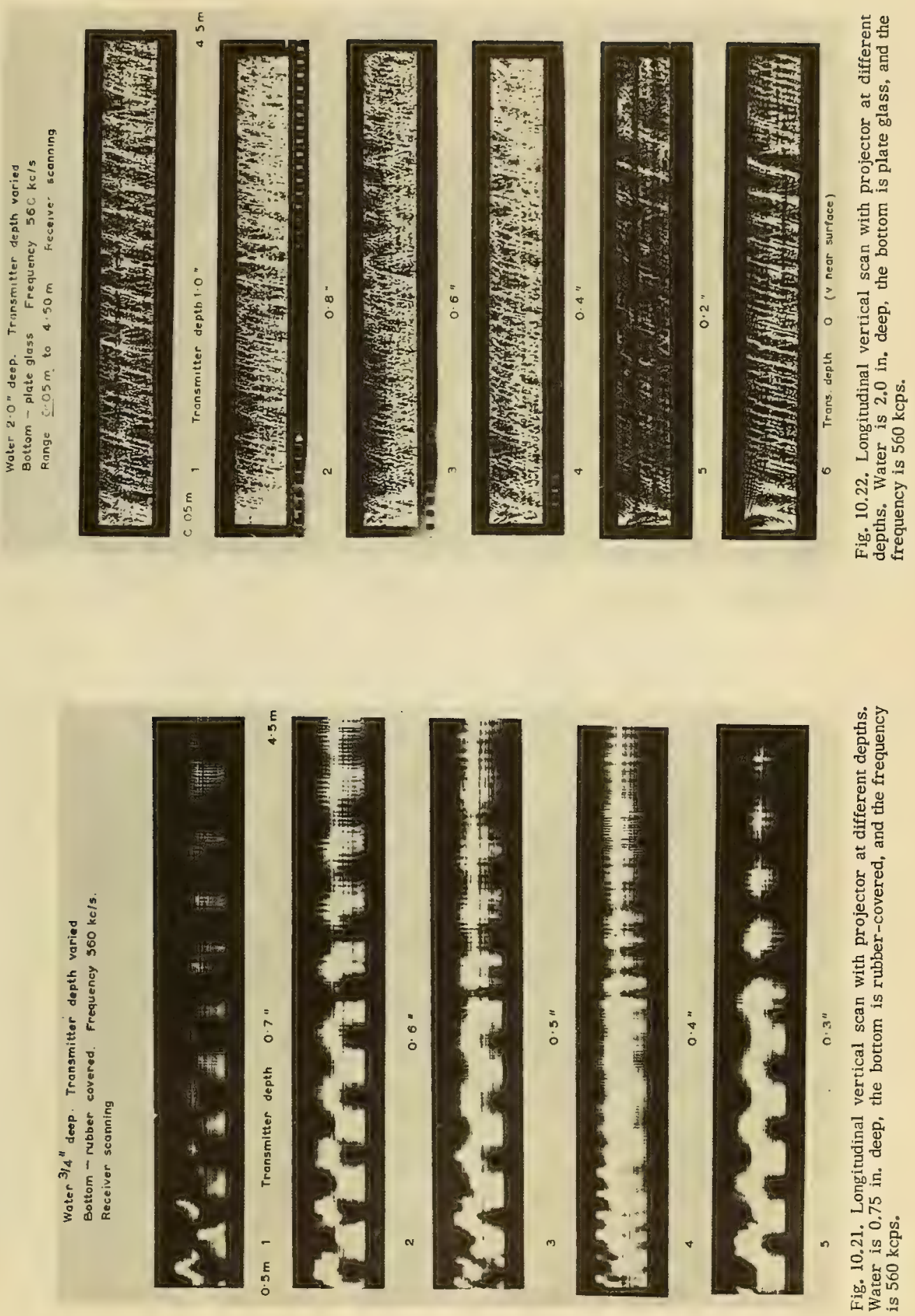

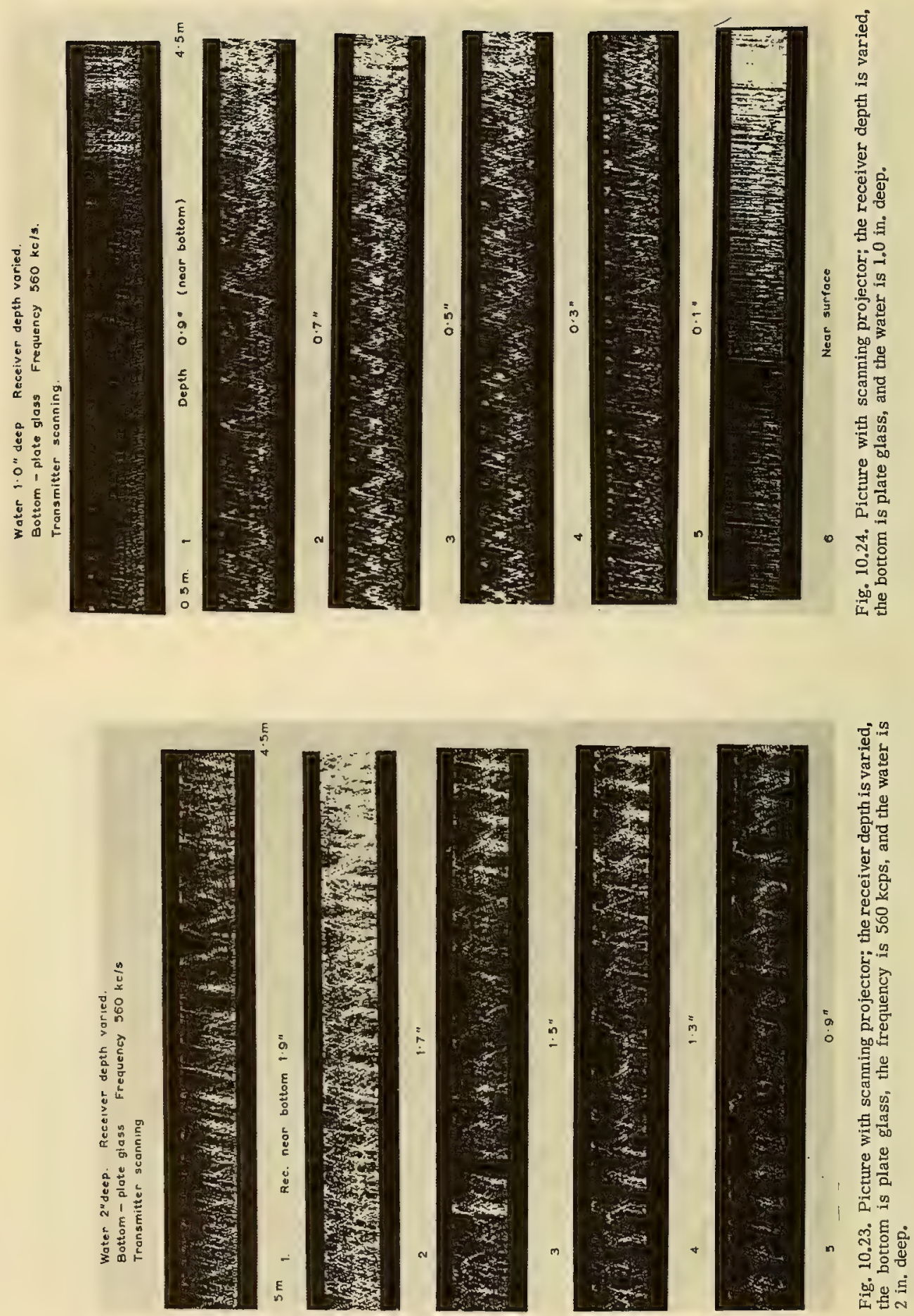


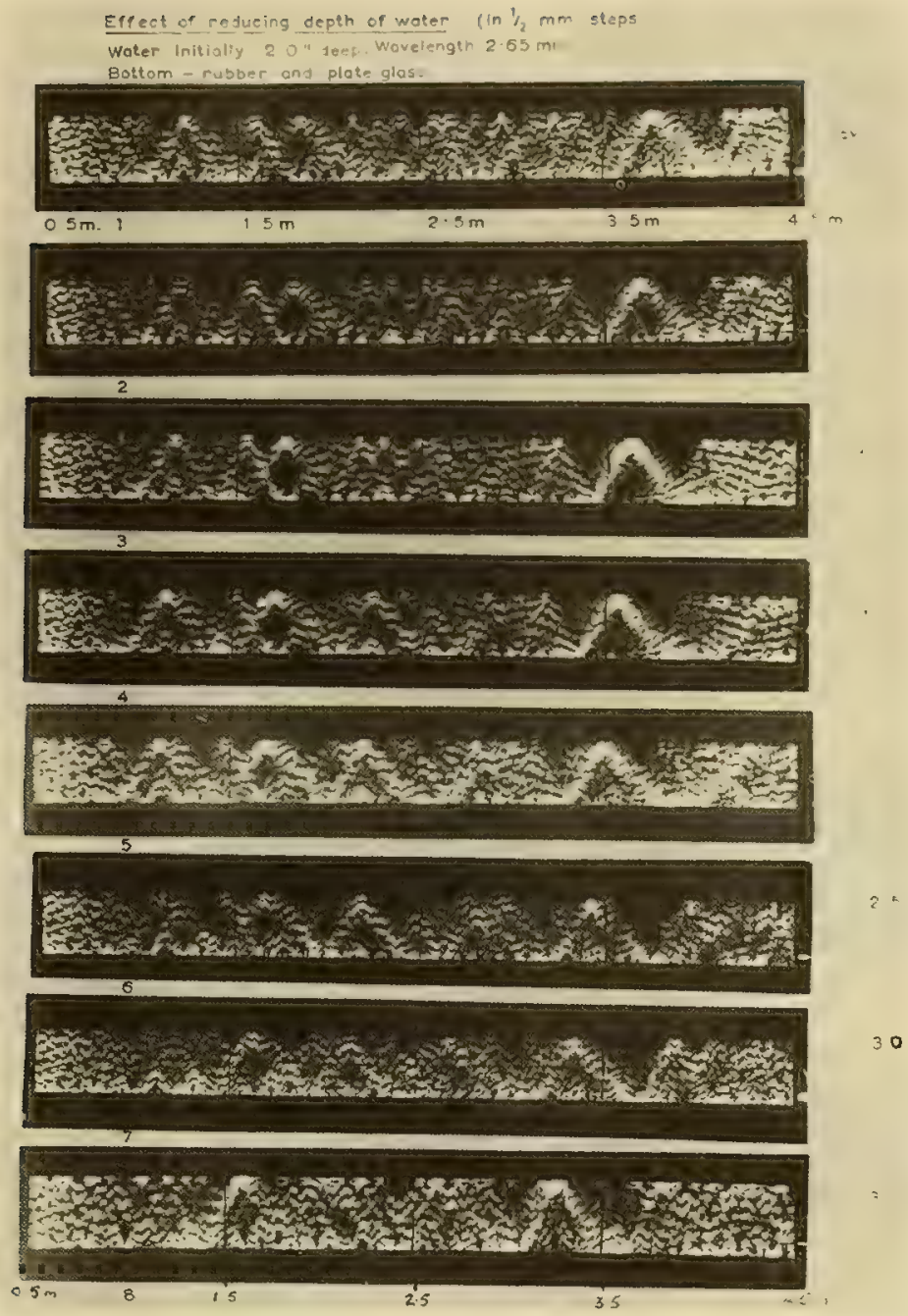

Fig. 10.25. Picture showing the effect of small changes in depth of water. The top picture is for $50 \mathrm{~mm}$ depth, and successive pictures from top to bottom made at decrements of $0.5 \mathrm{~mm}$. The bottom is plate glass and the frequency is $560 \mathrm{kcps}$.

depths being $2.0,1.0$, and 0.75 in., respectively, when the bottom was rubber covering plate glass on steel. The frequency in this case was $560 \mathrm{kcps}$ and the wavelength $2.65 \mathrm{~mm}$, approximately. Compared with the pattern over a plate glass or steel bottom the 2 -in.-depth record over rubber is comparatively simple, but it is nevertheless much more complicated than the records in shallower water, the ratio of depth-to-wavelength in the three cases being roughly 20,15 , and 10 . The relatively simple "corrugated" structure of the records in the last two cases is striking and is at present the subject of a mathematical analysis. In another series of records also at $560 \mathrm{kcps}$ the bottom is plate glass overlying the steel of the tank, the depth of water being 2 and 1 in. The charac- 
teristic $V$ pattern of the hard bottom is evident in the deeper water. In the records shown in Figs. 10.22 and 10.23, the water is in both cases 2 in. deep and the bottom plate glass. In the first series (Fig. 10.22), the records cover the range of depth from midwater to surface, while in the second series they extend from bottom to midwater. It should be noted that in the first series the range extends from 0.05 to $4.5 \mathrm{~m}$, and the last record (transmitter near the surface) shows Lloyd's fringes very clearly at the short ranges. In the shallower water record (1 in. deep) shown in Fig. 10.24, the pattern is still complex but shows signs of more regularity than that in the deeper (2 in.) water.

It is interesting to note the changes in the pattern when s mal1 depth changes are made. This is shown in Fig. 10.25, where records have been made in water of depth $50 \mathrm{~mm}$ reduced by steps of $1 / 2 \mathrm{~mm}$. If a particular feature of the records is selected, for example the inverted $V$ near the $3.5-\mathrm{m}$ mark on the records, it will be observed that this moves slightly to the left with each $1 / 2-m m$ decrease of depth. Similar drifts to the left are noticeable on other outstanding features, the drift decreasing with decrease of range from the transmitter. A comparable effect is seen in Fig. 10.26, where the wavelength (frequency) is varied in steps. Such records emphasize the relationship which must exist between depth and wavelength in delineation of the sound field.

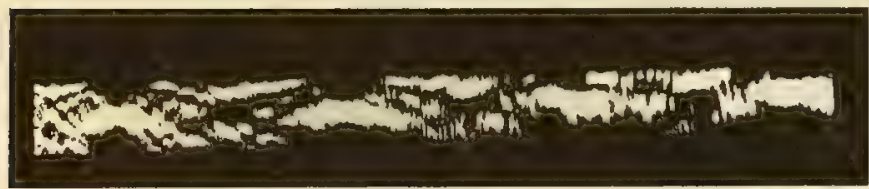
$0.6 \mathrm{M}$
(1) $450 \mathrm{KC}$
4. $3 \mathrm{M}$

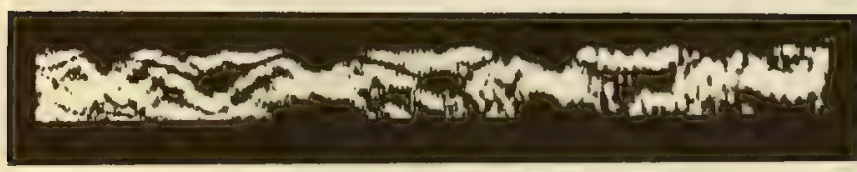

Fig. 10.26. Picture showing the effect of small frequency variations. Water 2.0 in. deep, the bottom is (2) $425 \mathrm{KC}$ rubber-covered, and the frequency is $560 \mathrm{kcps}$.

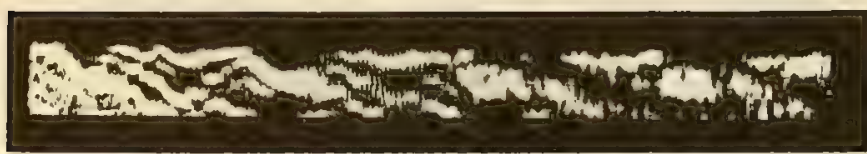

(3) $400 \mathrm{KC}$

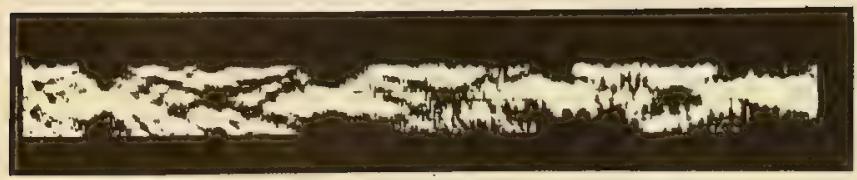

(4) $450 \mathrm{KC}$ 


\subsubsection{Wovelength of Sound}

Assuming the velocity of sound in the water is constant, variation of frequency will result in a corresponding variation of wavelength of the sound. Many of the earliest picture scan records [9] showed the effect of varying frequency, e.g. in steps of $100 \mathrm{kcps}$ over a range 640 to $400 \mathrm{kcps}$. Such records, particularly those made over a rubber (sound-absorbent) bottom, showed a marked similarity in general appearance, but it was difficult to trace the transition from one frequency to the next in the series (see Fig. 10.17). When the frequency steps are smaller however, e.g., 400,425, $450 \mathrm{kcps}$ as in records shown in Fig. 10.26, the change in position on the record of certain clearly marked features can easily be observed. These changes are comparable to those of Section 10.4.2 where the results of small depth changes were discussed.

\subsubsection{Temperature of Water}

The question of the temperature of the water, isothermal and with gradients, was discussed briefly in Section 10.2.5. Many bottom-to-surface records were made by the point-by-point oscillographic technique which showed that small temperature changes had the same effect as small changes of frequency. Thus, around $400 \mathrm{kcps}$ a change in temperature of $3.5^{\circ} \mathrm{C}$ could be expected to produce the same effect as a change of frequency of $3.2 \mathrm{kcps}$ (i.e., $1^{\circ} \mathrm{C}$ is approximately equivalent to a 1 -kcps change of frequency at $400 \mathrm{kcps}$ ). Variation of temperature in the water results in a change of velocity of propagation of the sound and, the irequency being assumed constant, is just another way of varying the wavelength. The frequency (wavelength) effects shown in Fig. 10.26 should therefore be reproducible by a sufficient change of temperature of the water.

\subsubsection{Directional Transmission}

In all the scan records mentioned hitherto, the transmitter has been of the omnidirectional type permitting the propagation of modes or rays in almost all directions. When the transmitter is directional, however, relatively few modes are propagated and the scan picture of the sound field along the model tank becomes much simpler. A series of four such records, using a $2 \mathrm{-cm}$-diameter transmitter at a frequency of $560 \mathrm{kcps}$ (semiangle of primary beam, $9.3^{\circ}$ ) in water $5 \mathrm{~cm}$ deep, is shown in Fig. 10.27. In record 1 the bottom is plate glass and the directional transmitter is in midwater. It will be seen that this record resembles that of a point source transmitting over a rubber (mud) bottom (see for example Fig. 10.19) rather than that over plate glass (rock). In both cases, of course, the higher modes are suppressed, but in different ways. In records 2,3 , and 4 the plate-glass bottom is rubber-covered, the directional transmitter being in midwater, near bottom and near surface, respectively. Changes in pattern due to these changes in depth of transmitter are apparent, but the general character of the record is much the same with all three cases, and indeed with that of record 1 when the bottom was plate glass.

In Fig. 10.28 the directional characteristics of a transmitter are shown at $1-m$ range (a) by rotating the transmitter, the point receiver scanning vertically at 1-m range, and (b) by rotating the transmitter, the receiver at $1-\mathrm{m}$ being fixed in midwater. Method (b) is of course the conventional method of recording beam characteristics. The record in (b) represents the intensity variations 
5OUMB FIELO OF OMECTIOHAL TRAMSMITTEA IH SHALLOW WATER

TTransmitter $2.0 \mathrm{ems}$ did. $\theta=9.30$ Frequeney $560 \mathrm{hc} / \mathrm{s}$

Woter Sems. deep.?

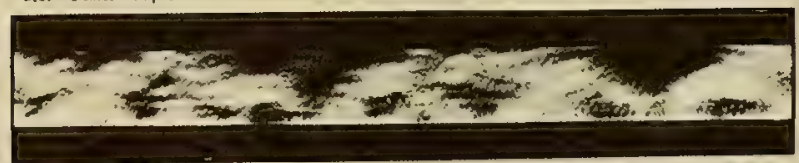

1. Bottom plote glass

ironsmitter in mid-woter.

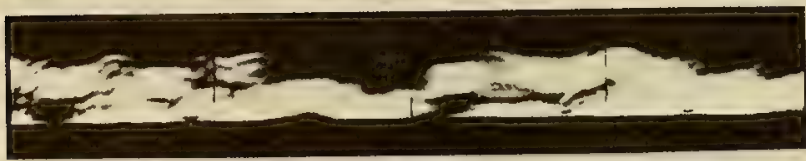

2. Sottom rubber covereo

Transmitter in mid-water.

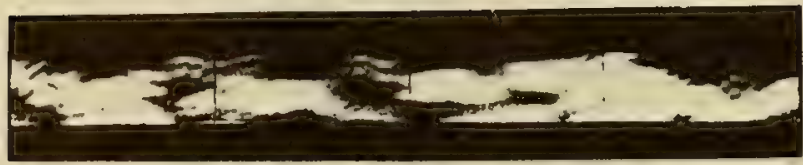

3. Bottom rubber conered

ironsmilter aeor boltom

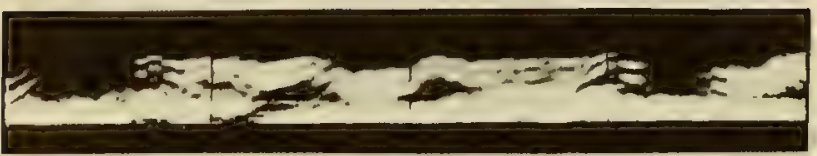

4. Boltom rubbep covered

isansmitter neor surlace
Fig. 10.27. Picture made with a directional transmitter. The bottom is plate glass in the top picture and rubber-covered in others. Water 2 in, deep.
Fig. 10.28. Directional characteristics of projector. (a) Vertical scan record. (b) Pressure amplitude record at midwater depth.

\section{OIREGTIOHAL CHARACIERISIICS}

of Ba Ti tronsmitter $12 \mathrm{cms}$. dio.l

|Tronsmitter sototing uniform $|y|$

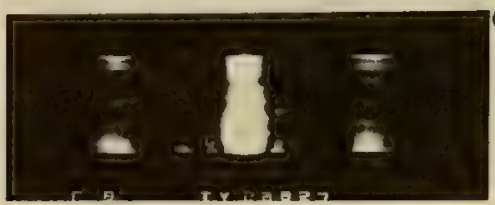

(a)

Point Receiver

sconning verticolly ot 1 melre ronge.

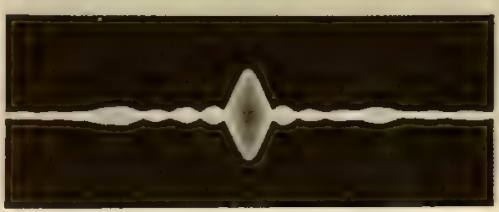

(b)

Point Receiver lixed in mid-water. Sound pressure amplitude recorded. 
shown by a horizontal midwater line of the scan record (a). The latter shows not only this, but the whole pattern of sound distribution between surface and bottom of the water layer. The stratified character of the transmitted beam and their relative intensities and angular spacings are clearly shown in the original scan records. Figure 10.29 shows a series of nine scan records, the first eight of which show sound fields in vertical sections across the tank at various ranges between 5 and $400 \mathrm{~cm}$ from a $2 \mathrm{~cm}$-diameter barium titanate transmitter operating at a frequency of $568 \mathrm{kcps}$, the semiangle of beam in this case being $9.3^{\circ}$, approximately. The water depth was 2 in. and the bottom rubbercovered. The last (9th) record shows a scan picture along the axis of the primary beam from one end of the tank range ( 5 to $400 \mathrm{~cm}$ ) to the other. It is of interest to identify the transverse section scans at the corresponding ranges of the longitudinal scan. The eight transverse records show very clearly the spreading of the primary beam and its tendency to stratification in horizontal planes, varying in spacing between the layers according to range from the transmitter. While it is appreciated that the directional transmitters used in these experi-

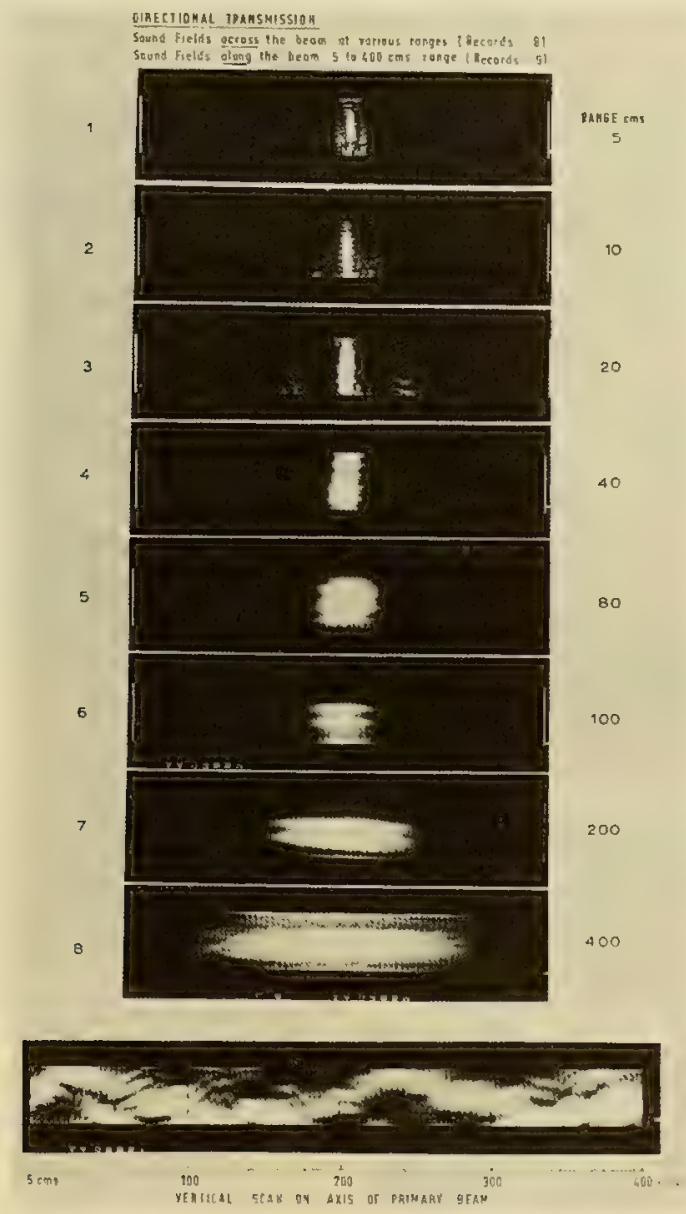

Fig. 10.29. Sound fields across the beam of the directional projector at various ranges. 
ments have excessively large diameters on the scale under consideration, they have proved very useful in providing known directional characteristics for certain propagation studies.

\subsubsection{Calibration of Scan Pictures of Sound Fields}

The recorded sound fields illustrating this paper show light and shade patterns representing the variations of sound intensity in vertical cross sections of the water. The areas of high intensity are seen as white (bright) spots while those of very low intensity appear dark on the records. There are two possible sources of error in regarding the brightness of the spots as a measure of sound intensity: (1) the motion of the scanningtransducer is SHM, and as a consequence undue emphasis is given to the brightness of the CRO spot and to the photographic exposure at the turning points of the motion near the surface and bottom of the water layer, and (2) there is also a possibility that the brightness modulation by the sound signal applied to the grid of the CRO may not be linear. In the method of intensity calibration to be described, these two unknowns are taken into account to some extent. The record brightness in this method is varied by introducing known changes in the transmitter voltage in $2 / 1(6-\mathrm{db})$ steps or by introducing a db attenuator in the receiver circuit. Typical records of such calibrations are shown in Fig. 10.30. The upper record shows a scan at constant range reduced in strength by $6-\mathrm{db}$ steps, the lower intensities being thereby gradually eliminated from the record. The two lower records show the effect of repeating the same longitudinal scan of the tank with a 6 -db reduction of signal strength in the second record. The predominant features and over-all character of the records of course remain the same.

\subsection{THEORETICAL WORK - FULL-SCALE TRIALS}

As indicated at the outset of this paper, the theoretical treatment of the complete problem of sound transmission in shallow water, even under idealized conditions, is very difficult. A brief reference was made to the work of C. L. Pekeris, particularly in regard to his development of the mode theory in 1948. More recently T.G. Weale [8] has dealt with the mathematical aspect of the
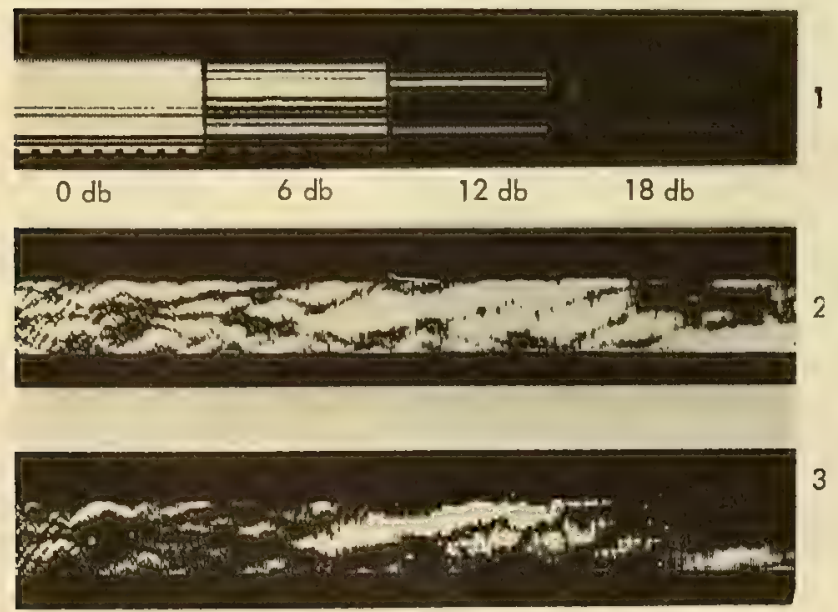

Fig. 10.30. Intensity calibration records. (1) Constant range intensity reduced in 6-db steps. (2) and (3) Longitudinal scan with a $6-d b$ reduction in record 3 . 


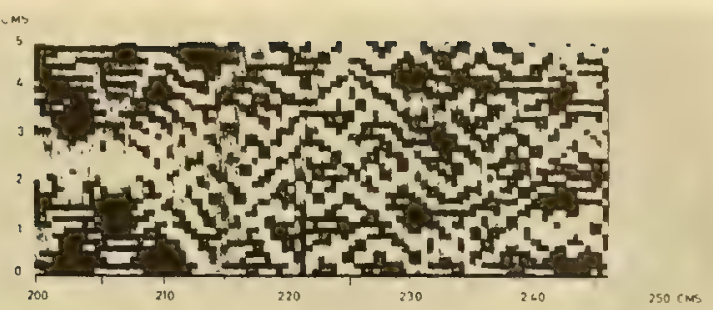

(a) Computed Chart (for Range $200-250 \mathrm{cms}$ ) (Mand Plotting)

ems

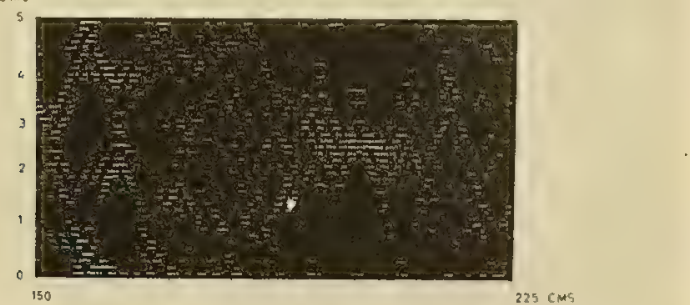

(b) Computed Chart (for Range $150-225 \mathrm{cms}$ )(Automatic Platting)
Fig. 10.31. Comparison of computed record with measured record. Water is $5.0 \mathrm{~cm}$ deep, the bottom is plate glass, and the frequency is $560 \mathrm{kcps}$.

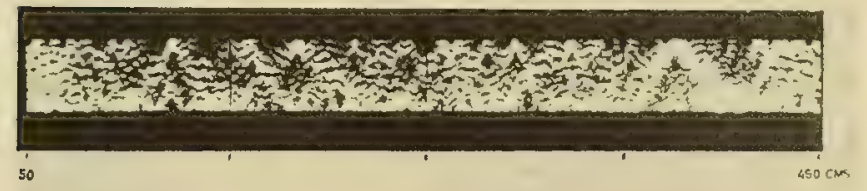

(c) Record made in Model Tank (for Ranqe $50-450 \mathrm{cms}$ )

problem and employing Hankel functions has derived expressions for the spatial distribution of sound intensity in a shallow water layer. Using Weale's theoretical results, K.W. Harrison has, by means of computer techniques, plotted the sound field in a part of a record actually made in the course of the experiments here described. The actual record and computed sound fields are shown in Fig. 10.31. In (a) the theoretical sound field is plotted by hand in the range irom 200 to 245 $\mathrm{cm}$ while in (b) the theoretical sound field is plotted automatically in the ranges from 150 to $225 \mathrm{~cm}$. The actual record obtained under the conditions assumed in the theoretical computation is shown in (c), covering the range from 50 to 450 $\mathrm{cm}$. The diamond and $\mathrm{V}$ structure of the recorded sound field is observable in all the records, and the slope of the $\mathrm{V}$ lines is approximately the same in the computed and recorded records when allowance is made for the relative scales of the $X$ and $Y$ axes in the various cases. In this record the bottom was plate glass partly covered by rubber (to remove some of the higher modes), the water was $5 \mathrm{~cm}$ deep, the frequency of the sound $560 \mathrm{kcps}$, and the transmitter situated about $2 \mathrm{~mm}$ above the bottom.

As a first attempt, this theoretical computation is very encouraging. It has, however, taken a long time and much effort to obtain, and there is clearly much more to do.

Full-scale trials are in preparation to try out the scanning technique in the sea. For this purpose suitable transducers and scanning equipment have to be designed and a suitable area chosen for the experiments. All this is taking much time and manpower, not to mention expense, all of which tends to emphasize the advantages of small-scale model experiments. 


\section{REFERENCES}

1. F. C. Johansen, "Research in Mechanical Engineering by Small Scale Apparatus," Proc. Inst. Mech. Engrs., 151-272 (1929).

2. R.W. Pohl, "Acoustics" and "Optics" (Springer Verlag, Göttingen).

3. D. E. Weston, "Moire Fringe Analogue of Sound Propagation in Shallow Water," J. Acoust. Soc. Am., Vol. 32 , No. 6, 647-654 (June, 1960).

4. A. B. Wood and F. B. Young, "On the Acoustic Disturbances Produced by Small Bodies in Plane Waves Transmitted Through Water with Speical Reference to the Single Plate Direction Finder," Proc. Roy. Soc. (London), Vol. A 100, 261-288 (1921).

5. F.H. Sanders and R.H. Stewart, "Image Interference in Calm, Near-Isothermal Water," Can. J. Phys., Vol. 32, 599-619 (1954).

6. R.W. Young, "Image Interference in the Presence of Refraction," J. Acoust. Soc. Am., Vol. 19, No. 1, 1-7 (January, 1947).

7. C. L. Pekeris, "Theory of Propagation of Explosive Sound in Shallow Water," Geo. Soc. Am., Mem. No. 27 (October, 1948).

8. T. G. Weale, unpublished theoretical paper, A.R.L., Report R5/Maths 2.26.

9. A. B. Wood, "Model Experiments on Propagation in Shallow Seas," J. Acoust, Soc. Am., Vol. 31, No. 9 , 1213-1235 (September, 1959). 


\title{
LECTURE 11
}

\section{NONSPECULAR SCATTERING OF UNDERWATER SOUND BY THE SEA SURFACE}

\author{
H. Wysor Marsh \\ Marine Electronics Office \\ AVCO Corporation \\ New London, Connecticut \\ U.S.A.
}

\subsection{INTRODUCTION}

Marsh et al. [1,2] developed a general theory of sound scattering by the sea surface, and considered the specular reflection in detail, in connection with an analysis of propagation in a surface isothermal layer.

Nonspecular reflection can also be important in influencing propagation, and is especially significant in studying reverberation; or back scattering.

\subsection{NONSPECULAR SCATTERING}

For the theory of nonspecular scattering we have a point of departure following Marsh et al. [2], employing their notation throughout, and using their equation:

$$
\Lambda(\lambda, \mu)=\left[1-4 \gamma \sigma^{2} \iint \nu(l, m) S(l-a, m-\beta) d l d m\right] \delta(\lambda-a) \delta(\mu-\beta)+4 \gamma^{2} \sigma^{2} S(\lambda-a, \mu-\beta)
$$

The specular term is that involving the brackets, which they denote by

$$
\Omega(\lambda, \mu) \delta(\lambda-\alpha, \mu-\beta)
$$

We now define the nonspecular term to be $\Gamma(\lambda, \mu)$ so that

$$
\Gamma(\lambda, \mu)=4 y^{2} \sigma^{2} S(\lambda-\alpha, \mu-\beta)
$$

To reduce this result, we need the following additional results from the work cited:

$$
S(l, m)=\frac{1}{2 \pi} \int_{0}^{\infty} \rho(r) J_{0}\left[r\left(1-\nu^{2}\right)^{1 / 2}\right] r d r
$$

and

$$
h^{2} \rho(r)=\frac{\pi}{2} \int_{0}^{\infty} J_{0}\left(\frac{\omega^{2} r}{\delta}\right) A^{2}(\omega) d \omega
$$

and

$$
A^{2}(\omega)=C \omega^{-6} \exp \left(\frac{-2 \beta^{2}}{\omega^{2} s^{2}}\right)
$$


Writing

we then have

$$
\nu^{\prime 2}=1-(\lambda-a)^{2}+(\mu-\beta)^{2}
$$

$$
\Gamma(\lambda, \mu)=C \gamma^{2} k^{2} \int_{0}^{\infty} \int_{0}^{\infty} J_{0}\left(\frac{\omega^{2} r^{2}}{k g}\right) J_{0}\left[r\left(1-\nu^{\prime 2}\right)^{1 / 2}\right] \exp \left(\frac{-2 g^{2}}{\omega^{2} s^{2}}\right) \omega^{-6} \tau d r d \omega
$$

Let

$$
\frac{\omega^{2}}{k \xi}=v
$$

Then

$$
\begin{gathered}
\Gamma(\lambda, \mu)=\frac{C \gamma^{2}}{2 k^{1 / 2} g^{5 / 2}} \int_{0}^{\infty} \int_{0}^{\infty} J_{0}(u r) J_{0}\left[r\left(1-\nu^{\prime 2}\right)^{3 / 2}\right] \exp \left(\frac{-2 g}{k s^{2} u}\right) u^{-9 / 2} r u d r d u \\
=\frac{C}{2} \gamma^{2} k^{-1 / 2} g^{-5 / 2}\left(1-\nu^{\prime 2}\right)^{-9 / 4} \exp \left[\frac{-2 g}{k s^{2}\left(1-\nu^{12}\right)^{1 / 2}}\right]
\end{gathered}
$$

This result may be more conveniently written as

$$
\Gamma(\lambda, \mu)=\frac{C \gamma^{2} s}{2^{3 / 2} g^{3}\left(1-\nu^{\prime 2}\right)^{2}} \exp \left(-\frac{1}{a^{\prime}}\right) a^{1-1 / 3}
$$

where

$$
a^{\prime}=\frac{k}{2} s^{2}\left(1-\nu^{\prime 2}\right)^{1 / 2} g^{-1}
$$

In terms of convenient units, we have finally

$$
\begin{gathered}
\Gamma(\lambda, \mu)=0.098 \gamma^{2} H^{2 / 5}\left(1-\nu^{\prime 2}\right)^{-2} \exp \left(-\frac{1}{a^{1}}\right) a^{\prime 1 / 2} \\
a^{\prime}=6.33 H^{-1 / 5} b\left(1-\nu^{\prime 2}\right)^{1 / 2}
\end{gathered}
$$

Let $\psi$ be the angle between the speculardirection and the direction of scattering. Then evidently,

$$
1-\nu^{\prime 2}=2-(\nu-\gamma)^{2}-2 \cos \psi
$$

\subsection{BACK SCATTERING}

In the case of back scattering, $\nu=\gamma$, and the angle between the vertical and the direction of the incident wave is $\psi / 2=\theta$. Thus, for back scattering,

and

$$
1-\nu^{\prime 2}=4 \sin ^{2} \theta
$$

$$
\begin{gathered}
\Gamma_{r}=0.0061 H^{2 / 5} \cos ^{2} \theta \sin ^{-4} \theta \exp \left(-\frac{1}{a^{\prime}}\right) a^{\prime}-1 / 2 \\
a^{\prime}=12.66 H^{-1 / 5} b \sin \theta
\end{gathered}
$$

where we have written $\Gamma=\Gamma_{\mathrm{r}}$ for this special case (reverberation). In applying this equation to the calculation of reverberation intensities, a number of technical factors, including the geometry of the situation, must be considered. How- 


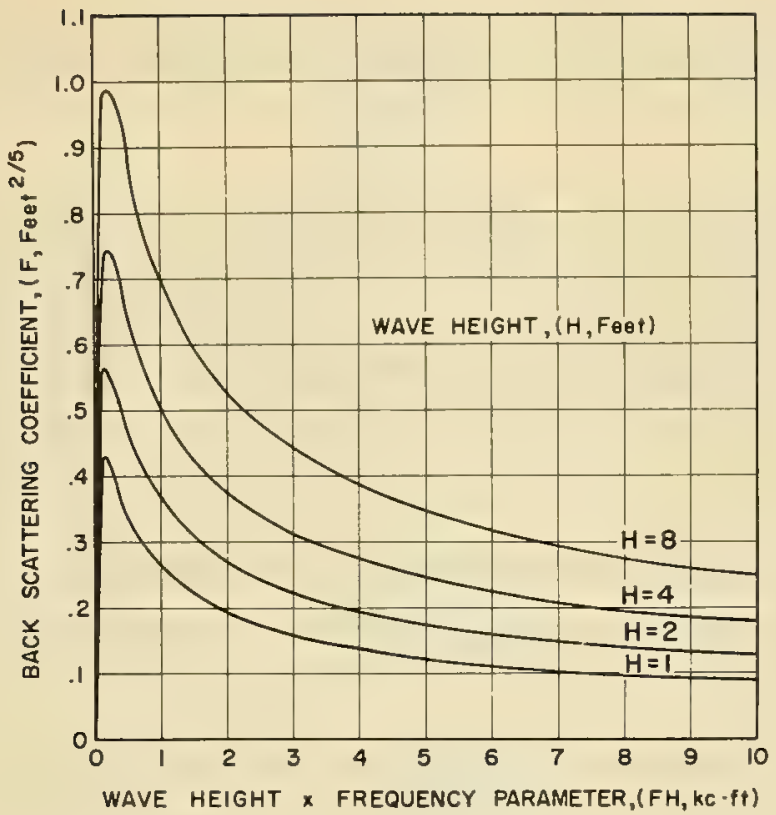

Fig. 11.1. Back scattering vs wave height and wave height times frequency.

ever, certain general conclusions can be drawn. The behavior of reverberation with changes in acoustic frequency and changes of sea condition is completely controlled by the expression

$$
F=H^{2 / 5} \exp \left(-\frac{1}{a^{\prime}}\right) a^{\prime-1 / 2}
$$

where $\Gamma_{z}=0.0061 \cos ^{2} \theta \sin ^{-4} \theta F$.

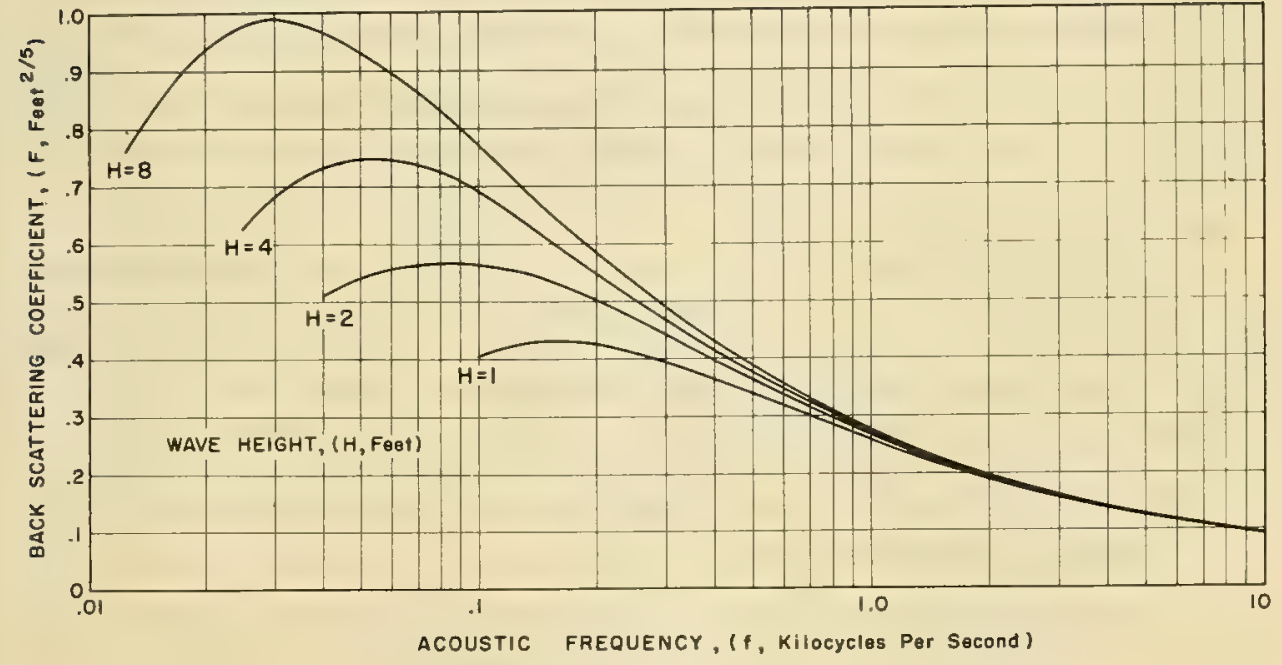

Fig. 11.2. Back scattering vs acoustic frequency and wave height. 
Using a value $\sin \theta=1$ for convenience, we have plotted $F$ against $b$ for fixed $H$ in Fig. 11.1, and $F$ against $f$ for fixed $H$ in Fig. 11.2. Figure 11.1 permits a comparison of back scattering with specular reflection.

\subsection{CONCLUSIONS}

Figure 11.2 shows the comparative insensitivity of reverberation to wave height and frequency, for the large value of $\sin \theta$ which corresponds to grazing incidence of the incident radiation. Both of these phenomena have been well known experimentally for some years. We are entitled, therefore, to have some confidence in the theory of sea surface scattering. In addition, the essential features of the sea surface spectrum are contained in $\Gamma$. We, therefore, have some additional confirmation that the Neumann-Pierson spectrum is correct.

\section{REFERENCES}

1. H.W. Marsh, "Exact Solution of Wave Scattering by Irregular Surfaces," J. Acoust, Soc. Am., Vol, 33 , 330-333 (1961).

2. H.W. Marsh, M. Schulkin, and S.G. Kneale, "Scattering of Underwater Sound by the Sea Surface," J. Acoust. Soc. Am., Vol. 33, pp. 334-340 (1961).

\section{DISCUSSION}

MR. A. G.D. WATSON thought the work most important and valuable and he was sorry the lecture was so condensed. In his talk, Dr. Marsh had raised many problems in mathematics, physics, oceanography, and underwater sound, but he was only going to comment on the mathematical aspect. He doubted the validity of the lecturer's use of Wiener's theory in the resolution of the reflected field into plane waves, but the final results are unaffected as Dr. Marsh does not employ Wiener's formula in his calculations. The justification of the resolution into plane waves could be, perhaps, carried out by considering a Fourier analysis of the field over a plane on the positive side of the scattering surface. It is not, however, necessary to use Wiener's formula for this purpose, if we follow Dr. Marsh and employ delta functions. Mr. Watson, then, spoke of the second step in the development of the coefficients of the power series in sigma which was also followed, without justification, by Rayleigh and others. Thirdly, there is the evaluation of the coefficients in terms of the correlation function of the surface and this is where, in Mr. Watson's opinion, Dr. Marsh has made a great contribution. He had, himself, made a similar approach and obtained the same result.

Mr. Watson said that it was difficult to reconcile Dr. Marsh's formula for his function $\Lambda$ with results of other workers. Using Dr. Marsh's notation and following a crude argument, assuming a Gaussian distribution of heights on the rough surface, one obtains a specular reflection coefficient of $\exp \left(-4 \gamma^{2} \gamma^{2}\right)$ and this agrees, to terms in $\sigma^{2}$, with Rayleigh's result, for a sinusoidal surface, in terms of Bessel functions. Finally, Mr. Watson asked Dr. Marsh if he would indicate how his formula is reconciled with the conservation of energy, when the film is integrated over all angles.

DR. MARSH: Wiener's method is formally applicable to any functions which are invariant under time translation. It is formally applicable to the resolution 
of any wave field into plane waves. Wiener's formulas are, in fact, used throughout my development, although a delta function is used for convenience in the final stages, since the power spectrum is more easily utilized than the integrated spectrum.

The question of rigor in the use of Wiener's method is another matter. Without doubt, sufficient qualifications could be made to guarantee validity, except for circumstances of vanishing probability. The fact is that the results are formally valid.

It is not surprising that it is difficult to reconcile my results with those of others. Only Rayleigh has results which are comparable, and, then, only for a sinusoidal surface. My results agree with Rayleigh's when compared under equivalent conditions. My results are not comparable to calculations based on the distribution of heights because the height distribution is, in general, not determined by the surface spectrum, and vice versa. A Gaussian surface cannot be sinusoidal and, therefore, any agreement between a Gaussian surface and Rayleigh's results is fortuitous.

The important point, as Eckart pointed out, is that the low-frequency scattering is determined by the surface spectrum, and not by the height distribution.

My formula shows that energy is conserved, since the negative integral of the nonspecular scattering over all direction cosines plus the specular term is unity.

DR. H. CHARNOCK asked the lecturer whether there was any evidence that the so-called "equilibrium sea" is horizontally isotropic.

DR. MARSH: I do not know whether the equilibrium sea is isotropic. However, the mean scattering of sound waves, for random orientation of the incident wave, is surely isotropic in azimuth. This is the situation developed in my lecture. 



\title{
LECTURE 12
}

\section{THERMAL MICROSTRUCTURE IN THE SEA AND ITS CONTRIBUTION TO SOUND LEVEL FLUCTUATIONS}

\author{
E.J. Skudrzyk \\ Ordnance Research Laboratory \\ The Pennsylvania State University \\ University Park, Pennsylvania \\ U.S.A.
}

\subsection{THE TEMPERATURE STRUCTURE OF THE SEA}

Bodies of water such as the sea are acoustically inhomogeneous because of the dissolved substances, suspended particles, turbulent motion and water currents, temperature gradients, and inherent microscopic temperature fluctuations. Temperature, which has the greatest effect on sound propagation, varies with daily and seasonal changes in the radiation from the sun. Variations in solar radiation cause temperature changes that take place over long distances and vary slowly with time. The turbulent motion of the water, on the other hand, generates microscopic temperature fluctuations from one point in the medium to another.

The sea surface is coldest at midnight and warmest at noon, and these surface temperature variations penetrate deeply into the medium as Fig. 12.1 illustrates. The curves of Fig. 12.1 are interesting because they have been computed on the basis of some very reasonable assumptions (concerning the radiation from the sun, evaporation at the sea surface, and turbulent heat conduction of the water). The unknown constants have been selected so that the curves coincide with a set of experimental curves measured at Key West, Florida. The only discrepancy occurs when the slopes become negative. These parts of the curves are not realized experimentally: the upper surface layers are heavier than the lower layers, and turbulent motion sets in. This surface-temperature instability generates strong turbulent motion in the sea, causing equalization of the temperature and generation of the so-called isothermal layer. The temperature gradients and long-scale temperature fluctuations lead to bending of the sound rays; they have been thoroughly investigated in recent years.

Figure 12.2 shows the microscopic variations of the temperature for various depths, as recorded by Urick and Searfoss [1] with a resistance thermometer (the temperature fluctuates by a few thousandths up to a tenth or over a full degree). These readings exhibit a surprising space periodicity, as if the temperature distribution had been generated by a resonance phenomenon. In the. 


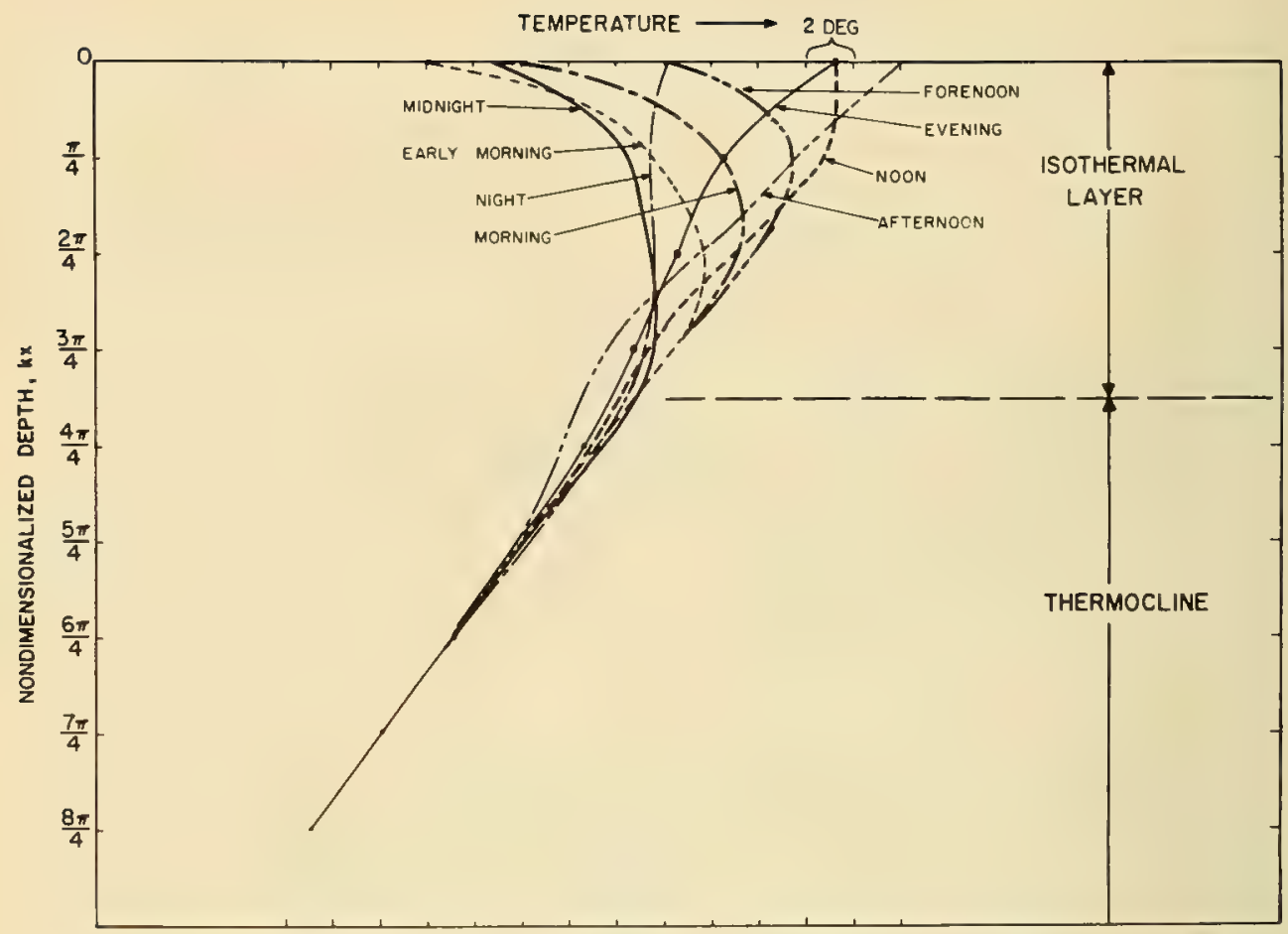

Fig. 12.1. Computed daily variations of the mean temperature of the sea.

literature on sound scattering half of this space wavelength is always identified with the diameter of the so-called temperature patches. An extended study (Fig. 12.3) shows that the patch radius is approximately equal to the depth at which the measurement was taken; the points of the "patch radius vs depth" lie as close to the line "patch radius equal to depth" as can be expected for statistical measurements. This result is very strange and hard to understand, but

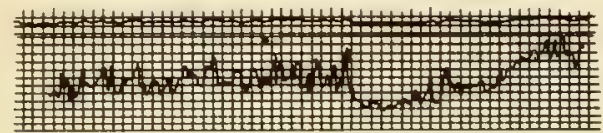

DEPTH: $25 \mathrm{FT}$

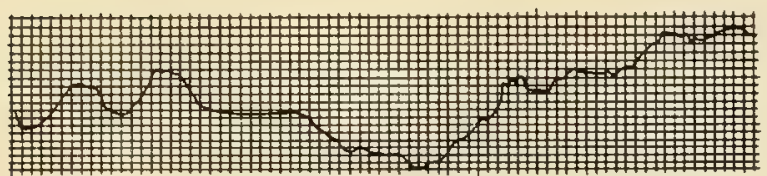

DEPTH: IIOFT

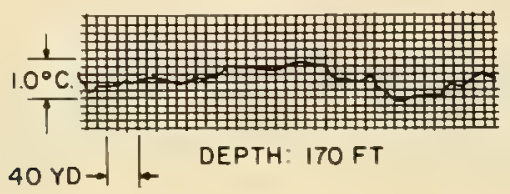

Fig. 12.2. Microthermal variations at various depths. 


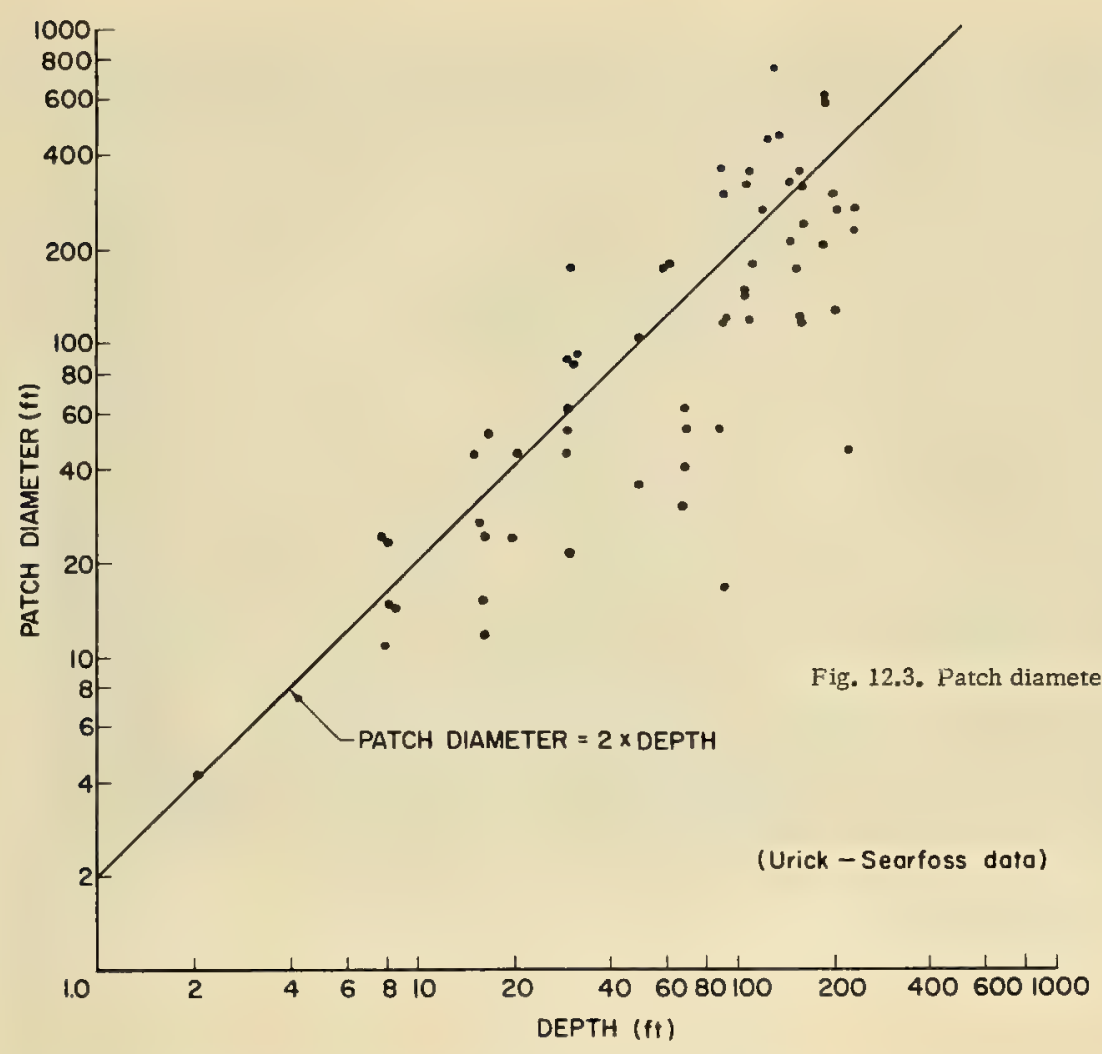

there is consolation in the fact that a similar result has been obtained for patches of turbulence. The classical mixing length, which is equivalent to the radii of the turbulent patches, has been found to be 0.4 times the distance from the wall. The classical theory of turbulence did not satisfactorily explain this strange relationship, and this is probably the main reason for the rejection of this theory in recent years. The measurements of the temperature structure of water indicate that the classical mixing length is not just a mathematical artifice; it has physical significance and can be determined experimentally.

The next important step in the study of the temperature structure of water is to determine the average temperature deviation between two points as a function of their distance. This has been determined by L. C. Pharo [2] (Ordnance Research Laboratory), who used the thermistor bar shown in Fig. 12.4 to measure temperature fluctuations. This bar, which is equipped with thermistors at distances of $1,2,4,8,16,32,64$, and $128 \mathrm{in}$, led to impressive results. The temperature fluctuation increases very little with the spacing between the thermistors. A closer study reveals that the rms temperature fluctuation increases with the cube root of the spacing (Fig. 12.5). If, for instance, the fluctuation is $0.001^{\circ}$ for a spacing of $10 \mathrm{~cm}$, the rms temperature fluctuation increases to only $1^{\circ}$ for a spacing of $100 \mathrm{~km}$. Again, a law that is similar to a well-known law in the theory of homogeneous turbulence has been confirmed. The rms fluctuation 


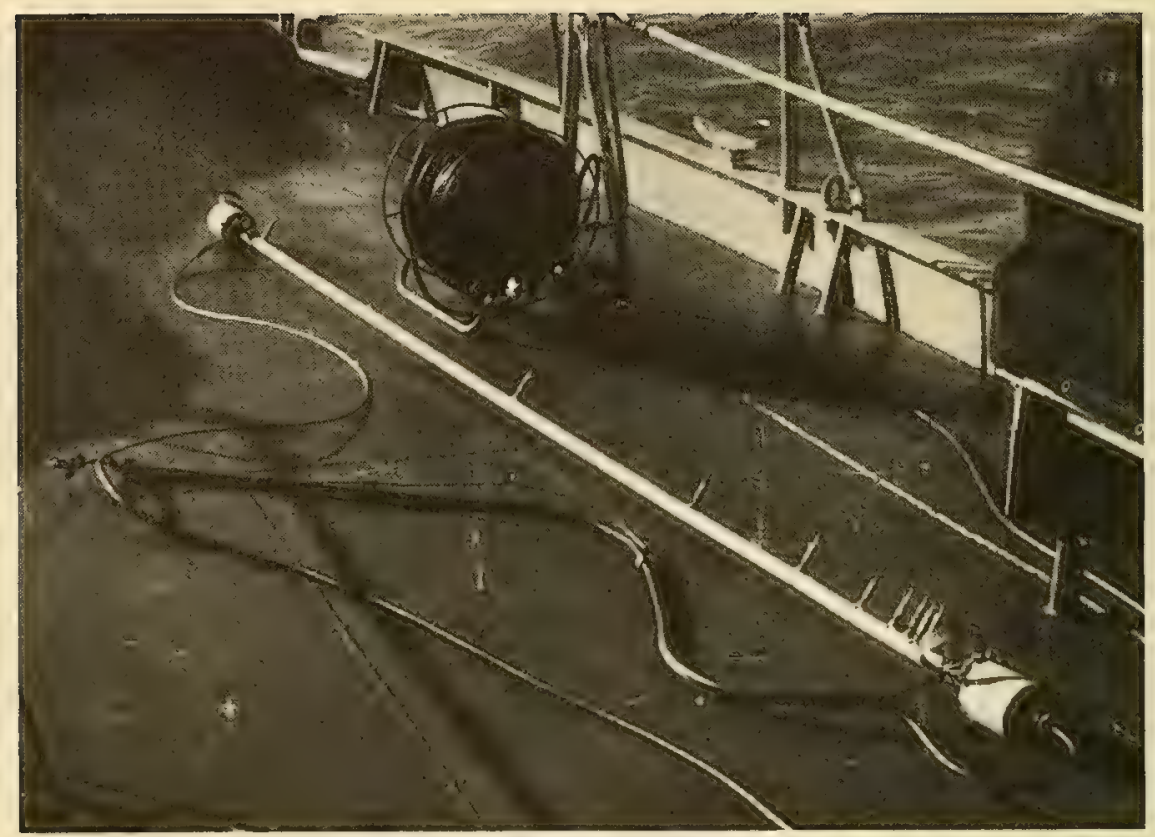

Fig. 12.4. Thermistor bar for measuring thermal microstructure.

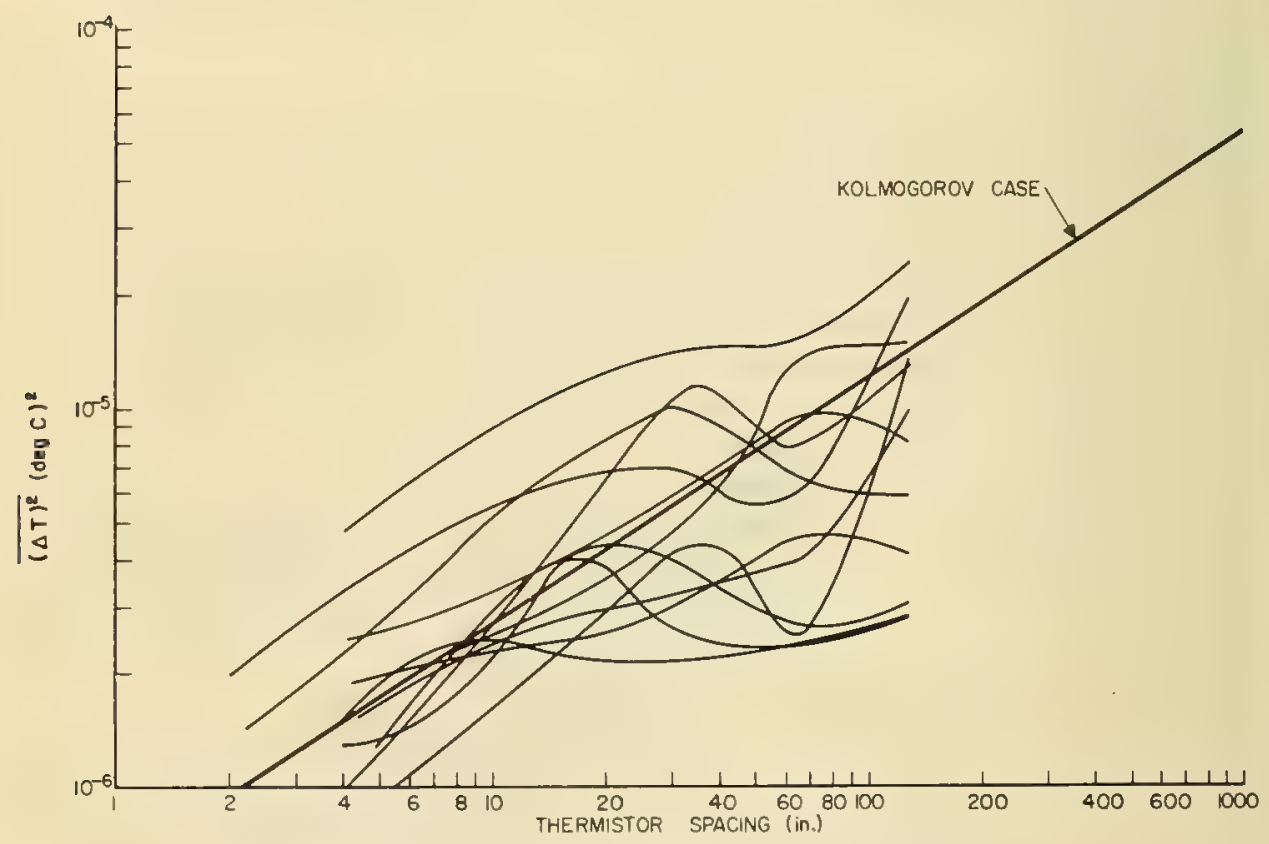

Fig. 12.5. Horizontal temperature distributions as measured in the sea. 
of the turbulent velocities is found to vary in exactly the same manner, and is explained by the Kolmogorov equilibrium law [3,5] for the spectral space distribution of the turbulent velocity fluctuations. This law is of the form

$$
E(\kappa)=K \kappa^{-5 / 3}
$$

where $K$ is a constant and $k$ is the space wave number. Such a power spectrum leads to the cube-root distance law, $\left\langle(\lambda)^{2}\right\rangle$ is equal to constant times $\rho^{1 / 3}$, where $\rho$ is the distance; and, vice versa, a cube-root distance law leads to such a power spectrum.

The Kolmogorov equilibrium law then turns out to be a general law of physics that applies not only to turbulence, but to many different phenomena. This law applies whenever the following sequence takes place: energy is introduced into a fluid at a constant rate, is redistributed until a state of equilibrium is attained, and is eventually dissipated by friction or heat conduction. The only other as sumption necessary is that the fluid be infinitely extended. Dimensional consideration then leads directly to this law in the following manner: the energy $D_{0}$ introduced per unit mass per unit time has the dimensions

$$
D_{0}=\frac{m v^{2}}{m t}=\frac{l^{2}}{t^{3}}
$$

where the expression derivable from the kinetic energy $\left(m v^{2}\right)$ has been used to arrive at the dimensions of energy. The magnitude of interest is the power spectrum $E(\kappa)$ of the phenomenon to be investigated. The power spectrum has the dimensions of energy per unit mass per unit space wave number, or

$$
E(\kappa)=\frac{m v^{2}}{m \kappa}=\frac{l^{2}}{t^{2}(1 / l)}=\frac{l^{3}}{t^{2}}
$$

where $\kappa=2 \pi / \lambda_{\mathrm{sp}}\left(\lambda_{\mathrm{sp}}\right.$ is the space wavelength and has the dimension $\left.l^{-1}\right)$. The power spectrum must be a function of the parameters that are available for the description of the phenomenon. Since the fluid has been assumed to be infinitely extended, the only available parameters are $D_{0}$ and $\kappa$. Hence, $E(\kappa)$ must be a function of $D_{0}$ and $\kappa$ :

$$
E(\kappa)=f\left(D_{0}, \kappa\right)
$$

which, in the simplest case, when written as a power product, is of the following form:

$$
E(\kappa)=D_{0}^{m} \kappa^{n}
$$

Since the dimension of the right hand sides of Eq. (5) and Eq. (3) must be the same, the following relation must hold for $m$ and $n$ :

$$
E(\kappa)=\frac{l^{3}}{t^{2}}=D_{0}^{m} \kappa^{n}=\frac{l^{2 t n}}{t^{3 m}} l^{-n}
$$

When we equate the exponents

$$
3=2 m-n \quad 2=3 m
$$


Hence,

and

$$
m=\frac{2}{3} \quad n=-\frac{5}{3}
$$

$$
E(\kappa)=D_{0}^{2 / 3} \kappa^{-5 / 3}
$$

which is the well known Kolmogorov law. Because of the generality of the assumption, it is not surprising that this law also describes the temperature fluctuations in the sea.

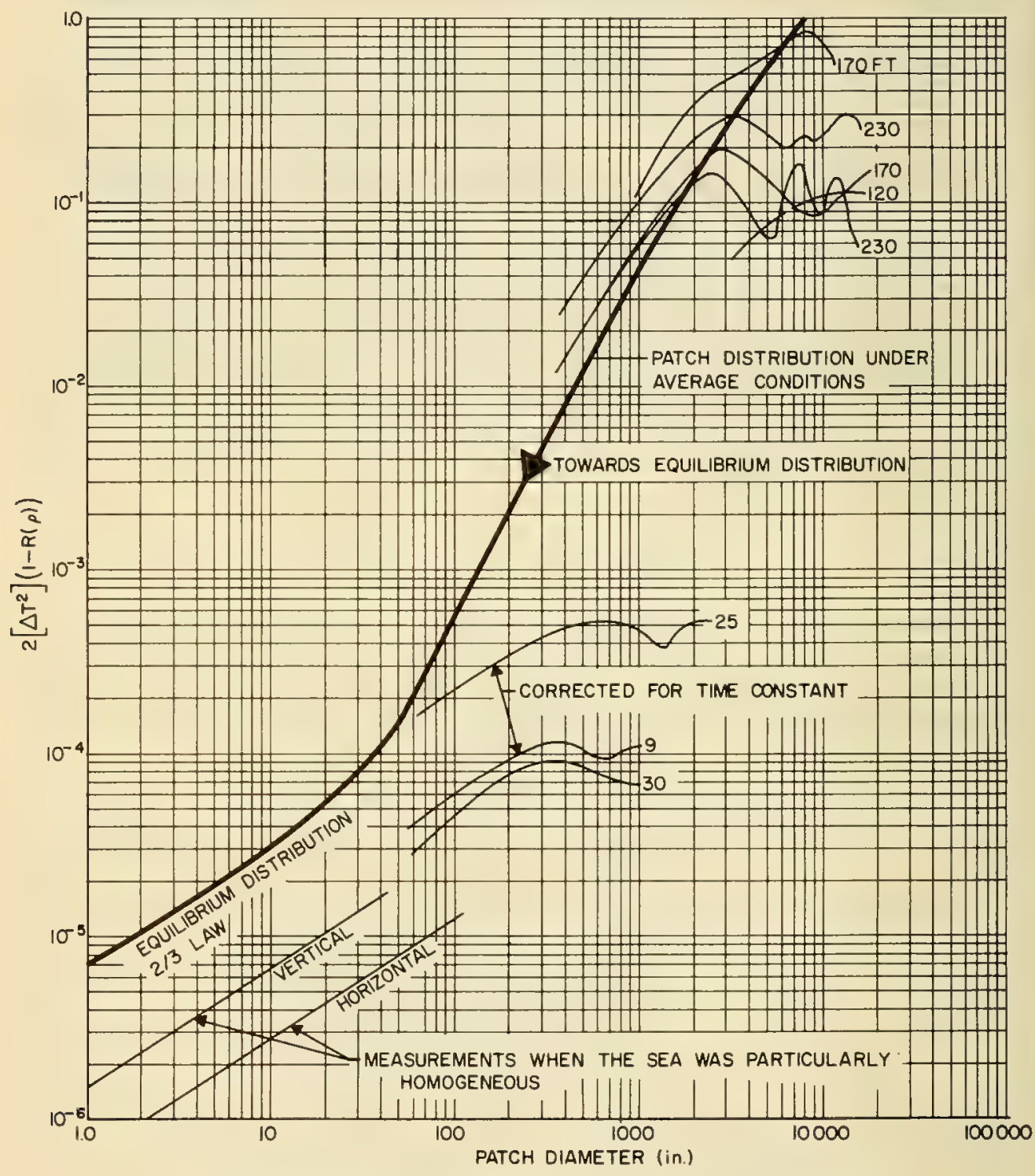

Fig. 12.6. Establishment of the equilibrium law for the range of small patches. 
The simple form of the Kolmogorov law applies for the equilibrium range of the phenomenon, which, by definition, is unaffected by the mechanism of the redistribution and the energy dissipation [4]. If the fluid is not infinitely extended-if, for instance, the receiver is at a finite distance from the water surface-an additional parameter is available to describe the phenomenon. In altering the Kolmogorov law for this case, let us be guided by the experimental results.

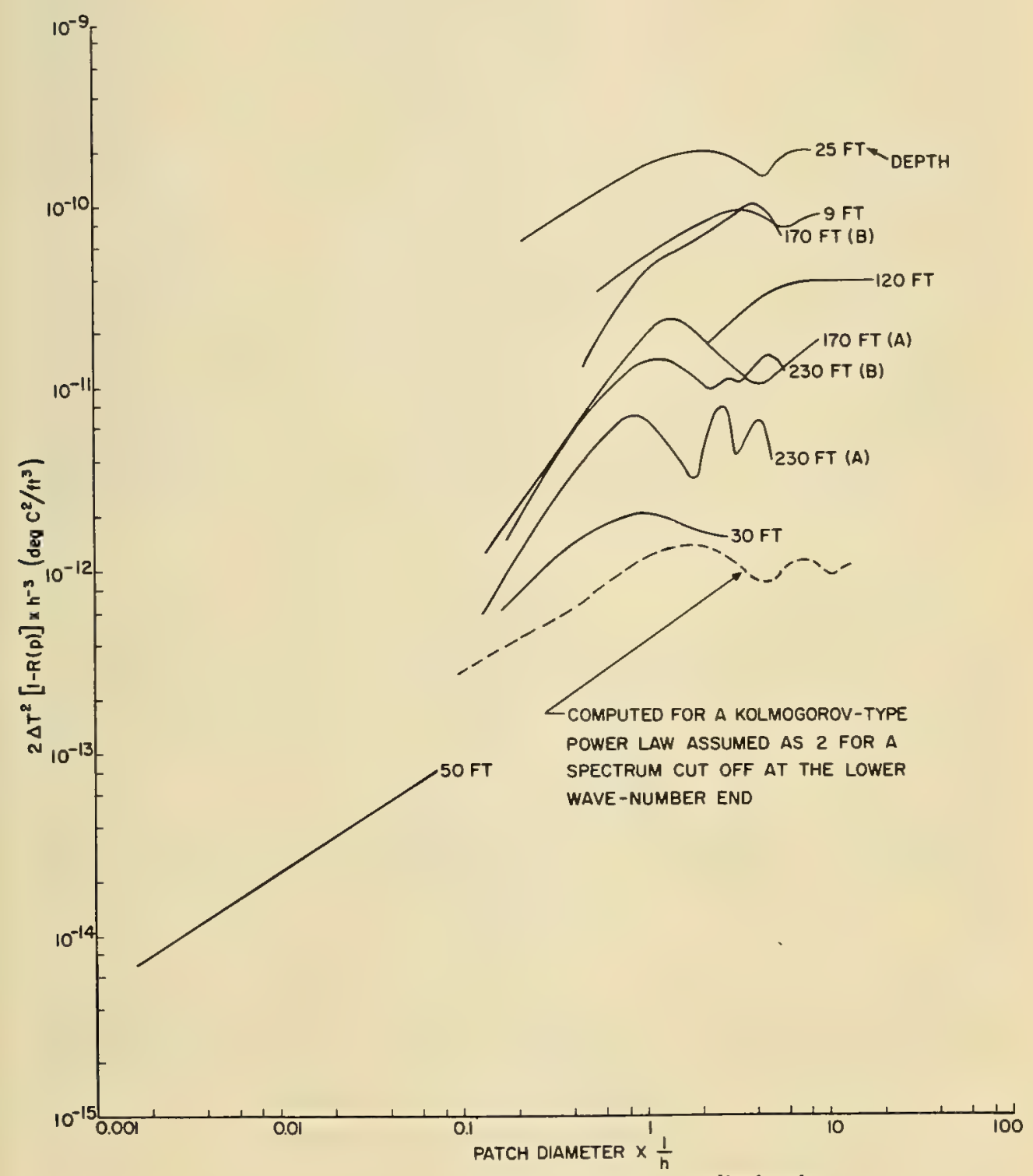

Fig. 12.7. Curves of Fig. 12.6 represented to a normalized scale. 
Figure 12.6 shows an extension of the rms temperature fluctuation measurements for long distances. As the distance is increased, the slope increases above the value given by the one-third power law. However, when the distance between the two points becomes equal to the depth of the measurements, the curves become practically horizontal. It is relatively easy to show that this discontinuity in the slope of the measured curve is due to a similar discontinuity in the slope of the power-spectrum frequency curve (or that it is even due to a cutoff of the power-spectrum) at a wavelength equal to one-fourth the depth of the measurement. Figure 12.7 shows a curve that is computed on the assumption of a Kolmogorov-type power spectrum and cutoff. This curve is similar to the experimental curves shown in the same figure. The discontinuity in the slope of the power spectrum also can be found directly from a Fourier analysis of the temperature fluctuations (Fig. 12.8), or from a Fourier analysis of the correlation function of the temperature fluctuations (Fig. 12.9). The results shown in Fig. 12.8 are of an orientative nature only, and the measurements are still affected by the time constants of the recording equipment. The frequency spectrum shown in Fig. 12.9 is practically constant up to a wave number equal to the depth, and decreases with increasing frequency. Figure 12.10 shows similar results obtained for the turbulent velocity fluctuations in a boundary layer.

The last result makes it possible to prove that the approximate constancy of the thermal patch diameters in all the temperature recordings (see, for instance, Fig. 12.2) is not due to a predominant spectral space component of the temperature fluctuation, but is due to a cutoff of the spectral distribution. For instance, let us consider a Fourier distribution with slowly varying amplitudes $A(t)$ and slowly varying phase angles $\psi(t)$. (The introduction of $A(t)$ and $\psi(t)$ elimi-

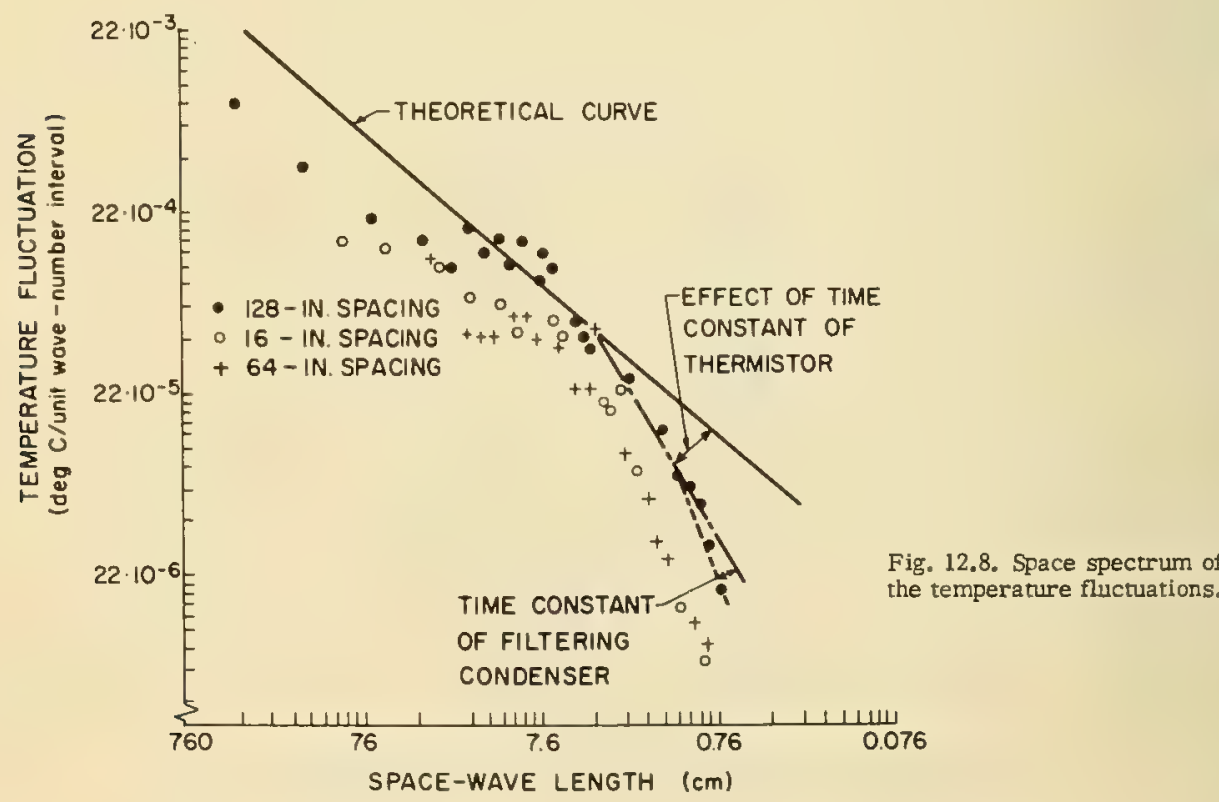




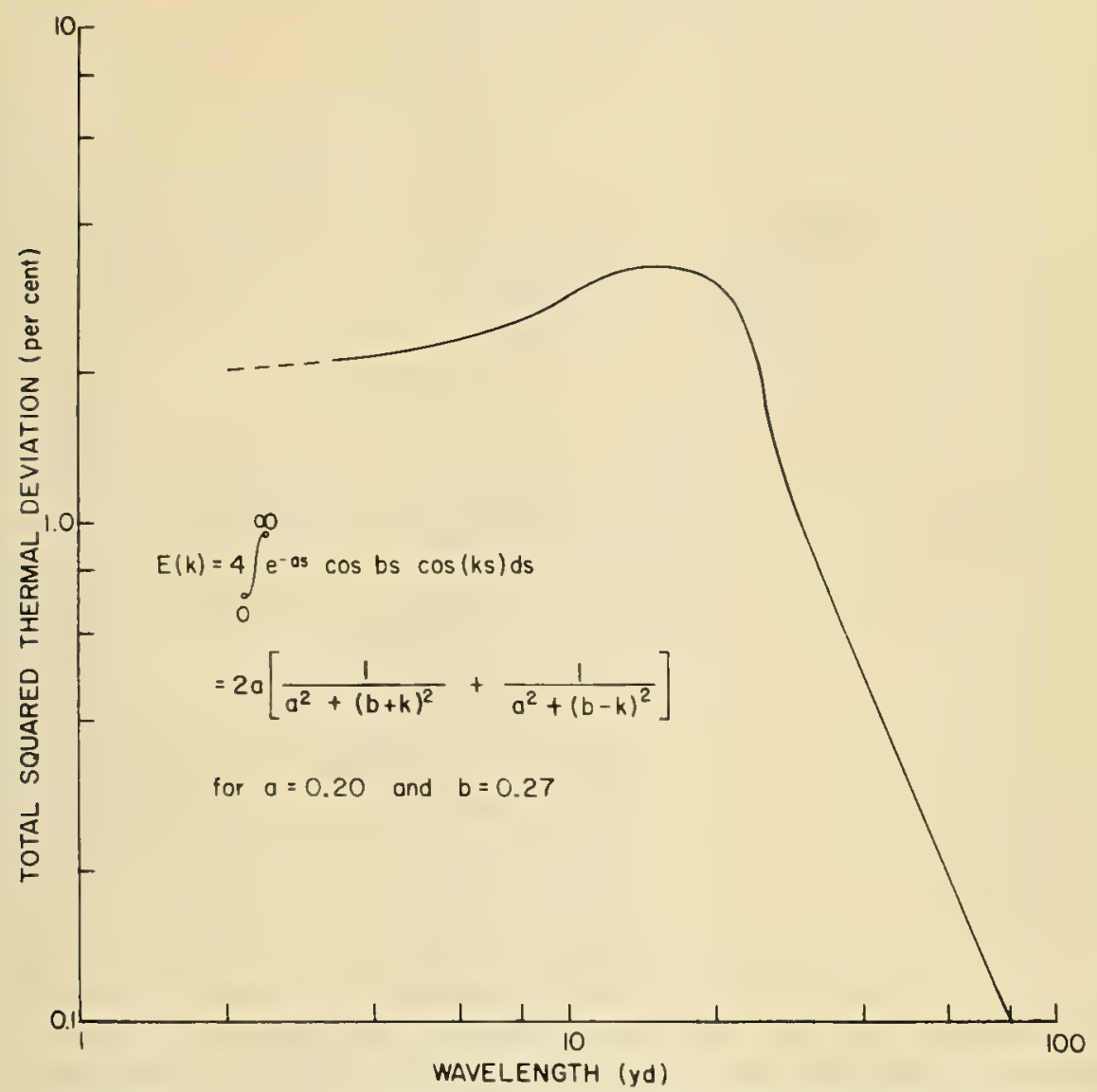

Fig. 12.9. Power spectrum obtained from an analysis of the correlation function $R(\rho)=e^{-0.20 \times} \cos 0.27 \mathrm{x}$ which represents a good fit to the experimental results [1].

nates the necessity to perform the Fourier integration over an infinite interval of time.) The time function, then, is given by

$$
\int A(t) \cos [\omega t+\psi(t)] d \omega
$$

A maximum is attained whenever $\omega t+\psi(t)=0$ for most of the spectral components of the distribution. In the vicinity of such a maximum, the above integral may be crudely approximated by the following expression:

$$
\int_{0}^{\omega_{0}} A \cos \omega t d \omega=A \omega_{0} \frac{\sin \omega_{0} t}{\omega_{0} t}=A \omega_{0} \frac{\sin x}{x}
$$

It has been assumed that the spectrum is of constant amplitude $A$, and that it is cut off at the frequency $\omega_{0}$. The time function is thus of the form $(\sin x) / x$. The amplitude fluctuates, and the period of the fluctuation is equal to the cutoff period. Actually, the spectrum is not constant, but is of the Kolmogorov type. It can be shown that for such a spectrum the fluctuations are still larger.

The above conclusions can be easily illustrated by experiments in which the space coordinate is replaced by the time. For instance, curves very similar 


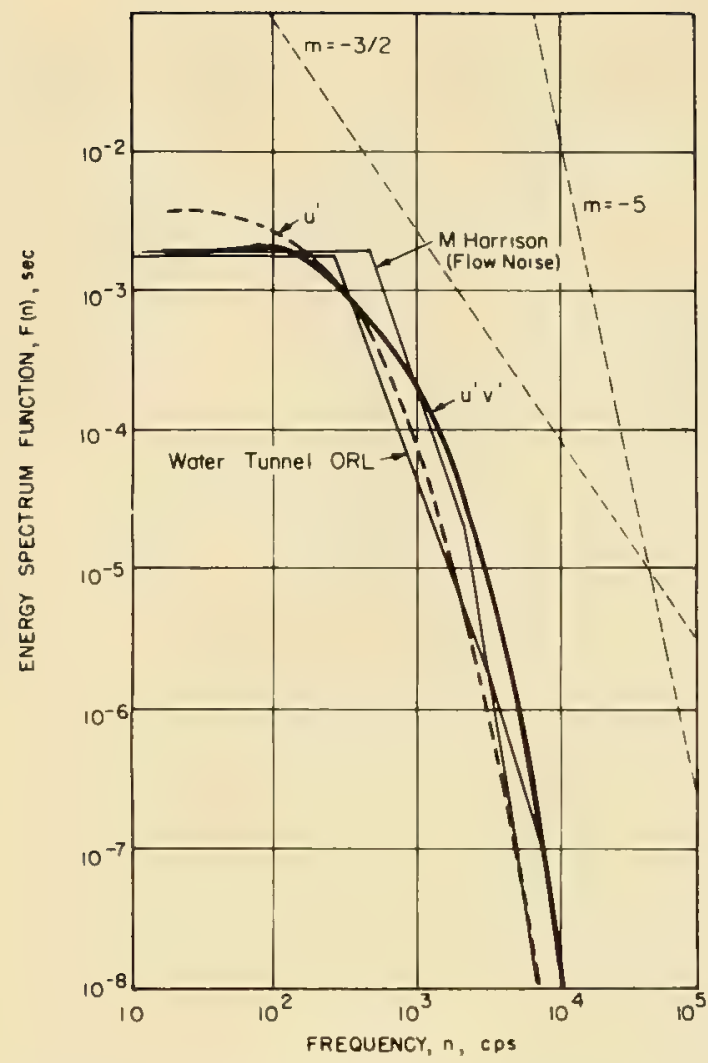

Fig. 12.10. Power spectrum of boundary layer turbulence.

to the temperature recordings are obtained when white noise is passed through a high-pass or low-pass filter and the filtered output is observed with an oscilloscope. The cutoff frequency can always be identified by the predominant periodicity of the output, which has a period equal to the cutoff period of the filter. Changing the cutoff frequency of the filter changes the periodicity of the output.

Another very similar phenomenon is observed in the detection of sound by an early type of condenser microphone. The transients of the sound generate decaying vibrations of a period equal to that of the cutoff period of the microphone. The ear, which integrates over only a short interval of time, perceives these transients as a hiss at the frequency of the microphone.

In a long-time Fourier analysis, the fluctuations cancel, but they always appear in a short-time analysis of the noise, such as that performed by the human ear. This fact is illustrated by Fig. 12.11 in which the left half of the curve is in antiphase to the right half of the curve. The contributions of the two halves of the curve cancel in the Fourier integral. If, however, the Fourier integration is limited to the right or left half of the curve, Fourier analysis leads to a predominant component of a period approximately equal to the period of the fluctuation of the curve.

The generation of the cutoff in the power spectrum at a wavelength equal to about four times the depth of the measurement still needs explanation. Un- 


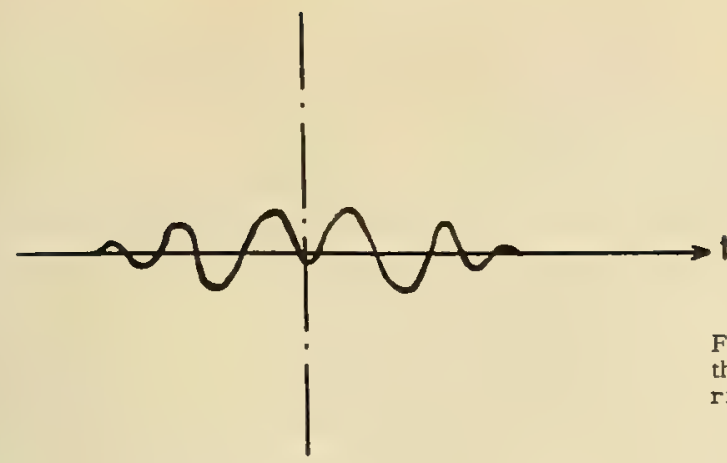

Fig. 12.11. Fluctuations in which the left half are in antiphase to the right half.

fortunately, no satisfactory explanation has yet been found, but a plausible explanation can be derived with the aid of a statistical method [6] that was introduced by Lande in 1913. This method, which represents an expansion of the procedures used in thermodynamics today, analyzes the physical system by counting the number of its degrees of freedom and by attributing a certain energy to each of them. The degrees of freedom are identified with the number of parameters that are required to describe the system, such as the number of the Fourier coefficients that are finite. This method has been very successful in recent years [7], although it does not seem to be fully understood at present. The temperature fluctuation within the finite layer of water between the surface and the point of measurement can be represented by a Fourier series. If isotropy is assumed in this layer, no consideration need be given to what happens at greater depth, and the layer can be assumed to be repeated periodically. Because of the continuity of the temperature distribution, the lower boundary has to be a plane of symmetry in this periodic pattern of layers, and the longest wavelength required to represent the temperature pattern is therefore equal to four times the depth. Since the lower boundary of the layer is not plane but distorted and fuzzy, the phenomenon is not strictly represented by a Fourier series but by a Fourier integral. However, the envelope of the spectrum may still be considered as approximately that of the series, with the low frequencies also filled in (because of the nonperiodicity of the actual temperature pattern).

\subsection{THE CHARACTERISTICS OF THE TEMPERATURE STRUCTURE OF THE SEA}

The static thermal conductivity of water is extremely small. Temperature patches would have a lifetime of many months if they were not split up and deformed by turbulent motion. There is, therefore, little doubt that these patches are originally generated by turbulent motion and represent "frozen turbulence."

The energy that is fed into the water by the radiation of the sun and by the wind acting on the sea surface seems to be predominantly used for the generation of very large turbulence patches and temperature patches (see Fig. 12.6). The large patches eventually split up into very small patches; thus, the turbulent and the thermal energies are passed down by a kind of cascade process to the range of the small and very small patches (or space wave numbers). The intensity of the large-diameter patches depends on the weather and on the depth 
of the measurement, and is considerably greater than the intensity predicted by the Kolmogorov equilibrium law (see Fig. 12.6). However, as the patch size becomes smaller, most of the fluctuations caused by the weather and by the daily variations of the temperature seem to average out, and a stable Kolmogorov-type space spectrum is generated. The slope of the curves in Fig. 12.5, then, is exactly one third. When the space wavelength becomes very small, the effects of viscosity and heat conduction predominate, and the temperature fluctuation eventually decreases inversely to the seventh power of the space wavelength [4]. This range does not appear in Fig. 12.5.

The space spectrum of the temperature fluctuations of the sea can be divided into three different regions: (1) the long wave number range, (2) the equilibrium Kolmogorov range, and (3) the dissipation range. The long wave number range, which depends greatly on the depth of the measurement, and which also seems to depend greatly on the weather and its history, as will be discussed in the next section, has practically no effect on sound scattering or the fluctuations of the transmitted signal. These are predominantly determined by the spectral components of the temperature pattern that have a wavelength $\lambda_{\text {sp }}$ equal to, or greater than, half the sound wavelength up to wavelengths somewhat above that given by $r=k R^{2}$, where $r$ is the range, and $R=\lambda_{\text {sp }} / 4$ represents the radii of the temperature patches. For the sound frequencies of practical interest, the spectral components of the temperature structure that affect sound propagation are always within the Kolmogorov equilibrium range.

The spectral distribution curve of the temperature fluctuations in water, as far as they affect sound propagation, can be assumed to be independent of the weather or the external conditions of the Kolmogorov type, $E(\kappa)=K \kappa^{-5 / 3}$. This assumption leads to a considerable simplification in the theories of sound scattering and of the fluctuations of the transmitted signal. All that needs to be determined in the Kolmogorov law is the constant $K$ as a function of the depth and of the variations in the weather. This can be done by measuring the rms temperature fluctuation between two points at a constant distance from one another. The results obtained in the past by acoustic measurements seem to indicate that the value of this constant decreases with depth, but depends only slightly on the weather.

The constant $K$ can be expressed as a function of the mean-square deviation of the sound velocity. If the power spectrum is constant up to a space wave number $\kappa_{0}$, and if it obeys a simple power law for the higher space wave numbers,

$$
\begin{array}{ll}
E(\kappa)=K \kappa^{-m} & \kappa \leq \kappa_{0} \\
E(\kappa)=K \kappa_{0}^{-m} & \kappa \geq \kappa_{0}
\end{array}
$$

the mean-square value of the velocity fluctuations then becomes

$$
a^{2}=\int_{0}^{\kappa_{0}} E(\kappa) d \kappa=K \kappa_{0}^{1-m}+\int_{\kappa_{0}}^{\infty} K \kappa^{-m} d \kappa=\frac{m}{m-1} K \kappa_{0}^{1-m}
$$

The constant $k$, therefore, is given by

$$
K=\frac{m-1}{m} \kappa_{0}^{m-1} a^{2}
$$


Actually, $m$ and $K$ are not constant, but increase toward the long wave number end. A more accurate determination of $K$ can be obtained for the Kolmogorov range by measuring the rms temperature difference between two points separated by a distance that is of the same magnitude as the diameter of the acoustically active thermal patches (about $10 \mathrm{ft}$ apart; see next section). It can be shown [8] that the equation

$$
[c(x+\rho)-c(x)]^{2}=\frac{4}{3} \int_{0}^{\infty} E(\kappa)\left(1-\frac{\sin \kappa \rho}{\kappa \rho}\right) d \kappa=\beta K \kappa^{-5 / 3}
$$

depends predominantly upon the value of $E(\kappa)$ for $\kappa \rho=1$, and that the constant $\beta$ is equal to $4.8 / 3$ in the range $\kappa \rho \cong 1$, even if the Kolmogorov law is only an approximation.

\subsection{THE SCATTERED PRESSURE AND SCATTERED INTENSITY}

\subsubsection{The Rayleigh Integral}

The classical as well as the modern theories of scattering are all based on the Rayleigh integral [9]. This integral represents the solution of the wave equation for the case in which the properties of the medium deviate slightly from their average values. This solution shows that the density changes caused by the natural temperature fluctuations have a negligible effect on sound propagation in comparison to that of the changes in sound velocity. But if the local variations of the density are neglected, the derivation of the wave equation is the same as that for a homogeneous medium except that the sound velocity now is locally variable. Therefore, for periodic vibrations, the wave equation is

$$
\nabla^{2} p+k^{2} p=0
$$

where

$$
k^{2}=\frac{\omega^{2}}{c_{0}^{2}}\left(1-\frac{2 \Delta \mathrm{c}}{\mathrm{c}}+\cdots\right)=k_{0}^{2}(1-2 a)
$$

In Eq. (16), $c=c_{0}+\Delta c$, the magnitude $c_{0}=\langle c\rangle$ represents the space-average value of the sound velocity, $k_{0}=\omega / c_{0}$ is the wave number of the undisturbed medium, and

$$
\underline{a}=\Delta \mathrm{c} / \mathrm{c}_{0}
$$

is the relative deviation of the sound velocity from the space-average value. The variable part of the term that contains the sound velocity may then be trans ferred to the right-hand side, thus:

$$
\nabla^{2} p+k_{0}^{2} p=2 k_{0}^{2} \underline{a} p
$$

Since only a small fraction of the incident energy is scattered per unit volume of the scatterer, the right-hand side is small and the sound pressure on the right-hand side may be replaced by the sound pressure, $p_{i}$, of the incident wave. The solution of the resulting equation is well known; it is given by

$$
p=p_{i}+p_{\mathrm{sc}}
$$

where the scattered pressure 


$$
p_{\mathrm{sc}}=\frac{k_{0}^{2}}{2 \pi} \int \underline{\alpha} p_{i} \frac{e^{-j k_{0} r}}{r} d \tau
$$

is represented by the Rayleigh integral [9] taken over the total scattering volume and the distance $r$ is given by

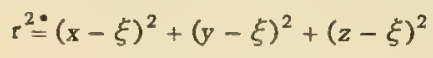

No assumptions other than $\Delta c / c_{0} \ll 1$ have been required in the derivation of this formula. To simplify the notation, $k_{0}$ has been replaced by $k$, where $k$ represents the average wave number in the inhomogeneous medium. As is standard practice in acoustics, the solutions here and in the following pages are represented in complex forms. The actual solution is given by the real part of the complex solutions.

Physically, the Rayleigh integral denotes the sound pressure radiated by a source distribution that is excited by the incident sound. Every elementary volume of the fluid turns into a sound generator that, at the expense of the energy of the incident sound, radiates (scatters) sound in all the different directions.

The simplest and most efficient way to study scattering problems is to consider a parallel beam of sound $p_{i}=p_{0} e^{-j k \zeta}$ propagated in the direction of the positive $\xi$ axis. The scattering volume may be assumed to be small in comparison to its distance, $\tau_{0}$, from the receiver and to be centered at $(\xi, \eta, \zeta)=0$. Let the point of observation have the coordinates $x, y$, and $z$. The scattered pressure then is given by the Rayleigh integral

$$
p_{s c}(x, y, z)=\frac{-k^{2}}{2 \pi} \int\left[\underline{a}(\xi, \eta, \zeta) \frac{e^{-j k r}}{r} p_{0} e^{-j k} \zeta\right] d \xi d \eta d \zeta
$$

where $\underline{a}=\Delta c / c_{0}$ is the relative change in sound velocity and $r$ [see Eq. (21)] is the length of the radius vector. It is standard practice to develop $r$, the exponent, into a Taylor series:

$$
r=r_{0}-\frac{x \xi}{\varepsilon_{0}}-\frac{y \eta}{r_{0}}=\frac{z \zeta}{\varepsilon_{0}}+\frac{\xi^{2}+\eta^{2}+\zeta^{2}}{2 r_{0}}+\therefore=t_{0}-(a \xi+\beta \eta+y \zeta)+\frac{\xi^{2}+\eta^{2}+\zeta^{2}}{2 r_{0}}+\ldots
$$

In this equation $r_{0}$ is the distance of the receiver from the center of the scatter and $a=x / r_{0}, \beta=y / r_{0}$, and $\gamma=z / r_{0}$ denote the direction cosines of the scattered sound. The Rayleigh integral then simplifies to

$$
\rho_{\mathrm{sc}}(x, y, z)=\frac{k^{2} p_{0}}{2 \pi} \int \frac{a e^{-j k{ }_{0}\left[r_{0}-\xi a-\eta \beta-\zeta(\gamma-1)\right]}}{r_{0}} d \xi d \eta d \zeta
$$

In the denominator, $t$ has been replaced by $r_{0}$ on the assumption that the dimensions of the scatterers are small in comparison to the distance of the scatterer from the sound receiver.

For forward scattering $\left(\alpha=\beta=0, \gamma=1\right.$, and $\left.\theta_{0}=0\right)$, the scattered pressure becomes

$$
p_{s c}(0)=\frac{k^{2} p_{0} e^{-j k r_{0}}}{r_{0}} \quad 2 \underline{a}(\rho) \rho^{2} d \rho=\frac{-k^{2} p_{0}}{2 \pi r_{0}}\left\langle a>r e^{-j k r}=p_{0} \frac{s}{r} e^{-j k r}\right.
$$


where

$$
s=\frac{2 \kappa^{2} \rho_{0} \alpha R^{3}}{3}
$$

is a measure of the strength of the scatterer; the magnitude $s$ is equal to the amplitude of the scattered pressure at unit distance from the scatterer. Thus, the pressure scattered in the forward direction becomes proportional to the product $a r$, where $a$ is the average deviation of the sound velocity from its mean value over the volume $\tau$ and $r$ is the volume of the scatterer. Polar coordinates $\rho, \theta$, and $\phi$ have been introduced to replace $\xi, \eta$, and $\zeta$.

\subsubsection{Spherical Scatterers}

A great deal of information can be obtained by considering the simple case of a spherical scatterer and assuming that the sound velocity is a function of the distance from the center of the scatterer only. The angular integration can be easily performed as follows. The quantities $\alpha, \beta$, and $\gamma-1$ can be considered components of a vector $A$ of magnitude

$$
A=\left[\alpha^{2}+\beta^{2}+(\gamma-1)^{2}\right]^{2 / 2}=[2(1-\gamma)]^{2 / 2}=\left[2\left(1-\cos \theta_{0}\right)\right]^{1 / 2}=2 \sin \frac{\theta_{0}}{2}
$$

$\theta_{0}$ is the angle between the direction of the incident sound (that of the $z$ or $\zeta$ axis) and that of the scattered sound ( $\theta_{0}=0$ for forward scattering). The magnitude $\dot{A}$ and the direction $(\alpha, \beta$, and $\gamma-1)$ of the vector $\mathbf{A}$ depend upon the scattering angle only. In the integration over the scatterer, $A$ is constant; therefore, the vector A may be assumed to be the axis of a system of polar coordinates $\rho, \theta$, and $\zeta$. The exponent in the integrand in Eq. (24) can then be written $\rho A \cos \theta$; and the integral becomes independent of $\zeta$ :

$p_{\mathrm{sc}}=\frac{k^{2} p_{0} e^{-j k r_{0}}}{\tau_{0}} \int_{0}^{\infty} \int_{0}^{\pi} a(\rho) e^{j k A \rho \cos \theta} d(-\cos \theta) \rho^{2} d \rho=\frac{k^{2} p_{0} e^{-j k r_{0}}}{\tau_{0}} \frac{2}{\Gamma} \int_{0}^{\infty} a(\rho) \sin (\Gamma \rho) \rho d \rho$

where $\Gamma=2 k \sin \left(\theta_{0} / 2\right)$. The Rayleigh integral now becomes solvable for several different velocity distributions.

In the classical theory of scattering, the dimensions of the scatterers are small compared to the wavelength of the radiation and the distance from the scatterer, $r_{0}$. The phase change of the incident pressure, $p_{l}$, and the scattered pressure, $p_{\mathrm{sc}}$, over the volume of the scatterer, $\tau=4 \pi R^{3} / 3$ (where $R$ is the radius of a sphere that has the same volume as the scatterer), may then be neglected; the scattered pressure

$$
p_{\text {se }}=\frac{k^{2}}{2 \pi} p_{0} \alpha \tau \frac{e^{-j k r_{0}}}{r_{0}}=\frac{8 \pi^{2} R^{2} p_{0}}{\lambda^{2}} \frac{R}{r_{0}} e^{-j k r_{0}}
$$

becomes independent of the scattering angle and is proportional to the square of the ratio of the diameter to the wavelength. Particles that are small compared to the wavelength of the radiation are, therefore, very inefficient scatterers.

If the scatterer is a finite sphere of constant sound velocity, the integral for scattered pressure in Eq. (27) simplifies to

$$
p_{s c}=\frac{-3 s \cdot e^{-j k r_{0}}}{R^{3} \Gamma_{\tau_{0}}} \int_{0}^{R} \sin (\Gamma \rho) \rho d \rho=\frac{3 s e^{-j k r_{0}} \sin \zeta-\zeta \cos \zeta}{t_{0}} \frac{\zeta^{3}}{\zeta^{3}}
$$


where $\zeta=\Gamma R=2 k R \sin \left(\theta_{0} / 2\right)$. The scattered pressure has decreased to half its maximum value when $\zeta=2.50 \mathrm{rad}$. The corresponding scattering angle is given by

$$
\theta_{0}=2 \sin \frac{\theta_{0}}{2}=\frac{2.5}{k R}=0.74 \frac{\lambda_{0}}{2 R}
$$

Outside this region, where $\sin \zeta \ll \zeta \cos \zeta$, the scattered pressure becomes

$$
p_{s c}=-\frac{3 s e^{-j k s_{0}}}{r_{0}} \frac{\cos \zeta}{\zeta^{2}}
$$

For small values of $\zeta$, that is, for scattering in the forward direction or for low frequencies, the Taylor development leads to the classical solution [Eq. (28)]. For a large scatterer, the scattered pressure fluctuates rapidly with changing scattering angle, and the envelope of these fluctuations decreases inversely proportional to the square of the sine of half of the scattering angle and to the square of the frequency. The phase of the scattered pressure is given by that of the incident wave at the center of the scatterer and the distance of the point of observation from the center; therefore, it corresponds to the distance the sound has actually traveled. However, this result applies only if the scatterer is very small in comparison to the distance from the scatterer, so that the higher-order terms in the Rayleigh integral can be neglected.

It is also interesting to study the effect of a continuous transition of the sound velocity from the undisturbed medium to the center of the scattering patch. Four cases of a gradual velocity transition are easily soluble $[10,11]$. They are represented in Table 12.1 as cases 3 to $6:$

$$
\begin{aligned}
& 3 \ldots \ldots \ldots \ldots a(r)=a_{0} e^{-r / R} \\
& 4 \ldots \ldots \ldots a(r)=\left\{\begin{array}{l}
a_{0}(1-r / R)^{2} \text { for } r<R \\
0 \text { for } r>R
\end{array}\right. \\
& 5 \ldots \ldots \ldots a(r)=\frac{a_{0}}{\left[1+(r / R)^{2}\right]^{2}} \\
& 6 \ldots \ldots \ldots a(r)=a_{0} e^{-(r / R)^{2}}
\end{aligned}
$$

Figure 12.12 shows a graphical comparison of the scattered pressure for the cases represented in Table 12.I. The ordinate represents the scattered pres sure; the abscissa, the quantity $\Gamma R$, which is twice the product of the undisturbedmedium wave number with the effective radius $R$ of the scatterer, as defined by Eqs. (32) and the sine of half the scattering angle. Forward scattering is, thus, described by points on the vertical axis, and backward scattering is described by points that, proportionally, are more to the right as the wave number and the radius of the scatterer become greater. Since the same scattering strength has been assumed [see Eq. (25)], forward scattering is the same in every case. For a given scattering power or a given volume of scatterers, the classical scatterer is by far the most effective. The scattered pressure is independent of the angle and has the maximum value possible. If the scatterer 
TABLE 12.I

\begin{tabular}{|c|c|c|c|c|}
\hline $\begin{array}{r}\text { Case } \\
\text { no. }\end{array}$ & $\begin{array}{l}\text { Relative deviation } \\
\text { of } \\
\text { sound velocity, } \\
\alpha\end{array}$ & $\left(2 \pi / k^{2} p_{0}\right) s$ & $\begin{array}{l}\text { Scattered pressure } \\
\text { referred to classical } \\
\text { scatterer of same s, } \\
\qquad D\left(\theta_{0}\right)\end{array}$ & Remarks \\
\hline 1 & $a_{0}, R \ll \lambda$ & $a_{0} d t$ & 1 & Classical scatterer \\
\hline 2 & $a_{0}, r \leq R$ & $\frac{4 \pi R^{3}}{3} \alpha_{0}$ & $3 \frac{\sin \Gamma R-\Gamma R \cos \Gamma R}{(\Gamma R)^{3}}$ & $\begin{array}{l}\text { Spherical scatterer } \\
\text { of constant sound } \\
\text { velocity }\end{array}$ \\
\hline 3 & $\alpha_{0} \mathrm{e}^{-r / R}$ & $8 \pi R^{3} a_{0}$ & $\frac{1}{\left(1+\Gamma^{2} R^{2}\right)}$ & $\begin{array}{l}\text { Exponential increase } \\
\text { of sound velocity }\end{array}$ \\
\hline 4 & $\begin{array}{c}a_{0}(1-r / R)^{2} ; r \leq R \\
0 ; r>R\end{array}$ & $2 \pi a R^{3} / 15$ & $30 \frac{(4-(6 \sin \Gamma R / \Gamma R+2 \cos \Gamma R)}{(\Gamma R)^{4}}$ & $\begin{array}{l}\text { Parabolic velocity } \\
\text { increase }\end{array}$ \\
\hline 5 & $\frac{a_{0}}{\left[1+(r / R)^{2}\right]^{2}}$ & $\pi^{2} R^{3} a$ & $e^{-\Gamma_{R}}$ & \\
\hline 6 & $a_{0} e^{-(r / R)^{2}}$ & $\pi \sqrt{\pi} R^{3} a_{0}$ & $e^{-\Gamma^{2} R^{2} / 4}$ & $\begin{array}{l}\text { Gaussian increase } \\
\text { of sound velocity }\end{array}$ \\
\hline
\end{tabular}

is large compared to the wavelength of the radiation, the scattered pressure decreases at least as $(\Gamma R)^{-2}$ (see Table 12.I). It fluctuates above and below this value for a sphere of constant sound velocity and approaches this value asymptotically if the change in sound velocity is exponential. The pressure scattered backwards by a large scatterer decreases at least inversely proportional to the square of the frequency and the square of the radius of the scatterer. Sharper transitions than the exponential, such as those of cases 4 and 5 in Table 12.I, lead to considerably less backscattering. Scatterers that exhibit a Gaussian velocity distribution produce a particularly small amount of backscattering.

Forward and backward scattering, then, are almost completely independent phenomena. Forward scattering, as will be shown later, essentially describes the phase change of the wave caused by the scatterer. This phase change is proportional to the average change of the sound velocity over the scatterer, and consequently it does not depend upon whether the change is abrupt or continuous. Thus, all scatterers that exhibit the same average change of sound velocity over their volume (i.e., they have the same scattering strength) generate the same amount of forward scattering. The scattered pressure reaches a maximum in the forward direction and decreases with increasing scattering angle. Backward scattering, on the other hand, is essentially a reflection phenomenon; therefore, it depends greatly on the details of the variation of the sound velocity across the scattering patch.

The results given above can be generalized to fit the case of a fluid containing a large number of scatterers. Because of the small deviation of the sound velocity from the average value, there is equal probability that this deviation may be either plus or minus; therefore, the energies add, and the intensity scattered becomes directly proportional to the average of the intensity scattered 


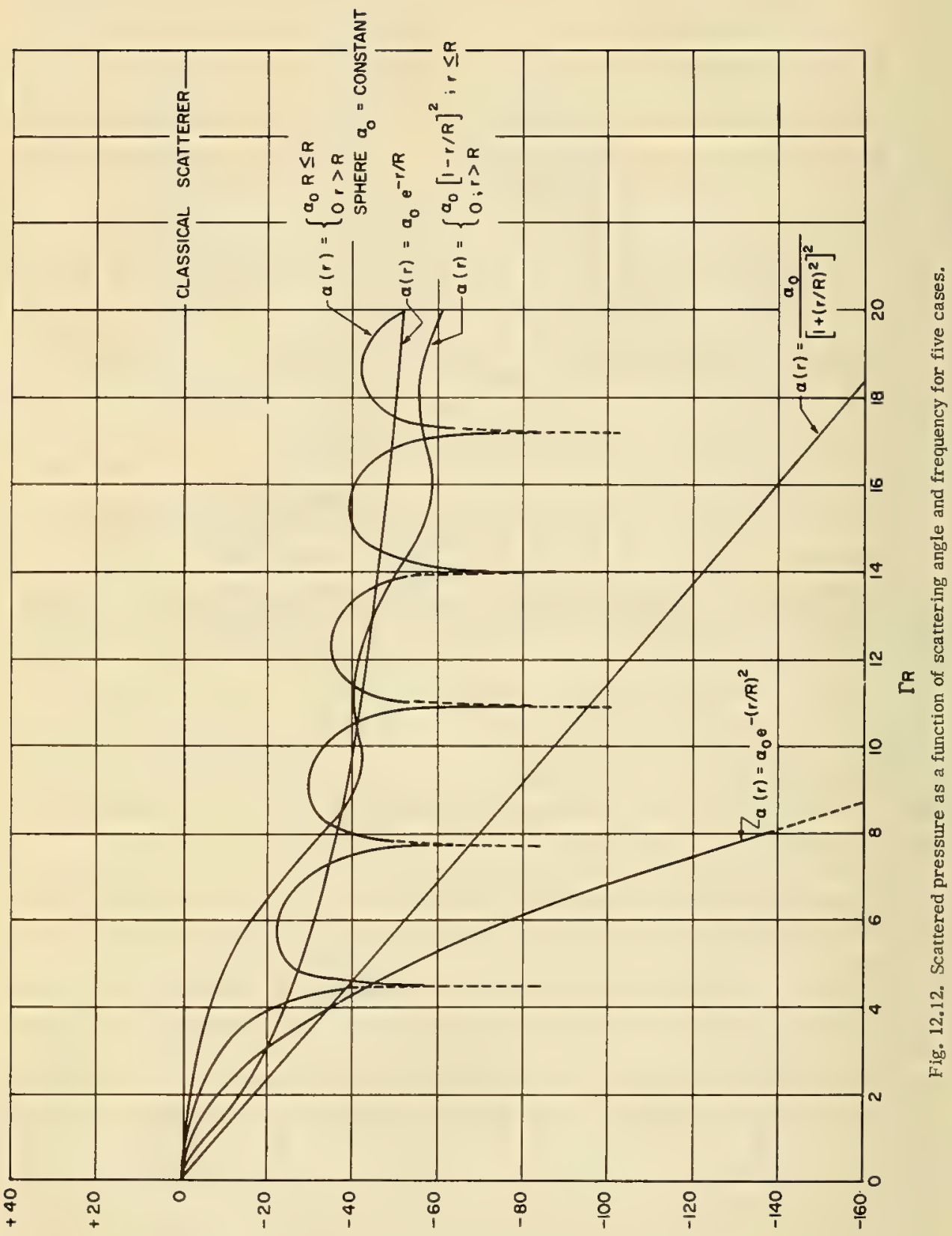

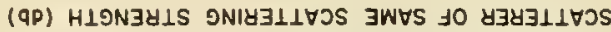

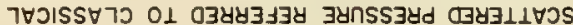


by a single scatterer and the number of scatterers. For constant density of the scatterers, the scattered intensity is proportional to the total scattering volume. A further elaboration of this procedure, however, is of little practical value since the true temperature field is of a statistical nature and cannot be described by a collection of individual scatterers.

\subsubsection{Scattering Described by the Correlation Function}

If the temperature field is not made up of simple temperature patches, or if a detailed description of the scattering inhomogeneities is not available, the scattering properties of the fluid can be described by the correlation function $R(\rho)$ or the space power spectrum $E(\kappa)$ of the sound velocity fluctuations, $\kappa$ being the Fourier space wave number. The Rayleigh integral can be transformed into a form based on the correlation function by multiplying $p_{\mathrm{sc}}$ by its conjugate complex value, $p_{s c}^{*}$, and taking the time average. If the variables are denoted by $\xi^{\prime}$, $\eta^{\prime}$, and $\zeta^{\prime}$ in the first integral and by $\xi^{\prime \prime}, \eta^{\prime \prime}$, and $\zeta^{\prime \prime}$ in the second, the product of the integrals can be written as

$$
\begin{aligned}
& \left\langle\left|p_{\mathrm{sc}}^{2}\right|\right\rangle=\left\langle p_{\mathrm{sc}} p_{\mathrm{sc}}^{*}\right\rangle \frac{k^{4} p_{0}^{2}}{4 \pi^{2} \tau_{0}^{2}} \int_{0}^{L} \int_{0}^{L} \int_{0}^{L}\left\langle a^{\prime} a^{\prime \prime}\right\rangle \\
& x e^{-j k\left[\left(\xi^{\prime}-\xi^{\prime \prime}\right) a+\left(\eta^{\prime}-\eta^{\prime \prime}\right) \beta+\left(\zeta^{\prime}-\zeta^{\prime \prime}\right)(\gamma-1)\right]} d \xi^{\prime \prime} d \eta^{\prime \prime} d \zeta^{\prime \prime} d \xi^{\prime} d \eta^{\prime} d \zeta^{\prime}
\end{aligned}
$$

where the integral sign denotes triple integrations over the primed and doubleprimed coordinates, and $L$ is the linear extension of the scattering medium in the three coordinate directions. If the medium is statistically homogeneous, the time average

$$
\overline{a^{\prime}\left(\xi^{\prime} \eta^{\prime} \zeta^{\prime}\right) a^{\prime \prime}\left(\xi^{\prime \prime} \eta^{\prime \prime} \zeta^{\prime \prime}\right)}=\alpha^{\overrightarrow{2} \underline{R}}(\rho)
$$

is a function of the distance $\rho$ of the two points $\xi^{\prime} \eta^{\prime} \zeta^{\prime}$ and $\xi^{\prime \prime} \eta^{\prime \prime} \zeta^{\prime \prime}$ only. This time average is then assumed to be the same as the space average $\left\langle a^{2}\right\rangle$ (Ergodic hy pothesis). The time average $\overline{a^{\prime} a^{\prime \prime}}$ has therefore been replaced by the space average $\left\langle a^{\prime} \alpha^{\prime \prime}\right\rangle$ in the above integral. The magnitude $\underline{R}(p)$ is called the normalized correlation function. If the two points are coincident, $\rho=0,\left\langle a^{\prime} a^{\prime \prime}\right\rangle=\left\langle\alpha^{2}\right\rangle$, and $R(0)=1$. If the points $\xi^{\prime} \eta^{\prime} \zeta^{\prime}$ and $\xi^{\prime \prime} \eta^{\prime \prime} \zeta^{\prime \prime}$ are far away, $a^{\prime}$ and $a^{\prime \prime}$ can be assumed to be independent of one another; then $\left\langle a^{\prime} a^{\prime \prime}\right\rangle=\left\langle a^{\prime}\right\rangle\left\langle a^{\prime \prime}\right\rangle=0$ because of a wellknown theorem of statistics. The above result can then be written as follows:

$$
\left\langle\left|p_{s c}^{2}\right|\right\rangle=\frac{k^{4} p_{0}^{2}}{2 \pi^{2} r_{0}^{2}}\left\langle\alpha^{2}\right\rangle \int_{0}^{L} \int_{-L}^{L} \underline{R}(\xi, \eta, \zeta) e^{j k[\alpha \xi+\beta \eta+(\gamma-1) \zeta]} d \xi d \eta d \zeta d \xi^{\prime} d \eta^{\prime} d \zeta^{\prime}
$$

where $\xi^{\prime}-\xi^{\prime \prime}, \eta^{\prime}-\eta^{\prime \prime}$, and $\zeta^{\prime}-\zeta^{\prime \prime}$ have been replaced by $\xi, \eta$, and $\zeta$. The $\xi^{\prime}, \eta^{\prime}, \zeta^{\prime}$ integration leads to a factor equal to the volume $r$ of the scattering region. The $\xi, \eta, \zeta$ integration is performed in exactly the same way as in Eq. (27). The result is

$$
\left\langle\mid o_{\mathrm{sc}}^{2}\right\rangle=\frac{k^{4} p_{0}^{2}}{r_{0}^{2}} \cdot \tau \frac{\left\langle a^{2}\right\rangle}{\pi \Gamma} \int_{-L}^{L} \underline{R}(\rho) \sin (\Gamma \rho) \rho d \rho
$$


The last integral is very similar to that which applies to the scattered pressure. The deviation, $a$, of the sound velocity from the mean value has been replaced by $\left\langle a^{2}\right\rangle \underline{R}(\rho)$. The number of scatterers is proportional to the volume $T$ of the scattering media, which therefore appears as factors in front of the integral.

The results obtained for a number of interesting cases are summarized in Table 12.II and plotted as curves in Fig. 12.13. They will be discussed later and compared with the corresponding results for the Kolmogorov case.

\subsubsection{Scattering Described by the Power Spectrum of the Sound Velocity Fluctuations}

Frequently the correlation function is unknown or it is too complex to be useful in integrals. It is then expedient to describe the fluctuations of the sound velocity of the medium by its Fourier space spectrum. The relation between the correlation function and the power spectrum $\phi\left(\kappa_{1}, \kappa_{2}, \kappa_{3}\right)$ of the velocity fluctuations is given by

$$
\left\langle a^{2}\right\rangle \underline{R}\left(\xi_{1}, \xi_{2}, \xi_{3}\right)=\iiint_{-\infty}^{\infty} \phi\left(\kappa_{1}, \kappa_{2}, \kappa_{3}\right) e^{j\left(\kappa_{1} \xi_{1}+\kappa_{2} \xi_{2}+\kappa_{3} \xi_{3}\right)} d \kappa_{1} d \kappa_{2} d \kappa_{3}
$$

If the fluctuations are isotropic, $\phi\left(\kappa_{1}, \kappa_{2}, \kappa_{3}\right)=\phi(\kappa)$, where $\kappa$ is the magnitude of the wave number, and

$$
\phi\left(\kappa_{1}, \kappa_{2}, \kappa_{3}\right) d \kappa_{1} d \kappa_{2} d \kappa_{3}=\phi(\kappa) 2 \pi \kappa^{2} d \kappa \sin \theta d \theta=\frac{E(\kappa)}{2} d \kappa \sin \theta d \theta
$$

where $E(\kappa) d \kappa=\Phi(\kappa) 4 \pi \kappa^{2} d \kappa(\kappa \geq 0)$. The exponent in the integral in Eq. (37) can be written as $k \cdot \varrho=\kappa \rho \cos \theta$, and the angular integration yields

$$
\underline{R}(\rho)=\int_{0}^{\infty} E(\kappa) \frac{\sin (\kappa \rho) d \kappa}{(\kappa \rho)}
$$

If this value is introduced into the scattering integral in Eq. (36),

$$
\left\langle\left|p_{\mathrm{sc}}^{2}\right|\right\rangle=\frac{k^{4} p_{0}^{2} r}{\pi t_{0}^{2}} \int_{0}^{\infty} \int_{-L}^{L} \frac{E(\kappa) \sin (\kappa \rho)}{\Gamma \kappa} \sin (\Gamma \rho) d \rho d \kappa
$$

and if $L$ is very large $(L \rightarrow \infty)$, the results becomes [11]

$$
\frac{\Delta p^{2}}{p_{0}^{2}}=\tau \frac{k^{4}}{2 r_{0}^{2}} \frac{E(\Gamma)}{\Gamma^{2}}=\tau \frac{k^{4}}{2 r_{0}^{2}} \frac{E\left[2 k \sin \left(\theta_{0} / 2\right)\right]}{\left[2 k \sin \left(\theta_{0} / 2\right)\right]^{2}}
$$

Thus, the scattered intensity becomes proportional to the spectral intensity $E(\kappa)$ of the temperature field for a wave number $\kappa=2 k \sin \left(\theta_{0} / 2\right)$. A similar result was obtained in the analysis of $\mathrm{X}$-ray scattering over forty years ago. The structure factor was found to be equal to the corresponding Fourier coefficient of the atomic structure.

For back scattering, $\theta_{0}=\pi$ and $\kappa=2 k$. The patch diameter may be crudely identified with half the space wavelength of the temperature fluctuations, i.e., $2 R=\lambda_{\mathrm{sp}} / 2=\pi / \kappa \quad($ where $\kappa=\pi / 2 R) ;$ backscattering is thus caused by patches of a diameter

$$
2 R=\frac{\pi}{\kappa}=\frac{\pi}{2 k}=\frac{\lambda_{\mathrm{sp}}}{2}
$$


TABLE 12.II

\begin{tabular}{|c|c|c|}
\hline Case & $\begin{array}{l}\text { Correlation function } R(\rho) \\
\text { or power spectrum } E(\kappa)\end{array}$ & $\begin{array}{l}\frac{\left\langle\left. p_{\mathrm{gc}}\right|^{2}\right\rangle}{\left|p_{0}\right|^{2}}=\frac{k^{4} r}{2 \pi r^{2}}\left\langle a^{2}>I\right. \\
\text { The above factor } I\end{array}$ \\
\hline 1 & $R(\rho)=e^{-\tau / R}$ & $8 \pi R^{3} /\left[1+(\Gamma R)^{2}\right]^{2}$ \\
\hline 2 & $R(\rho)=\mathrm{e}^{-(\boldsymbol{r} / R)^{2}}$ & $\pi^{3 / 2} R^{3} e^{-(T R / 2)^{2}}$ \\
\hline 3 & $R(\rho)=\left[1+(r / R)^{2}\right]^{-2}$ & $\pi^{2} R^{3} \mathrm{e}^{-\Gamma R}$ \\
\hline 4 & $\begin{array}{l}R(\rho)=(1-r / R)^{2} ; r \leq R \\
R(\rho)=0 ; r>R\end{array}$ & $\frac{4 \pi R^{3}}{(\Gamma R)^{4}}\left[4-\frac{6}{\Gamma R} \sin \Gamma R+2 \cos \Gamma R\right]$ \\
\hline 5 & $\begin{array}{l}E(\kappa)=\frac{a^{2}(m-1)}{\kappa_{0}}\left(\kappa / \kappa_{0}\right)^{-m} \\
\text { for } \kappa=k \sin (\theta / 2)>\kappa_{0}\end{array}$ & $\frac{\pi(m-1)}{\Gamma^{m+2}} \frac{\left\langle a^{2}\right\rangle k_{0}^{m-1}}{\left\langle\alpha^{2}\right\rangle}$ \\
\hline 6 & $E(\kappa)=K \kappa^{-5 / 3}$ & $\pi \frac{\tau K}{2 r^{2}} k^{1 / 3}\left[2 \sin \left(\theta_{0} / 2\right)\right]^{-11 / 3}$ \\
\hline
\end{tabular}

As the angle between the direction of incident sound and the direction of scattering becomes smaller, the scattered intensity is determined by larger patch sizes.

The spectral intensity of the thermal fluctuations increases with decreasing $\kappa$; scattering, therefore, increases toward the direction of the traveling wave. Scattering in the forward direction is described by the very large patches; the first-order approximation, Eq. (20), that was obtained by retaining only the linear powers in the Rayleigh integral, then breaks down. A better approximation (see Section 12.4) shows that the pressure scattered by very large patches is $90^{\circ}$ out of phase with the transmitted sound and affects the phase rather than the amplitude of the signal. The scattered pressure, then, is no longer coherent with the incident sound; the scattering integral degenerates to a mathematical correction that has no physical significance, and its square is no longer a meas ure of the divergence of the energy. The scattering integral $p_{\mathrm{sc}}$ primarily describes the change in phase because of the variation of the sound velocity in the medium.

If the power spectrum of the sound velocity fluctuations is of a Kolmogorov type, Eq. (41) leads to

$$
\frac{\left\langle p^{2}\right\rangle}{p_{0}^{2}}=\frac{\tau k^{4} K}{2 r_{0}^{2}} \frac{\left[2 k \sin \left(\theta_{0} / 2\right)\right]^{-5 / 3}}{\left[2 k \sin \left(\theta_{0} / 2\right)\right]^{2}}=\frac{\tau K}{2 r_{0}^{2}} k^{1 / 3}\left[2 \sin \left(\theta_{0} / 2\right)\right]^{-11 / 3}
$$

Scattering, then, increases with the cube root of the frequency. For forward scattering, $\theta_{0}=0$, and the above solution breaks down because of the assumption $L \rightarrow \infty$ in the evaluation of the integral in Eq. (40). If the $\rho$ integration is performed for finite $L$, the solution becomes

$$
\left\langle p_{\mathrm{sc}}^{2}\right\rangle=\frac{k^{4} p_{0}^{2} \tau}{\pi r_{0}^{2}} \int_{0}^{\infty} \frac{E(\kappa)}{\Gamma \kappa}\left[\frac{\sin (\kappa-\Gamma) L}{\kappa-\Gamma}-\frac{\sin (\kappa+\Gamma) L}{\kappa+\Gamma}\right] d \kappa
$$




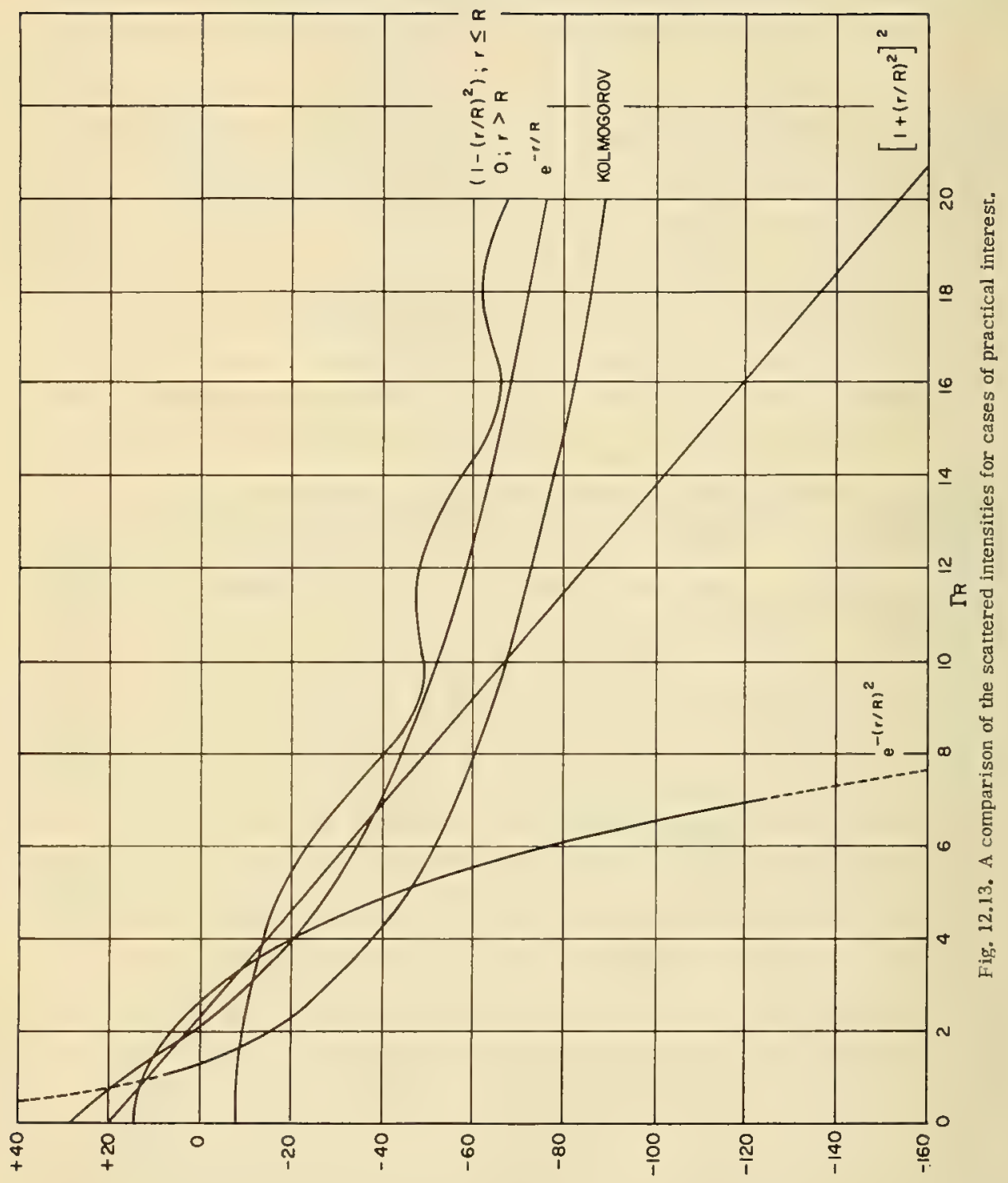


and the integral approaches a finite limit. It is possible to derive approximate expressions for this limiting value. However, there is not much point in doing so, since the computation would be equivalent to a computation of the phase change of the signal because of its different velocity in the medium.

In the Kolmogorov case, $m \doteq 5 / 3$ and the resulting solution is represented in the last line of Table 12.II; the Kolmogorov equivalent for the patch radius $R$ is the depth $h$ of the measurement, since the depth is the only dimensional parameter that is available and $\kappa_{0}=\pi / 2 h$. In the curve for the Kolmogorov case in Fig. 12.13, therefore, $R$ has to be interpreted as $h$.

Figure 12.13 compares the scattered intensities for the case of practical interest. The most frequently used correlation function is the exponential $e^{-r / R}$ since integrals based on this function can usually be evaluated. Also, the exponential correlation function seems to lead to good agreement with the experimental results in a great number of practical cases. Figure 12.13 shows that the exponential function generates about the same amount of scattering as the function $\underline{R}(\rho)=1-(\rho / R)^{2}, \mathrm{r}<R$. Unfortunately, the exponential correlation function leads to divergent integrals for the ray-theory limit of scattering. The curve for the Kolmogorov case is slightly broader; therefore, the corresponding correlation function is slightly steeper. This slight increase in steepness in the Kolmogorov case seems to be sufficient to ensure convergence of all the scattering integrals. Frequently, the Gaussian correlation function is used as a substitute for the exponential one to enforce convergence of the scattering integrals. Figure 12.13 shows that the Gaussian function represents a poor approximation to cases where an exponential or a Kolmogorov correlation function would be expected to apply.

\subsection{FLUCTUATION OF THE TRANSMITTED SIGNAL}

\subsubsection{Elementary Theory}

The amplitude of the transmitted signal fluctuates continuously because of the focusing and defocusing of the incident sound by the continuously moving patches of inhomogeneity and because of interferences caused by the scattered pressure. These signal fluctuations can be computed with the aid of the Rayleigh integral.

The transmitted sound is given by the vector sum of the directly transmitted pressure and the scattered pressure. Therefore, the phase of the scattered pressure must be taken into account. Forvery large scatterers and at great distances from the scatterers, the scattered pressure is found to be displaced in phase by $90^{\circ}$ with respect to the transmitted sound and affects only the sound velocity in the medium. The change in amplitude is then a second-order effect. For large scattering patches, it can be shown that the second-order terms have to be retained in the exponent of the scattering integral [Eq. (20)] to obtain the correct value of the scattered pressure phase. This fact complicates the computation of the transmitted signal considerably.

Up to the present, all the computations that have been made have been based on a Gaussian correlation function, or on the assumption of a definite patch size, both of which are very unrealistic assumptions. Since the mathematical theories of the transmitted signal fluctuation are so extremely complicated, they cannot 
be reviewed here; this has been done elsewhere $[12,13,58]$. However, it is possible to derive practically the same results by elementary considerations exclusively.

In Section 12.3, forward scattering was shown to be practically independent of the model assumed for the scatterers. Therefore, we can expect to obtain all the necessary information about the fluctuations of the amplitude and the phase of the transmitted signal by assuming scatterers that are as simple as possible, such as parallel discs having sound velocities slightly different from the mean value and thicknesses and radii of $d x$ and $R$, respectively. The scattered pressure, then, is given by the integral

$$
p_{\mathrm{sc}}=p_{0} \frac{k_{0}^{2} e^{-j k x^{\prime}}}{2 \pi} \int \frac{\alpha \mathrm{e}^{-j k r}}{r} d x 2 \pi \rho d \rho
$$

Integration can be performed in cylindrical coordinates setting

$$
r^{2}=\rho^{2}+x^{2} \quad r d r=\rho d \rho
$$

If $x^{\prime}$ is the distance between the sound source and the scatterer, and $x$ the distance of the point of observation, the pressure scattered by such a disc is given by

$p_{\mathrm{sc}}=p_{0} d x k^{2} e^{-j k x^{\prime}} \int_{\sqrt{\left(x-x^{\prime}\right)}}^{\sqrt{\left(x-x^{\prime}\right)^{2}+R^{2}}} a e^{-j k t} d r=p_{0} d x k^{2} \frac{e^{-j k x^{\prime}} a}{-j k} e^{-j k \sqrt{\left(x-x^{\prime}\right)^{2}+R^{2}}}-e^{-j k\left(x-x^{\prime}\right)}$

The disc radius $R$ can be considered as small in comparison to the range. Taylor development of the exponent then leads to the result

$$
p_{s c}=j p_{0} d x k e^{-j k x} \alpha\left[1-e^{-j k R^{2} / 2\left(x-x^{\prime}\right)}\right]
$$

If $R$ is very large, the second term in the brackets of Eq. (48) can be set equal to zero (as can be proved by the assumption of a very small attenuation). The scatterer pressure

$$
\delta p_{s c}=-j k a d x p_{0}
$$

then has a phase lagging $90^{\circ}$ behind that of the incident sound velocity of the slab.

The scattered pressure is a maximum if the second term in the bracket of Eq. (48) is -1 ; that is, if

$$
\frac{k R^{2}}{2\left(x-x^{\prime}\right)}=\frac{k R^{2}}{2 r}=\pi
$$

or

$$
R^{2}=\lambda r \text { or } k R^{2}=2 \pi r
$$

where $r$ has been written for $x-x^{\prime}$. If this condition is fulfilled, the disc will have exactly the same area as half the central zone in the Huygens zone construction.

The average pressure that is scattered in the forward direction by such a disc is given by

$$
p_{\mathrm{sc}}=2 j k \alpha R p_{0}
$$




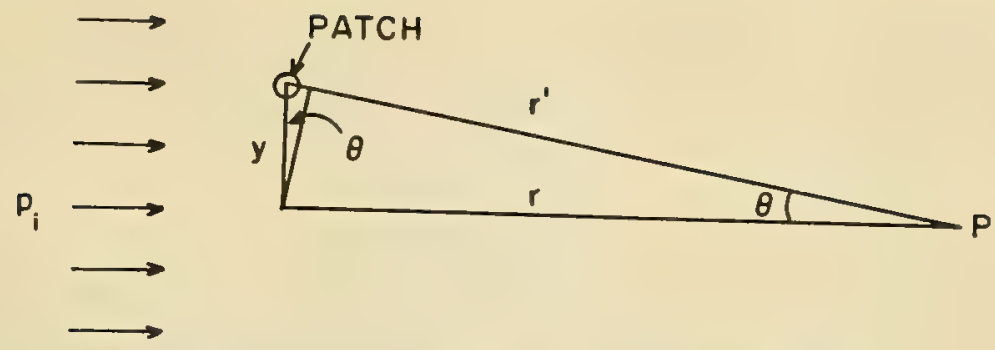

Fig. 12.14. Path difference because of disalignment of patch.

where $2 R$ has been substituted for the thickness $d x$ of the disc. In reality, the scattering discs are not all arranged in a line above the axis of propagation but are randomly distributed. Because of the large diameter of the scatterers in comparison to the acoustic wavelength, the scattered pressure is highly collimated in the forward direction and practically all the scattered energy is contained within a cone of an apex angle [see Eq. (30)]:

$$
\theta \cong \frac{2.5}{k R}
$$

where $R$ is the patch radius. The patches that contribute to the intensity at the point of observation are therefore contained within a similar cone having its apex at the point of observation. Because of the misalignment of the patches, the scattered sound reaches the point of observation with different path differences (Fig. 12.14), which, on the average, are not greater than

$$
r^{\prime}-r=\sqrt{y^{2}+r^{2}}=\tau\left(1+\frac{y^{2}}{2 r^{2}}+\cdots\right)-r \doteq \frac{y^{2}}{2 r}=\frac{r^{2} \theta^{2}}{2 r} \cong \frac{1}{2} \frac{r}{k R^{2}}
$$

where $\theta=1 / k a$ has been assumed as the average value of the angle $\theta$. The pressure scattered by the average patch is therefore out of phase by an angle

$$
\psi=k \frac{r}{k^{2} R^{2}}=\frac{r}{k R^{2}}
$$

with respect to the pressure scattered by a patch that is located on the axis of the beam of sound. The fluctuation of the transmitted signal is given by the component of the scattered pressure that is in phase with the incident sound. This component is

$$
p_{\mathrm{sc}} \sin \psi=p_{\mathrm{sc}} \sin \frac{r}{2 k R^{2}}=2 k \alpha R p_{0} \sin \frac{r}{2 k R^{2}}
$$

The deviations of the sound velocity in the various patches are statistically dis tributed. Therefore, the squares of the contributions of the various patches add, and the average fluctuation of the signal becomes proportional to the square root of the number of patches $n=\sqrt{r / 2 R}$ :

$$
\Delta p=\sqrt{\pi} p_{\mathrm{sc}}=\sqrt{2} k a \sqrt{R \mathrm{r}} \sin \left(\frac{\mathrm{r}}{2 k R^{2}}\right)
$$

At low frequencies, or for long ranges or small patches, the argument of 
the sine in the last expression may amount to many multiples of $\pi$. The phase of the contributions from the various patches of inhomogeneity may, therefore, be considered to be random too, and the sine may be replaced by its rms value, $1 / \sqrt{2}$. The result, then, reduces to

$$
V_{p}=a k \sqrt{R L}
$$

Practically the same formula has been derived by Mintzer [30] on the basis of a Gaussian correlation function. The range of validity of this solution is usually called the "interference" or "wave-theory" range of forward scattering.

As the frequency increases, the scattered radiation becomes more and more concentrated in the forward direction, and the phase differences between the extreme contribution left and right of the axis of the main beam become negligibly small. Essentially, then, forward scattering is a focusing or defocusing effect. Within this range, $L / k R^{2} \ll 1$; and the sine above can be replaced by its argument

$$
\frac{p_{\mathrm{sc}}}{p_{i}}=\left\langle\alpha^{2}\right\rangle^{3 / 2} \sqrt{2 R L} \frac{L}{2 R^{2}} \doteq\left\langle\alpha^{2}\right\rangle^{1 / 2}\left(\frac{L}{R}\right)^{3 / 2}
$$

Except for an insignificant factor, the last expression is identical with the Bergmann formula for ray-theory limit of scattering [16]. Within the ray-theory range, the fluctuations of the signal are mainly caused by focusing and defocusing effects. They increase with the $3 / 2$ power of the range and are independent of the frequency. For large values of the range (see Fig. 12.15), the solution passes over into the wave-theory result. The phase differences between the various scattered rays accumulate, and the focusing and defocusing are destroyed by the random phases of the scattered pressure contributions; the signal fluctuations are due entirely to interferences. The transition between ray- and wavetheory range occurs when

$$
L=L_{0} \approx k R^{2}
$$

If this condition is fulfilled, the diffraction cones generated at the boundaries of the scattering patches cover the entire cross section of the beam. The receiver becomes surrounded by an interference region, and the scattering patches as seen from the receiver have radii approximately equal to the central zone in the Huygens zone construction; patches of this diameter are particularly effective in generating signal fluctuation.

\subsubsection{Computation of Amplitude and Phase of Fluctuations for Spherical Patches}

The fluctuations of the amplitude and the phase of the transmitted signal depend not only upon the magnitude but also upon the phase of the scattered pressure. The computations in Section 12.3 seem to lead to the result that the scattered pressure is always in phase with the incident sound. This conclusion, however, is correct only for distances from the scatterer that are very large compared to the diameter of the scatterer, so that the square and the higherorder terms in the exponent of the Rayleigh integral can be neglected. At smaller distances the scattered pressure may have to be $90^{\circ}$ out of phase, as has been demonstrated for the infinitely large scattering slab [Eq. (49)] or any phase between zero and a multiple of $2 \pi$, as has been found for the finite scattering 


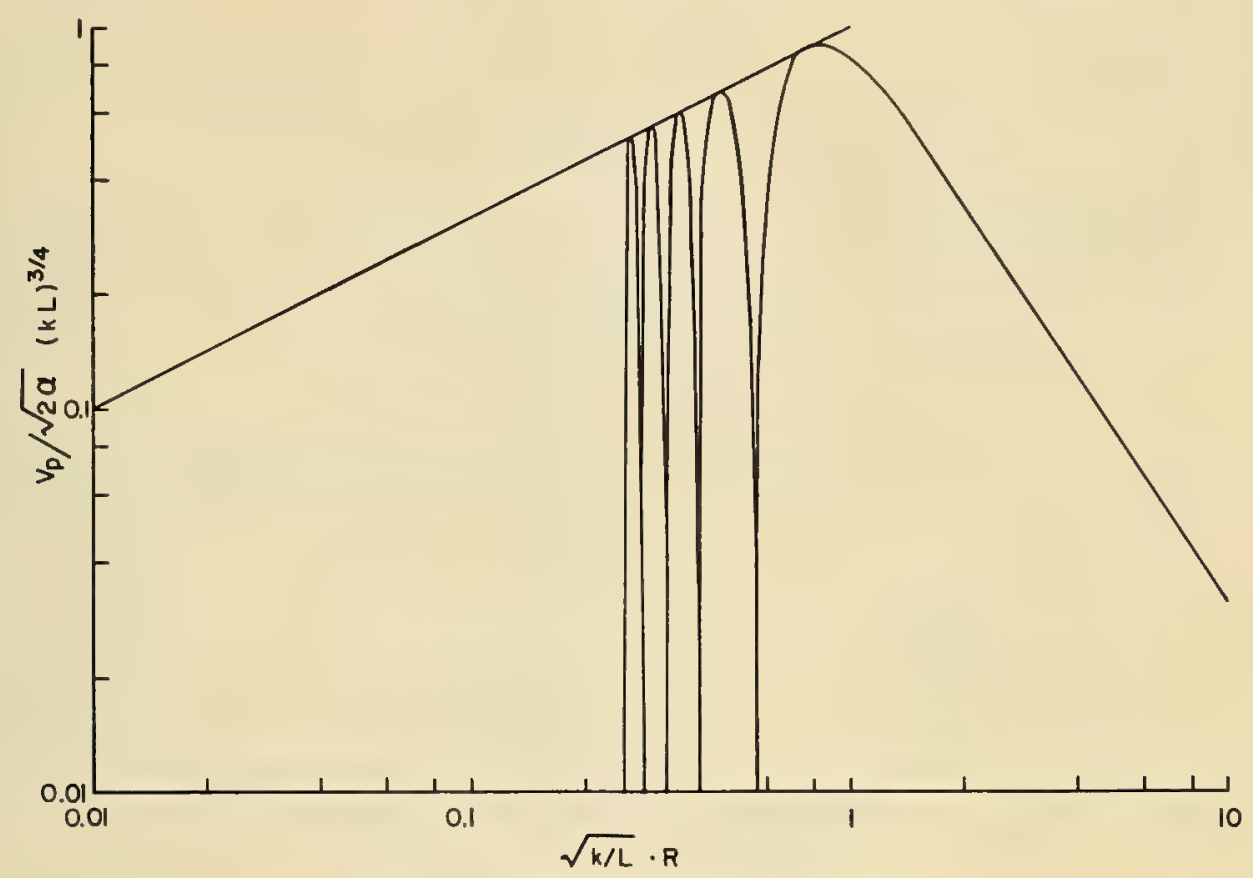

Fig, 12.15. Scattering as a function of patch size.

slab. For a computation of the fluctuations of the transmitted signal, at least the squares have to be retained in the exponent of the Rayleigh integral.

In computing the amplitude of the transmitted signal, the receiver may be assumed to lie on the $\xi$ axis at a distance $L$ from the sound source. If the squares are retained in the exponent in the Rayleigh integral, the following expression is obtained for the mean-square scattered pressure after a laborious computation $[58, \mathbf{1 2}]$ :

$$
\frac{\left\langle\Delta p^{2}\right\rangle}{p_{0}^{2}}=\frac{1}{2}\left(l_{1}-I_{2}\right)
$$

The magnitudes $I_{1}$ and $t_{2}$ are given by

$$
I_{\nu}=\frac{k^{3}\left\langle a^{2}\right\rangle}{2 \pi} \int_{-0}^{L} \int_{-\infty}^{\infty} a_{\nu}^{2} \sin \frac{a_{\nu}^{2} \rho^{2}}{2} R(r) d \eta d \xi d \zeta_{1} d \zeta_{2}
$$

For a parallel incident beam of sound, and for a Gaussian correlation function

$$
\begin{aligned}
\left\langle a^{2}>e^{-(z / R)^{2}}\right. & =\left\langle a^{2}>e^{-\left[\left(\zeta_{1}-\zeta_{2}\right)^{2}+\rho^{2}\right] / R^{2}}\right. \\
l_{1} & =\sqrt{\pi}<a^{2}>k^{2} R L
\end{aligned}
$$

and

$$
I_{2}=\sqrt{\frac{\pi}{4}}<a^{2}>k^{3} R^{3} \tan ^{-1} \frac{4 L}{k R^{2}}
$$




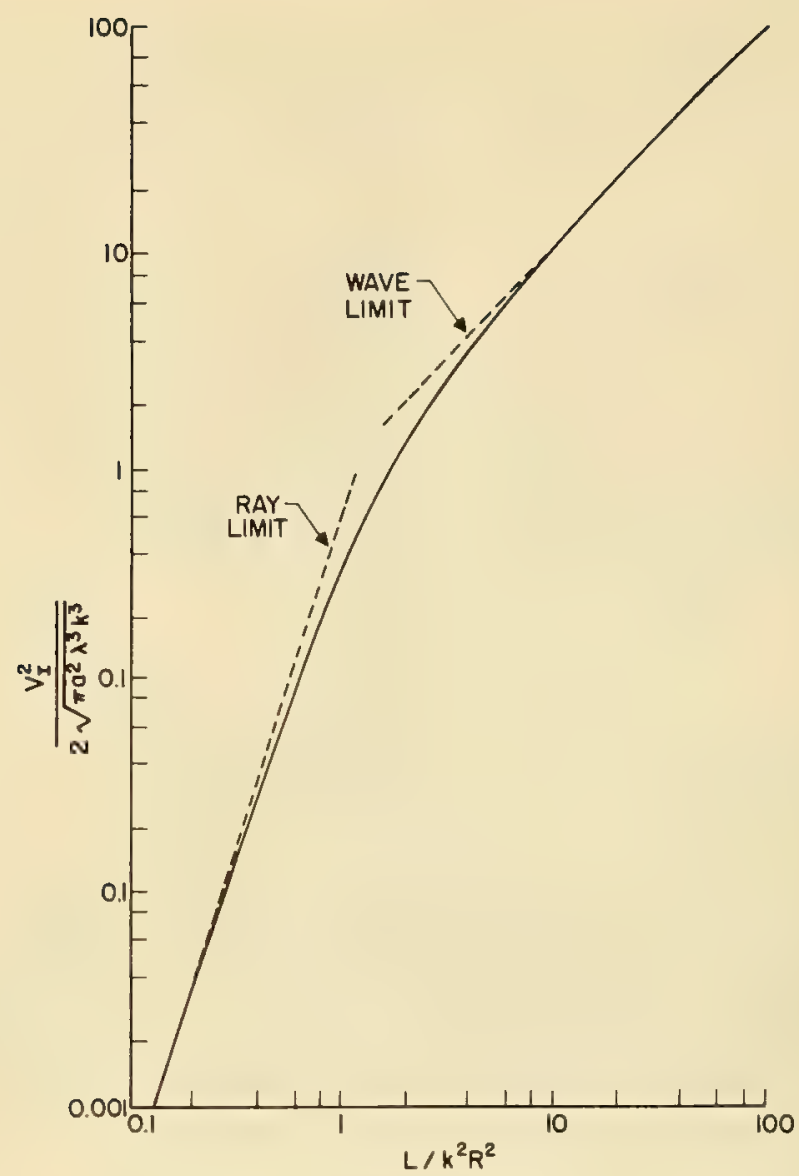

Fig. 12.16. Scattering as a function of frequency [51].

With the abbreviation

$$
D=\frac{4 L}{k R^{2}}
$$

the mean-square scattered pressure becomes

$$
V_{p}^{2}=\frac{\left\langle p_{\mathrm{se}}^{2}\right\rangle}{p_{i}^{2}}=\frac{1}{2}\left(I_{1}-I_{2}\right)=\sqrt{\frac{\pi}{2}}\left\langle a^{2}\right\rangle k^{2} R L\left(1-\frac{1}{D} \tan ^{-1} D\right)
$$

This solution is represented in Fig. 12.16. For large values of range, this solution passes over in the wave-theory expression for interference scattering.

\subsubsection{Continuous Patch Distribution}

In the general cases, the patches of inhomogeneity are not adequately represented by a simple Gaussian correlation function. The two experimental Gaussian terms yield a poor representation of the experimental results. It then becomes expedient to replace the correlation function by the power spectrum 
of the velocity fluctuations. The relation between the correlation function and the power spectrum is

$$
R(r)=\int_{-\infty}^{+\infty} \int_{-\infty}^{+\infty} \int_{-\infty}^{+\infty} \phi(\kappa) e^{J\left(\kappa_{1} \xi+\kappa_{2} \eta+\kappa_{3} \zeta\right)} d \kappa_{1} d \kappa_{2} d \kappa_{3}
$$

The scattering integrals have been represented in cylindrical coordinates. It is expedient, therefore, to express the correlation function in cylindrical coordinates too as

$$
R(r)=\int_{-\infty}^{\infty} \int_{-\infty}^{\infty} \int_{0}^{2 \pi} \phi(\kappa) e^{j \kappa \rho \cos \theta+j \kappa_{1} \xi} d \theta \kappa^{\prime} d \kappa^{\prime} d \kappa_{1}
$$

where $\theta$ is the angle between the $\rho$ vector and the projection of the wave-number vector on a plane perpendicular to the $\xi$ axis and

$$
\begin{gathered}
\kappa_{3}=\kappa^{\prime} \cos \theta \quad \kappa_{2}=\kappa^{\prime} \sin \theta \quad \kappa_{1}=\kappa_{1} \\
\kappa^{\prime 2}=\kappa_{2}^{2}+\kappa_{3}^{2} \quad \kappa^{2}=\kappa_{1}^{2}+\kappa^{\prime 2}
\end{gathered}
$$

The $\theta$ integration can then be performed:

$$
R(\rho)=2 \iint_{0}^{\infty} \phi(\kappa) \cos \left(\kappa_{1} \xi\right) \kappa^{\prime} d \kappa^{\prime} d \kappa_{1} 2 \pi J_{0}\left(\kappa^{\prime} \rho\right)
$$

where the lower limit of integration of $\kappa_{1}=-\infty$ has been replaced by zero, and correspondingly, the exponential by the cosine.

The integral $I_{\nu}$ [Eq. (61)] becomes

$$
l_{\nu}=2 k^{2} \int_{0}^{L} \int_{0}^{L} \int_{0}^{\infty} \int_{0}^{\infty} \phi(\kappa) \cos \left(\kappa_{1} \xi\right) \kappa^{\prime} d \kappa^{\prime} d \kappa_{1}\left(a_{\nu}^{2} \sin \frac{a_{\nu}^{2} \rho^{2}}{2} J_{0}\left(\kappa^{\prime} \rho\right) \rho d \rho\right) d \xi_{1} d \xi_{2}
$$

where

$$
\xi=\xi_{1}-\xi_{2} \text { and } a_{1}^{2}=k /\left(\xi_{1}-\xi_{2}\right) \text { and } a_{2}^{2}=k\left[\left(2 L-\left(\xi_{1}-\xi_{2}\right)\right]\right.
$$

The $\rho$ integral parenthesis is known $[10,11]$. Its value is $\cos \left(\kappa^{\prime 2} / 2 a_{\nu}^{2}\right)$. Hence

$$
I_{\nu}=2 k^{2} \int_{0}^{L} \int_{0}^{L} \int_{0}^{\infty} \int_{0}^{\infty} \phi(\kappa) \cos \left(\kappa_{1} \xi\right) \kappa^{\prime} d \kappa^{\prime} d \kappa_{1} \cos \frac{\kappa_{1}^{2}}{2 a_{\nu}^{2}} d \xi_{1} d \xi_{2}
$$

The value of these integrals depends greatly on the small-wave-number power spectrum of the fluctuations of the sound velocity where the Kolmogorov law no longer applies. For a square law as a first approximation rather than a $5 / 3$ power law, and on the assumption that the spectrum is zero at wave numbers $\kappa<\kappa_{0}$,

$$
E(\kappa)=\begin{array}{ll}
K \kappa^{-2} & \text { for } \kappa>\kappa_{0} \\
0 & \text { for } \kappa<\kappa_{0}
\end{array}
$$

The integrals become soluble and integration yields

$$
l_{1}=\frac{\hbar^{2} L}{16} \quad l_{2}=0
$$




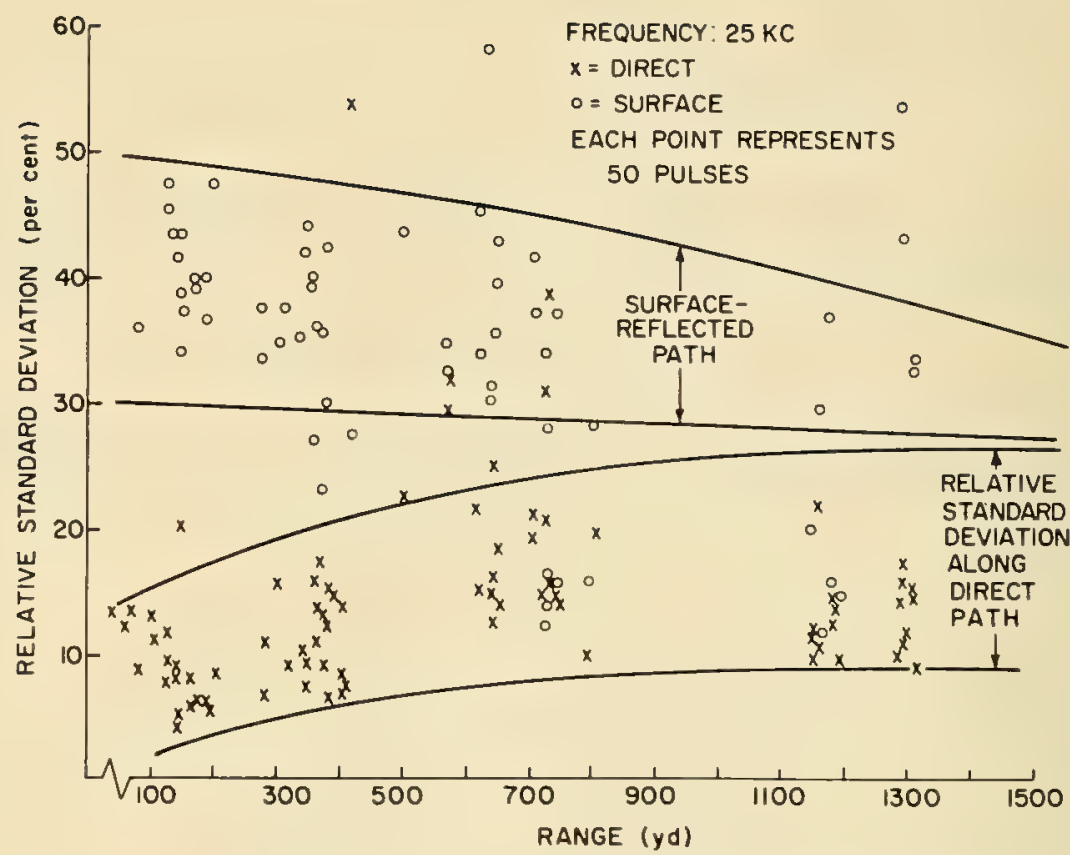

Fig. 12.17. Fluctuation measurements made by the Ordnance Research Laboratory at The Pennsylvania State University.

where $K=\left\langle\alpha^{2}\right\rangle \kappa_{0}$ and

$$
V_{p}^{2} \approx V_{\text {phase }}^{2} \approx \frac{\left\langle a^{2} \kappa_{0}\right.}{32} L
$$

The coefficient of variation $V_{p}$ is thus proportional to the root of the range and independent of the frequency. The $L$-range dependence seems to be certain, no matter what power has been assumed in the power spectrum; the frequency dependence, however, in only approximate. A still closer approximation to the Kolmogorov law (assuming $m=2$ in the integrand so that integration becomes possible and $m=5 / 3$ in the factor in front of the integral) leads to the result that scattering is proportional to the cube root of the frequency which is in good agreement with the experimental result.

\subsection{EXPERIMENTAL RESULTS}

\subsubsection{Standard Deviation of the Transmitted Signal}

The fluctuations of the transmitted signal are usually described by their standard deviation and by their distribution law. The standard deviation is defined as the ratio of the square root of the mean-square deviation from the mean value and the mean value of the pressure

$$
V_{p}=\sigma=\frac{\left\langle\left(p-p_{0}\right)^{2}\right\rangle^{1 / 2}}{p_{0}}=\frac{\left\langle\Delta p^{2}>1 / 2\right.}{p_{0}}
$$

The square of this quantity is called the relative coefficient of variation. 
Signal fluctuations at 25 and $60 \mathrm{kc}$ have been measured by the Ordnance Research Laboratory; Fig. 12.17 is an example of the data obtained during the month of July under a wide range of conditions. This figure indicates the fluctuation of the signal, traveling by way of the direct path and the surfacereflection path, as a function of range. The lines indicate the trend of the data.
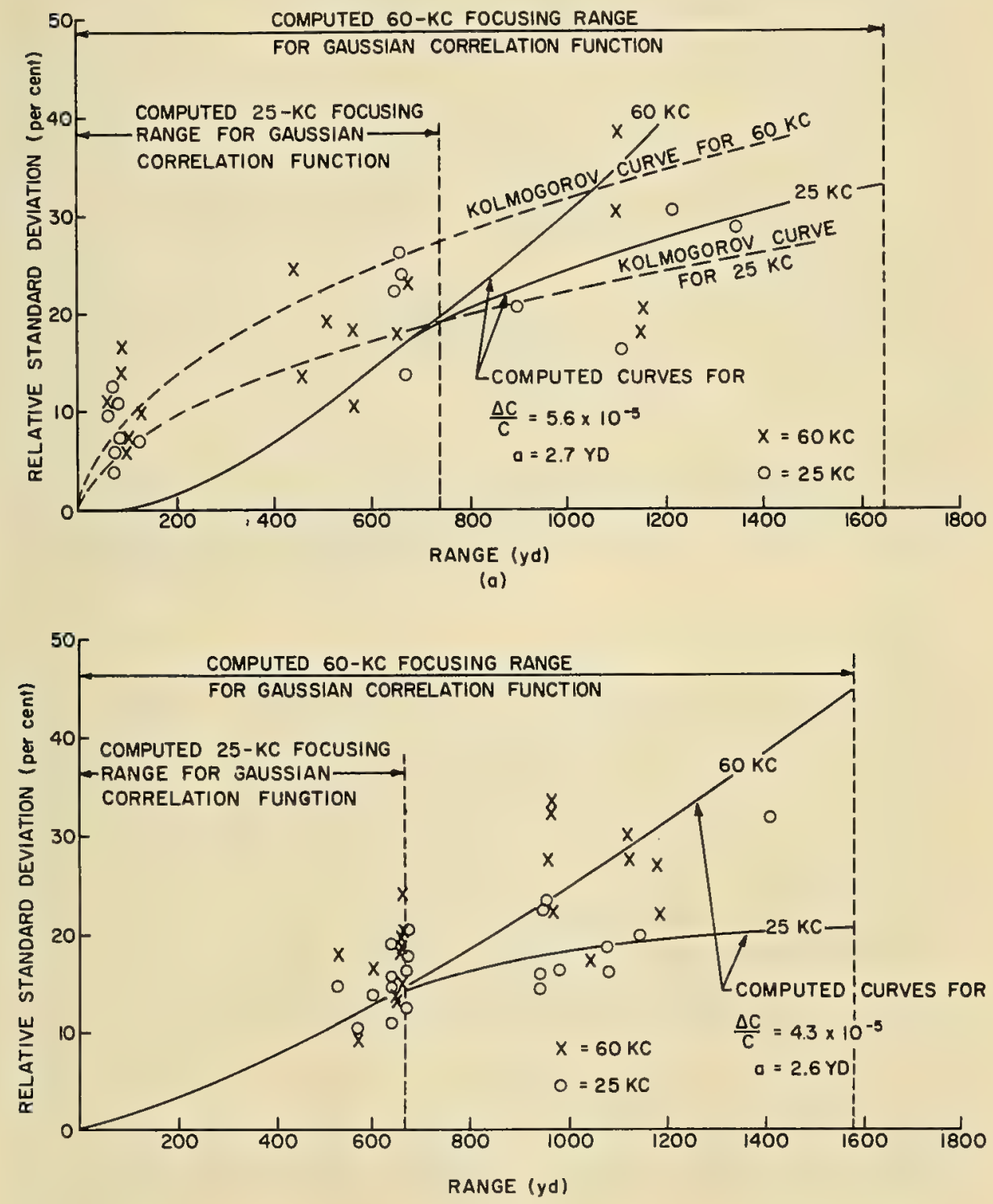

(b)

Fig. 12.18. Direct-signal fluctuations measured during one day; (a) in January, (b) in March. 


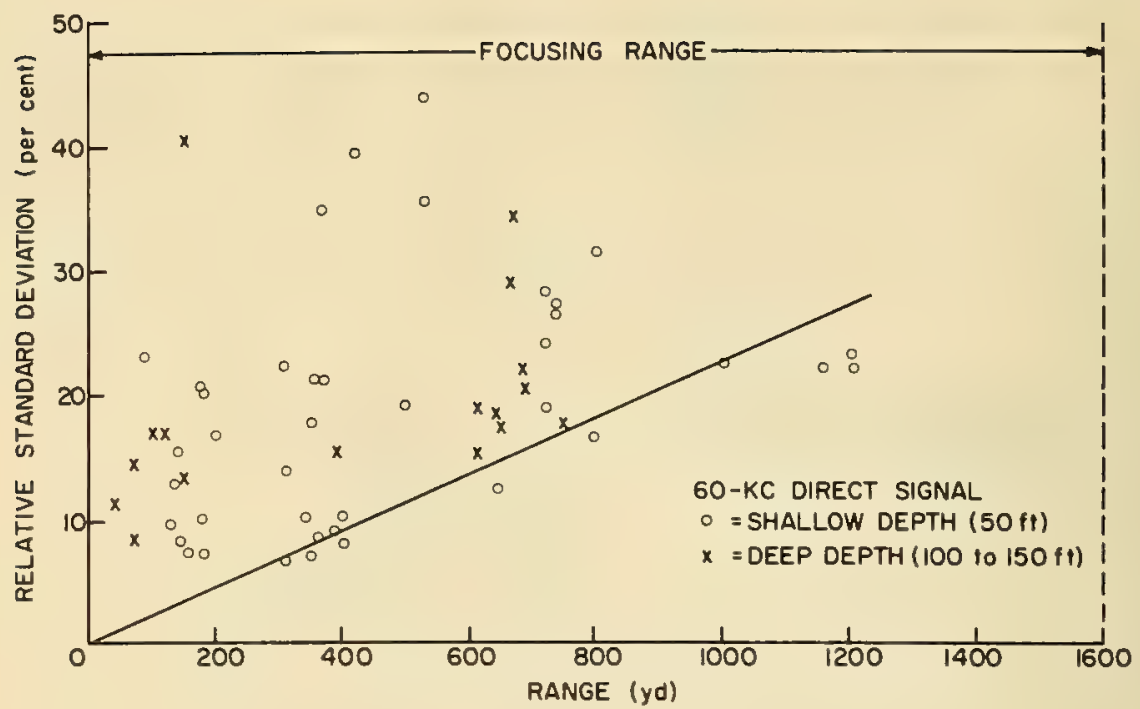

(o)

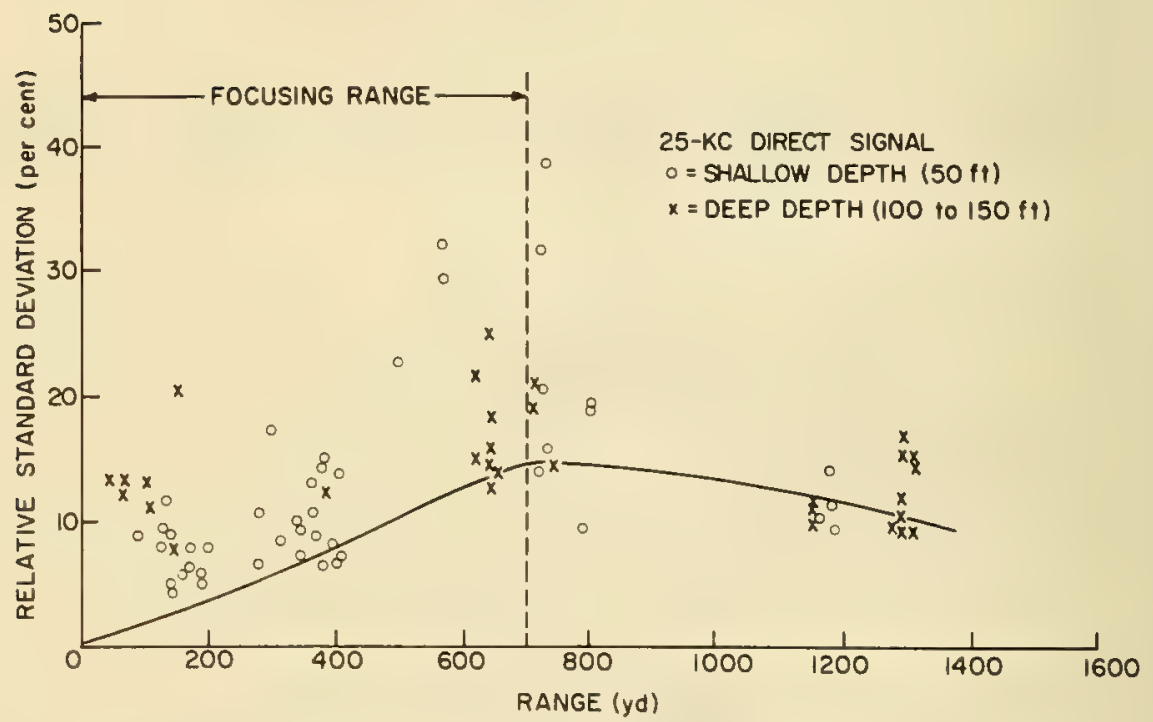

(b)

Fig. 12.19. Direct-signal fluctuations measured in one day in July; (a) at $60 \mathrm{kc}$, (b) at $25 \mathrm{kc}$.

As can be seen, the variability of the surface reflection is relatively high, $52 \%$ at short distances, but tends to decrease somewhat with increasing range. The variability of the direct transmission increases with distance, until at long ranges the same variability may be expected for the two paths. The slight decrease in variability of the surface-reflected signal may be attributed to the fact that the sea surface becomes more like a perfect mirror and less like a 
diffuse scatterer within the small grazing angles existing at the longer ranges. At long ranges, where the signal is specularly reflected, the fluctuations in both the direct and the reflected signal have the same causes; at short ranges, the diffuse reflection from the surface is an additional and more important source of variability.

Figure 12.18 shows two plots of the direct-signal fluctuations for one day. The solid lines represent the solution that would be computed if a Gaussian correlation function were assumed. By assuming a suitable value of the correlation distance $R$ and of the rms fluctuation of the temperature, lines can be drawn that pass through the measured points with reasonable accuracy. To obtain a better fit for the low values of range, the correlation function would have to be assumed to consist of two exponential terms. Figure 12.19 shows the results of measurements made in July, when measurements of the temperature microstructure were made simultaneously. The results of the measurements make it possible to test the prediction of Gaussian correlation functions and the Kolmogorov theory.

The natural way to deal with scattering is to assume a continuous distribution of patch sizes from the beginning, such as is given by the Kolmogorov law.

This theory leads to considerably better agreement with the experimental results than the procedures based on the experimental or Gaussian correlation function. The values predicted on the basis of this theory are of the right magnitude, and the frequency and range variations are reproduced in a better manner than with the Gaussian correlation-function theory. Future measurements will, therefore, have to be based exclusively on a Kolmogorov-type power spectrum of the sound velocity fluctuations.

\section{REFERENCES}

1. R. J. Urick and C.W. Searfoss, "The Microthermal Structure of the Ocean Near Key West, Florida," Part I-Description, Naval Research Laboratory Report No. S-3392 (December 7, 1948); Part II-Analysis, Nayal Research Laboratory Report No. S-3444 (April 12, 1949).

2. D. C. Whitmarsh, E. J. Skudrzyk, and R. J. Urick, "Forward Scattering of Sound in the Sea and Its Correlation with the Temperature Microstructure," J. Acoust. Soc. Am., Vol. 29, 1124-1143 (1957).

3. A. N. Kolmogorov, "Local Turbulence Structure in an Incompressible Liquid for Very Large Reynolds Numbers," Doklady Akad. Nauk SSSR, Vol. 30, 299-303 (1941).

4. W. Heisenberg, "Zur Statistischen Theorie der Turbulenz," Z. Tech. Phys., Vol. 124, 628 (1948).

5. D. I. Blokhinstev, Acoustics of an Inhomogeneous Moving Medium (Gostekhizdat, Moscow, 1946).

6. Lande, Geiger Scheel, Handbuch der Physik, Bd, XX, 453.

7. L. C. Kober, "Störung und Störbefreiung von Rückstrahlung in Wellenfeldern," 217-225; "Rückstrạhlung von Reflexions Körpern in Wellenfeldern," 217, Osterr. Ing.-Arch. (1951).

8. C.K. Bachelor, Theory of Homogeneous Turbulence (Cambridge University Press, 1956) p. 123; the formula in the text is obtained by correcting for the scalar nature of the temperature fluctuations.

9. Lord Rayleigh, The Theory of Sound (Dover Publications, Inc., New York, 1945).

10. Wolfgang Gröbner and Nikolaus Hofreiter, Integraltafel (Springer-Verlag, Vienna, 1949).

11. Bierens De Haan, Nouvelles Tables D'Integrales Definies (G. E. Stechert and Co., New York, 1939).

12. E. J. Skudrzyk, "The Scattering of Sound in an Inhomogeneous Medium," Pennsylvania State Univ. (May 10,1960 ).

13. V. I. Tatarski, Wave Propagation in a Turbulent Medium (McGraw-Hill Book Co., Inc., New York, 1961).

14. A. M. Obukhov, "On the Scattering of Sound in a Turbulent Flow," Doklady Akad. Nauk SSSR, 30, 611 (1941).

15. V.A. Krasilnikov, "On the Propagation of Sound in a Turbulent Atmosphere, " Doklady Akad. Nauk SSSR, Vol. 47, 486 (1945).

16. P. G. Bergmann, "Propagation of Radiation in a Medium with Random Inhomogeneities," Abstract, Phys. Rev., Vol. 69 (1946).

17. V. A. Krasilnikov, "On Amplitude Fluctuations of Sound Propagating in a Turbulent Atmosphere," Doklady Akad. Nauk SSSR, Vol. 58, 1353 (1947). 
18. C. L. Pekeris, "Note on the Scattering of Radiation in Inhomogeneous Media," Phys. Rev., Vol. 71 (1947).

19. A. M. Yaglom, "On the Local Structure of the Temperature Field in a Turbulent Flow," Doklady Akad. Nauk SSSR, Vol. 69, 743 (1949). German translation in Sammelband zur Statistischen Theorie der Turbulenz, 141 (Akademie-Verlag, Berlin, 1948).

20. V.A. Krasilnikov, "On Fluctuations of the Angle of Arrival in the Phenomenon of Twinkling of Stars," Doklady Akad. Nauk SSSR, Vol. 65, 291 (1949).

21. V. A. Krasilnikov and K.M. Ivanov-Shyts, "Some Experiments on the Propagation of Sound in the Atmosphere," Doklady Akad, Nauk SSSR, Vol. 67, 639 (1949).

22. A. M. Obukhov, "Structure of the Temperature Field in a Turbulent Flow," Izvest. Akad. Nauk SSSR, Ser. Geograf. Geofiz., Vol. 13, 58 (1949). German translation in Sammelband zur Statistischen Theorie der Turbulenz, 127 (Akademie-Verlag, Berlin, 1958).

23. H. G. Booker and W. E. Gordon, "A Theory of Radio Scattering in the Troposphere, " Proc. I.R.E., Vol. 38, 401-412 (1950); see also, Scatter Propagation Issue, Vol. 43 (1955).

24. M. J. Sheehy, "Transmission of 24-kc Underwater Sound from a Deep Sour ce," J. Acoust. Soc. Am., Vol. 22, 22-24 (1950).

25. M. L. Levin, "Sound Scattering in a Slightly Inhomogeneous Medium," Zhur. Tekh. Fiz., Vol, 21, 937939 (1951).

26. L. Liebermann, "The Effect of Temperature Inhomogeneities in the Ocean on the Propagation of Sound," J. Acoust. Soc. Am., Vol, 23, 563-570 (1951).

27. S. I. Krechmer, "Investigations of Microfluctuations of the Temperature Field in the Atmosphere," Doklady Akad. Nauk SSSR, Vol, 84, 55 (1952).

28. H. Staras, "Scattering of Electromagnetic Energy in a Randomly Inhomogeneous Atnosphere," J. Appl. Phys., Vol. 23, 10 (1952).

29. V. A. Krasilnikov, "On Phase Fluctuations of Ultrasonic Waves Propagating in the Layer of the Atmosphere Near the Earth," Doklady Akad. Nauk SSSR, Vol. 88, 657 (1953).

30. D. Mintzer, "Wave Propagation in a Randomly Inhomogeneous Medium," Part I, J. Acoust. Soc. Am., Vol. 25, 922-927; Part II, Vol. 25, 1107-1111 (1953).

31. A. M. Obukhov, "On the Influence of Weak Atmospheric Inhomogeneities on the Propagation of Sound and Light," Izvest. Akad. Nauk SSSR, Ser. Geofiz., No. 2, 155 (1953). German translation in Sammelband zur Statistischen Theorie der Turbulenz, 157 (Akademie-Verlag, Berlin, 1958).

32. V.I. Tatarski, "On the Theory of Propagation of Sound Waves in a Turbulent Flow," Zhur. Eksp. i Teoret. Fiz., Vol, 25, 74 (1953).

33. V.1. Tatarski, "Phase Fluctuations of Sound in a Turbulent Medium," Bull. Acad. Sci. USSR, Geophysics Series, No. 3, 252-258 (1953).

34. D. Mintzer, "Wave Propagation in a Randomly Inhomogeneous Medium," Part III, J. Acoust. Soc. Am., Vol. 26, 186-190 (1954).

35. J. Van Isacker, "The Analysis of Stellar Scintillation Phenomena, " Quart. J. Roy. Meteorol. Soc., Vol. 80,251 (1954).

36. F. Villars and V.F. Weisskopf, "The Scattering of Electromagnetic Waves by Turbulent Atmospheric Fluctuations," Phys. Rev., Vol. 94, 232 (1954).

37. L. A. Chernov, "Correlation of Amplitude and Phase Fluctuations for Wave Propagation in a Medium with Random Irregularities," Soviet Physics-Acoustics, Vol. 1, 94-101 (1955).

38. J.H. Chrisholm, P. A. Portman, J. T. de Bettencourt, and J.F. Roche, "Investigations of Angular Scattering and Multipath Properties of Tropospheric Propagation of Short Radio Waves Beyond the Horizon," Proc. I.R.E., Vol, 43, 1317 (1955).

39. D. S. Potter and S. R. Murphy, "Acoustic Fluctuation," Part 1, Univ. of Washington, Applied Physics Laboratory, Report APL/UW/TE/55-12 (April 7, 1955).

40. G. Keller, "Relation Between the Structure of Stellar Shadow Band Patterns and Stellar Scintillation," J. Opt. Soc. Am., Vol. 45, 845 (1955).

41. F.H. Sager, "Fluctuations in Intensity of Short Pulses of 14.5-ke Sound Received from a Source in the Sea," J. Acoust. Soc. Am., Vol. 27, 1092 (1955).

42. R.W. Stewart, H. L. Grant, W. N. English, and C.D. Maunsell, "The Fluctuation of Sound Transmitted in the Ocean," Pacific Naval Laboratory, Esquimalt, British Columbia, Interim Report No. PIR-7 (August, 1955).

43. L. A. Chernov, "Correlation Properties of a Wave in a Medium with Random Inhomogeneities," Soviet Physics-Acoustics, Vol. 2, 221-227 (1956).

44. V.A. Krasilnikov and A.M. Obukhov, "Propagation of Waves in a Medium with Random Inhomogeneities of the Index of Refraction," Soviet Physics-Acoustics, Vol. 2, 103-110 (1956).

45. R. A. Silverman, "Turbulent Mixing Theory Applied to Radio Scattering," J. Appl. Phys., Vol. 27, 699 (1956).

46. V.I. Tatarski, "Microstructure of the Temperature Field in the Layer of the Atmosphere Near the Earth," Izvest. Akad. Nauk SSSR, Ser. Geofiz., No. 6, 689 (1956).

47. V. I. Tatarski, "Pulsations of the Amplitude and Phase of a Wave which is Propagated in Weakly Inhomogeneous Atmosphere," Doklady Akad, Nauk SSSR, Vol. 107, 245-248 (1956).

48. G. R. Garrison, S. R. Murphy, and D. S. Potter, "Underwater Acoustic Transmission Variations Caused by Thermal Layers," Abstract, J. Acoust, Soc, Am., Vol, 29, 186 (1957).

49. V.N. Karavainikov, "Fluctuations of Amplitude and Phase in a Spherical Wave," Soviet Physics-Acoustics, Vol. 3, 175-186 (1957).

50. E.R. Pinkston, M. J. Pollack, and J. R. Smithson, "Sound Fluctuations and Related Oceanographic Parameters in an Estuary," Chesapeake Bay Institute, Johns Hopkins Univ. Report No, 57-6 (September, 1957). 
51. D. S. Potter and S. R. Murphy, "On Wave Propagation in a Random Inhomogeneous Medium," J. Acoust. Soc. Am., Vol. 29, 197 (1957).

52. E. J. Skudrzyk, "Scattering in an Inhomogeneous Medium," J. Acoust. Soc. Am., Vol. 29, $50-60$ (1957).

53. V.1. Tatarski, "Microinhomogeneities of the Temperature Field and Fluctuation Phenomena of Waves Propagating in the Atmosphere," Dissertation, Akust. Inst. Akad. Nauk SSSR, Moscow (1957).

54. R. Bolgiano, "The Role of Turbulent Mixing in Scatter Propagation," IRVE Trans, Anten, Prop., AP-6, 161 (1958).

55. B. A. Suchkov, "Amplitude Fluctuations of Sound in a Turbulent Medium," Akust. Zhur., Vol, 4, 85 (1958). 56. F. Villars and V.F. Weisskopf, "On the Scattering of Radio Waves by Turbulent Fluctuations of the Atmosphere," Proc. I.R.E., Vol. 43, 1232 (1958).

57. M. A. Kallistratova, "Experimental Investigation of the Scattering of Sound in the Turbulent Atmosphere," Doklady Akad. Nauk SSSR, Vol. 125, 69 (1959).

58. L. A. Chernov, Wave Propagation in a Random Medium (McGraw-Hill Book Co., Inc., New York, 1960). 

LECTURE 13

\title{
AMBIENT NOISE IN THE SEA AND ITS MEASUREMENT
}

\author{
P. M. Kendig \\ Ordnance Research Laboratory \\ The Pennsylvania State University \\ University Park, Pennsylvania \\ U.S.A.
}

\subsection{AMBIENT NOISE}

\subsubsection{Introduction}

It has been found that acoustic waves afford the best means for detecting underwater objects in the ocean. However, in any detection system there is an interfering source of noise or signal that sets a lower limit to the level of the signal it is desired to detect.

There are several different types of interference. Very often this limitation is the self-noise of the detection system, which may be caused by the hydrophone, the electronic system, or the vehicle with which the observations are made. In an active sonar detection system, the underwater object is detected by means of an echo due to transmitted acoustic energy reflected from the object. However, some of the transmitted acoustic energy is returned to the listening hydrophone, even if the sound waves do not encounter a target, because sound is scattered back to the hydrophone from the surface, the bottom, and even from scatterers in the medium itself. This may be, and often is, the principal source of interference.

There is still a third general type of interference, the only one to be dis cussed here, known as the ambient noise. This is the interfering noise that is due to natural conditions or sources in the ocean. It will be considered a property of the medium itself at the time and place of observation, irrespective of the hydrophone and the platform used to observe it. It is the composite noise from all sources present in a given environment; desired signals and noise inherent in the measuring equipment and platform are excluded. The ambient noise level is expressed in terms of the level of an "equivalent" isotropic noise field at the observing hydrophone. Such an equivalent field is one that would produce, at the output of the measuring system, a response equal to that produced by the noise actually present. Following is a list of the commonly recognized sources of ambient noise:

1. Thermal noise due to molecular agitation of the medium is especially 
important at high frequencies in deep water, since it limits the hydrophone threshold above about $50 \mathrm{kc}$

2. Sea-surface noise associated with waves is the dominant source of ambient noise in open-sea deep water in a frequency range from about 100 cps to $50 \mathrm{kc}$ and varies in level with sea state

3. Biological noise caused by snapping shrimp and other soniferous sea creatures occurs locally in shallow water when these organisms are present

4. Man-made noise, including that from distant ships and from industrial sources in and near busy harbors, is often the dominant source below $1 \mathrm{kc}$

5. Rain noise in and near storms

6. Shore noise produced by surf on coasts or reefs

7. Flow noise caused by current flow over rocky bottoms and hydrostatic pressure changes produced by waves

8. Terrestrial noise caused by earthquakes, volcanoes, microseisms, and distant storms

Noises from sources (7) and (8) are normally of very low frequencies.

\subsubsection{Deep Water-Thermal and Surface Noise}

Since there exists such a variety of sources of ambient noise, one would expect a considerable variability in level as a function of frequency and conditions related to the production of the ambient noise and indeed this is the case. It is found that in any given region of the spectrum, one or more sources are dominant and the output of the remainder is so low by comparison as to be entirely insignificant. Thus, for example, the thermal agitation due to molecular motion in the water provides a lower limit for the ambient noise at all frequencies, but it is dominant only in the upper frequency region, above about 50 to $200 \mathrm{kc}$, depending upon the sea state. The thermal noise level that sets this limit has been shown by R. H. Mellen [1] to be

$$
L=-115+30 \log f
$$

in $\mathrm{db}$ relative to $1 \mathrm{~d} / \mathrm{cm}^{2}$ in a 1 -cps band at a temperature of about $15^{\circ} \mathrm{C}$, and at a frequency $f$ in $\mathrm{kc}$. In the complete absence of all other sources of noise, this is the equivalent noise pressure that would be detected by an omnidirectional hydrophone with an efficiency of $100 \%$.

At lower frequencies, roughly 1 to $50 \mathrm{kc}$ and higher, depending upon sea state the most important source of noise in the ocean is sea-surface noise, which appears to depend upon the speed of the wind and on sea state. The first extensive measurements of ambient noise over this frequency region appear to have been made during World War II. The results of the measurements made during these studies were summarized in a series of curves known as the Knudsen curves [2]. In these curves, shown in Fig. 13.1, the spectrum of deep-water noise is plotted as a function of sea state and frequency. An extrapolation of the experimental data extends them to the thermal noise limit, which is also indicated.

The principal characteristic of deep-water ambient noise is its variation with sea state and wind speed. At all sea states it is noted that the spectrum level decreases about $5 \mathrm{db} /$ octave. It is also found that the intensity of ambient 


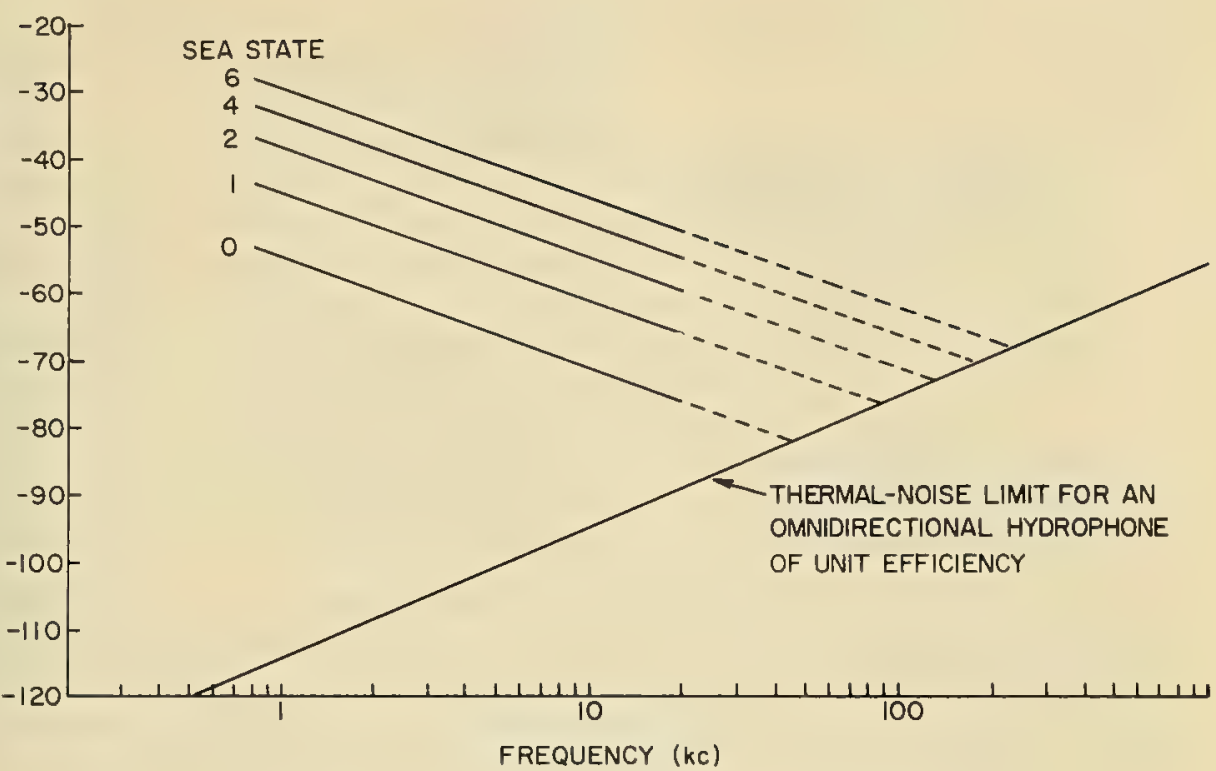

Fig. 13.1. Deep-water ambient noise levels (Knudsen curves).

noise over this frequency range varies approximately as the 1.8 power of the wind speed. This variation indicates that the noise originates at the surface, but very little is known about the mechanism by which it is generated. The breaking of waves may be the primary contributor to this noise; but, for sea states below those at which waves break, some other mechanism for the production of noise must be postulated. In the absence of peculiar occurrences such as biological noise cr the noise due to falling rain, this type of noise is dominant. At lower frequencies, somewhere below $1 \mathrm{kc}$, the variation with frequency is decreased and the levels are no longer greatly dependent upon wind force. However, at still lower frequencies, the ambient levels appear to increase more rapidly as the frequency is lowered.

The variation of ambient levels with depth in deep water was obtained by Lomask and Frassetto [3] with the Bathyscaphe Trieste. These measurements, which covered only the low frequency range up to about $300 \mathrm{cps}$, indicated a rather strong depth dependence except in calm seas. The level was shown to decrease by almost $16 \mathrm{db}$ from the surface to a depth of $3000 \mathrm{~m}$ for a sea state of 2 and a relatively narrow band centered at $68 \mathrm{cps}$. The levels decreased with depth at other frequencies in this range but to a lesser extent, the decrease being only about $6 \mathrm{db}$ at $10 \mathrm{cps}$. The measurements also indicated some stratification effects, but it did not seem that these were associated with the sound channel.

Wenz [4] has recently shown a very interesting variation of low-frequency acoustic ambient noise levels in the ocean. His studies reveal a periodic variation in underwater acoustic ambient noise levels at frequencies between 20 and $100 \mathrm{cps}$ which has the following characteristics: 
1. Frequency. The fundamental frequency is seven cycles perweek, i.e., one cycle per day. The fundamental period agrees closely with the solar day.

2. Amplitude. Amplitudes are relatively small in most cases, the overall change in level being 1.5 to $5 \mathrm{db}$, and the phenomenon is often masked by other sounds such as ship traffic noise. However, changes of 10 to $20 \mathrm{db}$ at one location during the summer solstitial period were observed. This is an extreme case.

3. Phase. Maxima occurred at approximately midnight, local-zone standard time, and did not shift from day to day as do the tidal maxima.

4. Wave form. The harmonic content varies with location. A second harmonic (14 cycles per week) was relatively strong and so phased as to give a second maximum at approximately noon, local time. In some cases, as many as seven harmonics were found.

The periodic variation has been observedeach season of the year. No marked seasonal dependence was evident except at one location. Here the variation was a maximum at approximately the time of the summer solstice.

The periodic variation has been observed at six locations, including sites in both deep and shallow water, both near to and remote from shore, and extending over three time zones $\left(45^{\circ}\right.$ longitude).

Wenz has no explanation for this variation, although he has apparently explored every possibility that comes to mind. He has considered wind-speed time patterns, tidal changes, variation in system sensitivity of performance, ship traffic noise, biological noise, and seismic activity. None of these seem to provide any logical clues. The close agreement of the period of these variations with the period of the earth's rotation suggests the possibility of an extraterrestrial connectior. For example, the intensity of cosmic radiation has a diurnal variation with a maximum near noon. But what mechanisms can account for an increase in noise level when there is a decrease in the intensity of cosmic radiation?

\subsubsection{Shallow Water-Man-Made and Biological Noise}

In contrast with the deep-water ambient noise levels, which are comparatively well defined, the ambient noise levels in coastal waters vary widely: For this reason, only very rough predictions of expected ambient levels can be made. However, the deep-water levels do define a lower limit for the shallow-water levels.

Except for those that also occur in deep water, the two most important sources of noise in coastal waters are soniferous marine life and man-made disturbances, such as those caused by ships and industrial installations on the adjoining shores.

The noise' produced by marine organisms has been studied extensively since the beginning of World War II. More recently, the occurrence and acoustic characteristics of soniferous life in the Atlantic and Pacific oceans have been studied and the results summarized by M.D. Fish $[5,6]$. Many types of marine organisms are known to produce sound, but it has not been possible to associate 


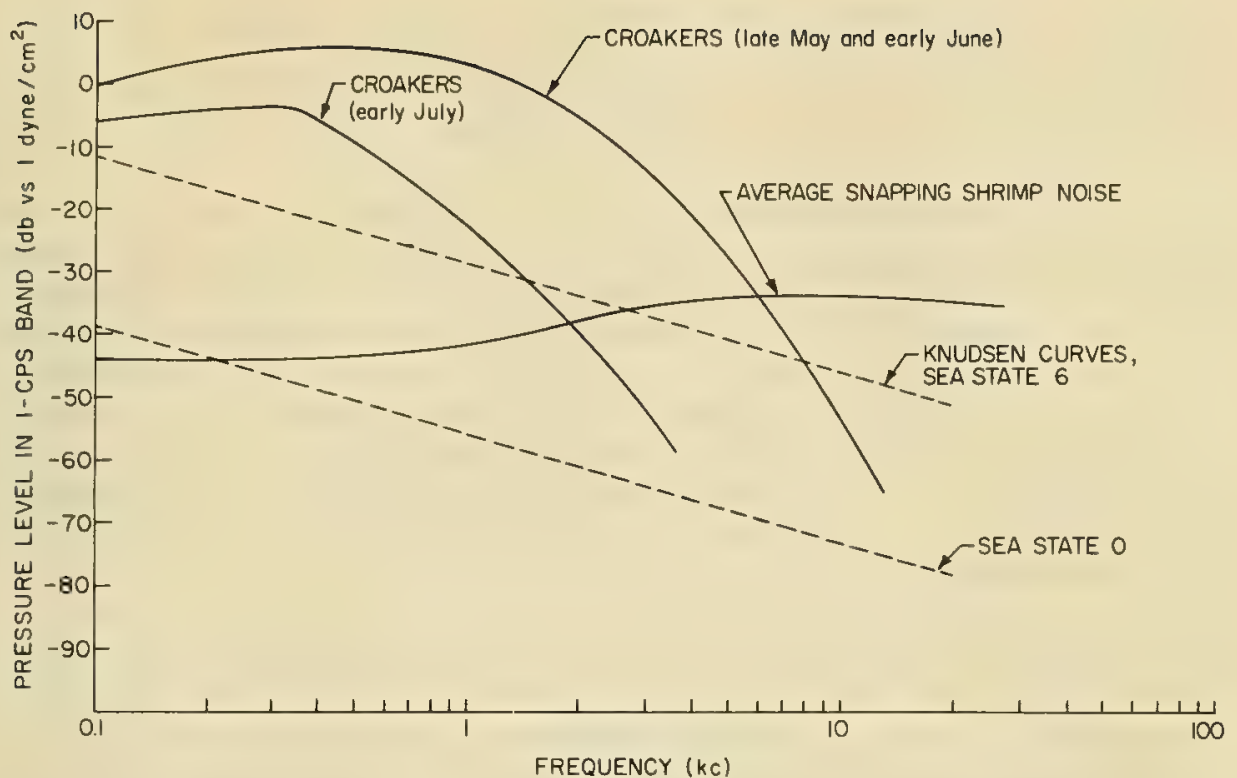

Fig. 13.2. Ambient noise levels produced by croakers and snapping shrimp.

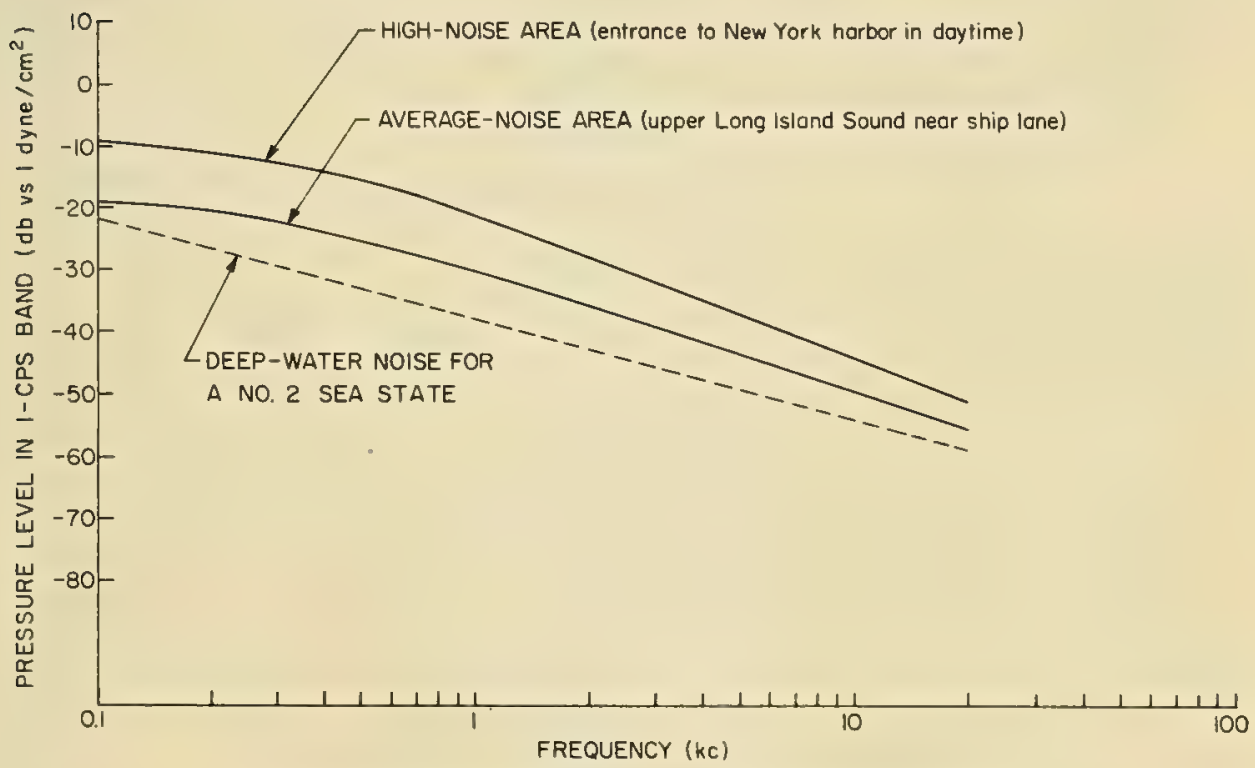

Fig. 13.3. Ambient noise levels in the presence of ship sounds.

all observed sounds with the particular marine animals that make them. Two types of marine animals, snapping shrimp and croakers, are known to produce a chorus of sound that can mask desired signals. Shrimp are common in shallow, 
hard-bottomed tropical waters, whereas croakers occur predominantly in Chesapeake Bay and other East Coast bays of the United States. Figure 13.2 shows typical ambient noise levels produced by croakers and snapping shrimp. It can be seen that biologically produced noise does extend into the frequency range of interest to sonar.

Man-made noise may be predominant in busy harbors, shipping lanes, and many coastal locations, particularly at lower frequencies. During the war, many measurements were made of ambient levels in bays and harbors in the United States and Great Britain and near some Pacific Islands. The results of these measurements are summarized in NDRC Division 6 Report No. 3 of the Survey of Underwater Sound. The outstanding characteristic of this coastal ambient noise is its great variability from place to place in the same harbor and from time to time at the same place. Figure 13.3 shows typical ambient noise spectra for both a noisy and an average location in comparison with the deep-water ambient noise level for a No. 2 sea state.

\subsubsection{Rain Noise}

Falling rain, hail, and snow may be expected to increase ambient noise levels. Teer [7] observed an increase of from -62 to $-50 \mathrm{db}$ relative to $1 \mathrm{~d} / \mathrm{cm}^{2}$ in a 1 -cps band in a No. 2 sea state at $19.5 \mathrm{kc}$ due to steady rain.

Heindsmann, Smith, and Arneson [8] observed the noise changes caused by the passage of two heavy rainstorms over a hydrophone system located $4 \mathrm{ft}$ above the bottom in $120 \mathrm{ft}$ of water near the eastern end of Long Island. During these observations, a recording was made and analyzed for the fall of 0.52 in. of rain in $90 \mathrm{~min}$; the results are shown in Fig. 13.4.

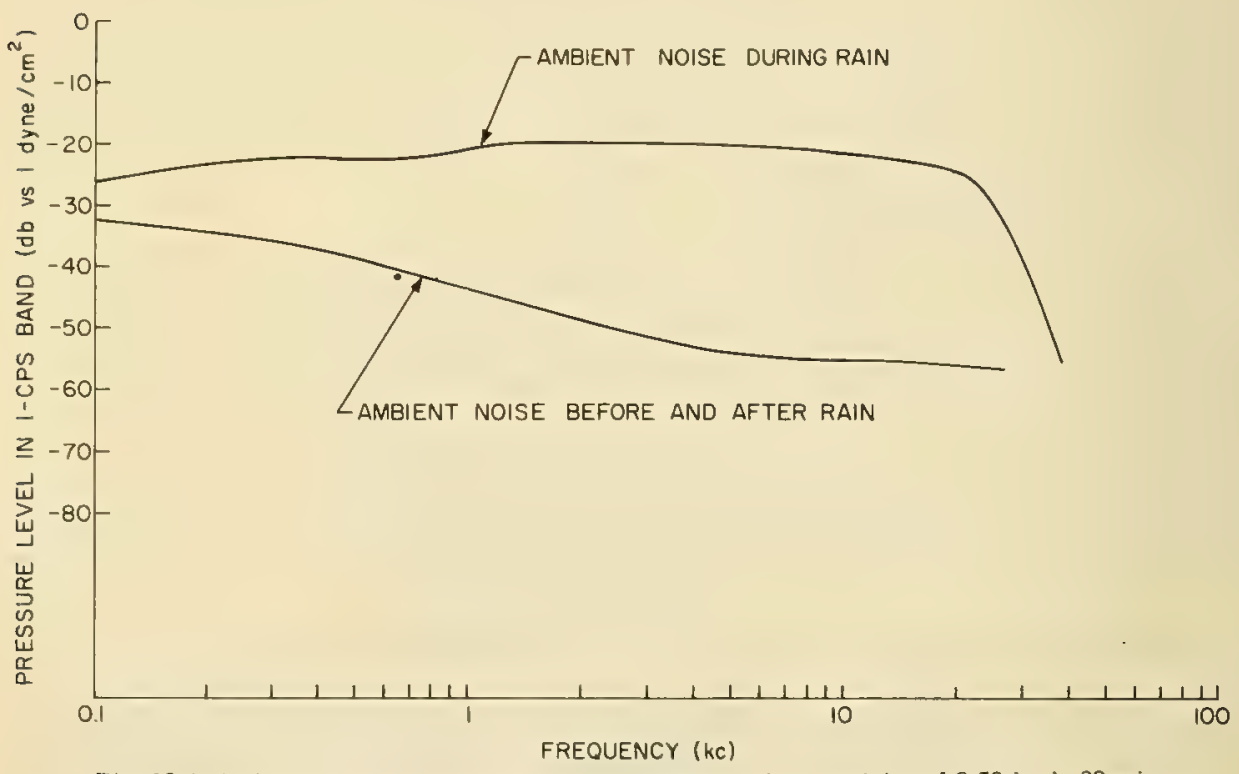

Fig. 13.4. Ambient noise levels produced by a rainstorm that precipitated $0.52 \mathrm{in}$, in $90 \mathrm{~min}$. 
The available information indicates that, at a frequency of $10 \mathrm{kc}$, rain can raise the average underwater noise level 15 to $25 \mathrm{db}$ above the level indicated by the Knudsen curves for a given sea state.

\subsubsection{Directional Characteristics}

It has been commonly assumed that ambient noise is isotropic; that is, the orientation of a directional hydrophone would have no effect on the detected level. However, R. J. Urick [9] has shown that for sounds originating at the surface, the variation of sound level with tilt angle will depend upon the directional characteristics of the hydrophone and the laws governing the radiation from the sea surface. He assumed the radiation to be distributed according to some power $n$ of the cosine of the angle of radiation measured from the vertical. This analysis showed that for $n$ equal to 0,1 , and 2 , the ambient levels measured by a searchlight hydrophone will vary with tilt angle.

The directional characteristics of ambient noise at ultrasonic frequencies in water depths of $600 \mathrm{ft}$ and hydrophone depths of $100 \mathrm{ft}$ have been studied at the Ordnance Research Laboratory of The Pennsylvania State University. Meas urements made with a searchlight hydrophone indicate that the ambient noise level measured in a vertical plane has two different characteristic distributions that appear to be functions of sea state. The data at sea states 1 and higher show the ambient noise level to be highest in the quadrant between the horizontal axis and the vertical axis, normal to the surface. The data taken at sea-state zero show that the peak in the ambient noise level usually occurs in the horizontal plane. A few exceptions to this do show a distribution at zero sea state similar to that obtained at the higher sea states. It would appear then that a transition between these two characteristic distributions occurs within the range of zero sea state. It was found that the distribution in the vertical plane was not strongly dependent on hydrophone depth at 60 or $100 \mathrm{ft}$.

The maximum levels were sometimes as much as $10 \mathrm{db}$ higher than the levels obtained with the hydrophone axis directed horizontally. These measurements also indicate that the levels are in close agreement when the hydrophone is directed either horizontally or vertically upward. This behavior did not fit any cosine law very well, but in some cases it tended to agree with the $\cos ^{n} \theta$ law where $n$ has a value somewhere near unity.

The directional distribution of ambient noise in the ocean has been studied recently and more extensively by the Marine Physical Laboratory of the Scripps Institute of Oceanography. This study is reported by B. A. Becken [10]. A 32 element, three-dimensional receiving array of hydrophones was employed, arranged in the form of a great stellated icosahedron and operating in the frequency range of 750 to $1500 \mathrm{cps}$. The 9 - $\mathrm{ft}$-diameter array was suspended from floats in from 1000 - to 2000-fathom deep ocean and at array depths between 150 to $1000 \mathrm{ft}$. The digital multibeam steering (DIMUS) technique used in conjunction with this array provided 32 simultaneous searchlight patterns with principal axes distributed uniformly over all possible directions ( $4 \pi \mathrm{sr}$ ). All 32 hydrophones were used in forming each beam. More detailed discussions of this sys tem are given by V.C. Anderson $[11,12]$. 
The results of these measurements indicated both an azimuthal and a vertical variation in the ambient noise. Azimuthal variation was observed principally in the outputs of array beams directed in the upper hemisphere. The distributions normally were elliptical in shape. It was observed that the maximum beam output for a given elevation angle usually aligned itself with the wavefronts of the swells while minimum outputs were perpendicular to the swells. This fact suggests a nonuniform radiation pattern of the whitecap or wavelet source; transmission in directions normal to the wavefront is impeded by the troughs, while lateral transmission along the ridges is less affected.

The variation of the ambient in the vertical plane was generally found to be much greater than that in azimuth. Except for very low sea states, for which the array output was nearly constant for all directions, the array output was generally much larger when the beam was directed vertically upward. Figure 13.5 is one example of the true-field vertical ambient distribution at a depth of $560 \mathrm{ft}$ in 2000 fathoms of water for a No. 3 sea state. In this figure, corrections have been made for the finite beamwidths of the arrays.

From the nature of the distribution shown in Fig. 13.5 and other similar results, the following picture of the mechanism of noise generation and distribution in the ocean has been constructed. Noise in the deep ocean is presumed to be a superposition of two fields. The first is that which exists at sea-state zero prior to the development of the wave motion and/or whitecaps. Its distribution

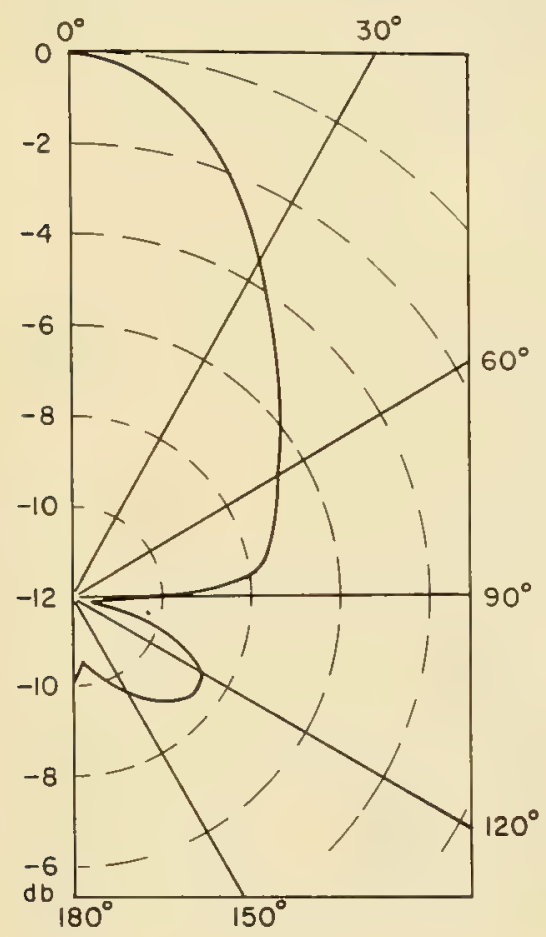

Fig. 13.5. True-field vertical distribution (No. 3 sea state; three-array depth, $560 \mathrm{ft}$ ). 
is isotropic and its origin is not yet established. The second source of noise is generated by wave motion and whitecaps at the ocean surface. The equivalent surface radiator is highly directional in the vertical plane when azimuthal effects are averaged. The directionality can possibly be attributed to the ocean waves and swells functioning in a manner analogous to angular focusing reflectors. In addition, the ocean swells impede transmission at shallow transmission angles in directions normal to the wavefronts of the swells.

It is further postulated that the noise intensity at a given point in the ocean caused by the equivalent surface radiator arrives by both a direct-ray path and single-bounce path experiencing specular reflection at the ocean bottom.

By taking into account the bottom-reflected energy and the attenuation due to spherical spreading and absorption occurring over the separation between radiator and receiver, it is possible to evaluate $S(\theta)$ which is the radiated-noise intensity due to unit incremental radiating surface at one yard. The quantity $S(\theta)$ is markedly directional in the downward direction and can be represented approximately by the expression:

$$
S(\theta)= \begin{cases}(\cos \theta)^{(90-\theta) / 10} \text { for } 0 \leq \theta \leq 70^{\circ} \\ \cos ^{2} \theta & \text { for } 70^{\circ} \leq \theta \leq 90^{\circ}\end{cases}
$$

Figure 13.6 is a plot of a reasonable approximation of $s(\theta)$ superimposed on the experimental data.

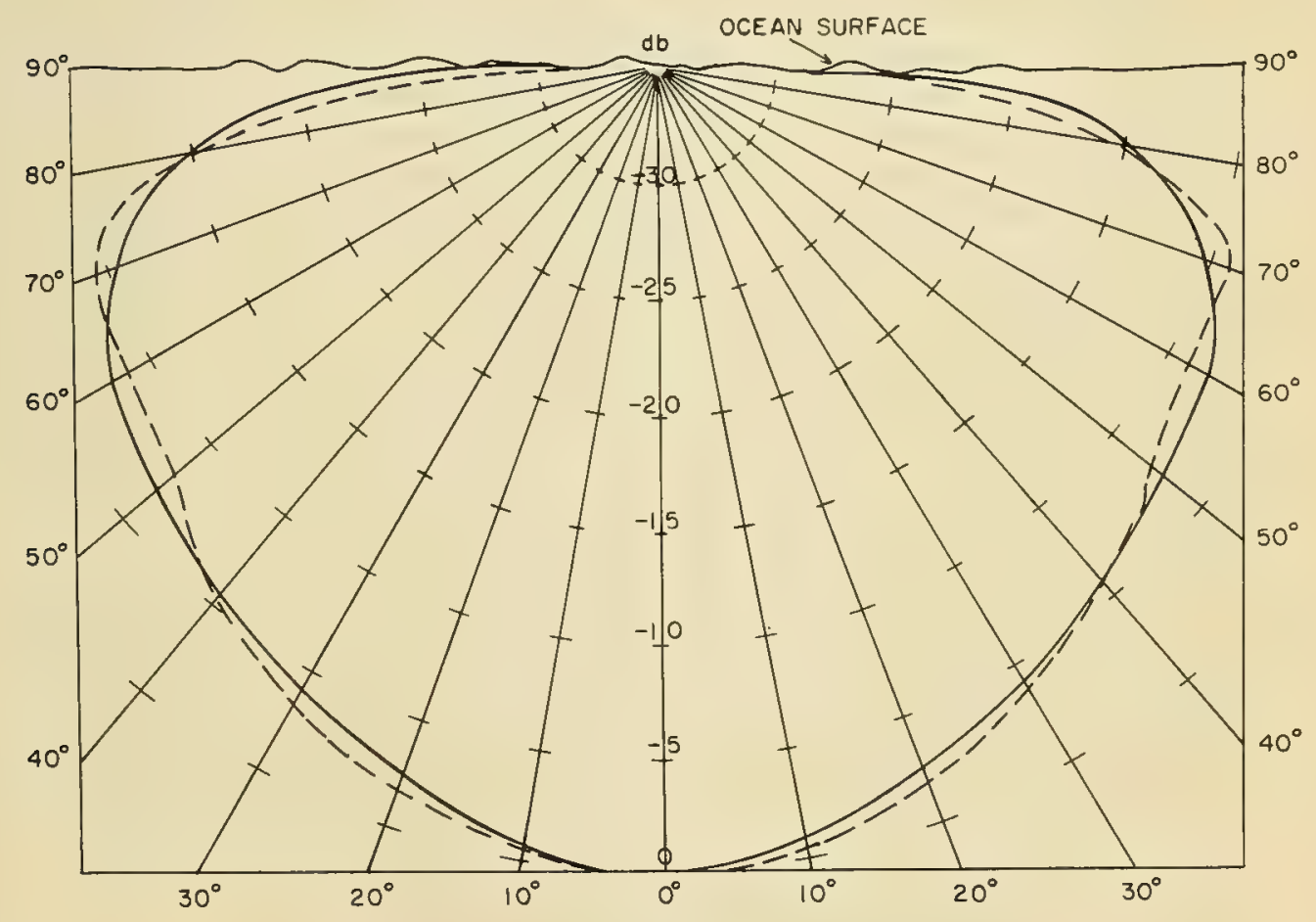

Fig. 13.6. Surface source level re normal to ocean surface. 


\subsection{THE MEASUREMENT OF AMBIENT NOISE}

We have seen that at high frequencies the ambient noise is dominated by thermal noise and represents the lowest detectable signal possible with an omnidirectional hydrophone of $100 \%$ efficiency. However, at lower frequencies (below about $40 \mathrm{kc}$ ), the sea ambient due to other causes and that due to thermal noise diverge about 11 or $12 \mathrm{db} /$ octave as the frequency is reduced. Thus, it should become increasingly easy to measure the sea ambient as the frequency is reduced below $40 \mathrm{kc}$. However, there are other factors which tend to counter this advantage. The principal one is the reduction in the acoustic loading on the hydrophone for those cases where the wavelength is much greater than the dimensions of the hydrophone. Since this loading varies as the square of the frequency in the low frequency range, it is quite incapable of measuring the thermal noise. But with proper attention paid to all the design parameters, the equivalent noise pressure may be kept well below the sea-surface ambient in the low frequency region.

The equivalent noise pressure of a hydrophone is that acoustic pressure due to plane sound waves which would produce the same open-circuit voltage across the terminals of the hydrophone as is produced by the thermal noise of the hydrophone in a 1 -cps band.

A hydrophone for broad-band underwater sound measurements should be essentially omnidirectional and have a flat, free-field voltage response over the frequency range. Section 13.3 indicates those factors which determine the equivalent noise pressure of a linear hydrophone that obeys the reciprocity relation, that is small compared with the operating wavelength, and that operates in a frequency range well below that which would permit any self-resonances. These are the characteristics required for omnidirectionality and flat response, and are quite easily attained with several shapes of piezoelectric transducer elements-especially those made of electrostrictive ceramic materials. Three shapes will be considered: a hollow spherical shell, a hollow circular cylinder loaded on the cylindrical surface, and a flat circular disk loaded on both circular surfaces. A number of approximations were made to better exhibit the significance of each of the parameters. Details of the development are given only for the spherical shell, but the results are given for all three in Table 13.I.

The results shown in Table 13.1 do not include mechanical losses; but in a well-designed transducer these losses should have negligible effect at these low frequencies, which are well below all hydrophone resonances.

The expressions for the efficiency show that it is strongly dependent upon the frequency and physical size of the transducer. For any present piezoelectric ceramic transducer, the second term in the denominator is small compared with the first. Thus, the efficiency is, approximately, in direct proportion to the square of the electromechanical coupling coefficient and inversely proportional to the loss tangent. Note that $1-k_{c}^{2}$ does not differ greatly from unity, since $k_{c}^{2}$ is always less than unity and is usually fairly small. However-and this is significant-if the coupling coefficient approaches unity, or if the loss tangent approaches zero, the efficiency approaches $100 \%$. This demonstrates the important role played by each of these parameters. 
Table 13.I. Electroacoustical Relationships for Three Types of Piezoelectric Transducers

\begin{tabular}{c|c|c|c}
\hline $\begin{array}{c}\text { Transducer } \\
\text { shape }\end{array}$ & Electroacoustical efficiency & Square of equivalent noise pressure & $\begin{array}{c}\text { Square of free-field } \\
\text { voltage response }\end{array}$ \\
\hline Sphere & $\frac{\rho \omega^{3} a^{4}(1-\sigma) k_{c}^{2}}{2 v b E \tan \delta\left(1-k_{c}^{2}\right)^{2}+\rho \omega^{3} a^{4}(1-\sigma) k_{c}^{2}}$ & $\frac{k T\left[2 b E \tan \delta\left(1-k_{c}^{2}\right)^{2}+\rho \omega^{3} a^{4}(1-\sigma) k_{c}^{2} / v\right]}{\pi \omega a^{4}(1-\sigma) k_{c}^{2}}$ & $\frac{a^{2}(1-\sigma) k_{c}^{2}}{2 E \epsilon \epsilon_{0}}$ \\
Cylinder & $\frac{\rho \omega^{3} a^{3} l k_{c}^{2}}{2 v b E \tan \delta\left(1-k_{c}^{2}\right)^{2}+\rho \omega^{3} a^{3} l k_{c}^{2}}$ & $\frac{k T\left[2 b E \tan \delta\left(1-k_{c}^{2}\right)^{2}+\rho \omega^{3} a^{3} l k_{c}^{2} / v\right]}{\pi \omega a^{3} l k_{c}^{2}}$ & $\frac{a^{2} k_{c}^{2}}{E \epsilon \epsilon_{0}}$ \\
Circular disk & $\frac{\rho \omega^{3} a^{2} b k_{c}^{2}}{v E \tan \delta\left(1-k_{c}^{2}\right)^{2}+\rho \omega^{3} a^{2} b k_{c}^{2}}$ & $\frac{k T\left[E \tan \delta\left(1-k_{c}^{2}\right)^{2}+\rho \omega^{3} a^{2} b k_{c}^{2} / v\right]}{\pi a^{2} \omega b k_{c}^{2}}$ & $\frac{4 b^{2} k_{c}^{2}}{E \epsilon \epsilon_{0}}$
\end{tabular}

Since the square of the equivalent noise pressure varies inversely with the efficiency, it depends upon the same physical properties and transducer dimensions as does the efficiency. For all three transducer types, if the second term in the numerator is neglected, which may be done without materially affecting the results, it is seen that the square of the equivalent noise pressure varies inversely as the frequency. This assumes that $\tan \delta$ is a constant for all frequencies, which is not necessarily true. Again, note that if $\tan \delta$ approaches zero or if $k_{c}$ approaches unity, $P_{t}^{2}$ approaches the value

$$
P_{t}^{2}=\frac{k T \rho \omega^{2}}{\pi v}
$$

which is just the thermal-noise limit of the ocean.

The last group of expressions presents the free-field voltage response in terms of the same parameters. It should be noted that $\eta_{0}$ does not depend upon either the frequency or the medium; it does depend upon the modulus of elas ticity, the dielectric constant, and the electromechanical coupling coefficient. It also depends upon some dimension of the transducer: for the hollow sphere and cylinder, it depends only upon the radius; and for the flat disk, it depends only upon the thickness.

When using these relationships, any single consistent system of units may be used for the efficiency and the equivalent noise pressure. Since $M_{0}$ is usually expressed with a mixed system of units-namely, volts per microbar-a conversion factor must be used. For example, if the cgs-esu system is used, the results must be multiplied by 300 to obtain the usual volts per microbar.

It seems worthwhile to re-emphasize the point that the free-field voltage response is definitely not an indication of the ability of a hydrophone to measure low-level signals, but that either the efficiency or the equivalent noise pressure is an essential indication of this ability. This is not to say that the free-field voltage response is unimportant, because low-level signals must be amplified to be measured. Now, the free-field voltage response depends upon the hydrophone impedance, and this impedance, relative to the input impedance of the amplifier, is important if one is to take full advantage of the threshold sensi- 
tivity. This is especially significant if one wishes to detect signals over a broad band of frequencies.

If a low equivalent noise pressure is the principal consideration in the design of a low-level, low-frequency listening hydrophone, then the cylindrical hydrophone should be as large as possible, and have a wall as thin as possible. However, the size will be limited by the requirements of omnidirectionality and absence of resonances. The wall must not be so thin as to be crushed by the hydrostatic pressure to which it will be subjected.

A hydrophone, shown in Fig. 13.7, and intended for operation at frequencies up to $3 \mathrm{kc}$ was designed in accordance with these principles. It consists of four barium titanate cylinders that are coaxially mounted with acoustic isolation spacers between the elements. All four cylinders are encased in a rubber boot which is closed at both ends with heavy metal plates. Each cylinder is 2 in. long, 6 in. in diameter, and has a 0.2-in. wall. The barium titanate cylinders are

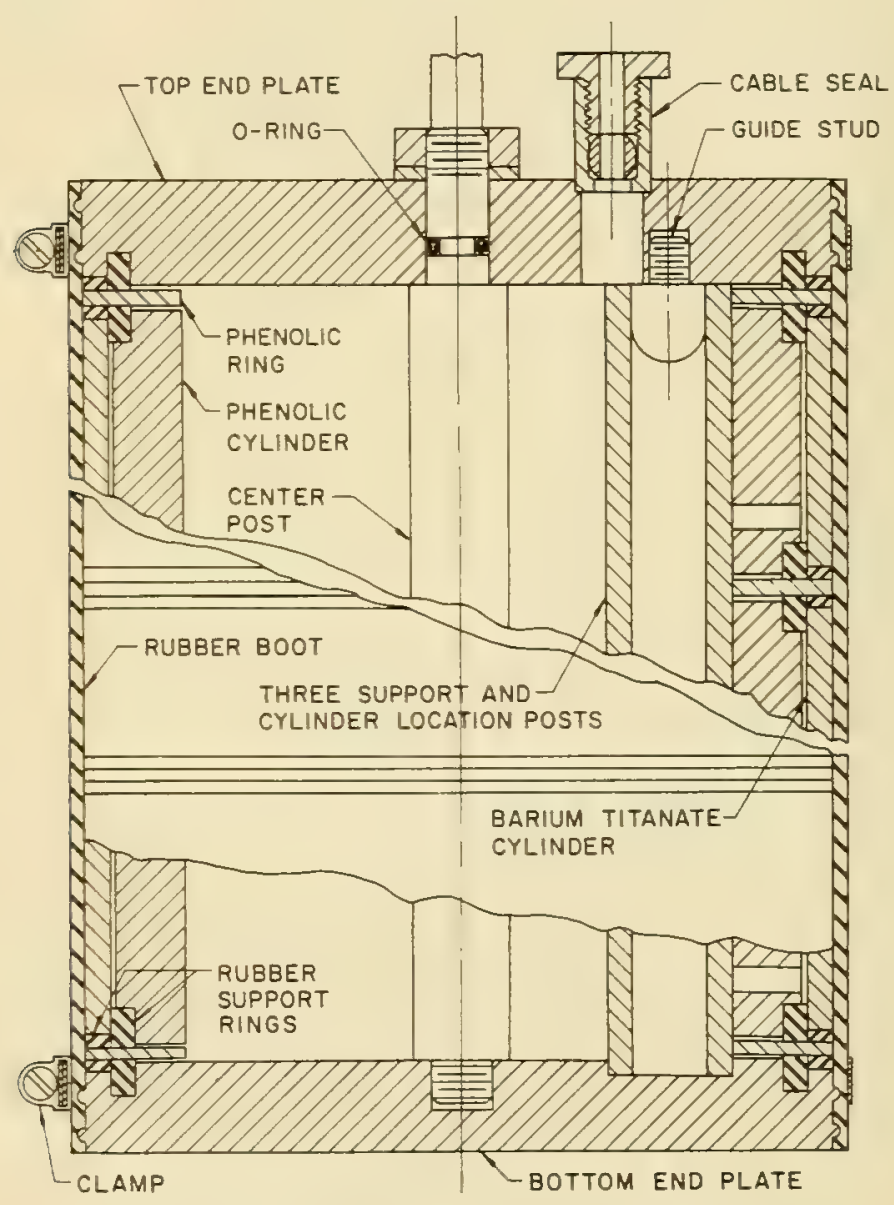

Fig. 13,7. Sectional view of hydrophone. 
connected in series in order to achieve a high value of the sensitivity and consequently a high impedance. (The hydrophone has successfully withstood hydrostatic pressures of $300 \mathrm{psi}$.) The four cylinders could be connected in several different series-parallel combinations which would provide various other impedances. Since the free-field voltage response varies as the square root of the impedance, this device may be used for adjusting the response. However, all such combinations will still give the same equivalent noise pressure. In this case, the cylinders were all series connected so as to achieve a high response and a high signal-to-noise ratio for the hydrophone amplifier combination. One very versatile means of controlling the impedance is to polarize the cylinder circumferentially in sections by means of electrical conducting stripes inside and outside the cylinder parallel to the cylinder axis. Since both the polarization and the field are now in the same direction (circumferential), the transducer also has a higher coupling coefficient than when it is plated inside and outside in the more usual manner (as was the case for the transducer described here).

Following is a summary of the most significant acoustic characteristics of the hydrophone shown in Fig. 13.7, all of which appear to meet the design requirements over the specified range of 100 to $3000 \mathrm{cps}$.

1. Free-Field Voltage Response. $-78 \pm 1.5 \mathrm{db}$ re $1 \mathrm{v}$ per $\mu$ bar over the frequency range of 100 to $8000 \mathrm{cps}$.

2. Directionality. Omnidirectional within $\pm 1 \mathrm{db}$ in all planes for frequencies up to $3000 \mathrm{cps}$.

3. Equivalent Noise Pressure. Varies from -91 to $-102 \mathrm{db}$ re 1 $\mu$ bar for a 1-cps band from 100 to $3000 \mathrm{cps}$, which is about 52 to $38 \mathrm{db}$, respectively, below the ambient noise levels of zero sea state.

4. Hydrophone Impedance. The impedance is essentially that due to a capacity of approximately $0.01 \mu \mathrm{f}$.

This section presented guide lines for designing a hydrophone with a low equivalent noise pressure and means for varying the hydrophone impedance in order to match the amplifier.

Now the question is, can one detect a target that has a level over a band of frequencies lower than the ambient noise over the same band ? Here we will not consider correlation techniques or other similar devices, but just straight listening. The answer to this question is definitely affirmative, provided the target is localized in space. Hydrophones with radiating areas whose dimensions are large compared with the wavelength of sound in the medium have directional properties. The best example is the circular piston which has the so-called searchlight pattern. The directivity factor is the ratio of the intensity on the main beam of such a transducer at a fixed distance $r$ to the average intensity over a sphere with a radius equal to $r$. For a circular piston with a diameter greater than the wavelength the directivity factor is approximately

$$
D=\left(\frac{\pi d}{\lambda}\right)^{2}
$$

where $d$ is the diameter of the circular piston and $\lambda$ is the wavelength of the sound. If one substitutes this value in Eq. (10) of Section 13.3, this rather remarkable expression is obtained: 


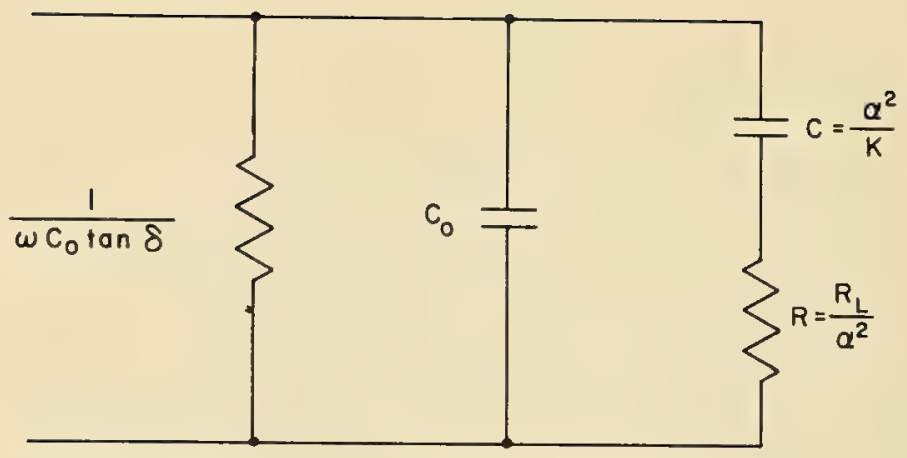

Fig. 13.8. Equivalent transducer circuit for low frequencies.

$$
\left(\frac{P_{t}^{2}}{\rho C}\right)\left(\frac{\pi d^{2}}{4}\right) \eta=k T
$$

The first factor on the left is the equivalent sound intensity of the ambient thermal noise for a 1 -cps band and the second factor is the area of the radiating face. Therefore, this expression states that for a $100 \%$-efficient transducer, the ambient thermal noise contained in a 1 -cps band is equivalent to a rate of flow of acoustic energy equal to $k T$, which, as we know, is the energy of one degree of freedom and is less than the kinetic energy of a single gas molecule.* Note especially that this is true no matter how large the radiating face of the transducer. On the other hand, Eq. (9) of Section 13.3 shows that the sensitivity or free-field voltage response of a hydrophone varies as the square root of the directivity, and hence it does increase with the size of the radiating face. Thus, we see that, at least for ambient thermal noise and indeed for any isotropic noise, an increase in the directivity provides an equivalent increase in the signal to ambient-noise ratio when the main beam of the receiving hydrophone is on the target.

\subsection{FACTORS THAT DETERMINE THE EQUIVALENT NOISE PRESSURE, FREE-FIELD VOLT. AGE RESPONSE AND EFFICIENCY OF A TRANSDUCER AT LOW FREQUENCIES [13]}

\subsubsection{The Equivalent Circuit and the Efficiency}

Consider a thin-walled hollow sphere of radius a and wall thickness $b$ that is vibrating in the radial mode at a frequency well below resonance. At these frequencies, the equivalent circuit may be represented by the circuit diagram shown in Fig. 13.8, where $C_{0}$ is the clamped capacitance, $C$ is the motional capacitance, $R$ is the resistance resulting from the mechanical load $R_{L}, K$ is the stiffness, $a$ is the electromechanical transformation ratio, $\tan \delta$ is the loss tangent (ratio of clamped resistance to clamped reactance), and $\omega$ is the angular frequency. The purely mechanical losses will be omitted in this discussion.

At the low frequencies under consideration, the impedance is almost entirely capacitive. Indeed, from impedance considerations alone, the resistance

*This expression appears to have the units of energy, because the bandwidth which has dimensions of reciprocal time was taken to be unity. 
$R$ is perhaps the least significant; yet, as a sound projector, it is the most important because the energy delivered to this element represents the radiated acoustic energy. Therefore, the value of this element, relative to the other resistive elements, determines the efficiency and, hence, the equivalent noise pressure of the transducer.

Examination of the equivalent circuit of Fig. 13.8 shows that the electrical input admittance is

$$
Y=\omega C_{0} \tan \delta+j \omega C_{0}+\frac{R(\omega C)^{2}+j \omega C}{(R \omega C)^{2}+1}
$$

and since $R \omega C \ll 1$ at these low frequencies, *

$$
Y=\omega C_{0} \tan \delta+R(\omega C)^{2}+j \omega\left(C+C_{0}\right)
$$

Again, since $\omega\left(C+C_{0}\right) \gg\left[\omega C_{0} \tan \delta+R(\omega C)^{2}\right]$,

$$
R_{T}=Z_{\text {real }}=\frac{\omega C_{0} \tan \delta+R(\omega C)^{2}}{\omega^{2}\left(C+C_{0}\right)^{2}}
$$

Since the second term is that portion of the series resistance produced by acoustic radiation, the efficiency is simply this term divided by $R_{T}$, or

$$
\eta=\frac{R \omega^{2} C^{2}}{\omega C_{0} \tan \delta+R \omega^{2} C^{2}}
$$

Now, $R_{L}$ depends upon the specific acoustic impedance of the medium $(\rho v)$ and the dimensions of the transducer. At low frequencies, where the radius is considerably smaller than the wavelength, the expression for the real part of the mechanical radiation impedance is approximately

$$
R_{L}=\frac{4 \pi \rho a^{4} \omega^{2}}{v}
$$

where $\rho$ is the density and $v$ is the sound velocity of the medium.

For a thin-shelled hollow sphere, the stiffness is approximately

$$
K=\frac{8 \pi b E^{\prime}}{1-\sigma}
$$

where $E^{\prime}$ is Young's modulus of elasticity and $\sigma$ is Poisson's ratio. The modulus $E^{\prime}$ is the short-circuit or zero-field modulus, and it is related to the open-circuit or zero-electric-displacement modulus $E$ by the relation

$$
E^{\prime}=E\left(1-k_{c}^{2}\right)
$$

where $k_{c}$ is the electromechanical coupling coefficient. This may be expressed approximately by

$$
k_{c}^{2}=\frac{C}{C+C_{0}}
$$

For a thin-walled hollow sphere, the clamped capacity is approximately

$$
C_{0}=\frac{\epsilon \epsilon_{0} 4 \pi a^{2}}{b}
$$

*The equality sign $\Leftrightarrow$ will be used throughout, even though most expressions are not exact equalities. 
where $\epsilon$ is the dielectric constant and $\epsilon_{0}$ is a constant whose value depends on the choice of units. Expressions involving Eqs. (3) through (6) and the electromechanical transformation ratio a may be substituted in Eq. (2) to give the following expression for the efficiency,

$$
\eta=\frac{\rho \omega^{3} a^{4}(1-\sigma) k_{c}^{2}}{2 v b E\left(1-k_{c}^{2}\right)^{2} \tan \delta+\rho \omega^{3} a^{4}(1-\sigma) k_{c}^{2}}
$$

\subsubsection{The Free-Field Voltage Response and Equivalent Noise Pressure}

It is now desired to obtain expressions for the free-field voltage response and the equivalent noise pressure in terms of the same parameters. As a consequence of the reciprocal properties of the transducer, the former is given by

$$
\eta_{0}=\lambda \sqrt{\frac{R_{T} \eta D}{\pi \rho v}}
$$

where $\lambda$ is the wavelength of sound in the medium and $D$ is the directivity factor, which will be taken equal to unity for the assumed conditions.

It is usually necessary to amplify the low-level output of a hydrophone, and no matter how well an amplifier is designed, there still remains a residual output produced by thermal noise in the conductors of the input circuit. In the ideal case, this residual output will be entirely due to the hydrophone.

The open-circuit voltage in the hydrophone circuit developed by thermal agitation in a 1 -cps band is given by Johnson [14]:

$$
E_{N}=\sqrt{4 k T R_{T}}
$$

where $k$ is the Boltzmann constant and $T$ is the absolute temperature.

Now, the equivalent noise pressure is defined as the equivalent acoustic pressure produced by sound energy, in a 1-cps band, that will generate an opencircuit voltage in the hydrophone just equal to that produced by the thermal noise at the same frequency, also for a 1 -cps band. Since the sensitivity in Eq. (9) is $E_{0}, P$, where $E_{0}$ is the open-circuit voltage across the terminals of the hydrophone resulting from an acoustic pressure $P$, it is possible to solve for $E_{0}$ and to equate this solution to the expression for the noise voltage above. Solving this equation for the pressure, we obtain the equivalent noise pressure

$$
P_{t}=\sqrt{\frac{4 k T \pi \rho v}{\lambda^{2} \eta D}}
$$

The desired expression for the equivalent noise pressure is obtained by substituting Eq. (8) in this expression. This result is

$$
P_{t}=\sqrt{\frac{k T\left[2 b E\left(1-k_{c}^{2}\right)^{2} \tan \delta+\rho \omega^{3} a^{4}(1-\sigma) k_{c}^{2} / v\right]}{\pi \omega a^{4}(1-\sigma) k_{c}^{2}}}
$$

From Eq. (1) it is easily seen that the real part of the series impedance that is due to radiation is

$$
R_{s}=\frac{R C^{2}}{\left(C+C_{0}\right)^{2}}
$$


and this is simply the product of the total series resistance and the efficiency. This would be true even if the purely mechanical losses were not neglected. Therefore, substituting this expression for $R_{T} \eta$ in Eq. (9) and making use of Eqs. (3) through (7), the free-field voltage response becomes

$$
u_{0}=\sqrt{\frac{a^{2}(1-\sigma) k_{c}^{2}}{2 E \epsilon \epsilon_{0}}}
$$

The three quantities, $\eta, M_{0}$, and $P_{t}$, have also been obtained for two other shapes of piezoelectric transducers: a hollow, thin-walled cylinder loaded radially and a flat, circular disk radiating from both flat circular faces. For these cases, the radiation resistance per unit area was assumed to be the same as that of a sphere having the same radiating area. Further, the length $l$ and the diameter $2 a$ of the cylinder were assumed to be roughly the same order of magnitude. The results are given in Table $13 . \mathrm{I}$.

\section{REFERENCES}

1. R. H. Mellen, "The Thermal-Noise Limit in the Detection of Underwater Acoustic Signals," J. Acoust. Soc. Am., Vol. 24, 478-480 (1952).

2. V.O. Knudsen et al., "Ambient Noise," from Report No. 3, Survey of Underwater Sound (OSRD Report 4333, NDRC Report 6.1-1848), PB 31021 (September 26, 1944).

3. M. Lomask and R. Frassetto, "Acoustic Measurements in Deep Water Using the Bathyscaphe," J. Acoust. Soc. Am., Vol. 32, 1028-1033 (1960).

4. G. M. Wenz, "Some Periodic Variations in Low-Frequency Acoustic AmbientNoise Levels in the Ocean," J. Acoust. Soc. Am., Vol. 33, 64-74 (1961).

5. M.D. Fish, "Marine Mammals of the Pacific with Particular Reference to the Production of Underwater Sounds," Woods Hole Oceanographic Institution, Report 49-30 (1949).

6. M.D. Fish, "An Outline of Sounds Produced by Fishes in Atlantic Coastal Waters, " Narragansett Marine Laboratory, Special Report No. 1, 53-1 (January, 1953).

7. C. A. Teer, "The Influence of Hydrophone Directivity upon the Measured Value of the Ambient Noise Level in the Ocean," Underwater Detection Establishment, England, Report 65 (May, 1949).

8. T. E. Heindsmann, R.H. Smith, and A.D. Arneson, "Effect of Rain upon Underwater Noise Levels," J. Acoust. Soc. Am., Vol. 27, 378-379 (1955).

9. R. J. Urick, "Some Directional Properties of Deep Sea Ambient Noise," U.S. Naval Research Laboratory Report 3796 (January, 1951).

10. B. A. Becken, "The Directional Distribution of Ambient Noise in the Ocean," Univ. of California, Marine Physical Laboratory of the Scripps Institution of Oceanography, S10 Reference 61-4 (March 7, 1961).

11. V.C. Anderson, "Arrays for the Investigation of Ambient Noise in the Ocean," J. Acoust. Soc. Am., Vol. 30, 470-477 (1958).

12. V.C. Anderson, "Digital Array Phasing," J. Acoust. Soc. Am., Vol. 32, 867-870 (1960).

13. P. M. Kendig, "Factors that Determine the Equivalent Noise Pressure, Free-Field Voltage Response, and Efficiency of a Transducer at Low Frequencies," J. Acoust. Soc. Am., Vol. 33, 674-676 (1961).

14. J.B. Johnson and F.B. Llewellyn, "Limits to Amplification," Bell System Techn. J., Vol. 14, 85-96 (1935).

\section{DISCUSSION}

DR. F.T. DIETZ, by way of augmenting the lecturer's remarks, mentioned the results of ambient noise studies being carried out at the University of Rhode Island in a shallow-water location. The main objective in the systematic measurements made was to find any possible seasonal variations and to deduce any possible correlations between the ambient noise spectrum and environmental parameters, such as wind speed and direction, tidal currents, etc.

Some 864 spectrum determinations were made on a $1 / 3$-octave basis in the range $40 \mathrm{cps}$ to $10 \mathrm{kc}$ using a "bottomed" hydrophone in a water depth of approximately $30 \mathrm{ft}$. The results revealed that after a critical value of the wind speed is exceeded, then the acoustic pressure levels are linearly related to the 
logarithm of the wind speed and there is a frequency dependence with a maximum effect at approximately $600 \mathrm{cps}$. These results are similar to observations made by N.E.L. and groups in Canada and Britain.

The data below $150 \mathrm{cps}$ showed variations which could be associated with tidal current flow around the hydrophones. More recent measurements, in the same locality but at $25 \mathrm{cps}$ using a 3 -axis seismometer, can be related to the lunar tides, but also display a sensitivity to the solar tides. In order to eliminate the noise arising from water flow, a systematic series of low-frequency measurements are planned with the seismometer buried in the bottom.

DR.W.N. ENGLISH said that he would like to draw attention to the ambient noise measurements made by A. R. Milne of the Pacific Naval Laboratory under ice in the Canadian Arctic. The initial measurements* were made under smooth new ice and the continuous noise level was extremely low and well below the Knudsen sea-state zero shown in one of Dr. Kendig's slides. Below $30 \mathrm{cps}$, some noise effects were obtained, attributed to wind and seismic influence, but observations generally were limited by instrument noise. A second operation was carried out in the spring of this year under old, rough, shore-fast ice in a water depth of $1300 \mathrm{ft}$, using greatly improved instrumentation. As before, the ambient noise level was found to be extremely low provided the ice was not undergoing cooling. On calm clear nights and on other occasions when the ice was contracting, very sharp transients were heard which echoed back and forth in spectacular fashion before disappearing. Depending on the rate of cooling, the transients were infrequent or gave rise to almost continuous noise. Dr. English thought that this form of impulsive noise was sufficiently different from anything found in the open ocean to provide some interesting problems.

DR.D.E. WESTON doubted the existence of any connection between ambient sea noise and turbulent density variations and thought a more likely cause to be the variations of water height, i.e., surface waves, ignoring the effect of breaking waves which could occur at higher sea states. Although the first-order pressure amplitudes due to surface waves will fall off exponentially with depth, yet, if standing waves exist, the second-order (or Miche) pressures will not show appreciable amplitude diminution. At the time of the maximum displacement in a standing wave, quantities of water have been raised from below to above the mean water level and expressed physically the Miche forces correspond to the vertical acceleration of these water masses, at a frequency double that of the surface wave. Longuet Higgins ${ }^{\dagger}$ has given a theory of microseisms based on the Miche pressure variations.

Dr. Weston then said that, for the explanation of ambient noise, it was necessary to consider the high-frequency or capillary waves and to demonstrate the occurrence of standing wave ripples. The latter may arise from the large beamwidth (approaching $180^{\circ}$ ) of waves generated by wind, especially in gusty conditions of variable wind direction. Reflection of ripples at the sharp crests of the gravity waves could also give a standing wave ripple "riding" on a gravity *J. Acoust. Soc. Am. (August, 1960).

†Phil. Trans. Roy. Soc, London, Ser, A, Vol, 243, 857 (1950). 
wave. This explanation is, of course, by no means a quantitative theory as yet, and such factors as the energy loss due to viscosity have to be considered, but Dr. Weston pointed out that it was encouraging to hear Professor Skudrzyk state (Lecture 12) that the Kolmogorov spectrum law is quite general and is applicable to both ripples and ambient noise.

DR. H. A. J. RYNJA commented upon the statement concerning the efficiency of hydrophones and said that their efficiency approaches $100 \%$ only at resonance, and below the resonant frequency will drop to a much lower value determined by the square of the coupling factor $(k)$. The maximum value of $k$ for the ma terials now available is between 0.50 and 0.60 so that the efficiency of the hydrophone below resonance cannot be more than $30 \%$. This means that for maximum sensitivity in the measurement of thermal noise a set of hydrophones with different resonance frequencies should be employed.

DR. KENDIG: At low frequencies, which are well below any resonance and for which the wavelength of sound in the medium is large compared to the hy drophone's dimensions, the efficiency will generally be low. In the case of typical ferroelectric materials, the low efficiency in this low-frequency region is due primarily to the dielectric losses. Thus, the loss tangent (dielectric losses) is just as important as the electromechanical coupling coefficient. On the other hand, if pure mechanical losses are large compared to the radiation losses, the efficiency will be low no matter what values exist for the loss tangent and the coupling coefficient, because the ratio of pure mechanical losses to radiation losses is independent of the loss tangent and the coupling coefficient.

At low frequencies, it is usually possible to measure the ambient noise of the sea even if the hydrophone efficiency is quite low, because the sea ambient, even for zero sea state, is very much greater than the thermal noise. For example, at $1000 \mathrm{cps}$ the zero sea-state ambient is about $60 \mathrm{db}$ higher than that due to the thermal noise. Thus, it is quite possible to measure the sea ambient at all sea states with a nonresonant hydrophone up to at least several thousand cycles per second. 



\title{
LECTURE 14
}

\section{FLOW NOISE, THEORY AND EXPERIMENT}

\author{
E. J. Skudrzyk and G. P. Haddle \\ Ordnance Research Laboratory \\ The Pennsylvania State University \\ University Park, Pennsylvania \\ U.S.A.
}

\subsection{INTRODUCTION}

Flow noise is defined as the noise that is produced by the hydrodynamic flow around a rigid body. Thus, flow noise is produced by the turbulence in the boundary layer, by the eddies generated by the surface roughnesses, by the eddies shed at the tail and the fin of the moving vehicle, and by flow-excited shell and cavity vibrations. Flow noise is primarily due to the centrifugal forces generated by the rotating eddies, to the Coriolis forces, and to various kinds of microscopic stagnation pressures caused by the eddies as they move towards or away from one another. All these phenomena generate great fluctuations in pressure. The pressure fluctuations are carried along with the flow. When they travel over the sensitive area of a hydrophone, they are recorded as flow noise. The flow noise that is recorded inside the region where it is generated is called the nearfield flow noise. Thus, the nearfield flow noise is predominantly due to the steady and continuous transport of pressure ripples as if they were frozen. These pressure ripples are carried along with the flow but they do not generate a pressure that propagates to greater distances. The nearfield flow noise is therefore not connected with the production of a true sound pressure nor with the production of sound energy.

However, turbulence is a very unsteady phenomenon. The pressure fluctua tions are not frozen in the flow; they decay because of viscosity effects and are regenerated by the energy fed into the fluidthrough the surface drag at the walls of the vehicle. They move towards and away from one another. The intermittent nature and the unsteadiness of the turbulence and of the nearfield pressure lead to the generation of a true sound that propagates all over the disturbed region and is radiated to greater distances. However, turbulence is a very poor sound radiator, and the radiation-field sound pressure is usually much smaller than the nearfield pressure.

At low speed the flow noise is much weaker than the machinery noise. But flow noise increases greatly with the speed and eventually masks all other components of noise. 
The fundamental work on flow noise was performed by Lighthill as early as 1951 in connection with the noise produced by jets. The Ordnance Research Laboratory became interested in flow noise in 1954. During an experimental test run of a vehicle, the main motor was shut off and the recording continued. Analysis of the recording indicated that the dead-interval noise-the flow noisewas appreciable. This incident gave rise to a continuous study of flow noise at this laboratory. Three aspects of flow noise were studied:

1. The flow noise generated by a turbulent boundary layer (nearfield and radiation field)

2. The flow noise produced by the surface roughnesses of the vehicle

3. The flow noise produced by cavity resonance and by wall vibrations

\subsection{THE NEARFIELD FLOW NOISE GENERATED BY A TURBULENT BOUNDARY LAYER}

Probably the most interesting of the three components of flow noise is that produced by the turbulence in the boundary layer. The velocity fluctuations caused by the turbulent eddies increase the transportation of momentum and generate the surface drag. The surface drag can be computed with the aid of the Stokes-Navier equations [1,2]; it is found to be

$$
r=\rho\left\langle u^{\prime} v^{\prime}\right\rangle=\rho v^{*}{ }^{2}
$$

where $u^{\prime}$ and $v^{\prime}$ are the components of the fluctuating velocity in the direction of the main flow and transverse to it. The magnitude $v^{*}=\sqrt{\left\langle u^{\prime} v^{\prime}\right\rangle}$ is defined as the root-mean-square average of the velocity components and is the shear velocity. This velocity $v^{*}$ determines the shear force near the wall or surface drag.

The surface drag has been thoroughly studied for channels [2], plates [1], and even for rotating cylinders [3]. The experimental results show that the surface drag is approximately proportional to the square of the free-stream velocity $u_{0}$. The surface drag can, therefore, be expressed as the product of the coefficient of drag $c_{d}$ and the square of the free-stream velocity:

$$
\tau=\frac{1}{2} c_{d} \rho u_{0}^{2}
$$

where $\rho$ denotes the density of the fluid. The coefficient of drag proves to be practically constant; it is approximately equal to $3 \cdot 10^{-3}$ whenever the flow is turbulent. It changes by only a factor of three when the velocity is changed by a factor of as much as 5,000. Since the surface drag is generated in the inner part of the boundary layer, we might expect that it would not directly depend on the curvature of the surface nor on the size of the body that generated it. This conclusion is verified by measurements with rotating cylinders [3]. No dependence on the ratio of diameter to height was found.

The fluctuating velocity $v^{*}$ can be estimated by equating the theoretical and the experimental results:

or

$$
\frac{1}{2} c_{d} \rho u_{0}^{2}=\frac{1}{2} 3 \cdot 10^{-3} \cdot \rho u_{0}^{2}=\rho v^{*^{2}}
$$

$$
v^{*}=0.04 u_{0}
$$


The shear velocity $v^{*}$ turns out to be very nearly equal to $4 \%$ of the free-stream velocity. This result is in good agreement with, for instance, Laufer channel measurements [2]. The shear velocity is $4 \%$ at the outside of the laminar sublayer and then decreases linearly with the distance until it becomes zero at the outer limit of the boundary layer.

The shear velocity $v^{*}$ is connected with the nearfield noise pressure by an equation very similar to the well-known Bernoulli equation, the only difference being a modified constant:

$$
p=a \rho v^{2}
$$

Computations have been performed by Batchelor [4] for a region of homogeneous turbulence, and by Kraichnan [5] for a simplified model of a boundary layer. For the boundary layer, the factor $1 / 2$ in the Bernoulli equation turns out to be the factor $a$ of the order of magnitude 7 . The constant $a$ may be expected to depend slightly on the Reynolds number. The nearfield sound pressure that is generated by the velocity fluctuations is similar to the acoustic radiation pressure, or the stagnation pressure on a moving body, except that stagnation now is generated not by solid bodies but by the velocity gradients of the moving eddies. This pressure is proportional to the square of the velocity fluctuations and is, therefore, a second-order phenomenon in terms of the velocities.

The spectral distribution of the nearfield pressure can be estimated in a very simple manner. The turbulent eddies are correlated over distances of the same order of magnitude as the boundary-layer thickness. Each one of the turbulent eddies that moves over the sensitive area of the hydrophone represents a pressure pulse whose magnitude is constant as long as the pulse is in full contact with the hydrophone and then decreases rapidly to zero when contact is lost. The spectrum of such a pulse is well known. It is practically constant up to a frequency $\omega$ whose semiperiod is equal to the pulse duration $t_{i}$. From then on, it decreases as

$$
\frac{\sin \left(\omega t_{i} / 2\right)}{\omega t_{i} / 2}=\frac{\sin \left(f / f_{0}\right)}{f / f_{0}}
$$

where $t_{1}=\delta / u_{0}=1 / f_{0}, u_{0}$ being the flow velocity, $\delta$ the space length of the pressure pulse, and $f_{0}$ the repetition frequency of the pulses. The low-frequency part of the spectrum is practically independent of the details of the pressure distribution inside the pulse. It is proportional to the average value of the pressure during this interval. In contrast, the high-frequency part of the spectrum depends on the details of the turbulence. It is practically impossible therefore to predict the high-frequency spectrum with adequate accuracy. However, the experimental results show that the spectrum decreases inversely proportional to the second or third power of the frequency.

Figure $12.10^{*}$ shows the power spectra of the longitudinal velocity fluctuations $u^{\prime}$ of the turbulence and of the Reynolds stress $u^{\prime} v^{\prime}$ as given in [6] and [7] (for a point close to the wall, a flow velocity of $49 \mathrm{ft} / \mathrm{sec}$, and a displacement thickness of the boundary layer of approximately $0.24 \mathrm{in}$.). The frequency $f_{0}$ is therefore $200 \mathrm{cps}$. As predicted, the spectral amplitudes are practically constant at low frequencies (particularly those of $v^{\prime}$ and $u^{\prime} v^{\prime}$ ); they decrease ac*See page 208 of this volume. 
cording to $a-5 / 3$ power law (Kolmogorov) [8] for high frequencies and according to a -7 power law (Heisenberg law) [9] for very high frequencies. The curves show that, apart from relatively unimportant deviations at very low frequencies (or space wave numbers), the velocity fluctuations and the Reynolds stresses (that is, the velocity products $u^{\prime} v^{\prime}$, etc.) have approximately the same spectral distribution. Similar results have been obtained for the power spectrum of the flow noise by M. Harrison [10], by W.W. Willmarth [11], and by the Ordnance Research Laboratory [12].

The spectral density of the turbulence noise may thus be considered to be constant up to a frequency $u_{0} / \delta$ determined by the ratio of the free-stream velocity to the thickness of the boundary layer. From there on, it may be expected to decrease inversely proportional to a certain power $m$ of the scale of the turbulence or the space wave number; a different power law may then be expected to apply in the viscous range. This power law may be deduced from the known laws of turbulence or deduced from the experimental results.

The power spectrum of the pressure referred to unit frequency interval is thus known by

$$
\begin{gathered}
P(\omega)=p_{0}^{2} \text { for } \omega \leq \omega_{0} \\
P(\omega)=p_{0}^{2}\left(\frac{\omega_{0}}{\omega}\right)^{m} \text { for } \omega \geq \omega_{0} \\
\omega_{0}=\frac{2 \pi u_{0}}{\delta}=\frac{2 \pi u_{0}}{5 \delta^{*}}
\end{gathered}
$$

In Eq. (8), the frequency $\omega_{0}$ at which the noise spectrum starts to decrease is expressed by the ratio of the free-stream velocity to a quantity $\delta$ which is of the same order of magnitude as the boundary-layer thickness. The relation $\delta=5 \delta^{*}$, where $\delta^{*}$ is the displacement thickness of the boundary layer, seems to lead to very good agreement with the experimental results. This relation has therefore been assumed in all the following computations.

The preceding considerations in conjunction with the pressure, Eq. (5), lead to the prediction of the spectral distribution of the noise pressure. The constant $p_{0}^{2}$, however, remains unknown. This constant can be determined by computing the rms noise pressure (neglecting the viscous range):

$$
p^{2}=a^{2} \tau^{2}=\int_{0}^{\infty} p(\omega) \frac{d \omega}{2 \pi}=\int_{0}^{\omega_{0}} p_{0}^{2} \frac{d \omega}{2 \pi}+\int_{\omega_{0}}^{\infty} p_{0}^{2}\left(\frac{\omega_{0}}{\omega}\right)^{m} \frac{d \omega}{2 \pi}
$$

and by equating the result to the value given by the Kraichnan equation:

$$
p_{0}^{2}=0.75 \cdot 10^{-5} \alpha^{2} \rho^{2} u_{0}^{3} \delta^{*}\left[\frac{3}{2}\left(m-\frac{1}{m}\right)\right]
$$

If this value is substituted above, the power spectrum $P(\omega)$ of the noise becomes

$$
P(\omega)=0.75 \cdot 10^{-5} a^{2} \rho^{2} u_{0}^{3} \delta^{*}\left[\frac{3}{2}\left(m-\frac{1}{m}\right)\right] \quad \text { for } \quad \omega<\omega_{0}
$$


and

$$
P(\omega)=1.5 \cdot 10^{-5}\left(\frac{a^{2} \rho^{2} u_{0}^{6}}{\omega^{3} \delta^{* 2}}\right)\left[\frac{3}{2}\left(m-\frac{1}{m}\right)\right]\left(\frac{2 \pi u_{0}}{5 \omega \delta^{*}}\right)^{m-3} \quad \text { for } \quad \omega>\omega_{0}
$$

Measurements with a small receiver lead to a value $m=3$ for the slope of the curves in the descending part of the spectrum. For $m=3$ the expression in square brackets reduces to 1 , and the power spectrum of the flow noise at low frequencies becomes proportional to the third power of the velocity and to the boundary-layer thickness; and proportional to about the sixth power of the velocity and inversely proportional to the square of the boundary-layer thickness at high frequencies. These predictions and the numerical values computed by the foregoing equation are in very good agreement with the experimental results, as will be shown later.

\subsection{THE RADIATED FLOW NOISE}

Lighthill's theory [13] leads to the computation of the farfield sound pressure. Unfortunately, the magnitude of this pressure can only be given in terms of higher order correlations, which have not yet been studied in detail. It has, therefore, not yet been possible to derive a correspondingly simple formula for radiation-field sound pressure generated by the turbulent velocity fluctuations.

It will subsequently be shown that the sensitivity of a hydrophone to the flow noise nearfield decreases greatly with the diameter of the hydrophone. A large hydrophone is, therefore, practically insensitive to the flow noise nearfield. But a large hydrophone is sensitive to the radiation-field pressure that is generated by the unsteadiness and the decay of the turbulence. This sound field propagates inside and outside the boundary layer and is correlated over distances of about half a sound wavelength. Since the hydrophone is usually much smaller than the sound wavelength, it is fully sensitive to the radiated sound and records it as flow noise. A large hydrophone measures almost exclusively the true sound that is produced by the unsteadiness of the turbulence.

\subsection{EFFECT OF SIZE AND SHAPE OF SOUND RECEIVER ON AMPLITUDE-VS-FREQUENCY CURVE OF FLOW NOISE}

Flow-noise pressure is a local, rapidly varying quantity and only a hydrophone that is small in comparison to the scale of the local pressure fluctuation measures the true value of pressure generated by the turbulent velocity fluctuations. If this hydrophone is tuned to a narrow frequency band, its response becomes proportional to the Fourier component of the noise pressure in the received frequency band. It has been shown by Taylor, Proudman [14], and others that the decay of the turbulence has only a minor effect on the nearfield noise pressure. It can be assumed that the turbulence is carried along by the flow, with a mean velocity $U$ not very different from the free-stream velocity of the flow. The frequency $f$ of noise pressure received by the small hydrophone, and the space wave number $\kappa$ of the turbulence are therefore connected by the relation,

$$
f=\frac{U}{\lambda_{\mathrm{sp}}}=\frac{1}{2 \pi} \frac{2 \pi}{\lambda_{\mathrm{sp}}} \cdot U=\frac{\kappa}{2 \pi} \cdot U
$$


where $\lambda_{\text {sp }}$ is the space wavelength of the pressure pattern that produces the noise band. Thus, the pressure fluctuations that give rise to a definite spectral component of the flow noise are no longer uncorrelated but correspond to a sinusoidal pressure pattern $p=p(\kappa) \cos \kappa(x-U t)$ that moves in the direction of the flow. Thus, tuning the hydrophone destroys the randomness of the received signal.

A hydrophone of finite size measures the average pressure over its area. If the hydrophone is infinitely narrow, but of a length. $l_{1}$, this average pressure fluctuation is given by the integral

$$
\begin{array}{r}
\frac{F(\omega)}{l_{1}}=\frac{1}{l_{1}} \int_{l_{1} / 2}^{l_{1} / 2} p(\kappa) \cos \kappa(\xi-U t) d \xi=\frac{p(\kappa)}{\kappa l_{1}}\left[\sin \kappa\left(\frac{l_{1}}{2}-u t\right)+\sin \kappa\left(\frac{l_{1}}{2}+u t\right)\right] \\
=\frac{2 p(\kappa)}{\kappa l_{1}} \sin \frac{\kappa l_{1}}{2} \cos \kappa U t=\frac{p(\kappa) \sin \left(\kappa l_{1} / 2\right)}{\kappa l_{1} / 2} \cos \omega t
\end{array}
$$

where $\omega=\kappa U$. The average value of spectral amplitude of the noise pressure over the hydrophone area is thus proportional to the infinite space spectrum $p(\kappa)$ of the pressure fluctuations in the boundary layer; $p(\kappa)$ alone determines the frequency component $F(\omega)$ where $\omega=U \kappa$, whether the hydrophone is finite or infinitely small. The effect of the finite dimension of the hydrophone in the direction of the flow on the received pressure amplitude is represented by the factor

$$
\frac{\sin \left(\kappa l_{1} / 2\right)}{\left(\kappa l_{1} / 2\right)} \cong\left(\frac{\kappa l_{1}}{2}\right)^{-1}
$$

The maxima and minima in the pressure distribution cancel one another over the hydrophone area and the contribution of one pressure maximum, on the average, remains, irrespective of the length of the hydrophone; the contribution of this maximum is represented by the above formula. The longitudinal dimensions of the hydrophone become important as soon as the length of the hydrophone is greater than one third the space wavelength $\lambda_{\mathrm{sp}}$ of the turbulence that generates the noise. Doubling the length of a tuned hydrophone should then reduce its relative flow-noise output by $6 \mathrm{db}$. This cancellation of successive maxima and minima introduces the factor $1 / \kappa^{2}=(U / \omega)^{2}$ in the frequency curve of the measured flow-noise power spectrum.

If the hydrophone is of variable width, but of a width that is always less than the transverse correlation length of the pressure fluctuations, the integrand must be multiplied by the width. If the hydrophone is symmetrical with respect to its midpoint, the mean force on its membrane becomes

$$
\frac{F(\omega)}{l}=p(\kappa) \int_{0}^{l} \cos \kappa(\xi-U t) y(\xi) d \xi
$$

The mathematics, then, is exactly the same as that for a shaded hydrophone array, the magnitude $\kappa l \sin \theta$ being replaced by $\kappa l$, and the shading function being 
replaced by the width $y(\xi)$ of the hydrophone. By properly shaping the hydrophone, the factor $1 / \kappa l_{1}$ of the preceding formulae can be changed into a factor $\left(1 / \kappa l_{1}\right)^{2}$, and the flow-noise sensitivity of the hydrophone can be made considerably smaller (as long as the nearfield pressure determines the received level). The flow-noise sensitivity of a hydrophone is thus a function of its shape, and the experiences acquired in shading hydrophone arrays can be used to reduce the received flow-noise level.

For a circular hydrophone whose radius $R$ is small in comparison to the transverse correlation length, the force on the hydrophone can be given by the following integral:

$$
F=R \int_{-R}^{R} p(\kappa) \cos \kappa \xi \frac{d \xi}{R} \sqrt{R^{2}-\xi^{2}}=p(\kappa) R^{2} \int_{-1}^{1} \cos (\kappa R y) d y \sqrt{1-y^{2}}=\frac{p(\kappa) R^{2} 4 J_{1}(\kappa R)}{\Gamma\left(\frac{1}{2}\right) \Gamma\left(\frac{3}{2}\right) \kappa R}
$$

where $\Gamma$ is the gamma function, and $J_{1}$ is the Bessel function of the first order. At higher frequencies, when $\kappa R \gg 1$, the Bessel function can be replaced by its asymptotic expression, and

$$
F=\text { constant } \cdot \frac{p(\kappa) R^{2}}{\kappa R \sqrt{\kappa R}}=\text { constant } \cdot F_{0} \frac{1}{(\kappa R)^{3 / 2}}
$$

Doubling the radius now decreases the narrow-band noise level by $9 \mathrm{db}$. If the transverse correlation length of the eddies were independent of their longitudinal wavelength, the effect of the finite diameter of the hydrophone would reduce the flow-noise output by $9 \mathrm{db}$ per frequency octave.

A hydrophone whose width increases as the sine of the distance from its end,

$$
y=\sin \frac{\pi x}{l_{1}}
$$

would yield an output

$$
F=l_{2} \int_{0}^{l_{1}} p(\kappa) \cos (\kappa x) \sin \frac{\pi}{l_{3}} d x=2 \frac{p(\kappa)}{\left(\pi^{2} / l_{1}^{2}\right)-\kappa^{2}}\left[-\frac{\pi}{l_{1}} \cos \kappa_{1}\right]_{0}^{l_{1}}=\frac{p(\kappa) l_{1} l_{2} \pi}{\kappa^{2} l_{1}^{2}}=\frac{F_{0}}{\kappa^{2} l_{1}^{2}}
$$

The hydrophone shape shown in Fig. 14.1 is interesting from a practical point of view. Its flow-noise sensitivity is proportional to the integral

$$
\begin{aligned}
& \int_{0}^{a} x \cos \kappa x d x+\int_{a}^{a+b} a \cos \kappa x d x+\int_{a+b}^{2 a+b}(2 a+b-x) \cos \kappa x d x \\
&=\frac{1}{\kappa^{2}}-2 \sin ^{2} \frac{k a}{2} \sin \kappa\left(\frac{3 a}{2}+b\right) \approx \frac{2}{\kappa^{2}}
\end{aligned}
$$

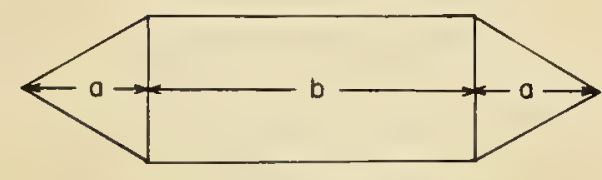

Fig. 14,1. Special hydrophone shape. 
The results of the preceding paragraph can also be deduced from the statistical theory given by Corcos, Cuthbert, and Von Winkle [15]. The integral of Eq. (15) is identical with the solution of the equation given in [15], if the transverse correlation length is small in comparison to the width of the hydrophone. But the statistical solution cannot be evaluated in closed form when the width of the hydrophone becomes larger than the correlation length.

However, it is easy to see that the hydrophone area can be subdivided into strips of width equal to the transverse correlation length of the pressure fluctuations. The contributions of all the strips will then be uncorrelated and random in phase, and they will add energy. The sensitivity of a rectangular hydrophone that is broad in comparison to the transverse correlation length will, therefore, be given by

$$
F \cong F_{0} \frac{\sin \kappa l_{1}}{\kappa l_{1}} \frac{\delta_{2}}{l_{2}} \sqrt{\frac{l_{2}}{\delta_{2}}}=F_{0} \frac{\sin \kappa l_{1}}{\kappa l_{1}} \sqrt{\frac{\delta_{2}}{l_{2}}}
$$

Doubling the dimensions of the hydrophone then decreases its flow-noise output by $9 \mathrm{db}$, and doubling the frequency decreases the flow-noise output by $6 \mathrm{db}$. For minimum flow-noise sensitivity, the strips should be of optimum shape; but none of them should be rectangular, and it is immaterial whether they are symmetrical or not. The strips can be of the shape shown in Fig. 14.2, but the length $l_{1}$ of each strip has to decrease by more than a space wavelength $\lambda_{\mathrm{sp}}$ of the turbulence per each unit increase of correlation length $\delta_{2}$. For a frequency of $20 \mathrm{kc}$, a correlation length $\delta_{2}$ equal to $0.1 \mathrm{in.}$, and a velocity of $600 \mathrm{in.} / \mathrm{sec}$,

$$
\frac{\partial l_{1}}{\partial y}=\frac{600}{20,000 \cdot 0.1} \doteq \frac{1}{3}
$$

Thus, the angle $\theta$ should be $45^{\circ}$ at the greatest. The ends of the hydrophone should be somewhat tapered in the direction of the flow, as indicated. A properly

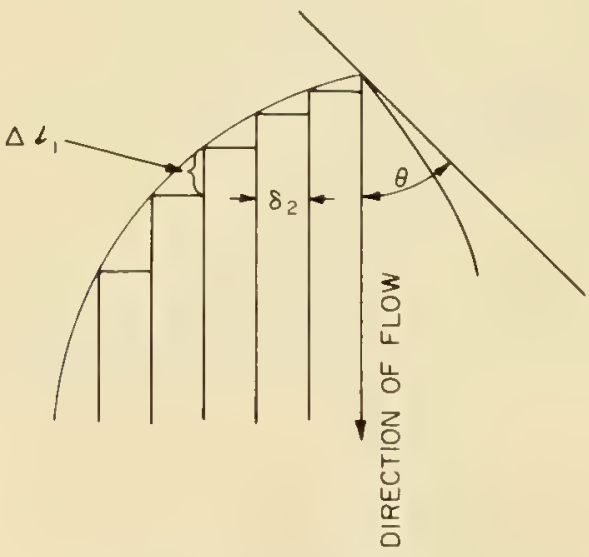

Fig. 14.2. Optimum shading of a hydrophone. 
shaped hydrophone can then be expected to have a flow-noise response proportional to

$$
F(\omega) \approx \frac{F_{0}}{\kappa^{2} l_{1} l_{2}}
$$

The power-spectrum decrease will be proportional to the fourth power of the frequency and to the square of the product of the linear dimensions of the hydrophone.

A circular hydrophone, as a first approximation, may be resolved into a central strip and a number of shaded strips, $n-1 \approx n$, the strips being all of a width equal to the transverse correlation distance. The resulting force on the receiver, then, is given approximately by

$$
F \approx \frac{1}{\kappa} \delta_{2}+\frac{1}{\kappa}(n-1) \cong \frac{1}{\kappa l_{1}} l_{1} \delta_{2}+\left[\frac{1}{(\kappa 1)}\right]^{2} \sqrt{\frac{l_{2}}{\delta_{2}}} l_{1}^{2}=\frac{1}{\kappa l_{1}}\left(l_{1} \delta_{2}+\frac{1}{\kappa l_{1}} \sqrt{\frac{l_{2}}{\delta_{2}}} l_{1}^{2}\right)
$$

If the width of the receiver is much greater than the correlation length, the second term predominates as long as the frequency is not too high. The circular hydrophone can therefore be expected to behave like a perfectly shaped hydrophone at the high, but not at the very high, frequencies. At very high frequencies, the flow-noise sensitivity of the circular hydrophone will probably increase again.

A rectangular hydrophone is particularly sensitive to flow noise. The flownoise sensitivity of a hydrophone can be considerably reduced (as long as the nearfield pressure determines the received level) by varying its width from a small value to a maximum in the middle and to a small value again at its end. In a hydrophone of this shape, the pressure maxima and minima of the noise pressure (Fourier components of the noise pressure) cancel one another to a higher degree as they are transported by the flow across the hydrophone area. The effect of varying the width of the hydrophone is similar to the shading of arrays of hydrophones. Its response outside the main maxima-that is, for the higher frequencies of the turbulence-decreases considerably. Because of the approximate isotropy of the eddies, the flow-noise sensitivity of a perfectly shaped hydrophone can be expected to be reduced by a factor of

$$
S=\left[\frac{\sin \left(\kappa l_{1} / 2\right)}{\kappa_{1} l_{1} / 2}\right]^{2}
$$

Figure 14.3 shows a graphical representation of the square of this factor, which determines the received power spectrum, for a hydrophone that has a diameter 4.3 times the boundary-layer thickness. This curve is very nearly the same as the curve determined for the hydrophone sensitivity from Willmarth's [11] measurements. The range of these measurements, however, extends over only 1.5 decades (energy levels); therefore, this apparent agreement does not mean too much. However, the theoretical curve explains the steep slope over the range $0.5<2 \pi f \delta^{*} / U_{0}<0.8$ that has been observed by Willmarth and Harrison and that has not been observed at frequencies greater than those of the buoyant-unit runs to be described later. Doubling the lineardimensions now reduces the flow-noise level by $12 \mathrm{db}$, and doubling the frequency reduces the spectral level by $12 \mathrm{db}$. 


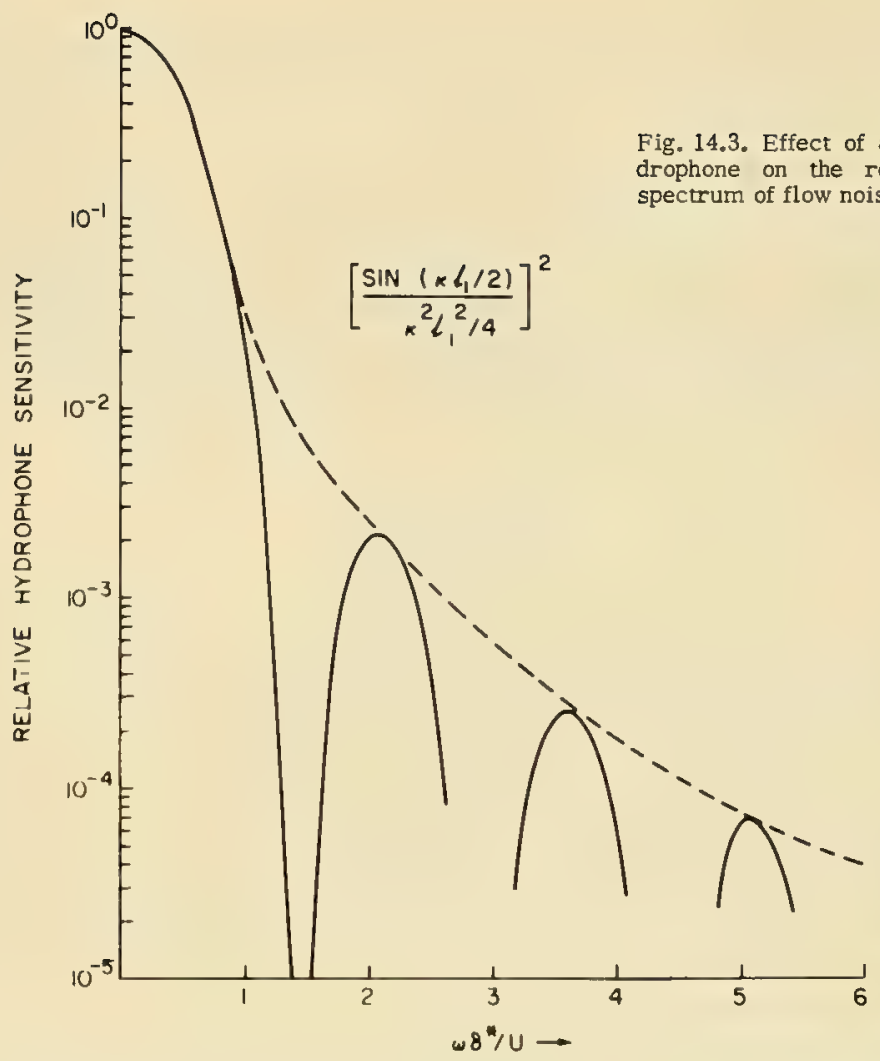

The above theory is only partially borne out by the experiments as will be shown later. It has been assumed that the turbulence is homogeneous, so that it can be described by its space- and time-average microscopic properties. However, for the frequency analysis, filters are used that have a relatively great bandwidth and consequently a very short integrationtime. The frequency analysis therefore yields results that correspond to averages over very short time integrations. For such short integrations the turbulence is far from being homogeneous. Every individual bump of turbulence is recorded separately: the filters have forgotten all about the first bump of turbulence when they receive the second, and the cancelling-out effect described above seems to be limited, on the average, to the volume of one such bump of turbulence. This volume is approximately the same as the volume over which the turbulent velocities are correlated.

The analysis of turbulence may usually be designated as an analysis of the macrostructure of the turbulence; in contrast, an analysis of the flow noise seems to correspond to an analysis of the microstructure of the turbulence.

\subsection{NOISE GENERATED BY THE SURFACE ROUGHNESSES}

As the velocity of the vehicle increases, the roughnesses become more effective. Experimental results show the roughnesses shed eddies whenever they 
penetrate the laminar boundary sublayer [16], that is, when the fluctuating velocity $v^{*}$ exceeds the value five. This Reynolds-number condition leads to the equivalent result that in a turbulent boundary layer, the surface roughnesses become effective whenever this height in inches becomes equal to $6 \cdot 10^{-2}$ divided by the velocity of the vehicle $U_{0}$ in knots:

$$
h \text { [inches }]=6 \cdot \frac{10^{-2}}{U_{0}}[\mathrm{knots}]
$$

If the boundary layer is not turbulent, $v^{*}$ has to be replaced by the true velocity $U_{0} h / \delta$ at the peaks of roughnesses, and the corresponding Reynolds-number condition becomes

$$
5 \approx h \frac{U_{0} h}{\delta \nu}
$$

or

$$
\frac{h^{2}}{\delta^{2}}=\frac{5 \nu}{U_{0} \delta}=\frac{4.2 \cdot 10^{-4}}{U_{0} \delta}
$$

where $U_{0}$ is in knots and $\delta$ is in inches. For a boundary layer of a thickness of $10^{-3}$ in. (as on the spherical head of a buoyant unit) and a speed of 42 knots, $h / \delta=0.1$, or $h=10^{-4}$ in., which is in good agreement with the experimental results.

The eddies shed by the surface roughnesses may be expected to depend on the velocity gradient near the wall. They will be independent of whether the

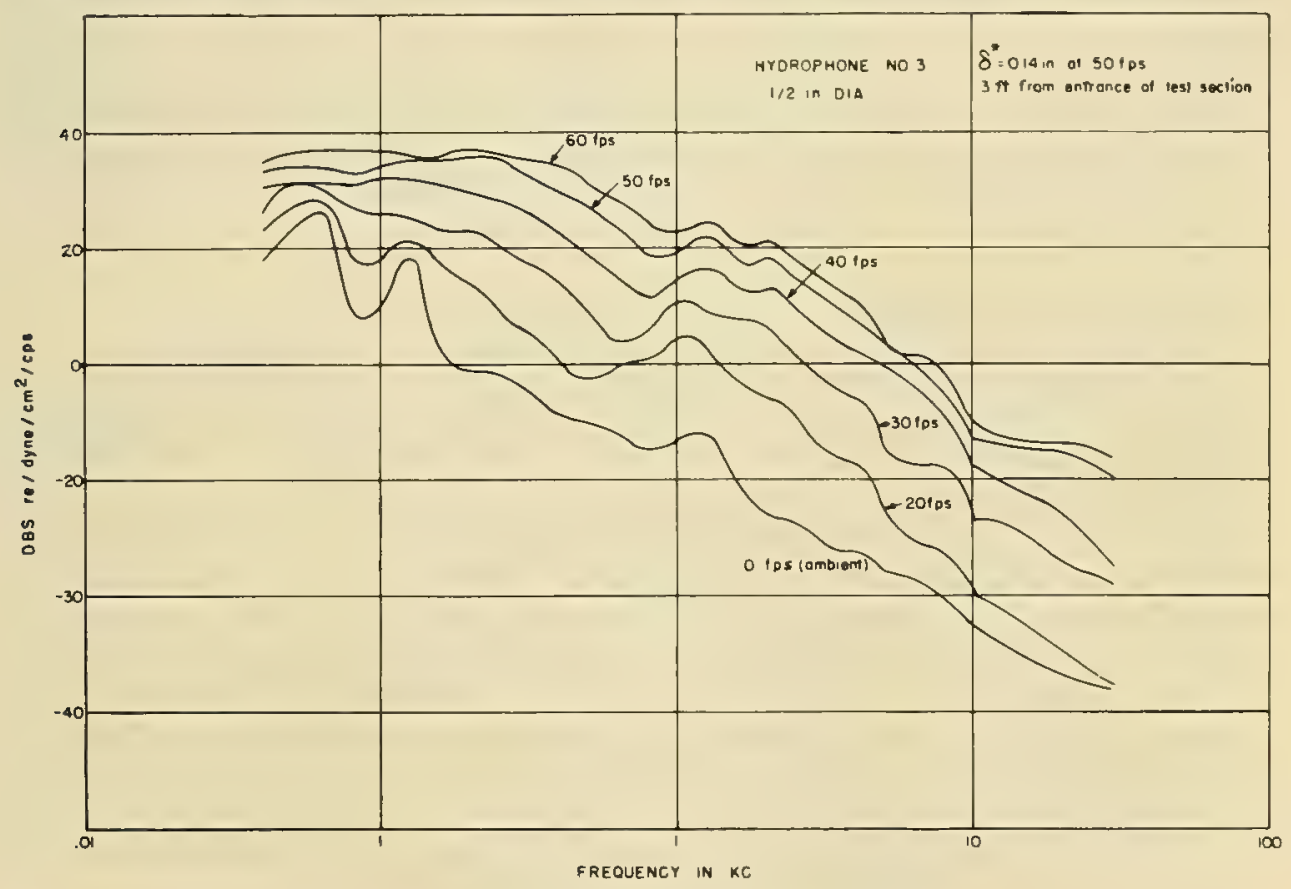

Fig. 14.4. Flow-noise spectra as a function of speed. 


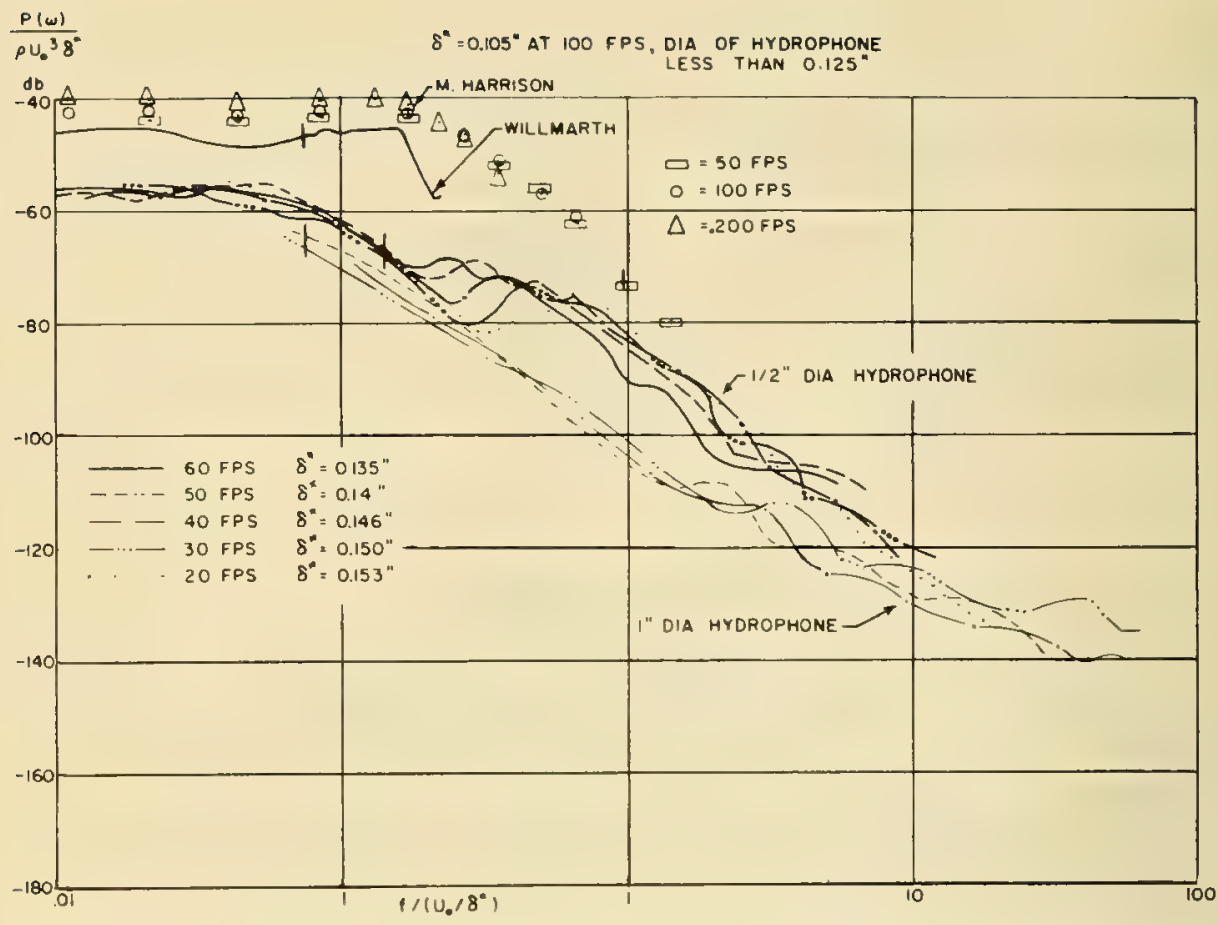

Fig. 14.5. Flow-noise spectra in dimensionless variables showing the effect of hydrophone diameter.

boundary layer is turbulent or laminar. However, the eddies decay rapidly in the laminar boundary layer, whereas in the turbulent boundary layer the eddies shed by the surface roughnesses increase the intensity of the turbulence in the boundary layer.

\subsection{EXPERIMENTAL RESULTS}

Measurements of the boundary-layer noise have been performed in the test section of the Garfield Thomas Water Tunnel at the Ordnance Research Laboratory and in Key West with the aid of two cigar-shaped buoyant units. Because of the great intensity of the flow noise, machinery noise is masked by flow noise and measurements can be performed in water tunnels. The curves shown in Fig. 14.4 were obtained in the test section of the Garfield Thomas Water Tunnel, which has a diameter of $122 \mathrm{~cm}$. Hydrophones were mounted flush with the wall and the noise levels were analyzed in a frequency range from $60 \mathrm{cps}$ to about 30 kc. Figure 14.4 shows the noise levels as functions of the frequency for various speeds. Figure 14.5 shows the same curves and additional ones for a larger hydrophone diameter represented in dimensionless variables. The curves obtained for a particular hydrophone size are almost coincident and the difference in the levels obtained for the two hydrophone sizes is about $12 \mathrm{db}$ as predicted by the theory. Figure 14.6 reproduces the result of a narrow-band analysis of the noise. Machinery noise and wall vibrations would have generated spectral lines; however, such lines do not appear in the spectrum. 


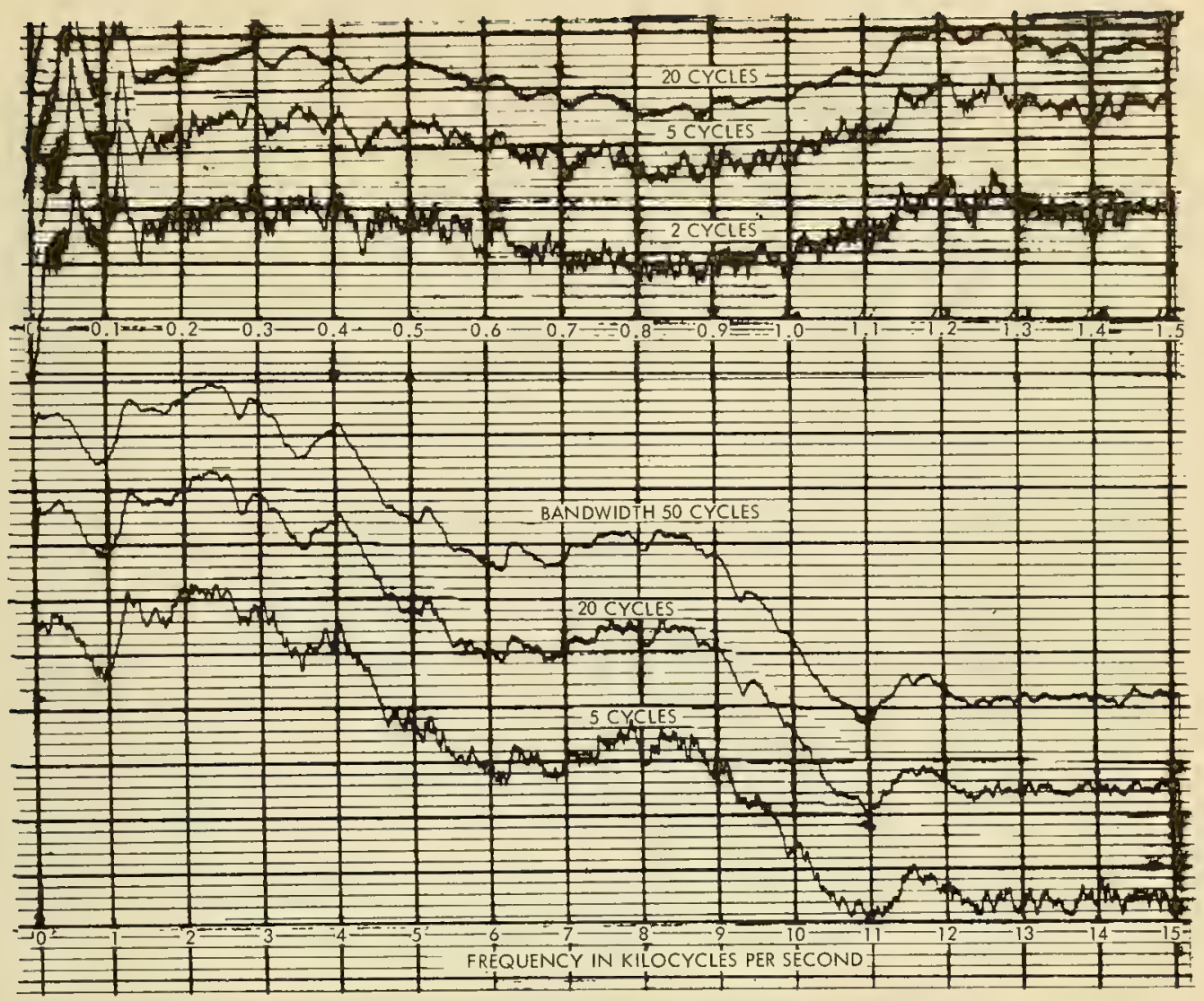

Fig. 14.6. Narrow-band spectrum analysis of flow noise.

The noise generated in a frequency band around $24 \mathrm{kc}$ by the surface roughnesses has been studied with the aid of a rotating cylinde: of a height of $42 \%$ in. and a diameter of $17.2 \mathrm{in}$. Such a cylinder has a very thick boundary layer, and consequently generates little high-frequency boundary-layer noise. A rotating cylinder is, therefore, particularly suited to study the effect of roughnesses. *

Figure 14.7 shows a number of curves that have been obtained for various types of roughnesses on the surface of the cylinder with a 2.5-in.-diameter hy drophone. The noise level now exceeds that of the ambient noise at a much slower speed than in the previous case. For the same velocities, the flow noise produced by the rough surface is 20 to $50 \mathrm{db}$ greater than that produced by the smooth, painted surface. Since nothing else has been changed, this greater noise must be attributed to the effect of the surface roughnesses. There is thus no doubt that roughnesses generate flow noise. For Grit No. 180, $80 \%$ of the particles have a height of about $5 \cdot 10^{-3}$ in. The critical speed [Eq. (26), where the roughnesses become effective] is therefore 2 knots or $1.08 \mathrm{~m} / \mathrm{sec}$. If we extrapolate the curve for this kind of grit down to a speed of 2 knots (from whence the roughnesses *Rotating cylinder measurements have also been performed by L. N. Wilson [17]. 


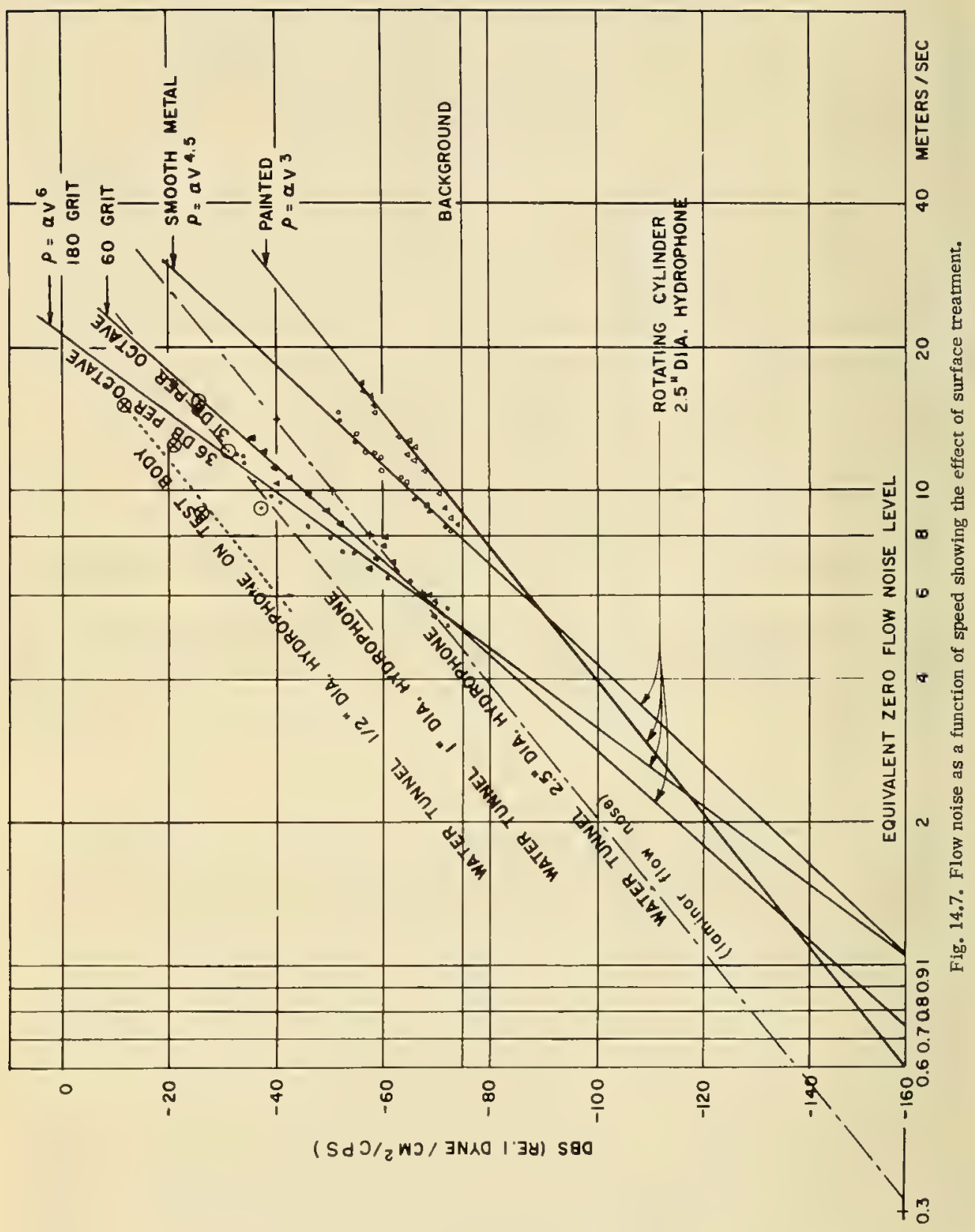


would be expected to become acoustically effective), the noise level turns out to be $-158 \mathrm{db}$ relative to $1 \mathrm{~d} / \mathrm{cm}^{2}$. This value may be considered as the equivalent of zero flow-noise level for the condition of the experiment. The other curves should then intersect with the zero flow-noise level, $-158 \mathrm{db}$, at speeds equal to the corresponding critical values; and this indeed seems to be what happens if we allow for a small experimental error and for the fact that the energy of the background and that of the flow noise add up to the resultant noise level so as to change the shape of the curves at lower noise levels. Paint covers the smaller roughnesses, but in doing this seems to increase the larger roughnesses with paint streaks. Yet the density of the larger roughnesses is relatively small and the roughness noises seem to be masked by the boundary-layer noise. This conclusion follows from the slope of the curve, which is only $18 \mathrm{db}$ per speed octave.

We may conclude that the roughness noise equals the boundary-layer noise at $24 \mathrm{kc}$ at a speed that is about six times as great as the critical speed if the surface is densely covered with roughnesses, and at a correspondingly higher speed if the roughnesses are widely spaced from one another.

Figures 14.8 and 14.9 show measurements obtained with buoyant units. The units were about 5 yards in length and $19 \mathrm{in.}$ in diameter. Each was equipped with six hydrophones and a seven-channel tape recorder. Theory predicts that the spectral level of the low frequencies is proportional to the thickness of the boundary layer and is borne out by the experiments. The low-frequency noise level increases with the distance from the head of the buoyant unit. The highfrequency noise level, on the other hand, is considerably weaker at the rear of the buoyant unit where the boundary layer is very thick. The boundary layer of the buoyant units or of a vehicle of a similar shape is very thin at its head. For a buoyant unit the thickness is about a thousandth of an inch. The flow is therefore laminar. The turbulence starts when the head merges into the cylindrical section. The noise that is received at a stagnation point is entirely due to the sound radiated from the turbulent zones into this area and to the eddies shed by the surface roughnesses. If the head of the unit is not highly polished, then the flow velocities become large enough an inch or two from the stagnation point, and the surface roughnesses generate eddies. Because of the laminar nature of the boundary layer in this part of the unit, the eddies decay very rapidly, but they do generate flow noise. This can easily be tested by performing measurements with hydrophones of different sizes. The received flow noise depends on the size of the hydrophone and consequently is at least partially nearfield noise. The flow becomes turbulent at the minimum pressure point which is very near to the joint between the cylindrical section and the head of the unit. In this region the velocities are $50 \%$ larger than the velocity of the vehicle and the turbulence is still very unstable and bumpy. The noise levels are therefore considerably larger than those which would correspond to the stable turbulence of the boundary layer. The region near this joint is particularly critical for the generation of flow noise. Toward the rear, one to two yards away from this joint, the tur bulence has already decreased to its normal value and the noise level is approximately the same as the noise level in the test section of the water tunnel. 


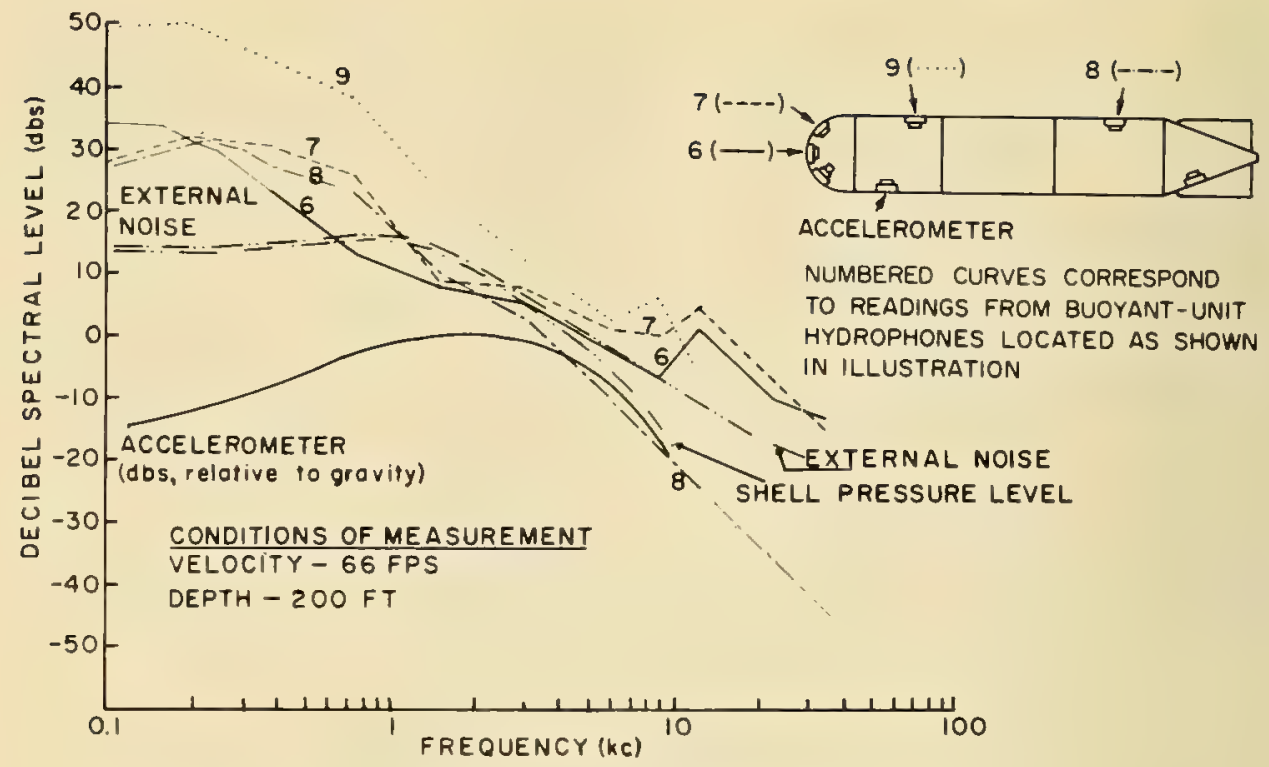

Fig. 14.8. Flow-noise spectrum of buoyant unit when covered with grit.

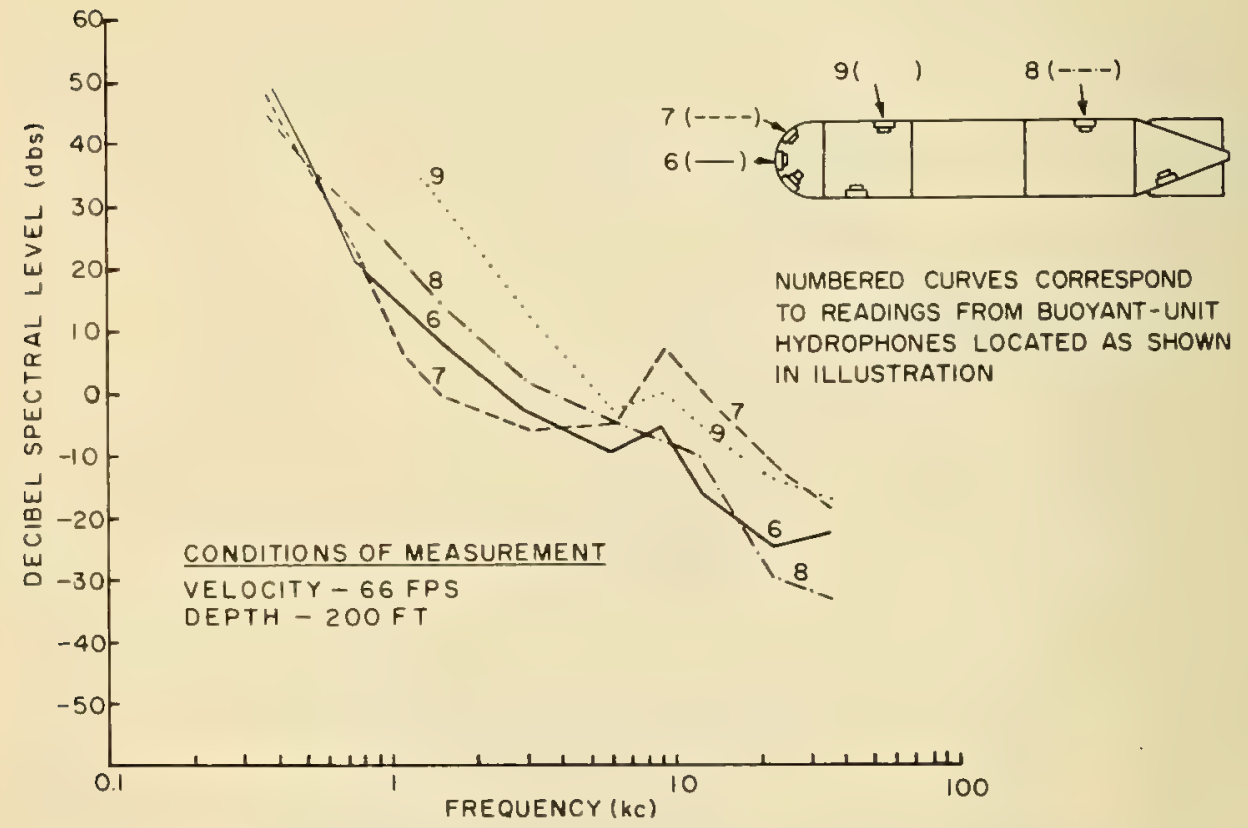

Fig. 14.9. Flow-noise spectrum of buoyant unit with highly polished finish and waxed joints. 
Figure 14.10 shows the noise spectrum recorded at hydrophone 8, located at the rear end of the cylindrical section. The boundary layer there is much thicker and the noise level is, therefore, more intense at the flow frequencies, as predicted by the theory. Conversely, the high-frequency noise levels are correspondingly lower. The curves for the polished surface, for instance, slope down very steeply ( $20 \mathrm{db}$ per frequency-doubling). The readings obtained with hydrophone 8 were practically the same as those obtained with hydrophone 10 , located just beyond the rear of the cylindrical section; therefore, only a limited number of readings was taken with hydrophone 10 .

Not filling the joint between the nose and the cylindrical section increases the low-frequency noise level recorded by the nose-section hydrophones by as much as $20 \mathrm{db}$ and also increases the vibration level of the shell. Uncovered joints apparently generate larger eddies and, consequently, considerable lowfrequency noise (Strouhal noise, for instance). But these large eddies also seem to increase the effective thickness of the boundary layer and reduce the gradients near the wall, lowering the high-frequency noise level considerably. The large eddies produced by the unwaxed joints apparently absorb a considerable fraction of the energy that otherwise would be available for the excitation of the highfrequency noise spectrum.

Figure 14.11 summarizes the effects of various surface finishes (high polish, spray paint, fine grit, and coarse grit) at low frequencies (frequency range of $700 \mathrm{cps}$ to $1.5 \mathrm{kc}$ ) and at high frequencies (frequency range of 20 to $40 \mathrm{kc}$ ). Grit can be seen to reduce the low-frequency noise level by as much as 10 to $16 \mathrm{db}$. Grit seems to have the effect of splitting up the larger eddies into smaller ones

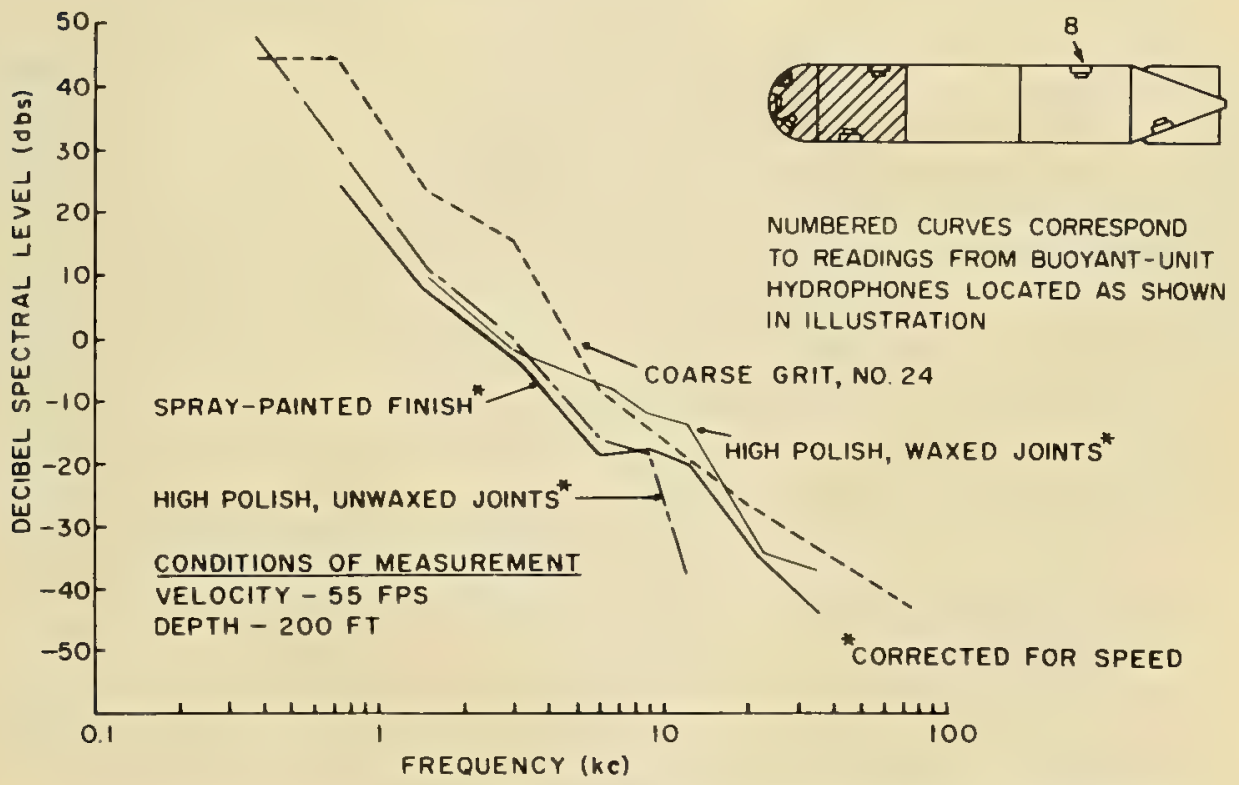

Fig. 14.10. Flow noise measured by hydrophone No. 8 on the buoyant unit for various finishes. 


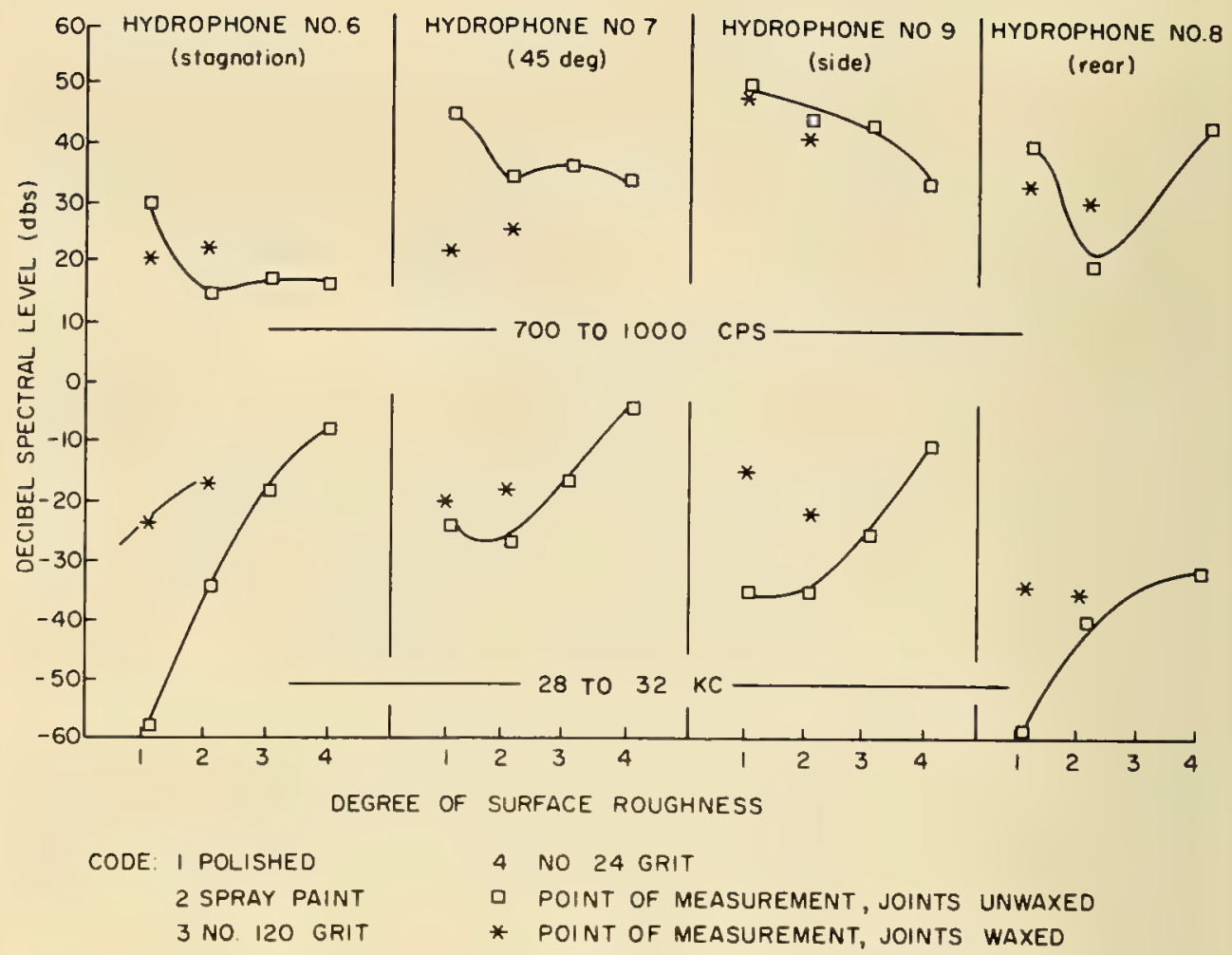

Fig. 14.11. Comparison of flow noise for various degrees of surface roughness at high and low frequencies.

and increasing the high-frequency noise spectrum, at least to some extent, at the expense of the low-frequency spectrum. At high frequencies the nose hydrophone (No.6) is particularly sensitive to surface roughness, and a high polish gives much better results than spray paint. The flow velocity at the circumference of the nose hydrophone is about $2 \mathrm{ft} / \mathrm{sec}$. The Reynolds number obtained by the height of the roughnesses $(0.001 \mathrm{in}$.) and the velocity that would be expected at the edges of the hydrophone is 25 and, hence, five times larger than the values required for shedding eddies. This Reynolds number would correspond to a noise level of roughly $-60 \mathrm{db}\left(\right.$ re $\left.1 \mathrm{~d} / \mathrm{cm}^{2}\right)$, as could be concluded from the rotating-cylinder measurements. Hydrophone 7 , the $45^{\circ}$ hydrophone, is not very sensitive to very small surface roughnesses over its area; the noise level is mostly radiated noise which masks the noise that would be produced by small surface roughnesses. However, the noise generated by coarser roughnesses over the hydrophone area seems to mask the radiated noise completely. At hy drophone 8, on the other hand, the boundary layer is already relatively thick, and, as a consequence, the high-frequency boundary-layer noise is very weak. Therefore, most of the high-frequency noise observed at this hydrophone is due to the surface roughness, and polishing leads to much better results than the spray-paint finish. 


\subsection{RADIATED PRESSURE AND SHELL VIBRATION LEVEL}

A large hydrophone has been shown to be very insensitive (by a factor of $1 / 1000$ or more) to the small-scale turbulence that generates the high-frequency noise. Such a hydrophone measures essentially only those pressure fluctuations that are correlated over greater distances, such as the true sound pressure produced by the generation and decay of the eddies in the turbulent boundary layer. The hydrophone gives an indication of the true radiation field only; the validity of this indication has already been verified by the rotating-cylinder experiment and, again, by all the buoyant-unit runs, where the pressure inside the boundary layer, and outside at distances of about 100 yards from the buoyant unit, has been recorded. At the higher frequencies, the external pressure, when corrected for the geometrical decrease of its amplitude with distance, is practically the same as the pressure recorded inside the boundary layer. The radiated pressure is usually greater than that which would be deduced from the vibration amplitude of the shell even under the most favorable conditions (as suming $\rho c$ as the value of the radiation resistance for the shell vibrations; see Fig. 14.8, double-dot-dash curve).

In the frequency range of the measurements, the bending wavelength of the shell is always smaller than the sound wavelength. The very-large-scale pressure fluctuations that are responsible for the true sound radiation are, therefore, alternately in phase and out of phase with the bending modes of the shell and do not excite these modes very much. (A bending mode can be excited only if the pressure pattern shows variations similar to the mode function.)

\subsection{EFFECT GF SIZE AIND SHAPE OF HYDROPHONE ON THE RECEIVED NOISE LEVEL}

It has been shown above that the level of the nearfield flow noise that is picked up by a hydrophone greatly depends on the size of the hydrophone. However, the experimental results show that this phenomenon is considerably more complex than was assumed initially. No area effect was found at the very low frequencies in the test section of the water tunnel; all the hydrophones were equally sensitive to flow noise in the frequency range 50 to $600 \mathrm{cps}$. This unexpected result leads to the conclusion that the correlation of the flow, in the test section of the water tunnel, in the transverse direction, is considerably greater than the boundary-layer thickness. That this conclusion is reasonable can be illustrated by smoke photographs of the turbulence around an airplane wing. Such photographs show that the turbulence is stratified in the direction transverse to the flow and that it is, therefore, correlated over great distances in this direction. The stratification seems to be particularly pronounced near the leading edge of the wing, where the turbulence is generated first, and is less pronounced toward the rear. At the frequencies above $1 \mathrm{kc}$, the area effect was very pronounced in the measurements in the water tunnel, whenever the hydrophone diameter was not very much larger than the boundary-layer thickness (see Fig. 14.5). A very pronounced area effect was also found for the rotatingcylinder measurements. At a frequency of $20 \mathrm{kc}$, a 5-in.-diameter hydrophone was $12 \mathrm{db}$ less sensitive to flow noise than a 2.5-in.-diameter hydrophone. The noise level outside the boundary layer of the cylinder ( $3 \mathrm{ft}$ distant from it) was 


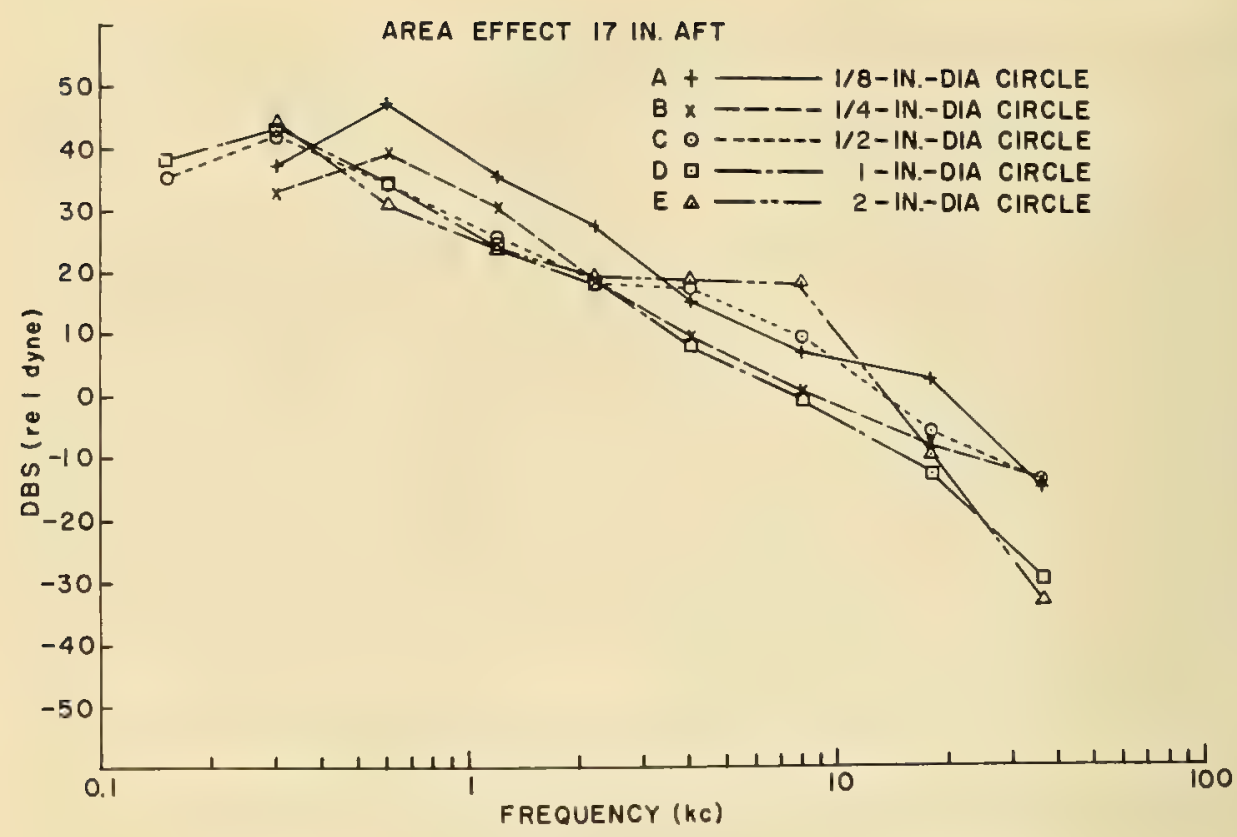

Fig. 14.12. Hydrophone-area effect on buoyant unit.

about the same as that recorded inside with the larger hydrophone. It was therefore concluded that the area effect occurred whenever the hydrophone diameter was smaller than $5 \mathrm{in}$. The buoyant units exhibit the theoretical area effect at the very low and middle frequencies up to about $20 \mathrm{kc}$ for hydrophones of, at the most, $1 / 2$ in.-diameter (see Fig. 14.12). At the higher frequencies the recorded noise seems to be essentially radiation-field noise and the recorded noise is therefore practically independent of the diameter of the hydrophone.

The area effect is greatly dependent on the ratio of the diameter of the hydrophone to the boundary-layer thickness and vanishes whenever the hydrophone becomes large. In contrast, the shape effect seems to persist irrespective of the hydrophone size.

A rectangular hydrophone turns out to be particularly flow-noise sensitive, whereas a circular hydrophone is much less sensitive. Figure 14.13 illustrates this for a cylindrical hydrophone, which is similar in its behavior to a rectangular hydrophone. A fish-shaped hydrophone indicates a small noise level if the head of the fish points in the direction of the flow, and the noise level is $15 \mathrm{db}$ larger if the fish points transverse to the main flow (Fig. 14.14a). The square shape shows a similar result when the corner is pointed in the direction of the flow (Fig. 14.14b). At frequencies above $8 \mathrm{kc}$, the nearfield sensitivity of most of the hydrophones used in the experiments became so poor that they indicated almost nothing but radiation-field noise (Fig. 14.14c). As a consequence, area and shape effects vanish. 


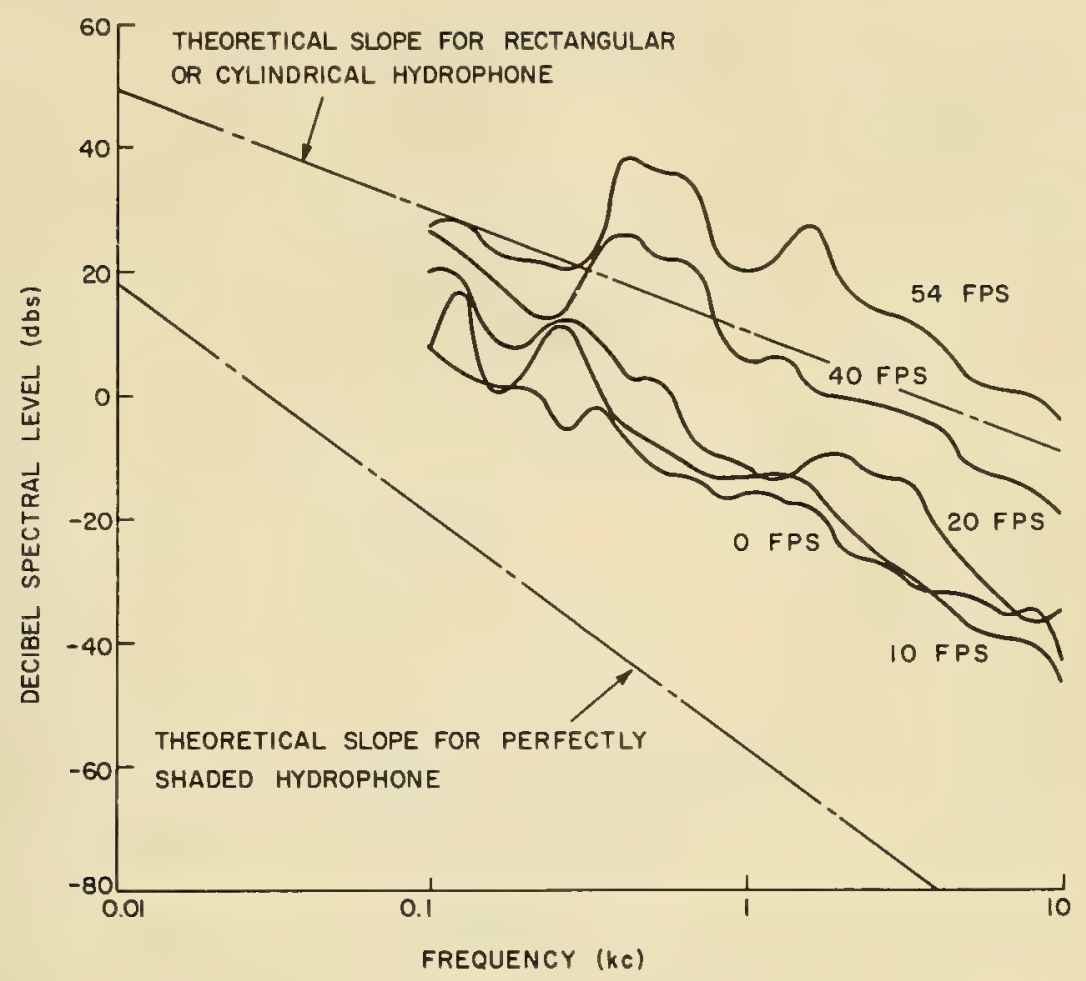

Fig. 14.13. Hydrophone-shape effect.

\subsection{THE REDUCTION OF FLOW NOISE}

The flow-noise level depends greatly on the position of the hydrophone. The intensity of the low frequencies is relatively small at the front of the unit where the boundary layer is laminar, or turbulent and very thin. The high-frequency noise is very weak in the stagnation region, which is nonturbulent, and even weaker towards the rear of the unit where the boundary layer is very thick. The high-frequency level (above $15 \mathrm{kc}$ ) received by the head hydrophone can be reduced considerably by the use of a flat head instead of a hemispherical head. Most of the noise that is received by this hydrophone is generated near the joint between the cylindrical section and the head. A flat head increases the shadow effect, and less noise is diffracted into the stagnation region. Turbulencesuppressing varnishes proved effective as noise reducers. A vaseline coating, for instance, reduced the noise level above $3 \mathrm{kc}$ by almost $20 \mathrm{db}$. The noise level also depends greatly on the condition of the joint between the head and the cylindrical portion. If this joint is not filled in, the boundary layer becomes thick, as if it were tripped, or oscillates. Not filling in the joint reduces the highfrequency noise level but increases the low-frequency noise level. Methods are being examined that may lead to a further reduction in the noise level. 

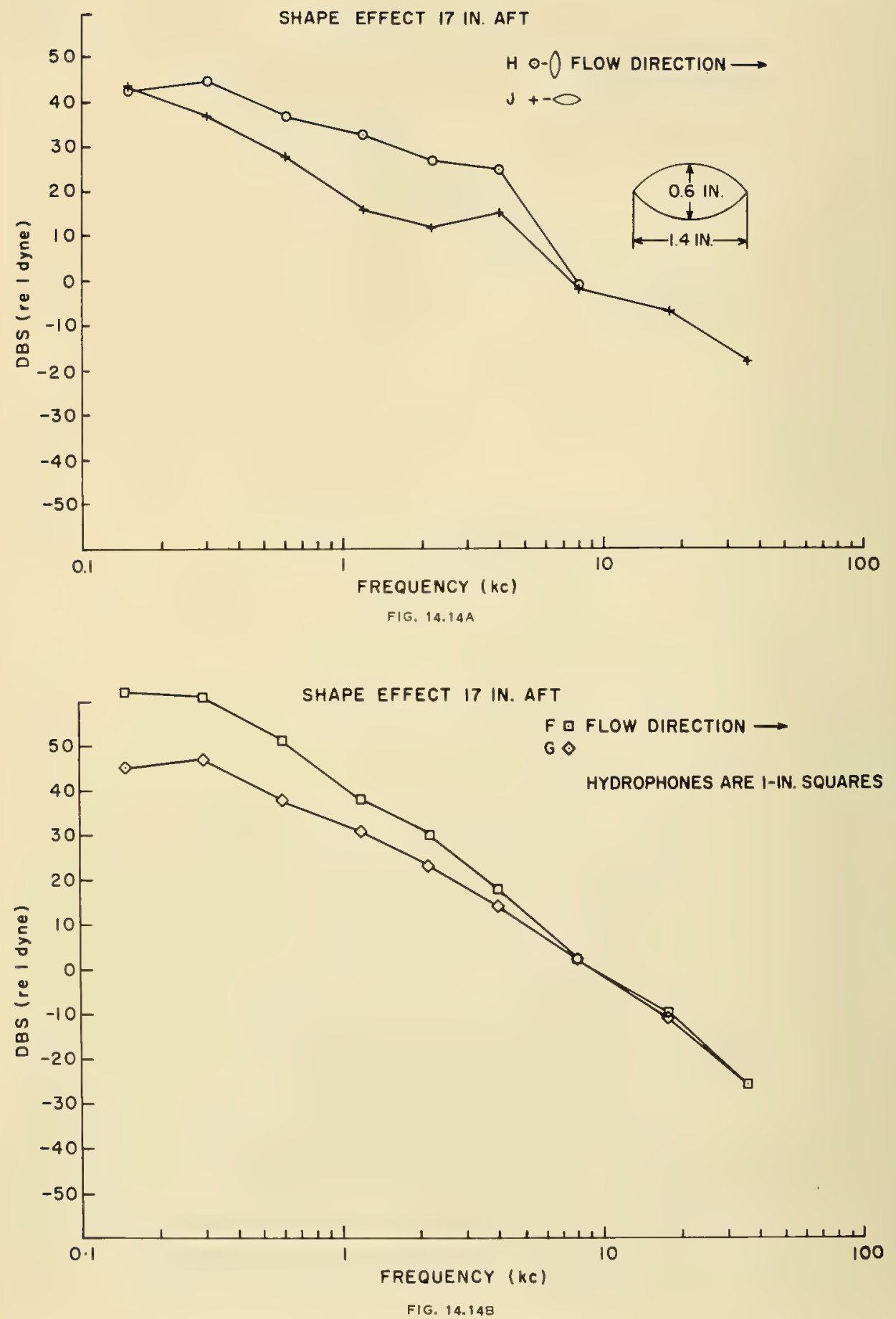


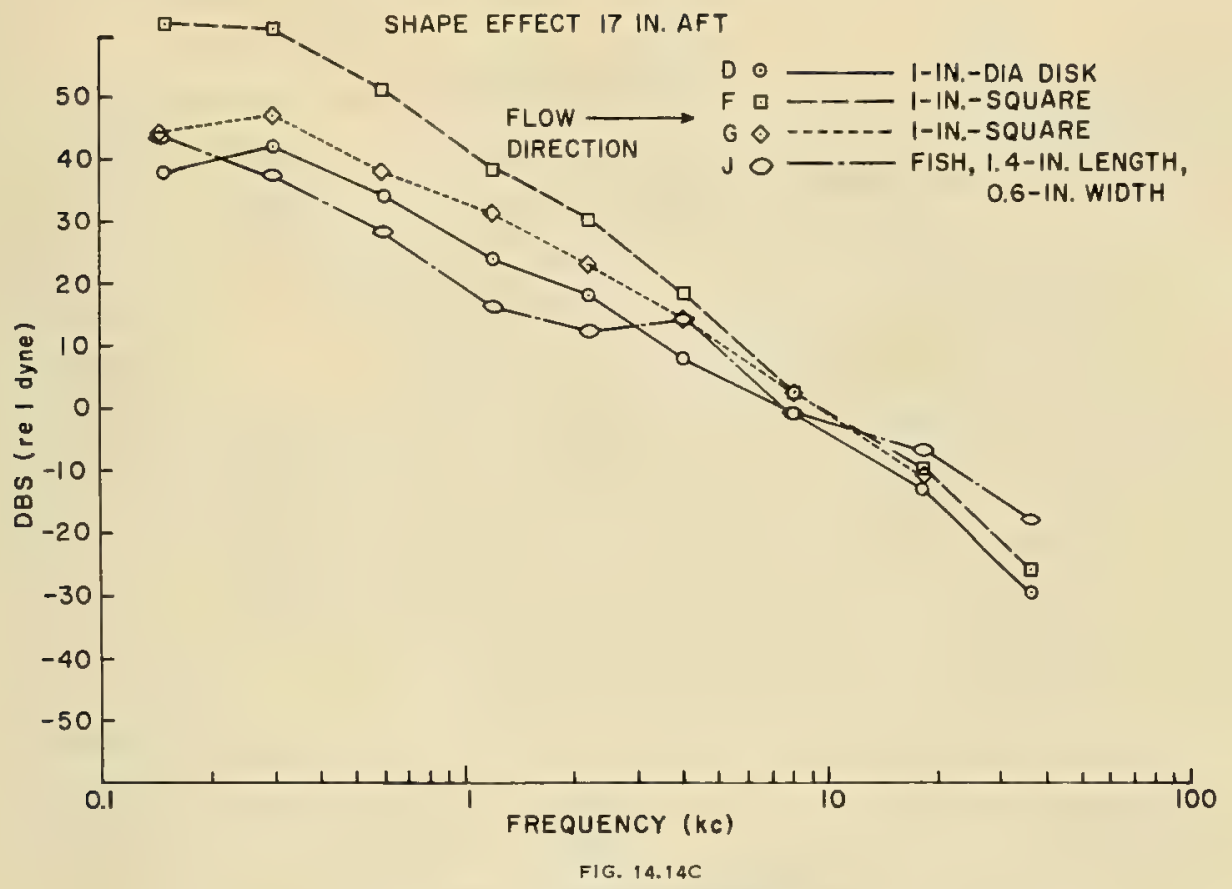

Fig. 14.14. Hydrophone-shape effect.

14.10. THE REPRODUCIBILITY OF THE MEASUREMENTS AND THE EFFECT OF SHELL VIBRATIONS ON THE RESULT

The results were obtained on different days and normally did not vary by more than two or three decibels. There were times when greater discrepancies were observed, however-discrepancies that could usually be attributed to temperature discontinuities or to great changes in the temperature structure of the sea. Some of the more important buoyant-unit runs were repeated five times; two runs were reserved for the recording of the low and middle frequencies and the remainder for the middle and high frequencies. Thus, five different recordings of the middle frequencies $(1 \mathrm{kc}$ to $20 \mathrm{kc}$ ) were available to check the accuracy of the recordings.

The measurements were not affected by shell vibrations. The driving-point impedance of the shell was about the same as the impedance of a $5 \mathrm{-g}$ mass at $1000 \mathrm{cps}$. The hydrophones had a mass of $800 \mathrm{~g}$. Therefore, only $1 / 160$ of the shell amplitude was transmitted to the hydrophones. This conclusion is in agreement with the experimental results obtained when the shell was driven in air with the same amplitude that normally would have been excited by the flow noise. The hydrophone readings were more than $40 \mathrm{db}$ below the readings that would have been obtained if the shell had been excited by flow noise. In a second investigation, a heavy damping layer was applied to the shell, but the damping had no effect on the hydrophone responses. 


\section{REFEREHCES}

1. H. Schlichting, Boundary Layer Theory (McGraw-Hill Book Co., 1955).

2. J. Laufer, "Investigation of Turbulent Flow in a Two-Dimensional Channel," NACA Report V, 1053 (1951).

3. T. Theodorsen and A. Regier, "Experiments on Drag of Revolving Disks, Cylinders, and Streamline Rods at High Speeds," NACA Report No, 793 (1945).

4. G. R. Batchelor, The Theory of Homogeneous Turbulence (Cambridge Univ. Press, New York, 1956).

5. R.H. Kraichnan, "Pressure Field Within Homogeneous Anisotropic Turbulence," J. Acoust, Soc. Am., Vol. 28, 64-72 (1956); "Pressure Fluctuations in Turbulent Flow over a Flat Plate," J. Acoust. Soc. Am., Vol. 28, 378-390 (1956).

6. V. A. Sandborn and W. H. Braun, "Turbulent Shear Spectra and Local Isotropy in the Low Speed Boundary Layer," NACA TN 3761 (September, 1956).

7. V.A. Sandborn and R. J. Slogar, "Longitudinal Turbulent Spectrum Survey of Boundary Layers in Adverse Pressure Gradients," NACA TN 3453 (May, 1955).

8. A.N. Kolmogorov, "The Local Structure of Turbulence in Incompressible Viscous Fluid for Large Reynolds Numbers," C. R. Acad., Sci., Vol, 30, 301, U.R.S.S.

9. W. Heisenberg, "Zur statistischen Theorie der Turbulenz," Z. angew. Phys., Vol. 124, 628-657 (1948).

10. Mark Harrison, J. Acoust. Soc. Am., Vol. 29, 1252A (1957); also appeared in full as Hydromechanics Laboratory Research and Development Report 1260 (December, 1958), Dept. of the Navy, David Taylor Model Basin.

11. W.W. Willmarth, "Space-Time Correlations and Spectra of Wall Pressure in a Turbulent Boundary Layer," NASA Memorandum 3-17-59W (March, 1959).

12. E. J. Skudrzyk and G. P. Haddle, "Noise Production in a Turbulent Boundary Layer by Smooth and Rough Surfaces," J. Acoust. Soc. Am., Vol. 32, 19-34 (January, 1960).

13. M. J. Lighthill, "On Sound Generated Aerodynamically," Part I, Proc. Roy. Soc. (London) A, Vol. 211, 564 (1952); Part II, Vol, 221, 1 (1954).

14. 1. Proudman, "The Generation of Noise by Isotropic Turbulence, "Proc. Roy. Soc. (London) A, Vol. 214 , $119-132$ (1952).

15. G. M. Corcos, J.W. Cuthbert, and W. A. Von Winkle, "On the Measurement of Turbulent Pressure Fluctuations with a Transducer of Finite Size," Univ. Calif., Ser. No. 82, Contract No, N-oar-222(30), (November, 1959).

16. M. Goldstein, "A Note of Roughness," A.R.U., Report and Memo No. 1763 (1936).

17. L. N. Wilson, "Experimental Investigation of the Noise Generated by the Turbulent Flow around a Rotating Cylinder," J. Acoust. Soc. Am., Vol. 32, 1203-1207 (October, 1960). 


\title{
LECTURE 15
}

\section{SOME CONTRIBUTIONS FROM AERONAUTICS TO THE FIELD OF UNDERWATER NOISE}

\author{
E.J. Richards, J.L. Willis, and D.J.M. Williams \\ Department of Aeronautics and Astronautics \\ University of Southampton \\ Southampton, England
}

\subsection{INTRODUCTION}

During the last fifteen years, the introduction of jet engines into civil aircraft operation has accentuated the problem of noise in aviation, and many investigations have been made which are of distinct significance in the apparently distant field of underwater noise. For example, with the growth of aircraft engine power, the speeds of aircraft have increased to such a degree that the rough boundary flow along the fuselage gives rise to the majority of the roise inside the cabin; it gives rise to fuselage vibrations which can in certain circumstances cause skin cracks and fuselage and wing failures, and it is suspected that the radiated noise from the fuselage movement may have some effect on transition from laminar flow on neighboring surfaces. The noise radiated from such fuselages is very much a function of the modes of oscillation and the degree of damping, both structural and acoustic, involved in these particular modes. In the field of engine design also, such problems arise. It is known that compressor noise has a large random content which is related to the boundary-layer pressure fluctuations on the blades and is caused by the unsteadiness of the flow incident upon them. This problem of noise radiated from blades is now arousing major interest in engine design.

The knowledge gained from investigation of these various factors is often directly applicable to analogous problems in underwater noise. For instance, the problem of the boundary-layer noise radiated from the hull of a vessel is closely related to that of radiation from a fuselage boundary layer; the effects of pressure gradients, waviness, rivets, roughness, skin discontinuities, and so on, have their obvious equivalents. Similarly, the skin response to boundary-layer pressure fluctuations has an exact analogy in the self-noise within an Asdic dome, and in the structural vibration of a submarine. Still further, the use of correlation techniques and fuselage damping to identify and supress fuselage vibrations must also be of interest to naval personnel seeking to reduce the reradiation of noise from their craft. 
It is impossible to describe here all the investigations of interest to naval research workers. Therefore discussion will be confined to three aspects of our work at Southampton University, viz:

1. The nature of boundary-layer pressure fluctuations and the effect of single roughnesses

2. The radiated sound from a small flat plate

3. The modes of oscillation of structures, the acoustic damping achieved, and the reradiated noise problem

In addition, the significance of the investigations to the field of underwater noise will be illustrated by some simple calculations of radiated and self-noise based on the above and other investigations.

\subsection{BOUNDARY-LAYER PRESSURE FLUCTUATIONS}

We are interested in measuring the pressure fluctuations on the surface of an aircraft both at subsonic and supersonic speeds in order to ascertain the fluctuating loads occurring on it and in order to obtain the information necessary to calculate the noise radiated from the surface as a result of these fluctuating forces. It has been necessary to measure not only the rms pressure at the skin, but also the areas over which these pressures are correlated and the power spectrum of the fluctuating pressures relative to a convected frame of reference.

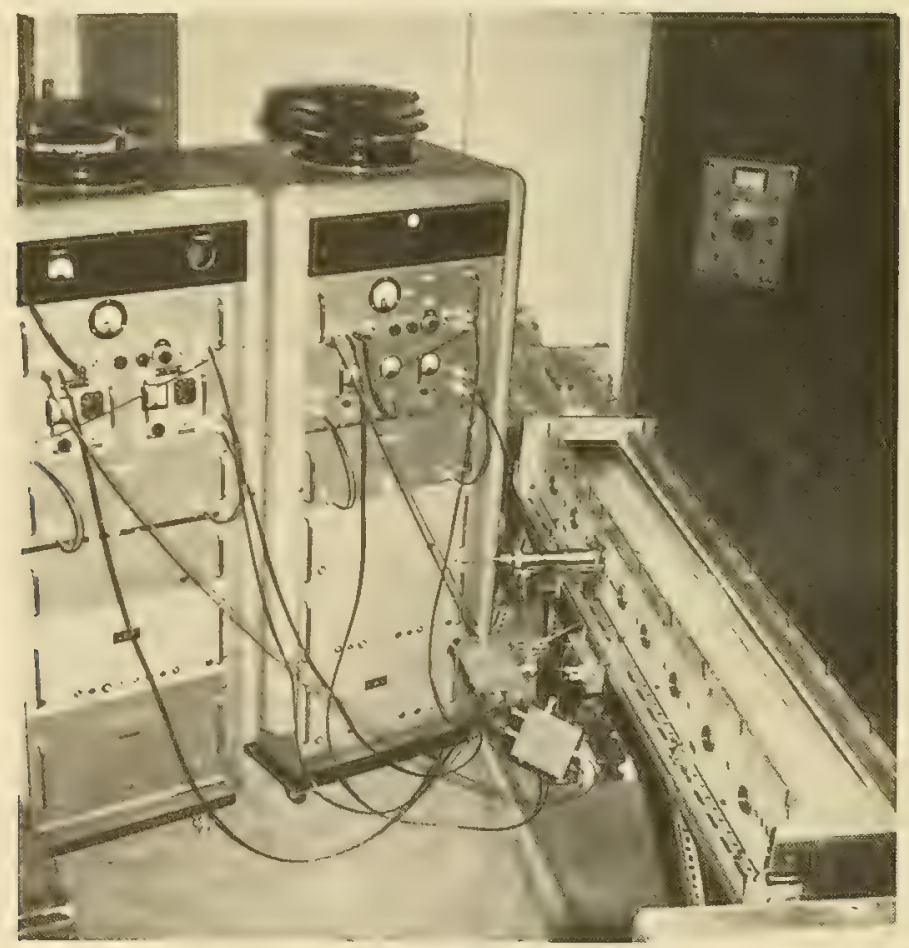

Fig. 15.1. Boundary-layer wind tunnel. 
Two experimental rigs have been used to do this; these are shown in Figs. 15.1 and 15.2. Figure 15.1 shows an induced-flow air channel of massive construction incorporating two measurement areas, one subsonic, the other supersonic. The supersonic region acts incidentally as a choke to prevent the radiation of any injector noise forward into the subsonic working section and results in a low level of background noise against which the boundary-layer pressure fluctuations are measured. Some difficulties have arisen, however, in eliminating lowfrequency vibration [1] and, consequently, the results are tentative in this frequency region.

The second facility (Fig. 15.2) is a water rig. Water flows vertically from an overhead tank via a series of gauzes and a well-designed contraction and into a base tank. The headertank and the pipe are isolated from any outside vibrations of the building. The elimination of background machinery noise by the use of a gravity feed has proved to be useful and measurements may be made in boundary layers of up to one inch in thickness. Again, some low-frequency vibration occurred, but much of this has been traced to a vibration of the pipe and has been reduced by the addition of a damping material to the outside of the pipe. Even so, it is suspected that some acoustical interference at frequencies of 200

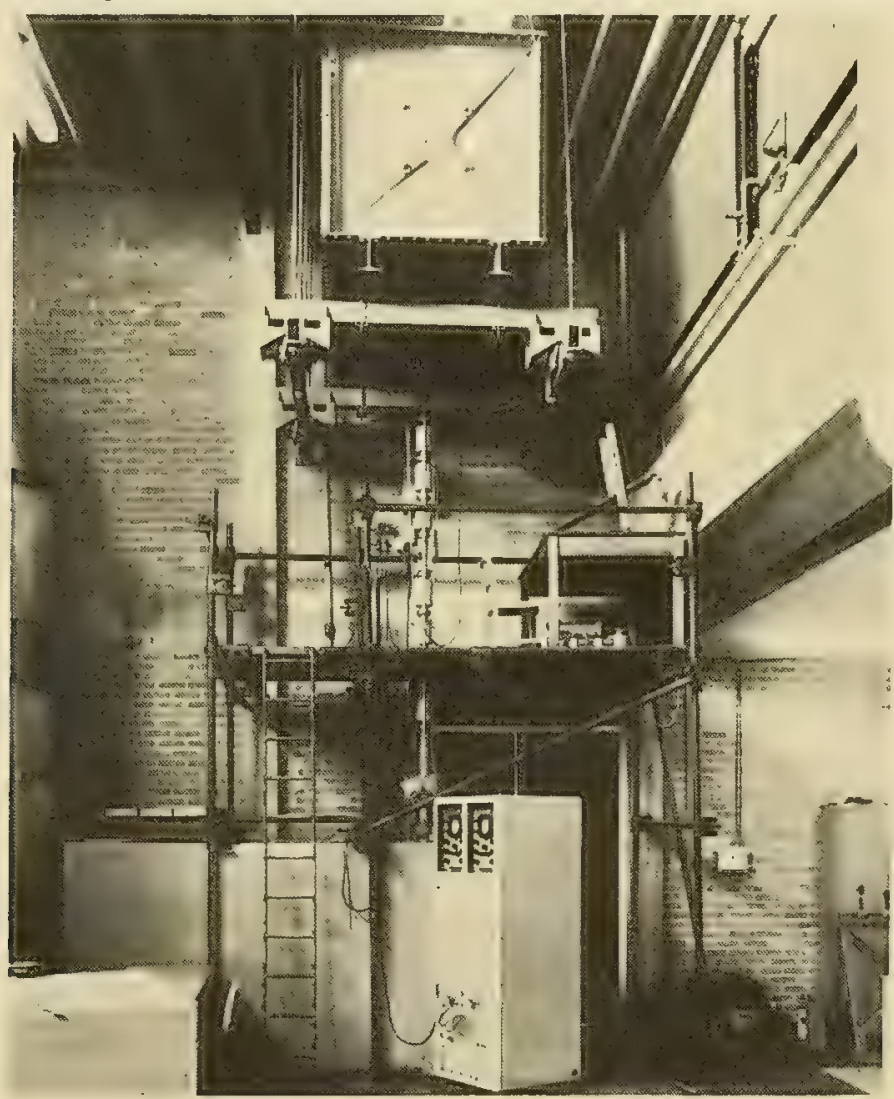

Fig. 15.2. The water rig. 


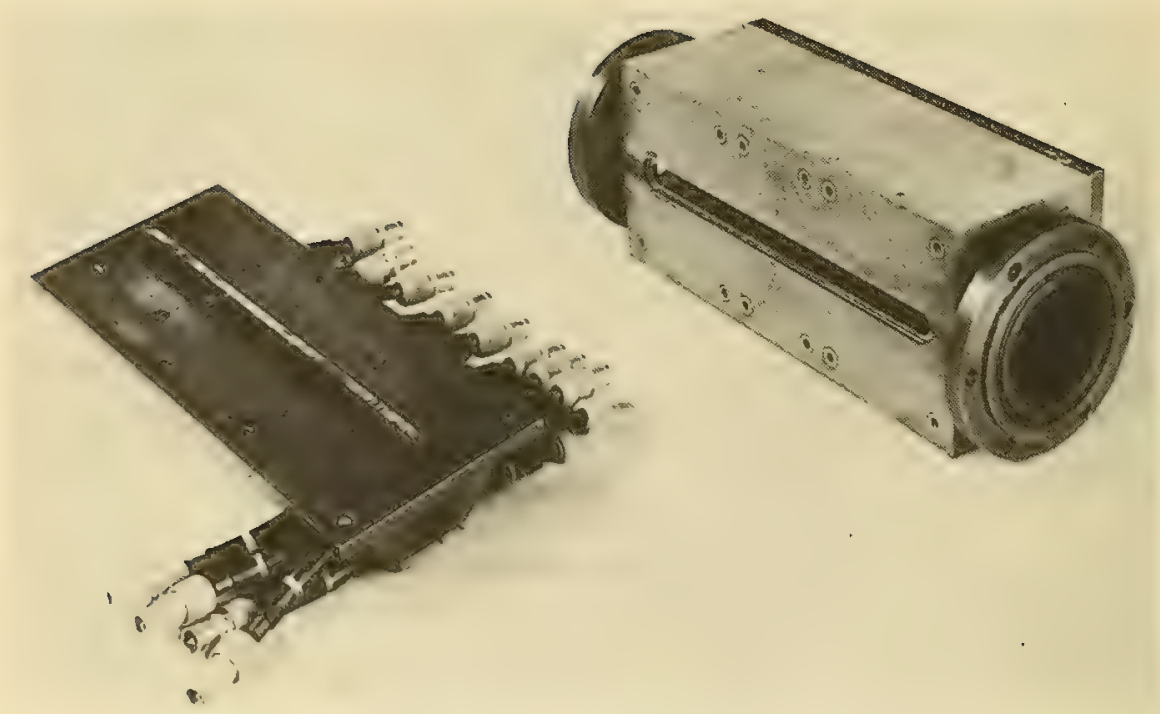

Fig. 15.3. Transducer bank.

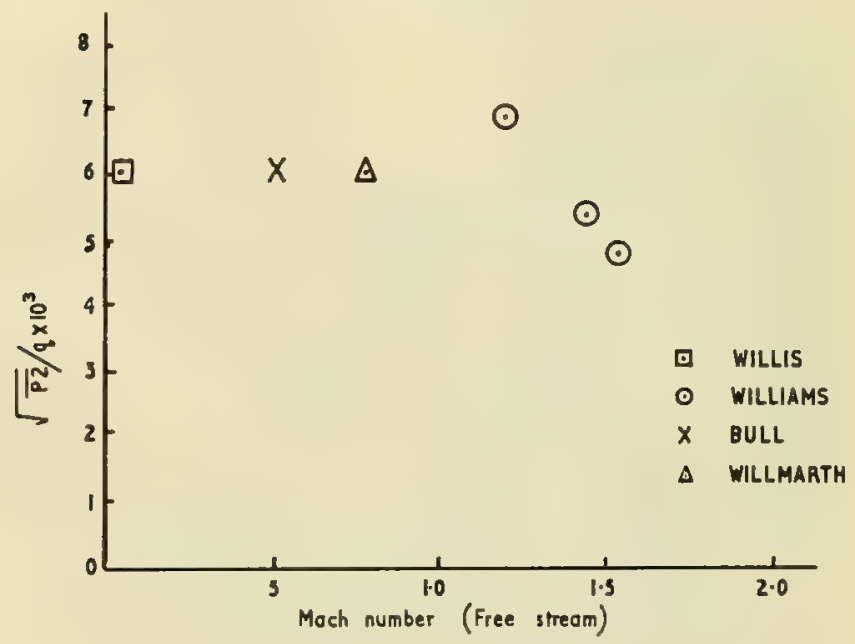

Fig. 15.4. Over-all rms pressures.

cps and below occurs, and this aspect is being further investigated.

In view of the smallness of the pipe and our interest in pressure fluctuations at a point rather than the fluctuating loads over an area, Willis [2] at Southampton developed extremely small lead-zirconate-titanate pressure transducers in a bank of twenty or more (Fig. 15.3) to determine the relative properties of the pressures at neighboring points.

Figure 15.4 indicates the over-all pressure levels obtained in the various experiments. It is seen that both Willis [2] and Bull [1] agree very well with the 


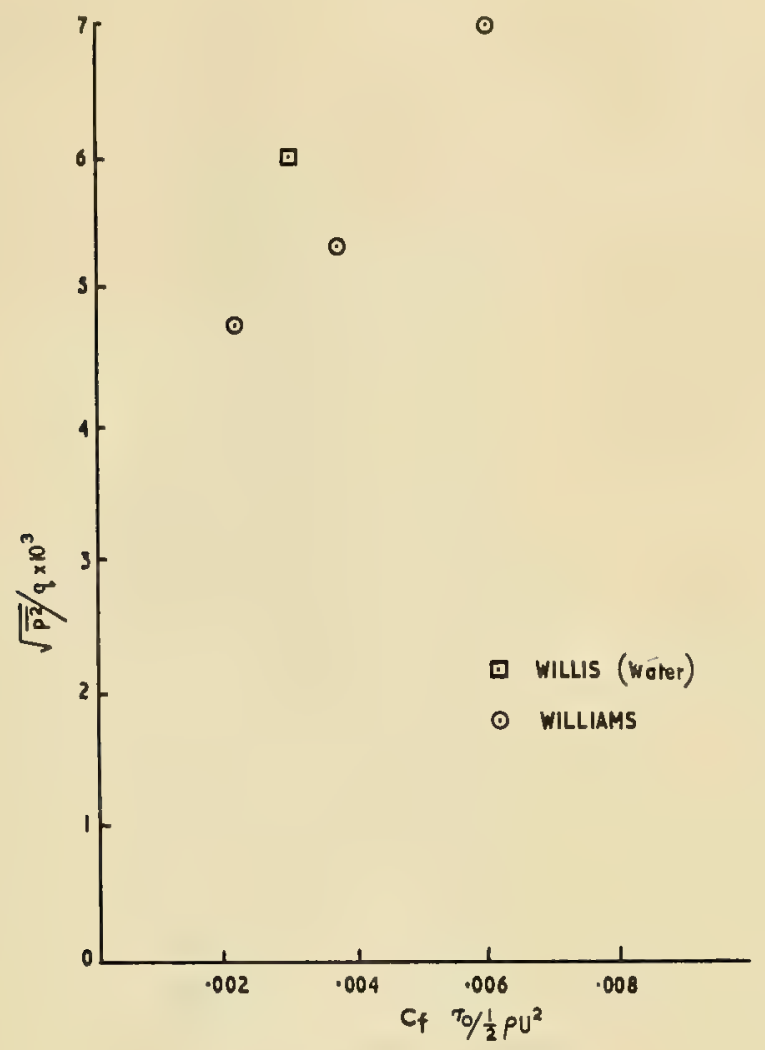

Fig. 15.5. Over-all rms pressures.

smooth-flow over-all rms pressure level of $0.006 q^{*}$ obtained by Willmarth [3], while Williams [4] in his experiments at low supersonic speeds has pressure fluctuations at the walls which fall with forward Mach number but which are in general agreement with the low subsonic answers. If, as is more logical, the pressure fluctuations are plotted against the local skin friction at the walls, we get a level of $3 \tau_{0} \dagger$ in the subsonic work, and a variation between $2.4 \tau_{0}$ and $1.2 \tau_{0}$ in Williams' supersonic investigations; see Fig. 15.5.

To evaluate the spectral content of these pressure fluctuations, it is necessary to bear in mind the purpose for which these spectra are to be used. If they are to be incorporated into structural-response calculations, the normal spectral measurement methods are satisfactory; if, however, it is necessary to calculate the radiated noise from the pressure fluctuations at the surface, it is usual to carry out the calculations in terms of turbulence being convected along the surface with some mean speed of flow of the turbulence. Thus, the significant spectra are those of turbulence as seen by an observer moving along the surface with the mean velocity of convection, not those at a stationary point. Such spectra

* $q$ is the mean flow in the dynamic head.

$t_{0}$ is the wall shearing stress or skin friction. 


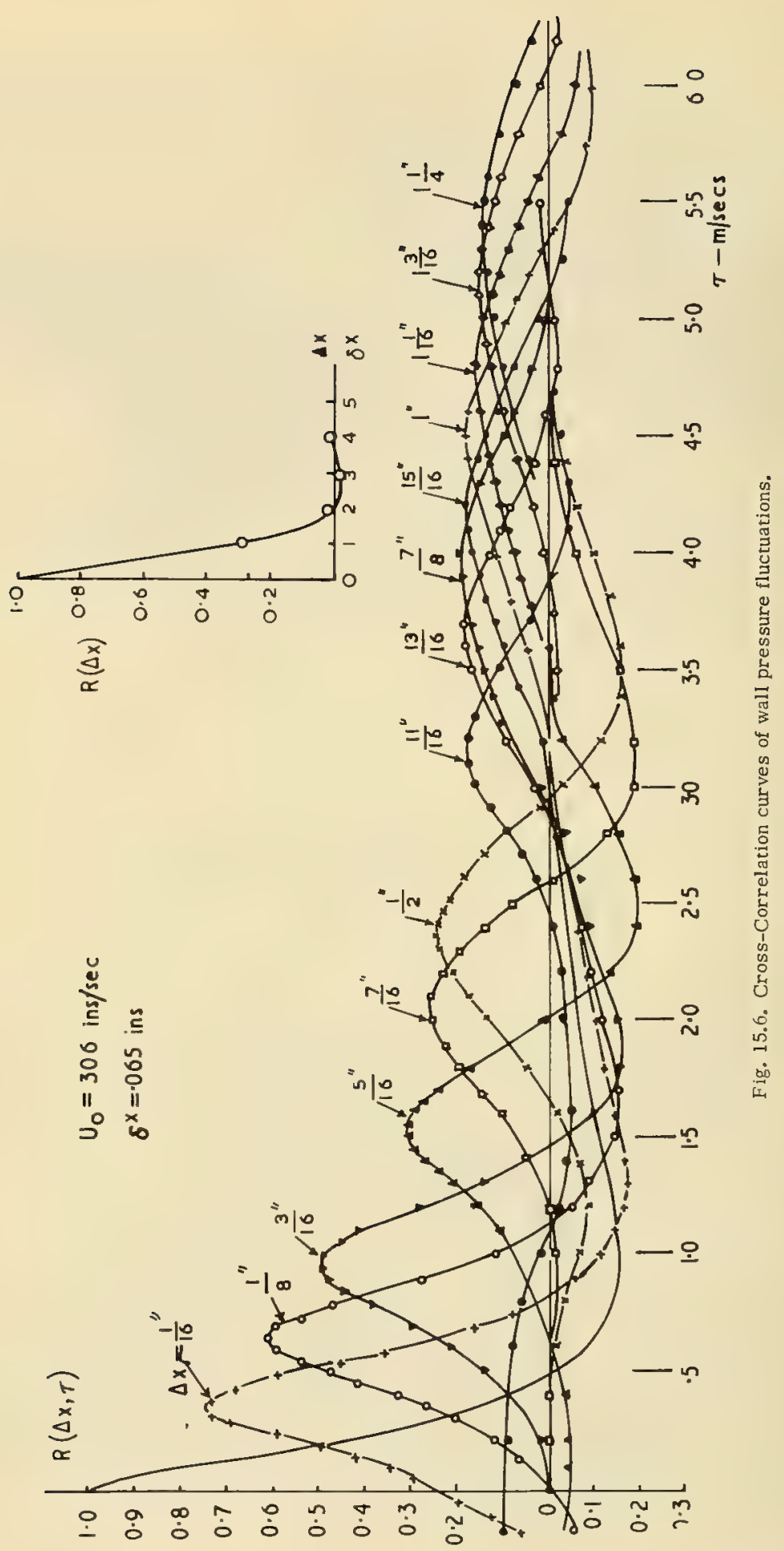

Lecture 15 
can be obtained using space-time correlation methods; the curves of Fig. 15.6 indicate typical results obtained by Willis and Bull in their work for various transducer spacings. The optimum convection speed can be obtained from the delay time giving the best correlation for the various spacings, or from the time delay for envelope tangency; the results differ very little. Figure 15.7 shows the convection speed based on different separations of the microphones. The variation of velocity so obtained is significant, indicating, as would be expected, that the large eddies in the boundary layer are convected at higher speeds than the smaller eddies. The convection speed of interest is clearly that of the noiseradiating eddies (i.e., the highest frequency at which the energy level is high). The fixed and moving frame spectra of pressure fluctuations can be obtained from the cross-correlation curves, the Fourier cosinetransform of the envelope to the fixed-point cross-correlation curves giving the moving-frame power spectra. Figure 15.8 indicates these two spectra and the essential differences between them also shown, but to an arbitrary scale, is the spectrum of the pressure-time derivative $\partial p / \partial t$, a parameter directly related to the radiated noise from the wall pressure fluctuations [17].

In aviation, these pressure fluctuations are modified in many ways by the specific environment of the flow. For example, in separated flow over a narrow delta wing, Jones and Judd [5] have shown that wing pressure fluctuations beneath the cast-off vortex are increased sometenfold and are well correlated over quite large areas of the wing. Thus, the noise radiated in such instances is presumably far greater than that calculated from normal turbulent boundary layers. Other, though smaller, increases occur, however, behind discontinuities in the surface or in regions of roughness, and these are presently being studied at Southampton [2]. For example, the variation of rms pressure behind a rectangular ridge of $1 / 4$-in. length and $0.1-i n$. height in a boundary-layer flow is shown in Fig. 15.9. It is seen that the rms pressure increases with distance behind the ridge to a value

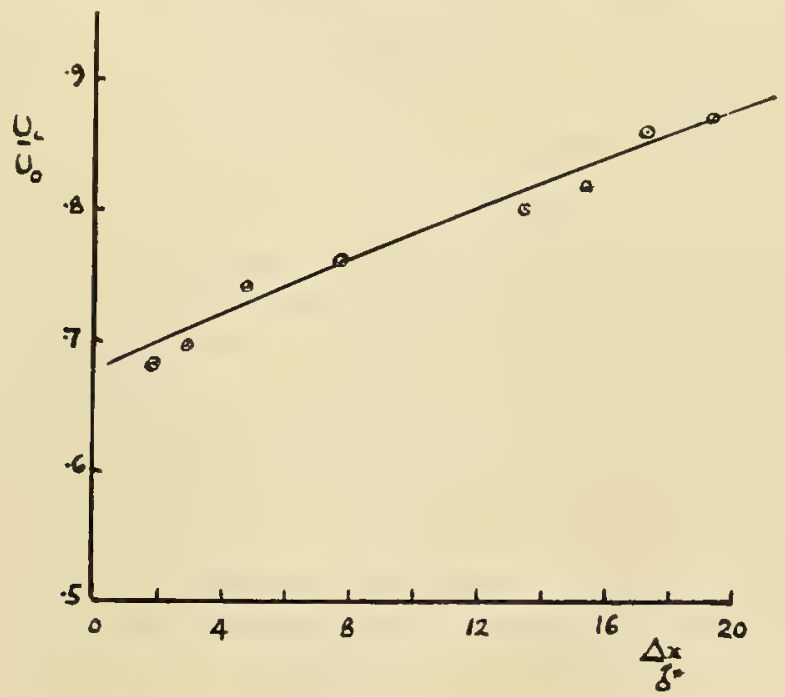

Fig. 15.7. Variation of convection velocity with distance along the tunnel wall. 
twice that of the smooth-flow condition and ceases to have an effect some 20 heights downstream. Further studies of the detailed effects of discontinuities, roughness, pressure gradients, and waviness of surface are proceeding at Southampton and will be reported later. The work is clearly of great interest in a wide variety of contexts as will be shown later by an elementary attempt to illustrate the significance of these results.

\subsubsection{The Radiated Sound from a Small Flat Plate}

It is difficult to check the theory of radiated noise from these pressure fluctuations since it is impossible to place a microphone, shielded from the flow,

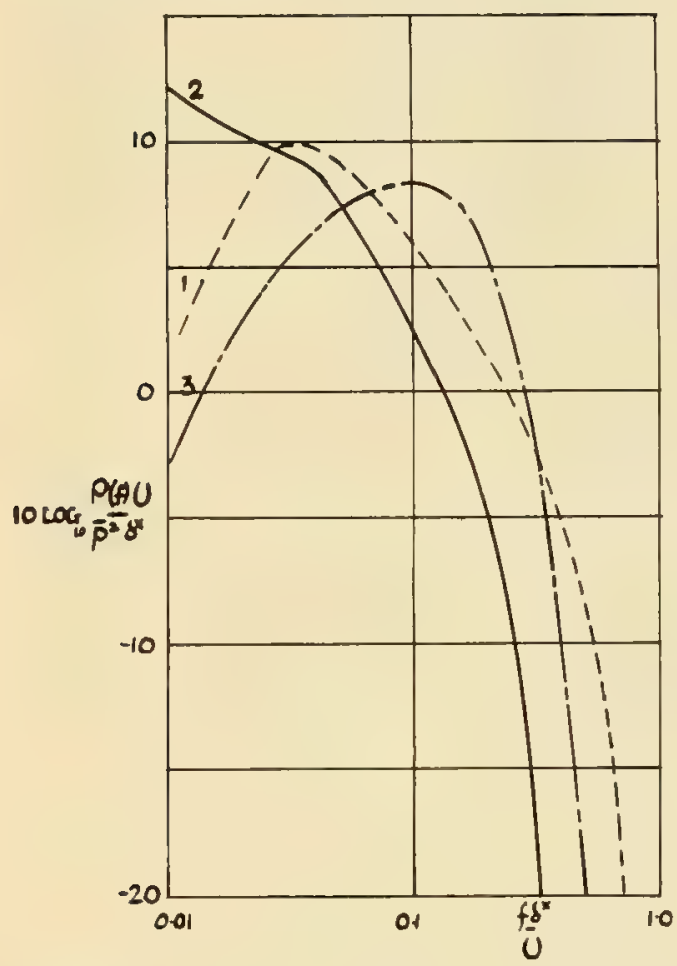

Fig. 15.8. Comparison of spectra of pressure fluctuation on the wall adjacent to a turbulent boundary layer. Curves: (1) fixedaxis, (2) moving axis, (3) moving axis + 6 db/octave (arbitrary ordinate scale).

within the restricted space of a wind-tunnel working section and also in the far field in which reactive effects are absent. Some attempts, however, have been made at Southampton to obtain the radiation from flat plates in jet flows. The agreement between the measured and predicted far-field noise [6], which is quite good, justifies the use of existing theory in the prediction of radiated noise from a boundary layer. The only reference necessary at this point is to the directional pattern to be obtained. Figure 15.10 shows a plot of the noise variation circumferentially around a small aerofoil placed at zero incidence in a jet compared with the theoretical pattern obtained from a single dipole with its axis at right angles to the surface. Since the noise radiated along the surface is theoretically zero for this type of wall pressure radiation, we cannot obtain any true indication of the noise radiated along the surface without introducing theories of scatter and diffraction. With present knowledge, therefore, the best method of calculating 


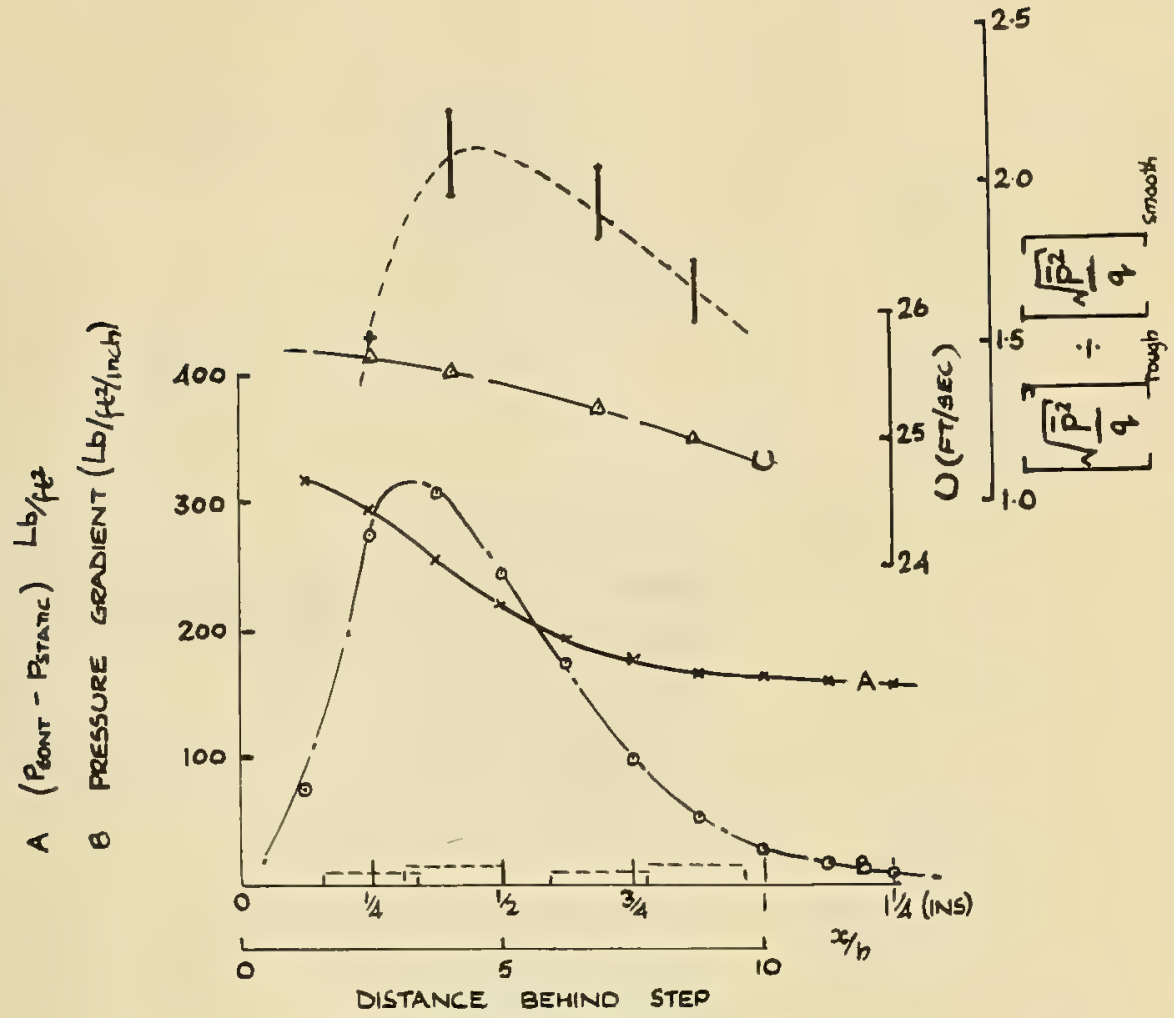

Fig. 15.9. Measurements taken behind 0.10-in. step.

the noise radiated from one part of a body to another is to relate it to the theoretical total acoustic power output, modified to allow empirically for the directional pattern as, for example, obtained in Fig. 15.10. Thus, some idea of the noise environment of a laminar region of a wing produced by the radiated noise from the turbulent region downstream can be obtained from the theoretically computed total acoustic output reduced by some $6 \mathrm{db}$ (see Fig. 15.10) to allow for the directionality of the sound field. This distribution of the sound field is of course a function of the boundary-layer thickness, but this may not introduce a great error since the frequency at which the wall pressure fluctuations fall off (and therefore the frequency of greatest noise emission) is also related to the boundary-layer thickness.

\subsection{STRUCTURAL RESPONSE AND RERADIATION}

A structure as complicated as that of an airplane or submarine has a large number of possible modes of vibration, all of which can conceivably be excited in any particular case. Clarkson, however, has indicated by careful crosscorrelation techniques that at least in the Caravelle [7] and the Comet [8] the number of modes excited by jet noise is quite small and manageable. For example, Fig. 15.11 shows the root-mean-square stress level in one plane of the Caravelle while Fig. 15.12 shows the cross correlation at each frequency 


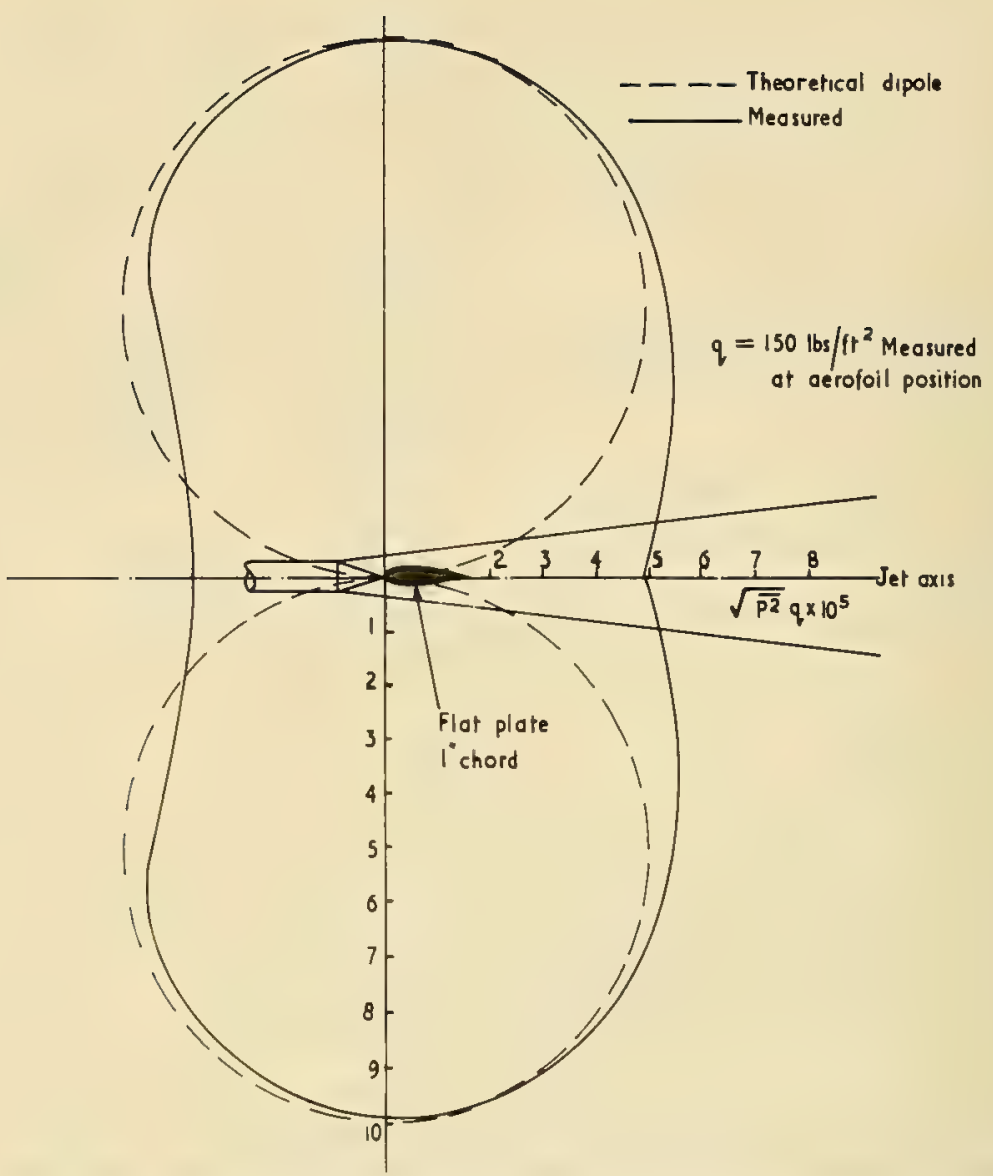

Fig. 15.10. Polar plot of noise from small flat-plate aerofoil at zero incidence measured at three-foot radius.

between the stresses in two adjacent panels around the periphery of the fuselage. In this case, at least, the actual motion is independent between frames but takes on an approximate "hourglass" or "eggtimer" mode around the periphery, adjacent panels being antiphase.

The reradiation from such a motion can be calculated and the acoustic damping of such an array estimated. Calculations have been carried out by Mead [9] of the acoustic damping ratio of flat, rectangular, simply supported panels of various length-to-breadth ratios, mounted in a rigid structure and vibrating in their fundamental modes (see Fig. 15.13). The reradiation is simply calculated in such cases and can be used to indicate the noise radiated once the panel movements are known.

Preliminary tests on the acoustic damping of arrays of panels have commenced at Southampton [10] by testing a single panel as shown in Fig. 15.14. As shown in Fig. 15.13, the agreement with theory in this particular case is good. 


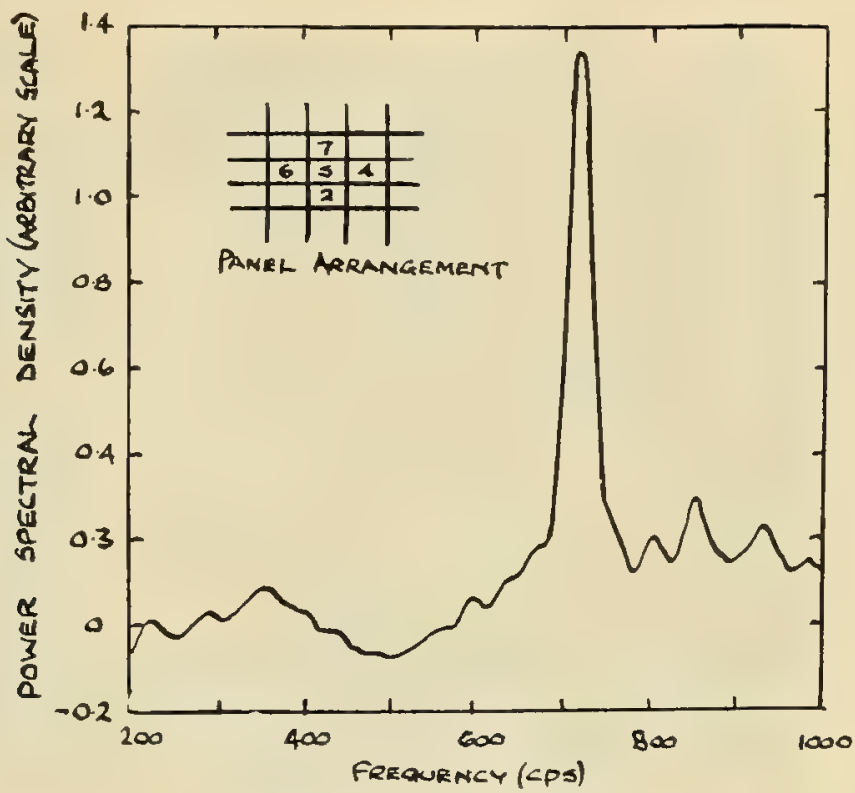

Fig. 15.11. Srain spectrum at panel 5.

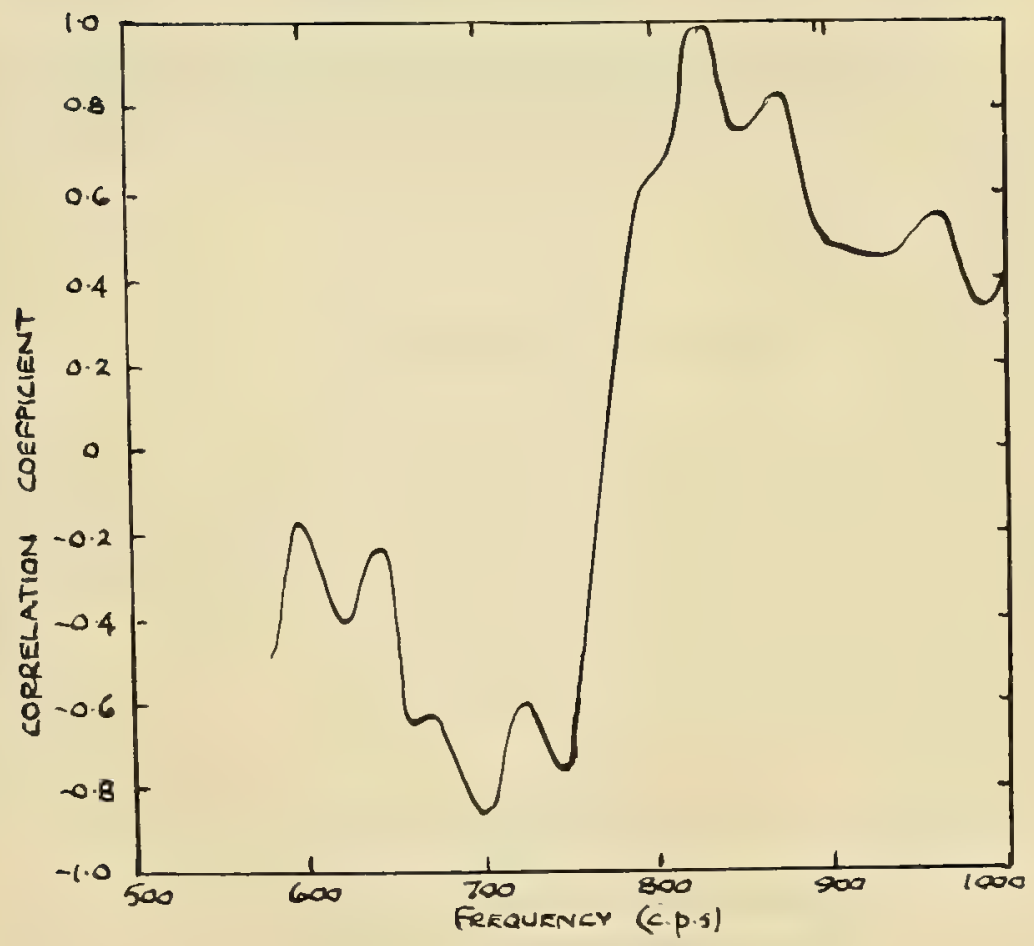

Fig. 15.12. Correlation spectrum between panels 2 and 5 . 


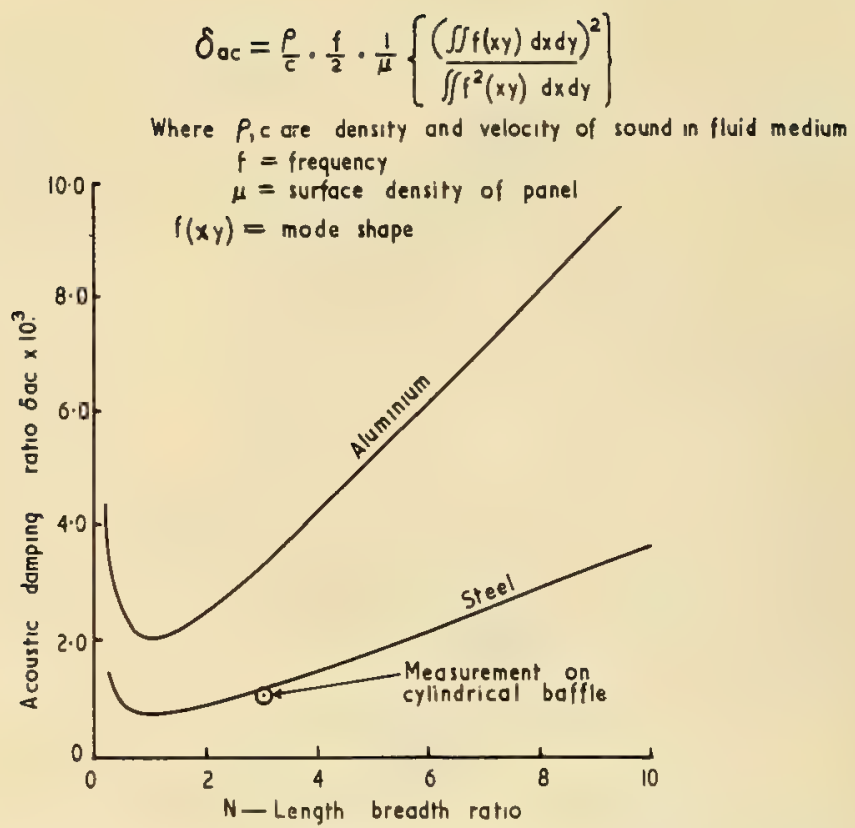

Fig. 15.13. Comparison of variation of acoustic damping ratio with length-tobreadth ratio calculated from uniform and nonuniform pressure theories.

The radiation due to over-all modes of a cylinder, in which the whole surface vibrates in a sinusoidal mode, has been calculated by Junger [11]. He shows that if the lengthwise distance between nodal points is less than the wavelength of the radiated sound, then a cutoff in radiation occurs, and the acoustic damping is then zero (Fig. 15.15). Thus the amount of reradiation in the underwater case can be assessed in qualitative terms, once the modes of oscillation and excitation are determined.

\subsection{APPLICATIONS TO UNDERWATER NOISE PROBLEMS}

\subsubsection{Rodiated Noise}

The radiated noise from a ship's hull or submarine has several components some of which are clearly not of hydrodynamic origin. Thus, propeller noise and noise originating from machinery inside the hull are excluded from the discus sions of this paper, even though the mechanism of machine noise radiation is similar to that discussed here. Thus, we are interested in four separate mechanisms of underwater noise excitation:

1. Noise from the turbulence in the boundary layer itself, in the absence of surfaces;

2. Noise from the fluctuations of force exerted by the structure on the fluid and arising from the constraint imposed on the turbulent boundary layer by the presence of the structure which is assumed rigid;

3. As in No. 2, but due to the roughnesses of the surface;

4. The reradiation of sound from the structural deflections arising from the above forces. 


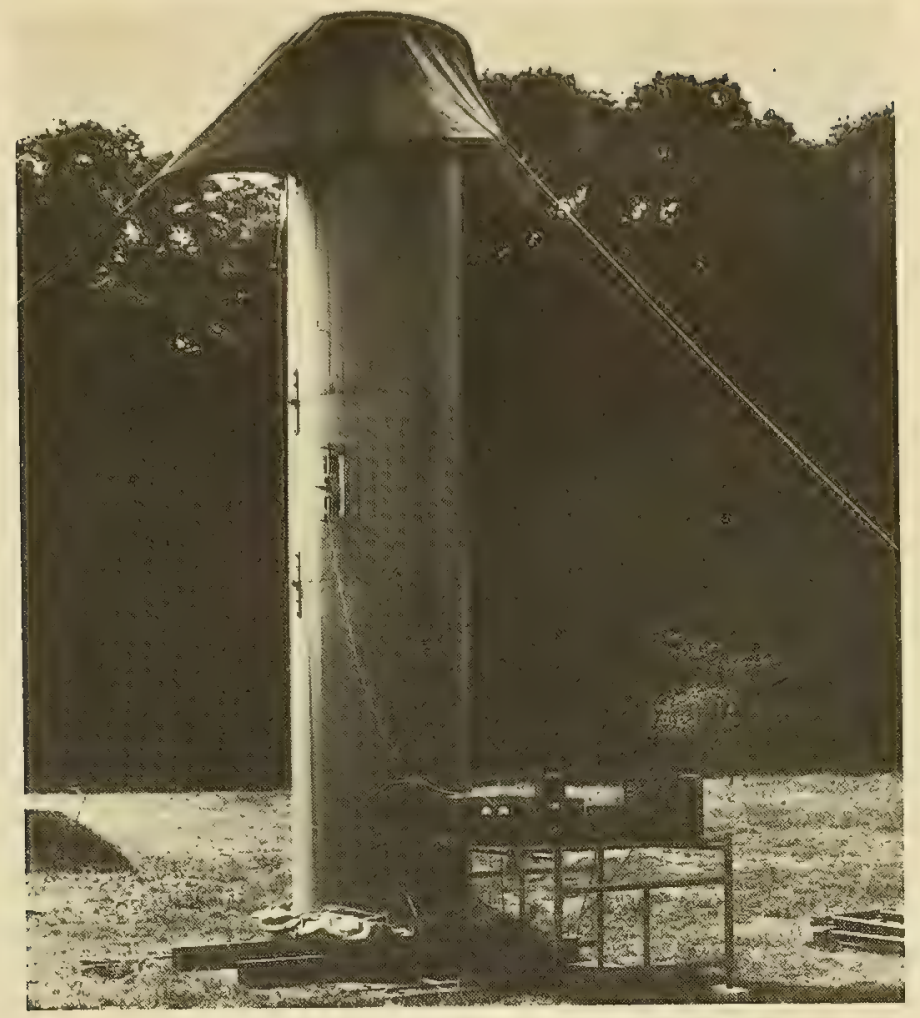

Fig. 15.14. Rig for experimental measurement of $\delta$ ac.

\subsubsection{Noise from the Turbulence}

Noise from turbulence in the absence of solid surface is quadrupole in nature and is very inefficient at the low speeds occurring in water vehicles; hence, it is unlikely to be significant. This may not be so if the boundary layer separates from the surface, but even in this condition the noise radiated by the other mechanisms above are likely to predominate.

\subsubsection{Turbulent Boundary-Layer Wall Pressure Noise}

The radiated noise from a modern submarine has been calculated using our own measured levels of wall pressure fluctuations and correlation areas taken in conditions of a small pressure gradient. The results are shown in Fig. 15.16 for a series of distances and submarine speeds. Comparison with data on radiated submarine noise available in the literature suggests that these levels are some 30 to $40 \mathrm{db}$ below the noise actually radiated, and it must be presumed that the noise is certainly not simply that due to the fluctuating force field arising from the pressure fluctuations on a nominally smooth rigid wall. We must, therefore, investigate other effects, including the influence of roughness on the force field, the possibility of structural vibrations which in turn reradiate noise, and, presumably, other effects with which we ourselves have had little experience in the underwater noise field, such as cavitation and separated and vortex flows of the kind studied in [5]. 


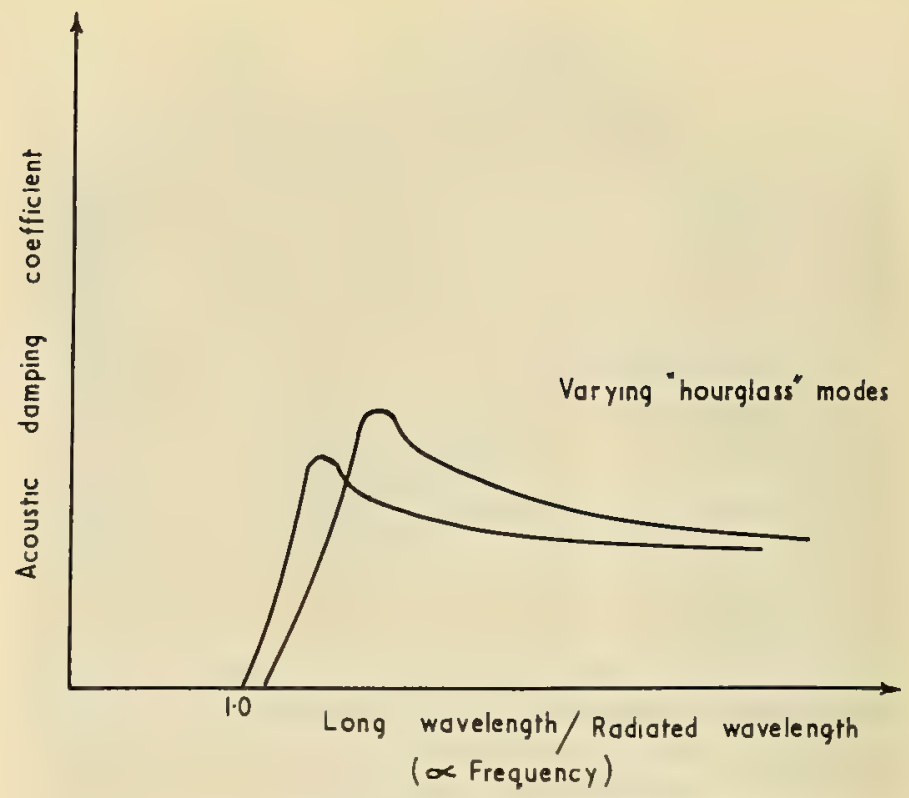

Fig. 15.15. Junger's "cutoff" frequency.

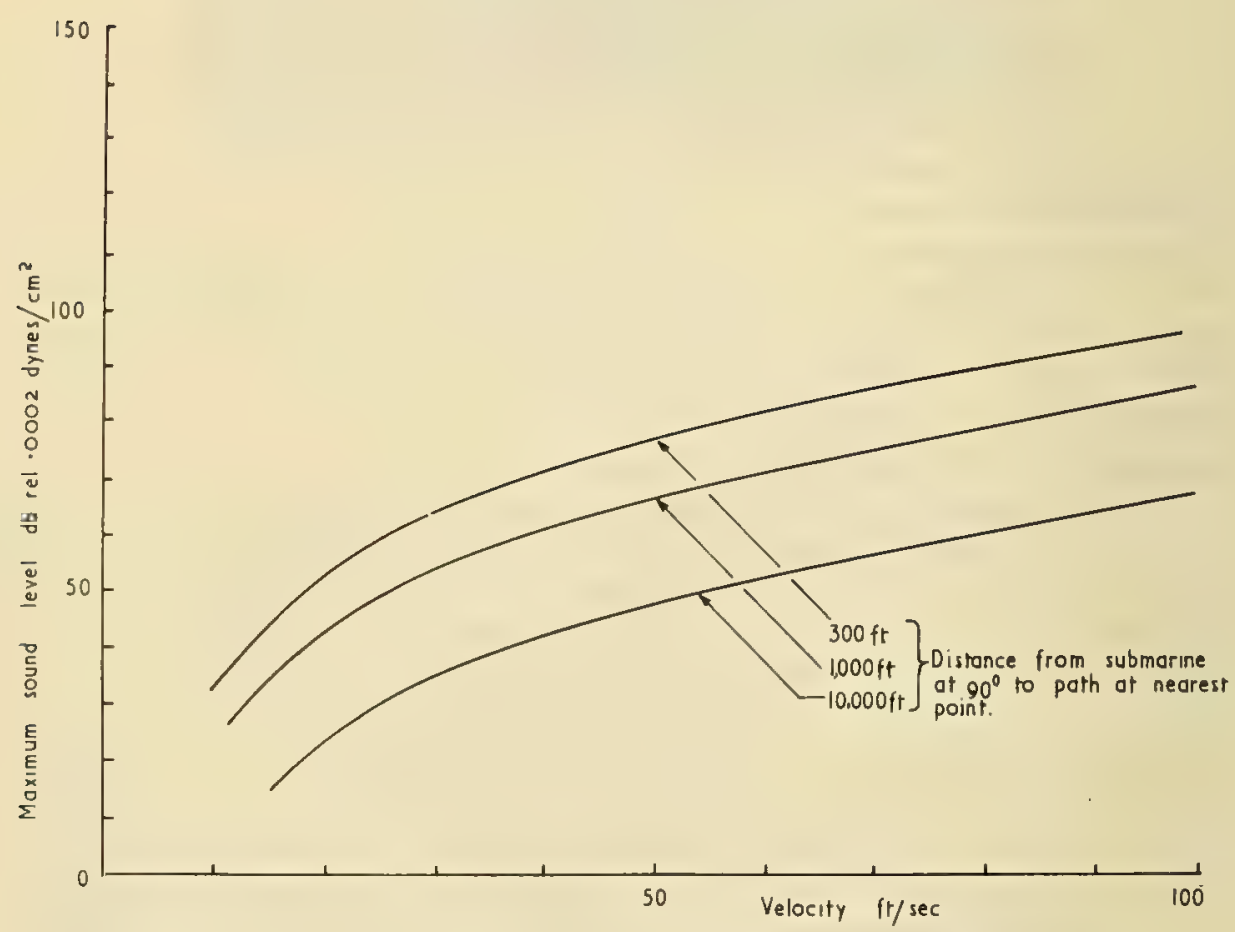

Fig. 15.16. Radiated sound from smooth rigid submarine (surface area, $18,000 \mathrm{ft}^{2}$ ). 


\subsubsection{Noise from Roughness}

Willis' results on the effect of single roughnesses are still in a preliminary stage [2] and it would be unwise to use these results other than qualitatively at the moment. It is interesting, however, to note (Fig. 15.9) that the rms pressure from even a large single roughness apparently does not exceed twice the smoothsurface result. Thus, at first sight, the effect of roughness would seem small. If, however, we assume that the influence of each ridge is independent of the next one, that ridges are separated by distances equal to their height, and that the areas over which these pressures are correlated are similar to those measured in the single-roughness experiments, then a rather different picture emerges. In Fig. 15.17, the noise measured at a typical distance from the submarine is estimated for varying degrees of roughness. It must be emphasized that the effect of roughness will depend critically on the height of the protrusion on the boundary layer or its viscous sublayer and that these results are purely illustrative. It is seen, nevertheless, that in an extreme case of roughness, we can explain the high observed noise environment, although these figures are unquestionably extremes and other possibilities should not be excluded.

It is difficult to obtain practical observations of the effects of roughness. The buoyant-body tests [12] of Haddle and Skudrzyk give spectra, but no over-all levels. However, if the calculations of radiated noise are made on the above basis, we obtain an estimated over-all level increase of $11 \mathrm{db}$ for a 0.1 -in. step. This compares well with the increases of about $10 \mathrm{db}$ given in [12].

\subsubsection{Reradiated Noise from Body Vibrations}

As with an aircraft fuselage, the possible modes of vibration of a submarine hull fall into two categories. Firstly, there are the over-all modes of vibration of the whole cylinder; these have been considered by Arnold and Warburton [13] ignoring frames or stiffeners and later by Miller and Junger [14, 15] who have included stiffeners. Secondly, there are the localized vibrations of the panels, the boundaries of which are defined by the stiffener spacing. Some symmetric modes of vibration of these have been considered theoretically, but it has been found from testing of practical structures that the modes which are excited are not necessarily symmetrical and are therefore extremely difficult to define.

If the stiffeners in the submarine hull are ignored, the frequencies of the over-all modes of vibration may be obtained (Fig. 15.18) directly from Arnold and Warburton's work. Junger's paper [11] on radiation considers these over-all modes of a circular cylinder and shows that there is no resultant radiation if the sound wavelength is greater than the axial structural wavelength. This "cutoff" line is also shown in Fig. 15.18. It is clear in this case, therefore, that the only over-all modes of vibration which can radiate are those which have long axial wavelengths and relatively short circumferential wavelengths (e.g., when $n=12$, the circumferential wavelength is $5.24 \mathrm{ft}$, and the axial wavelength is greater than $44 \mathrm{ft}$ ). It is felt, without mathematical backing it must be confessed, that a boundary layer is unlikely to excite this type of mode.

Over-all flexural modes of the submarine will not cause any resultant radiation because the frequencies will be low, the sound wavelength relatively long, and complete cancellation will occur. The only other mode of interest is the 


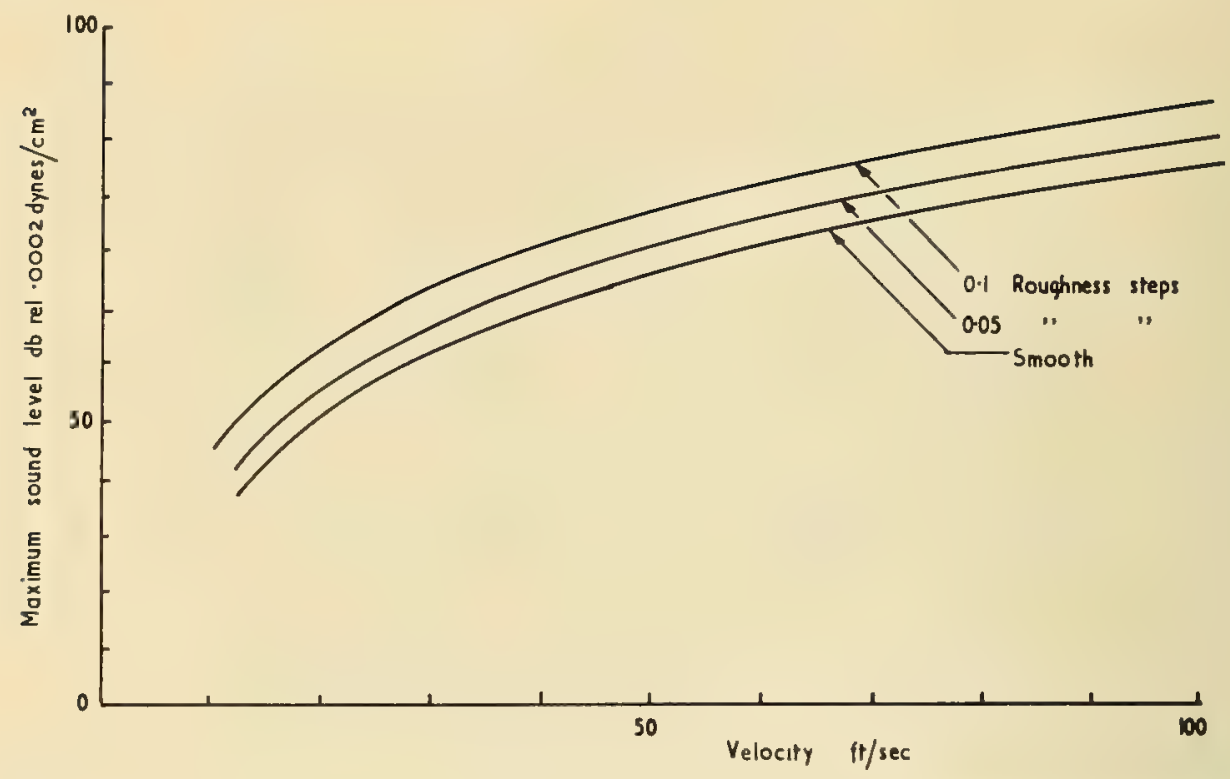

Fig. 15.17. Radiated sound from typical rigid submarine measured at 100 yards for various surface roughnesses.

over-all dilatory mode, i.e., that mode in which the whole shell either expands or contracts at the same time. Simple calculations, however, indicate that the damping in this mode would be overcritical and it cannot therefore be excited.

The localized panel vibrations must now be considered, and since the possible modes of vibration are difficult to define, a panel 30 by 24 by $1 \frac{1}{2} \mathrm{in.}$., as sumed to be flat and vibrating in a fundamental mode, is used to give some indication of the possible radiation. The undamped natural frequency of such a panel is $398 \mathrm{cps}$ if simple supports are assumed at the four edges and $760 \mathrm{cps}$ if full fixation is assumed. As mentioned earlier, Mangiarotty [10] has both calculated and measured the damping ratio on one side of such a panel in air and, if his figures are used, the damping ratio of the panel in water is of the order of 0.13 . If the submarine is assumed to be traveling at $45 \mathrm{ft} / \mathrm{sec}$ ( $25 \mathrm{knots}$ ) and the boundary-layer thickness to be $1 \mathrm{ft}$, the power spectral density at 400 cps is, at the most, $4.8 \cdot 10^{-5}(\mathrm{psi})^{2} / \mathrm{cps}$, and the correlation area of fluctuating pressures of this frequency is approximately $0.25 \mathrm{in}^{2}$. When subjected to this field, the panel will vibrate with an rms amplitude of $4.3 \cdot 10^{-6} \mathrm{in}$. in the fundamental mode simply supported at four edges. If the energy from such a vibration is assumed to be radiated evenly over one hemisphere, the sound pressure level at 100 yards is approximately $70 \mathrm{db}\left(\right.$ re $\left.0.0002 \mathrm{~d} / \mathrm{cm}^{2}\right)$.

If all such panels vibrate randomly with respect to each other, then for a typical submarine the radiated level from the whole structure could be 90 to 100 $\mathrm{db}$ ( $\mathrm{re} 0.0002 \mathrm{~d} / \mathrm{cm}^{2}$ ) in the case considered and is of the order of level actually observed.

In practice, the panels will be neither fully fixed nor simply supported and their disposition will in any case depend on the details of attachment to frames, 


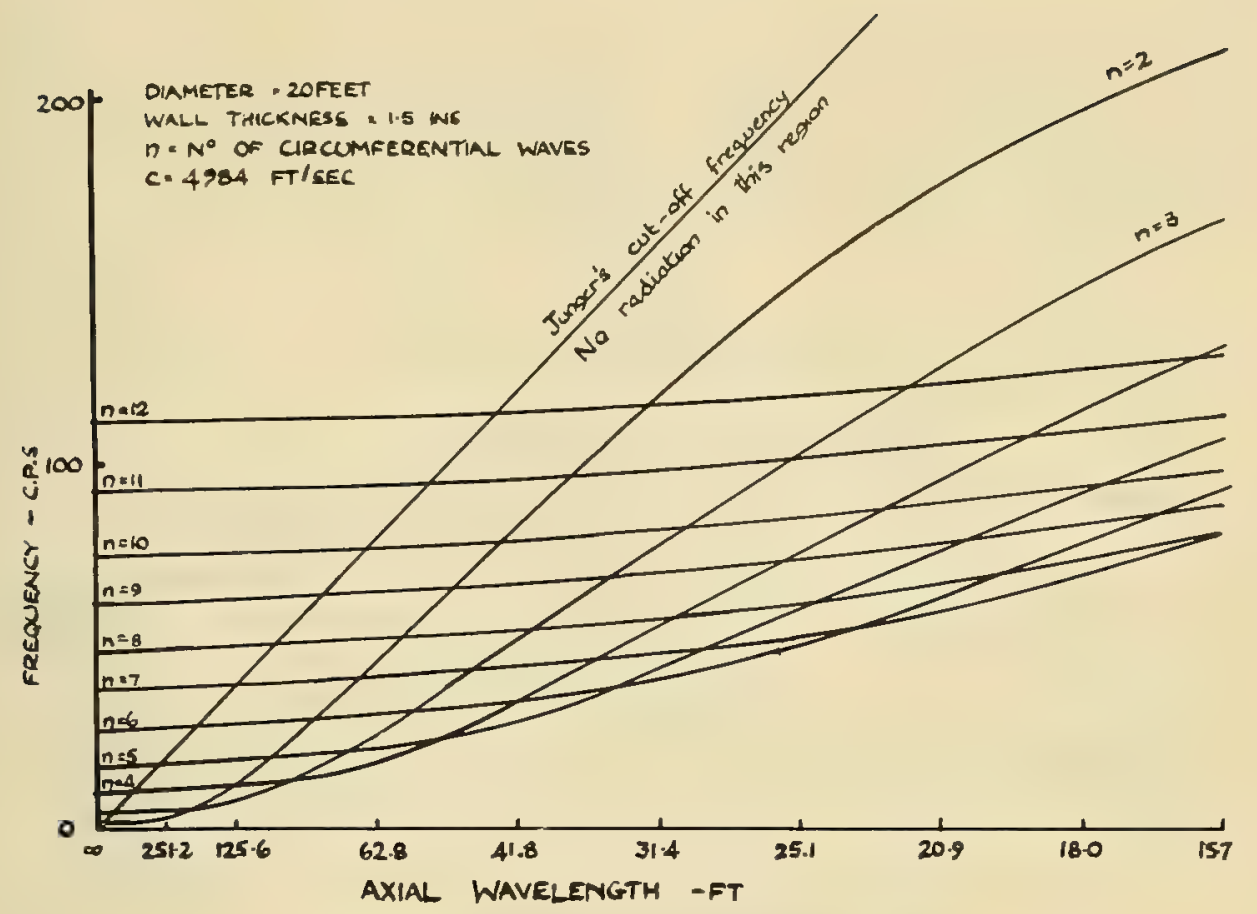

Fig. 15.18. Frequencies of vibration of submarine hull.

etc., and may well involve coupling with other panels which will tend to reduce the radiation. All that can be said at this stage is that panel vibration can be a major contributor to the radiated noise from a submarine and that careful studies of the detailed vibratory modes of typical parts of the structure, with the techniques we have used $[7,8]$ on the Caravelle and Comet, would be well worth while.

\subsubsection{Self-Noise}

Boundary-layer pressure fluctuations also provide the level of self-noise against which underwater listening devices have to act. This can be calculated from the level of the pressure fluctuations, $0.006 q$, and from the area over which these pressures are correlated. This information is available from our experiments and can be used to estimate the self-noise in the various applications.

Obviously the self-noise will depend on the free-stream velocity outside the boundary layer and will, of course, be much reduced if the flow at that point is laminar. If this level has been sufficiently reduced, then presumably the background level will be that of the noise radiated from adjacent surfaces with their higher free-stream velocities and turbulent boundary-layer conditions. It seems wise, therefore, to place the listening area in a low-speed laminar region as far away as possible from the free-flow turbulent boundary layer further downstream.

A type of body design comes to mind in this context which is based on experimental work widely discussed in aeronautical circles some years ago. It is 
possible [16] to maintain extensive laminar flow over aerofoils if they are designed to have a favorable velocity gradient over the region involved. Dr. A.A. Griffith put forward a suggestion, that if a wing were designed (Fig. 15.19) to have a favorable gradient at all points except one, where a discontinuity in velocity occurred, and if suction were applied to remove the boundary layer just forward of this point, then laminar flow would occur everywhere.

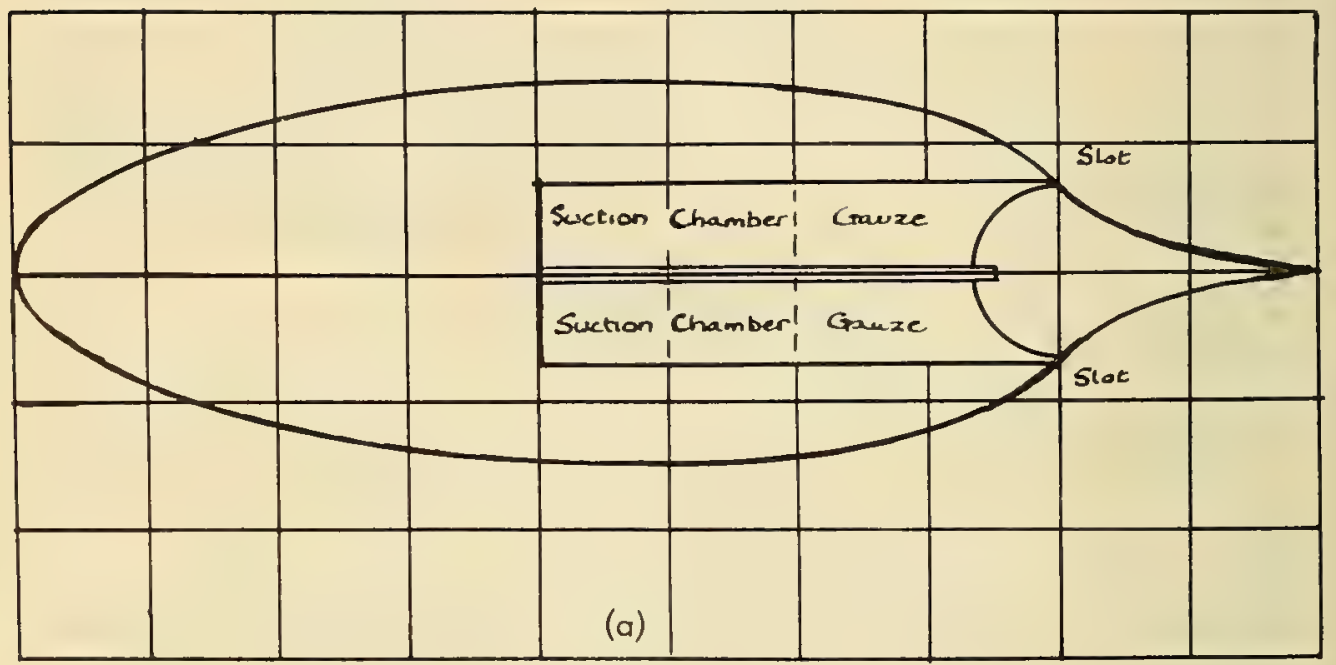

Fig. 15.19. Thirty-per-cent-suction wing and theoretical velocity distribution: (a) wing with suction chamber, (b) theoretical velocity distribution.

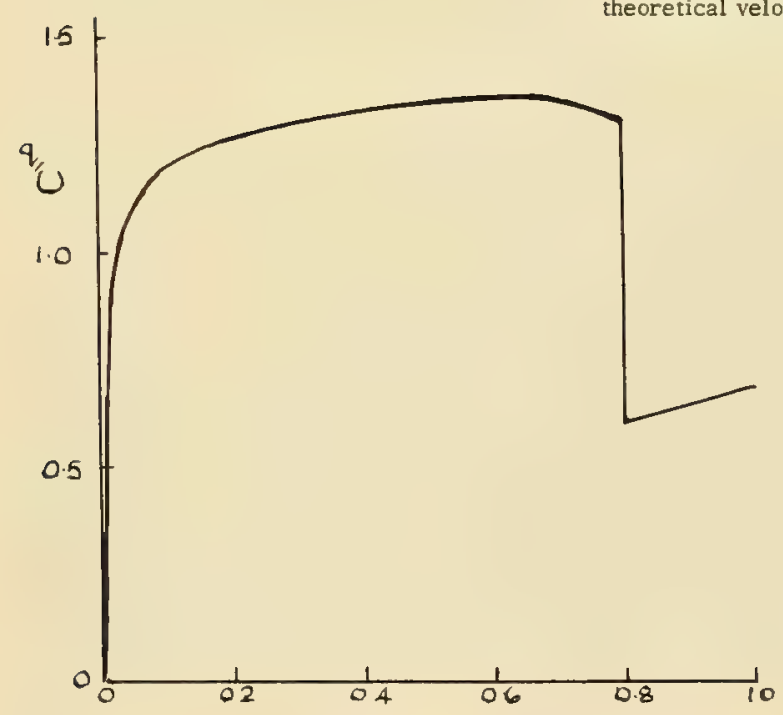

(b) 

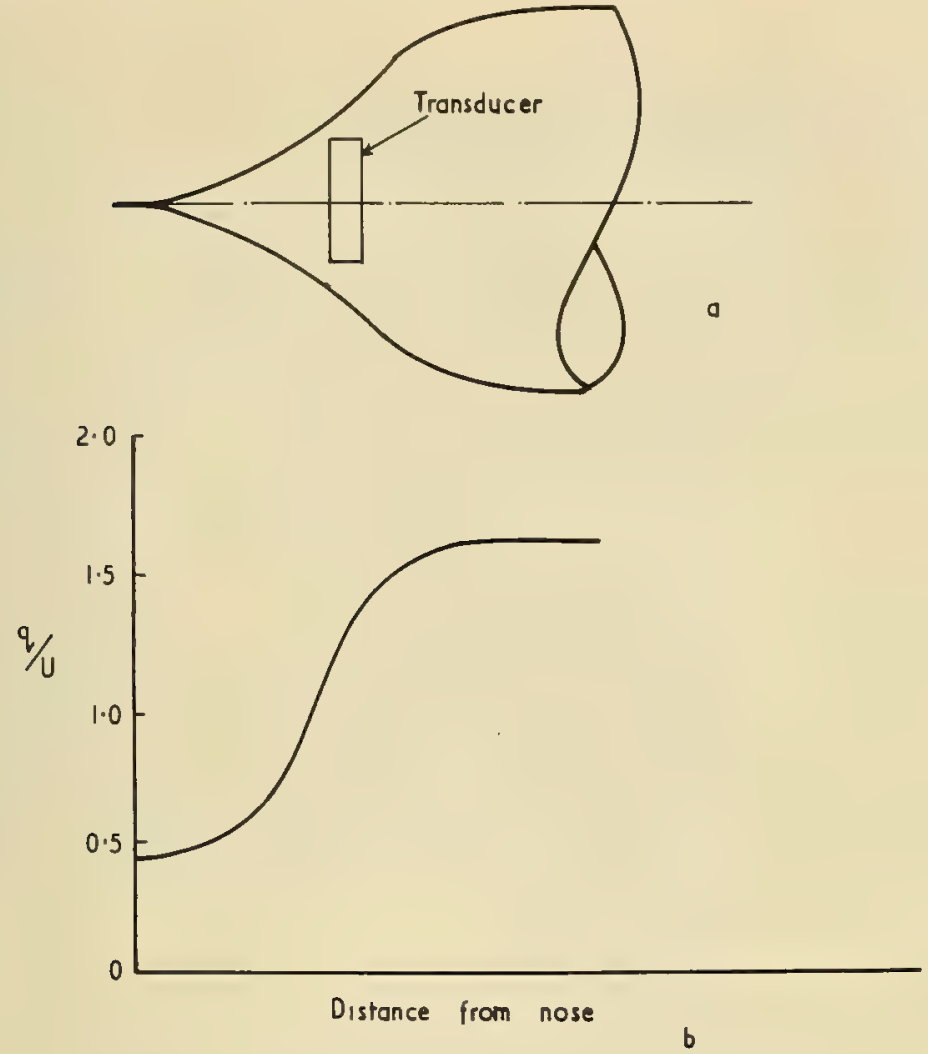

Fig. 15.20. Suggested shape for submarine nose: (a) nose shape; (b) velocity distribution.

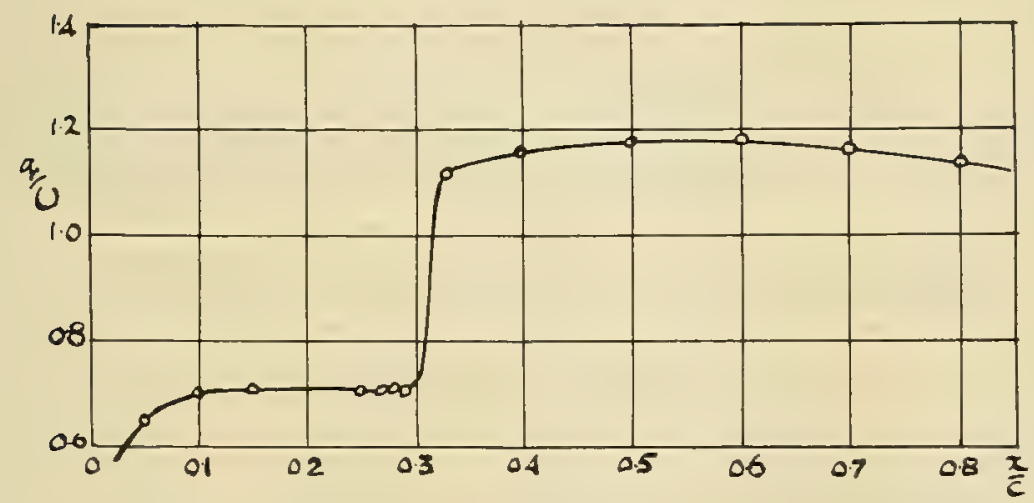

Fig. 15.21. Velocity distribution of thirty-per-cent-suction wing with tail foremost; six degrees incidence. 
Since such a shape (but designed for a body of revolution) is designed on a potential-flow basis, the direction of flow can be reversed without influencing the pressure or velocity distribution. Thus it is perfectly feasible to design a body containing a fairly long region of low-velocity free-stream flow, a velocity gradient strongly favorable to the maintenance of extensive laminar flow, and a neck aft of this region which would screen at least some of the radiated noise from the body aft of this neck. Such a shape has not been designed in detail since it is not known to be of any practical interest, but it could well be similar to that shown in Fig. 15.20, and it does indicate an application of aeronautics to underwater problems. It should be emphasized that suction is not necessary in this particular case since the discontinuity in velocity here is a favorable one, guarantees no separated flow and consequent high drag. In fact, some pressure measurements of such a wing, reversed and at incidence, were made many years ago by the principal author and are shown in Fig. 15.21.

The extent to which such a design is worthwhile depends on the strength of the radiated sound from the other parts of the structure, and this is difficult to calculate since we still do not know the basic source of such noise.

We have implied that the radiated noise is not likely to be that of the fluctuating forces on the body alone, but is more apt to be due to roughness or reradiation from panel oscillations. There is, therefore, little point at the moment in calculating the radiated noise from the pressure field in the way suggested earlier in the paper, but in any specific case such a calculation of possible magnitude would be enlightening and worth carrying out.

\subsection{CONCLUSION}

The foregoing paragraphs have shown in a qualitative manner that the radiation of the boundary layer under smooth wall conditions is by no means the largest contribution to the over-all level. Roughness and vibrating panel radiation are likely to be the largest components which can, in the widest sense, be esti- mated at the present time. Skudrzyk's studies show that roughness will considerably enhance the high-frequency portions of the spectrum, while panel response might be expected to contribute largely to the low-frequency region.

The authors appreciate that the estimates of cylinder vibration given herein ignore the more recently reported studies. It is hoped, however, that measurements of the actual response of submarine and torpedo structures on the lines indicated will be made available shortly, as they are obviously of considerable interest.

Other aerodynamic sources, whose effects have not been taken into consideration, are fluctuating transition and separated and vortex flows. The latter two have received attention, as indicated earlier, from the point of view of aircraft structural design, but no measurements of radiation have yet been made. These details are shortly to be investigated at Southampton.

Finally, it is hoped that the suggestion for a particular configuration of laminar flow noise might be of some merit in the reduction of noise received by acoustic homing sets while also providing a low-drag body acceptable also purely from the point of view of performance. 


\section{REFERENCES}

1. M. K. Bull, "Instrumentation for and Preliminary Measurements of Space-Time Correlations and Convection Velocities of the Pressure Field of a Turbulent Boundary Layer," AASU Report 149.

2. J. L. Willis and M.K. Bull, "Progress Report on Flow Noise Investigation," AASU Report 182.

3. W. W. Willmarth, "Statistical Properties of the Pressure Field in a Turbulent Boundary Layer," WADC Conf, on Acoust. Fatigue (October, 1959).

4. D. J.M. Williams, "Measurements of the Surface Pressure Fluctuations in a Turbulent Boundary Layer in Air at Supersonic Speeds," AASU Report 162.

5. M. Judd and J.P. Jones, "Surface Pressure Fluctuation Correlation Measurements on a $70^{\circ}$ Delta Wing," ACR. 22428 (December, 1960).

6. D. J.M. Williams and 1.J. Sharland, "An Experiment on the Noise from a Flat Plate Aerofoil in a Turbulent Subsonic Jet," Southampton Univ., Internal Note.

7. B. L. Clarkson and R.D. Ford, "Random Excitation of a Tailplane Section by Jet Noise," AASU Report 171.

8. B. L. Clarkson and R.D. Ford, "Experimental Study of the Random Vibrations of an Aircraft Structure Excited by Jet Noice," AASU Report 128.

9. D. J. Mead, "The Damping, Stiffness and Fatigue Properties of Joints and Configurations Representative of Aircraft Structures," WADC Tech. Report 56, 616.

10. R. Mangiarotty, M. Sc. Thesis, Univ, of Southampton (1961).

11. M. Junger, "The Physical Interpretation of the Outgoing Wave," J. Acoust. Soc. Am., Vol, 25, 40-47 (1953).

12. E.J. Skudrzyk and G.P. Haddle, "Buoyant Body Experiments," The Ordnance Research Laboratory Report, The Pennsylvania State Univ.

13. Arnold and Warburton, "Flexural Vibrations of the Walls of Thin Cylindrical Shells Having Freely Supported Ends," Proc, Roy. Soc., A 197, 238-256 (1949).

14. P. R. Miller, "Free Vibrations of a Stiffened Cylindrical Shell," ACR. 19338 (May, 1957).

15. M. Junger, "Dynamic Behavior of Reinforced Cylindrical Shells in a Vacuum and in a Fluid," J. Appl. Mechanics, Vol. 76, 35-41 (1954).

16. E. J. Richards and W.J. Walker, "Wind Tunnel Tests on a Thirty Per Cent Suction Wing."

17. P.E. Doak, "Acoustic Radiation from a Turbulent Fluid Containing Foreign Bodies," Proc. Roy. Soc., A $254,129-145$ (1960).

\section{DISCUSSION}

MR. A. KENDRICK asked the lecturer whether he had reconciled his calculation of total noise power with results obtained by the methods of N. Curle and O. M. Phillips.

PROFESSOR RICHARDS: Phillips has calculated the noise radiated by relating it dimensionally to the skin friction at each point and, therefore, to the drag coefficient of the plate. It was, naturally, necessary to assume some relationship between the fluctuating pressure field and the local skin friction, and this he did using the known theory of flowing turbulence. The advantage of our calculations lies in the use of measured fluctuations and correlation areas and, therefore, eliminates any uncertainties (and there are plenty) in using isotropic homogeneous-turbulence theories in a shear region. In practice and as far as my recollection goes, Phillips's results also give noise levels which are some 30 $\mathrm{db}$ lower than in practice, a result in agreement with ours. Needless to say, Phillips does not go on to study the effects of roughness nor of panel oscillations. 



\title{
UNDERWATER ACOUSTICS AS A TOOL IN OCEANOGRAPHY
}

\author{
M. J. Tucker and A. R. Stubbs \\ National Institute of Oceanography \\ Wormley, Surrey \\ England
}

\subsection{INTRODUCTION}

Electromagnetic waves are severely attenuated in sea water, but fortunately sound travels well enough to be used for many of the purposes served by radio and radar in the atmosphere. Historically, the principle was first applied to find the depth of the sea; a sound impulse was made in the water and the time it took for the echo from the sea bed to return to the ship was measured. As techniques for this "echo-sounding" were developed, it became apparent that echoes were also being obtained from objects in midwater. These turned out to be fish shoals, and nowadays no fishing boat of any size would put to sea without an echo-sounder for locating fish.

Probably the most important scientific application has been the examination of the geology of the sea bed. In suitable areas it has been possible to determine the geological structure of the sea floor in more detail than is possible in many land areas, and much useful information is being obtained about the transport of sediments.

In the realm of biology, the habits of fish shoals have been determined using a scanning asdic to follow their movements, and something has been learned about the habits and composition of plankton. This is a fascinating field in which acoustics should be a powerful tool, but the application is still in its infancy. The telemetering of information using the acoustic wave as a carrier is also in its infancy, but is potentially of wide application.

\subsection{MARINE GEOLOGICAL APPLICATIONS}

\subsubsection{Precision Echo-Sounding}

The method of measuring the depth of the sea by transmitting a pulse of sound from the ship and measuring the echo-time is well known. In the last few years, major improvements in the technique of echo-sounding in the deep sea have been made, notably at the Woods Hole Oceanographic Institution [1] and at the Lamont Geological Observatory, and it is now possible to measure depths of 3000 fathoms or more to an accuracy of one fathom in any weather in which 


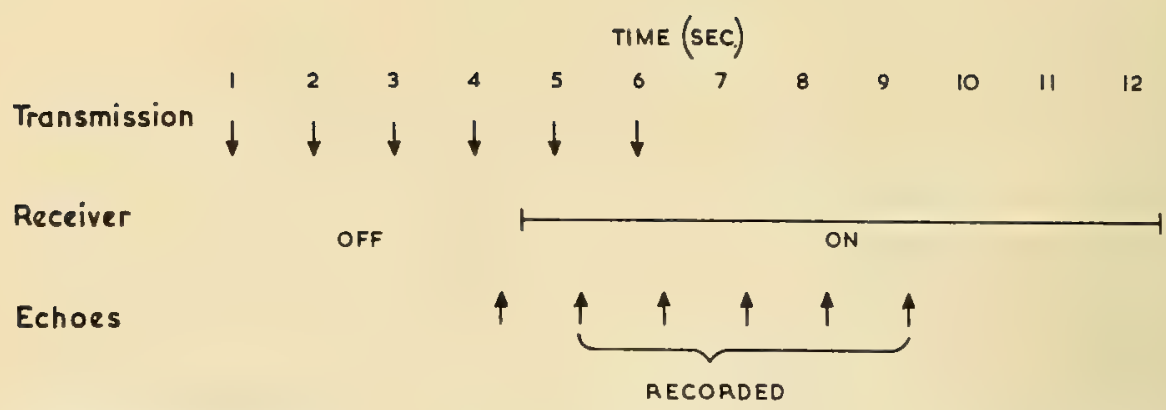

Fig. 16.1. Coding system of the precision echo-sounder. The diagram is drawn for an echo from approximately 1300 fathoms.

the ship can operate. In the recording system used, the stylus records continuously, so there is no chance of missing a bottom echo because the stylus is on the wrong phase.

Precision has been obtained by using a precision timed recorder with an open scale. Since these recorders are used in many oceanographic instruments, including two to be discussed below, they will be described here in some detail. They are usually modified facsimile picture receivers; the ones used by N.I.O. and described here are part of the Mufax system. The stylus consists of a single turn helical blade rotating against a knife edge with chemically impregnated paper in between. The point of contact between the helix and the blade starts at one edge of the chart, moves across to the other edge, and then immediately enters at the first edge again, so that it is always on the chart. Passage of current between the helix and the stylus dissolves some iron from the stylus which reacts with the chemicals in the damp paper to form a black mark. The helix is driven by a synchronous motor supplied from a tuning fork, so that its rate of rotation is precisely controlled. The usual speed is one revolution per second.

A contact on the helix shaft closes as the writing point moves onto one side of the chart and initiates the transmission of the acoustic pulse into the water. In deep water, the echo from the sea bed may not return until after several

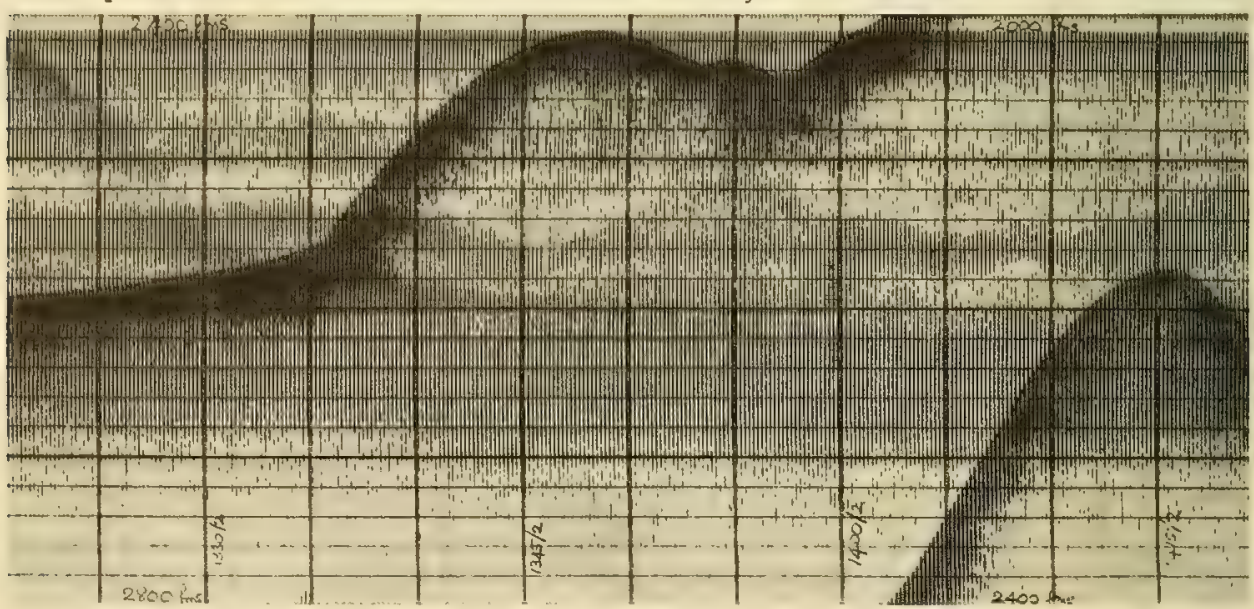

Fig. 16.2. A typical record taken with the precision echo-sounder. 
revolutions of the helix, and if no precautions were taken might well be lost in the transmission mark or in the reverberation due to scatterers in midwater. To overcome these difficulties and to find out how many complete revolutions of the helix elapse before the echo returns, a switching system is used. Figure 16.1 shows a typical arrangement using twelve sweeps of the helix. Pulses are transmitted on only the first six and the receiver is switched off for the first four sweeps. Thus, apart from the fifth and sixth sweep, there is negligible local reverberation and the number of echoes received allows the number of revolutions between transmission and echo to be determined. This number is usually determined by ear, as successive sweeps are not well separated on the record.

To overcome errors due to shrinkage of the paper in drying, range marks are put on the paper by counting down the tuning-fork frequency to produce suit-

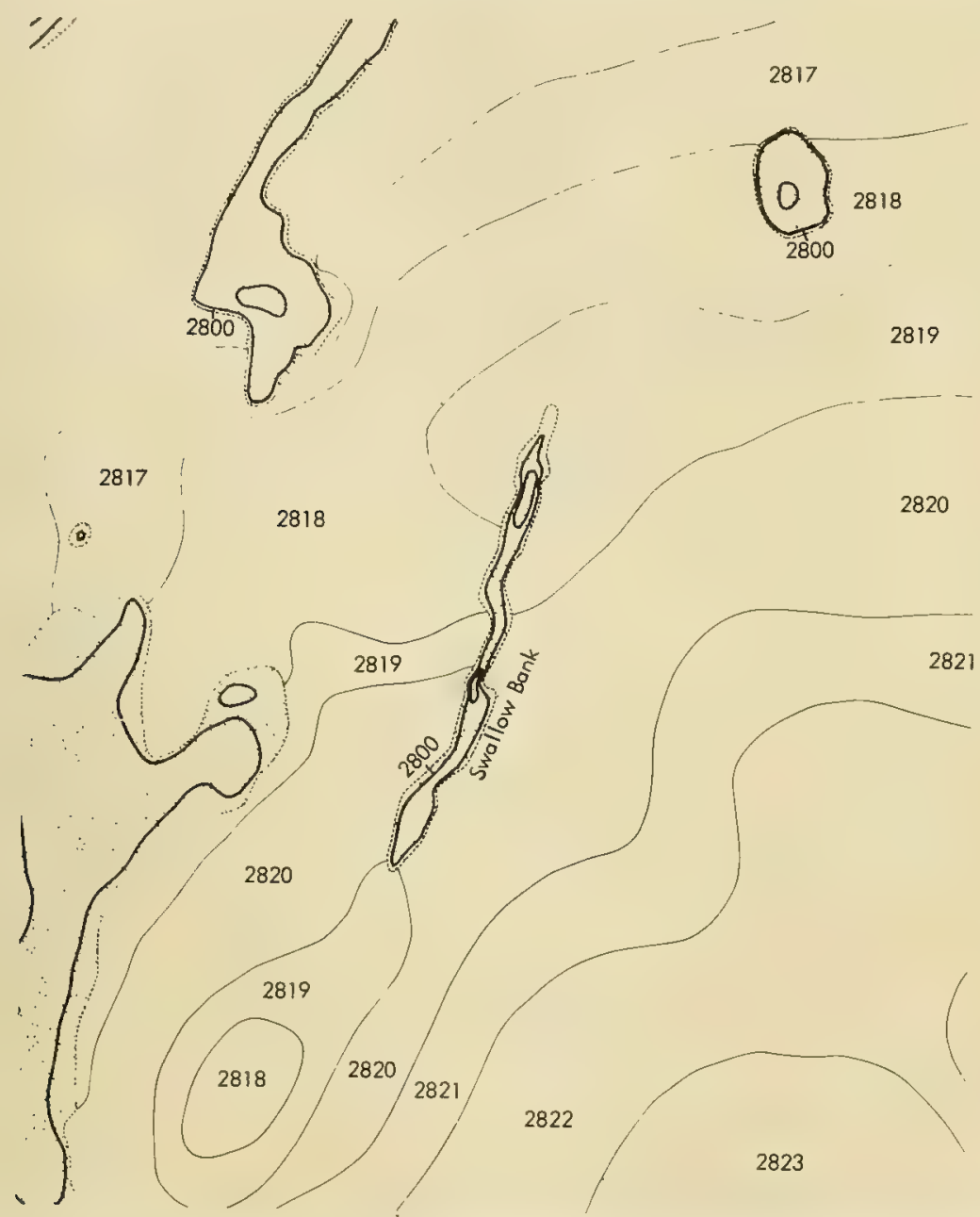

Fig. 16.3. Part of a bathymetric chart of the Swallow Bank; area, approximately 30 by 35 miles. The depths are marked in fathoms. This figure shows the remarkable consistency which can be obtained using the precision echo-sounder. (Chart from A.S. Laughton.) 
ably spaced pulses which are superimposed on the echoes as shown in Fig. 16.2. The counters are reset by the transmission. Assuming a velocity of sound of 800 fathoms $/ \mathrm{sec}$, and with a helix rate of $1 \mathrm{rps}$, the chart width represents 400 fathoms and the position of the echo can be readily determined to the nearest fathom. Part of a chart produced from a precision echo-sounder survey is shown in Fig. 16.3 and demonstrates the remarkable consistency with which the depth can be measured.

Among other things, the use of precision echo-sounders has enabled geologists to understand much more about the sedimentation processes in the sea. Laughton [2], for example, has surveyed a channel between two abyssal plains, formed by the passage of turbidity currents. An old-fashioned echo-sounder could have given the precision to show the feeder channels and the general slope of the plain only if its timing were checked every few minutes.

An echo-sounder using a transducer mounted on the hull of a ship usually fails even in moderately rough seas due to "blanketing" of the transducer by air bubbles dragged under the ship as she pitches. This has been overcome by mounting the transducer in a streamlined body towed on a short warp (typically $40 \mathrm{ft}$ ) from a boom alongside the ship. This arrangement will give satisfactory results in all weathers.

\subsubsection{Multiple-Beam and Scanning Echo-Sounders}

To quote Professor Brackett Hersey, the conventional echo-sounder (even the precision echo-sounder!) is a blunt instrument. With a typical beam angle of $30^{\circ}$, it "illuminates" a large area of the sea floor and misses all the fine detail. To obtain narrower beam angles involves using large transducers and stabilizing them against the roll of the ship; but even if such a system is achieved, a large number of runs is required to survey an area in detail. Thought is being devoted to these problems and various systems have been tried, but none is really operational yet.

Howson and Dunn [3], following suggestions by D. G. Tucker [4], have experimented with multiple-beam echo-sounders in which the beams are formed by an interferometer using two strip transducers, and also with high-speed electronic beam-scanning techniques. These enable a comparatively wide strip of sea floor to be surveyed on a single run. In the multiple-beam system, identification of the beams is a problem, since the records from all of them appear on the same chart; M. J. Tucker [5] has suggested that this can be overcome to some extent by tilting the transducer so that the pattern is unsymmetrical and port and starboard beams are distinguishable by their intensities. Figure 16.4 shows an example of a record taken with such a system. The beams were very narrow in the fore-and-aft direction $\left(1.3^{\circ}\right)$ and separated by about $15^{\circ}$ athwartships. The trans ducer was tilted to the angle shown so that the central (most intense) beam pointed to starboard. This record shows that the bed of the small canyon crossed tilted downwards $2.5^{\circ}$ from the horizontal in a direction of $110^{\circ} \mathrm{T}$.

The present difficulty with the electronic sector-scanning technique is that the sea-bed profile athwartships is presented on a CRO, and no means of making a permanent record has been devised except photography of the traces, which would not be satisfactory in practical use. The basic difficulty is, of course, that three dimensions have to be recorded. 


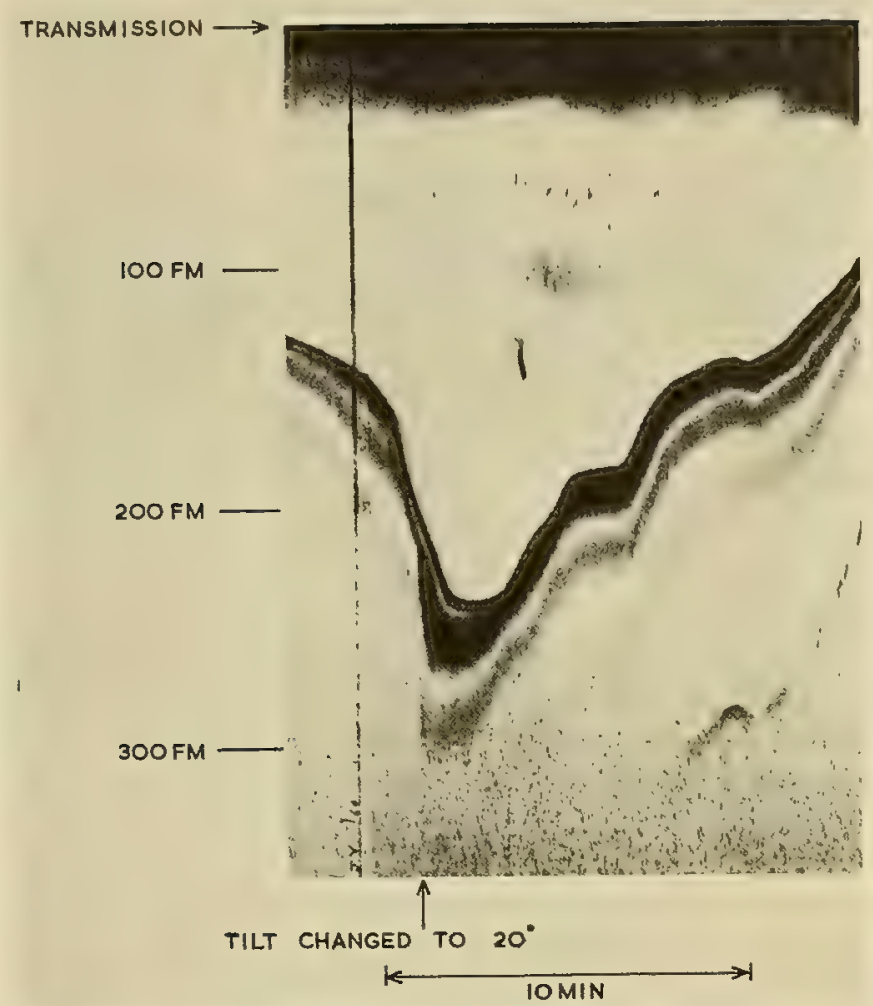

Fig. 16.4. Record from an echo-sounder employing multiple beams. From the record it can be shown that the bed of the canyon slopes at $2.5^{\circ}$ in a direction of $110^{\circ} \mathrm{T}$.

\subsubsection{Echo-Sounding for Subbottom Strata}

Ordinary echo-sounders do not usually show reflections from strata below the sea bed. This is probably due mostly to the high attenuation of these frequencies in the sea-bed material, but is probably also due in part to the high reverberation level from irregularities in this material. Under very favorable conditions, 10 -kcps sounders will penetrate at least $100 \mathrm{ft}$ of mud to show the bedrock underneath (Fig. 16.5), and where they will do so they are most useful because they have higher resolution than the devices described below. In order to get deeper penetration, and in less favorable conditions, lower frequencies are necessary, since these show much less attenuation and reverberation.

The use of these low frequencies presents major design problems. Firstly, the ambient noise level in the sea rises rapidly at low frequencies, as does also ship's noise, and high pulse powers of the order of $1 \mathrm{Mw}$ are therefore required from the transmitter. Owing to the long wavelength, the number of waves in the transmitted pulse must be kept small if useful resolution is to be obtained. A half wave would be ideal, but this requires wide-band receivers, which again increase the noise level. The types of transducers used at supersonic frequencies are thus not suitable. 


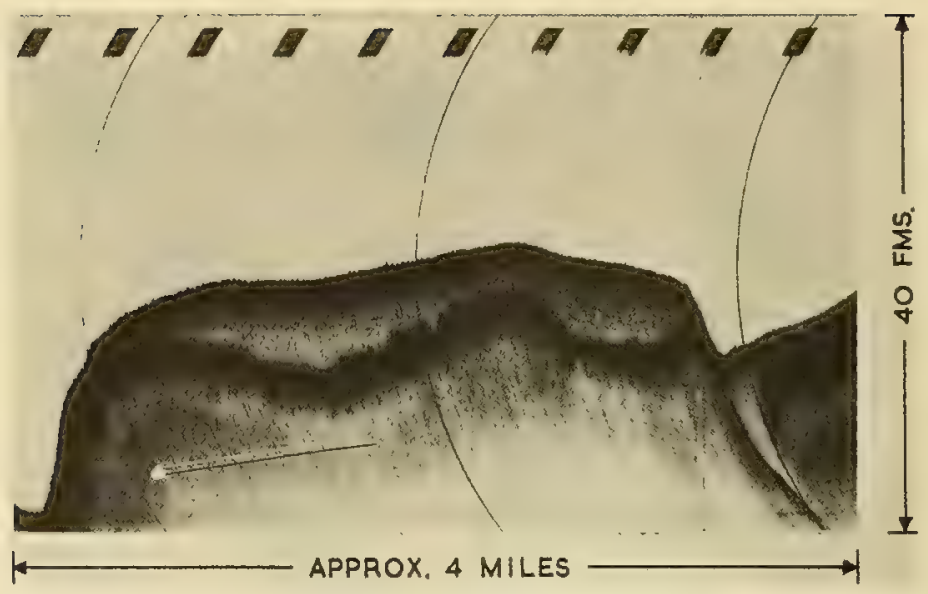

Fig. 16.5. Ten-kcps echo-sounder record showing penetration of soft mud over bedrock.

Several kinds of sound source have been designed for this application. The "Sonoprobe" developed by the Magnolia Oil Co. produces approximately a half wave of $3.8-k c p s$ sound with a peak power of about $10 \mathrm{Mw}$. This is still rather a high frequency for deep penetration, but it has high resolution. Underwater sparks have been used with considerable success [6] but always produce a double pulse due to the collapse of the gas bubble, and this reduces their effective resolution.

Perhaps the most interesting and promising device is the Edgerton "Thumper" [7]. This is extremely simple in principle, consisting of a flat coil of wire with a circular aluminum plate 20 in. in diameter held loosely against it. A pulse of current passed through the coil produces a strong magnetic field which induces eddy currents in the aluminum plate. The interaction of these eddy currents with the magnetic field produces a force which strongly repels the plate from the coil, and thus produces an acoustic pulse in the water. The standard unit uses a peak current of about $2000 \mathrm{amp}$ from the discharge of a 160 - $\mu \mathrm{f}$ capacitor charged to about $4 \mathrm{kv}$ and produces a peak pressure of about $250,000 \mathrm{~d} / \mathrm{cm}^{2}$ at 1 yard. The initial pulse consists of a clean unidirectional pressure pulse lasting about $0.5 \mathrm{msec}$, but unfortunately this was followed in the original design by two or three waves of lower frequency caused by various factors. N.I.O. has made major modifications to the mounting arrangements of the coil and plate, and it seems that this has considerably improved the waveform, but at the time of writing the system is still being tested at sea, and the precise waveforms are not available.

N.I.O. is now using as a receiver a line array of hydrophones towed behind the ship so that the array has almost zero sensitivity in the forward direction and thus does not hear the noise generated by the ship. The received signal is passed through a band-pass filter with a range of $300 \mathrm{cps}$ to $1 \mathrm{kcps}$, which has to be specially designed to have a good impulse response. Using this system towed at $7 \frac{1}{2}$ knots, echoes trom strata arriving $0.16 \mathrm{sec}$ after the bottom echo have been obtained in water depths of about $900 \mathrm{ft}$. We understand (private communication) that considerably better results have been obtained in the United 


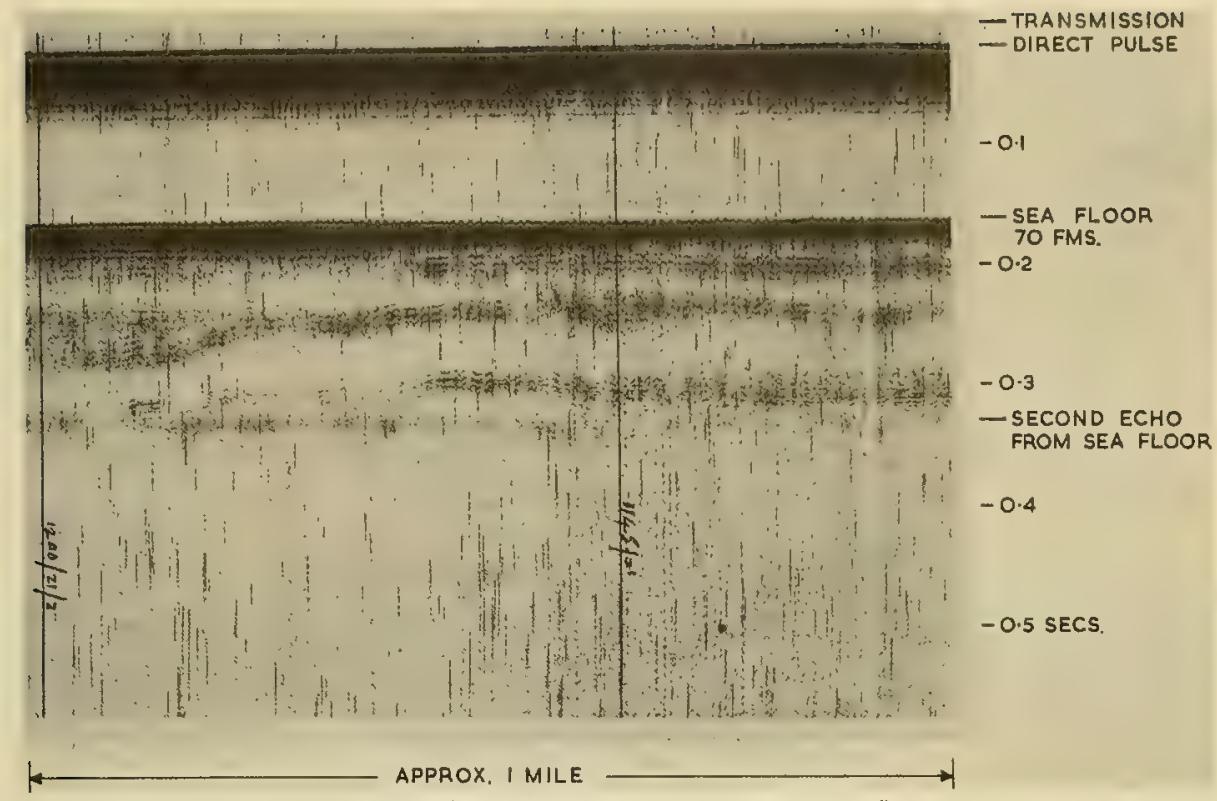

Fig. 16.6. Record taken using the "Thumper."

States using a more powerful Thumper. A typical record is shown in Fig. 16.6; this was obtained using the original Thumper and a nondirectional hydrophone.

\subsubsection{Seismic Refraction Shooting}

When the very deep rocks below the sea bed are to be examined, a system known as seismic refraction shooting is used. The principle of this method is shown in Fig. 16.7. An explosive charge, which may vary from a $1 / 2-1 b$ charge to a depth charge, is set off nearthe ship, A. The sound travels by various paths to a number of sono-radio buoys which transmit the received sound back to the ship by radio. The travel time of the first sound to arrive at the receiver is plotted against the horizontal distance given by the travel time of the direct water wave to give a diagram as in Fig. 16.8. At close ranges, the first sound to arrive has traveled through the direct water path, but at longer ranges the higher velocity of sound in the sediment layer means that sound through this path arrives first. At even longer ranges, the shortest path is through the deeper but even higher velocity layers. Thus, the diagram consists of a series of straight lines, each representing a stratum, whose slopes give the velocity of sound in that stratum. From the intercepts with the axis, the depth of the stratum can be determined. Thus, not only the true depth, but also some clue as to the composition of the stratum can be obtained, by comparing the observed velocities with those of known rocks. This technique can penetrate several miles below the sea bed.

\subsubsection{Sea.Bed Survey Using Asdic}

Chesterman, Clynick, and Stride [8] provided the first published account relating certain bottom reverberation patterns on an asdic record to the sea-bed geology. The equipment in use at N.I.O. was designed primarily for fish-detection 


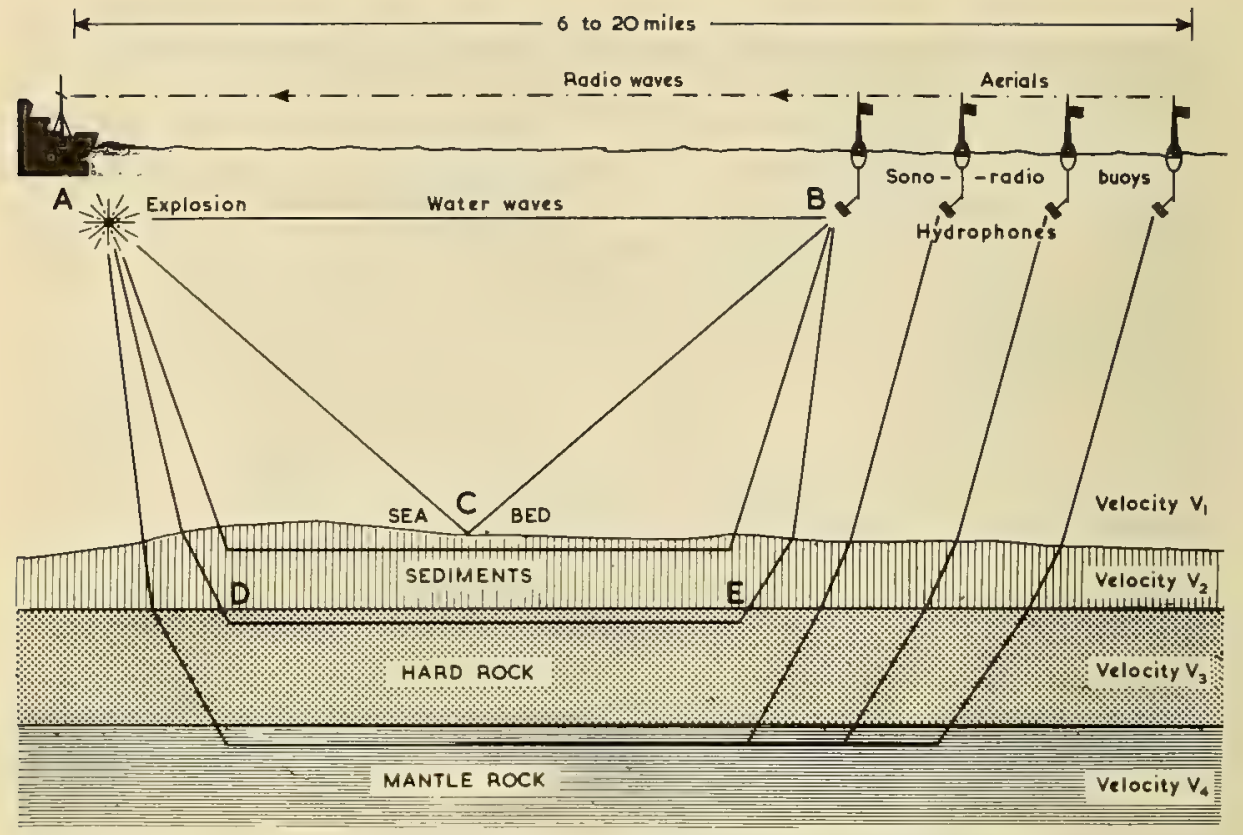

Fig. 16.7. Principle of seismic refraction method showing different paths by which waves can travel from explosion to sono buoy.

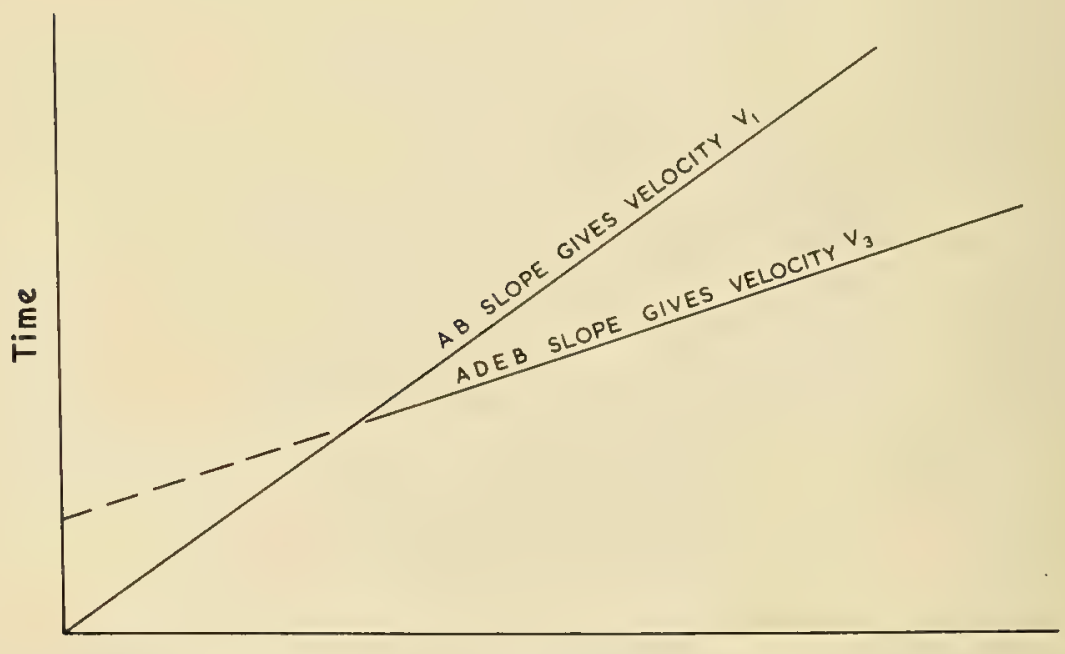

Distance

Fig. 16.8. Graph of travel times against distance for seismic refraction shooting. 


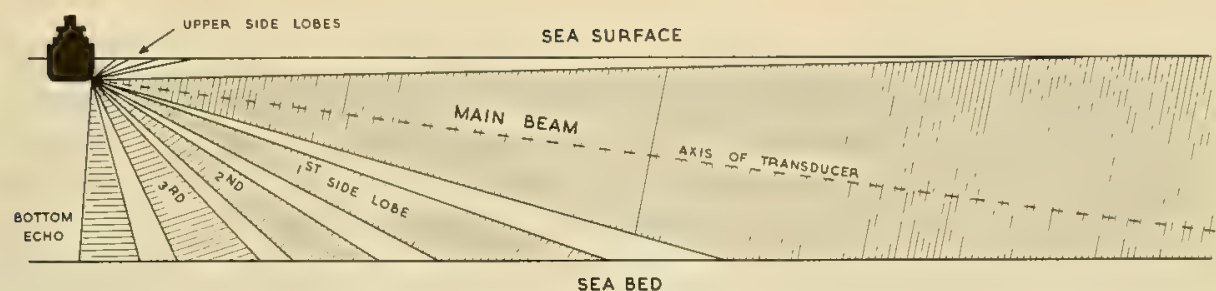

Fig. 16.9. Diagram showing vertical arrangement of beams of the asdic mounted in the R.R.S. "Discovery II."

studies, but also with the geological application in mind, and has in practice become a most powerful geological tool. (See Tucker and Stubbs [9] for a detailed description of the instrument and Stride [10] for a survey of the geological uses.)

The asdic points sideways from the ship, with a narrow beam of $1.3^{\circ}$ in plan view and a wider beam in the vertical plane (approximately $11^{\circ}$ in the usual arrangement). The main particulars are:

Frequency .................... $36 \mathrm{kcps}$

Pulse length............... 0.3 to $3 \mathrm{msec}$ (normally used at $1 \mathrm{msec}$ )

Pulse power........... $250 \mathrm{w}$

Repetition rate......... 1 pulse per sec

Maximum recorder range.... 800 yards

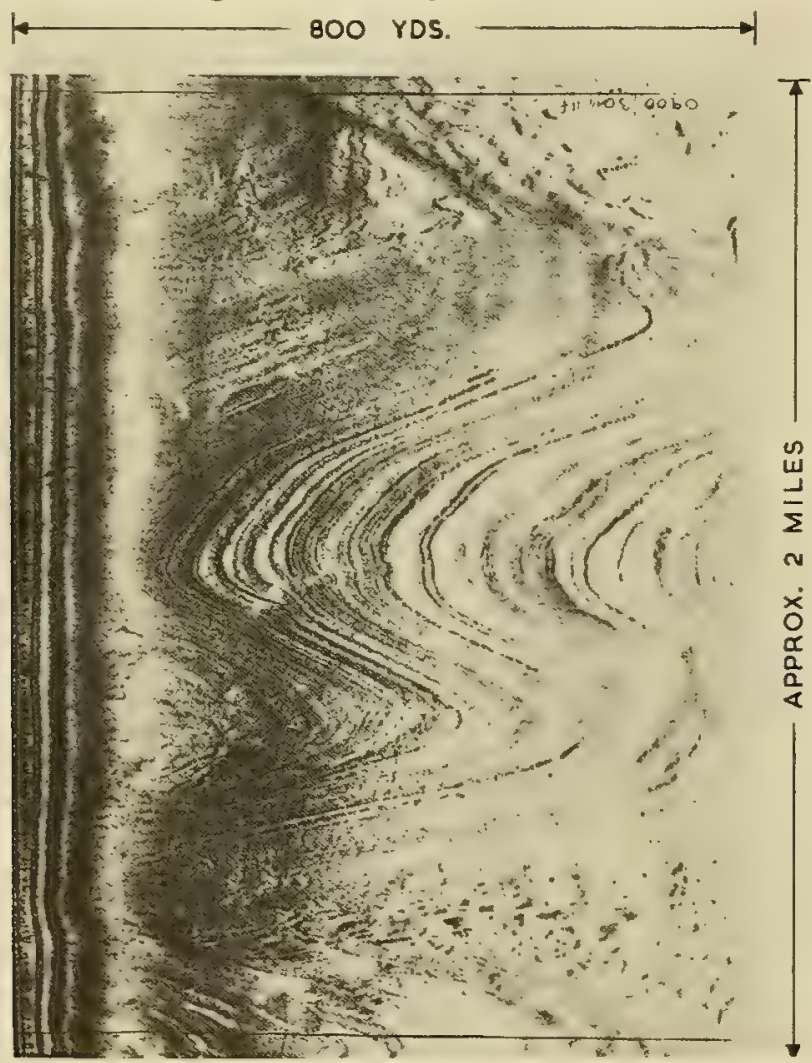

Fig. 16.10. Asdic record showing strata on the sea floor in the Bristol Channel. 
The side lobes in the vertical plane are important (Fig. 16.9) and give useful information. Figure 16.10 shows a record taken with this equipment. Starting from the left-hand side of the record and referring also to Fig. 16.9, there is first the transmission mark (somewhat ragged because the blade has been eaten away at this point). Then comes a hard line which is the bottom echo from the vertical side lobe, followed closely by the echo from the third side lobe, then by those from the second and first side lobes, and finally the echoes from the main beam extending across the rest of the record.

Figure 16.10 shows an area where the bottom has been largely swept clean of sediment and the bedrock is exposed. Figure 16.11 shows a sediment-covered area where sand waves have been formed. It will be seen that the side lobes give a quantitative measure of the bottom relief and aid in the interpretation of the acoustic picture.

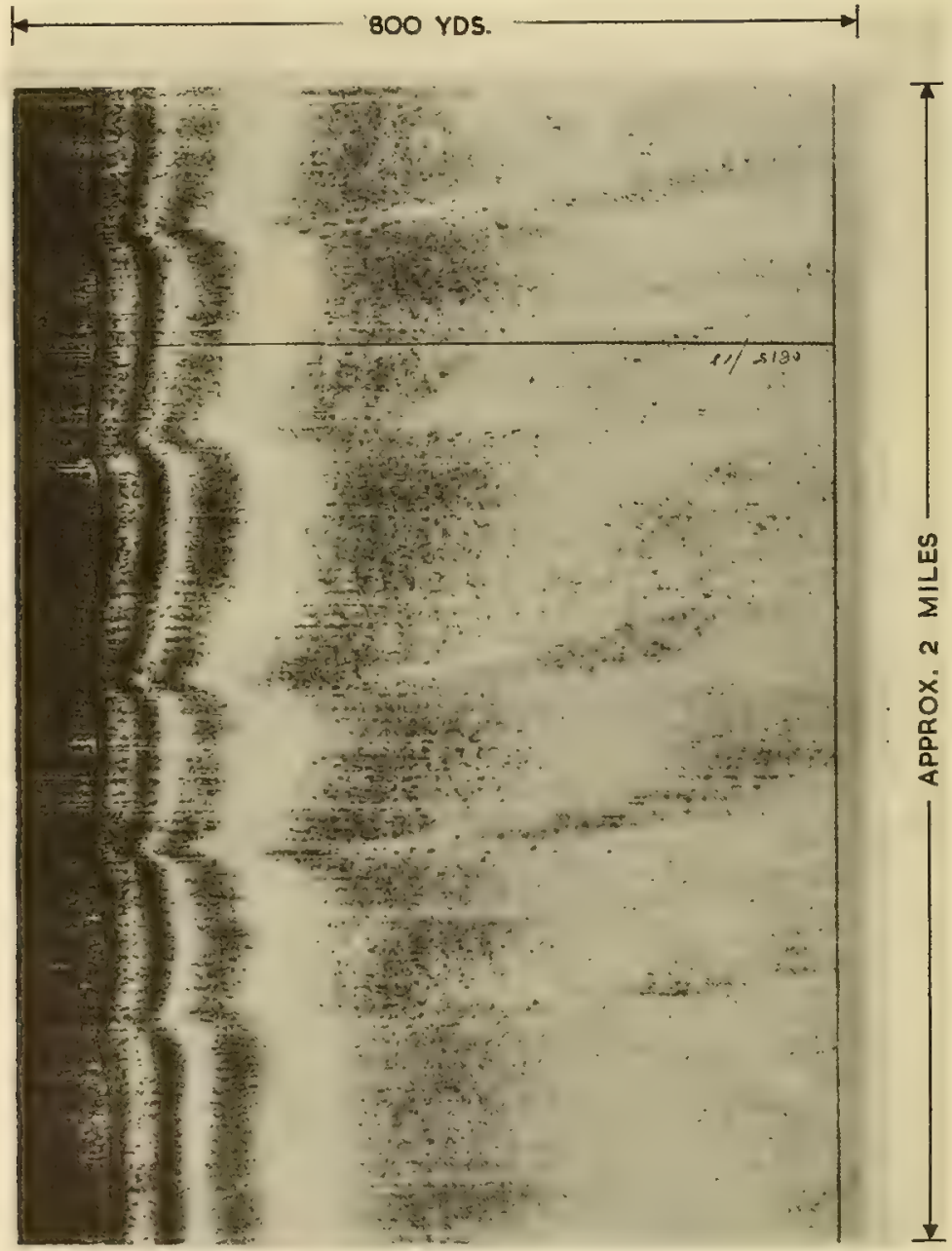

Fig. 16,11. Asdic record showing sand waves in the Irish Sea. 
It is advantageous to be able to alter the relative amplitude of the side lobes so that they produce recordings of roughly equal density, and for this purpose the transducer is divided into three horizontal strips whose relative sensitivities can be altered. The most satisfactory arrangement for geological survey work is to have the relative sensitivities in the ratio 3 to 2 to 3 .

High resolution in the recorder and good correlation between adjacent traces is required, so a Mufax recorder as described above is used. The transducer is stabilized against the roll of the ship; otherwise the side-lobe patterns become confused in rough weather. The instrument has given useful results in depths of up to 100 fathoms.

\subsection{BIOLOGICAL APPLICATIONS}

\subsubsection{Fish Detection}

Although this subject comes under the field of fishery research rather than oceanography, a brief account of it should be of interest here.

An echo-sounder of adequate sensitivity will show echoes from midwater organisms. The most powerful echoes come from shoais of fish, and the vast majority of the larger fishing boats nowadays are equipped with echo-sounders specially designed for finding fish shoals.

One of the most difficult problems for the fisherman is to decide what kind of fish he is seeing. At present, this is largely a matter of experience and local knowledge. He knows that at a particular time of year in a particular position, a particular type of pattern on the recorder usually means a certain kind of fish. Even an experienced fisherman often makes mistakes, however, and will catch valueless fish; thus, there is much interest in finding some means of identifying the fish. Present ideas suggest either using high-resolution echo-sounders with narrow acoustic beams [11], which will show more detail of the structure of the shoal, or measuring the frequency dependence of the echo strength. This latter idea is attractive in principle, but presents severe practical problems which have not yet been overcome.

A second problem which has been solved with a fair measure of success is the detection near the sea bed of fish which can be caught by bottom trawls. The vertical height of the opening of such a trawl is about $12 \mathrm{ft}$, and it is fish within this distance of the sea bed which have to be detected. Most important is cod, a fairly large fish with an acoustic cross section of up to about $1000 \mathrm{~cm}^{2}$ at typical echo-sounding frequencies. Echo-sounders can be made which will detect even individual cod up to the maximum trawling depth (say, 300 fathoms), the difficulty being to obtain sufficient resolution to separate these echoes from the bottom echo. Currently, the system most commonly used is to present the echo trace on a cathode-ray oscilloscope using an "A scan," but with the time base expanded and its initiation controlled so that only the last 50 or $60 \mathrm{ft}$ above the sea bed is presented (Fig. 16.12). This is, however, rather a trying display to watch, and systems have been developed to separate fish and sea-bed echoes on a paper recorder [12].

The area examined by an echo-sounder is, of course, a comparatively small lane directly beneath the path of the ship; thus, horizontal echo-rangers (asdics) are beginning to be used to look for fish over a wider area. There are several 


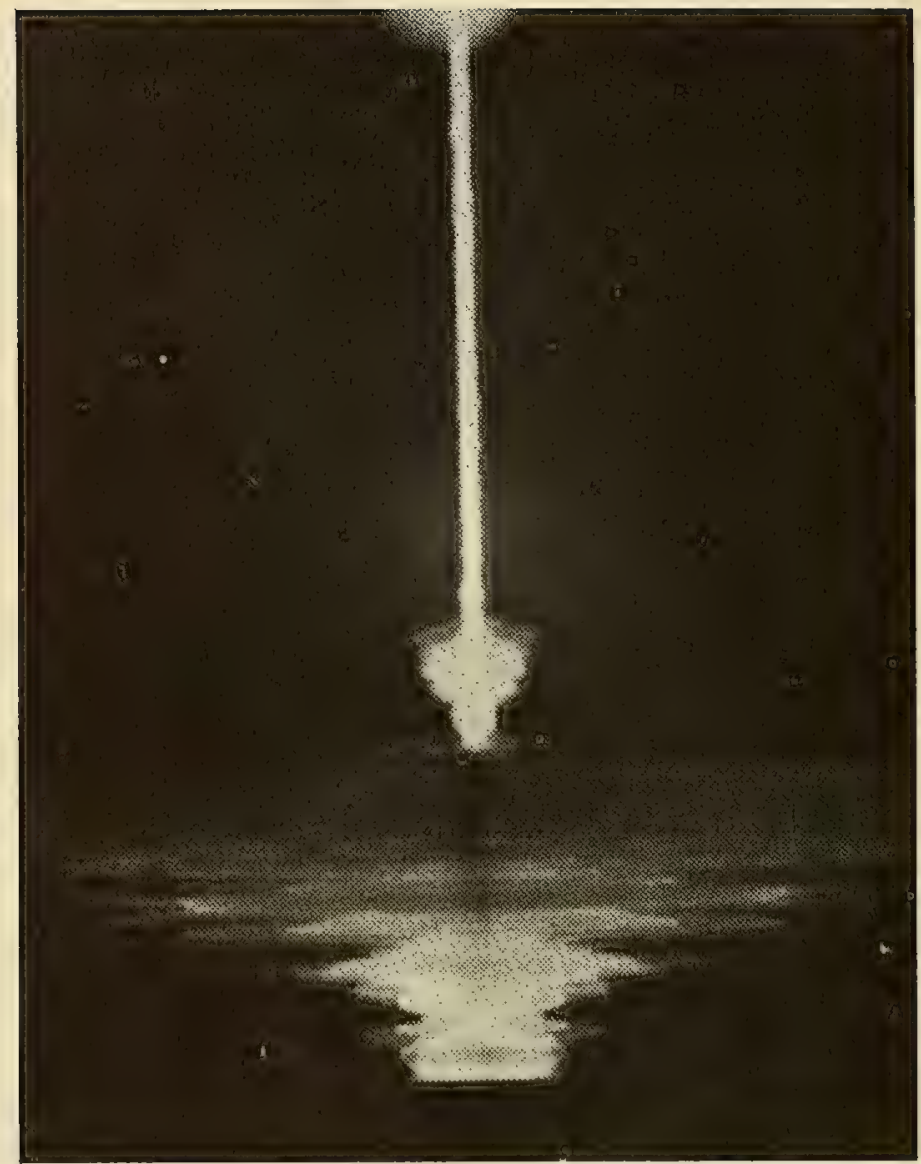

Fig. 16.12. Cod echoes displayed on Fish-lupe. Expanded portion of trace is about seven fathoms.

models commercially available, ranging from a short-range, high-resolution instrument used by purse-seine fishermen to models with ranges up to 2000 yards. A record taken with the N.I.O. asdic described in Section 16.2.5 is shown in Fig. 16.13. It will be seen that fish close to the ship appear very clearly, but at longer ranges where the main beam hits the sea bed, the fish echoes have to compete with bottom reverberation and detection is more difficult. The long ranges of detection are only obtained where the sea bed is smooth and has comparatively low reverberation.

\subsubsection{Scattering Layers}

Apart from the dense shoals of fish, a sensitive echo-sounder also records reverberation from diffuse and more or less continuous layers in the sea. A narrow-beam, high-resolution sounder operating in deep water will usually show a complex structure of layers, some of which migrate to the surface at night and return to deeper water at dawn. A record taken with a narrow-beam sounder (the N.I.O. asdic turned with the beam axis vertical) is shown in Fig. 16.14. 


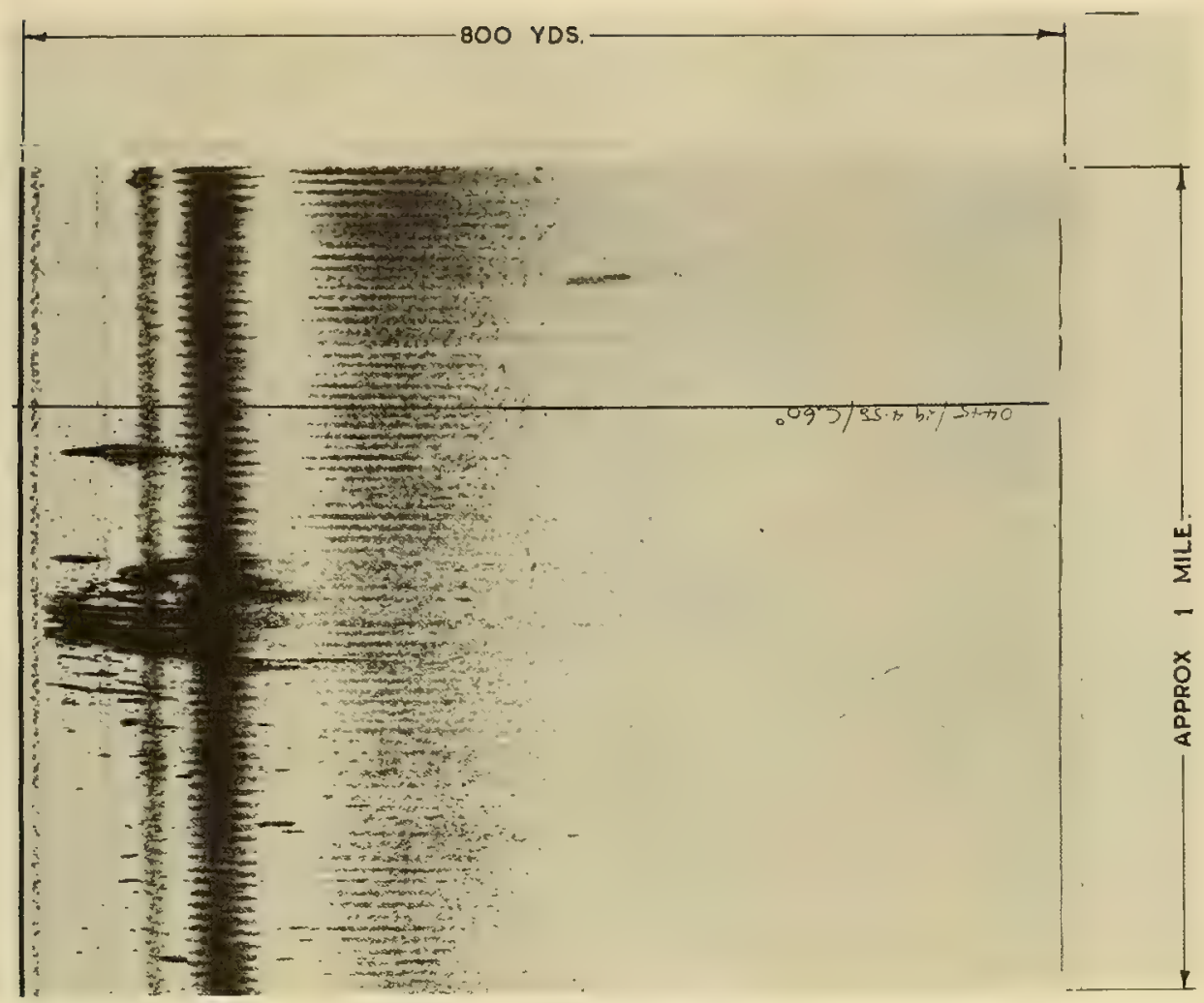

Fig. 16.13. Asdic record showing large fish concentration in midwater.

Surprisingly little is known about the composition of these layers. One can tow a plankton net through them and usually catch nothing which apparently could cause the level of reverberation actually observed. With the aid of acoustic techniques, it is sometimes possible to identify the organisms in one or two layers with a fair degree of certainty. In the record shown, for example, which was taken with a $36-k c p s$ sounder, the diffuse layer between 250 and 350 fathoms did not appear on the record from a 16 -kcps sounder. A net haul through this layer caught a number of small fish (Cyclothone sp.) 2 or $3 \mathrm{~cm}$ long, whose air bladders would resonate at a frequency in the region of $36 \mathrm{kcps}$; and though calculation showed that the number caught, compared with the volume swept out by the net, was hardly sufficient to cause the observed level of reverberation, it is known that active animals can avoid the net, and it seems reasonably certain that this layer was composed of these fish. We have occasionally managed to identify other layers by a similar technique, and Hersey [13] has managed to identify a scattering layer with some certainty using a more refined technique for measuring the frequency characteristics.

From the acoustic results, it seems reasonably certain that the nets used at present by biologists are very inefficient for sampling certain types of organisms in the sea, and we may well have a completely false idea of the relative abundance of the different species. 


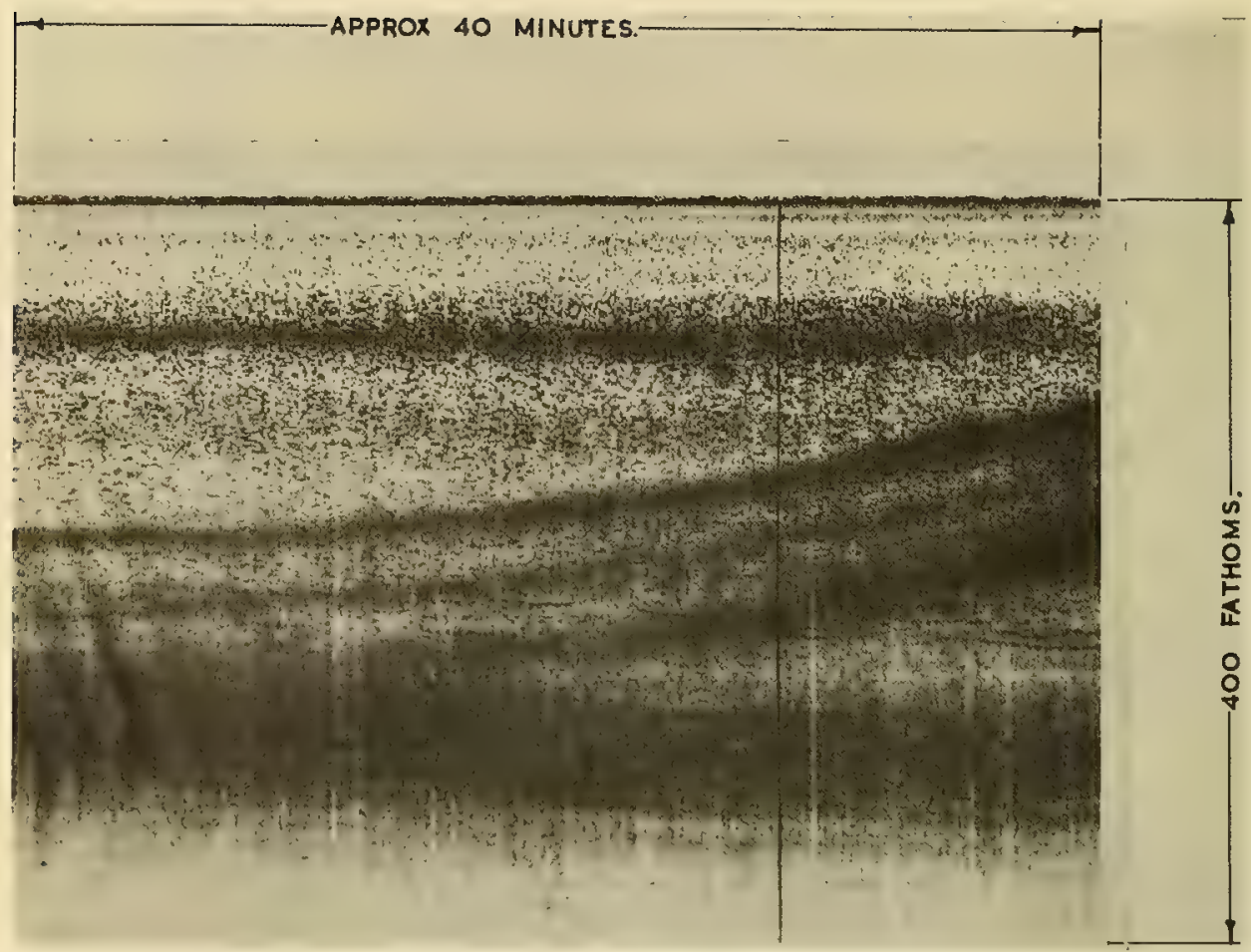

Fig. 16.14. Record from asdic equipment used as an echo-sounder showing migrating scattering layers.

\subsection{ACOUSTIC TELEMETERING}

\subsubsection{General}

Compared with radio telemetering in the atmosphere, acoustic telemetering in the sea is in its earliest infancy. Marine scientists and engineers are constantly agitating for all kinds of telemetering devices: depth-of-net meters, devices for telemetering the behavior of dredges, telemetering temperature meters, and so on, but the number of people available in civilian establishments with the rather specialized knowledge necessary for the development of these devices is so small that progress is slow.

It is perhaps relevant to ask way the information is not brought up via electric cables. Oil-well engineers, for example, lower equipment to similar depths in oil wells on strain-bearing cables incorporating one or more conductors, as a matter of routine. Oceanographers do in fact use similar cables, but they are not really satisfactory for several reasons. In many ways marine use is more severe than use in oil wells; in particular, because of the vertical motion of the point of suspension due to wave-induced pitching and rolling of the ship, and because of the extra strain, vibration, and twisting due to towing the cable through the water: even the normal drift of the ship or differential currents may be significant in this respect.

Perhaps the difficulties can best be illustrated by considering a particular cable designed specifically for marine use by a specialist in the field. Briefly, 
the specifications of this cable are:

Over-all diameter..... 0.270 in.

Weight in water. ...... $150 \mathrm{lb}$ per 1000 yards

Breaking load........ $2500 \mathrm{lb}$

Resistance per core. ... 40 ohms per 1000 yards

An 8000 -yard length would be required to operate gear near the bottom in deep water. The weight of the cable alone is half its breaking strain, which is not a good factor of safety, even with no equipment on the end. At a towing speed of one knot, the wire would stream out at an angle of about $45^{\circ}$, so that only very low towing speeds would be practicable, and of course the wire is expensive, costing $£ 5,760$ for the 8000 yards.

This is, however, an extreme example, and it is often a practical proposition to use such cables at shallower depths. There are also applications, such as underwater television, where it would not be possible to bring the information back over an acoustic link.

\subsubsection{The "Pinger"}

Though in most applications the "Pinger" is perhaps not used as a telemeter in the most conventional sense, it is a device widely used for obtaining infor mation about equipment in the sea. It consists, in its simplest form, of a simple relaxation oscillator (Fig. 16.15) in which a capacitor is charged from a battery and discharged through a coil round a nickel "scroll" transducer using a gas tube as a switch. The pulse of current produces a momentary contraction of the ring by the magnetostriction effect and the ring then oscillates at its natural frequency, giving a damped train of waves. The circuit illustrated, using a 10kcps scroll, has a range of four miles in favorable circumstances.

In this simple, free-running form, it is used by Swallow [14] in his system for deep-current measurement. He has a neutrally buoyant float which is less compressible than sea water, so that if suitably weighted it will sink to a predetermined depth and then move around with the water at that depth. The float contains a Pinger, and is tracked using a directional hydrophone system aboard a ship. A float has been tracked for as long as six weeks in this way.

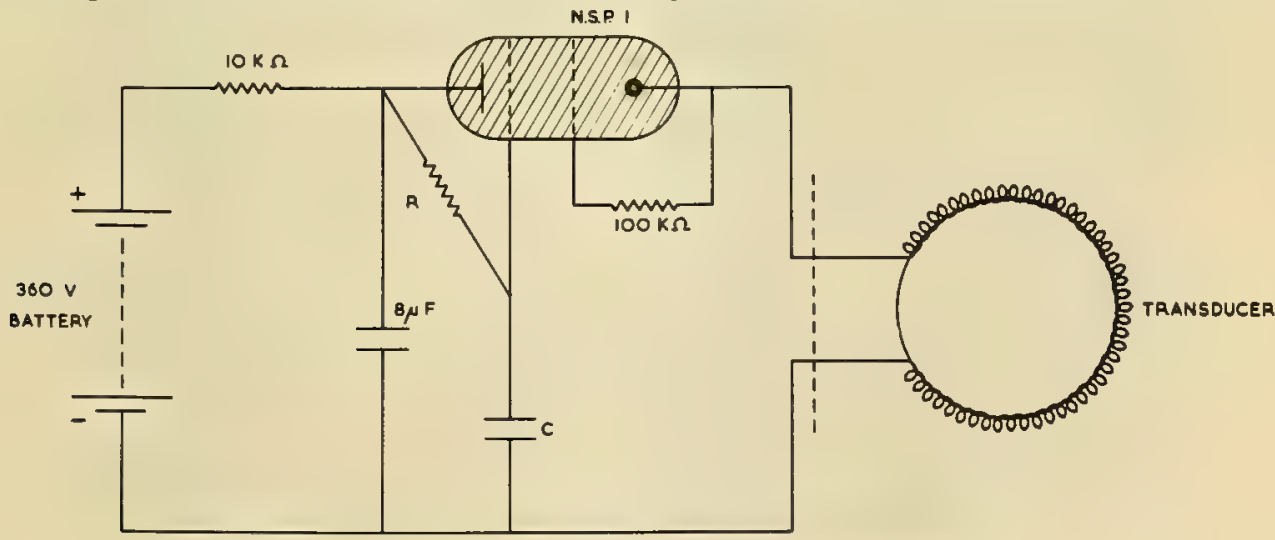

Fig. 16.15. The relaxation oscillator circuit of a "Pinger." 
A somewhat similar acoustic system working at $132 \mathrm{kcps}$ has been used by Trefethen, Dudley, and Smith [15] to follow the movements of salmon. In their system, the complete transistorized transmitter is only $2.4 \mathrm{in}$. long and $0.9 \mathrm{in}$. in diameter, which is so compact that it can be fixed to the fish as a "tag" and followed from a boat using a 4-element receiving array and a servomechanism which keeps the receiver pointing at the salmon by a phase-comparison system.

By controlling the repetition rate of a Pinger to precisely one per second, signals can be received and recorded on the precision echo-sounder receivers already described in Section 16.2.1. Edgerton and Cousteau [16] have used such a system for measuring the distance of a deep-sea camera from the sea floor by measuring the separation of the direct pulse from that reflected from the sea floor.

Laughton [17] with his camera arranges for the pulse repetition rate of a Pinger to change when the trigger-weight hits the sea bed and a photograph is taken. The signals are received by a hydrophone hung from the ship, and as soon as the change of rate of pinging is heard, the camera is raised a short distance, the ship is allowed to drift, and the camera is then relowered and another photograph taken.

\subsubsection{Acoustic Telemeters with Continuous Transmission}

A few telemetering systems have been built in which the information is transmitted over a continuous carrier. Perhaps the type of telemeter most in demand is one which transmits the depth of a piece of equipment, usually by measuring the external water pressure. Such an instrument is in demand, for example, by commercial fishermen who are becoming increasingly interested in catching shoals of fish in midwater using midwater trawl nets. Their echo-sounders tell them the depth of the shoal as they go over it and they want to make sure that the nets they are towing are at the same depth. The same problem is also encountered by biologists studying the scattering layers (see Section 16.3.2), and in other applications.

A successful device of this type has been described by Stephens and Shea [18]; it is based on an instrument designed by W. J. Dow. In this instrument, the pressure is made to vary the frequency of a supersonic carrier, and the temperature is also measured and varies an audio-frequency which is transmitted as an amplitude modulation on the carrier.

The physical arrangement of the system is shown in Fig. 16.16. The acoustic transducers have to have fairly wide beams, but even some directionality greatly

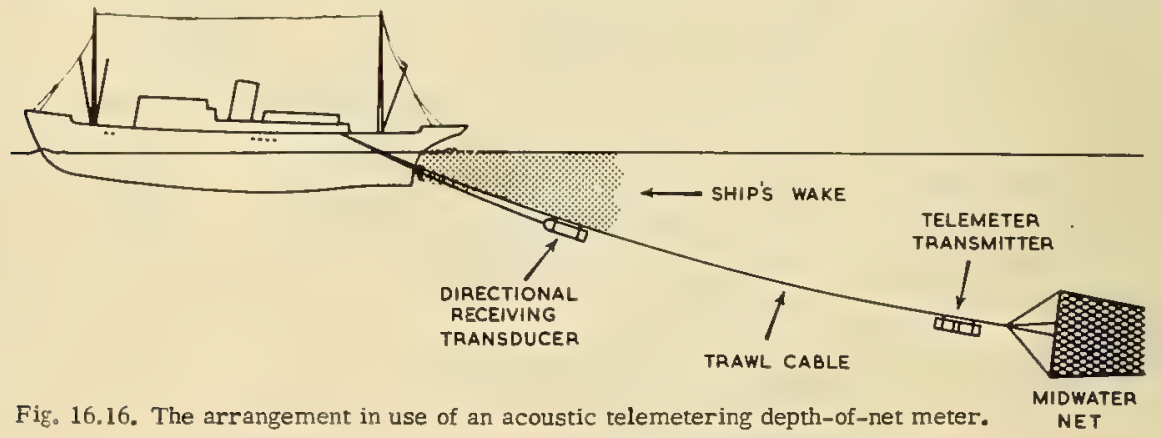


improves the signal-to-noise ratio. The electrical input to the transmitting transducer is between 1 and $2 \mathrm{w}$, the frequency deviation is 21 to $36 \mathrm{kcps}$, and the audio-frequency temperature signal varies between 200 and $800 \mathrm{cps}$.

The receiving transducer is slipped down the towing warp after this has been fully let out, and thus needs no extra men for its operation. After preamplification, the received signal is fed to a low frequency radio receiver whose dial reading is a measure of the depth. The output of the radio receiver is the audiofrequency temperature signal. A depth accuracy of $1 \%$ and a range of one mile is claimed for the instrument.

For some purposes, such as the seismic refraction shooting method described in Section 16.2.4 for examining sub-bottom geology, there are advantages in placing a hydrophone on the sea bed. Dow [19] has also designed an acoustic telemetering hydrophone for this application. It will receive sounds in the range $50 \mathrm{cps}$ to $5 \mathrm{kcps}$ and transmit these on an acoustic carrier of $21 \mathrm{kcps}$. It is capable of working at depths of up to 3000 fathoms, and uses a power input to the transducer of about $30 \mathrm{w}$.

\subsection{MISCELLANEOUS APPLICATIONS}

\subsubsection{Wave Recording}

An inverted echo-sounder placed on the sea bed and pointing upward at the surface may be used to record wave height. The height recorded is the distance

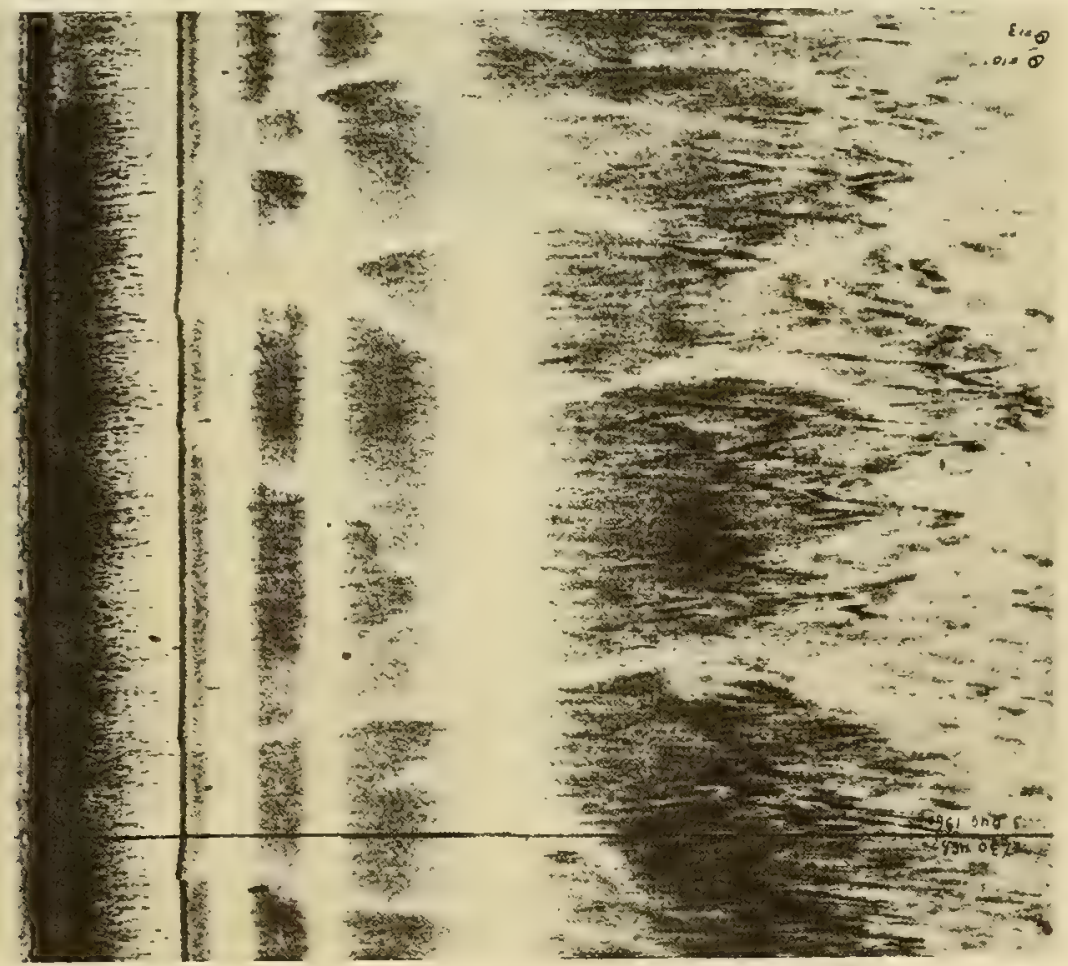

Fig. 16.17. Asdic record showing patterns at the right-hand side which are attributed to refraction by internal waves. 


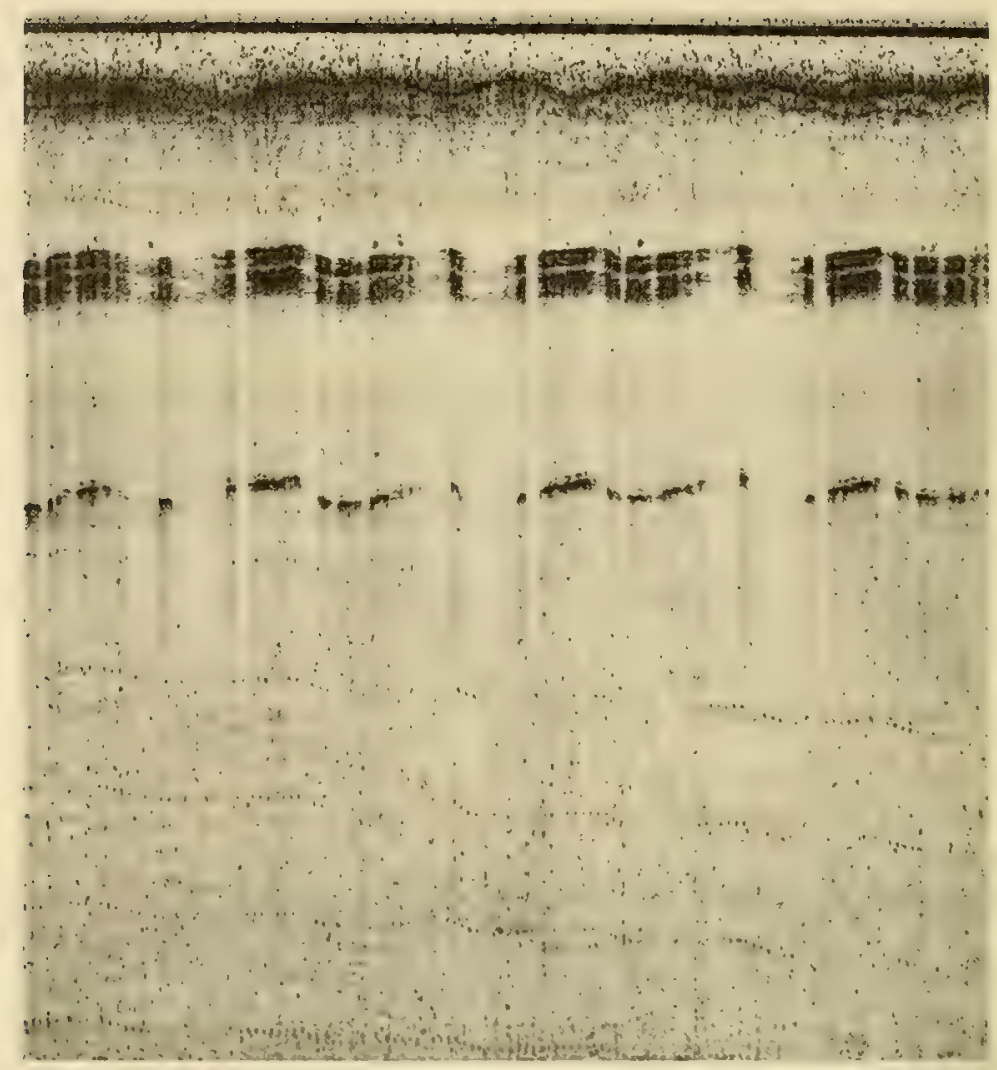

Fig. 16.18. Echo-sounder record showng movement of scattering layers caused by internal waves.

of the transducer from the nearest part of the water surface which is effectively in the beam, which may be considerably nearerthan the water surface vertically above the transducer. Such errors can be quite large with a wide-beam transducer, but if the beam is too narrow, there may be no reflecting facet in the surface area "illuminated" by the beam, and thus echoes may be lost.

The other major snag with this type of instrument is that in storms, when it is probably most important to record the waves, the water is often so aerated that the sound cannot penetrate it and noechoes may be obtained for long periods of time.

\subsubsection{Internal Waves}

Internal waves in the sea have both direct and indirect effects on underwater sound. The direct effect is refraction of sound, and this has a marked effect on asdic records (Fig. 16.17). Here, the sound is focused or dispersed so that some areas of the sea floor are more brightly "illuminated" than others and a marked pattern is produced on the record. This effect has been studied by Lee [20] and is mentioned in Lecture 8 ( $\mathrm{J}$. Crease), and might possibly give useful information on the waves themselves. The indirect effect is to produce movement of organisms in the sea which are then picked up on echo-sounders [21]. Figure 16.18, 
for example, shows a scattering layer which is being carried up and down by the internal waves and allows the measurement of them at the depth of the layer.

\section{REFERENCES}

1. S. T. Knott and J.B. Hersey, "Interpretation of High-Resolution Echo-Sounding Techniques and Their Use in Bathymetry, Marine Geophysics and Biology," Deep-Sea Research, Vol, 4, 36-44 (1956).

2. A. S. Laughton, "An Interplain Deep-Sea Channel System," Deep-Sea Research, Vol. 7, 75-78 (1960).

3. E. A. Howson and J.R. Dunn, "Directional Echo-Sounding," J. Inst. Navigation XIV, 348-359 (1961).

4. D. G. Tucker, "Directional Echo-Sounding," Intern. Hydrographic Rev. XXXVII, 43-53 (1960).

5. M.J. Tucker, "Beam Identification in Multiple-Beam Echo-Sounders," Intern. Hydrographic Rev. XXXVIII, 25-32 (1961).

6. W. C. Beckmann, "Geophysical Surveying for a Channel Tunnel," New Scientist, Vol. 7, 710-712 (1960),

7. J.B. Hersey, H.E. Edgerton, S. O. Raymond, and G. Hayward, "Sonar Uses in Oceanography, "Instrument Society of America, Conference Preprint 21-60, 5-9 (1960).

8. W.D. Chesterman, P.R. Clynick, and A.H. Stride, "An Acoustic Aid to Sea-Bed Survey," Acustica, Vol. 8, 285-290 (1958).

9. M. J. Tucker and A.R. Stubbs, "Narrow-Beam Echo-Ranger for Fishery and Geological Investigations," Brit. J. of Appl. Phys., Vol. 12, $103-110$ (1961).

10. A. H. Stride, "Geological Interpretation of Asdic Records," Intern. Hydrographic Rev. XXXVIII, 131 -I39 (1961).

11. R. E. Craig, "Some Successful Experiments with a Pencil-Beam Echo-Sounder," World Fishing, Vol. 8, 40-4l (1959).

12. Kelvin and Hughes, "The MS 28/29 Operating and Service Manual, " Publication 342.

13. J.B. Hersey and R.H. Backus, "New Evidence that Migrating Gas Bubbles, Probably the Swim Bladders of Fish, are Largely Responsible for Scattering Layers on the Continental Rise South of New England," Deep-Sea Research, Vol. 1, 190-191 (1954).

14. J. C. Swallow, "A Neutral-Buoyancy Float for Measuring Deep Currents," Deep-Sea Research, Vol. 3, $74-81$ (1955).

15. P.S. Trefethen, J.W. Dudley, and M.R. Smith, "Ultrasonic Tracer Follows Tagged Fish," Electronics, Vol. 30, 156-160 (1957).

16. H. E. Edgerton and J. Y. Cousteau, "Underwater Camera Positioning by Sonar," Rev. Sci. Instr., Vol. 30 , 1125-1126 (1959).

17. A. S. Laughton, "A New Deep-Sea Underwater Camera," Deep-Sea Research, Vol. 4, 120-125 (1957).

18. F.H. Stephens and F. J. Shea, "Underwater Telemeter for Depth and Temperature," U.S. Fish Wildlife Serv., Spec. Sci. Rept., Fisheries Ser. No. 181.

19. W. Dow, "A Telemetering Hydrophone," Deep-Sea Research, Vol, 7, 142-147 (1960).

20. O. S. Lee, "Effect of an Internal Wave on Sound in the Ocean," J. Acoust. Soc. Am., Vol. 33, 677-681 (1961).

21. V. Valdez, "Internal Waves on an Echo-Sounder Record, " Deep-Sea Research, Vol, 7, 148 (1960). 



\title{
LECTURE 17
}

\section{SOME EXPERIMENTS ON THE REDUCTION OF STRUCTURE-BORNE NOISE}

\author{
J.H. Janssen \\ Technisch Physische Dienst T.N.O. en T.H. \\ Delft, Netherlands
}

\subsection{INTRODUCTION}

"The acoustic stealth of a ship or torpedo is determined by the external noise it generates" [1]. Four different sources of underwater ship noise may be distinguished: (1) cavitation, (2) hydroelastic forces, (3) flow, and (4) machinery. This paper is concerned with some problems of machinery noise reduction.

Noise control is basically a system problem. The system consists of a ship, water, and detection apparatus. It contains a source, a path, and a receiver for sound signals. The aim of noise control in this instance is to prevent detection by the enemy. In underwater noise reduction, more often than not a restriction is imposed on the measures to be taken: only the sound paths are available for blocking.

It is for this reason that only the path is considered here, or rather the multiplicity of paths. Of course, not all types of ships, sources, and structures can be reviewed with respect to their acoustical behavior. A considerable simplification is obtained if only the structures of small ships like submarines or minesweepers are studied. Only two typical noise paths seem to be important then; viz., the path via the structure only and the path via the air and the structure. In the latter instance, for example, the noise is radiated by a diesel engine directly into the air and excites the shell, which in turn radiates sound into the water. The pure structure-borne noise follows the path through springs, foundation, and hull into the water. At large distances, hydrophones may detect the pressure fluctuations due to the radiated sound power anyway.

In Fig. 17.1 a noise path together with associated levels is illustrated. In this paper some experiments, both theoretical and practical, relating to the steps in the path (machinery to foundation, foundation to shell, and shell to water) will be discussed.

\subsection{UNITS AND LEVELS}

Sound power manifests itself by way of fluctuations in pressure and particle velocity. In fluids and gases, these pressure fluctuations are easily measured 


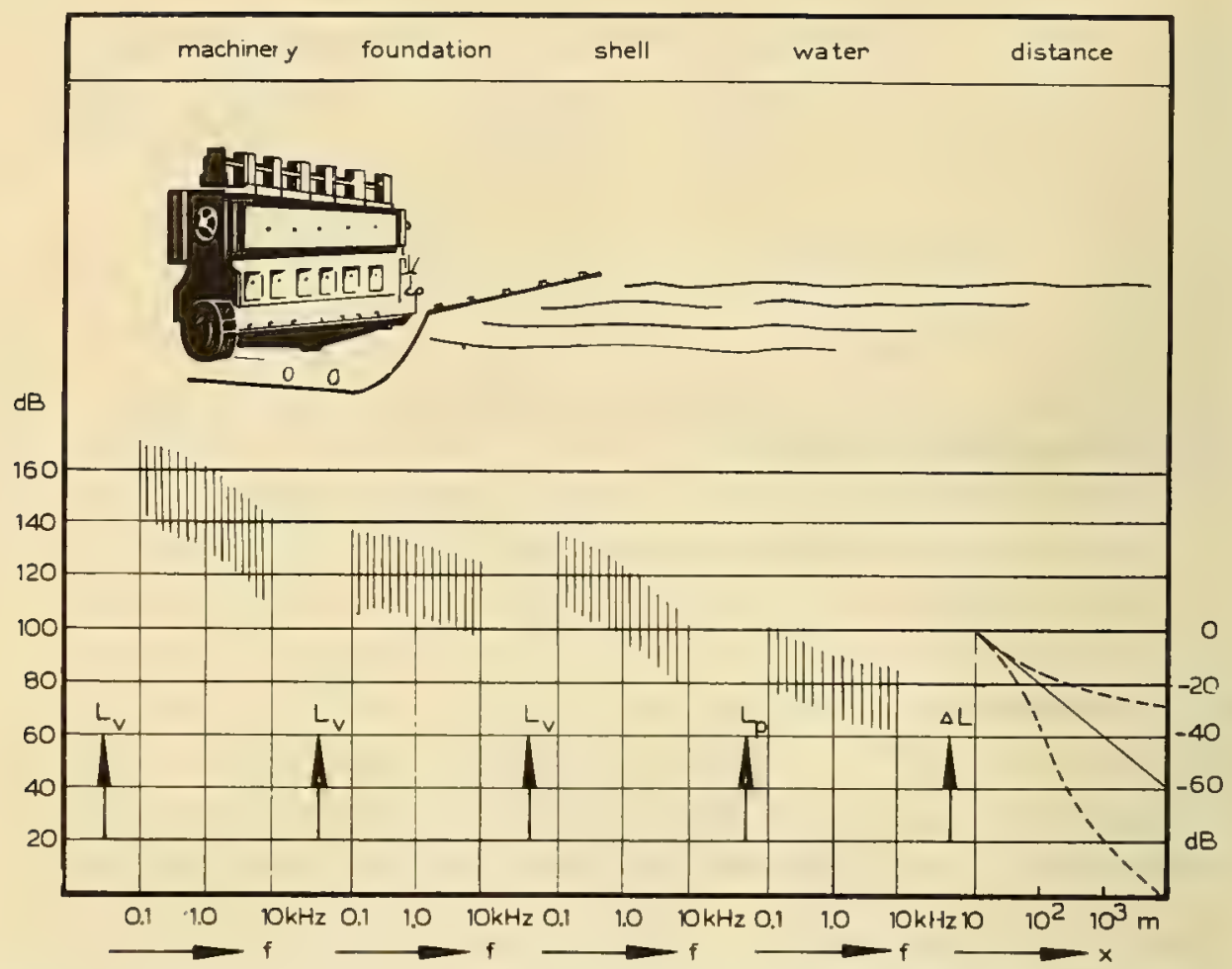

Fig. 17.1. Typical ranges for octave-band spectra measured during diesel engine operation. Left: velocity at engine footings: middle: velocity at substructure or foundation (ship's side of resilient mounts); right: velocity on shell plates, and pressure at $10 \mathrm{~m}$ from shell together with attenuation due to transmission in sea water, Reference values for db scale: velocity $13 \mathrm{pm} / \mathrm{sec}$, pressure $20 \mu \mathrm{N} / \mathrm{m}^{2}$.

by means of hydrophones or microphones. There is no other simple means of detecting or measuring sound signals. Statements about sound pressure measurements, whether in air or in water, are easily made when use is made of a decibel scale; the latter needs a reference pressure. The contents and conclusions of a technical memorandum by Dr. R. W. Young offer sufficient information and illustration to establish $20 \cdot 10^{-6} \mathrm{~N} / \mathrm{m}^{2}$ as the single reference pressure for both air-borne and water-borne sound measurements [2]. This reference pressure is in accordance with internationally standardized, albeit air-borne, practice [3].

A sound pressure level $\left(L_{p}\right)$ may be mathematically defined thus:

$$
L_{p}=10 \log \left(\frac{\int_{0}^{T} p^{2} d t}{p_{0}^{2} T}\right)
$$

where $p_{0}$ is the reference pressure mentioned, $T$ is an integrating period, and $p$ is the time-dependent sound pressure expressed in decibels (db).

In the same way a particle velocity level $L_{v}$ can be defined for a time-dependent $v$ :

$$
L_{v}=10 \log \left(\frac{\int_{0}^{T} v^{2} d t}{v_{0}^{2} T}\right)
$$


where now the reference value $v_{0}$ is given by

$$
v_{0}=\frac{p_{0}}{\rho_{w} c_{w}}=13 \cdot 10^{-12} \frac{\mathrm{m}}{\mathrm{s}}
$$

if underwater noise reduction is considered $\left(\rho_{w} c_{w}\right.$ is the characteristic impedance of sea water). It follows that $L_{p}=L_{v}$ for plane traveling waves. This convenient choice of $p_{0}$ and $v_{0}$ is applied in this paper.

The response of mechanical structures to alternating forces can be investigated either by means of discrete frequency excitation or by means of random frequency-mixture excitation (noise). The vast amount of information obtained from discrete frequency responses may be reduced in most instances by averaging over certain adjacent frequency bands. The same "data reduction" is performed at once if filtered noise bands are used. Bandwidths half an octave wide are very convenient: they offer sufficient information in only 16 bands for the audio-frequency range. Of course, the time required for an 8-octave-band response measurement is shorter still; too much detail is lost, however. The half-octave-band measurement, as reported here, seems to be a good compromise between discrete frequency (or one-third-octave messurements) and the octave-band method.

Noise may be supposed to consist of many random discrete frequency components. Within a given frequency band, the components of a "noise force" are numbered $1,2,3,4, \ldots, n$. For a half-octave band the highest frequency $f_{b}$ is equal to or lower than $\sqrt{2}$ times the lowest component frequency $f_{a}$. The "effective value" $F_{\text {eff }}$ (time rms) of a noise force is given by

$$
F_{\text {eff }}=\frac{1}{2}\left(\hat{F}_{1}^{2}+\hat{F}_{2}^{2}+\hat{F}_{3}^{2}+\cdots\right)
$$

where the sign $\wedge$ indicates the maximum value (amplitude) of a force component. If very many components are present, we may suppose that Eq. (4) can be written in the form of an integral

$$
F_{\text {eff }}^{2}=\int_{f_{a}}^{f_{b}} F_{f}^{2} d f
$$

where $F_{i}^{2}$ is called the spectral density of the force. If a force of this type acts upon a mechanical structure, the resulting velocity is of interest. For example, the velocity at the excitation point can be found from the usual mechanical input impedance defined as the complex ratio of sinusoidal force to velocity, $Z=R+j X$.

The expression for the effective value $v_{\text {efi }}$ of the "noise velocity" reads

$$
v_{\mathrm{eff}}^{2}=\int_{f_{a}}^{f_{b}} \frac{F_{i}^{2} d f}{R^{2}+X^{2}}
$$

In this way, a kind of averaged-out impedance-or in general system responsemay be obtained without going into all details 'of the discrete frequency analysis. A similar result could also be obtained, however, by such an analysis: if the system response, e.g., the impedance $Z_{n}$, were known for very many closely spaced frequencies (numbered $n$ ), then the ratio of Eq. (6) to Eq. (5) would be obtained by averaging all $1 /\left|Z_{n}\right|^{2}$. 
Thus, mechanical noise impedance $Z_{\mathrm{av}}$ is introduced as

$$
Z_{\mathrm{av}}=\sqrt{\frac{F_{\mathrm{eff}}^{2}}{v_{\mathrm{eff}}^{2}}}
$$

where $F_{\text {eff }}^{2}$ and $v_{\text {eff }}^{2}$ are taken from Eqs. (5) and (6), respectively. For measurement, computation, or graphical representation, moreover, it is convenient to define an impedance level $L_{z}$ as

$$
L_{z}=20 \log \frac{Z}{Z_{0}}
$$

where $Z$ equals $Z_{\text {av }}$ in Eq. (7) or the modulus of a complex impedance $Z$, and where $Z_{0}$ is taken as $1 \mathrm{~kg} / \mathrm{sec}$ for simplicity. Besides the levels for pressure already defined in Eq. (1), velocity in (2), and impedance in (8), a power level $L_{P}$ is used and defined as

$$
L_{P}=10 \log \frac{P}{P_{0}}
$$

where $P$ is the sound power (in watts) to be expressed in $\mathrm{db}$ and $P_{0}$ equals $10^{-12} \mathrm{~W}$, the convenient reference power for air-borne sound measurements.

Having defined some symbols, units and levels we proceed now to follow the sound path from a source into the water. Some of the steps in the path will be considered in more detail.

\subsection{RESILIENT MOUNTS}

A simplified classical system involving a resilient element or spring is shown in Fig. 17.2. An (electrodynamic) exciter or shaker causes one side of the spring to vibrate sinusoidally in a "normal" direction; normal refers to the direction normal to the two parallel contact planes at each side of the spring.

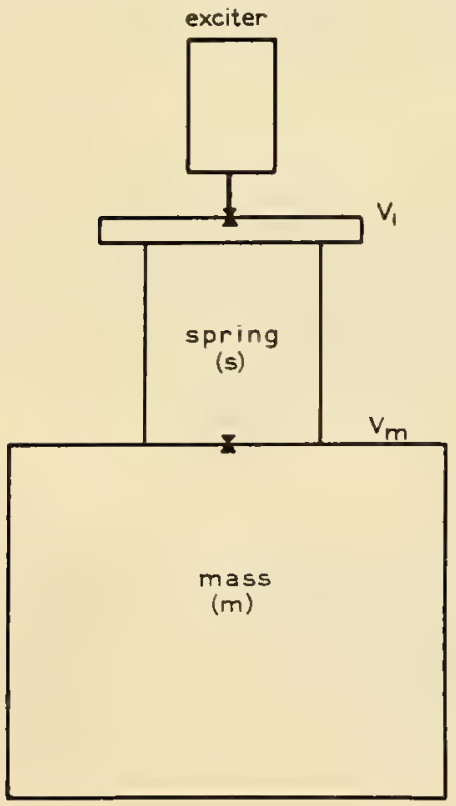

Fig. 17.2. Classical example of vibration isolation; the electromechanical analog shows clearly the purpose of the spring (condenser): acoustical uncoupling or release. The mass is hanging from a spring instead of resting on it; the two are not fundamentally different.

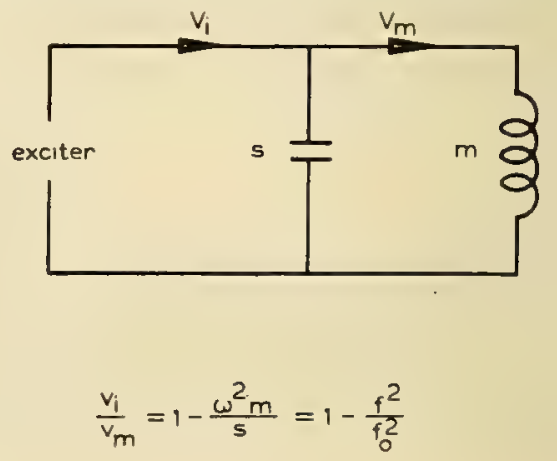


The spring is compressed more or less and in turn excites the mass. The wellknown equivalent electrical circuit is also shown. The ratio of $v_{t}$ (input velocity) to $v_{m}$ (velocity of the mass) depends only on the ratio: forcing frequency $f$ to resonance frequency $t_{0}$. Let us now call

$$
20 \log \left|\frac{v_{i}}{v_{m}}\right|=L_{v i}-L_{v m}
$$

the "isolation" for this situation.

Clearly this isolation equals, approximately, the difference in impedance level for the mass and the spring, provided the frequency $f$ is greater than $f_{0}$. For normal excitation and normal vibration, this statement turns out to be approximately valid if either the spring behaves less like a spring or the mass is replaced by a more general foundation; provided, again, that the impedance level of the spring is some $10 \mathrm{db}$ lower than the impedance level of the foundation.

Figure 17.3 shows the well-known wave effects $[4,5,6]$ in the spring, the mass still simulating "rigid inertia." Figure 17.4 shows the effect of the foundation impedance on the isolation; simple theory agrees sufficiently with practice. In Fig. 17.5, however, an unexpected difference between two isolation curves is shown. For both curves the excitation was "normal"; the vibration, however, was not. Clearly, this simple fact is of great importance to the isolation. Many other experimental results indicate that besides normal excitation other-parallel or rotational-excitation must also be taken into account $[7,8]$.

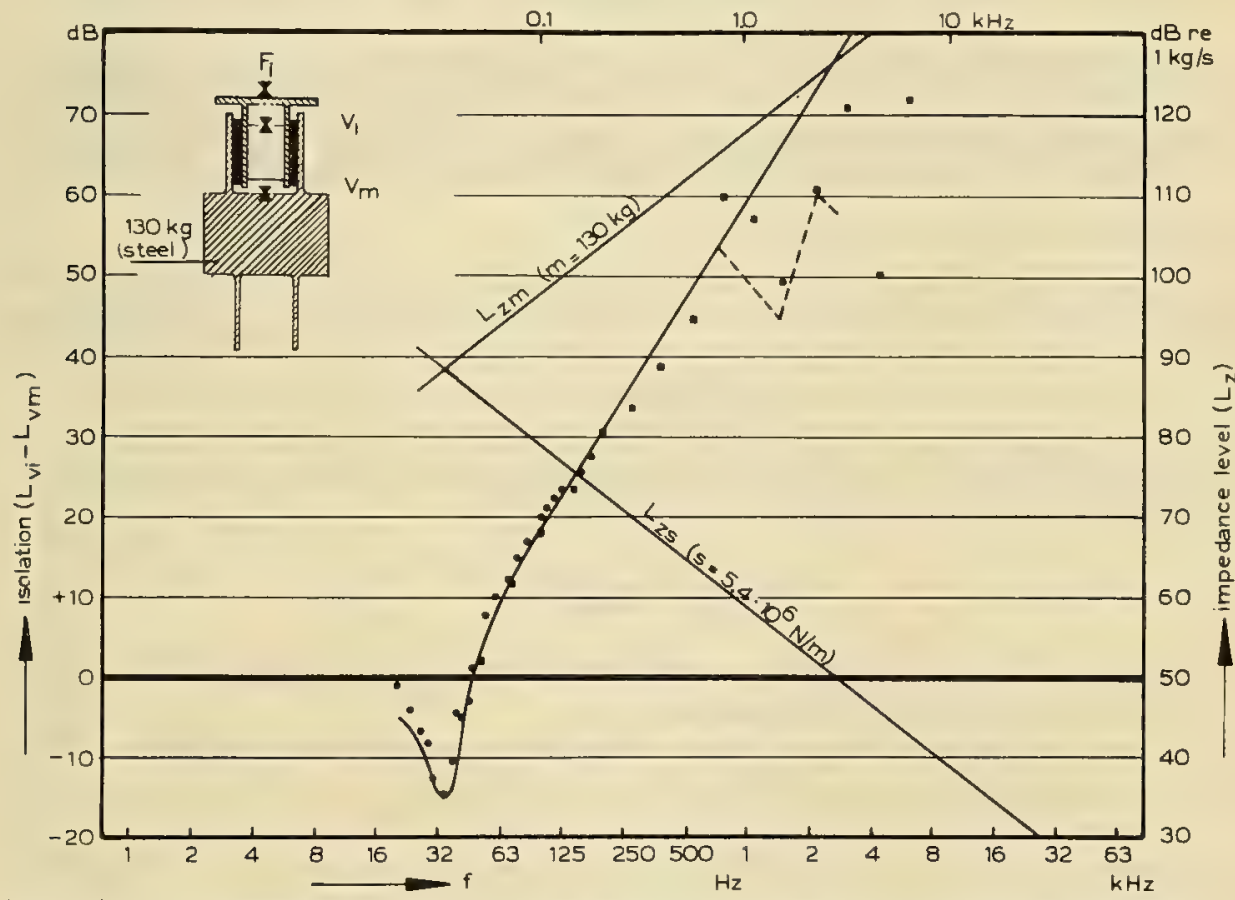

Fig. 17.3. While hanging on two battens, one side of a $400-\mathrm{kg}$ nominal load rubber mount was excited normally (cf. Fig. 17.2); the other side was connected as rigidly as possible to a $130-\mathrm{kg}$ steel mass, which was resiliently mounted as shown. Measured isolation figures for discrete frequency excitation (dots) and for half-octave-band noise excitation (squares) are shown in comparison with an approximate theoretical curve (solid line) including standing wave effects (dotted curve). Also shown are the computed impedance of mount and mass derived from their static stiffness and weight, respectively. 


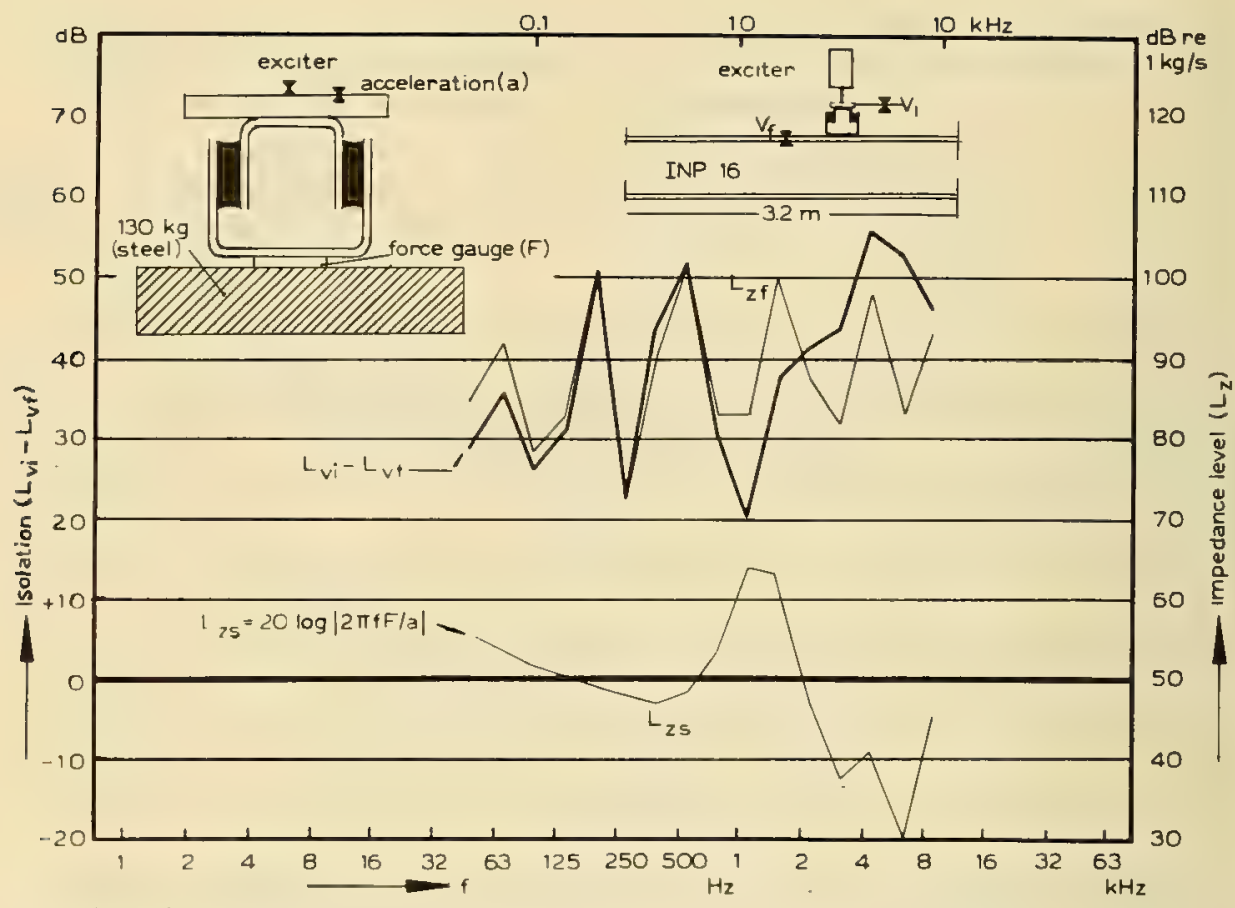

Fig. 17.4. The impedance level $L_{z s}$ of a spring was measured. (Metalastik double-U-shear mount $31 / 149 \mathrm{~B} / 7 ; 130-\mathrm{kg}$ "immobile" steel base, force gauge between mount and base, and "normal" excitation.) The impedance level $L_{z f}$ of a foundation was also measured (3.2-m I-NP-16-beam on rubber blocks). Moreover, the "normal" isolation $L_{v i}-L_{v f}$ was measuredfor the spring mounted on the beam (same point as for impedance measurement). The isolation is approximately equal to $L_{z f}-L_{z s}$ !

\subsection{BENDING WAVES IN STRUCTURES}

A simple model of what possibly happens when a mechanical structure is excited by alternating forces can be based upon the pure bending wave phenomenon in plates or in beams. This type of wave seems to be very important, moreover, for the radiation of sound by vibrating plates in direct contact with a fluid acoustic medium. A plane longitudinal wave in some such medium, e.g., water, can be described, as is well known, by a differential equation showing the balancing of inertia and elasticity forces; e.g.,

$$
\rho_{w} \frac{\partial^{2} u}{\partial t^{2}}-K_{w} \frac{\partial^{2} u}{\partial x}=0
$$

where $\rho_{w}$ is the fluid density, $u$ is the particle displacement in the $x$ direction, and $K_{w}$ is the fluid compression modulus.

A similar though largely different equation may be derived for the plane bending waves in plates or in beams [9]:

$$
\rho A \frac{\partial^{2} w}{\partial t^{2}}+E l \frac{\partial^{4} w}{\partial x^{4}}=0
$$

where $\rho$ is the density and $E$ the modulus of elasticity of the plate or beam material; $A$ is the area and $l$ is the axial moment of inertia typical of the cross section; and $w$ is the particle displacement in the $z$ direction, i.e., normal to the plate or beam. 


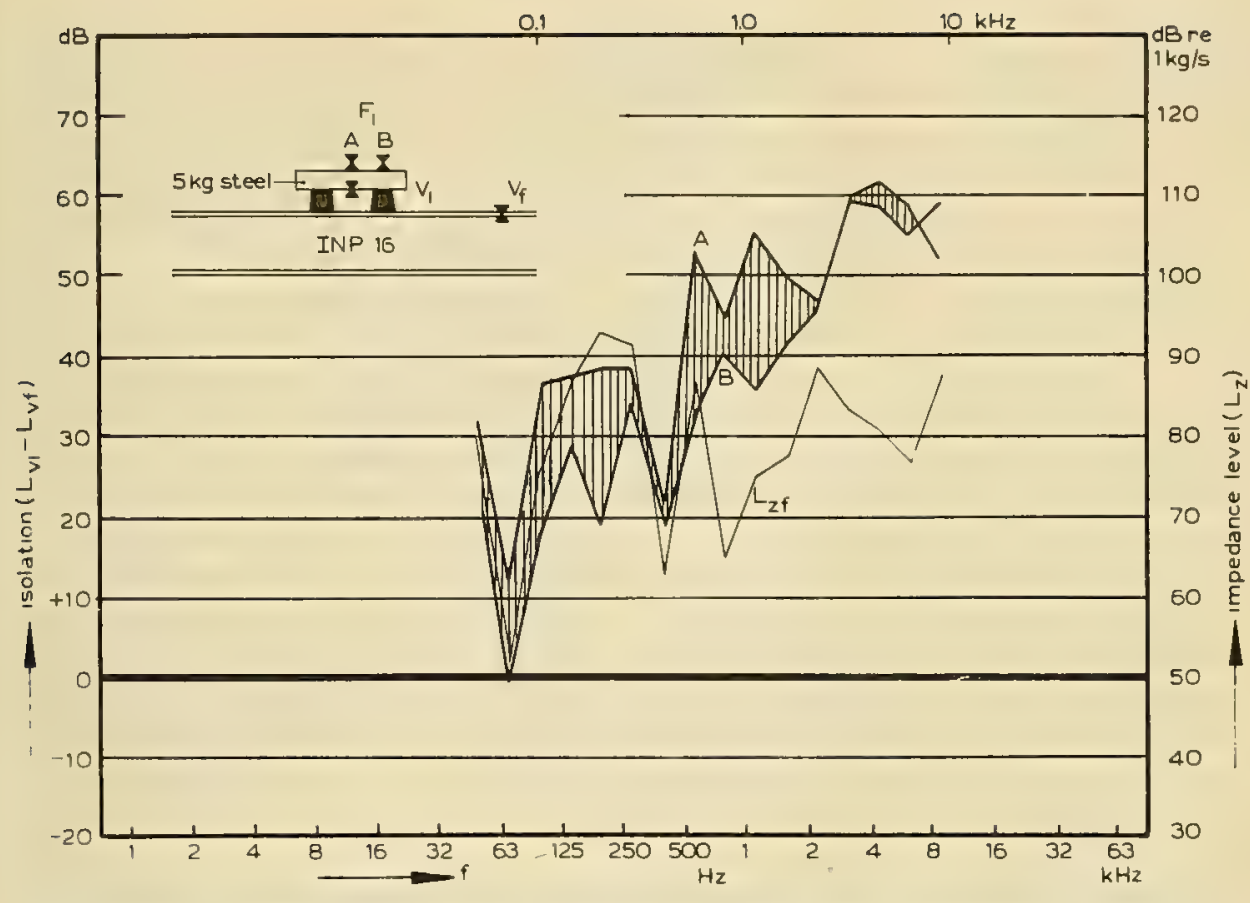

Fig. 17.5. For "normal" excitation, two isolation curves were determined: (A) exciter position in the middle of a steel bar resting on two rubber blocks on a 4-m I-NP-16-beam, and (B) exciter position above one of the rubber blocks. From the stiffness of the blocks $\left(\approx 2.5 \cdot 10^{4} \mathrm{~N} / \mathrm{m}\right.$ per block $)$ and the impedance level of the beam ( $L$, shown in figure), an approximate isolation curve may be computed which agrees fairly well with curve $A$. The exciter position clearly influences the experimental result; additional rotation of a spring contact plane presumably increases the velocity level $L_{v f}$ of the beam.

Sinusoidal plane waves may exist in the plate or beam. For the bending wave velocity $c_{B}$ one finds that

$$
c_{B}=\sqrt{2 \pi f c_{L} i_{y}}
$$

where $c_{L}=\sqrt{E / \rho}$, the longitudinal plane wave velocity in the material; and $i_{y}$ is the so-called radius of gyration, equal to $\sqrt{l / A}$. For plates, this radius of gyration is given by

$$
i_{y}=\frac{h}{2 \sqrt{3}}
$$

where $h$ is the plate thickness; for beams, $i_{y}$ may be found in tables or handbooks.

In Fig. 17.6 the bending wavelength $\lambda_{B}=c_{B} / t$ is shown as a function of the frequency $f$ and the plate thickness $h$ for three common building materials. The longitudinal wavelength for sinusoidal plane waves in air and in sea water is also shown. For the greater part of the audio-frequency range, $\lambda_{B}$ is smaller than $\lambda$ in sea water. This fact has an important bearing on the sound radiation by shell plates into water, and it can be understood in a qualitative way as follows. Suppose two loudspeakers are mounted close together in one baffle. They vibrate in opposite phase. If the distance between the speakers is less than the wavelength in the surrounding air, the sound radiation is very inefficient because of 


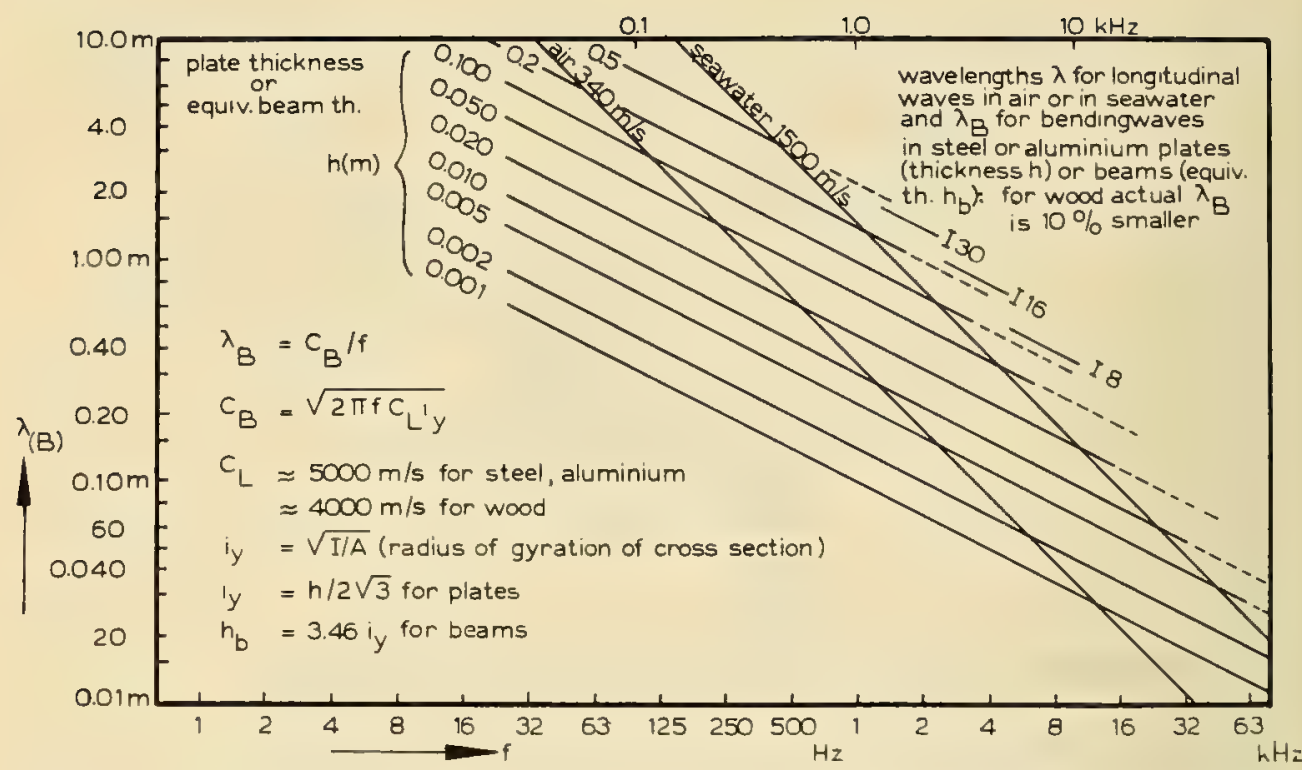

Fig. 17.6. Wavelengths for longitudinal waves in air or in sea water and for bending waves in plates of thickness $h_{i}$ some equivalent I-beam DIN-numbers are indicated. The lines for constant $h$ must not be extended to the right.

the "pumping-around" effect. A similar situation exists when the bending waves in a plate are of such a form that nearby parts of the plate vibrate in opposite directions within a distance of, say, half the wavelength associated with the sound radiated into the surrounding fluid medium. The frequency $f_{c r}$ for which $c_{B}=c_{w}$ is called the critical frequency; here $c_{w}$ is the velocity for longitudinal waves in the fluid. The critical frequency is given by

$$
t_{\mathrm{cr}}=\frac{c_{w}^{2}}{2 \pi c_{L} i_{y}}
$$

as may easily be derived. For frequencies $f>f_{\mathrm{cr}}$, the radiation is very efficient and shows pronounced directional effects. The frequency range discussed in this paper is such that $\lambda_{B}<2 \mathrm{~m}$, approximately, for the lower limit; and $t<f_{\mathrm{cr}}$ for the upper limit.

It is supposed that bending waves are easily excited in typical ship structures consisting of plates and beams. These waves are presumed primarily responsible for the radiation of sound. Thus it seems worthwhile to consider them in more detail, especially with respect to mechanical impedances and radiated sound power.

\subsection{MECHANICAL IMPEDANCE}

In describing the response of a mechanical structure exposed to alternating forces, it is almost impossible to avoid extremely simplified mathematical models of the physical reality. Thus, a ship will be considered as a composition of flat plates and straight beams in this paper. It may be hoped that conclusions drawn from theories for these simple configurations will, in broad outlines, also be valid for more complicated configurations. 
Moreover, only bending waves are tolerated in our model; i.e., it is supposed that Eq. (12) suffices to describe the wave phenomena in plates and beams. This is not necessarily a serious restriction for at least two reasons: (1) for very general mechanical structures similar statements can be made as those derived below) [10]; (2) results of measurements agree reasonably with the very simple theory.

Let us consider a specific example of a mechanical structure, a beam. It is well known that a beam can vibrate laterally at an infinite number of natural frequencies. For each frequency there is a definite shape in which the beam will deflect while vibrating harmonically; this shape is called a normal mode of vibration of the beam. Such a normal mode will be designated by $\hat{u}_{\nu}$, the sign $\wedge$ indicating the maximum value (amplitude) of the displacement of the beam at any point $x$, the instantaneous value $u$ varying sinusoidally with time $t ; \nu$ is the number of the mode.

The problem now is to describe the forced vibrations of this beam when excited by a general harmonic force. The directions of the force(s) and of the displacements are supposed to be parallel. In most instances, the force will be acting near a certain point A, e.g., a resilient mount; this point will be given some mathematical preference below. If $p$ is the force as a function of the coordinates on the surface $S$ of the beam, the total force $F$ is given by

$$
F=\int p d S=\hat{F} e^{j \omega t}
$$

The velocity of the beam caused at the point $C$ by the action of the force at $A$ can be written as the product of this force and the sum of an infinite series of "mechanical admittance" or mobility terms:

$$
v(C)=F \sum_{\nu} \frac{\hat{u}_{\nu}(C) / \hat{u}_{\nu}(A)}{q_{\nu}(A) \varphi_{\nu}(A) Z_{\nu}}
$$

The meaning of the various symbols is explained below. As is usual, the real part of the complex $v(C)$ is the measurable quantity, "velocity at C."

The numerator of Eq. (17) contains only the ratio of the values at $C$ and $A$ of the $v$ th natural mode. This ratio is equal to $\phi_{\nu}(C) / \phi_{\nu}(A)$, where $\phi_{\nu}$ is the characteristic function describing a natural mode. With the aid of tables of these functions $[11]$ this ratio can be determined.

In the denominator of Eq. (17), the so-called "quadratic amplitude transform factors" $q_{\nu}(A)$ are given by

$$
q_{\nu}(A)=\frac{\overline{\hat{u}_{\nu}^{2}}}{\overline{\widehat{\hat{u}}_{\nu}^{2}}(A)}
$$

This name suggests the same action so well known from electric transformers. These impedances are transformed in the ratio 1 to $n^{2}$, while currents or voltages are transformed in the ratio 1 to $n$. In $q_{\nu}(A)$, a more general seesaw action is described: the mechanical impedance felt by an exciting force at one end of a seesaw due to a load at the other end is proportional to the square of the ratio of the distance load-pivot to the distance force-pivot. The bar over $\hat{u}_{\nu}^{2}$ indicates the mean value over the homogeneous beam of $\hat{u}$ squared. In [11], the characteristic functions are normalized in such a way that 


$$
q_{\nu}(A)=\frac{\widehat{\hat{u}_{\nu}^{2}}}{\hat{\mathrm{u}}_{\nu}^{2}(A)}=\frac{1}{\phi_{\nu}^{2}(A)}
$$

thus allowing a quick determination of $q_{\nu}(A)$.

The factor $\varphi_{\nu}(A)$ in the denominator of Eq. (17) may be called a force distribution factor. It is equal to 1 for a point force applied in A. For a pressure distribution, it is given by

$$
\varphi_{\nu}(A)=\frac{\hat{u}_{\nu}(A) \hat{F}}{\int \hat{p} \hat{u}_{\nu} d S}
$$

where $F$ is the amplitude of the total force [cf. Eq. (16)]. For the ratio $\hat{u}_{\nu} / \hat{u}_{\nu}(A)$ under the integral sign, $\phi_{\nu} / \phi_{\nu}(A)$ may be substituted (cf. [11]).

The last term in the denominator of (17) is the most illustrative. It is given by

$$
Z_{\nu}=j \omega M+R_{\nu}+\frac{\omega_{\nu}^{2} M}{j \omega}=R_{\nu}\left(1+j v_{\nu} Q_{\nu}\right)
$$

the well-known expression for the complex impedance of a series circuit, where

$$
\begin{gathered}
R_{\nu}=2 \pi \eta f_{\nu}^{2} \frac{M}{f} \\
Q_{\nu}=\frac{f}{\eta f_{\nu}} \\
v_{\nu}=\frac{f}{f_{\nu}}-\frac{f_{\nu}}{f}
\end{gathered}
$$

and $\eta$ is the loss factor, $f_{\nu}$ is the $v$ th natural frequency, $f$ is the excitation frequency $(\omega / 2 \pi)$, and $Y$ is the total mass of the beam. Eventually, Eq. (17) turns out to be a complicated expression for what may be called a "parallel connection

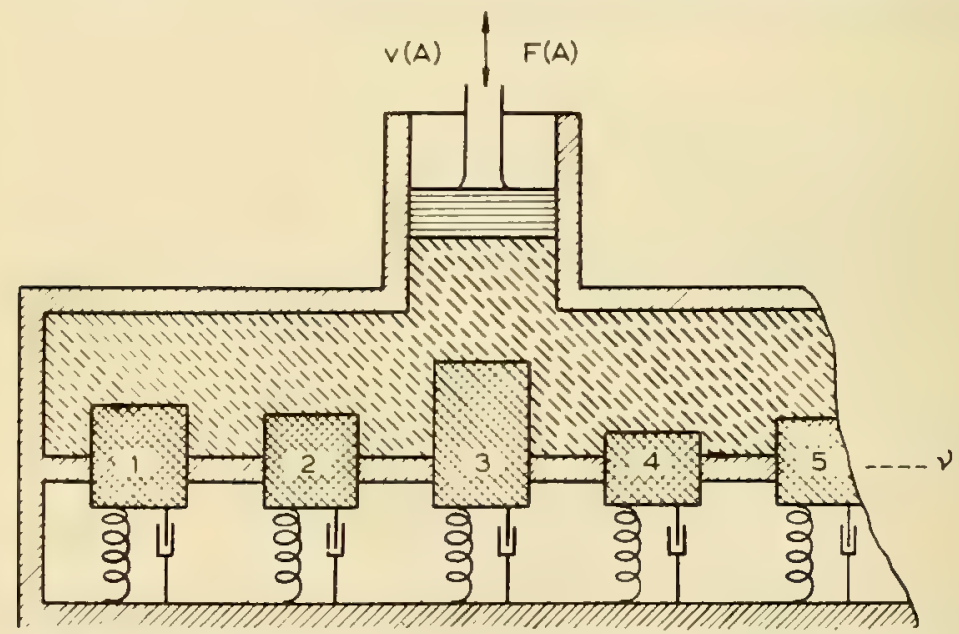

Fig. 17.7. Hydraulic analog of a mechanical structure (e.g., beam or plate) excited by a sinusoidal point force $F(A)$. The velocity $v(A)$ at $A$ depends upon the response of infinitely many "resonators" (mass on spring and mechanical resistance) numbered $1,2,3, \ldots \infty$. An electrical analog would be a parallel connection of series circuits. The values of the circuit components are given in Eqs. (17) through (24). 
of series circuits." The natural frequencies of the beam correspond to the "resonant frequencies" of the series circuits. A hydraulic analog for a pointforce excitation is shown in Fig. 17.7. The picture of "parallel connection of series circuits" for a beam seems to have a general validity for other mechanical structures as well. It agrees very well with experimentally observed resonance curves of mechanical structures which closely resemble those of the simple classic RLC series circuit.

The behavior of these circuits is well known, and the noise impedance $Z_{\mathrm{av}}$, if measured with half-octave band filters, is given by

$$
Z_{\mathrm{av}}=\frac{R_{\nu} \sqrt{Q_{\nu}}}{2.12}
$$

where the symbols of Eqs. (7), (22), and (23) are used. Of course, Eq. (25) can be only approximately valid for a mechanical structure and only for one resonant frequency within the frequency band of the exciting force noise. Assuming the various natural frequencies within a given frequency band to be sufficiently separated, it is possible to derive an expression for the noise impedance of a plate or a beam as soon as the total number of the "peaks" in that band is known.

For simply supported beams or plates, the noise irmpedances averaged over the beam or plate turn out to be given by

$$
\widetilde{Z^{2}} \approx 6.5 \eta t \frac{M^{2} c_{L} h}{S}
$$

for point excitation of a rectangular plate of thickness $h$, width $b$, and area $S=l b ;$ and,

$$
\overrightarrow{Z_{j}^{2}} \approx 23 \eta f^{3 / 2} \frac{M^{2} b \sqrt{c_{L} h}}{S}
$$

for line excitation along the width $b$ of a plate; and,

$$
Z^{2} \approx 42 \eta f^{3 / 2} \frac{M^{2} \sqrt{c_{L} i_{y}}}{l}
$$

for point excitation of a beam (length $l$ and radius of gyration $i_{y}$ ). In Fig. 17.8 the reasonable agreement between a measured and the corresponding computed impedance from Eq. (26) is shown. Especially interesting is the deviation from the theoretical curve for frequencies $f>f_{\mathrm{cr}}$. Presumably it is due to the increased load caused by the efficient radiation of sound into the surrounding air; the order of magnitude for this effect is correct.

A closer examination of the results given above raises at least two questions respecting their applicability to underwater noise control. Up to now, theory has introduced only structural data, but no coupling of the structure with a fluid medium. The first question, therefore, concerns the extra load due to water when in direct contact with a vibrating plate. The second has to do with the excitation. It was assumed that the exciting force acted in a direction parallel to the resulting velocity of the points on the beam. In principle, it must be possible to handle situations like that shown in Fig. 17.5 with the aid of the method given. However, it is too simple a supposition that a diesel engine vibrates in a "normal" direction only. It is equally probable that its contact planes with springs move "parallel" to the beam, or otherwise, of course. 


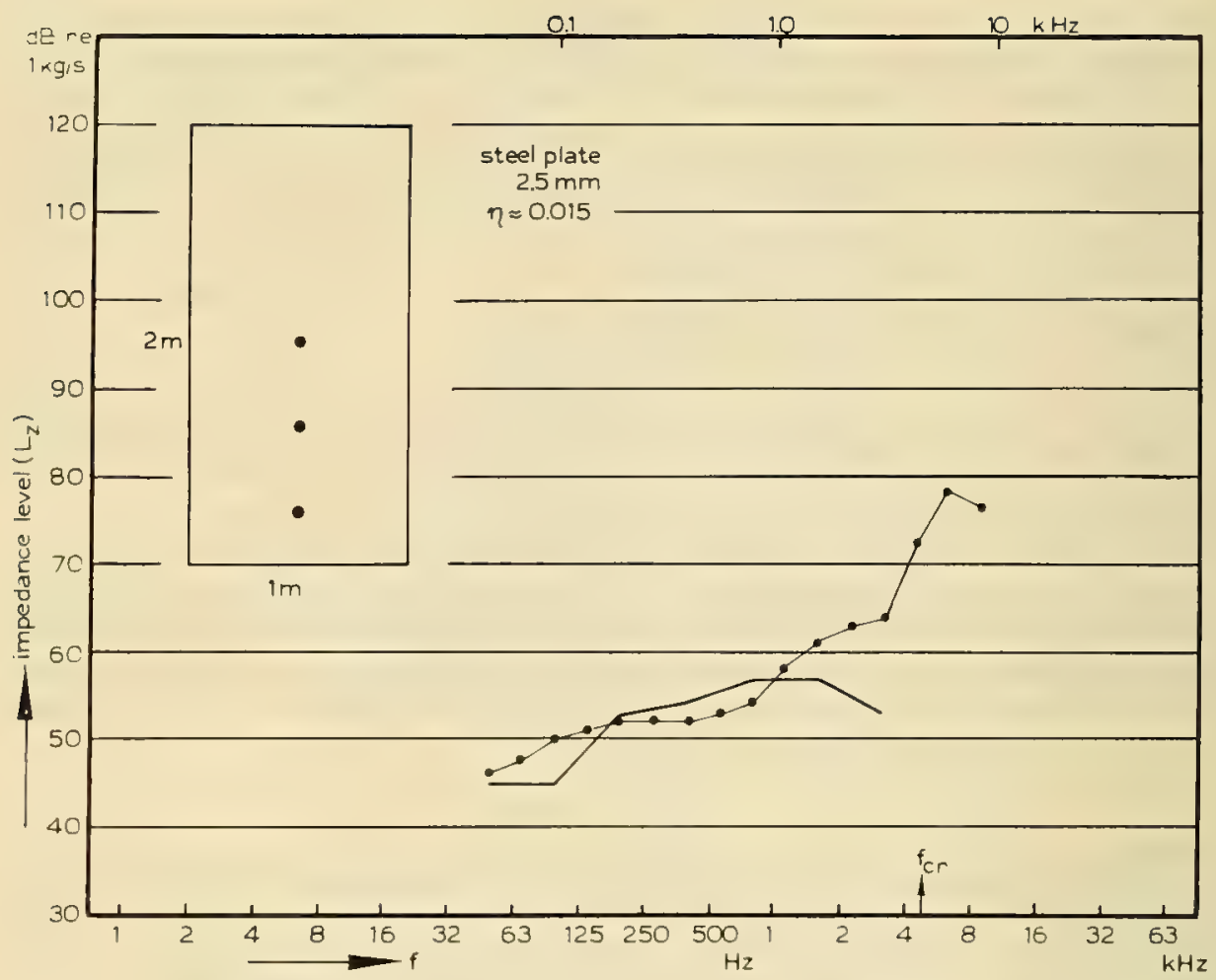

Fig. 17.8. Point-impedance level as measured (average of three points as shown; half-octave-band noise) on a steel plate boited to a steel doorframe in wall of reverberant room $\left(156.3 \mathrm{~m}^{3}\right.$. empty; reverberation time approximately two sec). The measured values are connected. For comparison with theory, a curve is shown (heavy line), computed according to Eq. (26) and using measured $\eta$-values (derived from reverberation times of plate; $\left.\eta=2.2 / T f_{\nu}\right)$.

A solution to the first problem can be estimated as long as $f<f_{\mathrm{cr}}$. For this frequency range, it may be assumed that the water presents a mass load only because of inefficient radiation. The magnitude of the extra vibrating mass may be assessed by taking the thickness of the vibrating water layer near the plate as $\lambda_{B} / 4$. Thence, the total mass of the plate plus water becomes approximately equal to

$$
M\left(1+\frac{\rho_{\mathrm{p}} \lambda_{B}}{4 \rho h}\right)
$$

which means a considerable increase of the impedance compared with the plate in vacuo, a $10 \%$ to $20 \%$ decrease of $\lambda_{B}$, and a factor 0.6 to 0.8 for the resonant frequencies of metal structures (e.g., ship propellers).

The second problem is very complicated as long as the source of structureborne noise is in "rigid" contact with the mechanical structure (e.g., the foundation). Figure 17.9 shows how a tendency might be estimated if the source is resiliently mounted. Again the model chosen to represent a practical situation is very simple. It is supposed that the contact plane at the source side of a spring is vibrating in two directions only, viz., causing a "normal" excitation and a 

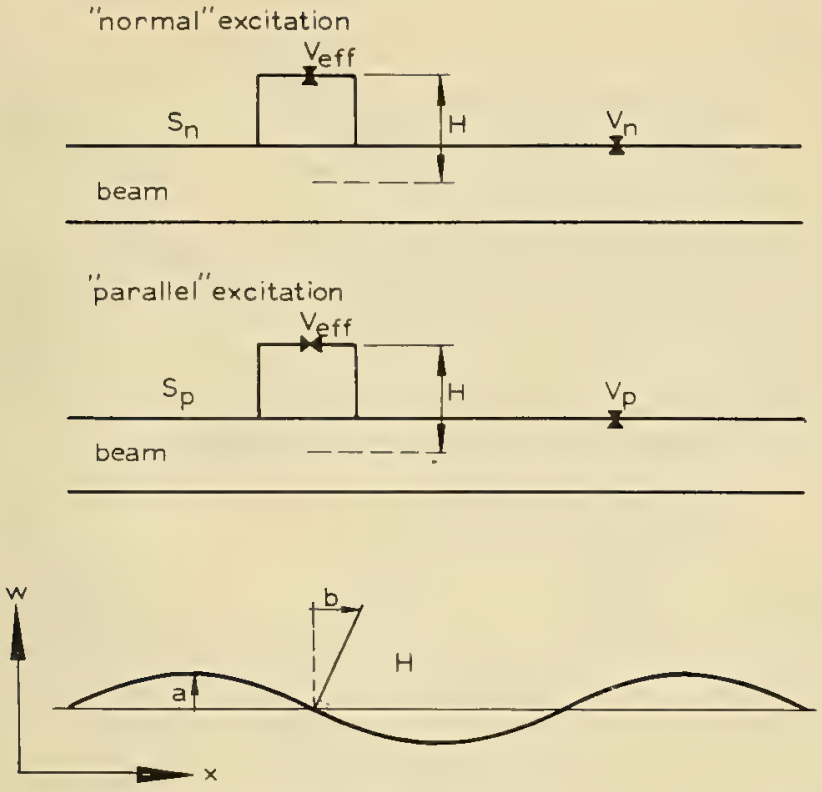

Fig. 17.9. "Normal" excitation (i.e., direction of force parallel to direction of particle displacement of beam) and "parallel" excitation (i.e., a bending moment is exerted on beam) are compared. It is supposed that a resilient mount is inserted between a beam and a source of structureborne sound. The sourceside contact plane moves in a "normal" or in a "parallel" direction. The height of this plane above the neutral layer of the beam is $H$. If the spring is mounted at a velocity antinode, (a) the "normal" excitation causes a velocity $v_{n}$ somewhere on the beam; or, if it is mounted at a node, (b) the "parallel" excitation causes $v_{p}$.

"parallel" excitation of the beam. Two cases are compared. The first is "normal" excitation acting at a velocity antinode; the resulting velocity at an arbitrary point of the beam is $v_{n}$. The second case shows the same spring at a velocity node, the excitation being "parallel." Again, the velocity $v_{p}$ at an arbitrary point is observed.

If the height of the upper contact plane of the spring above the "neutral layer" of the beam is $H$ and the stiffnesses are called $S_{n}$ and $S_{p}$, then the ratio $v_{n} / v_{p}$ is given by

$$
\frac{v_{\pi}}{v_{p}}=\frac{S_{n} \lambda_{B}}{2 \pi S_{p} H}
$$

For an I-beam it must be expected that considerably shorter bending wavelengths may exist in the upper and lower flanges separately, than in the longitudinal direction of the beam as a whole. It seems wise, therefore, to assume that $\lambda_{B}$ in this case equals approximately the value it would have if the flanges were free plates of the same thickness. The order of magnitude involved is $\lambda_{B} \approx 0.5 \mathrm{~m}$ and $H \approx 0.25 \mathrm{~m}$. The values of the stiffnesses $S_{n}$ and $S_{p}$ are approximately equal in magnitude. The extreme requirements of many practical applications for resilient mounts necessitate a higher degree of isolation for normal excitation alone than is often available. A value for $v_{n} / v_{p}$ in Eq. (29) smaller than 1 therefore cannot be tolerated.

If a foundation is constructed from steel profiles, the resilient mounts should be of such design as to render $S_{p}$ sufficiently less than $S_{n}$, unless very special precautions are taken. The same does not apply for wooden beams, because in general the bending wavelengths will be greater than for comparable steel structures. After the noise has reached the foundation, no appreciable attenuation during the propagation through the hull is generally found. 


\subsection{RADIATION OF SOUND}

In following the sound path of the present noise control problem we arrive at the vibrating shell plates. There are indications that only relatively small portions of the shell actually radiate sound into the surrounding water. The simplification, "flat plate with bending waves," may therefore represent a not too unrealistic approach.

We borrow at once two formulas for the radiated sound power from a paper by Heckl [12]. They were derived for radiation into a rectangular duct. This means that the radiating plate is slightly better loaded than a comparable plate radiating into an infinite half-space. This effect probably implies a factor of 2 in power. For the present purpose this order of magnitude may be neglected. The formula for a rectangular plate excited by a point force $F_{\text {eff }}$ reads

$$
P_{.} \approx F_{\text {eff }}^{2} \rho_{w} S^{2}\left(\frac{1+0.8 \lambda_{B}^{2} / \eta S}{2 \pi M^{2} c_{w}}\right)
$$

where $S$ is the area of the plate radiating into water (of density $\rho_{w}$ and sound velocity $c_{w}$ ), $\lambda_{B}$ is the bending wavelength, and $\eta$ is the loss factor of the plate. It is supposed that $t<t_{\mathrm{cr}}$. Moreover, it must be supposed that the mass $M$ of the plate is corrected to include the additional water load as mentioned before.

The same remarks apply to the formula for a plate excited along a line of length $b$ (the width of the plate); the power is given by

$$
P_{/} \approx F_{\text {eff }}^{2} \rho_{w} S^{2} \frac{1+\lambda_{B} b / \pi \eta S}{4 \pi M^{2} b f}
$$

In both instances the radiated power consists of two additive components, one due to the over-all vibrations of the plate-the corresponding term contains $\eta$ the other due to the deformation near the excitation point or line. Nearly always, 7) will be so small that the latter contribution to the total power may be neglected. It follows that the radiated power may be reduced by increasing the loss factor if the exciting force is kept constant.

Inserting the expressions for the point or line impedances from Eqs. (26) and (27), one finds for the radiated power as a function of the velocity $v_{\text {eff }}$ of the excitation point or line that

$$
P_{.} \approx 0.45 v_{\text {eff }}^{2} \rho_{w} \mathrm{c}_{w} \lambda_{c r}^{2}
$$

and

$$
P_{/} \approx 0.43 v_{\text {eff }}^{2} \rho_{w} c_{w} b \lambda_{c r}
$$

where $\lambda_{\mathrm{cr}}$ equals the wavelength in the plate at the critical frequency $f_{\mathrm{cr}}$ (the formulas given are derived for $\left.t<f_{\mathrm{cr}}\right)$. For constant-velocity excitation, clearly, radiated noise reduction cannot be achieved by increasing the loss factor $\eta$ ?

It is now worthwhile to discuss these formulas. The results of some laboratory measurements (in air) are shown in Figs. 17.10 and 17.11. In Fig. 17.10 the radiated sound power for half-octave-band noise excitation with constant force as measured in a reverberant room is compared with the theory of Eq. (30). The agreement is satisfactory. In Fig. 17.11 the agreement of the experimental data with the theory of Eq. (32) is not so good. For a velocity of the excitation point of $1 \mathrm{~m} / \mathrm{sec}$, Eq. (32) indicates a power level of $120 \mathrm{db}(\hat{\triangleq} 1 \mathrm{w})$, 


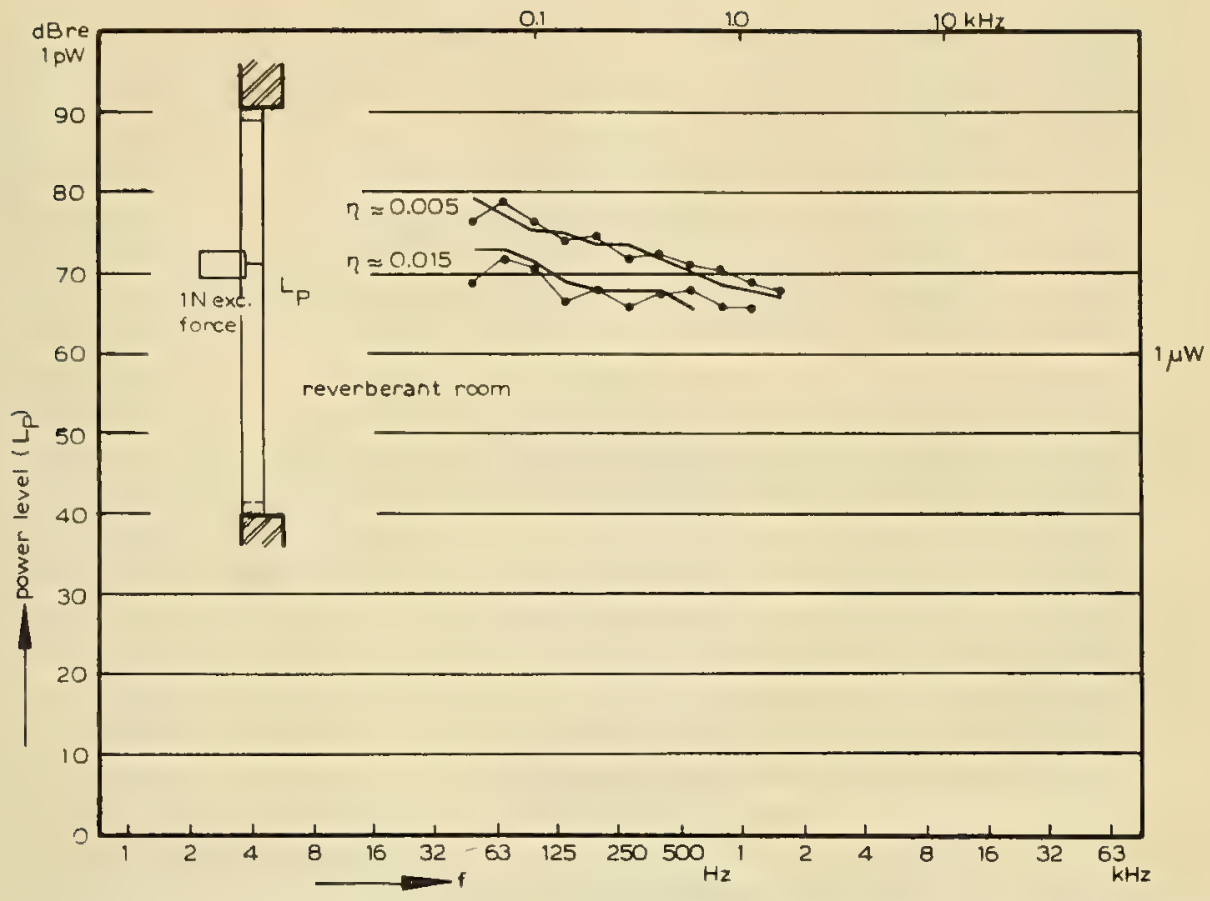

Fig. 17.10. The same plate used for Fig. 17.8 was excited by half-octave bands of noise. The airborne sound power (level $L_{P}$ ) radiated into the reverberant room was measured for two values of the loss factor $\eta$ of the plate, while the effective value of the exciting force was 1 newton. A factor of 3 increase in $\eta$ (obtained by an additional damping layer with negligible mass) corresponds to a decrease in $L_{P}$ of $5 \mathrm{db}$, approximately. Theory-heavy line, based on Eq. (30); actually measured $\eta$-values are inserted-agrees very well with experiment.

independent of frequency. The discrepancy is not understood; probably the plate was too small. It is clear, however, that increasing $\eta$ does not reduce appreciably the radiated power, contrary to constant force excitation.

In practice it is sometimes not quite clear which path is followed by the noise originating in a known source. It is, then, very useful to have at one's disposal a relation between the average velocity level of a radiating plate and the sound pressure level at a certain distance. It might be derived from Eq. (32) and particularly Eq. (33) as soon as the relation between the "average" velocity over the plate and the excitation-point velocity were known.

It turns out that the factor 0.45 in Eq. (32) becomes 1.0 and the factor 0.43 in Eq. (33) becomes 0.64 if $v_{\text {eff }}^{2}$ equals the value of the time-effective velocity squared and averaged over the plate. Formulas were given for the power radiated by a plate excited by a point force or by a line force. In general, for all practical purposes, for the same average $v_{\text {eff }}^{2}$ over the plate, line excitation radiates more sound power than point excitation. It would be interesting to know if area excitation forms an even more efficient method to radiate sound; area excitation occurs, for example, when the shell is excited by air-borne noise. In this respect Fig. 17.12 is interesting. Area excitation and point excitation are compared for the same value of $v_{\text {eff }}^{2}$ averaged. It is outside the scope of this paper to treat further the radiation of sound. 


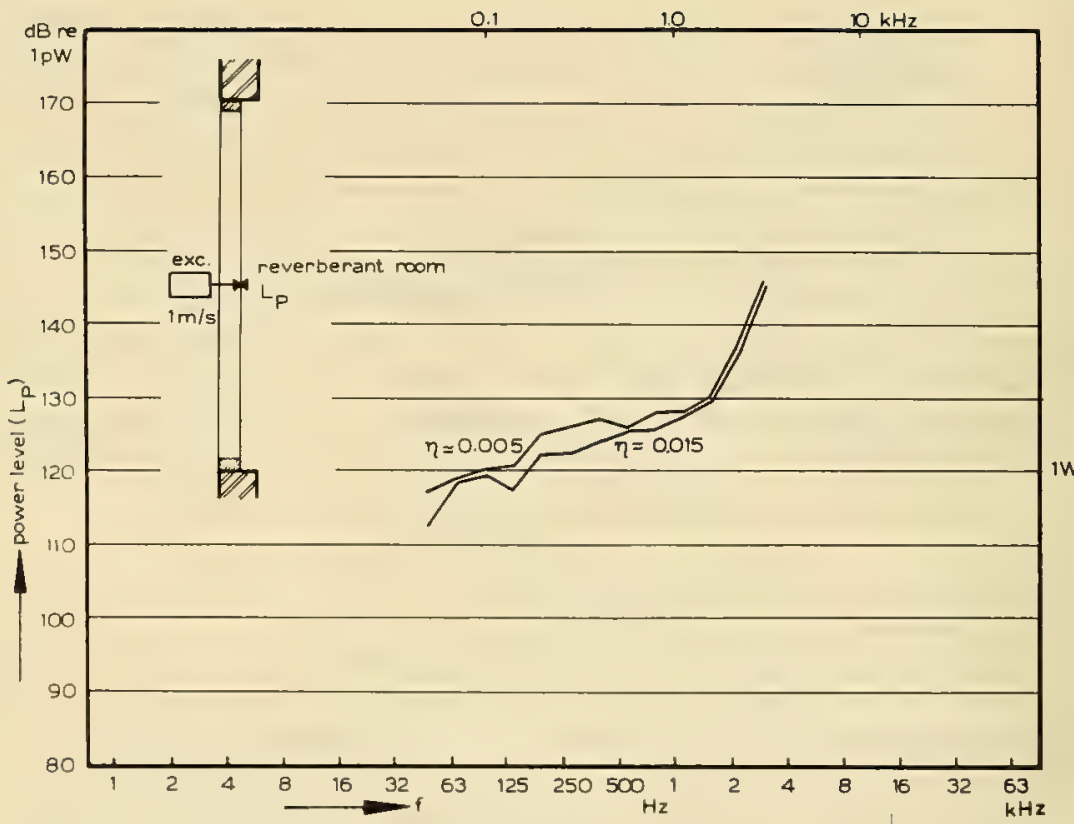

Fig. 17.11. The power levels $L_{P}$ due to the plate in $\mathrm{Fg} .17 .8$ were measured; however, the velocity of the point of excitation was $1 \mathrm{~m} / \mathrm{sec}$ for two values of the loss factor. For constant velocity excitation, damping layers do not reduce the radiated sound power appreciably (cf. Fig. 17.10).

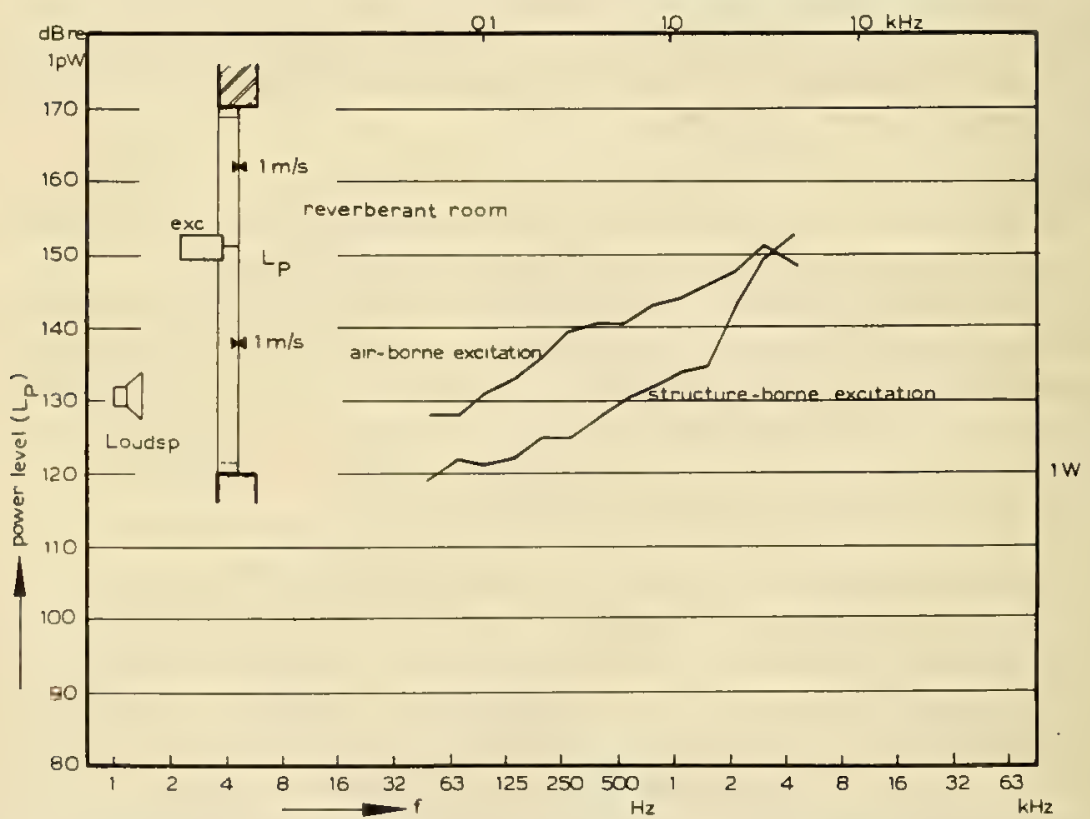

Fig. 17.12, Again the power levels $L_{P}$ due to the plate in Fig. 17.8 were measured. The velocity level averaged over various points on the plate was $1 \mathrm{~m} / \mathrm{sec}$ for point excitation and for area excitation (loudspeaker at $2 \mathrm{~m}$ from plate). 
Of course, many questions remain. Obviously, one of the first questions is whether the Eq. (32) or the Eq. (33) "model" is representative for a given underwater noise problem; also, how large is $b$, the width of the radiating part of the shell when excited by machinery vibrations? This raises another question, viz., can a shell with frames be considered as an orthotropic plate; or what is the direction of $b$ ? Although answers cannot be given in this paper, some experiments seem to indicate that the approach to the underwater noise reduction problem as presented here offers rather good qualitative and sometimes even quantitative insight.

\subsection{CONCLUSIONS}

It may be concluded that for the audio - frequency range, filtered noise measurements offer an efficient method for investigating the general response of a mechanical structure to vibratory excitation.

The isolation of structure-borne noise by resilient mounts is understood quantitatively as long as the "normal" excitation model is representative for a practical situation. For simple configurations like beams or plates, computation of direct or transfer impedances is accurately possible; results agree with measurements. Qualitatively, it is understood how the "parallel" stiffness of a spring may spoil the "normal" isolation for practical resilient mounts. Flanges of I-beams near springs should be stiffened.

The idealized point-force excitation model must be expected to fail if bending moments can be transmitted via a contact plane; assessment of improvement obtained by inserting a resilient mount between a source of structure-borne sound and a foundation is therefore very difficult.

For frequencies $f<f_{\mathrm{cr}}$, the simple radiation theory of flat vibrating plates with bending waves qualitatively describes experimental facts. It may be concluded that increasing the loss factor $\eta$ of the shell plates by applying damping layers is useless if the machinery is mounted rigidly, but it may reduce the radiated underwater noise considerably if resilient mounts are inserted (constant velocity vs constant-force excitation).

\section{CONVERSION FACTORS*}

\begin{tabular}{|c|c|c|c|}
\hline Length & $\begin{array}{l}1 \mathrm{in} . \\
1 \mathrm{~m}\end{array}$ & $\begin{array}{l}\hat{=} \\
\hat{=}\end{array}$ & $\begin{array}{l}0.0254 \mathrm{~m} \\
39.37 \mathrm{in} .\end{array}$ \\
\hline Force & $\begin{array}{r}1 \mathrm{lb}_{\mathrm{f}} \\
1 \mathrm{~N}\end{array}$ & $\hat{=}$ & $\begin{array}{l}4.448 \mathrm{~N} \\
0.223 \mathrm{lb}_{\mathrm{f}}\end{array}$ \\
\hline Pressure & $\begin{array}{l}1 \mathrm{~d} / \mathrm{cm}^{2} \\
\text { X db re } 20 \mu \mathrm{N} / \mathrm{m}^{2} \\
\text { Y db re } 1 \mathrm{~d} / \mathrm{cm}^{2}\end{array}$ & $\begin{array}{l}\hat{\equiv} \\
\hat{\bar{\equiv}} \\
\hat{=}\end{array}$ & $\begin{array}{l}0.1 \mathrm{~N} / \mathrm{m}^{2} \\
\mathrm{X}-74 \mathrm{db} \text { re } 1 \mathrm{~d} / \mathrm{cm}^{2} \\
\mathrm{Y}+74 \mathrm{db} \text { re } 20 \mu \mathrm{N} / \mathrm{m}^{2}\end{array}$ \\
\hline Impedance & $\frac{11 \mathrm{~b}_{\mathrm{f}}}{\mathrm{in} \cdot / \mathrm{sec}}$ & $\hat{=}$ & $175 \mathrm{~kg} / \mathrm{sec}$ \\
\hline $\begin{array}{l}* N=\text { newton. } \\
d=\text { dyne }\end{array}$ & $\frac{1 \mathrm{lb}_{\mathrm{f}}}{\mathrm{in} \cdot \mathrm{sec}}$ & $\hat{\approx}$ & $45 \mathrm{db}$ re $1 \mathrm{~kg} / \mathrm{sec}$ \\
\hline
\end{tabular}




\section{REFERENCES}

1. V.M. Albers, Underwater Acoustics Handbook, p. 208 (The Pennsylvania State Univ. Press, University Park, Pennsylvania, 1960).

2. R.W. Young, "Reference Sound Pressure for Navy Noise Measurements," Tech. Memo. No. T. M. 392, U.S. Navy Electronics Laboratory, San Diego 52, California (March 15, 1960).

3. "Expression of the Physical and Subjective Magnitudes of Sound or Noise," Intern. Organization for Standardization, Recommendation, ISO/R 131-1959 (October 30, 1959)

4. C. W. Kosten, "Over de Elastische Eigenschappen van Gevulcaniseerde Rubber, " Diss., Delft, 77 (1942).

5. M. L. Exner, "Schalldämmung durch Gummi und Stahlfedern," Acustica, Vol, 2, 213-221 (1952).

6. A.O. Sykes, "A Study of Compression Noise Isolation Mounts Constructed from Cylindrical Samples of Various Natural and Synthetic Rubber Materials," T.M.B. Report 845, Navy Department, U.S.A. (1953).

7. J.H. Janssen, "Toepassingen van Trillingsvrije Opstellingen voor Geraasbestrijding," De Ingenieur, Werktuig-en Scheepsbouw, 7, Fig. 9 (No. 13, 1959).

8. J.H. Janssen, "Einige Erfahrungen bei der Körperschallisolation Mittels Federn," Third International Congress on Acoustics, Stuttgart, 1175-1178 (1959)

9. L. Cremer, "The Propagation of Structure-Borne Sound," Department of Scientific and Industrial Research, U.K., Sponsored Research (Germany), Report No. 1, Series B.

10. W. Pechhold, "Zur Behandlung von Anregungs- und Störungsproblemen bei Akustischen Resonatoren," Acustica, Vol. 9, 48-56 (1959).

11. D. Young and R.P. Felgar, "Characteristic Functions Representing Modes of Vibration of a Beam," The Univ. of Texas, Publication No. 4913 (1949).

12. M. Heckl, "Schallabstrahlung von Platten bei Punktförmiger Anregung," Acustica, Vol. 9, $371-380$ (1959). 


\title{
LECTURE 18
}

\section{SIGNAL PROCESSING OF UNDERWATER ACOUSTIC FIELDS}

\author{
P. L. Stocklin \\ Office of Naval Research \\ Washington, D.C. \\ U.S.A.
}

\subsection{INTRODUCTION}

Within the Acoustics Branch of the Office of Naval Research, we have a program of applied research in underwater acoustic signal processing. Since a thorough treatment would require far more space than is at my disposal, I will attempt first to summarize the rationale behind this program, and then to treat in detail one research area which may be both novel and of interest to you, that of space-time decision-theory applications in acoustic signal processing.

\subsection{RATIONALE}

Signal processing is defined as the art of using physical information to make decisions. The basic physical fact of life around which modern signal-processing research is being built is the instantaneous acoustic field, in contrast to the basis of power averages of the field which has led in the past to such notions as power-pattern formation and correlation analysis. With a detailed knowledge of the acoustic field for situations of interest, we can make intelligent surmises about processor design and behavior and formulate from these a meaningful program. This detailed knowledge may in itself be only statistical; but the point is that, precisely to the extent we can describe the field in time and space, to that extent we can design an efficient processing system. The field description and the design of an efficient processor are in fact one and the same.

A useful starting point for this discussion is the well-known sonar equation for passive detection:

$$
\left(\frac{S}{N}\right)_{S D}=\left(L_{s}-N_{w}\right)-\left(L_{n}-N_{D I}\right)
$$

where

$$
\begin{aligned}
\left(\frac{S}{N}\right)_{S D} & =\text { signal differential } \\
L_{s} & =\text { radiated source level, db } / 1 \mu \mathrm{b} / 1 \text { yard } \\
N_{\mathrm{m}} & =\text { propagation loss, db } \\
L_{n} & =\text { ambient noise level (local), } \mathrm{db} / 1 \mu \mathrm{b} \\
N_{D r} & =\text { directivity index, db }
\end{aligned}
$$


Let us consider the sonar equation as a possible starting point or gauge for research in signal processing. Now, the central point concerning the sonar equation is that it is a power signal-to-noise relationship. It epitomizes what may be called the "signal-to-noise" approach to system performance in general and to signal processing in particular. In such a relationship, propagation phenomena and signal processing are linked only in terms of power and intensity loss. Several comments may be made concerning this signal-to-noise approach.

Since power is the parameter used, the processing techniques implied in this approach are those directly related to or of a lower order than power. Speaking statistically, for most cases of interest power is a second central moment of the basic physical data. Power can be used to measure directly two averages of the second-order joint probability density function of the basic physical data: the power spectrum and the cross-or autocorrelation function. Thus we find that such average processing can readily be incorporated into the sonar equation. Whether other processing principles bear a relation to power simple enough to permit such incorporation is another question.

A second comment is that variability and fluctuations due to the medium and other acoustic elements of the problem are taken into account only on an ad hoc basis. For many situations of interest, both waveform and wavefront variability and fluctuation may reasonably be expected to be of the same order of magnitude as, or much greater than, the deterministic elements of the problem, and so must certainly enter our thinking other than as an error estimate or a first-order correction.

Closely related to this point is the fact that since there are a number of single terms, each connected with a specific item of acoustic generation or propagation or processing in the sonar equation, there is no explicit treatment of interactions. Interaction between different sources of interference, between our instrumentation and the acoustic field, and generally between the acoustic and processing elements of the problem, cannot be treated on other than an ad hoc basis. In addition, the separability implied by these single terms leads directly to a point of view in which we assume we can take propagation loss from one set of measurements, source level from another, and so forth. In fact, however, the question of just what interactions are involved, whether they are negligible, and what degree of separability is possible and useful must be considered carefully from the very start of a processing study.

As to research in signal processing, however, the most serious comment I would like to present is that the signal-to-noise approach, as embodied in the sonar equation, does not guide us very effectively in improving our processing. What Eq. (1) tells us, for example, is that to improve the signal differential, and hence our decision-making ability, we must look for a noisier target, or a stretch of ocean with less transmission loss, or build a bigger array, or find an area with lower noise background, or somehow find a processor which gives us the same decision-making ability with a lower signal differential, but such comments are inevitably phrased in terms of what we have done in processing design-in the signal-to-noise approach, the interesting and heuristic physics has already been smoothed over. 
In light of these facts, we in ONR are seeking a fundamentally different approach, one which builds upon the sonar equation, but which, in addition, possesses those characteristics found lacking and leads us directly to answer basic questions of what processing we can do, how we can do it, and what its basic limitations are in terms of the physical acoustic situations with which we must deal. Let us recapitulate the shortcomings of the signal-to-noise approach discussed earlier by way of listing requirements for our new approach. We want, then, not to be restricted to power processing; we want to include, directly, variation, fluctuation, and interaction in our approach; and, most important, we want to have an effective guide to sustained improvement in our processing design. Finally, we want a direct relation between the acoustic field in its realistic complexity and the answers concerning processing limitations and processor design.

\subsection{SPACE-TIME DECISION THEORY APPLICATIONS}

\subsubsection{Introduction}

Space-time decision theory involves a direct attack upon the instantaneous acoustic field using decision theoretical methods. Here the point of view is that we have available for a given amount of time $T$ a local volume of acoustic field which we must use to make decisions concerning some event or series of events having possibly occurred at some distance from the local volume. To get information from the acoustic field, we sample it in space and time, then process these samples, ultimately making a decision. The basic ingredients for processor design, then, are: first, the description of our state of knowledge of the acoustic field in the local volume available to us; and second, the set of decisions, one of which we must make. In this view, any description of an acoustic event, however distant, must be in terms of the acoustic fields produced in the local volume.

Now, for many situations of interest, it is abundantly clear from experience at sea and from model studies, such as the excellent experiments described by Dr. A. B. Wood in Lecture 10, that we never know and probably never will know the local acoustic field in exact, deterministic detail. In fact, the best description of our state of knowledge appears to be a probabilistic one. I hasten to add that the term "probabilistic" is not taken to mean only random or stochastic processes-far from it. A probabilistic description properly includes, as a possible extreme, the deterministic descriptions with which we are most familiar, such as the wavefront and waveform.

A useful way in which to express a probabilistic state of knowledge is in terms of the conditional probability that, if a certain event occurs, then a certain set of acoustic field pressures will occur in the available volume $V$ during processing time $T$. In order to make these joint probabilities both tractable and realistic in terms of our actual field sampling in space and time, it is necessary to develop space-time sampling theorems, analogous to the temporal sampling approach of Peterson, Birdsall, and Fox [1]. In this paper, two such sampling theorems are stated, one treating the monochromatic acoustic field and the 
other the band-limited, time-limited acoustic field. Their importance lies in determining the number and the geometry of the samples in space and time necessary to reconstruct completely-or, in the case of finite $V$, almost completely-the space-time acoustic field. Using these theorems, a finite-order joint probability density function may be constructed over $V$ and $T$ for each pos sible (mutually exclusive) event. These density functions are in fact the state-ofknowledge descriptions we seek. To get on with efficient processor design, the possible events are considered two at a time. For each pair of events, the ratio is formed of their conditional-probability density functions and is called the likelihood ratio for this pair of events. The likelihood ratio for any pair of events is a mathematical description of the processor which is (a) optimum in the sense of minimun average risk and (b) most efficient statistically of all the processors that could be built given this state of knowledge.

The likelihood-ratio processor for the entire set of events is formed by the linear superposition of the processor for each pair of events. If each event is considered relative to a common event (such as noise alone), each individual processor receives a weight in the over-all processor corresponding to the a priori probability of occurrence of the noncommon event. For the examples at the end of this paper, we will consider only the simplest possible set of events, the binary set, and the two events will be denoted by $S N$ (the event is that both signal and noise are present) and $N$ (the event is that noise alone is present).

\subsubsection{Sompling Theorems}

Much of the subsequent material is developed from [2], where in particular the following two space-time sampling theorems are proved.

\subsubsection{Theorem I (Uniform Space Sampling of a Monochromatic Field}

If a sound field $p(x, y, z ; t)$ consists entirely of acoustic radiation of a single frequency $t$, formed from the superposition of ficlds from an arbitrary number of single-frequency sources of arbitrary phase and amplitude, then at any instant of time $t, p(x, y, z ; t)$ is given within the volume not containing these sources as a function of $x, y$, and $z$ by its complex values, at points spaced a half wavelength apart in $x, y$, and $z$, times a three-dimensional Nyquist-Shannon sampling function, such sampling extending throughout space:

$$
p(x, y, z ; t)=e^{i \omega t} \sum_{l,} \sum_{m, n}^{\infty} \sum_{n=-\infty} p\left(\frac{l \lambda}{2}, \frac{m \lambda}{2}, \frac{n \lambda}{2}\right) s(l, m, n ; x, y, z)
$$

where the function $p(l \lambda / 2, m \lambda 2, n \lambda / 2)$ is the complex amplitude of the sound field at $x=l \lambda / 2, y=m \lambda / 2, z=n \lambda / 2, t=0$; and

$S(x, y, z ; l, m, n)=$ spatial sampling function

$$
=\frac{\sin \pi(2 x / \lambda-l)}{\pi(2 x / \lambda-l)} \cdot \frac{\sin \pi(2 y / \lambda-m)}{\pi(2 y / \lambda-m)} j \frac{\sin (2 z / \lambda-n)}{\pi(2 z / \lambda-n)}
$$


18.3.2.2. Theorem II (Band-Limited Frequency Spectrum, Finite Time)

If a sound field $p(x, y, z ; t)$ of interest for finite time $T$ consists almost completely [3] of radiation within the band of frequencies $(0, W)$, then $p$ is given everywhere by its real amplitude values at discrete sampling points times a sampling function:

$$
\begin{aligned}
p(x, y, z ; t)=(2 W T)^{-1} \sum_{q=-W T}^{W T} e^{l \omega_{q} t} & \sum_{=-W T}^{W T} K(q, s) e^{-t q u / W T} \\
& \times \sum_{l, m, \pi} \sum_{=-\infty}^{\infty} \sum p\left(\frac{l \lambda}{2} q, \frac{m \lambda_{q}}{2}, \frac{n \lambda_{g}}{2} ; \frac{s}{2 W}\right) \cdot S_{q}(l, m, n ; x, y, z)
\end{aligned}
$$

where

$$
\begin{aligned}
& \omega_{q}=2 \pi q / T \\
& \lambda_{q}=2 \pi c / \omega_{q}
\end{aligned}
$$

and

$$
K(q, s)=\frac{1}{\pi} \int_{\pi(\sim-\alpha w T)}^{\pi(-s+W T)}\left[(\sin v / v) e^{-i q \nabla / W T}\right] d v
$$

$$
S_{q}(l, m, n ; x, y, z)=\frac{\sin \pi\left(2 x / \lambda_{q}-l\right)}{\pi\left(2 x / \lambda_{q}-l\right)} \cdot \frac{\sin \pi\left(2 y / \lambda_{q}-m\right)}{\pi\left(2 y / \lambda_{q}-m\right)} \cdot \frac{\sin \pi\left(2 z / \lambda_{q}-n\right)}{\pi\left(2 z / \lambda_{q}-n\right)}
$$

and $p\left(l \lambda_{q} / 2, m \lambda_{q} / 2, n \lambda_{q} / 2 ; s / 2 W\right)$ is the real amplitude of $p(x, y, z ; t)$ at point $\left(x=l \lambda_{q} / 2\right.$, $\left.y=m \lambda_{q} / 2, z=n \lambda_{q} / 2\right)$ at time $t=s / 2 W$.

\subsubsection{Space-Time Likelihood Ratio for Detection}

Let us consider the binary set of possible events $S N$ (signal and noise are present) and $N$ (noise alone is present). The decision to be made is, which of these events has occurred. This is usually called the detection decision or simply detection. Following the outline given in the Section 18.1 and using the space-time sampling theorem for a series band-limited, time-limited, volumelimited (actually, $M$ spatial sampling points) acoustic field, we have for the detection likelihood ratio:

$$
l(X)=\frac{f_{S N}(X)}{f_{N}(X)}=\frac{f_{S N}\left({ }_{1} x_{1},{ }_{1} x_{2}, \ldots,{ }_{1} x_{2 W T} ;{ }_{2} x_{1}, \ldots,{ }_{2} x_{2 W T} ; \cdots{ }_{M} x_{1}, \cdots{ }_{M} x_{2 W T}\right)}{f_{N}\left({ }_{1} x_{1},{ }_{1} x_{2}, \cdots,{ }_{1} x_{2} w T ;{ }_{2} x_{1}, \ldots{ }_{2} x_{2} w T ; \cdots{ }_{M} x_{1}, \cdots{ }_{M} x_{2} W T\right.}
$$

where ${ }_{i} x_{j}$ is the pressure at the th space sampling point and th time sampling point. Both $i_{S N}(X)$ and $t_{N}(X)$ have to do with the joint occurrence of the $2 W T^{\prime} M$ samples $\left({ }_{1} x_{1}, \ldots, M^{x_{2}}{ }_{W T}\right)$ in space-time. Under certain conditions [Eq. $\left.(2)\right], l(X)$ is separable into space and time components; generally, it is not.

Substitution of the specific probability density functions into Eq. (7) gives the specific processor design for efficient detection. This is done in the next section for one space-time and two space-only examples. While this is the primary application of the space-time likelihood ratio, a second and equally interesting use of the likelihood ratio is as a $\mathrm{gauge}$ of the efficiency of existing or proposed processors, or of the effect of different states of knowledge upon the capability to make accurate or, more generally, minimum average risk descriptions. 


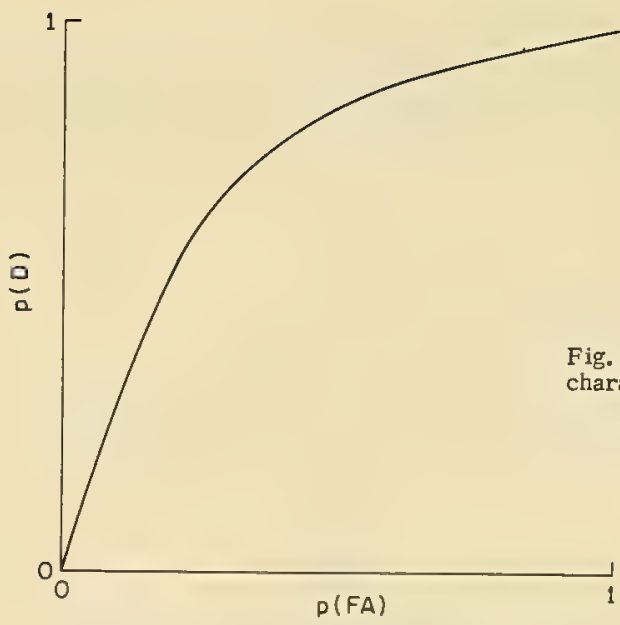

Let us imagine many independent successive trials using the detection likelihood-ratio processor in the $f_{3 N}(X)$ and $f_{V}(X)$ acoustic fields. The (a priori) probability of occurrence of $S N$ is $p(S N)$, and $p(N)$ is the a priori probability of occurrence of $N$ on any trial. A threshold constant $K$ is compared with the likelihood-ratio processor output at each trial. If the processor output exceeds $K$, the decision $S N$ is made $\left(\hat{\delta}_{S N}\right)$; otherwise, the decision $N$ is made $\left(\delta_{N}\right)$. There are four possible combinations of event $S N$ or $N$ and decision $\delta_{S N}$ or $\delta_{N}$ :

$$
\begin{aligned}
\left(S N, \delta_{S N}\right) & =\text { detection }(D) \\
\left(S N, \delta_{N}\right) & =\text { miss } \\
\left(N, \delta_{S N}\right) & =\text { false alarm }(F A) \\
\left(N, \delta_{N}\right) & =\text { correct dismissal }
\end{aligned}
$$

If $p(D)=p\left(S N, \delta_{S N}\right)$ is the probability of detection developed as the number of trials becomes infinite, and $p(F A)$ is the probability of false alarm similarly developed, then it is found that both are functions of $K$. If $K$ is large, both $p(D)$ and $p(F A)$ are small; if $K$ is small, both $p(D)$ and $p(F A)$ are large. Generally, if $K$ is varied, a receiver operating characteristic will be traced out for a given $f_{S N}(X)$ and $f_{N}(X)$, as shown in Fig. 18.1.

If $t_{S N}(X)$ ur $f_{N}(X)$ is changed, a family of operating characteristics will be generated. Further, if some processor other than $l(X)$ is used in the trials, a different $p(D)$ and $p(F A)$ will result, and will be to the right and below the operating characteristic; i.e., the processor is not as efficient as $l(X)$. From this brief discussion of operating characteristics, the mechanism for comparing different states of knowledge and/or different processors in terms of $p(D)$ and $p(F A)$ should be clear. A fuller discussion of the use of operating characteristic curves and receiver efficiency is given in [1].

\subsubsection{Examples}

Three examples will be given:

I. Signal is known exactly in time and space (waveform and wavefront); 
additive Gaussian noise is independent between sampling points in space and between sampling points in time.

II. (space only): Signal is known exactly in space. Noise is additive and Gauss-Markov between space sampling points.

III. (space only): Signal wavefront is Gauss-Markov perturbed between space sampling points; noise is Gauss-Markov between space sampling points.

Example I: Suppose there are $M$ hydrophones receiving either (a) a signal $S$ known exactly in time and space plus independent Gaussian (in time and space) noise, or (b) the noise alone. What is the optimum space-time processor? If $x_{i}$ is the input acoustic pressure amplitude at the $i$ th hydrophone at the $j$ th instant of time, ${ }_{i} s_{j}$ is the signal pressure amplitude of the $i$ th hydrophone at the $j$ th instant of time, and

$$
X=\left({ }_{1} x_{1},{ }_{1} x_{2}, \ldots,{ }_{1} x_{2}{ }_{W T} ; \ldots ;{ }_{M} x_{1}, \ldots,{ }_{M} x_{2 W T}\right)
$$

then

$$
f_{N}(X)=\left(2 \pi \sigma_{N}^{2}\right)^{-M W T} \exp \left(-\frac{1}{2 \sigma_{N}^{2}} \sum_{i=1}^{M} \sum_{j=1}^{2 W T} x_{j}^{2}\right)
$$

and

$$
f_{S N}(X)=\left(2 \pi \sigma_{N}^{2}\right)^{-M W T} \exp \left[-\frac{1}{2 \sigma_{N}^{2}} \sum_{i=1}^{M} \sum_{j=1}^{2 W T}\left(i, x_{j}-s_{j}\right)^{2}\right]
$$

where $f_{N}(X)$ is the probability that if noise alone is present then sample $X=\left({ }_{1} x_{1}, \ldots, M^{x_{2}}{ }_{W T}\right)$ will occur; and $f_{S N}(X)$ is the same for signal-plus-roise condition. The space--time likelihood ratio for this example, $l_{\mathrm{I}}(X)$, is

$$
l_{\mathbf{I}}(X) \equiv \frac{f_{S N}(X)}{f_{N}(X)}=\exp \left[-\frac{1}{2 \sigma_{N}^{2}} \sum_{j=1}^{M} \sum_{j=1}^{2 W_{T}}\left({ }_{i} s_{j}^{2}-2{ }_{j} x_{j l} s_{j}\right)\right]
$$

In Eq. (11), we see that

a. The basic processing operation is a space-time cross-correlation between the signal known exactly $i s_{j}$ and the inpur ${ }_{i} x_{j}$, since the double summation over ${ }_{1} s_{j}^{2}$ is just the total signal energy received at all the hydrophones, and so is a constant known exactly.

b. It is immaterial whether the space summation (usually called "integration") is done first.

c. By "signal known exactly in space" we mean that we know the wavefront location. Thus, our signal-known-exactly assumption means that we know both the waveform and the wavefront.

Two completely equivalent block diagrams for Eq. (11) are given in Fig. 18.2. As a final point, the ROC curve for this example is identical to Fig. 2 of [1], with

$$
d=\frac{2 M E}{N_{0}}
$$

where $E$ is the signal energy as measured at one hydrophone output. The spatial 

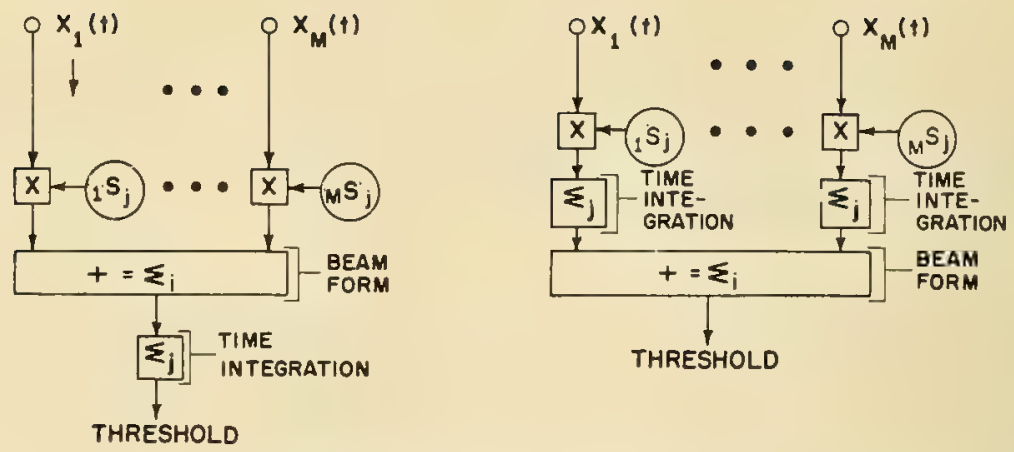

Fig. 18.2. Example I, space-time processor.

processing gain in $\mathrm{db}$, then, is $10 \log M$ for this case, and the temporal processing gain is $10 \log \left(2 E / N_{0}\right)$.

Example II: The following example is restricted to space statistics only, to bring out the idea that for several quite likely statistical situations pattern formation in the usual sense is not optimum likelihood-ratio processing in space. This example illustrates the departure from pattern formation due to a space-correlated noise field, specifically, a first-order Gauss-Markov noise field. In this example, a particular instant of time is chosen and space statistics only will be discussed. For the noise field alone, with $M$ (regularly spaced) point hydrophones, the $M$ th-order spatial-probability distribution function $\boldsymbol{t}_{N}(X)$ is, under the Markovian assumption,

$$
\begin{aligned}
f_{N}\left(X_{1}\right)=f_{N}\left({ }_{1} x_{1}, \ldots, M_{M} x_{1}\right) & \left.=f_{N}\left({ }_{1} x_{1}\right) \cdot f_{N, 1} x_{1}\left({ }_{2} x_{1}\right), \cdot f_{N},{ }_{2} x_{1},{ }_{3} x_{1}\right) \cdots f_{N}, M-1 \\
f_{N}\left(X_{1}\right) & =f_{N}\left({ }_{1} x_{1}\right) \prod_{i=2}^{M} f_{N, 1-1 x_{1}}\left({ }_{1} x_{1}\right)
\end{aligned}
$$

and together with the Gaussian assumption, Eq. (12), gives

$$
f_{N}\left(X_{1}\right)=\left[2 \pi \sigma_{N}^{2}\right]^{-M / 2}\left[1-\rho_{N}^{2}\right]^{-(M-1) / 2} \exp \left[-\frac{{ }_{1} x_{1}^{2}}{2 \sigma_{N}^{2}}-\sum_{i=2}^{M} \frac{\left({ }_{1} x_{1}-\rho_{N \cdot 1}-1 x_{1}\right)^{2}}{2\left(1-\rho_{N}^{2}\right) \sigma_{N}^{2}}\right]
$$

where $\sigma_{N}^{2}$ is the noise power per hydrophone, and $\rho_{N}$ is the normalized noise (spatial) correlation function between adjacent hydrophones.

Now, if signal is known exactly-in this example, such a state of knowledge means knowing the signal wavefront at the chosen instant of time-then $f_{S N}(X)$ is .

$$
f_{s N}\left(X_{1}\right)=\left[2 \pi \sigma_{N}^{2}\right]^{-M / 2}\left[1-\rho_{N}^{2}\right]^{-(M-1) / 2} \exp \left\{-\frac{\left(1 x_{1}-{ }_{1} s_{1}\right)^{2}}{2 \sigma_{N}^{2}}-\sum_{i=2}^{M} \frac{\left[\left(x_{1}-i s_{1}\right)-\rho_{N}\left(1-1 x_{i} \cdot 1-1 s_{1}\right)\right]^{2}}{2\left(1-\rho_{N}^{2}\right) \sigma_{N}^{2}}\right\}
$$

Using Eqs. (7), (13), and (14):

$$
\begin{gathered}
l_{\mathrm{II}}\left(X_{1}\right)=\frac{f_{S N}(X)}{f_{N}\left(X_{1}\right)}=\exp \left\{[ \frac { 1 } { 2 ( 1 - \rho _ { N } ^ { 2 } ) \sigma _ { N } ^ { 2 } } ] \left[-\left(1-\rho_{N}^{2}\right)_{1} s_{1}^{2}-\sum_{i=2}^{M}\left({ }_{i} s_{1}-\rho_{N} \cdot 1-1 s_{1}\right)^{2}+2\left(1-\rho_{N}^{2}\right)_{1} x_{1 \cdot 1} s_{1}\right.\right. \\
\left.\left.\quad+2{ }_{M} x_{1 \cdot M} s_{1}+2\left(1+\rho_{N}\right) \sum_{i=2}^{M}{ }_{1} x_{1 \cdot d} s_{1}+2 \rho_{N} \sum_{i=2}^{M}\left({ }_{i} x_{1 \cdot 1-1} s_{1}+i-1 x_{1 \cdot l} s_{1}\right)\right]\right\}
\end{gathered}
$$




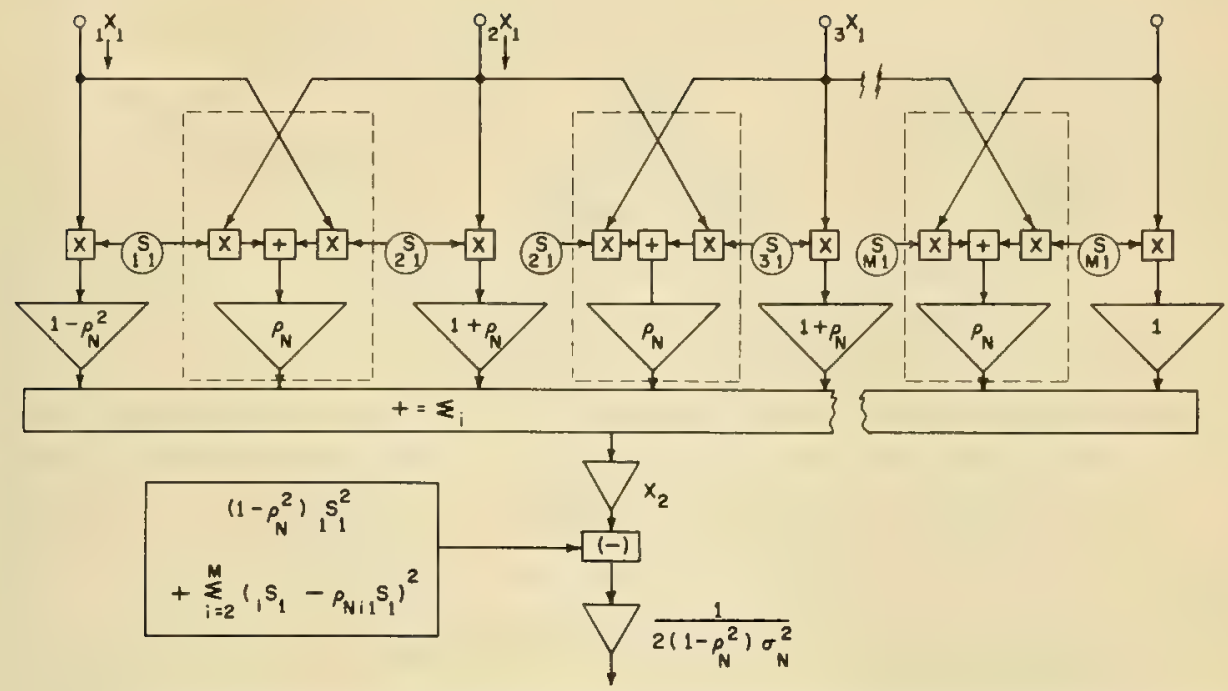

Fig. 18.3. Spatial likelihood-ratio processor for Gauss-Markov (spatial) possibility distribution of noise of Example III. Dotted lines enclose processing due to spatially correlated noise.

The first two additive terms on the right of Eq. (15) are signal-bias terms. The next three additive terms are those for normal pattern formation [see Example I, Eq. (11)]; however, the coefficients of the pattern terms for inputs ${ }_{1} x_{1}$ and $M^{\mathrm{x}_{1}}$ differ from the coefficient $\left(1+\rho_{N}\right)$ for the majority of the inputs. Finally, additional processing of the inputs beyond pattern formation is indicated by the last additive term. The spatial likelihood-ratio processor design for this example is drawn in Fig. 18.3.

Comparing Figs. 18.2 and 18.3 , we see that the essential difference in spatial processing due to spatially correlated noise (Example II) versus independent noise (Example I) is the multiplication of an input by both the succeeding and following values of the signal. For a plane wavefront, this operation would consist of delaying and advancing an input from the ith hydrophone as though it were received at the $(i-1)$ th and $(i+1)$ th hydrophones, respectively. If we allow the noise to become spatially independent, $\rho_{N}$ goes to zero and the Markovian processing vanishes.

Example III: The previous example may be generalized to include first-order Gauss-Markov perturbation of the signal wavefront about its ideal mean, in addition to first-order Gauss-Markov noise in space. The spatial likelihoodratio processor design for several interior $(i \neq 1, M)$ hydrophone outputs is given in Fig. 18.4, together with the values of the amplification coefficient in Table I. Comparison with Fig. 18.3 reveals two new processes: multiplication of hydrophone outputs and squaring of the individual hydrophone outputs. By juggling values of $\rho_{S}, \rho_{N}, \sigma_{S}^{2}$, and $\sigma_{N}^{2}$, a smooth transition from the case of classic pattern formation alone (Example I), through correlated noise processing (Example II), to completely incoherent spatial processing can be found. This latter 


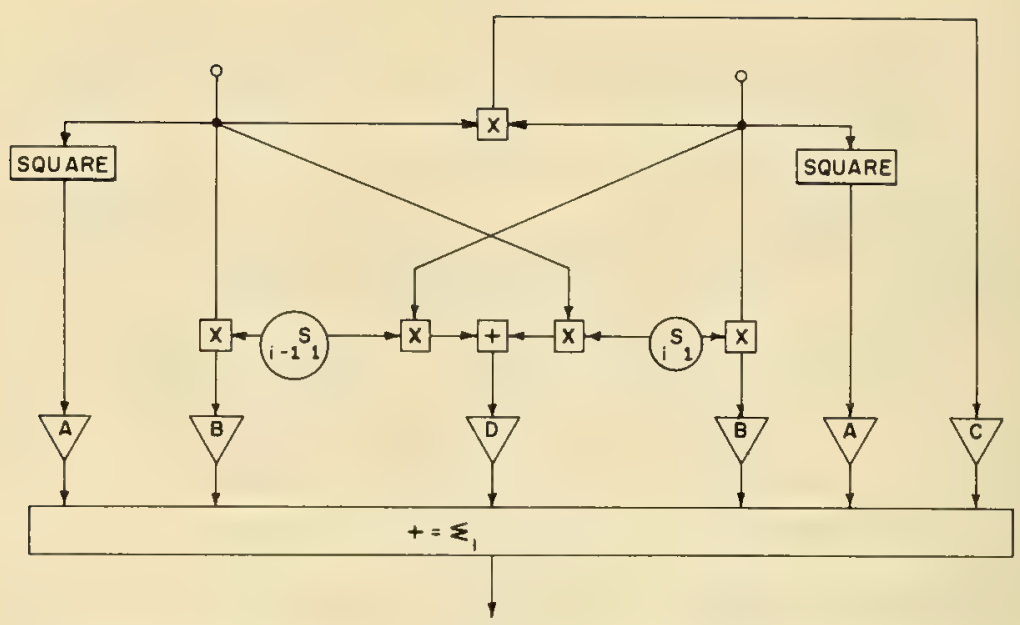

Fig. 18.4. Space processor for Gauss-Markov perturbed signal wavefront in GaussMarkov noise.

case corresponds to the conditions $\rho_{S}=0=\rho_{N}$ and $\sigma_{S}^{2} \gg \sigma_{N}^{2}$. As an intermediate example, for $\rho_{S}=1, \rho_{N}=0$, and $\sigma_{S}^{2}=\sigma_{N}^{2}$, the amplification factors become:

$$
\begin{array}{cl}
A=\frac{1}{2 \sigma_{N}^{2}}\left(1-\frac{17}{30}\right) & B=\frac{1}{2 \sigma_{N}^{2}}\left(\frac{17}{30}\right) \\
C=\frac{1}{2 \sigma_{N}^{2}}\left(\frac{2}{15}\right) & D=\frac{-1}{2 \sigma_{N}^{2}}\left(\frac{2}{15}\right)
\end{array}
$$

In Table 18.I, values of the amplification factors for the processor of Fig. 4 are given, where

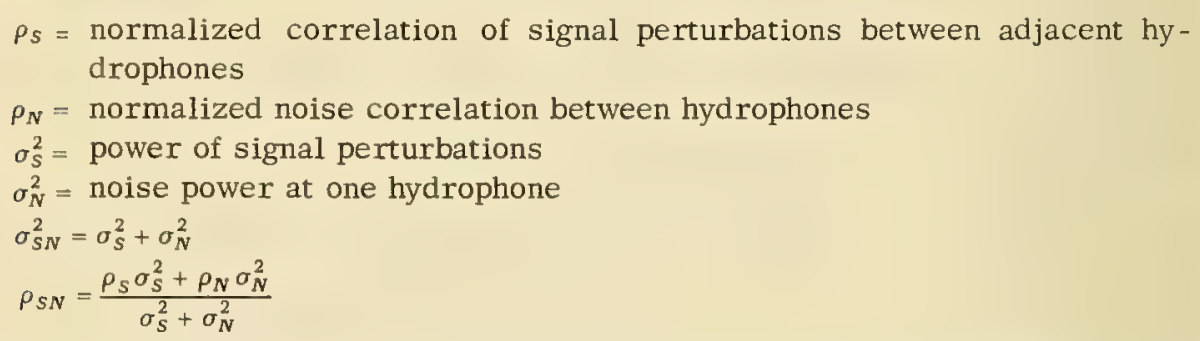

In these examples, previously developed space and time processing, such as pattern formation and cross correlation, have been related to novel space processing through inclusion in the general framework of space-time decision theory. The purpose of these few examples is to suggest the utility of this framework as a logically developed and heuristic guide to processor design and efficiency-that is, to space-time processor research. 
Table 18.I. List of Values of Amplification Factors for Spatial Likelihood-Ratio Processor of Fig. 18.4

\begin{tabular}{c|c|l}
\hline Symbol & Value & \multicolumn{1}{c}{ Process amplified } \\
\hline A & $-\frac{\left(1+\rho_{S N}^{2}\right)}{2 \sigma_{S N}^{2}\left(1-\rho_{S N}^{2}\right)}+\frac{\left(1+\rho_{N}^{2}\right)}{2 \sigma_{N}^{2}\left(1-\rho_{N}^{2}\right)}$ & $\begin{array}{l}\text { Squaring of individual hydrophone } \\
\text { outputs, followed by summation- } \\
\text { essentially, spatial incoherent } \\
\text { processing }\end{array}$ \\
\hline B & $+\frac{1+\rho_{S N}^{2}}{\sigma_{S N}^{2}\left(1-\rho_{S N}^{2}\right)}$ & $\begin{array}{l}\text { Classic pattern formation-essen- } \\
\text { tially, spatial coherent processing }\end{array}$ \\
\hline $\mathrm{C}$ & $\frac{\rho_{S N}}{\sigma_{S N}^{2}\left(1-\rho_{S N}^{2}\right)}-\frac{\rho_{N}}{\sigma_{N}^{2}\left(1-\rho_{N}^{2}\right)}$ & $\begin{array}{l}\text { Pair-wide multiplication of hydro- } \\
\text { phone outputs-related to un-nor- } \\
\text { malized interhydrophone correla- } \\
\text { tion of signal perturbations }\end{array}$ \\
\hline $\mathrm{D}$ & $-\frac{\rho_{S N}}{\sigma_{S N}^{2}\left(1-\rho_{S N}^{2}\right)}$ & $\begin{array}{l}\text { Offset pattern formation-related } \\
\text { to both noise and signal interele- } \\
\text { ment hydrophore correlations }\end{array}$ \\
\hline
\end{tabular}

\section{REFERENCES}

1. W. W. Peterson, T.G. Birdsall, and W.C. Fox, "The Theory of Signal Detectability," Trans. IRE, PGIT4, 171 (September, 1954).

2. P. L. Stocklin, "Limits of Measurement of Acoustic Wave Fields, " manuscript submitted for publication, J. Acoust. Soc. Am.

3. D. Van Meter and D. Middleton, "Detection and Extraction of Signals in Noise from the Point of View of Statistical Decision Theory. Part I," J. Soc. Ind. Appl. Math., Vol. 3, No. 4, 241 (December, 1955). 



\section{APPENDIX: ATTENDANCE AT THE INSTITUTE}

Professor Vernon M. Albers

Chairman of the Institute

Ordnance Research Laboratory

The Pennsylvania State University

University Park, Pennsylvania, U.S.A.

Mr. Homer R. Baker

North Atlantic Treaty Organization

Scientific Affairs Division

Paris, France

Dr. D. Schofield

Naval Research Establishment

Halifax, Nova Scotia, Canada

Professor D. G. Tucker

University of Birmingham

Birmingham, Warwickshire, England

Dr. D. E. Weston

Admiralty Research Laboratory

Teddington, Middlesex, England

Dr. G. G. Parfitt

Department of Physics

Imperial College of Science and Technology

Londor IIniversity

London S. W. 7, England

Dr. Martin Greenspan

National Bureau of Standards

Washington, D.C., U.S.A.

Dr. C.C.A.N. Foäche

Laboratoire de Detection

Le Brusc, (Var.) France

Dr. James Crease

National Institute of Oceanography

Wormley, Surrey, England

Professor Erwin Meyer

III. Physikalisches Institut

der Universität Göttingen

Göttingen, West Germany

Mr. Carl J. Anderson

Norwegian Defense Research Establishment Horten, Norway

Mr. Arne Aubell

Norwegian Defense Research Establishment

Horten, Norway
Dr. R. W. B. Stephens

Co-Chairman of the Institute

Department of Physics

Imperial College of Science and Technology

London University

London S. W. 7, England

Leeturers

Dr. A. B. Wood

Admiralty Research Laboratory

Teddington, Midřiesex, England

Dr. H. Wysor Marsh

Marine Electronics Office

Avco Corporation

New London, Connecticut, U.S.A.

Professor Eugen J. Skudrzyk

Ordnance Research Laboratory

The Pennsylvania State University

University Park, Pennsylvania, U.S.A.

Professor Paul M. Kendig

Ordnance Research Laboratory

The Pennsylvania State University

University Park, Pennsylvania, U.S.A.

Professor E. J. Richards

University of Southampton

Southampton, Hants, England

Mr. M. J. Tucker

National Institute of Oceanography

Wormley, Surrey, England

Mr. A. R. Stubbs

National Institute of Oceanography

Wormley, Surrey, England

Ir. J.H. Janssen

Technisch Physiche Dienst T.N.O. en T.H.

Stieltjesweg 1

Delft, Netherlands

Mr. P. L. Stocklin

Office of Naval Research

Washington 25, D.C., U.S.A.

Porticipants

Mr. G.J. Barber

Admiralty Underwater Weapons Establishment Portland, Dorset, England

Professor A. Barone

Instituto Nazionale di Ultracustica

Rome, Italy 
Professor J.F.W. Bell

Royal Naval College

Greenwich, England

Mr. G.C. Braysher

Admiralty Underwater Weapons Establishment

Portland, Dorset, England

Dr. A. E. Brown

Kings College

Durham University

Newcastle upon Tyne, England

Mr. S. Byard

Admiralty Research Laboratory

Teddington, Middlesex, England

Mr. I. J. Campbell

Admiralty Underwater Weapons Establishment

Portland, Dorset, England

Dr. H. Charnock

SACIANT ASW Research Center

La Spezia, Italy

Mr. B. M. Cheney

Society Alsacienne de Constructions Mecaniques 69 Rue de Monceau

Paris, France

Mr. R. C. R. Chesters

Marine Equipment Unit

Plessey Limited

Ilford, Essex, England

Mr. W. B. Coffman

Office of the U.S. Naval Attache

Landon, England

Mr. R. P. Coghlan

Admiralty Research Laboratory

Teddington, Middlesex, England

Mr. G. L. Connon

Admiralty Underwater Weapons Establishment

Portland, Dorset, England

Mr. M. J. Daintith

Admiralty Underwater Weapons Establishment

Portland, Dorset, England

Dr. F. T. Dietz

Physics Department

University of Rhode Island

Kingston, Rhode Island, U.S.A.

Dr. W. N. English

Pacific Naval Laboratory

Esquimalt, British Columbia, Canada

Professor Dr. Maurizio Federici

Viale Vittorio, 14

Milan, Italy

Mr. R. D. Finch

Department of Physics

Imperial College of Science and Technology

London University

London S. W. 7, England

Dr. F. M. V. Flint

Admixalty Underwater Weapons Establishment

Portland, Dorset, England
Mr. J.H. Foxwell

Admiralty Underwater Weapons Establishment Portland, Dorset, England

Mr. A. Freedman

Admiralty Underwater Weapons Establishment Portland, Dorser, England

Mr. I. A. Gatenby

Admiralty Underwater Weapons Establishment Portland, Dorset, England

Mr. W. K. Grimley

Admiralty Underwater Weapons Establishment Portland, Dorset, England

Mr. Harold Haegermark

Stockholm, Sweden

Dr. G. K. Hartman

Naval Ordnance Laboratory

White Oak, Silver Spring, Maryland, U.S.A.

Mr. A. Hobson

Admiralty Research Laboratory

Teddington, Middlesex, England

Mr. G. P. Horton

Admiralty Underwater Weapons Establishment Portland, Dorset, England

Mr. S. D. Hawkins

Department of Physics

Imperial College of Science and Technology

London University

London S. W. 7, England

Mr. Hub

Royal Naval College

Greenwich, England

Mr. L. Kay

Birmingham University

Birmingham, Warwickshire, England

Mr. A. L. Kendrick

Admiralty Research Laboratory

Teddington, Middlesex, England

Dr. T. S. Korn

Faculte des Sciences Appliquees

Ecole Polytechnique Universite Libre de Bruxelles Ave. F.D. Roosevelt

50, Bruxelles, Belgium

Mr. R. L. Laval

SACLANT ASW Research Center

La Spezia, Italy

Mr. D. R. Leah

Admiralty

Bath, Somerset, England

Dr. E. Rune Lindgren

Tekniska Hogskolan

Stockholm, Sweden

(Visiting Professor, Johns Hopkins University, Baltimore, Maryland, U.S.A.)

Mr. D. A. Linkens

Marine Equipment Unit

Plessey Limited

llford, Essex, England 
Mr. J.R. Littlefair

Admiralty Research Laboratory

Teddington, Middlesex, England

Mr. B. W. Lythall

Admiralty Underwater Weapons Establishment Portland, Dorset, England

Mr. H.H. Margary

Admiralty Research Laboratory

Teddington, Middlesex, England

Dr. H. Medwin

U.S. Office of Naval Research

London Branch

Keysign House

London, England

Dr. J.H. Mole

Admiralty Underwater Weapons Establishment Portland, Dorset, England

Mr. A. Nairn

Admiralty Underwater Weapons Establishment Portland, Dorset, England

Mr. G. W. B. Neal

Admiralty Underwater Weapons Establishment Portland, Dorset, England

Mr. E. Neppiras

Mullard Limited

London, England

Mr. R. B. Newman

Admiralty Underwater Weapons Establishment Portland, Dorset, England

Mr. T. O'Brien

H. M. Admiralty

Londor, England

Mr. Aubrey Pryce

Office of Naval Research

Washington 25, D.C., U.S.A.

Mr. E. D. Rabun

Bureau of Naval Weapons

Washington 25, D.C., U.S.A.

Mr. J. W. Ramsey

Admiralty

Bath, Somerset, England

Mr. J. D. Rands

Department of Physics

Imperial College of Science and Technology

London University

London S. W. 7, England

Professor R. E. H. Rasmussen

Tekniske Højskole

Østervoldgade 10

Kobenhavn K, Denmark

Mr. Charles Reis

Research Consultant

Hewlett-Packard Company

1501 Page Mill Road

Palo Alto, California, U.S.A.

Mr. R. W. Renson

Admiralty Underwater Weapons Establishment

Portland, Dorset, England
Mr. T. N. Reynolds

Admiralty Underwater Weapons Establishment

Portland, Dorset, England

Mr. H. Ritter

Admiralty Research Laboratory

Teddington, Middlesex, England

Lta. (I) G. Rittore

Italian Navy Mariperman

La Spezia, Italy

Mr. A, Y. Robin

Society Alsacienne de Constructions Mecaniques 69 Rue de Monceau

Paris, France

Dr. A. Roshiko

U.S. Office of Naval Research

London Branch

Keysign House

London, England

Mr. J.S. M. Rusby

Admiralty Research Laboratory

Teddington, Middlesex, England

Mr. D. L. Ryal!

Admiralty Research Laboratory

Teddington, Middlesex, England

Ir. H. A. J. Rynja

Physics Laboratory

National Defence Research Council

The Hague, Netherlands

Mr. J. Sands

Royal Naval College

Greenwich, England

Dr. Sauls

Royal Naval College

Greenwich, England

Dr. Daniele Sette

Department of Engineering

University of Rome

Rome, Italy

Mr. Stig Söderqvist

A. B. Akustikby ran

Atterbomsvägen 50

Stockholm, Sweden

Dr. D. Stansfield

Admiralty Underwater Weapons Establishment

Portland, Dorset, England

Mr. H. Sussman

U.S. Navy Underwater Sound Laboratory

Fort Trumbull

New London, Connecticut, U.S.A.

Professor G. B. Thurston

Department of Physics

College of Arts and Sciences

Oklahoma State University

Stillwater, Oklahoma, U.S.A.

Dr. W. C. Thompson

U.S. Office of Naval Research

London Branch

Keysign House

London, England 
Commandex Trendell

Royal Naval College

Greenwich, England

Dr. J. Tunstead

Admiralty Underwater Weapons Establishment Portland, Dorset, England

Mr. G. Voglis

Admiralty

London, England

Dr. R. V. Waterhouse

Airo, England

Dr. H. H. Waterman

SACLANT ASW Research Center

La Spezia, Italy

Mr. A. G.D. Watson

Admiralty Research Laboratory

Teddington, Middlesex, England

Mr. B. S. Webster

Department of Physics

Imperial College of Science and Technology

London University

London S. W. 7, England
Dr. V. G. Welsby

Birmingham University

Birmingham, Warwickshire, England

Dr. S. Wennerbery

Stockholm, Sweden

Dr, M. A. Williamson

Dean, College of Engineering and Architecture

The Pennsylvania State University

University Park, Pennsylvania, U.S.A.

Mr. Willis

Department of Aeronautical Engineering

Southampton University

Southampton, Hants, England

Mr. R. W. Windley

National Research Department Corporation

1 Tilney Street

London W. 1, England

Mr. Jan Zeibon

Navalkonsult

Orlogsvarvet

Stockholm 100, Sweden 



